This item was submitted to Loughborough's Research Repository by the author.

Items in Figshare are protected by copyright, with all rights reserved, unless otherwise indicated.

\title{
Selective laser melting of Inconel 625 using pulse shaping
}

PLEASE CITE THE PUBLISHED VERSION

PUBLISHER

(C) Kamran Mumtaz

\section{PUBLISHER STATEMENT}

This work is made available according to the conditions of the Creative Commons Attribution-NonCommercialNoDerivatives 4.0 International (CC BY-NC-ND 4.0) licence. Full details of this licence are available at: https://creativecommons.org/licenses/by-nc-nd/4.0/

\section{LICENCE}

CC BY-NC-ND 4.0

\section{REPOSITORY RECORD}

Mumtaz, Kamran A.. 2019. "Selective Laser Melting of Inconel 625 Using Pulse Shaping”. figshare. https://hdl.handle.net/2134/33630. 


\section{Selective Laser Melting of Inconel 625 using Pulse Shaping}

By

\section{Kamran Mumtaz}

BEng (Hons) MSc

Doctoral thesis submitted in partial fulfilment of the requirements of the award of Doctor of Philosophy of Loughborough University

October 2008

C by Kamran Mumtaz (2008) 


\section{Abstract}

Selective Laser Melting (SLM) is an additive manufacturing technology that consolidates layers of metal powder using a high power laser. The laser's small spot size and relative accuracy facilitates the production of high resolution parts with great complexity that would be otherwise difficult to manufacture using conventional manufacturing techniques (e.g. casting, machining etc.). The possibility to build thin wall high resolution parts complements the technology's main advantage and extends its manufacturing capabilities. The high heat input delivered by the laser and complex melt pool dynamics, requires that laser process parameters are carefully controlled in order to prevent solidified parts from exhibiting poor properties such as a high surface roughness and poor resolution.

Harnessing an extra degree of control over the melting process can regulate and improve part properties. This additional control can be made possible by employing a technique known as pulse shaping. Pulse shaping can precisely control the energy distribution throughout the duration of a single laser pulse and tailor the heat input delivered to a material. This can improve the control over melt pool formation and subsequent part properties.

This research studied the effects of pulse shaping on the properties of SLM parts, specifically evaluating whether the additional degree of control could minimise the wall thickness and lower the surface roughness of thin wall Inconel 625 parts. Further understanding was developed on how laser energy distribution affects melting behaviour and the development of melt pool instabilities. The study found that a compromise in surface finish between the top/side facing surfaces and resolution of parts often had to be made due to melt pool behaviour. A variety of different energy distributions demonstrated the ability to increase/decrease melt pool solidification time and vary melt pool characteristics. A gradual increase in the rate at which heat was imparted to a material was shown to generate a preheating effect that reduced the maximum power required to initiate melting as well as reducing the amount of material ejected from the melt pool. Inducing a gradual ramp down in laser energy prolonged the 
time for which the melt pool remained molten, allowing more time for gravity and recoil pressures to reshape and flatten the top surface profile of the melt pool.

A key development within this research was the generation of a "Suppressed" pulse shape, this possessed a high peak power with an especially low energy/duration. This pulse induced a rapid/aggressive melting of material and was able to generate large recoil pressures that exerted an external mechanical force on the melt pool reducing both top/side surface roughness of the solidified part. The low pulse duration limited the time available for melt pool instabilities to develop and the low pulse energy minimised further melt pool expansion subsequently reducing the width of thin wall parts. 


\section{Acknowledgments}

In the name of God the most gracious most merciful

I would like to extend my gratitude to my supervisor Neil Hopkinson, who first introduced me to the world of Rapid Manufacturing. I thank him for his guidance and encouragement throughout my research.

Thanks to technical staff Rod Springthorpe for his effort, Bob Temple and Peter Wileman for their advice on metallurgical and laser issues. Andy Sandaver and Jagpal Singh for their help in specimen preparation and testing. Thanks to all members of the Rapid Manufacturing Research Group at Loughborough who assisted me throughout my research.

My final thanks go to my family, especially Aboo, Ammi, Rabiah, Aanika, Giorgia and Danni. 


\section{Table of Contents}

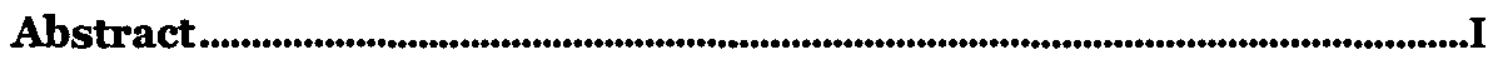

Acknowledgments............................................................................................................................. III

Publications.......................................................................................................................VIII

Presentations ..........................................................................................................................IX

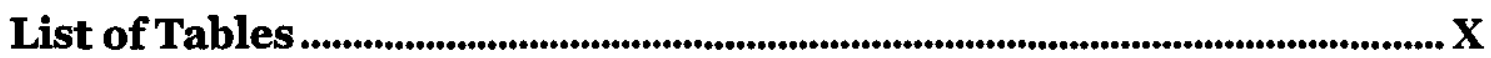

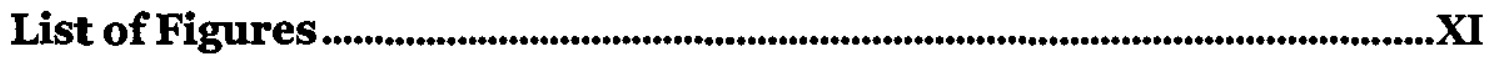

Abbreviations .............................................................................................................................XVI

Symbols ............................................................................................................................................ XVII

CHAPTER 1 INTRODUCTION ....................................................................................... 1

1.1 SCOPE OF RESEARCH ................................................................................ 1

1.1.1 Organisation of Thesis..................................................................................................2

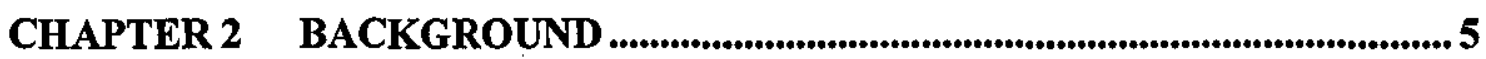

2.1 SOLID FREEFORM FABRICATION ......................................................................5

2.1.1 Solid Freeform Fabrication Technologies...............................................................................6

2.1.2 Rapid Manufacturing and the Production of Metal Parts...........................................................7

2.1.2.1 Key non laser based metal SFF processes...........................................................................................

2.2 DIRECT METAL LASER FABRICATION.............................................................9

2.2.1 Laser Material Interactions .....................................................................................................9

2.2.2 Sintering Mechanisms .......................................................................................................10

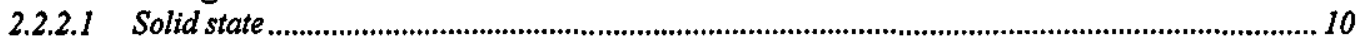

2.2.2.2 Liquid phase/partial melting............................................................................................................... 10

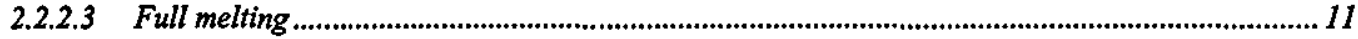

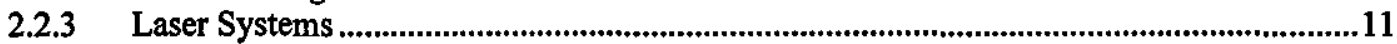

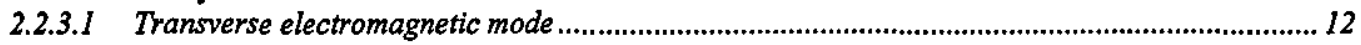

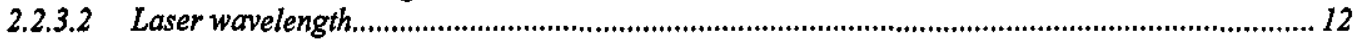

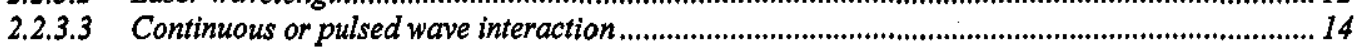

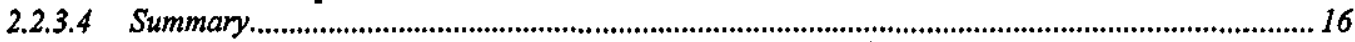

2.2.4 Overview of Material Deposition Techniques ...................................................................16

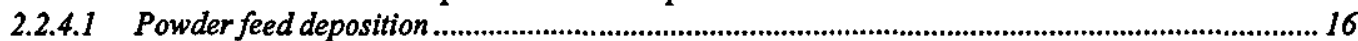

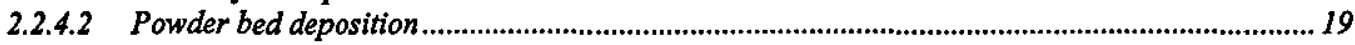

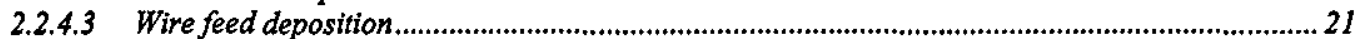

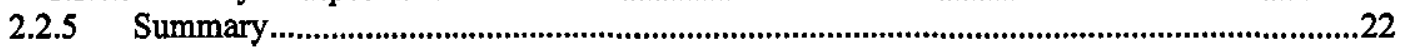

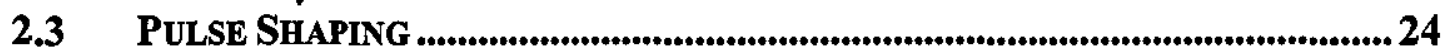

2.3.1 Standard Laser Pulse.........................................................................................................25

2.3.2 Improved Control over Laser Energy using Pulse Shaping ...................................................26

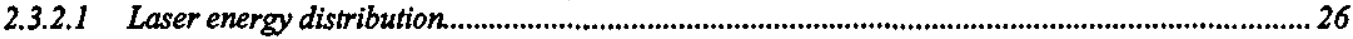

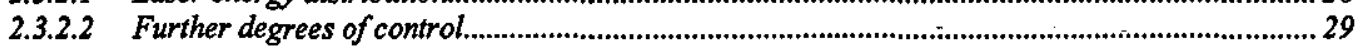

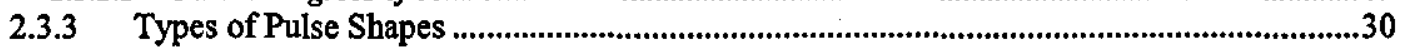

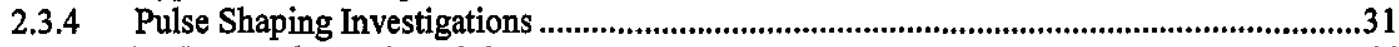

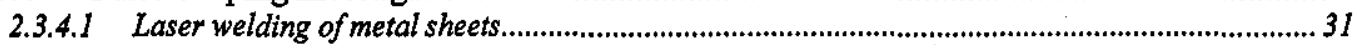

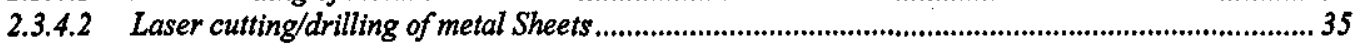

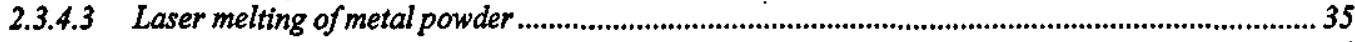

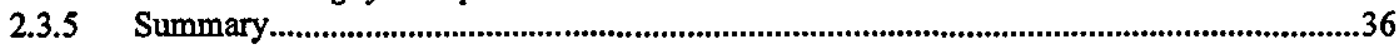


CHAPTER 3 SELECTIVE LASER MELTING .................................................. 37

$3.1 \quad$ SLM CONTROL FACTORS.................................................................................38

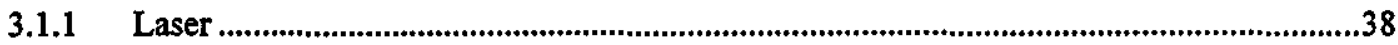

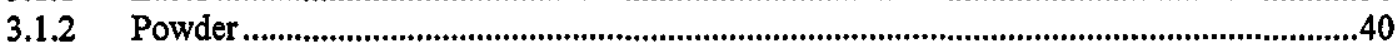

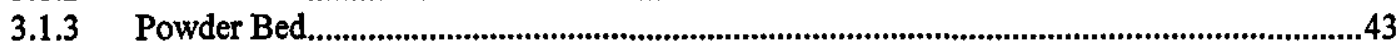

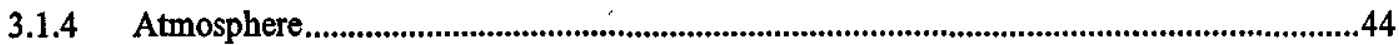

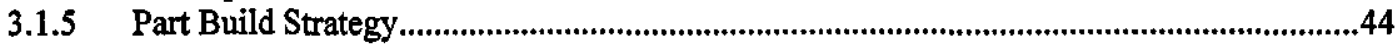

3.2 HEAT TRANSFER IN A POWDER BED............................................................ 45

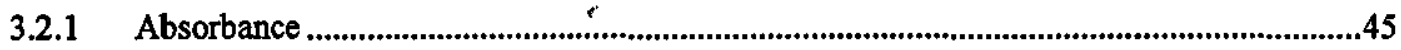

3.2.2 Thermal Conductivity and Melt Energy ...............................................................46

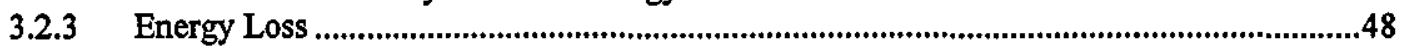

3.3 MELT POOL DYNAMICS ............................................................................. 48

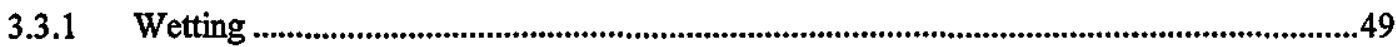

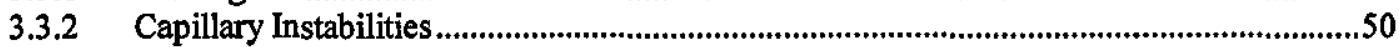

3.3.3 Thermocapillary Flows..................................................................................................52

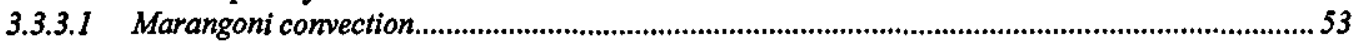

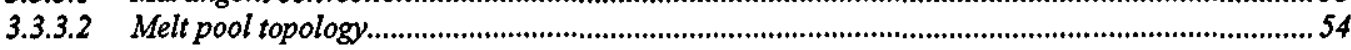

3.4 VAPORISATION AND RECOIL PRESSURES ................................................. 58

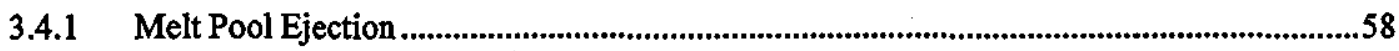

3.4.2 Melt Pool Shape Modification ..................................................................................60

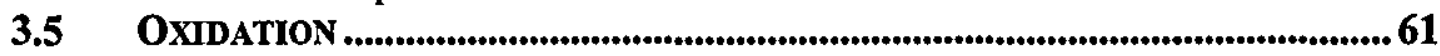

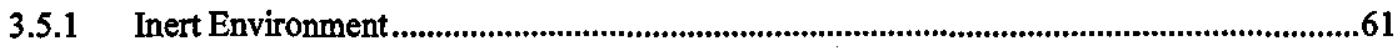

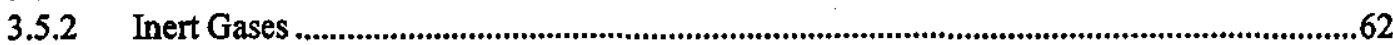

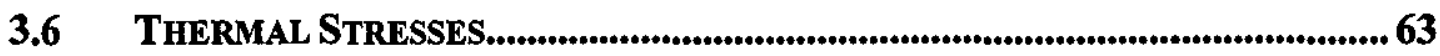

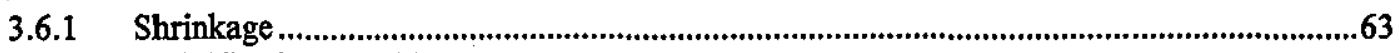

3.6.2 Solidification Cracking ........................................................................................64

CHAPTER 4 PRINCIPAL HYPOTHESIS \& RESEARCH OBJECTIVES ..... 65

4.1 RESEARCH NOVELTY .................................................................................................6 66

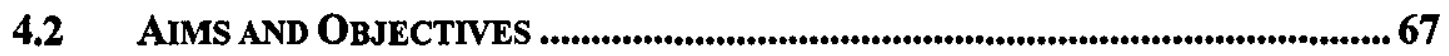

\section{CHAPTER 5 SYSTEM CHARACTERISATION ...........................................68}

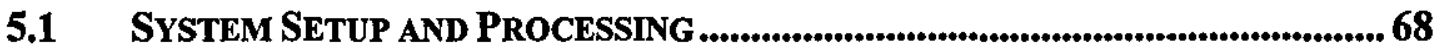

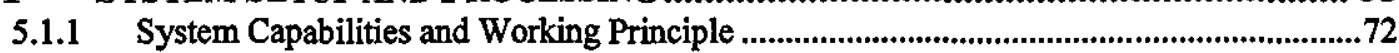

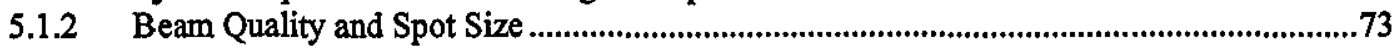

5.1.2.1 Focal distance adjustment ......................................................................................................... 73

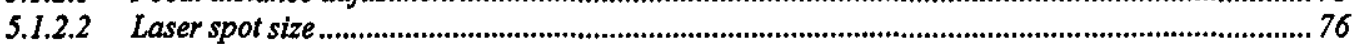

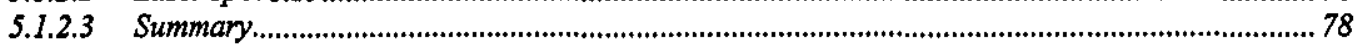

5.2 MATERIAL CHARACTERISATION ................................................................... 79

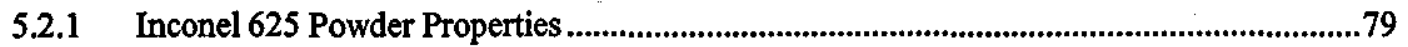

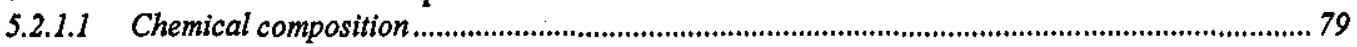

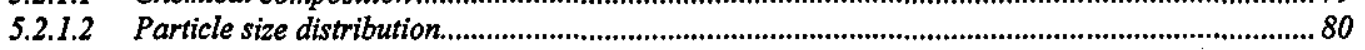

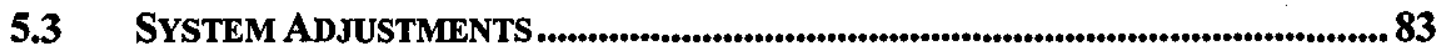

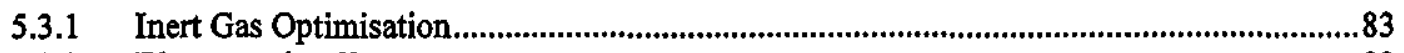

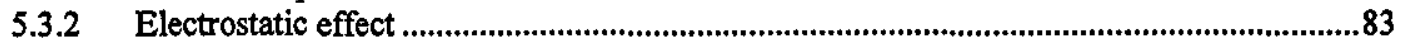

\section{CHAPTER 6 RECTANGULAR PULSE OPTIMISATION EXPERIMENTS . 84}

6.1 INCONEL 625 OPERATING WINDOW....................................................................... 85

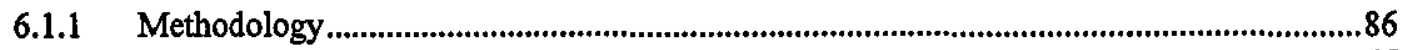

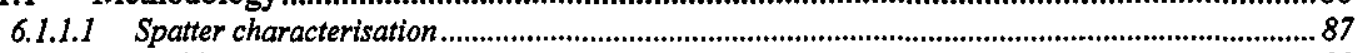

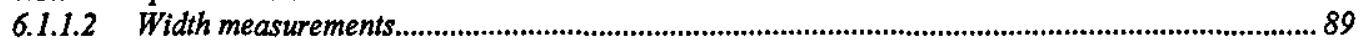

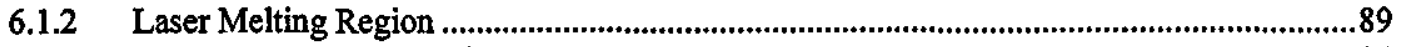

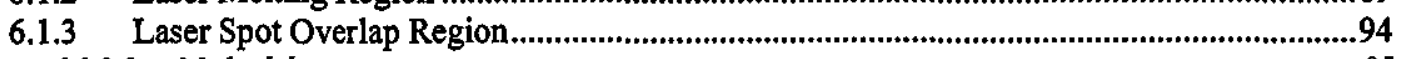

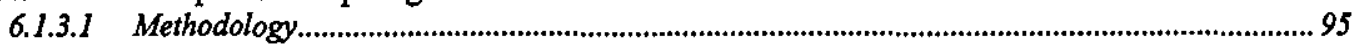

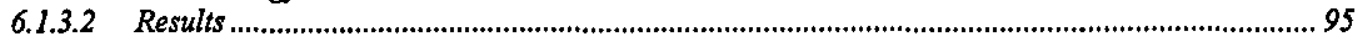

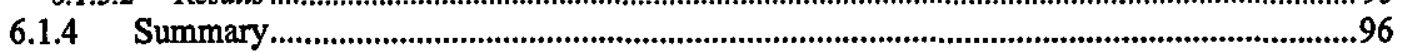




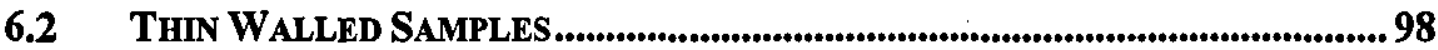

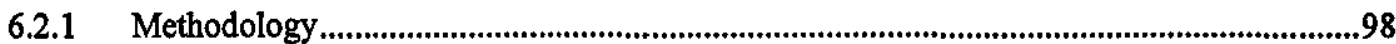

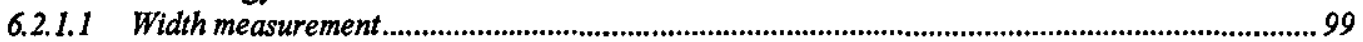

6.2.1.2 Surface roughness measurement .....................................................................................99

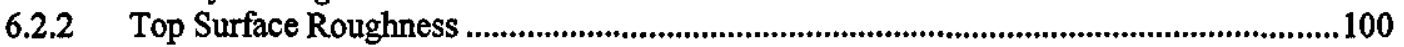

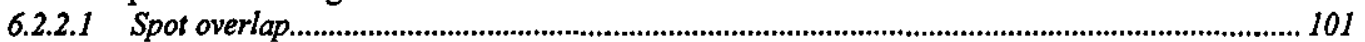

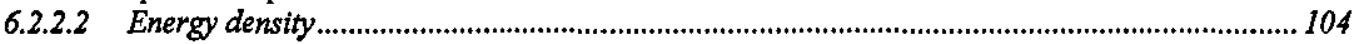

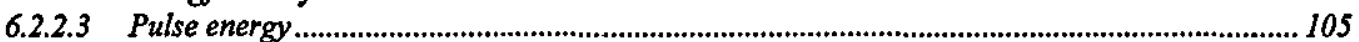

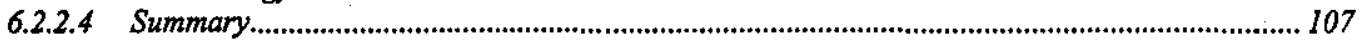

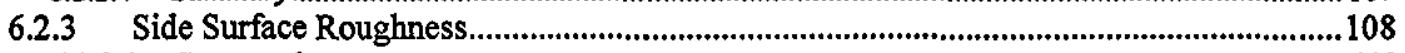

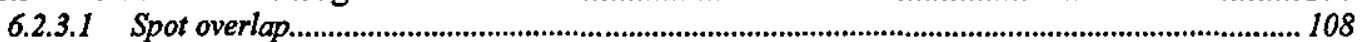

6.2.3.2 Energy density ........................................................................................................112

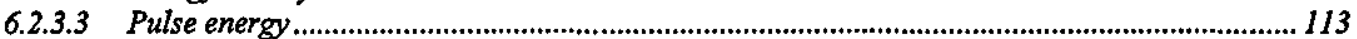

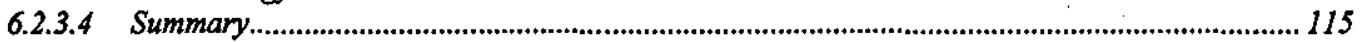

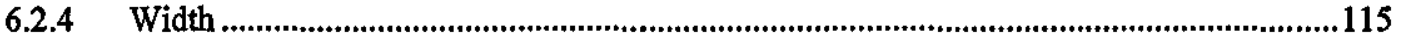

6.2.4.1 Spot overlap......................................................................................................................115

6.2.4.2 Energy density .................................................................................................................116

6.2.4.3 Pulse energy .................................................................................................................117

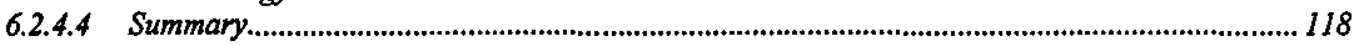

6.3 OPTIMISATION OF SURFACE ROUGHNESS AND WALL THICKNESS ............. 119

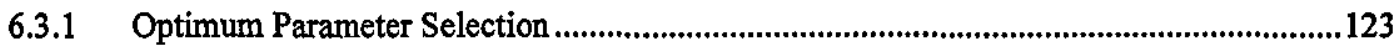

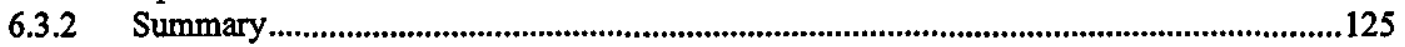

CHAPTER 7 PULSE SHAPING EXPERIMENTS................................................ 127

7.1 PUlSe ShaPing ChaRACTERISATION............................................................. 128

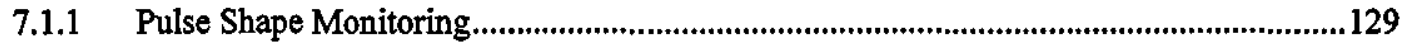

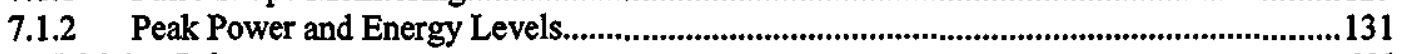

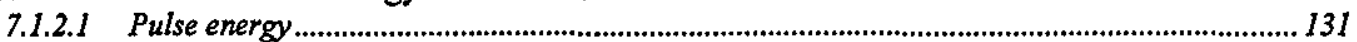

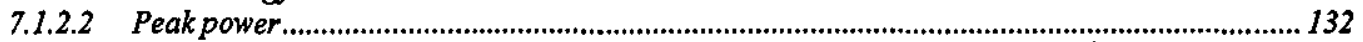

7.1.3 Characteristics of the Optimised Non Shaped Rectangular Pulse.........................................133

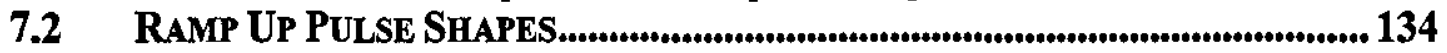

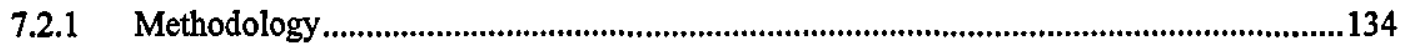

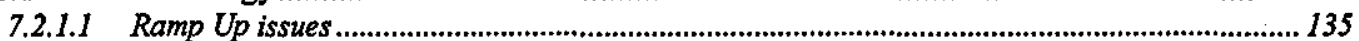

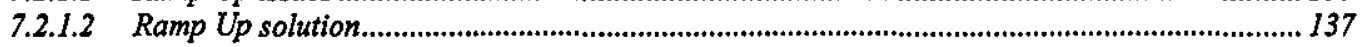

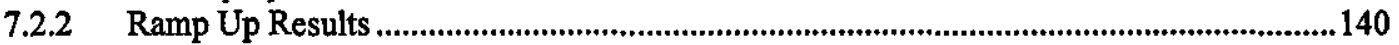

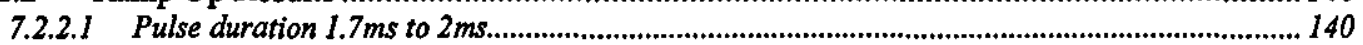

7.2.2.2 Pulse durations $4 m s$ to 6 ms................................................................................................... 146

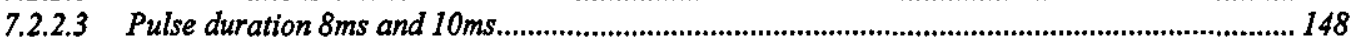

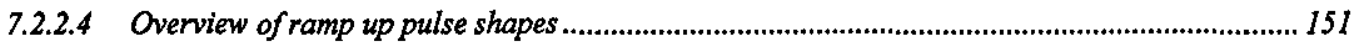

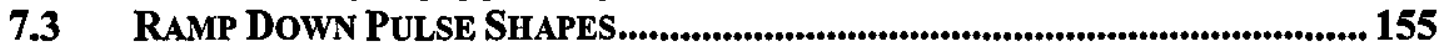

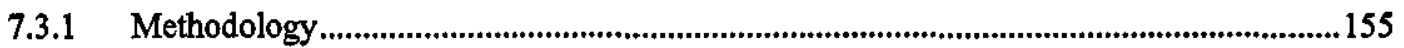

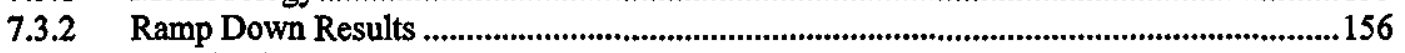

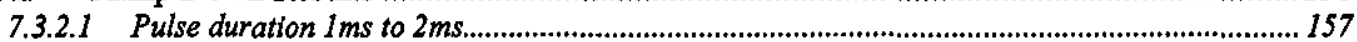

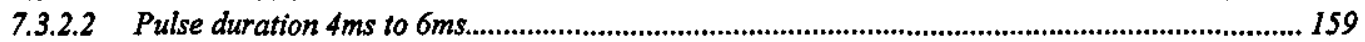

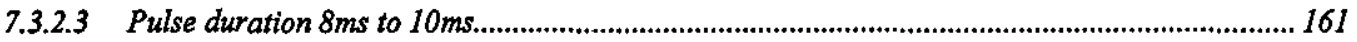

7.3.2.4 Unexpected "Suppressed Pulse Shape" generation .................................................................161

7.3.2.5 Overview of Ramp Down/Suppressed Pulse Shapes................................................................1.164

7.4 PULSE SHAPE TRENDS AND EFFECTIVENESS................................................... 167

7.4.1 Pulse Shaping Effectiveness ......................................................................................171

7.4.1.1 Ramp Up pulse shape .........................................................................................................171

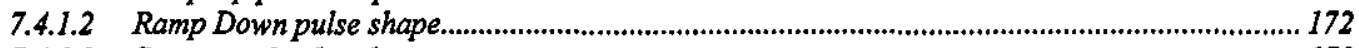

7.4.1.3 Suppressed pulse shape ....................................................................................................173

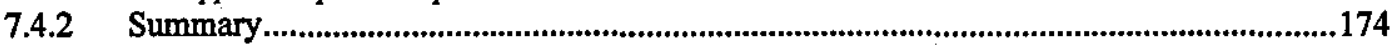

CHAPTER 8 TEST PART VALIDATION ................................................................ 176

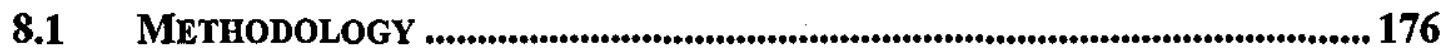

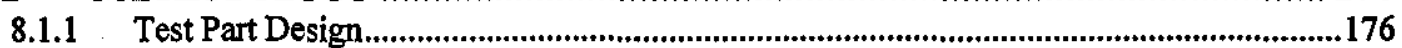

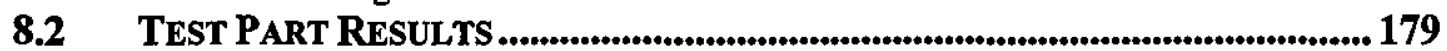

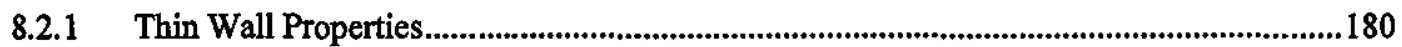

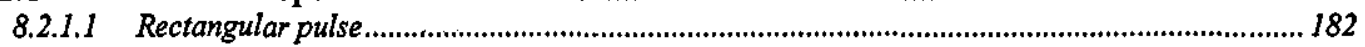




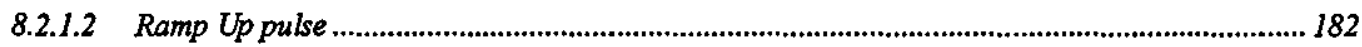

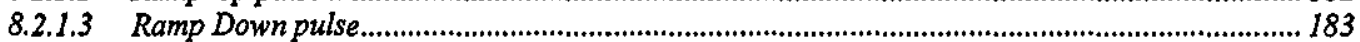

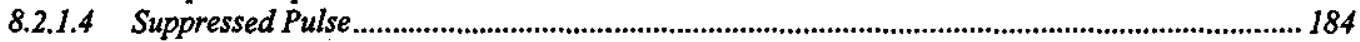

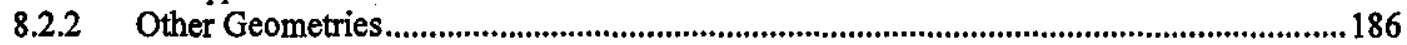

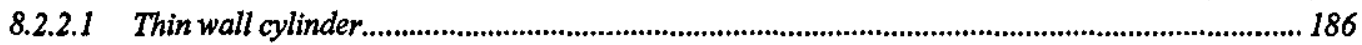

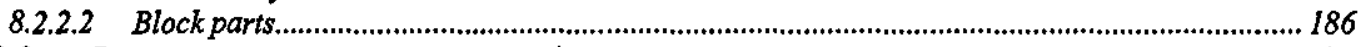

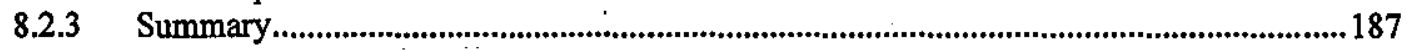

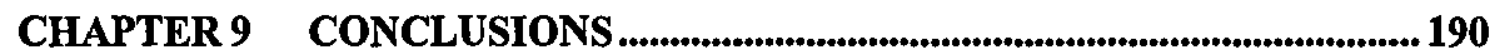

9.1 SUMMARY OF CONCLUSIONS ............................................................................. 190

9.2 SPECIFIC CONCLUSIONS.................................................................................... 192

CHAPTER 10 RECOMMENDATIONS FOR FURTHER STUDY ...................196

CHAPTER 11 REFERENCES............................................................................................. 198

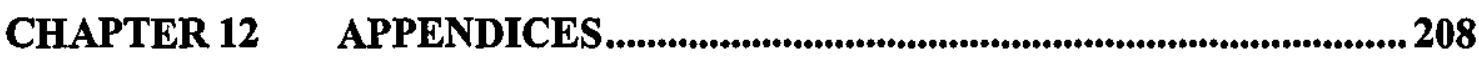

12.1 INERT GAS OPTIMISATION......................................................................208

12.1.1 Methodology ...........................................................................................................208

12.1.2 Results.....................................................................................................................209

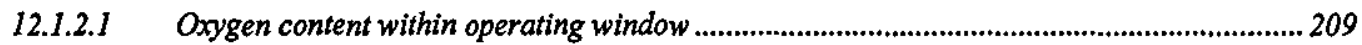

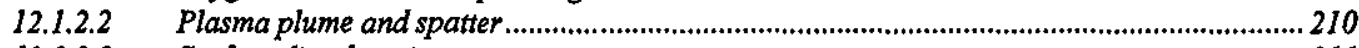

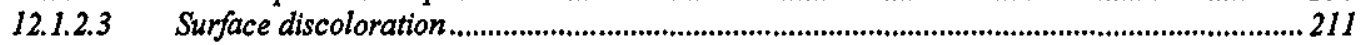

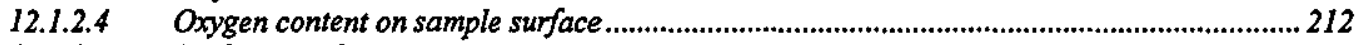

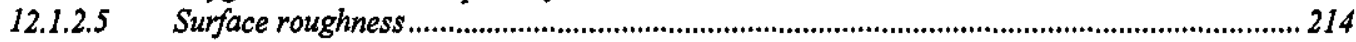

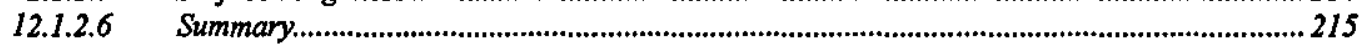

12.2 ELECTROSTATIC EFFECT ….............................................................................215

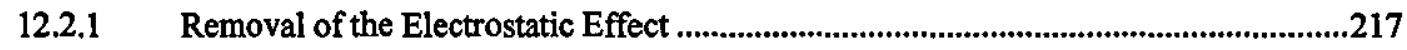

12.3 PUBLICATIONS BY AUTHOR .........................................................................219

12.3.1 Laser melting functionally graded composition of Waspaloy and Zirconia powders ..219

12.3.2 High density selective laser melting of Waspaloy .......................................................229

12.3.3 Top surface and side roughness of inconel 625 parts produced using pulse shaping...240 


\section{Publications}

[1] Mumtaz, K., Hopkinson, N., Erasenthiran, P., High Density Selective Laser Melting of Waspaloy, Proc. Solid Freeform Fabrication Symposium, Austin, TX, USA, August (2006), pp. 220-232.

[2] Mumtaz, K., Hopkinson, N., Laser Melting Functionally Graded Compositions of Waspaloy and Zirconia Powders, Journal of Material Science, 42 (2007) 7647-7656

[3] Mumtaz, K., Hopkinson, N., Erasenthiran, P., High Density Selective Laser Melting of Waspaloy, Journal of Material Processing Technology, 195 (2008) pp. 77-87.

[4] Mumtaz, K., Hopkinson, N., Selective Laser Melting of Inconel 625 using Pulse Shaping, Proc. Solid Freeform Fabrication Symposium, Austin, TX, USA, August (2008), Awarded Outstanding Paper.

[5] Mumtaz, K., Hopkinson, N., Top Surface and Side Roughness of Inconel 625 Parts Processed using Selective Laser Melting, Rapid Prototyping Journal, 2(15) (2009) pp. 96-103.

[6] Mumtaz, K., Hopkinson, N., Selective Laser Melting of Inconel 625 using Pulse Shaping, Rapid Prototyping Journal, accepted January (2009).

[7] Mumtaz, K., Hopkinson, N., Selective Laser Melting of Thin Wall Parts using Pulse Shaping, submitted to Journal of Material Processing Technology January (2009). 


\section{Presentations}

[1] Wolfson School Research Student Conference, November 2005, Loughborough University, Loughborough, UK.

[2] 17th Solid Freeform Fabrication Symposium, August 2006, University of Austin Texas, Austin, Texas, USA.

[3] Rapid Manufacturing Research Group, February 2006, Loughborough University, Loughborough, UK.

[4] 19th Solid Freeform Fabrication Symposium, August 2008, University of Austin Texas, Austin, Texas, USA. Awarded Best Presentation by the Rapid Prototyping Journal \& Emerald Publishing. 


\section{List of Tables}

Table 2-1 Reflectance and absorbance of metals at different wavelength [Glardon $e t$

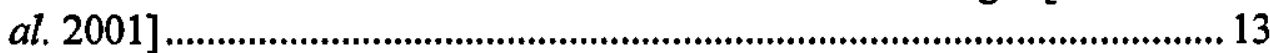

Table 2-2 Main DMLF powder feed deposition processes .............................................. 18

Table 2-3 Commercial direct DMLF powder bed processes ........................................ 20

Table 2-4 Advantages and disadvantages of various DMLF material deposition

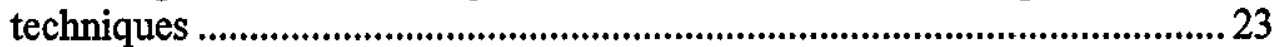

Table 2-5 Total number of control parameters for continuous and pulse wave lasers 29

Table 2-6 Pulse shaping effects on laser welded metal sheets....................................... 34

Table 5-1 Full range of GSI Lumonics JK701H Nd:YAG laser control parameters.. 73

Table 6-1 Chapter 6 optimisation experiments ........................................................... 85

Table 6-2 Laser processing parameters used within melting region experiments .......86

Table 6-3 Processing parameters used to identify useable laser spot overlap region.95

Table 6-4 Process parameters used for the fabrication of thin wall parts ..................... 98

Table 6-5 Top $R_{a}$ for a selection of samples produced using $0.5 \mathrm{~J} \& 400 \mathrm{~mm} / \mathrm{min} \ldots 101$

Table 6-6 Thin wall samples processed using 0.7J pulse energy............................... 109

Table 6-7 Process parameters that reduce part top/side $\mathrm{R}_{\mathrm{a}}$ \& wall width .................... 123

Table 7-1 Ramp Up pulse generation parameter range............................................... 135

Table 7-2 Ramp Down pulse generation parameter range..........................................156

Table 7-3 Benefits of selected pulse shapes to be used within test part validation... 174

Table 8-1 Laser parameters used to produced test parts ............................................. 179

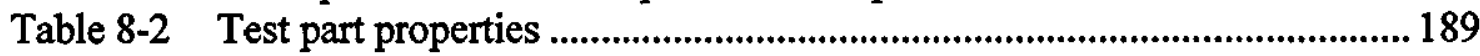

Table 8-3 Measured effectiveness of various pulse shapes when employed within

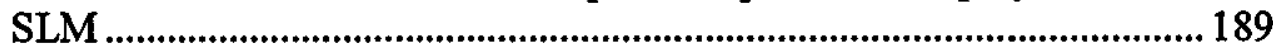

Table 12-1 Processing parameters used for gas flow rate optimisation study..............209 


\section{List of Figures}

Figure 1-1

Figure 2-1

Figure 2-2

Figure 2-3

Figure 2-4

Figure 2-5

Figure 2-6

Figure 2-7

Figure 2-8

Figure 2-9

Figure 2-10

Figure 2-11

Figure 2-12

Figure 2-13

Figure 2-14

Figure 2-15

Figure 2-16

Figure 2-17

Figure 2-18

Figure 2-19

Figure 2-20

Figure 2-21

Figure 3-1

Figure 3-2

Figure 3-3

Figure 3-4

Figure 3-5

Figure 3-6

Figure 3-7

Figure 3-8

Figure 3-9

Organisation of thesis 4

Sectors embracing SFF technologies [Wohlers 2008] ............................... 5

Solid freeform fabrication technologies ................................................... 6

Melt pool formation ................................................................................ 11

Radial intensity distribution of different TEM profiles adapted from [Steen 2003] .12

Schematic of powder feed deposition during laser processing................. 17

DLF process and fabricated nickel part [Peng et al. 2005] ...................... 18 Inconel 625 parts produced using Accufusion's Laser Consolidation [Xue 2006]. 18

DMLF powder bed deposition................................................................ 19 Inconel 625 parts produced using Phenix's PM250 system [Yadroitsev $e t$ al. 2007] \& EOS M270 [Shellabear \& Nirhylä 2004]

Benchmark model made from 316 stainless steel using MTT Realizer [Castillo 2005] 21

DMLF wire feed deposition.................................................................22

Generation of pulses of light using an Nd:YAG laser...............................24 Standard Rectangular pulse shape supplied as a current input to laser (a) $\&$ laser's actual output (b) 25

Approximate extended variations in average peak power and pulse energy capable using pulse shaping. 28

Laser output from standard laser pulse (a) \& modified pulse shaped laser output (b) ................................................................................................. 28

Ramp Up (a) and Ramped Down (b) pulse shapes...................................30

A complex/unconventional pulse shape ................................................30

Ramp Up and Ramp Down pulse shapes combined to reduce material spatter \& delay solidification [Katayama et al. 1993].

Removal of gas porosity with increased Ramp Down pulse duration [Katayama et al. 1993].

Welds made on aluminium using a Rectangular \& Ramp Up pulse shape (a) (5 ms, 1.75 J) \& Ramp Down/Up pulse shape with shorter pulse duration (b) 33

Welds on Magnesium plates, using Ramp Down (a) \& Ramp Up (b) pulse shapes [Pan et al. 2005].

Schematic of the selective laser melting process

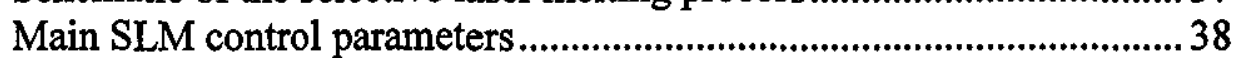

Laser spot overlap of two overlapping pulses ........................................... 40

Reflected, absorbed and transmitted wave intensities .............................. 45

Transfer of heat between powder particles via conduction (a) \& heat distribution within powder bed (b)

Energy loss leading up to and after melt pool formation........................... 48

Three phase equilibrium for wetting of a liquid on a solid (adapted from

[Eustathopoulos et al. 1999]).

Poor wetting behaviour \& complete wetting of liquid on a solid............50

Transition from half cylinder to sphere, adapted from Kruth et al. [2004] 
Figure 3-10

Figure 3-11

Figure 3-12

Figure 3-13

Figure 3-14

Figure 3-15

Figure 3-16

Figure 3-17

Figure 5-1

Figure 5-2

Figure 5-3

Figure 5-4

Figure 5-5

Figure 5-6

Figure 5-7

Figure 5-8

Figure 5-9

Figure 5-10

Figure 5-11

Figure 5-12

Figure 5-13

Figure 5-14

Figure 5-15

Figure 6-1

Figure 6-2

Figure 6-3

Figure 6-4

Figure 6-5

Figure 6-6

Figure 6-7

Figure 6-8

Figure 6-9

Figure 6-10

Figure 6-11

Figure 6-12

Figure 6-13

Figure 6-14

Figure 6-15

Marangoni convection within the cross-section of a melt pool. .54

Surface rippling along laser scan direction [Ramos et al. 2003] ............. 55

Relaxation mechanism restoring liquid to surface free level [Ramos et al. 2003] . .55

Melt pool formation and solidification by a pulsed laser ......................... 57

Peaks and troughs between solidified melt pools .....................................57

Melt pool ejection as a result of high recoil pressure on melt pool surface

.59

Melt pool modification influenced by external recoil pressure ................60

Inert gas shield fed from laser nozzle onto laser material interaction zone

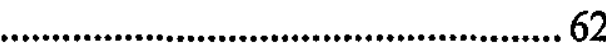

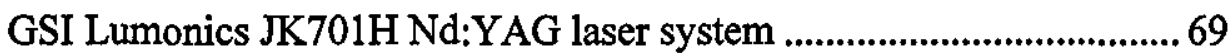

Main selective laser melting apparatus................................................... 70

Plan view of processing table ..................................................................... 70

Side view of processing table ………....................................................... 71

Side view of processing table with powder deposition \& levelling methodology 71

Substrate placement on processing table \& $0.1 \mathrm{~mm}$ powder deposition .72

Laser nozzle alignment with powder bed prior to processing ................. 72

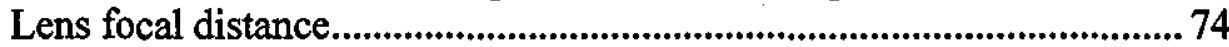

Spot size against focal distance for steel (a) \& Kapton/Steel at refined focal distance region (b) 75

Spot size against pulse energy on steel (a) \& Kapton film (b) .................77 Single laser shot on stainless steel at $20 \mathrm{~ms} 15 \mathrm{~J}$ ( $750 \mathrm{~W}$ ) (a) \& laser beam

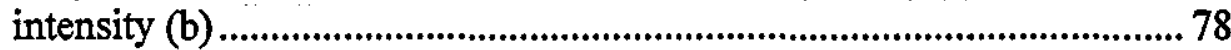

Inconel 625 chemical composition obtained from EDS ........................... 80 Inconel 625 particle size distribution..................................................... 81

SEM image of Inconel 625 powder at X200 (a) \& X1300 (b) ................. 82

SEM image of failed batch of Inconel 625 powder at X1300 (a) \& comparison between different powder sizes (b)

Plasma plume measurement $(a, b, c) \&$ powder bed contamination due to undesirable level of material spatter (d)

Screenshot of Flash optical measurement software measuring width of a single scan sample. 89 Inconel 625 single scans at $0.5 \mathrm{~ms}, 10 \mathrm{~Hz}, 168 \mathrm{~mm} / \mathrm{m} \&$ 0.1-2.5J............. 91 Inconel 625 melting region $(10 \mathrm{~Hz} \& 168 \mathrm{~mm} / \mathrm{min})$..................................92 Peak power against pulse duration........................................................93 Width of samples produced using variable pulse durations and energy. 94 Repetition rate/spot overlap against scan speed useable region ...............96 Thin wall part.......................................................................................98 Talysurf CLI 2000 apparatus used to measure surface roughness......... 100 Top/Side $R_{\mathrm{a}}$ measurement of thin wall part ............................................ 100 Solidified melt pool produced with a $70 \%$ laser spot overlap (a) \& $90 \%$ spot overlap (b) 101

Top $R_{a}$ of 4 layered samples processed using $0.5 \mathrm{~J}, 400 \mathrm{~mm} / \mathrm{min}$ at variable repetition rates.

Top $R_{\mathrm{a}}$ against spot overlap. 104

Top $R_{a}$ against energy density 105

Top $R_{a}$ against repetition rate at $100 \mathrm{~mm} / \mathrm{min}$ 106 
Figure 6-16 Lowest top $\mathrm{R}_{\mathrm{a}} 4 \mu \mathrm{m}$ achieved using $0.5 \mathrm{~ms}, 0.7 \mathrm{~J}, 50 \mathrm{~Hz}$ and $100 \mathrm{~mm} / \mathrm{min}$... 106

Figure 6-17 Thin walled parts processed at $0.7 \mathrm{~J}$ at varied repetition rates $\&$ scan speeds.

Figure 6-18

Figure 6-19

Figure 6-20

Figure 6-21

Figure 6-22

Figure 6-23

Figure 6-24

Figure 6-25

Figure 6-26

Figure 6-27

Figure 6-28

Figure 6-29

Figure 6-30

Figure 6-31

Figure 6-32

Figure 7-1

Figure 7-2

Figure 7-3

Figure 7-4

Figure 7-5

Figure 7-6

Figure 7-7

Figure 7-8

Figure 7-9

Figure 7-10

Figure 7-11

Figure 7-12

Figure 7-13

Figure 7-14

Figure 7-15

Figure 7-16

Figure 7-17

Figure 7-18

Figure 7-19

Figure 7-20

Figure 7-21

Figure 7-22

Figure 7-23

Figure 7-24

Figure 7-25

Figure 7-26

Four layer processed using $0.5 \mathrm{~J}, 40 \mathrm{~Hz} \&$ varying scan speeds ............ 111

Overlap against side roughness...................................................... 112

Side $\mathrm{R}_{\mathrm{a}}$ against energy density..................................................... 113

Side roughness against repetition rate at $400 \mathrm{~mm} / \mathrm{min}$........................ 114

Lowest side roughness of $10 \mu \mathrm{m}$ produced using $30 \mathrm{~Hz}$ (a) $\& 40 \mathrm{~Hz}$ (b) at

$0.5 \mathrm{~ms}, 0.7 \mathrm{~J} \& 400 \mathrm{~mm} / \mathrm{min}$. 114

Width against spot overlap 116

Sample width against energy density............................................. 117

Width against repetition rate for samples produced at $400 \mathrm{~mm} / \mathrm{min} \&$ variable pulse energies.

Plan view of melt pool formed by pulsed laser \& variations in surface tension due to temperature gradient

Variations in surface tension as a results of melt pool volume and width..

Reduction in surface tension via the theory of lateral balling

Surface $R_{a}$ at variable laser spot overlaps ............................................ 122

Surface $R_{a}$ at variable energy densities ........................................... 122

Side $R_{a}$ against top $R_{a}$ for thin wall parts........................................ 124

Lowest combined top \& side $\mathrm{R}_{\mathrm{a}}$ thin walled part $(0.7 \mathrm{~J}, 40 \mathrm{~Hz}$ at

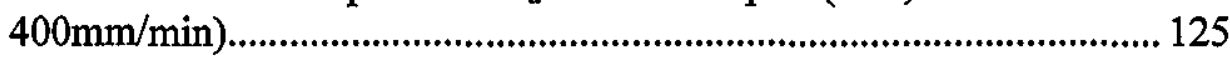

SLM laser control screen with pulse shaping control .......................... 129

PicoScope 3205 PC oscilloscope ....................................................... 130

Oscilloscope window in PicoScope PC software ................................ 131

Rectangular pulse shape with a 4ms durations \& 2J energy................. 132

Maximum/average peak power of a 5ms, 1J Rectangular pulse shape 133

Optimised Rectangular pulse shape .................................................... 133

Ramp Up pulse shape methodology ................................................ 135

Issues with Ramp Up pulses, 1ms \& 1J........................................... 136

Issues with Ramp Up pulses, effects of different sector heights .......... 136

Ramp Up issues, prolonged $1^{\text {st }}$ sector causes power drop off .............. 137

Successful Ramp Up pulse generation for pulses $1.7 \mathrm{~ms}$ in duration.... 138

Successful Ramp Up pulse generation employing three sectors .......... 139

Increased pulse duration \& power drop-off using three sectors ........... 139

Reduced power drop-off period with insertion of extra sector............. 140

Ramp Up pulse $1.7 \mathrm{~ms} \&$ 0.6 J........................................................... 141

Ramp Up pulse $1.7 \mathrm{~ms} \&$ 0.7J ........................................................... 141

Ramp Up pulse 1.7ms \& $0.8 \mathrm{~J}$........................................................ 142

Ramp Up pulse 1.7ms \& 0.9J ........................................................... 142

Ramp Up pulse 1.7ms \& 1J ............................................................. 142

Ramp Up pulse 2ms \& 0.6J .......................................................... 144

Ramp Up pulse 2ms \& 0.9J ........................................................... 144

Ramp Up pulse 2ms \& 1J ................................................................ 144

Comparison of the Rectangular \& Ramped Up pulse shape (2ms) ...... 146

Ramp Up pulse 4ms \& 1J .................................................................. 147

Ramp Up pulse 4ms \& 1.1J ........................................................ 147

Ramp Up pulse 8ms \& 1.6J ............................................................. 149 
Figure 7-27

Figure 7-28

Figure 7-29

Figure 7-30

Figure 7-31

Figure 7-32

Figure 7-33

Figure 7-34

Figure 7-35

Figure 7-36

Figure 7-37

Figure 7-38

Figure 7-39

Figure 7-40

Figure 7-41

Figure 7-42

Figure 7-43

Figure 7-44

Figure 7-45

Figure 7-46

Figure 7-47

Figure 7-48

Figure 7-49

Figure 8-1

Figure 8-2

Figure 8-3

Figure 8-4

Figure 8-5

Figure 8-6

Figure 8-7

Figure 8-8

Figure 8-9

Figure 12-1

Figure 12-2

Figure 12-3

Figure 12-4

Figure 12-5

Figure 12-6

Figure 12-7

Figure 12-8

Ramp Up pulse $8 \mathrm{~ms} \& 1.72 \mathrm{~J}$ 149

Ramp Up pulse $8 \mathrm{~ms} \& 3.4 \mathrm{~J}$ 150

Comparison of a Rectangular pulse \& a Ramp Up pulse (8ms) ........... 151

Ramp Up top/side $\mathrm{R}_{\mathrm{a}}$ 154

Standard Rectangular pulse shape, $0.5 \mathrm{~ms} \& 0.7 \mathrm{~J}$

Ramp Down pulse shape methodology

Ramp Down pulse $1 \mathrm{~ms} \& 0.78 \mathrm{~J}$

Ramp Down pulse $1 \mathrm{~ms} \& 0.92 \mathrm{~J}$ 158

Ramp Down pulse $1.5 \mathrm{~ms} \& 0.86 \mathrm{~J}$ 159

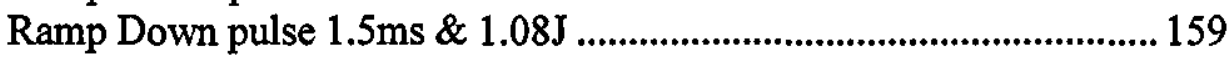

Ramp Down pulse $4 \mathrm{~ms} \& 1.37 \mathrm{~J}$

Ramp Down pulse $4 \mathrm{~ms} \& 1.51 \mathrm{~J}$

Ramp Down pulse 4ms \& 2.3J

Suppressed pulse shape $1 \mathrm{~ms} \& 0.4 \mathrm{~J}$

Suppressed Pulse shape $1 \mathrm{~ms} \& 0.5 \mathrm{~J}$

Suppressed Pulse shape $1 \mathrm{~ms} \& 0.6 \mathrm{~J}$

Ramp Down/Suppressed pulse shape top/side $R_{a} \&$ width

165

Top/Side $R_{a}$, width of samples and plasma plume height produced using

Ramp Up, Ramp Down/Suppressed pulse shapes

Side $R_{a}$ against Top $R_{a}$ for different pulse shapes.

Ramp Up\Ramp Down $\backslash$ Suppressed pulse Top $R_{a}(a) \&$ Side $R_{a}(b)$ at different peak powers.

Ramp Up pulse producing low wall width \& generating minimal spatter

generation during processing 172

Ramp Down pulse with lowest top $\mathrm{R}_{\mathrm{a}}$ 173

Suppressed pulse with low top/side $R_{a}$ \& low width............................ 174

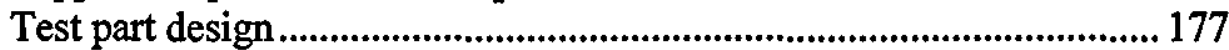

Final stages of test part fabrication .................................................... 178

Test parts made with different pulse shapes ........................................ 179

Pulse shapes used to produce test parts ............................................. 180

Thin wall part surface roughness and width ........................................ 181

Side view of thin wall parts .............................................................. 181

Side $\mathrm{R}_{\mathrm{a}}$ vertical for thin wall cylinder (including thin wall part results for comparison)

Side $\mathrm{R}_{\mathrm{a}}$ horizontal \& vertical average for all four sides of block parts (including thin wall part results for comparison)

Test part produced using the Suppressed pulse shape.

Oxygen content within processing region at different inert gas flow rates 210

Plasma Plume/spatter generated with \& without the assistance of an inert shielding gas ....

Three layers single scan processed with no shielding gas (a) $\& 10 \mathrm{ml} / \mathrm{s}$ shielding gas (b) (both processed at $0.5 \mathrm{~ms}, 1 \mathrm{~J}, 10 \mathrm{~Hz} \& 100 \mathrm{~mm} / \mathrm{m}$ )... 212 SEM image of parts surface measured for oxygen content Oxygen content on samples at different gas flow rates and processing scan speeds.

Surface roughness against repetition rate for specimens produced at varied shielding gas flow rates.

Plan view of single scan of Inconel 625 from powder bed.

Possible electrostatic effect generated by laser material interaction .... 217 
Figure 12-9 Revised earthing arrangement 218

Figure 12-10 Plan view of powder bed processed with improved earthing (a) \& without (b) (processed using $0.5 \mathrm{~ms}, 1 \mathrm{~J}, 60 \mathrm{~Hz} \& 100 \mathrm{~mm} / \mathrm{min}$ ) 218 


\section{Abbreviations}

$\begin{array}{ll}\text { 3DP } & \text { 3D Printing } \\ \text { BNC } & \text { Bayonet Neill Concelman } \\ \text { CNC } & \text { Computer Numerical Control } \\ \text { CW } & \text { Continuous Wave } \\ \text { DLF } & \text { Direct Laser Fabrication } \\ \text { DMD } & \text { Direct Metal Deposition } \\ \text { DMLF } & \text { Direct Metal Laser Fabrication } \\ \text { DMLS } & \text { Direct Metal Laser Sintering } \\ \text { EBM } & \text { Electron Beam Melting } \\ \text { EDS } & \text { Energy Dispersive X-ray Spectroscopy } \\ \text { FGM } & \text { Functionally Graded Material } \\ \text { HAZ } & \text { Heat Affected Zone } \\ \text { HIP } & \text { Hot Isostatic Pressing } \\ \text { IR } & \text { Infra Red } \\ \text { LC } & \text { Laser Consolidation } \\ \text { LENS } & \text { Laser Engineered Net Shaping } \\ \text { PSD } & \text { Particle Size Distribution } \\ \text { PW } & \text { Pulsed Wave } \\ \text { RDPS } & \text { Ramp Down Pulse Shape } \\ \text { RP } & \text { Rapid Prototyping } \\ \text { RPS } & \text { Rectangular Pulse Shape } \\ \text { RUPS } & \text { Ramp Up Pulse Shape } \\ \text { RM } & \text { Rapid Manufacturing } \\ \text { SEM } & \text { Scanning Electron Microscope } \\ \text { SFF } & \text { Solid Freeform Fabrication } \\ \text { SL } & \text { StereoLithography } \\ \text { SLM } & \text { Selective Laser Melting } \\ \text { SLS } & \text { Selective Laser Sintering } \\ \text { TEM } & \text { Transverse Electro Magnetic } \\ & \end{array}$




\section{Symbols}

$\begin{array}{ll}P_{e} & \text { Pulse energy [J] } \\ t_{p} & \text { Pulse duration [ms] } \\ f & \left.\text { Frequency [s }{ }^{-1}\right][\mathrm{Hz}] \\ V & \text { Scan speed [mm/m] } \\ P_{a v} & \text { Average laser power [W] } \\ P P_{a v} & \text { Average peak power [W] } \\ P P & \text { Maximum peak power [W] } \\ E_{d e n} & \left.\text { Laser energy density [J/mm }{ }^{2}\right] \\ S & \text { Theoretical spot diameter [m] } \\ S_{d} & \text { Laser spot diameter [m] } \\ S_{d m i n} & \text { Minimum laser spot diameter [m] } \\ S_{o v} & \text { Laser spot overlap [\%] } \\ D & \text { Beam diameter at laser head front plate [m] } \\ D_{I} & \text { Beam diameter at lens [m] } \\ M^{2} & \text { Beam quality } \\ f_{l} & \text { Focal length of processing lens [m] } \\ \lambda & \text { Laser wavelength [m] } \\ \Theta & \text { Beam divergence [rad/s] } \\ & \\ R_{f} & \text { Reflectance } \\ T & \text { Transmittance } \\ I_{o} & \text { Incident wave intensity } \\ I_{a} & \text { Absorbed intensity } \\ I_{r} & \text { Reflected intensity }\end{array}$

$T \quad$ Absolute temperature [K]

$\Delta T_{m} \quad$ Ambient temperature $[\mathrm{K}]$

$T_{m} \quad$ Melting temperature [K]

$T_{v} \quad$ Boiling temperature $[\mathrm{K}]$

$L_{f} \quad$ Latent heat of fusion [J/kg]

$K_{b} \quad$ Thermal conductivity [W/mK] 
$C_{p} \quad$ Specific heat capacity $[\mathrm{J} / \mathrm{kgK}]$

$\alpha$

Thermal expansion coefficient $[\mathrm{K}]$

$L_{v} \quad$ Latent heat of vaporisation $[\mathrm{J} / \mathrm{kg}]$

$\delta \quad$ Thermal Diffusivity $\left[\mathrm{m}^{2} / \mathrm{s}\right]$

$E_{m} \quad$ Melt energy []]

$\Delta H_{m} \quad$ Energy required to melt material [J]

$\begin{array}{ll}\gamma & \text { Surface free energy }\left[\mathrm{J} / \mathrm{m}^{2}\right] \\ \gamma_{l v} & \text { Surface free energy liquid-vapor }\left[\mathrm{J} / \mathrm{m}^{2}\right] \\ \gamma_{S V} & \text { Surface free energy solid-vapor }\left[\mathrm{J} / \mathrm{m}^{2}\right] \\ \gamma_{I S} & \text { Surface free energy liquid-solid }\left[\mathrm{J} / \mathrm{m}^{2}\right] \\ \gamma_{l o w} & \text { Low surface free energy }\left[\mathrm{J} / \mathrm{m}^{2}\right] \\ \gamma_{\text {high }} & \text { High surface free energy }\left[\mathrm{J} / \mathrm{m}^{2}\right] \\ L & \text { Length of melt pool }[\mathrm{m}]\end{array}$

$R \quad$ Universal gas constant [J/(mol.K)]

$\Delta G \quad$ Free energy of reaction $[\mathrm{J} / \mathrm{mol}]$

$A \quad$ Absorbance [W/mK]

$P_{b} \quad$ Bulk density $\left[\mathrm{kg} / \mathrm{m}^{3}\right]$

$P_{\rho} \quad$ Powder bed density $\left[\mathrm{kg} / \mathrm{m}^{3}\right]$

$r_{p} \quad$ Radius of particle [m]

$v_{p} \quad$ Volume of particle [m]

$R_{a} \quad$ Average arithmetic surface roughness [ $\left.\mu \mathrm{m}\right]$

$\eta \quad$ Dynamic viscosity [Pa's]

$\begin{array}{ll}\mathrm{O}_{2} & \text { Oxygen } \\ \mathrm{N}_{2} & \text { Nitrogen } \\ \mathrm{A}_{\boldsymbol{r}} & \text { Argon } \\ \mathrm{H}_{e} & \text { Helium } \\ \mathrm{PO}_{\mathrm{O}_{2}} & \text { Partial pressure of Oxygen }\end{array}$




\section{Chapter 1 Introduction}

\subsection{Scope of Research}

The main advantage of freeform fabrication processes such as SLM is the capability to build complex geometries that would otherwise be difficult to produce using conventional manufacturing processes. This is due to the versatility, accuracy and small spot size of a laser beam. The possibility to build thin wall parts to a high resolution complements the technology's main advantage and extends its manufacturing capabilities. However the production of metal parts via SLM is not without its difficulties. Many processing issues arise due to the use of a high power laser to fully liquefy material from a powder bed. The presence of a fully molten melt pool makes the process more difficult to control than liquid phase sintering due to increased capillary and thermocapillary effects [Rombouts 2006].

The surface roughness of a part is critical in many applications, with some applications requiring a surface roughness of $0.8 \mu \mathrm{m}$ or better to avoid premature failure due to surface initiated cracking [Dalgarno, 2007]. High part surface roughness is often an issue that arises from poor melt pool control due to instabilities and movement of molten material. Parts produced using commercial powder bed machines such as MTT Realizer, EOS M270 etc., often require post processing operations such as surface machining, polishing and shot peening to attain final part surface properties. These additional post processing operations incur further time and delay part completion. Therefore the surface roughness developed during powder processing is still a dominant issue and needs to be further improved and better controlled [Glardon et al. 2001; Ramos et al. 2006].

Part resolution can be affected by the volume of liquid present within a melt pool and its spreading behaviour. The minimum achievable feature size (resolution) has a great influence on part accuracy. The minimum resolution is of high importance in the design of functional parts, e.g. for thin walls, honeycomb and lattice structures or other open 
cell structures. Glardon et al. [2001] state that the accuracy and resolution of metal parts produced using powder bed processes must be enhanced to comply with specific features and details found in precise parts and moulds. Thin walls are an example of socalled positive minimum feature size (smallest solid part possible), while open cells are an example of negative minimum feature size (smallest gap size possible) [Rehme \& Emmelmann 2005].

Achieving a fine control over the melt pool properties is critical in producing high resolution parts with low surface roughness. Pulse shaping techniques have been shown to extend the capabilities and control over laser welded metal sheets by allowing the user to carefully tailor a specific laser heat input to the material. The use of pulse shaping within SLM may improve melt pool control and hence resolution and surface finish of parts. Therefore the use of pulse shaping within SLM potentially has great future promise as an advanced manufacturing technique capable of further improving part properties without a need to compromise on part surface roughness while maintaining a high resolution due to a strategic distribution of heat giving the process more refinement and optimisation potential. Due to the complexity of SLM there is a requirement to understand the issues that affect specifically the top and side surface roughness of parts and a demand to further improve these properties.

This research investigates the production of thin wall Inconel 625 parts using SLM and examines whether part width and surface roughness can be effectively controlled and improved using pulse shaping techniques.

\subsubsection{Organisation of Thesis}

This thesis is divided into four main stages as shown in Figure 1-1. It begins with Chapter 1 introducing the scope of research and organisation of thesis. Chapter 2 forms the first stage of research, namely the literature review that discusses various metal freeform fabrication techniques. The chapter ends with details on pulse shaping control, describing techniques, investigations, benefits and improvements that can be derived from its use. Chapters 3 discusses the SLM process in detail and describes melt pool dynamics and heat transfer. Chapter 4 details the research hypothesis, novelty and aims and objectives. Chapter 5 forms stage two and details the SLM apparatus and 
adjustments made to the system prior to main experimentation. The chapter also characterises the Inconel 625 powder used within the research. Chapter 6 forms stage three and begins preliminary optimisation experiments identifying a usable processing window for the fabrication of Inconel parts. Experiments included the identification of material bonding/melting region and required laser spot overlap to produce consistent high quality multilayered samples with low top and side surface roughness. Chapters 7 and 8 forms stage four and the main core of research focusing on pulse shaping. Chapter 7 introduces preliminary experiments using pulse shaping, including energy levels, pulse shaping effects on part geometry, surface roughness and spatter generation during processing. Chapter 8 focuses on validating information gathered in chapter 7 by producing larger test parts with variable geometries. Parts were produced with and without pulse shaping control and comparisons in part properties were made. Chapter 9 concludes the research discussing the findings of this investigation and reveals the benefits that can be derived from employing pulse shape control within the SLM process. Chapter 9 is followed by recommendations for further work. 


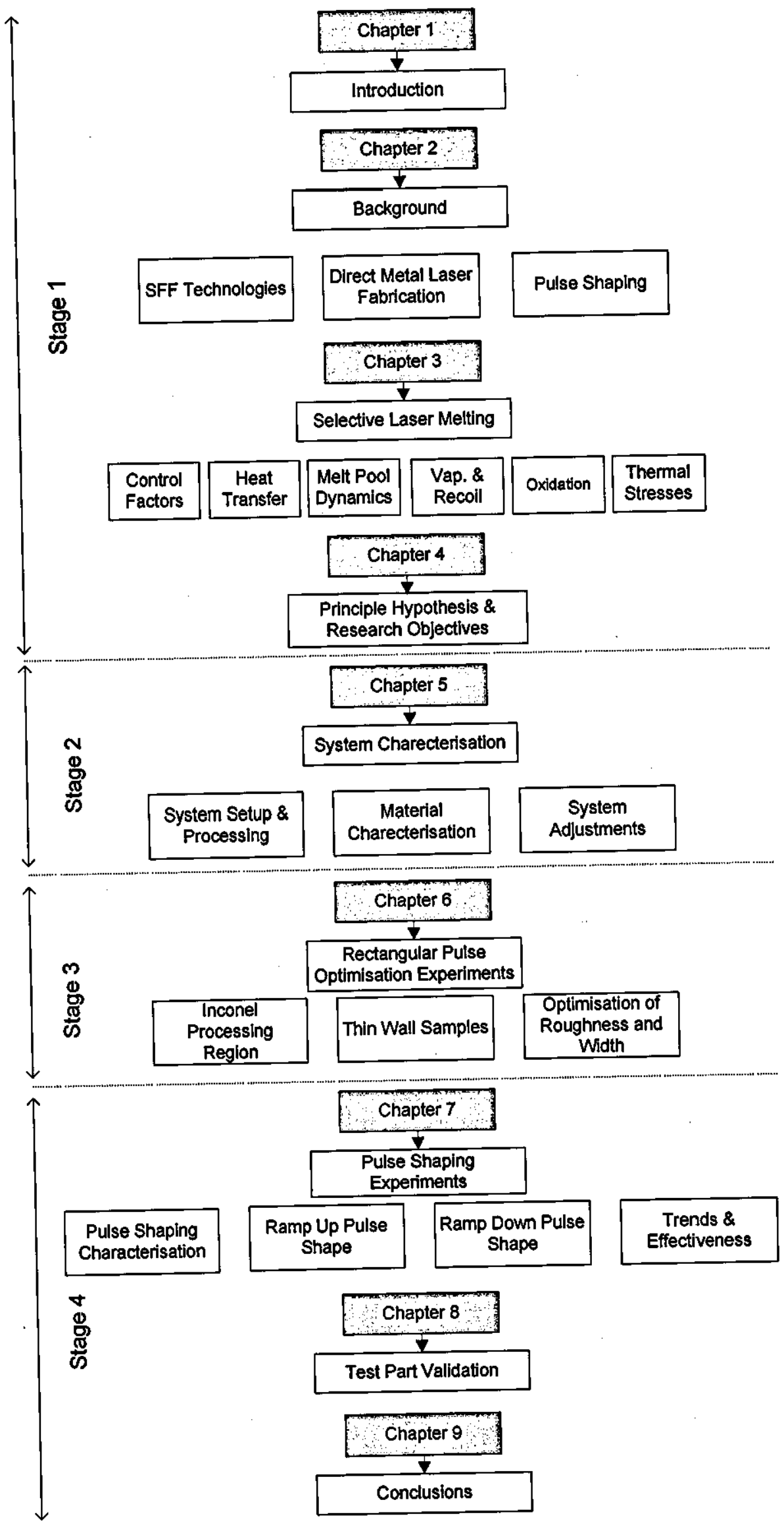

Figure 1-1 Organisation of thesis 


\section{Chapter 2 Background}

\subsection{Solid Freeform Fabrication}

Solid Freeform Fabrication (SFF) is a group of manufacturing technologies that are capable of producing 3D solid parts by a consecutive layer-wise shaping and consolidation of material. Part geometric data can be taken directly from a CAD model and processed without the need for any moulds or tools, allowing for greater design freedom [Hague et al. 2004]. The principal advantage of SFF processes is the ability to quickly manufacture parts of high complexity or geometry entirely without the need for tooling. SFF technologies seek to improve upon conventional processing technologies through a reduction in processing steps, reduction in use of materials and the reduction in fabrication time and cost. Due to the advantages associated with SFF, an entirely new industry is being created. In the last few years it has been commercialised by several enterprises [Choi \& Chang 2005]. Figure 2-1 displays the various sectors embracing SFF technologies.

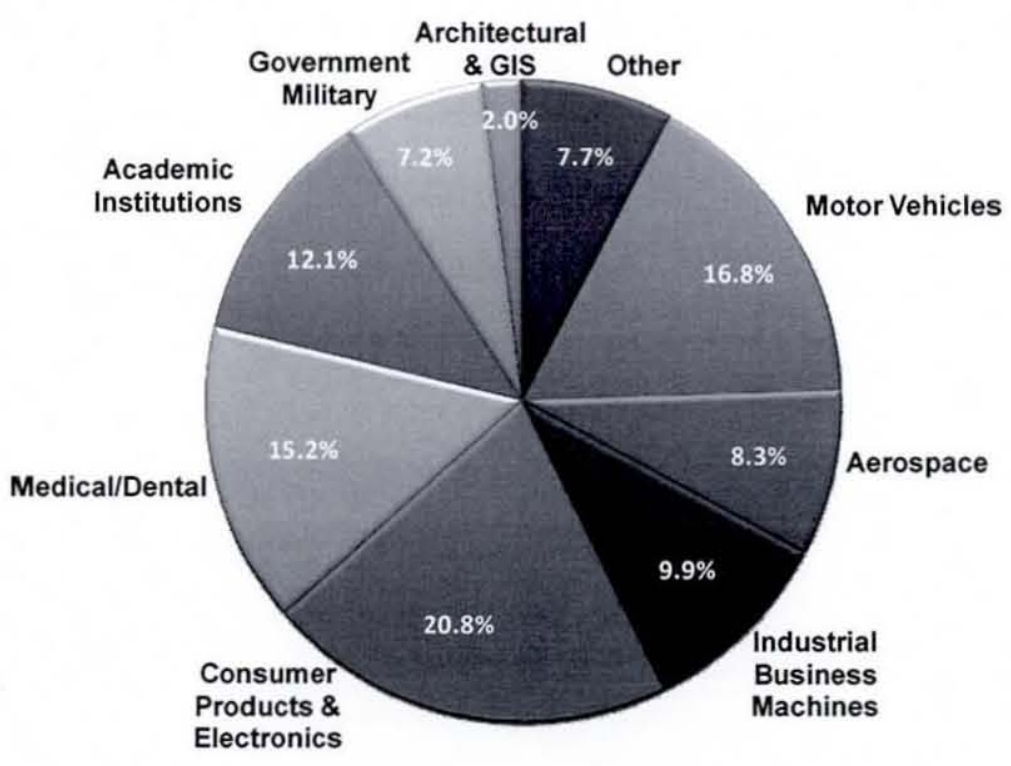

Figure 2-1 Sectors embracing SFF technologies [Wohlers 2008] 


\subsubsection{Solid Freeform Fabrication Technologies}

SFF technologies can process materials such as polymers, metals, ceramics and composites. The binding or fusion of material can be achieved through different chemical or physical reactions. Figure 2-2 displays the different types of SFF processes available.

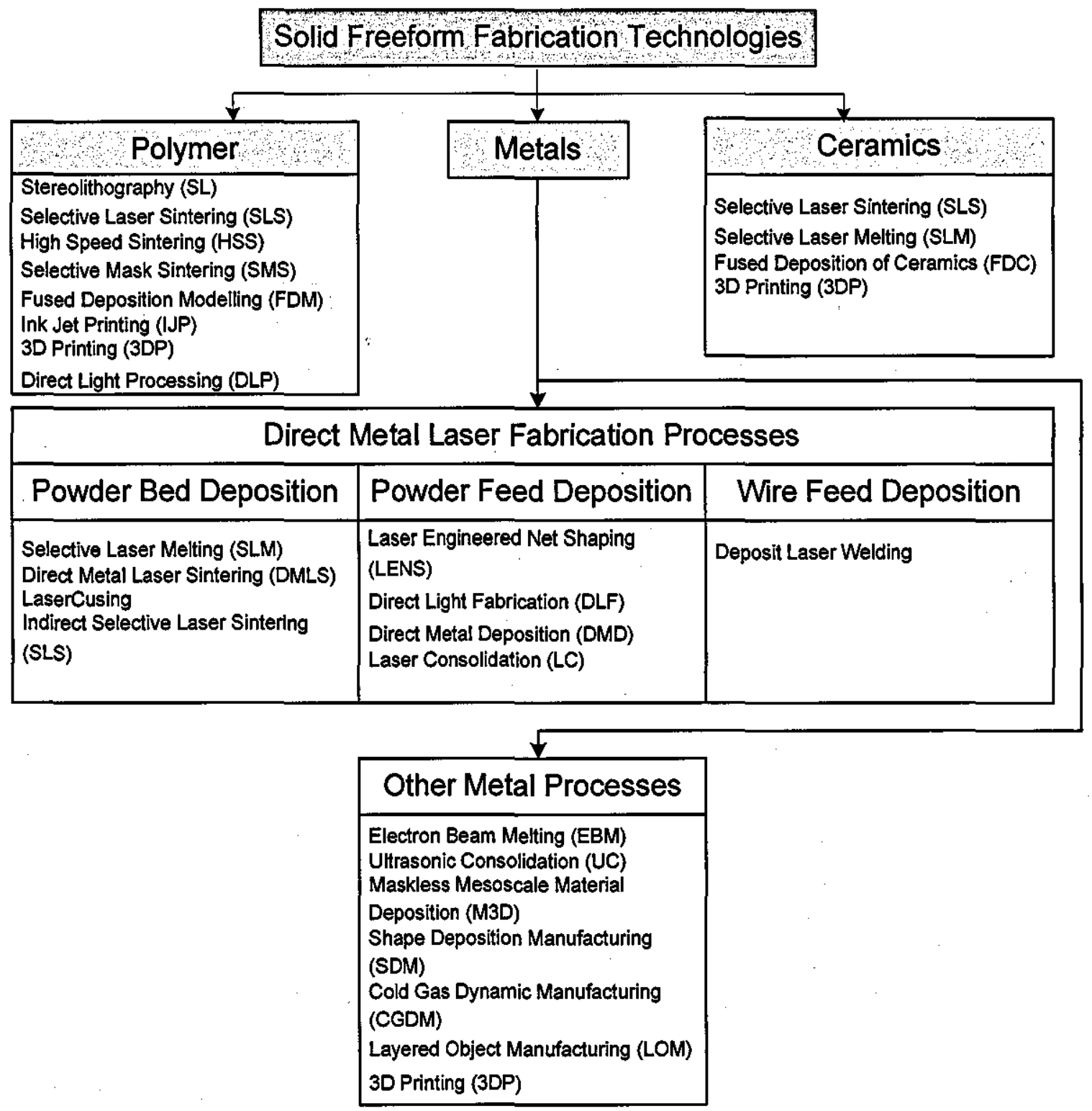

Figure 2-2 Solid freeform fabrication technologies 
Within the family of SFF processes there are terms such as Rapid Prototyping (RP), Rapid Manufacturing (RM) and Rapid Tooling (RT). These terms describe the way in which the SFF processes are used. RP uses SFF to manufacture prototypes, often using technologies such as 3DP, SL, SLS etc. RM has evolved from RP techniques and produces fully functional parts, e.g. dental implants [Kruth et al. 2005]. Though still limited in use, RM technologies are steadily growing. Kruth et al.[2005] expect that in the next decade RM will achieve the same level of acceptance as RP through a continuous improvement in material properties, accuracy and decrease in costs. RM offers great geometrical flexibility in manufacturing, which is a very attractive option for directly fabricating fully functional end use parts. RT is similar to RM, but instead pursues the production of tools for functional use.

\subsubsection{Rapid Manufacturing and the Production of Metal Parts}

Figure 2-2 showed that there are a variety of SFF processes available for the fabrication of metal parts. The use of polymer processing technologies within industry is currently more widely accepted and more extensively used than metals. The use of metal processes within RM is limited and is the subject of major research studies. The current metal processes include Electron Beam Melting (EBM), Ultrasonic Consolidation (UC), 3D Printing (3DP) and laser based processes known as Direct Metal Laser Fabrication (DMLF). DMLF processes include Laser Engineering Net Shaping Lens (LENS), Laser Consolidation (LC), Direct Metal Laser Sintering (DMLS) and Selective Laser Melting (SLM) processes. All these processes have their individual advantages, constraints, building procedures, post processing operations etc. Industry is naturally more inclined towards metal SFF processes that can produce high quality fully functional parts with minimum post processing operations or without significant breaks in the build cycle. This will allow various industries to cut down in machining effort, fabrication time, cost and material wastage. 


\subsubsection{Key non laser based metal SFF processes}

Electron Beam Melting - The EBM process was developed by Arcam, using an electron beam to melt compositions of metal powder from a pre-laid powder bed. The high heat intensity generated by the electron beam is capable of completely melting the powder together creating high density metal parts. The process is renowned for its quick fabrication times as the electron beam is capable of traversing at very high speed along the powder bed [Cormier et al. 2004; Williams et al. 2005]. Due to the high heat intensity and quick fabrication times EBM parts require surface finishing operations due to poor surface finish.

3D Printing - Prometal has licensed this process from MIT. A print head is used to print a polymer over a pre-laid powder bed. This polymer temporarily bonds the metal powder together forming a green part. This green part is then post-processed using furnace debinding, sintering and infiltration operations to attain a high density. Parts produced using 3DP typically have high surface roughness and require further post processing operations to attain final part properties [Feenstra et al. 2003; Williams et al. 2005]. Fcubic also uses inkjet technology but is capable of producing steel parts with extremely high resolutions of approximately $35 \mu \mathrm{m}$ [Fcubic 2008].

UItrasonic Consolidation - Solidica currently produces UC machines to fabricate metal parts. The process applies ultra sonic oscillations to metal foil under an applied load. This oscillation bonds the thin metal foil together with a very low heat. The process is particularly useful for embedding components within a part, however it has difficulty in producing overhanging features. 


\subsection{Direct Metal Laser Fabrication}

DMLF techniques allow metal to be joined in a liquid or partial liquid state using a focused laser beam. The material is deposited such that it comes directly under the path of the laser beam. The heat generated by the laser causes the material to sinter or completely melt. The laser source is then removed from the material allowing it to cool and solidify as a bulk mass. Most DMLF processes are based upon the same layer building, yet these processes may differ in the specific fabrication methodology. The material deposition methodology can vary (material feed or powder bed), material can also be in powder or wire form. Laser types can also vary within the system configuration [Lu.L et al. 2001].

Laser based DMLF processes may be some of the most viable technologies for the direct fabrication of metallic components [Schwendner et al. 2001]. This is due to the versatility and accuracy of a laser beam, it is able to produce parts to a high accuracy and resolution. High melting temperatures can be easily generated completely melting powders within a small Heat Affected Zone (HAZ) and reducing part distortion and surface roughness [Steen 2003]. Some DMLF processes have the capability to produce accurate fully functionally end use parts in a single step without the requirement of extensive post processing (SLM, DMLS, LENS, LC) [Milewski et al. 1999]. In addition these direct processes can produce parts with improved microstructural properties compared to those that are conventionally processed [Xue \& Islam 2000; Bourell et al. 2002]. Sections 2.2.4.2-2.2.4.3 discuss the different material deposition techniques and the DMLF processes that employ them.

\subsubsection{Laser Material Interactions}

Light is a form of electromagnetic radiation or energy. It can be considered as either an energy wave or moving particles with quantified energy values. Laser light is generated by a laser, which is an acronym standing for Light Amplification Stimulated by Emission of Radiation (LASER). The process of melting using a laser begins with the conversion of the laser's photons into kinetic energy [Webb \& Jones 2004]. Incoming photons (light) excite electrons in the outermost shell of surface atoms of a 
substrate/target material, including the release and excitation of conduction electrons. These transfer their energy as phonons or vibrational waves. Phonon excitation is revealed as rapid heating or thermalisation. This energy transfer train normally takes about $1 \mathrm{ps}$ in metals and slightly longer in ceramics [Chichkov et al. 1996]. For pulses shorter than the thermalisation time (femtosecond pulses), the energy transfer occurs firstly in a superficial layer [Dumitru et al. 2005]. As a surface is exposed to electromagnetic radiation three phenomena occur, a portion of the energy is reflected back (Reflectance $R$ ), the material absorbs another part (Absorbance $A$ ) and the rest is transmitted (Transmittance $T$, generally negligible [Karapatis 2001]) through the material (discussed further in section 3.2.1). The heat generated by the laser will travel through the surrounding powder particles via conduction and if sufficient, will completely liquefy powder particles forming a melt pool, see section 2.2 .2 .

\subsubsection{Sintering Mechanisms}

\subsubsection{Solid state}

When temperatures reach approximately $50-80 \%$ of the absolute melting temperature solid state sintering occurs [Cubberly \& Bakerjian 1989]. This binding of powder particles occurs without the presence of a liquid phase at contact areas along the particles surface to form "necks". This method of binding is rarely used within SFF technologies as diffusion of atoms is slow and not compatible with the desired high laser scan speed that would increase process productivity and economic feasibility [Kruth et al. 2007].

\subsubsection{Liquid phase/partial melting}

Liquid phase sintering involves the melting of material with a lower melting temperature (e.g. polymer binder), while the remaining material with a higher melting temperature remains solid. There is a limited degree of rearrangement of the solid particles under the action of capillary forces caused by melt formation and short interaction time. Therefore metal parts produced using partial melting contain high levels of porosity when directly processed [Rombouts 2006]. Partial melting can occur when no binder is used and only a shell at the grain border is melted with its core remaining solid. The heat supplied to a powder particle is insufficient to melt the whole 
particle [Kruth et al. 2007]. As with liquid phase sintering, the molten shell will form bonds between surrounding particles. Parts produced using liquid phase or partial sintering have a low structural integrity and may require post-processing operations in order to reduce part porosity and improve mechanical properties.

\subsubsection{Full melting}

The production of fully functional parts with high densities in a single step often requires the complete melting of powder particles. This can be achieved with the use of high laser energy densities that can generate temperatures capable of fully liquefying powder particles as shown in Figure 2-3. Once the heat has been removed from the melt pool the molten component solidifies forming a bulk mass with the generation of new grains within its structure. However full melting of material can induce large internal stresses and distortions of a part and induce melt pool instabilities (see section 3.3) resulting in poor surface finish [Aggarangsi \& Beuth 2006; Mercelis \& Kruth 2006; Kruth et al. 2007].

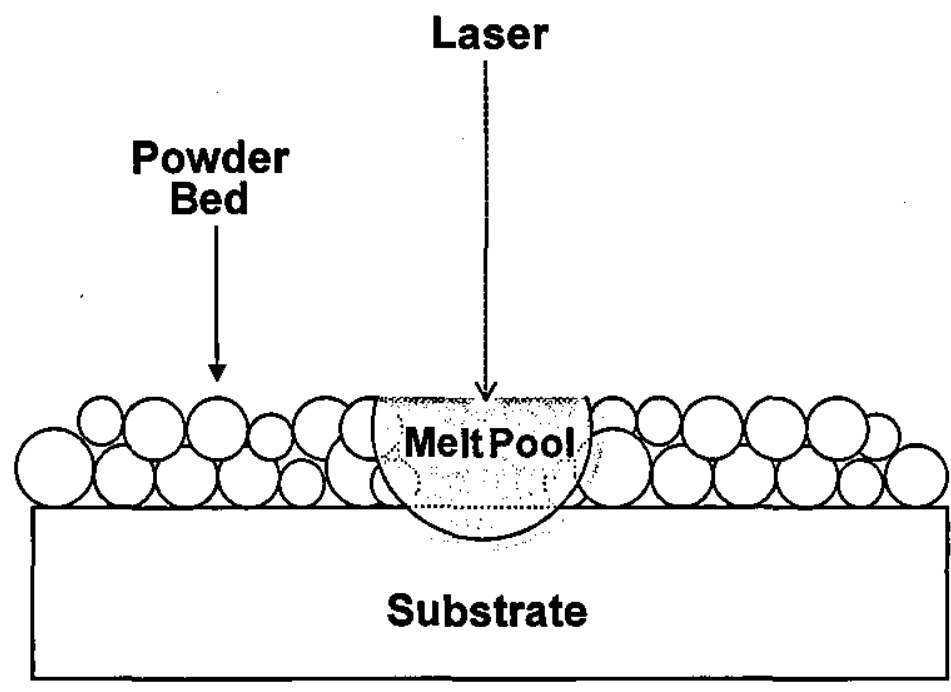

Figure 2-3 Melt pool formation

\subsubsection{Laser Systems}

DMLF processes can vary in regards to system configuration (laser type, preheating of powder bed, etc.). The type of laser used within the system is a significant factor that greatly affects the capabilities of the system. Chapters 2.2.3.1 and 2.2.3.2 will explain 
the differences between the main lasers used within the DMLF industry including the energy distribution profiles and wave interactions modes available.

\subsubsection{Transverse electromagnetic mode}

Three different Transverse Electromagnetic Mode (TEM) radial intensity distributions are shown in Figure 2-4. This describes the energy distribution of the laser relative to distance from the centre of the laser spot profile of the laser. A TEM 00 profile has the main portion of the laser energy distributed within the centre and diminishes further away from the centre. A TEM ${ }_{01}$ profile does not emit any energy from within the centre of the laser, instead it gradually increases and decreases in energy intensity further away from the centre. The TEM 10 profile is similar to $\mathrm{TEM}_{00}$, however it emits small peaks of energy after the first maximum energy point is emitted. The TEM $\mathrm{T}_{00}$ profile is best suited for laser welding and cutting due to high concentrations of energy being emitted within a precise and central area [Kanzler 2006].

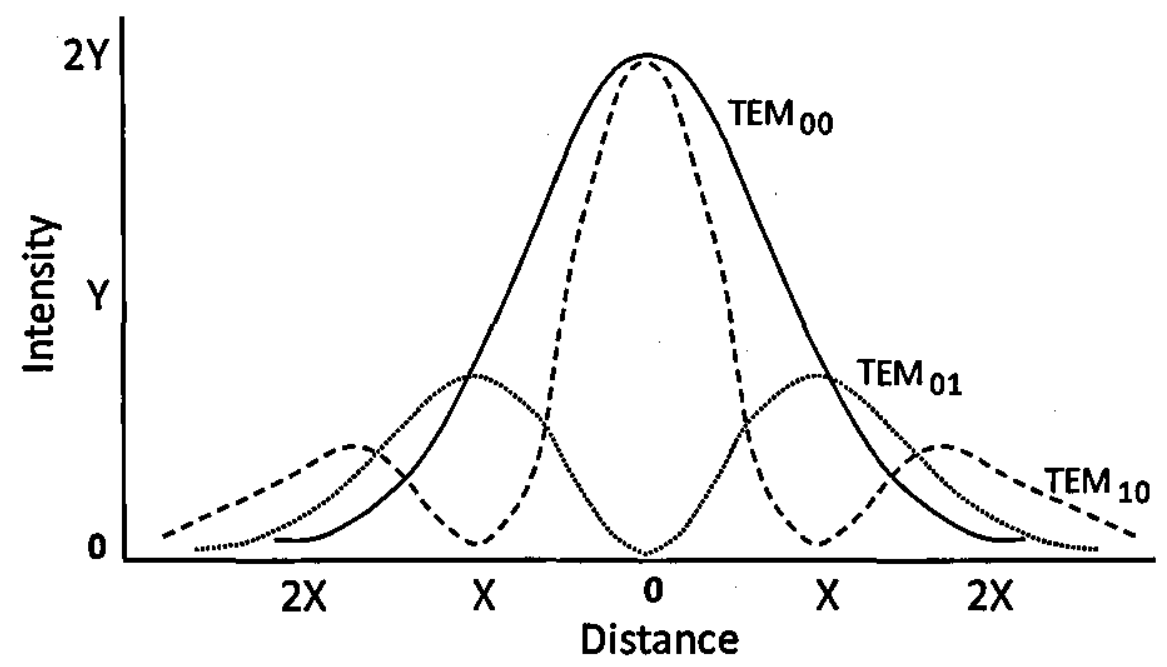

Figure 2-4 Radial intensity distribution of different TEM profiles adapted from [Steen 2003]

\subsubsection{Laser wavelength}

A variety of different laser sources are used within DMLF. Carbon Dioxide lasers $\left(\mathrm{CO}_{2}\right)$ and a Neodymium-Doped Yttrium Aluminium Garnet laser (Nd:YAG) were the most extensively used within laser processing. $\mathrm{CO}_{2}$ lasers operate at a long wavelength of $10.6 \mu \mathrm{m}$ within the Infrared Red (IR) region of the electromagnetic radiation spectrum. 
Nd:YAG lasers also operate within the IR region but at a lower wavelength of $1.06 \mu \mathrm{m}$. At longer wavelengths metallic materials generally have a large reflectivity and consequently the laser processing of metals is more difficult. Laeng et al. [2000] state that it is easier to process metals using lasers with shorter wavelength (Nd:YAG) as the material is able to absorb more energy from the laser. This is because of resonance and scattering effects of the laser's wavelength [Fankhauser \& Kwasniewska 2003]. The $\mathrm{Nd}: Y A G$ laser wavelength of $1.06 \mu \mathrm{m}$ is absorbed fairly well by conductive materials, with a typical reflectance of about $20-30 \%$ for most metals [Kruth et al. 2003]. Table 2-1 shows the typical reflectance and absorbance of metals at different wavelengths. Section 3.2.1 discusses absorption of laser radiation further.

\begin{tabular}{l|ll|ll}
\hline \multirow{2}{*}{ Material } & \multicolumn{2}{|c|}{ (Nd:YAG) $\lambda=\mathbf{1 . 0 6 \mu m}$} & \multicolumn{2}{c}{$\left(\mathbf{C O}_{2}\right) \mathbf{\lambda}=\mathbf{1 0 . 6 \mu m}$} \\
\cline { 2 - 5 } & Reflectance (\%) & Absorbance (\%) & Reflectance (\%) & Absorbance (\%) \\
\hline $\mathrm{Ti}$ & 60 & 40 & 92 & 8 \\
\hline $\mathrm{Co}$ & 73.3 & 26.7 & 95 & 5 \\
\hline $\mathrm{Ni}$ & 74.1 & 25.9 & 97 & 3 \\
\hline $\mathrm{Au}$ & 98 & 2 & 799 & $<1$ \\
\hline
\end{tabular}

Table 2-1 Reflectance and absorbance of metals at different wavelength [Glardon et al. 2001]

A further advantage of an Nd:YAG laser over that of a $\mathrm{CO}_{2}$ laser is the enhanced focusing ability and beam quality. This is because focus ability scales directly with the wavelength of the emitted radiation [Fischer et al. 2005]. The diffraction limited minimum spot diameter, $S_{d m i n}$ to which a Gaussian beam can be focused is given by Equation (2-1).

$$
S_{d \min }=\frac{4 M^{2} f_{l} \lambda}{\pi D_{I}}
$$

$M^{2}$ donates the beam quality factor (beam propagation factor), $f_{l}$ is the focal length of the lens, $\lambda$ is the laser wavelength and $D_{I}$ is the beam diameter at the lens. At comparable beam quality, beam diameter and focal distance values an Nd:YAG laser could be focused to a diameter 10 times smaller than that of a $\mathrm{CO}_{2}$ laser. However at higher laser power $\mathrm{CO}_{2}$ lasers can maintain a higher beam quality $\left(M^{2}\right)$ than Nd:YAG 
lasers due to the influence of defects in the Nd:YAG crystal (thermal lensing) [Karapatis 2001]. An argument for use of $\mathrm{CO}_{2}$ lasers stems from their lower purchasing cost, greater safety and improved efficiency in regards to power usage over that of an $\mathrm{Nd}: Y A G$ laser.

Another type of laser that is becoming popular for use within DMLF processes is the newly developed ytterbium fibre laser. This solid state laser operates at a similar wavelength to that of an Nd:YAG and uses a fibre doped with ytterbium as the active gain medium. Fibre lasers possess a higher beam quality than that of the Nd:YAG due to the fibre's wave guiding properties eliminating thermal distortions along the optical path [Photonics 2008]. Due to improved beam quality this type of laser is being used within the majority of commercial powder bed processes (EOS M270, MTT Realizer, Concept Laser's LaserCusing, etc.), however these fibre lasers can only operate in continuous wave mode and cannot exert pulse shape control. However some fibre lasers can be Qswitched (generating nanosecond pulses which cannot be pulse shaped) but are limited in attaining low peak powers due to the gain per unit length being smaller than that of an Nd:YAG laser [Photonics 2008].

\subsubsection{Continuous or pulsed wave interaction}

Lasers can operate in either continuous or pulsed wave mode. A continuous mode laser has a continuous laser energy output due to continuous excitation of reactive medium where the energy output is nominally constant. In this case the powder can be nearly homogeneously heated and melted [Fischer et al. 2002]. The energy of a pulsed wave laser varies with respect to time and is emitted in short bursts generating high peak powers (with millisecond, nanosecond or femtosecond durations). Pulsing can be achieved by a number of different techniques, such as gain pumping, Q-switching and modelocking. The energy and duration of the pulsed laser can be specified, this pulse can be repeatedly emitted at a set repetition rate $(\mathrm{Hz})$.

In continuous wave lasers the only control parameters required for the formation of

single scans are laser power and laser scan speed, spot size can also be adjusted but this is normally adjusted before a build. This makes the production of single scans and eventual multi-layered parts relatively simple. Newer commercial SLM technologies 
employ continuous wave lasers. The output of a continuous wave laser can be modulated such that laser output can be switched on and off periodically, creating short bursts of laser energy with nominally constant laser energy. The laser exposure time of the continuous wave laser can be set as well as the distance between exposures, dictating the amount of overlap between successive pulse spots. This method of laser energy delivery is carried out such that heat build up and melt pool width is minimised.

Pulsed lasers melt material by a series of discrete events that produce melt pools that are kept molten by the repeatedly emitted laser pulses [Yevko et al. 1998]. As the laser pulses move away from the molten material it cools to form overlapped solidified material. The degree of overlap is directly related to the laser spot overlap generated as each pulse raises the temperature profile of the melt pool. Pulsed lasers generally have more variables and adjustable parameters related to the laser's output (pulse energy, pulse duration, repetition rate) than a continuous wave laser and therefore a wider range of experimental conditions that can be applied with more control over thermal input [Tzeng \& Chen 2001; Laoui et al. 2006]. The use of varied pulse energies and pulse durations allow the user a greater degree of control over the melt pool produced. Pulsed lasers are capable of producing very good metallurgical bonds due to material compaction (recoil pressure) [ONeill et al. 1998] and tend to have a lower heat build-up in the powder and therefore lower heat-affected zone, dilution and tendency to crack than continuous wave lasers [Sun et al. 2005]. Pulsed wave interaction minimises the average power required for consolidation of metallic powder (up to $30 \%$ lower) compared to continuous wave lasers. This leads to a reduction in residual stresses within a part and reduction in the balling mechanism [Tzeng \& Chen 2001; Fischer et al. 2005], discussed further in section 3.3. The use of pulsed wave lasers are more efficient at reducing the defects associated with the balling effect due to higher peak powers suppressing this phenomenon [P. Fischer 2005]. Kruth et al. compared the density and mechanical properties of SLM iron powder when using a laser in continuous and pulsed wave mode. It was found that the use of pulse wave interaction increased the melting effect producing parts with a higher density, suppressed balling formation and improves part strength due to improved inter-layer connection [Kruth et al. 2004]. Pulsed lasers also have the ability to employ pulse shaping control (discussed in chapter 2.3), a feature not available to continuous wave lasers. The negative aspects of pulsed lasers include the potential to generate very high peak powers that can easily vaporise 
material. If pulsed laser settings are not applied correctly the laser may encounter difficulties maintaining a constant melt pool and suffer from hot cracking due to rapid solidification of the melt [Bagger et al. 1993].

Santos et al.[2006] believe that pulsed wave lasers are more suitable for powder bed processing then continuous wave lasers. The researcher draws these conclusions from studying work conducted at the Fraunhofer Institute and Osaka University. It was found that pulsed lasers were able to produce very good metallurgical bonds between tracks and layers with a lower HAZ by the use of high pulse energy and low pulse duration [Morgan et al. 2001; Santos et al. 2006]. This reduction in HAZ was found to reduce a structures tendency to crack [Sun et al. 2005].

\subsubsection{Summary}

Literature suggests that greater control over thermal input and improved metallurgical bonding can be achieved using pulsed Nd:YAG or modulated continuous wave Fibre lasers (pulsed wave mode as they become available) with a $\mathrm{TEM}_{00}$ profile when processing from a powder bed. There is also the potential to use pulse shaping techniques, further improving control over thermal input.

\subsubsection{Overview of Material Deposition Techniques}

\subsubsection{Powder feed deposition}

Within powder feed deposition, powder is injected/blown into the trajectory of a laser from a powder feed nozzle shown in Figure 2-5. The material that is consolidated into the melt pool generated by the laser forms a cladding line. As the laser moves away from the cladding line the molten material is given time to cool and form a solidified structure. For successful part fabrication, it is important to melt the powders and homogenise the melt pool [Lu et al. 2001]. The rate at which powder is fed into the melt pool is also critical. Laeng et al. [2000] state that other issues such as powder particle distribution and injection angle are factors that further complicate this type of material deposition. 


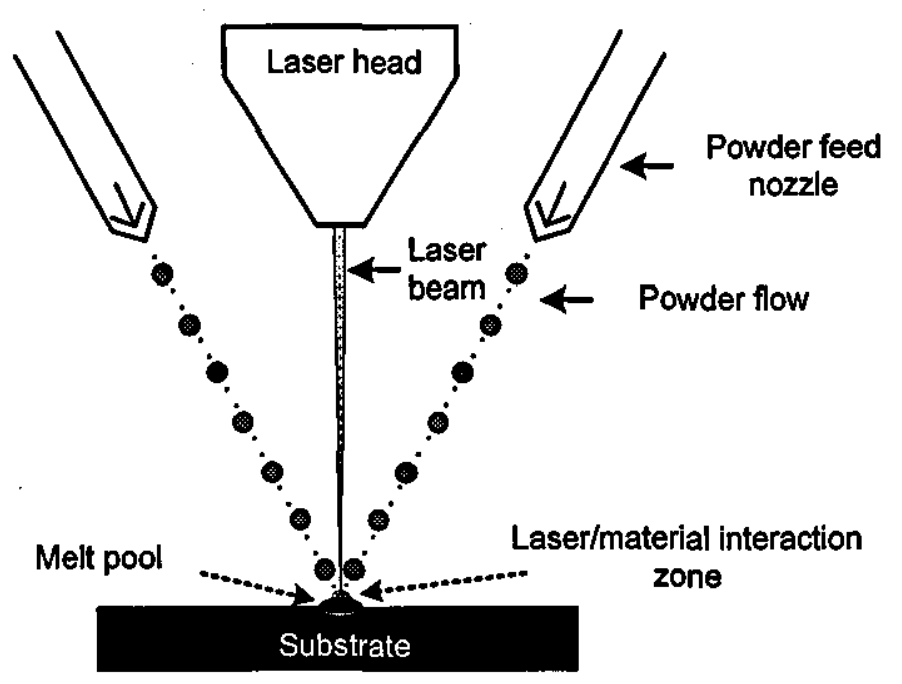

Figure 2-5 Schematic of powder feed deposition during laser processing

Table 2-2 shows the main laser based powder feed processes, the laser, materials processed and their developer (note "CW" and "PW" represent continuous wave and pulsed wave output respectively). Figure 2-6 and Figure 2-7 show parts created using various DMLF powder feed processes. Most of these processes encounter problems associated with high part surface roughness (with the exception of Laser Consolidation due unique split laser delivery and powder angle deposition design). It is known that not all the powder that flows through the nozzle is incorporated into the part. Schlienger $e t$ al.[1998] believe that this is due to some of the powder impinging upon the solidifying material and sticking to the surface due to over spray. This does not cause problems with the bulk of the part but only with the external surfaces and often requires surface machining to complete the part [Rangaswamy et al. 2003]. Another issue associated with this type of material delivery is material wastage and powder stream alignment [Laeng et al. 2000; Allen 2007]. After ejection from the delivery nozzle, powder not consolidated within the melt pool scatters away from the laser. This scattered powder is often difficult to collect and re-use. The scanning strategy within powder feed deposition should remain as continuous as possible without turning powder flow off. This is due to difficulty acquiring an instantaneous steady powder flow rate, this often requires time to regulate. 


\begin{tabular}{|c|c|c|c|}
\hline Process/Product & Developer & Laser & Metals Processed \\
\hline $\begin{array}{l}\text { Laser Engineered } \\
\text { Net Shaping } \\
\text { (LENS) MR-7 } \\
850-\mathrm{R}\end{array}$ & $\begin{array}{l}\text { Optomec, } \\
\text { US }\end{array}$ & $\begin{array}{l}\text { Fibre } 0.5 / 1 / 2 \mathrm{~kW} \\
\text { CW }\end{array}$ & $\begin{array}{l}\text { Ti-6-4, 316ss,Inco., H13 steel } \\
\text { [Blackwell 2005] }\end{array}$ \\
\hline $\begin{array}{l}\text { Direct Metal } \\
\text { Deposition (DMD) } \\
\text { 505/105D }\end{array}$ & $\begin{array}{l}\text { POM, } \\
\text { US }\end{array}$ & $\begin{array}{l}\mathrm{CO}_{2} / \text { Fibre } \\
6 \mathrm{~kW} / 1 \mathrm{~kW} \\
\mathrm{CW}\end{array}$ & $\begin{array}{l}\text { H13, Aluminium, Inco, Ti } \\
\text { [Sears 1999; Wohlers 2008] }\end{array}$ \\
\hline $\begin{array}{l}\text { Laser } \\
\text { Consolidation } \\
\text { (LC) }\end{array}$ & $\begin{array}{l}\text { Accufusion, } \\
\text { Canada }\end{array}$ & $\begin{array}{l}\text { Nd:YAG 500W, } \\
\text { PW }\end{array}$ & $\begin{array}{l}\text { Inco 625/718, Ti-6Al-4V, } \\
\text { 316L SS, Numerous [Xue \& } \\
\text { Islam 2000; Xue 2006] }\end{array}$ \\
\hline $\begin{array}{l}\text { Directed Light } \\
\text { Fabrication (DLF) }\end{array}$ & $\begin{array}{l}\text { Los Almos Nat. } \\
\text { Lab. US }\end{array}$ & $\begin{array}{l}\mathrm{Nd}: Y A G / 2 \mathrm{~kW} \\
\mathrm{CW}\end{array}$ & $\begin{array}{l}\text { Ti-6-4, 316ss,Inconel } 690 \text {, } \\
\text { H13 steel Lewis et al. 1997; } \\
\text { Kobryn et al. 2000] }\end{array}$ \\
\hline
\end{tabular}

Table 2-2 Main DMLF powder feed deposition processes
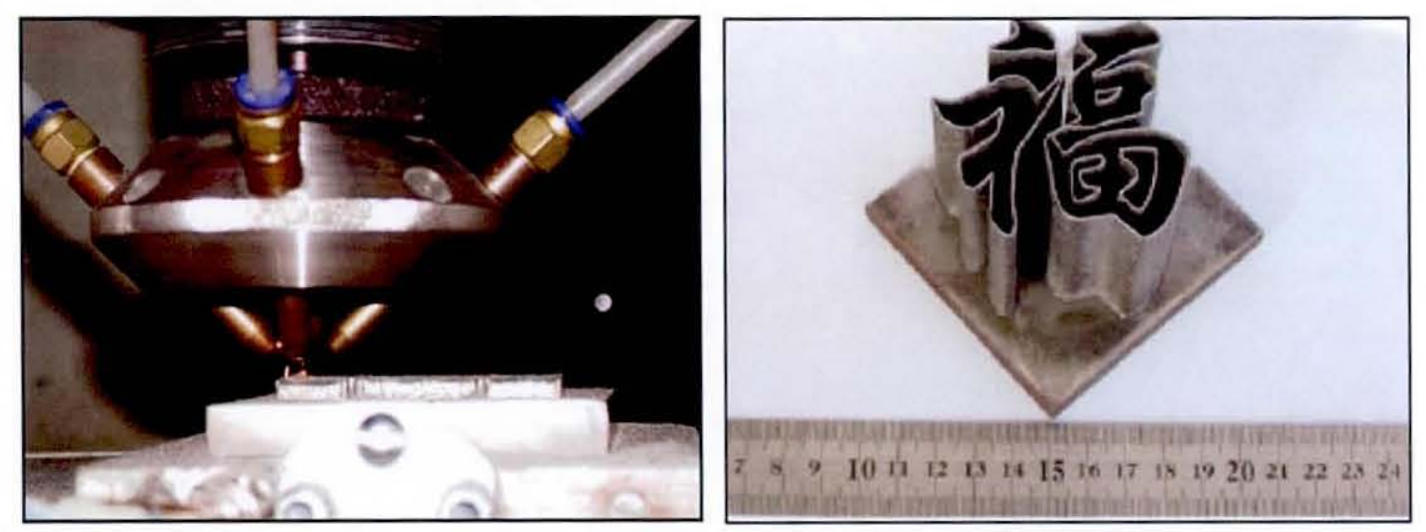

Figure 2-6 DLF process and fabricated nickel part [Peng et al. 2005]
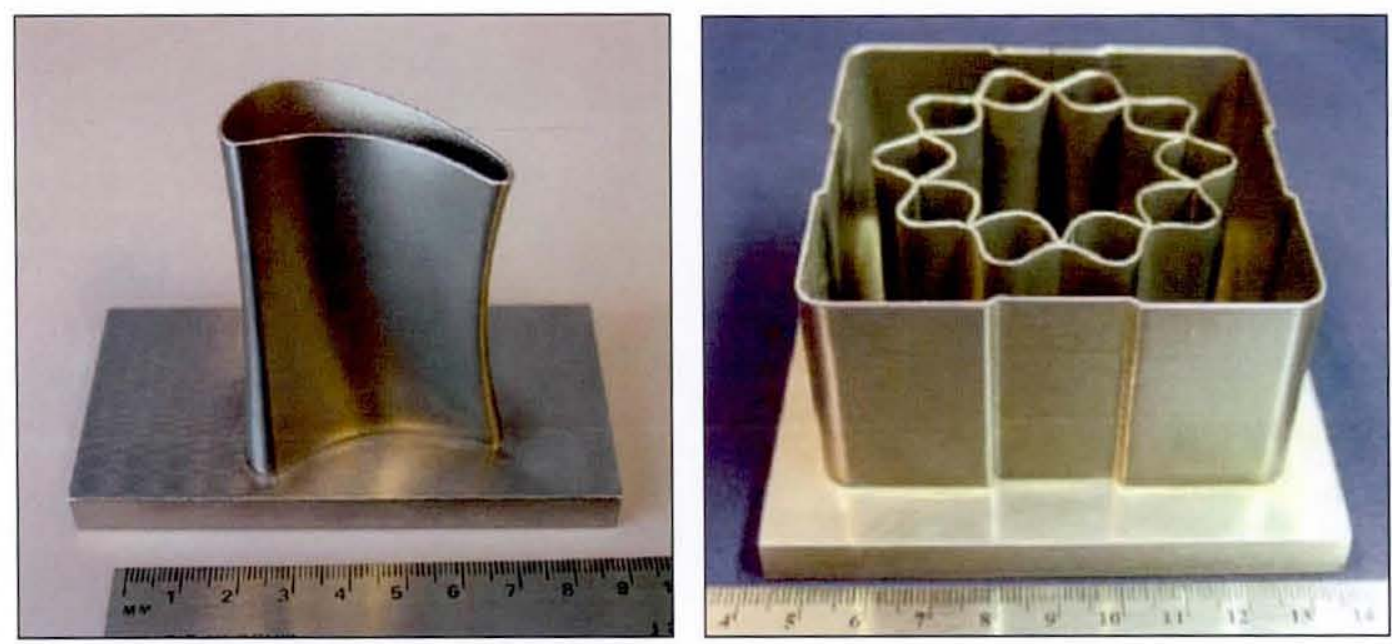

Figure 2-7 Inconel 625 parts produced using Accufusion's Laser Consolidation [Xue 2006] 


\subsubsection{Powder bed deposition}

A thin layer of powder is deposited onto a substrate which is held in place by an adjustable platform. A laser then raster scans the surface of the powder and the heat intensity generated by the laser causes localised bonding between powder particles and the substrate [Santos et al. 2004]. As the laser beam moves away from the melt pool, the molten material is solidified, forming a structure. Once the layer has been scanned the adjustable platform lowers in the z-axis, the next layer of powder is deposited onto the previous layer and is melted by the laser to the previously processed layer. Figure 2.7 shows a schematic of this process. Material particles that are not melted or fused remain loose and are removed once the component is completed. Supports are required to anchor down certain unsupported features due to shrinkage and curling of solidifying material. This restricts the processes' geometric freedom and incurs further post processing operations to remove supports.

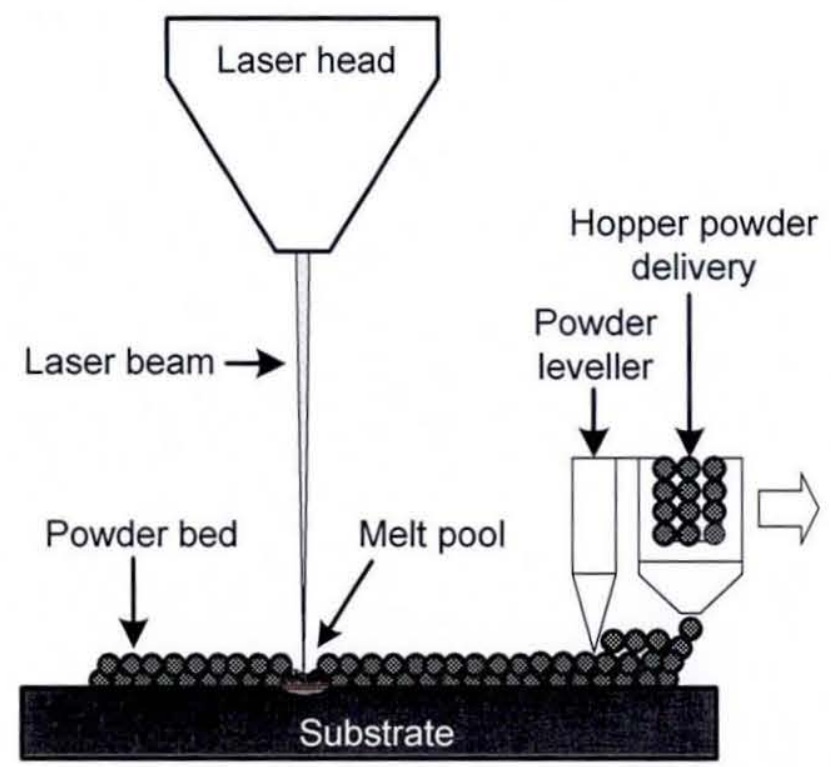

Figure 2-8 DMLF powder bed deposition

Several organisations have developed and commercialised DMLF powder bed processes. They use different terms to describe their processes, including DMLS, SLM and LaserCusing. The main commercial powder bed processes are listed in Table 2-3. All of these processes except 3D systems SLS, can directly fabricate high density parts in a single step, however post processing is often required. In SLS, metal powder is mixed with a plastic binder. The heat generated by the laser melts the plastic, which acts 
as a binder between the metal particles. This is called a "green part" which is then processed in a furnace to remove the binder and sinter the steel particles. At this stage the part is only $60 \%$ dense and is now referred to as a "brown part". This is then infiltrated with another metal such as bronze within further furnace cycle [Rossi et al. 2004]. After the final step the part is fully dense and often requires surface polishing. Other powder bed processes (EOS M270, MTT Realizer, Concept Laser's LaserCUSING etc.) are capable of producing fully dense parts in a single step by completely melting material from a powder bed (layer thickness of 20-100 $\mu$ m). Figure 2-9 and Figure 2-10 show the variety of parts that can be produced using these processes. Parts generally still require surface finishing operations such as polishing, shot peening and support removal. Some parts require furnace cycles to relieve stresses developed within parts during the fabrication process. SLM vendors claim that parts produced using their processes have surface roughness of approximately $10 \mu \mathrm{m}$ before post processing. Powder injection processes have a slightly higher surface roughness compared to powder bed processes (with the exception of Laser Consolidation) [Wohlers 2008].

\begin{tabular}{|c|c|c|c|}
\hline Process/Product & Developer & Laser & Metals Processed \\
\hline $\begin{array}{l}\text { DMLS EOSINT } \\
\text { M250/270 }\end{array}$ & EOS, Germany & $\begin{array}{l}200 \mathrm{~W} \\
\text { Fibre } \\
\mathrm{CW}\end{array}$ & $\begin{array}{l}\text { H13, Ni. Ti64, Co-Cr [Khaing } \\
\text { et al. 2001; Rossi et al. 2004; } \\
\text { Eos 2006] }\end{array}$ \\
\hline $\begin{array}{l}\text { SLM Realizer } \\
100 / 250\end{array}$ & $\begin{array}{l}\text { MTT/MCP, } \\
\text { UK/Germany }\end{array}$ & $\begin{array}{l}100 \mathrm{~W} \\
\text { Fibre } \\
\mathrm{CW}\end{array}$ & $\begin{array}{l}\text { 316, Al-Sil2, Al-Si10-Mg, Ti, } \\
\text { Co-Cr [Wohlers 2008] }\end{array}$ \\
\hline $\begin{array}{l}\text { LaserCUSING } \\
\text { M1,M2 \& M3 }\end{array}$ & $\begin{array}{l}\text { Concept Laser, } \\
\text { Germany }\end{array}$ & $\begin{array}{l}\text { 200W Fibre CW, } \\
\text { 100W Nd:YAG } \\
\text { CW/PW }\end{array}$ & $\begin{array}{l}\text { H13, Co-Cr, Ti-Al6-V4 Inco } \\
\text { 718, Al-Si12, Al-Si10-Mg } \\
\text { [Wohler 2006; Laser 2006] }\end{array}$ \\
\hline $\begin{array}{l}\text { SLM } \\
\text { PM 250/100 }\end{array}$ & $\begin{array}{l}\text { Phenix Systems, } \\
\text { France }\end{array}$ & $\begin{array}{l}200 / 50 \mathrm{~W} \\
\text { Fibre } \\
\mathrm{CW}\end{array}$ & $\begin{array}{l}\text { H13, Inco625, Co-Cr } \\
\text { [Yadroitsev et al. 2007; } \\
\text { Systems 2008] }\end{array}$ \\
\hline $\begin{array}{l}\text { SLM LUMEX } \\
25 C \text { (possibly } \\
\text { discontinued) }\end{array}$ & Matsuura, Japan & $\begin{array}{l}500 \mathrm{~W} \\
\mathrm{CO}_{2} \\
\mathrm{PW}\end{array}$ & [Santos et al. 2006] \\
\hline $\begin{array}{l}\text { SLM TrumaForm } \\
\text { LF250 } \\
\text { (discontinued) }\end{array}$ & $\begin{array}{l}\text { Trumpf, } \\
\text { Germany }\end{array}$ & $\begin{array}{l}250 \mathrm{~W} \\
\text { Disk laser } \\
\mathrm{CW}\end{array}$ & [Santos et al. 2006] \\
\hline
\end{tabular}

Table 2-3 Commercial direct DMLF powder bed processes 

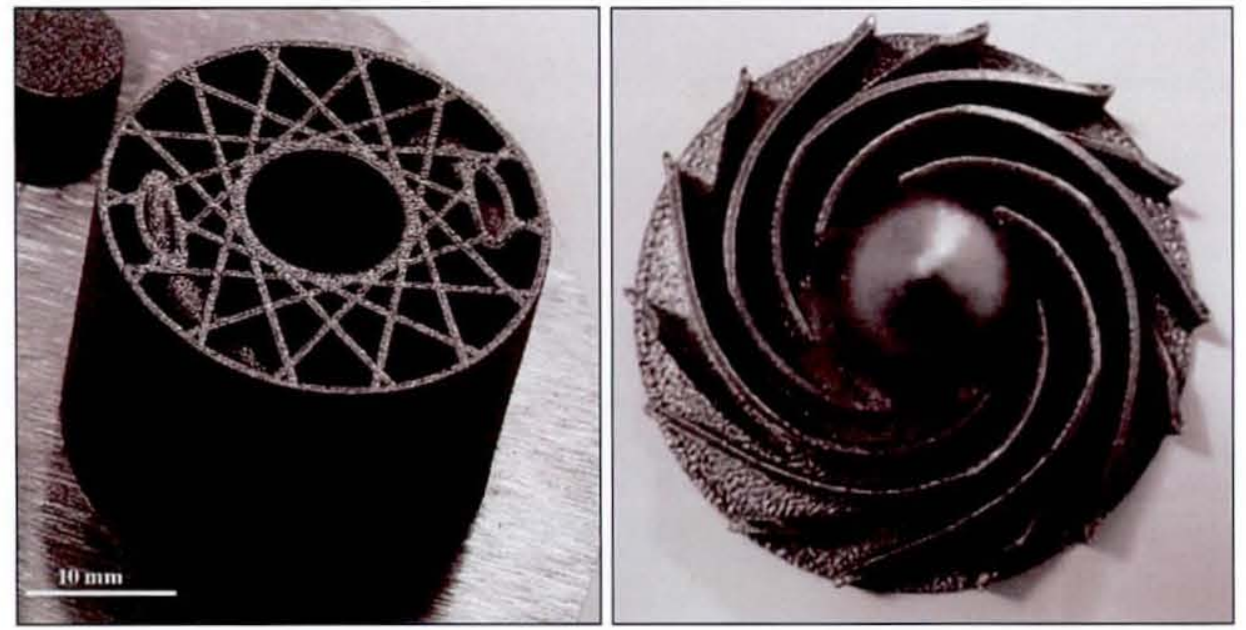

Figure 2-9 Inconel 625 parts produced using Phenix's PM250 system [Yadroitsev et al. 2007] \& EOS M270 [Shellabear \& Nirhylä 2004]
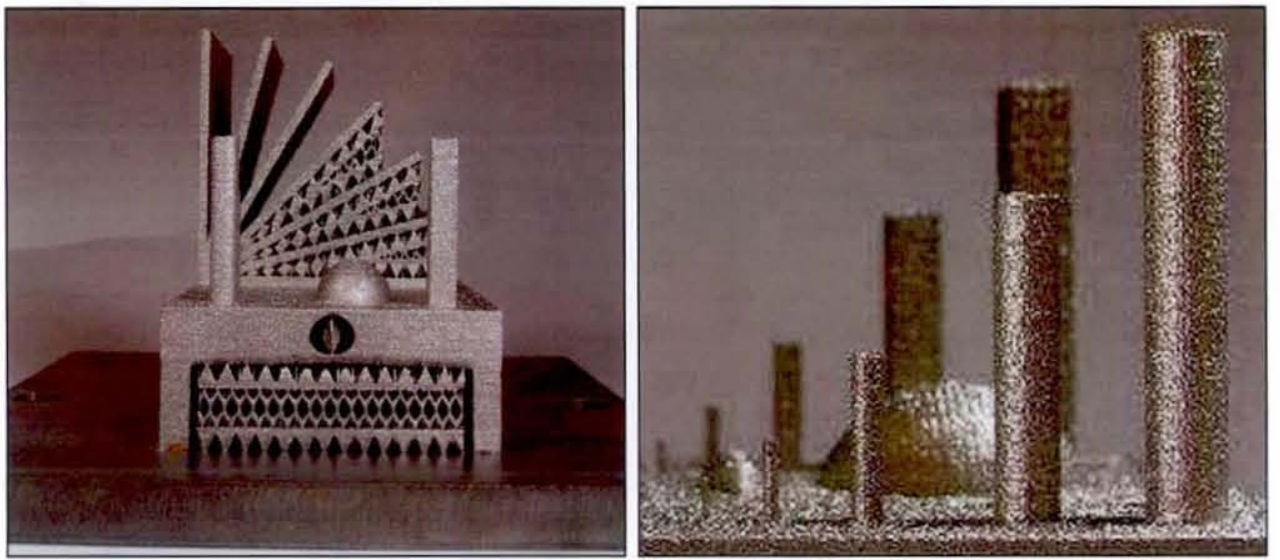

Figure 2-10 Benchmark model made from 316 stainless steel using MTT Realizer [Castillo 2005]

\subsubsection{Wire feed deposition}

This process is similar to laser welding and is best suited for surface treatment operations. Wire is fed directly under the path of a laser. The wire feed nozzle is positioned close to the melt pool to ensure accurate feeding of the material to the melt area. This method of material deposition is less frequently used to produce $3 \mathrm{D}$ metal parts. The laser spot size often has to be larger than the wire's diameter in order to successfully melt the material. 


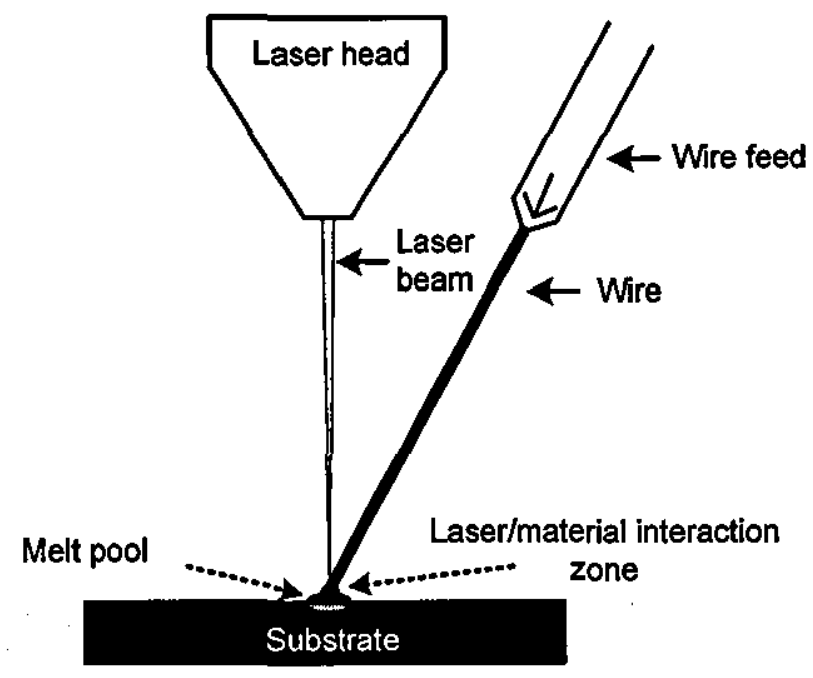

Figure 2-11 DMLF wire feed deposition

\subsubsection{Summary}

The technique selected for material deposition within DMLF is crucial as it can determine material utilisation efficiency, dictate the capabilities and processing efficiency of a system and ultimately the quality of parts. Table 2-4 lists the main advantages and disadvantages of wire feed, powder feed and powder bed deposition.

Wire feed and powder feed can be used for repair operations of components, however wire feed deposition is not often used for the production of 3D parts. Powder bed processing cannot be considered for part repair operations, however this type of powder deposition has advantages over powder fed deposition. These advantages include a higher material utilisation and avoidance of complications associated with powder flow during powder feed deposition. These complications include maintaining consistent powder feed rate and maintaining constant scanning strategy. Powder bed processes do not incur problems related to increased surface roughness due to over spray of powder impinging on the surface of the solidifying molten pool. Powder bed deposition also allows for the production of overhung geometries, which is something difficult to produce with powder fed deposition. 


\section{Wire Feed Processing}

Advantages
- Good at cladding rotationally symmetric
shapes[Schneider 1998]
- Material losses are negligible compared to
other powder material deposition
techniques[Laeng et al. 2000 ]
- $20-30 \%$ improvement in part surface
roughness compared to powder processed
samples[Syed et al. 2005 ]

\section{Disadvantages}

- Wire delivery and laser beam conflicts, wire partially shields laser from melt pool therefore requiring a larger beam spot of at least three times the wire diameter.

- Other powder deposition techniques generally have better control over melt pool compared to wire feed deposition samples[Syed et al. 2005

Powder Feed Processing

Advantages

- Blending different mixtures of material [Griffith ML et al. 1997; Hu \& Kovacevic 2003]

- Online variation of powder flow rate [Laeng et al. 2000]

- Can be used for part repair operations

- Large variety of materials available

- High deposition rate capability

\section{Disadvantages}

- Does not have an inherent support structure as is available in powder bed processing.

- Requires accurate delivery of powder flow rate, Material utilisation is between $30-80 \%$, [Ashley 1995; Sears 1999; Laeng et al. 2000]

- Difficult to maintain low surface roughness (apart from LC process) [Schlienger $e t$ al. 1998; Su 2002; Williams C.B et al. 2005]

- Not good for overhand surfaces [Allen 2007]

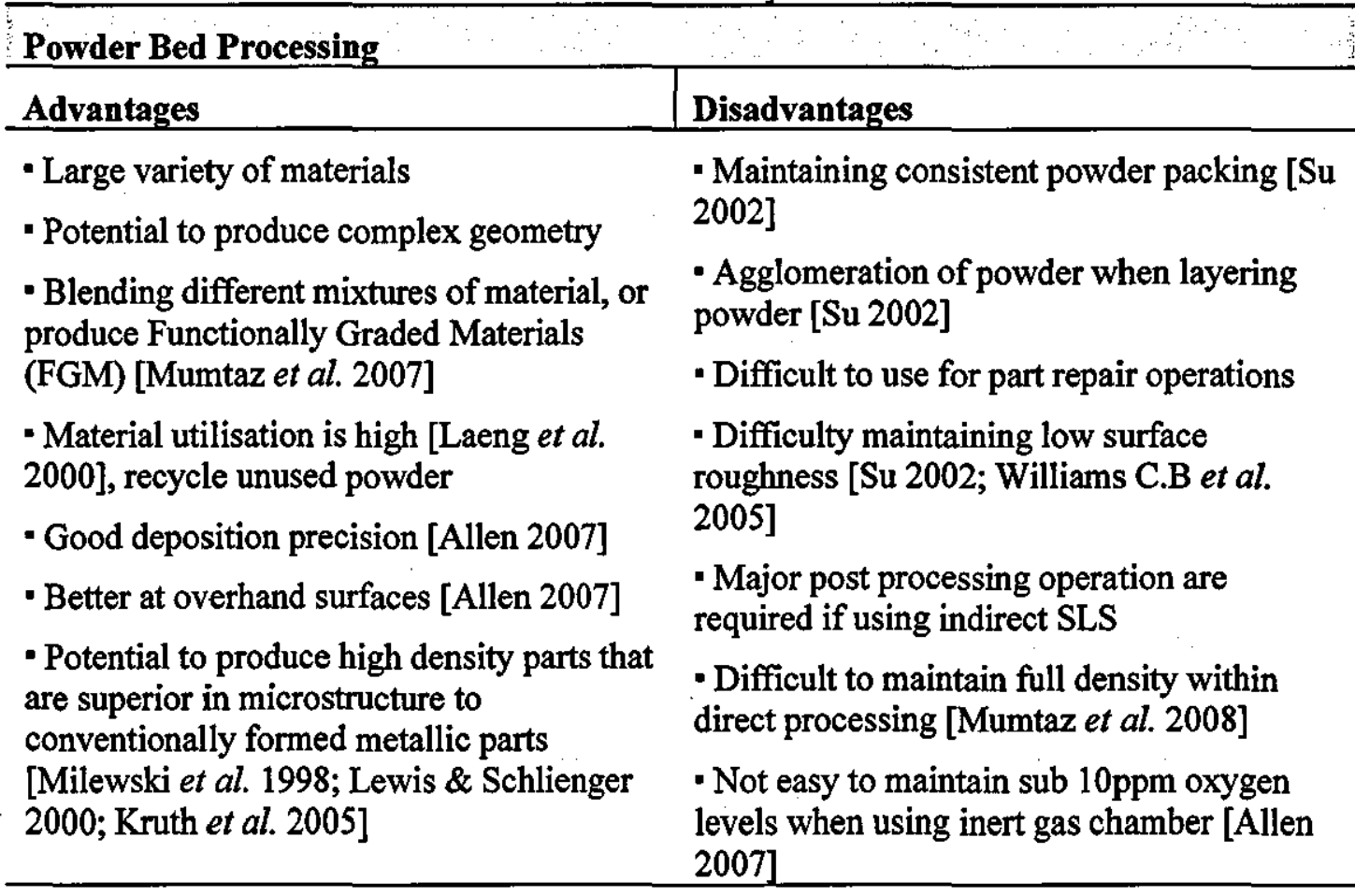

Table 2-4 Advantages and disadvantages of various DMLF material deposition techniques 


\subsection{Pulse Shaping}

A pulsed laser emits bursts of energy that consist of a fixed amount of energy for a specified duration. Pulse shaping is a technique used to strategically distribute energy within a single laser pulse. It can also be defined as a variation in power supplied to a laser to change the shape of the output pulse and subsequently the heat distribution within the pulse [Kanzler 2006 ]. Figure 2-12 shows a schematic of a typical Nd:YAG laser system and the method for pulsed laser generation. Pulse shaping can be accomplished by dividing the pulse's current used to excite a laser's flash lamp into as many as 20 individual sectors and specifying the duration and peak power of each sector. The current supplied by the electric power supply causes the flashlamps to emit light that is absorbed and amplified by the Nd:YAG crystal. This amplified light is emitted in short bursts/pulses through various focusing lenses and eventually onto a work piece. Therefore the electric power supply is one of the main factors that dictate the characteristics of a laser pulse.

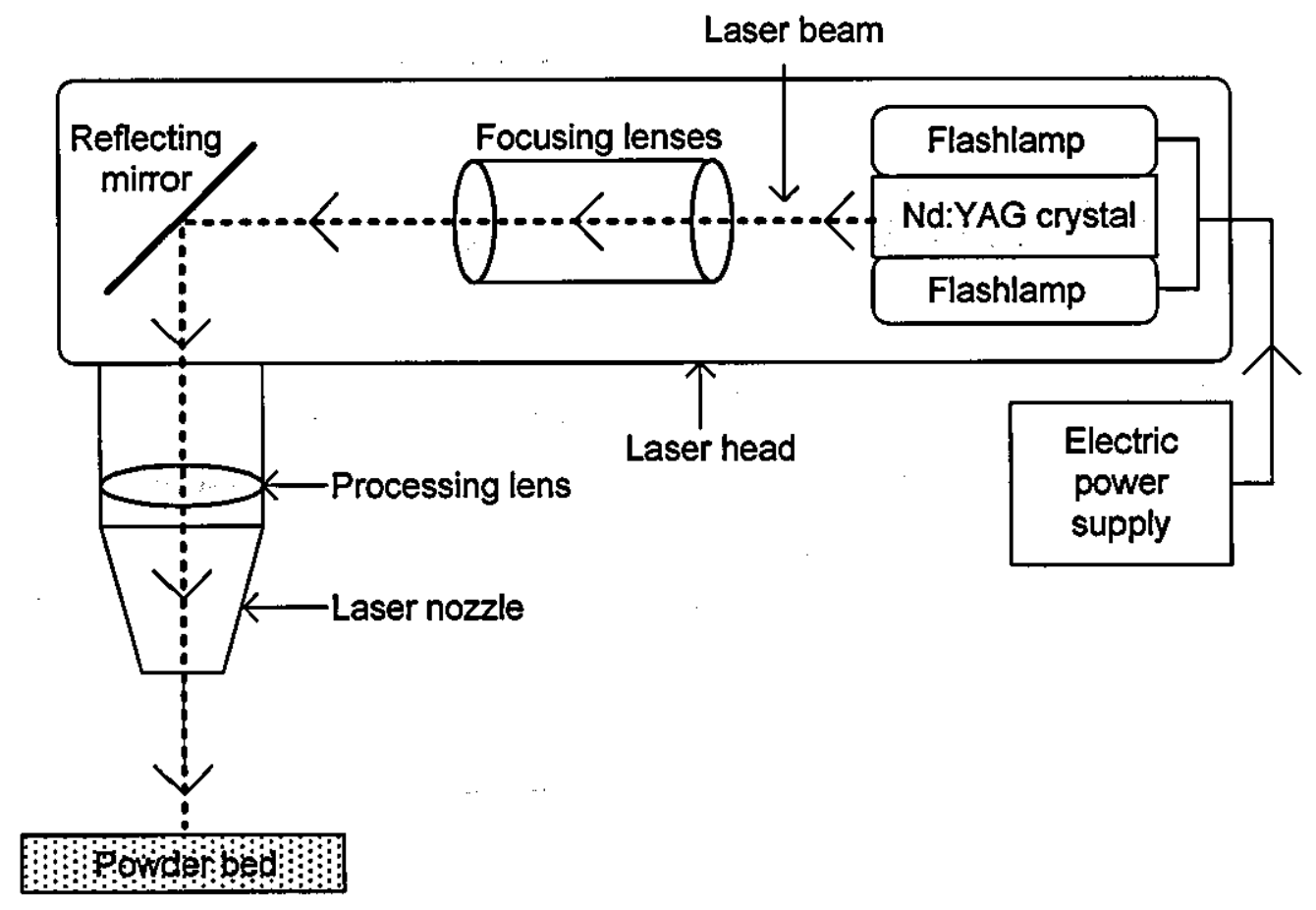

Figure 2-12 Generation of pulses of light using an Nd:YAG laser 


\subsubsection{Standard Laser Pulse}

Figure 2-13 (a) shows an example of a standard pulse's demand profile. This is known as a Rectangular pulse shape or "top hat" pulse shape with power densities remaining nominally constant throughout the input pulse. Rectangular pulse shapes are the simplest and most extensively used laser pulse shape within academia and industry containing only one energy sector. This pulse shape is fed to the flashlamps but due to the filter effect a more realistic output profile of the laser is represented in Figure 2-13 (b). With most solid state lasers such as the Nd:YAG, the use of a Rectangular pulse shape input creates a power overshoot that can be between $75-100 \%$ over the allocated peak power for the first $0.7-1.1 \mathrm{~ms}$ of the pulse and comprises of $2-5 \%$ of the pulse energy [Bransch et al. 1994]. The extent of the overshoot varies according to the laser parameters. This overshoot can be beneficial in regards to increased penetration in laser drilling [Cheng et al. 2000], however an over usage of energy within a very short space of time can increase material vaporisation and spatter generation during laser processing of metal [Petrov et al. 1992]. After the overshoot the laser power delivery normalises to its steady state value for the majority of the pulse's duration and is then followed by an abrupt loss in power that results in rapid cooling of the processed material. This laser output shape will characteristically remain the same even when using different pulse durations and pulse energies. The only variation will be with the scaled size of this pulse shape as different pulse energy/duration combinations are set.
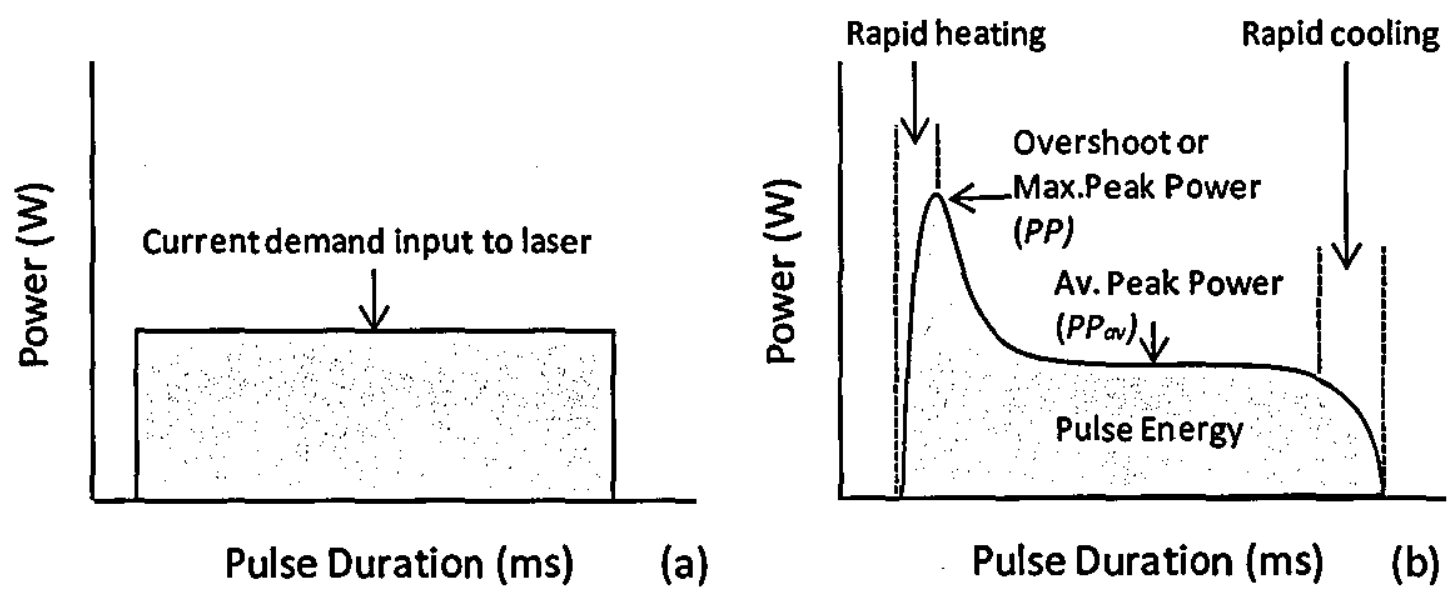

Figure 2-13 Standard Rectangular pulse shape supplied as a current input to laser (a) \& laser's actual output (b) 
The use of a Rectangular pulse shape results in rapid heating (with a large degree of power overshoot) and rapid cooling of material towards the end of the pulse's duration due to an abrupt termination of laser power. Katayama et al. [1993] believes that the use of standard Rectangular pulses within laser welding is a main contributor to porosity within a part. This is because a melt pool under certain conditions may drastically collapse due to the rapid reduction in power delivered by Rectangular pulses. The upper part of the melt solidifies to prevent gas bubbles from flowing up leaving a pore in the weld fusion zone (see section 2.3.4.1). Another disadvantageous phenomenon that can occur as a result of rapid cooling brought on by the use of Rectangular pulse shapes is hot cracking (see section 3.6.2), something that commonly occurs using pulsed lasers to process metal due to the melt pool high solidification rates [Bagger et al. 1993; Shannon $\&$ Li 2004].

Using simple, one sector Rectangular pulse shapes serves a purpose for many applications, however studies have shown that the effects of altering pulse shapes within laser metal processing has proven to be beneficial, particularly within laser welding of metal sheets [Weedon 1987; Badawi et al. 1989; Petrov et al. 1992; Katayama et al. 1993; Bransch et al. 1994]. These benefits include improved control over molten material solidification, subsequently reducing porosity formation. Pulse shaping can decrease cooling rates and subsequently reduce solidification cracking in crack sensitive material [Katayama et al. 1993]. Section 2.3.4 discusses pulse shaping advantages in more detail.

\subsubsection{Improved Control over Laser Energy using Pulse Shaping}

\subsubsection{Laser energy distribution}

Without the use of pulse shaping control, only a pulse's energy and pulse duration can be controlled. As a result of the pulse energy/duration selection the average peak power $P P_{a v}$ can be calculated. The degree of overshoot or maximum peak power $P P$ is unknown unless the pulse's actual output is measured using a power meter and digital oscilloscope (see section 7.1.1). Even when the maximum peak power is monitored it cannot be effectively controlled without pulse shape control and tends to intensify in 
value with increases in pulse repetition rate and pulse energy for a fixed duration [Lumonics 1995].

Figure 2-14 shows an example of how the use of pulse shaping extends the control over a non pulse shaped laser pulse. The dashed line is the average peak power/pulse energy range for a standard $1 \mathrm{~ms}$ pulse. Along the chart $1 \mathrm{~J}$ of energy will automatically produce an average peak power of $1000 \mathrm{~W}$ (see equation (3-2) in section 3.1.1). A $1 \mathrm{~ms}$ standard pulse with an energy of $1 \mathrm{~J}$ will always produce a peak power of $1000 \mathrm{~W}$. The average peak power pulse energy relationship is standardised with the use of standard Rectangular pulses, its relationship can be calculated by dividing the pulse's energy by its duration. According to this relationship it is not possible to produce a $1 \mathrm{~ms}$ pulse with an average peak power of $1000 \mathrm{~W}$ containing $0.5 \mathrm{~J}$ pulse energy. In order to achieve this average peak power/pulse energy combination the pulse duration would have to reduce to $0.5 \mathrm{~ms}$ in order to satisfy this relationship. If the pulse duration would remain constant at $1 \mathrm{~ms}$ with $0.5 \mathrm{~J}$ of pulse energy this would produce an average peak power of $500 \mathrm{~W}$ (see Figure 2-14). This fixed relationship between the pulse's average peak power, pulse energy and pulse duration can be seen to restrict the process. It may be deemed more suitable to process a material with a $1 \mathrm{~ms}$ pulse duration at a high average peak power (2000W) and low pulse energy (1J). However this combination is not physically possible using standard laser pulses. The use of pulse shaping can remove certain restrictions related to laser pulse energy allowing altered power/energy combination. An example of the extended control (approximated) possible using pulse shaping for a $1 \mathrm{~ms}$ pulse is represented by the shaded area in Figure 2-14. 


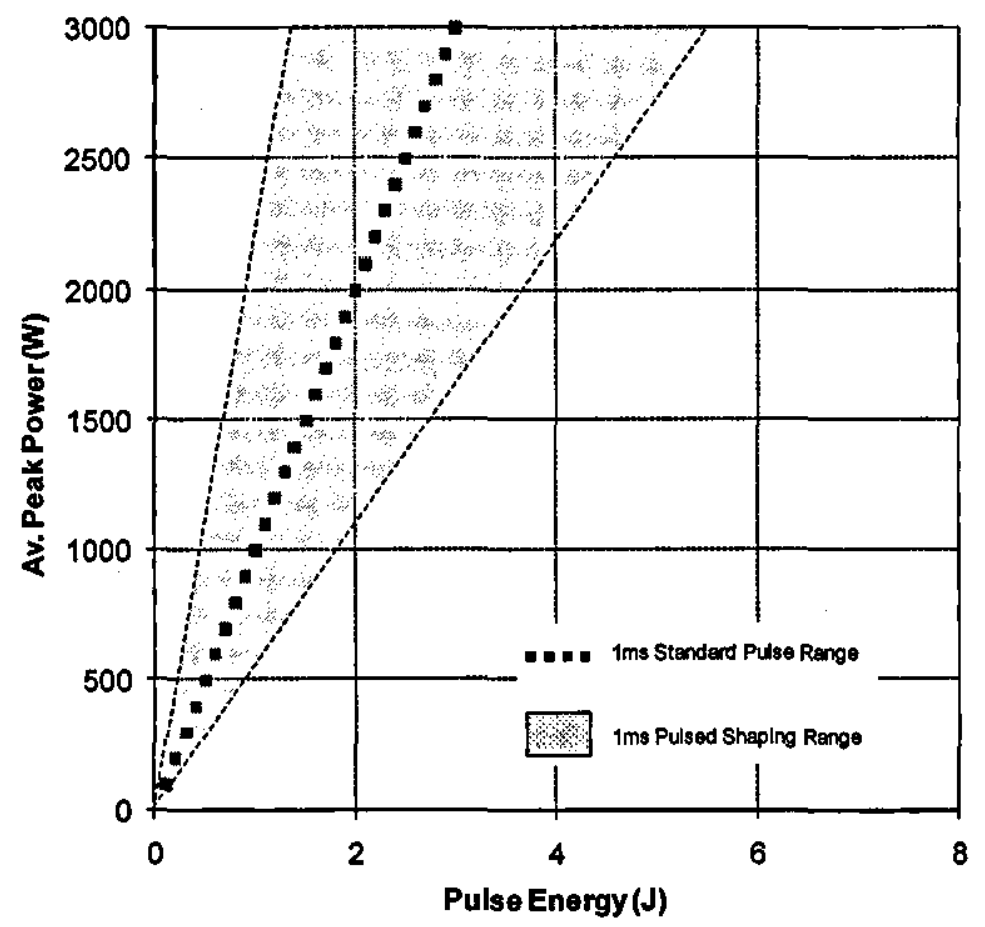

Figure 2-14 Approximate extended variations in average peak power and pulse energy capable using pulse shaping

Figure 2-15 (a) shows the output that would expected from a standard Rectangular 1ms laser pulse with $1 \mathrm{~J}$ of energy. Figure 2-15 (b) shows how the fixed relationship between pulse energy/duration and peak power can be modified using pulse shaping to produce a $1 \mathrm{~ms}$ laser pulse with a lower peak power, higher average peak power while a similar pulse energy and duration is maintained.
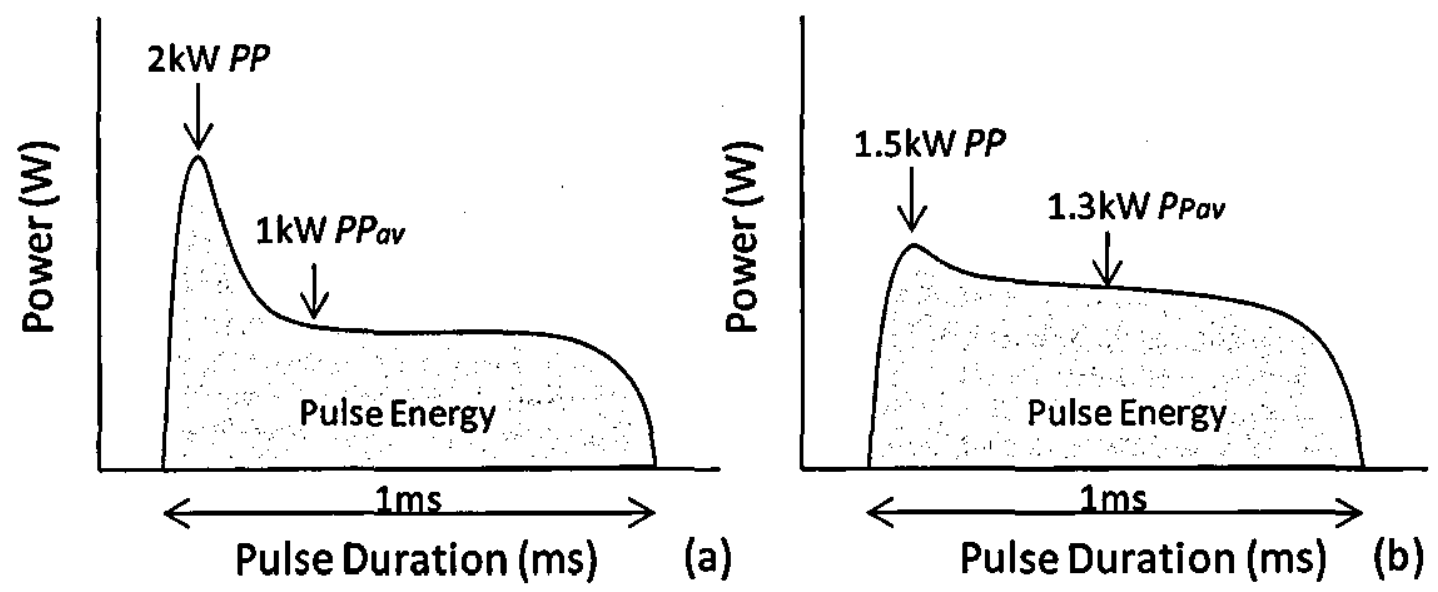

Figure 2-15 Laser output from standard laser pulse (a) \& modified pulse shaped laser output (b) 


\subsubsection{Further degrees of control}

Continuous wave lasers such as the ones used within MTT Realizer and EOS M270 have two main control parameters that relate to the laser (laser power and scan speed). However it is possible to edit the system's data file and adjust the laser's exposure time and time between each laser exposure to get a pulsing effect. The energy distribution within the single laser exposure would remain constant. By adjusting the laser exposure time, time between each exposure and scan speed a variable spot overlap can be set. The main benefit of operating a continuous wave laser in a pulse like mode would be to reduce heat build up within the powder bed. The majority of pulsed lasers have as standard four control factors, pulse duration, pulse energy, repetition rate and scan speed. A pulse may be split into a minimum of two sectors giving an extra pulse duration within the pulse to control and a second pulse sector height giving a minimum of six control factors. The further degree of control capable using pulse shaping is illustrated in Table 2-5. The pulse height for each sector controls the current delivered to each sector and combined will control the total pulse energy. The splitting of sectors and setting height control for pulse shaping are explained in more detail in section 7.1. As mentioned earlier in section 2.3 the pulse has the potential be split into a maximum of 20 sectors (only with pulse durations of $10 \mathrm{~ms}$ and higher with a minimum $0.5 \mathrm{~ms}$ sector duration) giving a total of 46 control parameters. This translates to more control being given over the energy/heat distribution with each laser pulse.

\begin{tabular}{|c|c|c|}
\hline Type of laser system & Control Parameters & Total No. of Control \\
\hline Continuous wave laser & $\begin{array}{l}\text { - Laser power } \\
\text { - Scan speed } \\
\text { - (Laser exposure time) } \\
\text { - (Time between exposure) }\end{array}$ & $2(4)$ \\
\hline Pulsed wave laser & $\begin{array}{l}\text { - Pulse duration } \\
\text { - Pulse energy } \\
\text { - Repetition rate } \\
\text { - Scan speed } \\
\end{array}$ & 4 \\
\hline $\begin{array}{l}\text { Pulsed wave laser with pulse } \\
\text { shaping control }\end{array}$ & $\begin{array}{l}\text { - Pulse duration sector } 1 \\
\text { - Pulse duration sector } 2 \\
\text { - Pulse height sector } 1 \\
\text { - Pulse height sector } 2 \\
\text { - Repetition rate } \\
\text { - Scan speed }\end{array}$ & $\begin{array}{l}6 \text { (minimum) } \\
\text { pulse can be split into a } \\
\text { maximum of } 20 \text { sectors } \\
\text { allowing a possible } 46 \\
\text { control parameters }\end{array}$ \\
\hline
\end{tabular}

Table 2-5 Total number of control parameters for continuous and pulse wave lasers 


\subsubsection{Types of Pulse Shapes}

Pulse shaping investigations have focused on the use and creation of three basic pulse shapes. The first is the standard Rectangular pulse shape previously shown in Figure 2-13(a). This is often used as a reference or benchmark to make future comparisons against when using variable pulse shapes. The other two modified pulse shapes are known as Ramp Up and Ramp Down pulse shapes. Figure 2-16(a) and (b) show an example of a typical Ramp Up and Ramp Down pulse shape when it's current demand input and output profile are viewed. As the names suggest these pulses either emit the majority of their power early or late within the pulse. This equates to either a gradual heating or gradual cooling of material. The majority of pulse shaping investigations have focused on using these pulse shapes when laser welding and drilling metal sheets. However pulse shapes can also be adjusted such that complex heating distributions are applied as shown in Figure 2-17.

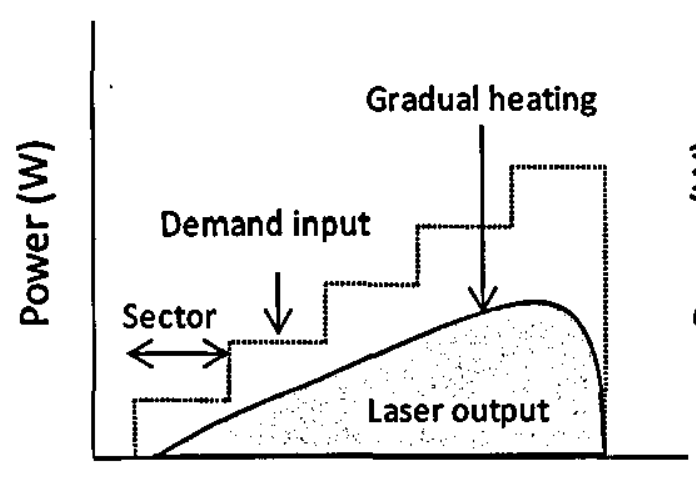

Pulse Duration (ms)

(a)

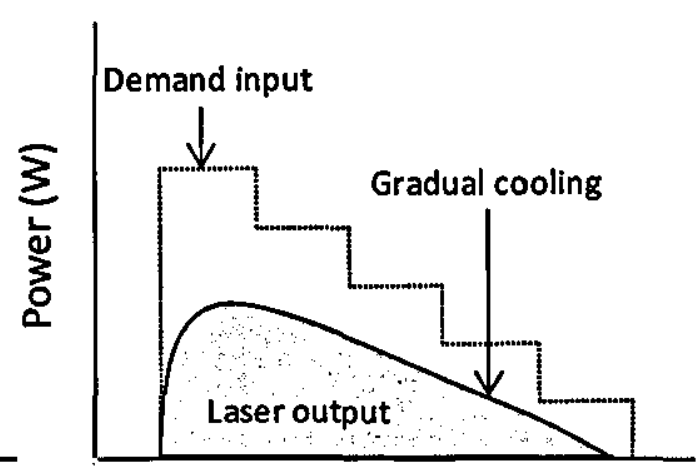

Pulse Duration (ms)

(b)

Figure 2-16 Ramp Up (a) and Ramped Down (b) pulse shapes

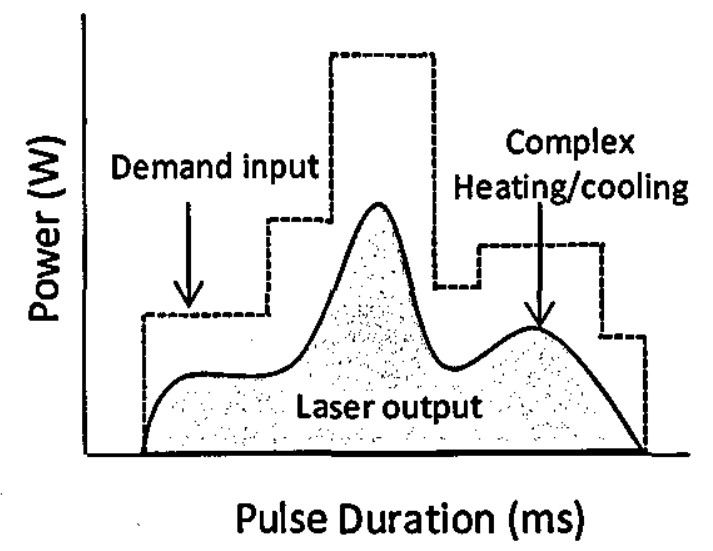

Figure 2-17 A complex/unconventional pulse shape 


\subsubsection{Pulse Shaping Investigations}

Pulse shaping investigations have mainly focused on the laser welding of metal sheets. However the use of pulse shaping has also covered laser drilling/cutting and very limited work on processing metal powders using powder feed processes.

\subsubsection{Laser welding of metal sheets}

Bransch et al.[1994] used a Lumonics JK702 Nd:YAG pulsed laser to produce spot welds on stainless steel sheets. The researchers examined the effects of different pulse shapes on the weld dimension and quality. It was found that porosity within the weld was at its lowest when Ramp Down pulses were used compared to Ramp Up pulses. The welds produced with Ramp Up pulses contained a higher level of porosity due to the abrupt finish of high power at the end of the pulse. This abrupt end caused the vapour cavities within the melt pool to collapse. The molten metal from the outer edge of the HAZ was drawn in by surface tension forces faster than the metal could flow back into the cavity, producing a gas pore. Katayama et al.[1993] welded stainless steel sheets using an Nd:YAG laser, the pulse shaping technique was used to reduce the defects found within welds. Figure 2-18 shows the pulse shape used within the investigation, a gradual increase in laser power (Ramp Up) was introduced before the main pulse. It was found that it reduced the amount of ejected molten material (spatter) generated during the welding process. The pulse also incorporated a tailing power (Ramp Down) that was found to delay the solidification process and reduced the amount of cracking and porosity within the weld [Weedon 1987; Watanabe et al. 1998; Gower et al. 2005]. Figure 2-19 shows the location of gas occluded porosity found within the steel welds as Ramp Down pulses were increased in time duration. The gas occluded porosity travels further up the weld as the solidification time is increased, eventually escaping the melt after a pulse duration of $16 \mathrm{~ms}$. 


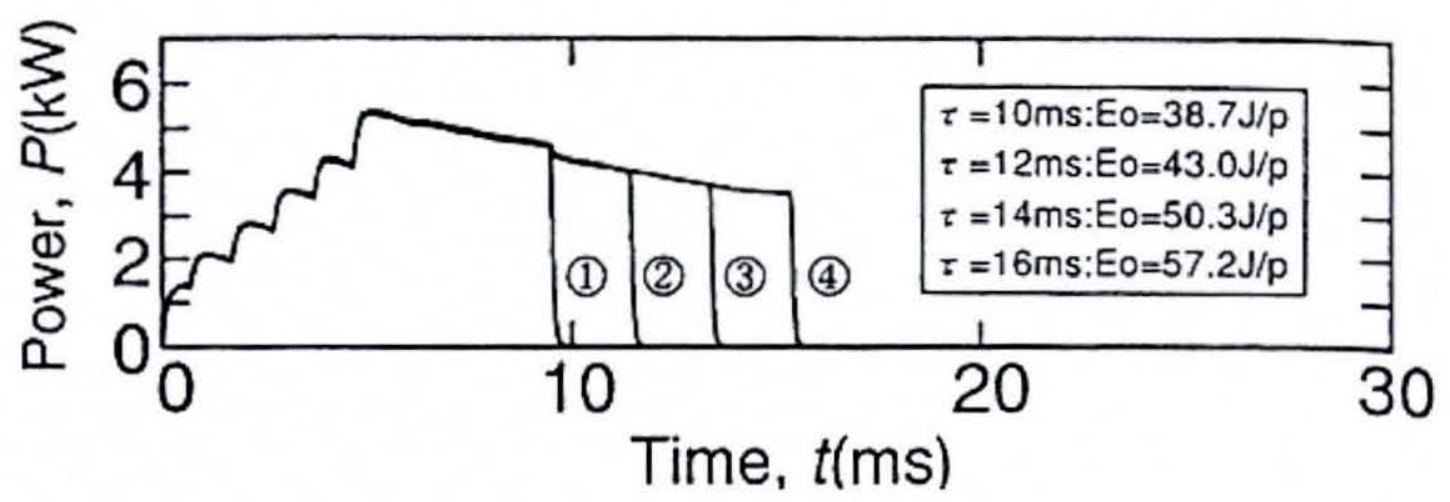

Figure 2-18 Ramp Up and Ramp Down pulse shapes combined to reduce material spatter \& delay solidification [Katayama et al. 1993]
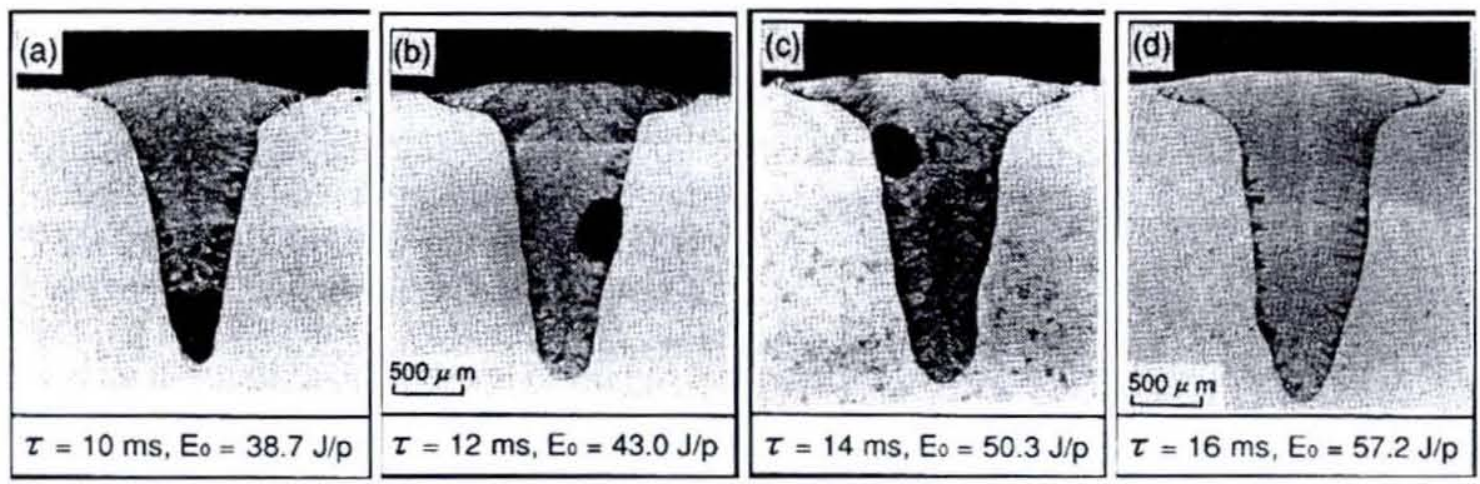

Figure 2-19 Removal of gas porosity with increased Ramp Down pulse duration [Katayama et al. 1993]

Badawi et al. [1989] welded sheets of stainless steel and aluminium using a $\mathrm{CO}_{2}$ laser, dividing pulses into two power sectors. It was found that the penetration and melt area of welds increased as the period of maximum power density shifted from the beginning to the end of the pulse (Ramp Up). This was due to the highest power density occurring at the end of the pulse when the absorbance of the material was at its highest. This is in contrast to a Ramp Down pulse, which emit their highest energy density at the beginning of the pulse when a metal's energy absorbance is at its lowest.

Gower et al.[2005] used a pulsed Nd:YAG laser to produce a series of spot welds on aluminium alloys. Welds were produced using Rectangular, Ramp Up and Ramp Down pulse shapes, these are shown in Figure 2-20(a). It was found that use of Ramped pulse shapes whether with increasing or decreasing power increased both the weld diameter 
and crater size compared to standard Rectangular pulses. Figure 2-20(b) shows welds produced using Ramp Up and Ramp Down pulse shapes with shorter pulse durations. Ramp Down pulses vaporised some of the oxide layers present, leaving a smoother weld. Ramp Up pulses removed all traces of oxide layers but caused a violent expulsion of molten metal, creating a very deep crater. $100 \%$ of welds formed using Ramp Up pulses contained signs of cracking.

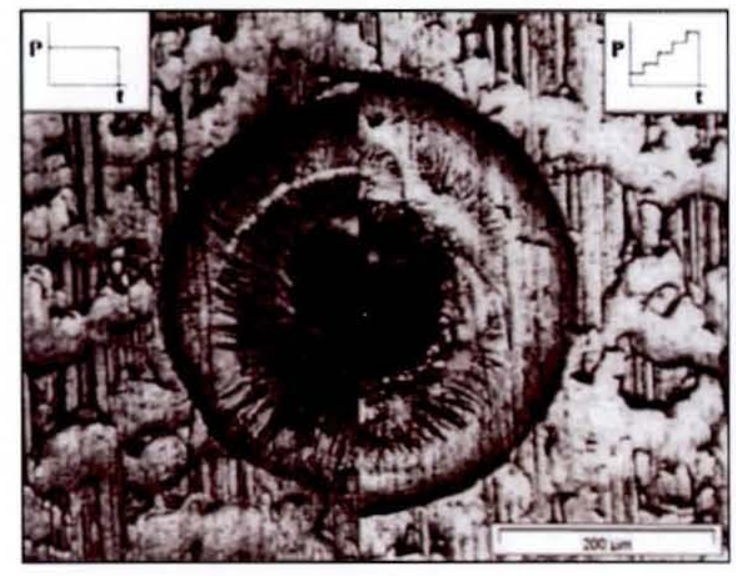

(a)

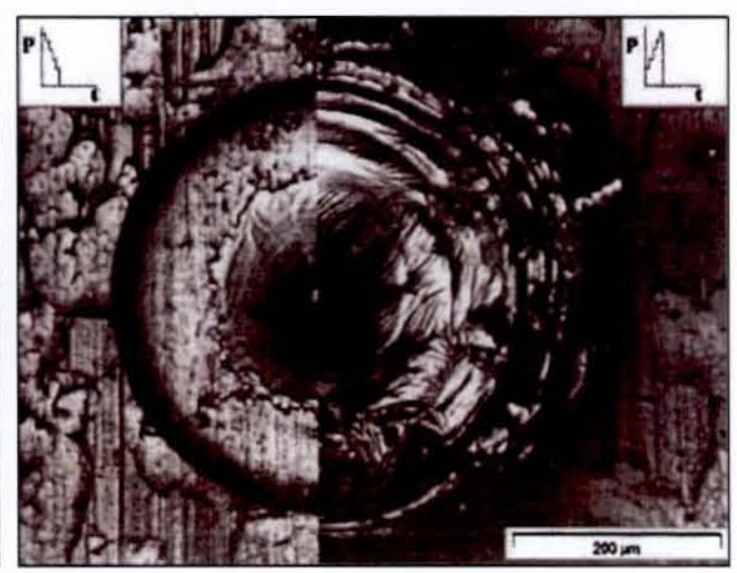

(b)

Figure 2-20 Welds made on aluminium using a Rectangular \& Ramp Up pulse shape

(a) (5 ms, $1.75 \mathrm{~J}) \&$ Ramp Down/Up pulse shape with shorter pulse duration (b)

(1.5 ms, $1.05 \mathrm{~J})$ [Gower et al. 2005]

Tzeng et al. [2000] used an Nd:YAG laser to seam weld electrogalvanised steel sheets. Welds produced with Ramp Down pulses generally had larger bend strengths compared to those produced with ramp up and rectangular pulses. This may be due to Ramp Down pulse shapes producing the largest weld profile owing to it having the strongest heating effect. Pan et al. [2005] used an Nd:YAG laser to weld magnesium plates. A Taguchi methodology was used to reveal the laser parameters that held the greatest influence on the weld's ultimate tensile stress. It was found that pulse shaping using a Ramp Down pulse shape held a greater influence on part tensile strength than laser power, repetition rate and scan speed. The authors also noticed that Ramp Up pulse shapes penetrated deeper into the magnesium plates than Ramp Down pulse shapes (shown in Figure 2-21). This was due to the highest peak power being emitted at the end of the pulse when the absorbance of the material was at its highest. Table 2-6 represents a summary of all major laser welding investigation employing pulse shaping. 

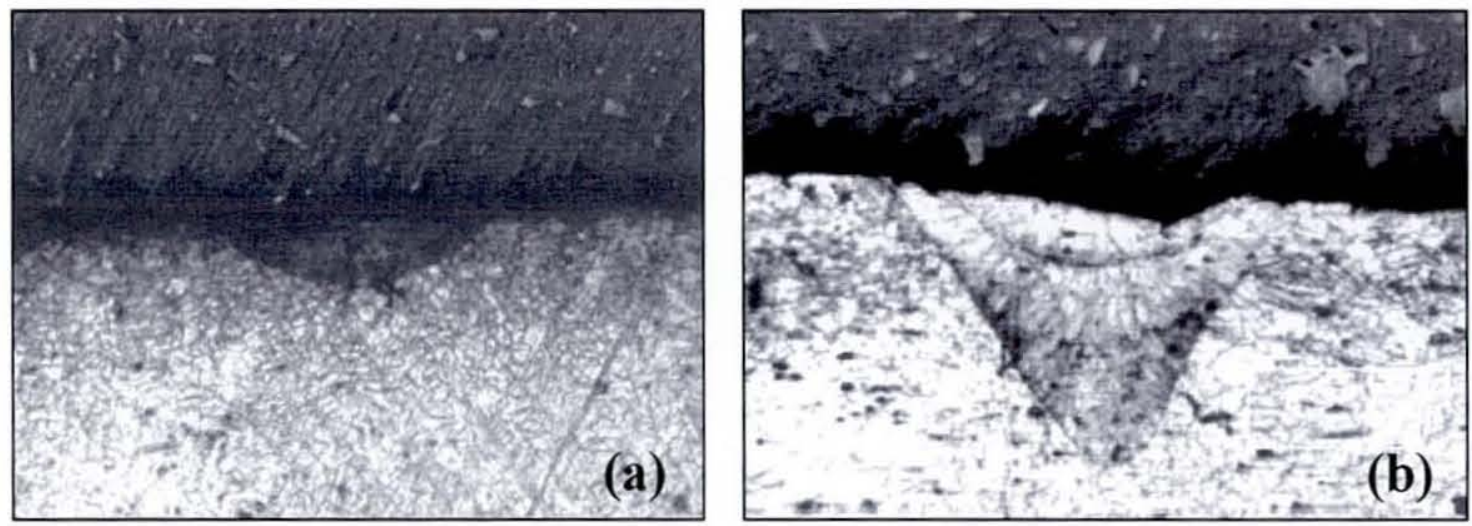

Figure 2-21 Welds on Magnesium plates, using Ramp Down (a) \& Ramp Up (b) pulse shapes [Pan et al. 2005]

\begin{tabular}{|c|c|c|}
\hline Ramp Down pulse & Ramp Up pulse & Material \\
\hline Large Crater Size & & Steel [Bransch et al. 1994] \\
\hline Increased penetration & Highest penetration & $\begin{array}{l}\text { Steel, Aluminium [Badawi et al. } \\
\text { 1989] }\end{array}$ \\
\hline Increased weld profile & Lower weld profile & $\begin{array}{l}\text { Steel, Aluminium [Badawi et al. } \\
\text { 1989; Tzeng \& Chen 2000; } \\
\text { Gower } \text { et al. 2005] }\end{array}$ \\
\hline Cracks shortest & Larger cracks & $\begin{array}{l}\text { Steel, Aluminium [Katayama et } \\
\text { al. 1993; De Lima 2005; Gower } \\
\text { et al. 2005] }\end{array}$ \\
\hline $\begin{array}{l}\text { Absorption lowest at } \\
\text { main pulse }\end{array}$ & $\begin{array}{l}\text { Absorption highest at } \\
\text { main pulse }\end{array}$ & Steel, Alum.[Badawi et al. 1989] \\
\hline $\begin{array}{l}\text { Least gas occluded } \\
\text { porosity }\end{array}$ & $\begin{array}{l}\text { Largest gas occluded } \\
\text { porosity }\end{array}$ & $\begin{array}{l}\text { Steel, Aluminium [Weedon 1987; } \\
\text { Katayama et al. 1993; Bransch et } \\
\text { al. 1994; Fujinaga et al. 2000; } \\
\text { Gower et al. 2005] }\end{array}$ \\
\hline Smoother weld & Smoother weld & Aluminium [Gower et al. 2005] \\
\hline Highest tensile stress & & Magnesium [Pan et al. 2005] \\
\hline Increased bend strength & & Steel [Tzeng \& Chen 2000] \\
\hline $\begin{array}{l}\text { Some oxide films } \\
\text { removed }\end{array}$ & Most oxide films removed & Aluminium [Gower et al. 2005] \\
\hline $\begin{array}{l}\text { Slowest solidification } \\
\text { rate }\end{array}$ & $\begin{array}{l}\text { Solidification rate similar } \\
\text { to rectangular pulse }\end{array}$ & Aluminium [Michaud et al. 1994] \\
\hline \multirow[t]{2}{*}{$\begin{array}{l}\text { Short time to initiate } \\
\text { melt pool }\end{array}$} & $\begin{array}{l}\text { Longest time to initiate } \\
\text { melt pool }\end{array}$ & Aluminium [Michaud et al. 1994] \\
\hline & $\begin{array}{l}\text { Reduced expulsion of } \\
\text { material/Increased metal } \\
\text { expulsion (dependant on } \\
\text { laser power) }\end{array}$ & $\begin{array}{l}\text { Steel, Aluminium [Petrov et al. } \\
\text { 1992; Katayama et al. 1993; } \\
\text { Fujinaga et al. 2000; Gower et al. } \\
\text { 2005] }\end{array}$ \\
\hline
\end{tabular}

Table 2-6 Pulse shaping effects on laser welded metal sheets 


\subsubsection{Laser cutting/drilling of metal Sheets}

Pulse shaping has generally not been as effective in cutting/drilling as in laser welding. Pulse train modulation rather than individual pulse shaping has been used when percussion drilling a nimonic alloy [Low et al. 2001]. Pulse train modulation is the adjustment of pulses over a period of time (inter-pulse shaping). Rather than creating a particular pulse shape for use within processing, the pulse's power is adjusted dependant on time and the frequency of pulses emitted. The researchers used a Rectangular pulse shape and gradually increased the pulse's power over a defined period of time. The authors state that pulse train modulation is more effective within laser drilling than conventional pulse shaping, when reducing spatter and increasing the depth of penetration.

\subsubsection{Laser melting of metal powder}

Very limited work to date has been conducted using pulse shaping techniques to laser process metal powder. Toyserkani et al. [2004] claimed to use the pulse shaping technique when laser processing powder feed iron [Toyserkani et al. 2004]. The researcher used a conventional Rectangular pulse shape and increased the power of the laser in steps. This technique fits the description of pulse train modulation used by Low et al. for laser percussion drilling and should technically not be referred to as pulse shaping. Within the investigation the laser's energy was increased every 5 seconds over a 20 second period and the effect of this increase in power on clad height was examined. It was found that increasing the pulse energy increased the height of the clad.

Sparks et al. [2006] used a $1 \mathrm{~kW}$ diode laser to melt powder fed H13 tool steel [Sparks et al. 2006]. The author varied the pulse energy, frequency, material feed rate and pulse duration of a Ramp Down pulse shape in an attempt to discover the parameter that held the greatest influence on the microstructural grain growth within the specimen. The author states that the work reported is not conclusive due to incomplete experimental runs. However from initial experimental results it was found that prolonged Ramp Down pulse durations increase the cooling time and therefore lengthen the grain sizes of H13 specimens. The researcher states that manipulating the microstructure using pulse shaping would allow improved properties such as material hardness or fatigue strength to be situated within optimised locations within the same part. 


\subsubsection{Summary}

Research suggests that pulse shaping has many benefits, particularly within laser welding of metal sheets. Its benefits include microstructural improvement such as porosity and crack reduction, reduced weld profiles, surface roughness improvements, oxide layer reduction, reduction in spatter during processing and tensile and bending strength improvements. Controlling the microstructure within a part would allow properties such as increased hardness, resistance to delamination or tensile strength to be strategically distributed within a single part.

The majority of pulse shaping investigations have focused on laser welding of metal sheets, focusing mainly on steels and aluminium. Minimal research has been conducted on the effects of pulse shaping on processing metal powder, with no research focusing on its use within powder bed processing. The additional process control achieved through pulse shaping was able to reduce weld profiles, reduce surface roughness and spatter generation of welded metal sheets. This addition control and improvement of part properties and processing conditions may be transferrable to other laser processing technologies such as SLM. 


\section{Chapter 3 Selective Laser Melting}

SLM as mentioned in section 2.2.4.2 is a powder bed process that uses a laser to selectively scan and melt pre-laid powder, as shown in Figure 3-1. Unlike indirect SLS, SLM uses a high powered laser capable of completely melting powder in a single step to produce high density fully functional metal parts.

This section examines the SLM process in more detail. The control factors that influence part formation are discussed including laser processing, powder bed properties, atmosphere and part build strategy. Topics that specifically attend to the heat transfer, fluid dynamics, melt pool instabilities and factors that affect part properties such as surface roughness are discussed. Other topics of discussion include material vaporisation, oxidation and residual stresses that build up within solidified parts.

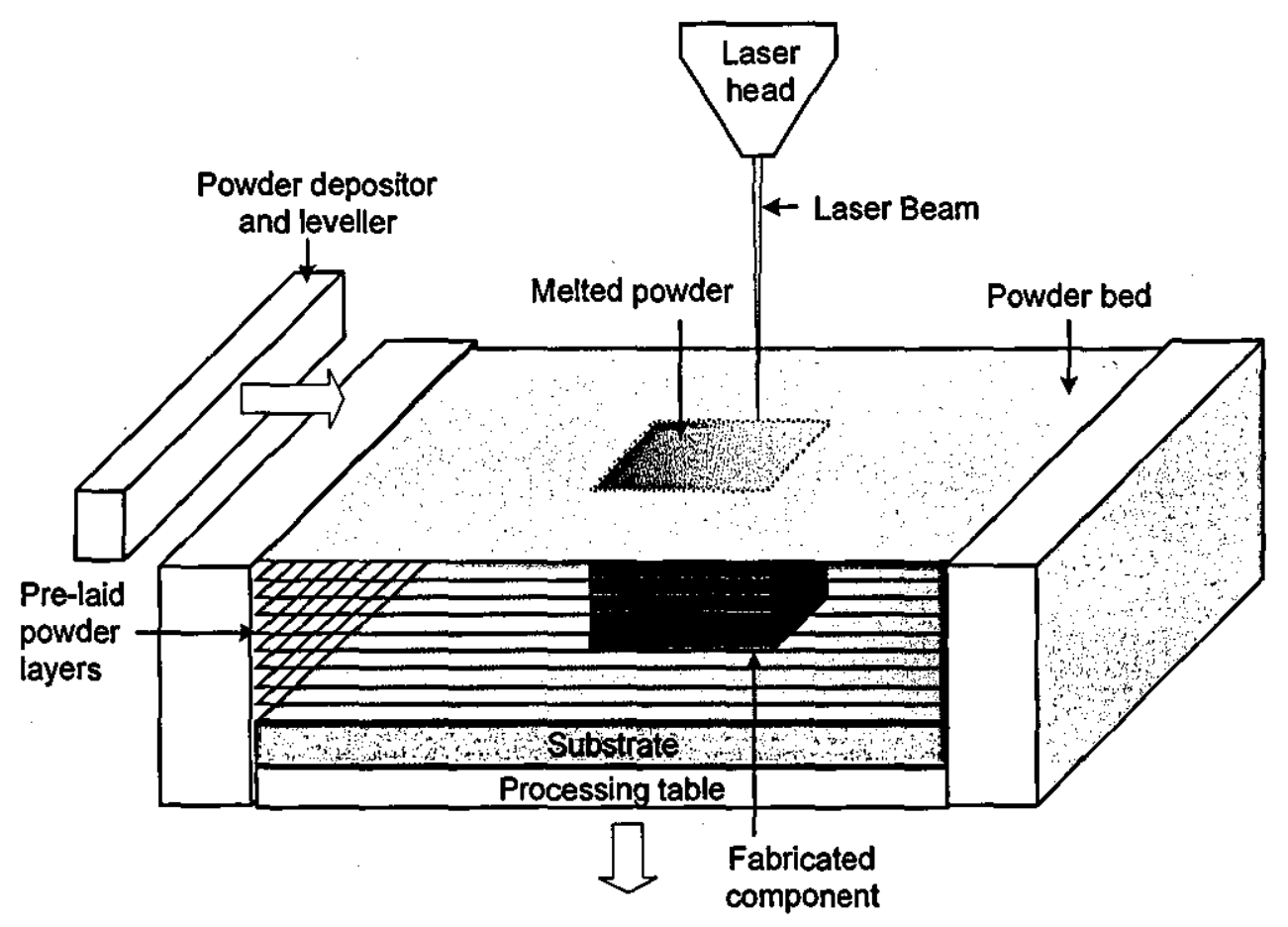

Figure 3-1 Schematic of the selective laser melting process 


\subsection{SLM Control Factors}

This section will highlight the main processing parameters used within SLM and describe how these parameters affect the formation of metal parts.

Various research performed using SLM has shown that important part features such as build height, wall thickness, surface roughness, porosity, microstructure are strongly dependent on the system's laser processing parameters such as laser power, laser scan speed, repetition rate, etc. [Morgan et al. 2004; Childs et al. 2005; Kruth et al. 2005; Mumtaz et al. 2006]. As well as the main laser control parameters there are also other controllable factors that can affect part formation, including powder size, layer thickness, scanning strategy, processing atmosphere etc. The main control parameters/factors within SLM are highlighted in Figure 3-2.

\begin{tabular}{|c|}
\hline Powder \\
\hline $\begin{array}{c}\text { Material } \\
\text { Powder size/distribution } \\
\text { Powder shape }\end{array}$ \\
\hline
\end{tabular}

\begin{tabular}{|c|}
\hline Powder bed \\
\hline Powder layering \\
Layer thickness \\
Substrate type/thickness \\
Pre-heat powder bed \\
\hline
\end{tabular}
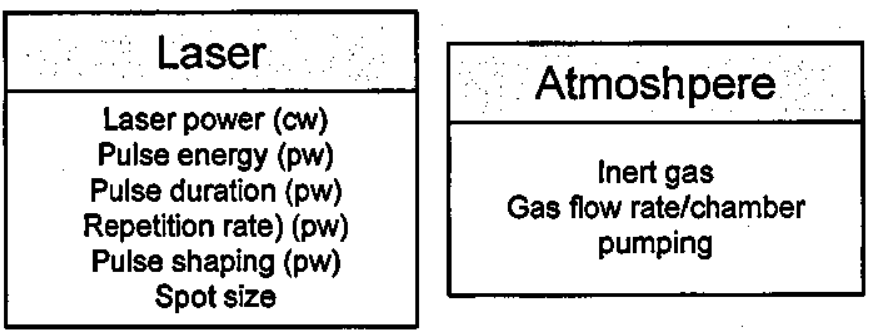

\begin{tabular}{|c|}
\hline Part build \\
\hline $\begin{array}{c}\text { Scan speed } \\
\text { Scan strategy } \\
\text { Hatch distance } \\
\text { Cross-hatching } \\
\text { Exposure time/dwell }\end{array}$ \\
\hline
\end{tabular}

Figure 3-2 Main SLM control parameters

\subsubsection{Laser}

The main laser processing parameters for a pulsed laser are discussed below. These processing parameters drive the SLM process generating heat that allows consolidation of material. 
Pulse Energy $P_{e}(J)$ is the amount of energy contained within each laser pulse. This is an important factor as it directly affects the amount of heat delivered to the material and will subsequently induce any phase changes (i.e. melting). An increase or decrease in energy will lead to the expansion or reduction of melt pool size as the local surrounding powder is drawn into the already generated melt pool.

Pulse Duration $t_{p}(\mathrm{~ms})$ also known as the pulse length or pulse width. This is the time for which a single laser pulse is emitted.

Pulse Frequency $f\left(\mathrm{~s}^{-1}\right)(\mathrm{Hz})$ is the number of cycles or rate at which the laser pulse is emitted per second.

Scan Speed $V(\mathrm{~mm} / \mathrm{min})$ is the rate at which the processing table moves relative to the fixed laser head or the rate at which the laser spot moves relative to the powder bed.

Average Power $P_{a v}(\mathrm{~W})$ is a function of pulse energy $(\mathrm{J})$ and repetition rate $(\mathrm{Hz})$ :

$$
P_{a v}=P_{e} f
$$

Average Peak Power $P_{p a v}(\mathrm{~W})$ is the average power within each pulse. It is one of the most important factors dictating the attribute of a laser pulse and the degree of melting. It can be approximated using the following equation:

$$
P P_{a v}=\frac{P_{e}}{t_{p}}=\frac{P_{a v}}{f t_{p}}
$$

This calculation does not take into consideration a pulse's maximum peak power $P P$ (overshoot or spiking) that occurs within the first millisecond of a pulse. This can be 75$100 \%$ over the allocated $P P_{a v}$ (as discussed in section 2.3.1). The maximum peak power value can only be accurately established through real-time pulse recording (e.g. with a digital oscilloscope) as will be shown in Chapter 7. 
Energy Density $E_{d e n}\left[\mathrm{~J} / \mathrm{mm}^{2}\right]$ defined as the energy per unit area, it is a function of average power $(\mathrm{W})$, scan speed $(\mathrm{mm} / \mathrm{min})$ and spot size $\left(S_{d}\right)$ :

$$
E_{d e n}=\frac{P_{a v}}{S_{d} V}=\frac{P_{e} f}{S_{d} V}
$$

Laser spot overlap $S_{\mathrm{ov}}[\%]$ is the amount a laser pulse overlaps the previous pulse. It is a key parameter dictating the level of heating and melt pool properties. In reality with the movement of the laser, the beam's circular profile may become more elliptical;

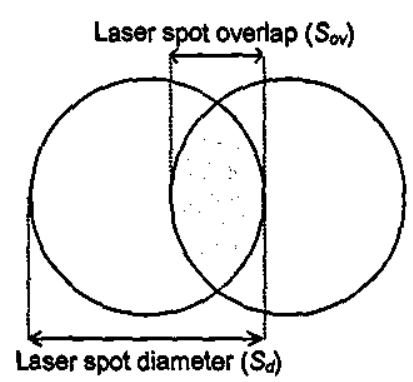

$$
S_{o v}=\left|1-\frac{V}{S_{d} f}\right| 100
$$

Figure 3-3 Laser spot overlap of two overlapping pulses

\subsubsection{Powder}

Metals and metallic alloys are the largest group of materials currently developed for the SLM process. The properties of the material will affect the ease or difficulty at which SLM processing takes place. Metals such as stainless steel can be processed with relative ease within SLM, encountering minimal processing issues to produce fully functional parts. However processing highly reactive metals, such as aluminium, pose more processing difficulties. There has been a variety of academic work using SLM to process aluminium, copper, iron, stainless and tool steel, chromium, nickel alloys, titanium and composites of these materials [Abe et al. 2001; Su 2002; Abe et al. 2003; Kruth et al. 2004; Pogson et al. 2004; Santos et al. 2004; Childs et al. 2005; Mumtaz et al. 2006; Yadroitsev et al. 2007]. Kruth et al.[2005] state that the range of commercially available metals for SLM is still limited due to the requirement of experimentally determining a processing-window for each new material. The properties of a particular material can be specified to suit the processing system and final application. Material 
properties that should be considered before processing include Particle Size Distribution (PSD), bulk density $\rho_{b}$, melting temperature $T_{m}$, boiling temperature $T_{b}$, Absorptivity $\alpha$, etc. [Rehme \& Emmelmann 2005]. These material properties are discussed below:

Particle Size Distribution (PSD) of a powder represents the frequency of powder particles at given diameters. The PSD can be specifically tailored to suit a particular application. Having a wide range of powder sizes within a batch of material can be beneficial in improving bulk powder packing density as smaller sized particles fill gaps in between larger particles. However a wide PSD may conversely lead to increased porosity as larger particles within a batch of material could melt or partially melt while smaller particles may vaporise [Morgan et al. 2004]. Using a very narrow range of PSD can improve the consistency of melt but may lose the benefits of improved powder packing density [Laeng et al. 2000]. The use of a narrower PSD tends to be more expensive than wider ranges as sifting is required and material is wasted. Research performed using powder bed processes generally use a powder size range of $20-50 \mu \mathrm{m}$ when producing fully functional high density metal parts [Das 1998; Khaing et al. 2001; Hauser et al. 2005]. Using larger or smaller powder sizes within a defined distribution has advantages and disadvantages. Use of smaller powder particle requires less heat input than larger particles to change state from solid to liquid. Smaller powder particles will generally form parts that have a lower side surface roughness as the stair stepping effect is reduced due to the ability of depositing thinner layers [Karapatis et al. 1998; Sears 1999; Syvanen et al. 2000; Lu et al. 2001; Carroll 2007]. Work conducted by Mazumder et al. [2000] showed that as powder layers become thicker the side roughness of parts increased. This is due to the beam diameter having a longer distance to diverge. Therefore, the width of melt is larger at the bottom of the melt in comparison with the top. By reducing powder size and layer thickness, this beam diameter variation is also reduced and therefore the side roughness of the part is minimised. Smaller powder particles can improve part accuracy and resolution if the laser beam size is reduced [Pinkerton 2007], however the efficiency of the laser reduces if the particle size becomes smaller than the laser's wavelength. Also with the use of smaller particle sizes there is more likelihood of powder agglomeration forces (Van der Waals) making the handling and deposition of powder more difficult [Boivie 2001]. The issue of agglomeration can easily be resolved by including larger particles within the batch of powder [Karapatis et al. 1998; Simchi et al. 2003]. Smaller particles (especially nanoparticles) are more of a health hazard and can be easily be inhaled or absorbed into 
the skin if improper handling and protection is used. Larger particles are less likely to be blown away by shielding gas or displaced by plasma plumes generated by lasers and therefore reduce the amount of material consolidated into the melt [Laeng et al. 2000]. Morphology is the physical shape of the powder particles and is determined by material preparation technique. Atomised powders are generally spherical in shape whereas milled powders exhibit a more irregular shape [Flemmer et al. 1993]. Particle shape can influence flow ability and powder bed packing density. Particles with a perfectly equixed shape display good flowing properties as motion is not hindered by angle contacts. Additionally they display a higher compaction ability and increase powder packing density [Das 1998; Karapatis 2001].

Bulk density $\rho_{b}\left[\mathrm{~kg} / \mathrm{m}^{3}\right]$ determines density of powder material and maximum final density of a processed component. Densities influence heat balance (see section 3.2) and expulsion of particles from melt pool. The density of the powder bed $\rho_{p}\left[\mathrm{~kg} / \mathrm{m}^{3}\right]$ compared to the bulk density gives an indication of the compaction required to obtain fully dense components [Elsen 2007].

Thermal conductivity $k_{b}[\mathrm{~W} / \mathrm{mK}]$ changes as material temperature varies. This property indicates the materials' ability to conduct heat and affects the heat balance (see section 3.2).

Specific heat capacity $C_{p}[\mathrm{~J} / \mathrm{kgK}]$ is the heat energy required to increase the temperature of a unit quantity of a material by a certain temperature. $C_{p}$ affects the heat balance (see section 3.2)

Latent heat of fusion $L_{f}[\mathrm{~J} / \mathrm{kg}]$ is the amount of energy required to change the state of a solid material to a liquid. This again affects the heat balance (see section 3.2). The amount of energy required to change the state of a liquid to gas is known as the latent heat of vaporisation $\left.L_{v} \mathrm{~J} / \mathrm{kg}\right]$.

Melting point $T_{m}[\mathrm{~K}]$ is the temperature at which a solid material will transform into a liquid state.

Evaporation point $T_{v}[\mathrm{~K}]$ is the temperature at which a material will transform into a gas.

Dynamic viscosity $\eta[P a . s]$ influences melt pool dynamics and is discussed further in section 3.3.

Thermal expansion coefficient $\alpha[\mathrm{W} / \mathrm{mK}]$ is the response to temperature change, affecting stress during solidification [Nickel et al. 1999]. 
Surface free energy $\boldsymbol{\gamma}\left[\mathrm{J} / \mathrm{m}^{2}\right]$ quantifies the disruption of chemical bonds when a surface is transformed [Elsen 2007]. Its influence within SLM is discussed in section 3.3.

Absorbance $A$ defined as the ratio between absorbed energy and reflected/transmitted (also known as laser/energy coupling). Its influence within SLM is discussed further in section 3.2.1.

\subsubsection{Powder Bed}

A substrate is a metal base plate to which the first layer of powder fuses onto during the SLM process. The material of the substrate is normally the same as material being processed. The substrate size has an effect upon part microstructure and heat build-up within the powder bed. Large substrates will have a large heat extraction capability and allow the material to cool substantially after each deposition step, whereas small substrates will result in a higher heat build-up within the part [L. Costa et al. 2005]. The powder bed can be pre-heated such that less heat input is required by the laser to change the material from a solid to liquid state [Watkins 2001]. This may also reduce surface roughness and improve part accuracy due to reduced thermal gradients and therefore reduced shrinkage [Sexton et al. 2002; Over 2003]. Preheating of the powder bed has been reported to improve spreading behaviour of a melt pool due to reduced temperature variation between solid and melt pool (see section 3.3.1). The majority of commercial powder bed processes including MTT Realizer, EOS M270 and Phenix Systems PM250/100 preheat their powder bed to reduce the thermal input required by the laser to liquefy powder particles and also to alleviate thermal stresses developing within the part. Concept LaserCUSING process does not employ powder bed preheating but instead uses a patented scanning strategy to alleviate thermal stresses building up within a part during fabrication. Research conducted by Das [1998] gave a conflicting report regarding the benefit of powder bed preheating. The research showed that preheating a powder bed does not necessarily improve part properties as preheating could only raise the temperature of a bed to $100-250^{\circ} \mathrm{C}$ before "caking" would occur [Das 1998; Elsen 2007]. This is the partial sintering or solid state sintering of smaller powder particles within the powder bed. The temperature required to fully melt most steels, titanium and nickels is above $1000^{\circ} \mathrm{C}$, therefore a reduction of $100-200^{\circ} \mathrm{C}$ heat input required by the laser was not shown to produce any obvious benefits or improve 
part properties (see 3.2.2 for energy reduction with powder bed preheating). However Phenix Systems claim to be able to preheat powder beds within their PM250/100 models up to $900^{\circ} \mathrm{C}$ [Wohlers 2008]. Maximum preheating temperature is also restricted in order to reduce any possible thermal deviations of the optical system [Elsen 2007]. The layering of powder can affect the accuracy of a part, inconsistent powder packing can increase variation in the final geometry [Crane et al. 2005].

\subsubsection{Atmosphere}

An inert atmosphere should be provided within the processing environment to prevent molten material reacting with non-inert gases such as oxygen $\left(\mathrm{O}_{2}\right) . \mathrm{O}_{2}$ reacts with molten material causing the surface to oxidise which in turn affects part properties. A gas chamber filled with an inert gas such as argon (Ar), nitrogen $\left(\mathrm{N}_{2}\right)$ or helium $(\mathrm{He})$ can be used to surround the build area or a shrouding gas can be fed directly onto the melt pool to reduce $\mathrm{O}_{2}$ content. Elsen [2007] states that the $\mathrm{O}_{2}$ level within a SLM processing environment should be below at least $0.3 \%$ to aid reduction in oxidisation during processing. Section 3.5 discusses issues surrounding material oxidation in more detail.

\subsubsection{Part Build Strategy}

Part build strategy influences the quality of a fabricated part as well as the speed at which it can be produced. Many variations of scanning strategy exist [Onuh \& Hon 1998] and can affect melt pool cooling rates, part porosity, accuracy, microstructure, stresses etc. Scanning strategies can include spiral, zig-zag and hatching formations when melting the inner portion of a component's geometry. The processing scan speed in combination with repetition rate (for pulsed lasers) are important parameters that will determine the speed of build. The processing scan speed will vary the average temperature within the operating window during the build-up process, leading to a progressive increase or decrease in melt pool size. Re-melt scanning strategies can also be used to re-melt the surface of a part to reduce surface roughness [Childs 2005]. 


\subsection{Heat Transfer in a Powder Bed}

Section 2.2.1-2.2.2 discussed the process of thermalisation as laser radiation interacted with powder particles. The different sintering mechanisms of solid state sintering, liquid phase sintering and full melting were also examined. This section discusses in more depth factors that initiate early stages of melt pool formation. This includes influence of material absorbance of laser radiation, energy required to initiate full melting and transfer of heat through solid powder particles via conduction.

\subsubsection{Absorbance}

When the surface of a material is exposed to electromagnetic radiation, a portion of the incident wave intensity $I_{o}$ is reflected $I_{r}$, another portion is absorbed $I_{a}$ and the remaining is transmitted $I_{t}$ through the material (generally negligible) [Karapatis 2001] and is shown in Figure 3-4. Absorbance and reflectance can be defined as ratios shown in Equation (3-5):

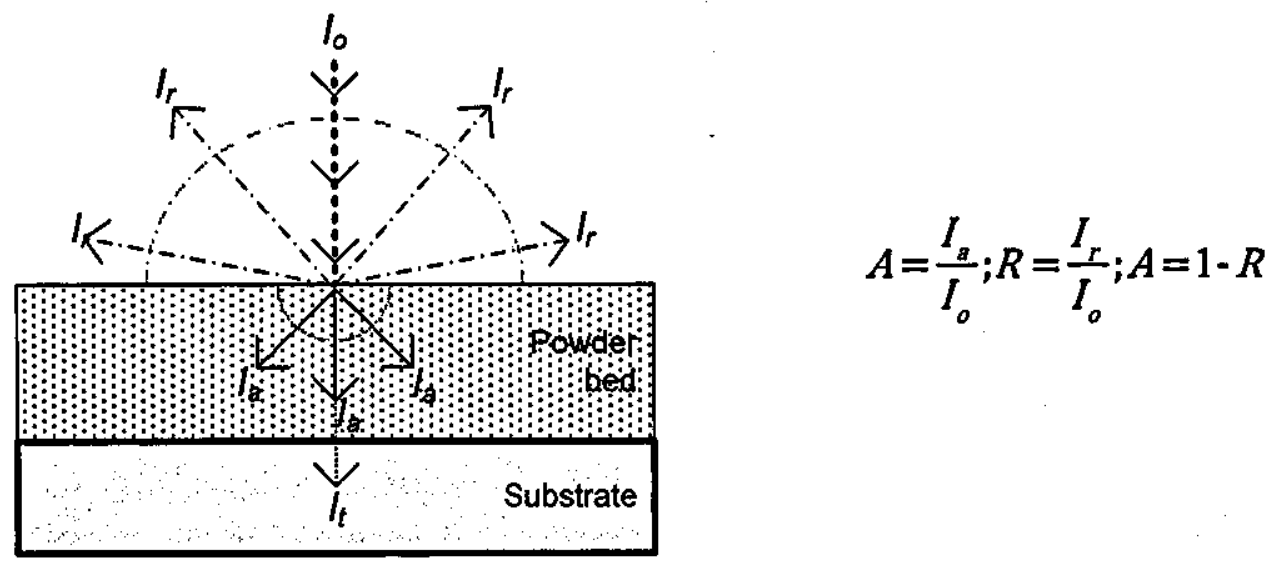

Figure 3-4 Reflected, absorbed and transmitted wave intensities

Absorbance and reflectance are determined by the properties of the material (surface roughness, oxides, etc.). The absorbed energy is the useful energy that initiates melt pool formation. As mentioned in 2.2.3.2 a material will have varying levels of reflectivity when processed using lasers of different wavelengths. Aluminium has a high reflectivity of approximately $90 \%$ when using an Nd:YAG laser $(1.06 \mu \mathrm{m} \lambda)$ and is more 
difficult to process than metals with lower reflectance such as nickel (70\% reflectivity) and stainless steel (60\% reflectivity) [Steen 2003].

As the temperature of a material increases the collision frequency between the heat carrying electrons and phonons increases due to increases in amplitude of vibration. This increase in collision frequency increases the energy absorbance of the material [Von 1987; Messler 1999]. Another reason for increase in metal conductivity at higher temperature is due to phase change of solid to liquid. The density of free electrons in liquid is higher than that in a solid state due to decrease in Fermi's energy [Karapatis 2002], this subsequently increases the absorbance of the material.

\subsubsection{Thermal Conductivity and Melt Energy}

Thermal conductivity influences heat transfer during SLM, its value changes significantly with material state (solid, liquid and gas) and temperature. The absorbed energy from the laser is transformed into heat and flows via conduction down thermal gradients according to Fourier's theory of heat transfer. Thermal properties of powders are not the same as bulk materials as they are not homogenous and continuous solids [Karapatis 2001]. The powder conductivity will depend upon the number of contacts made between powder particles with more contacts equating to an improved conductivity. Figure 3-5(a) shows that heat absorbed from the incident laser radiation $I_{o}$ is transferred via conduction $Q_{c}$ between particle contacts. The heat is conducted almost exclusively within the highly conductive particles, towards the core of particles [Fischer et al. 2003]. Once the temperature within the particle has homogenised the heat conducts into other conductive particles. Some heat will also be transferred between spaces between particles via radiation $Q_{r}$. The conductivity of metals generally increase with increasing temperature [Howard \& Koczak 1981]. The illustration has not included the optical penetration depth which occurs as laser radiation passes through gaps between particles causing multiple reflections [Fischer et al. 2003]. Heat conducts away from the centre of laser spot but the temperature profile of the powder bed reduces the further the region is from the heat source as shown in Figure 3-5(b). 


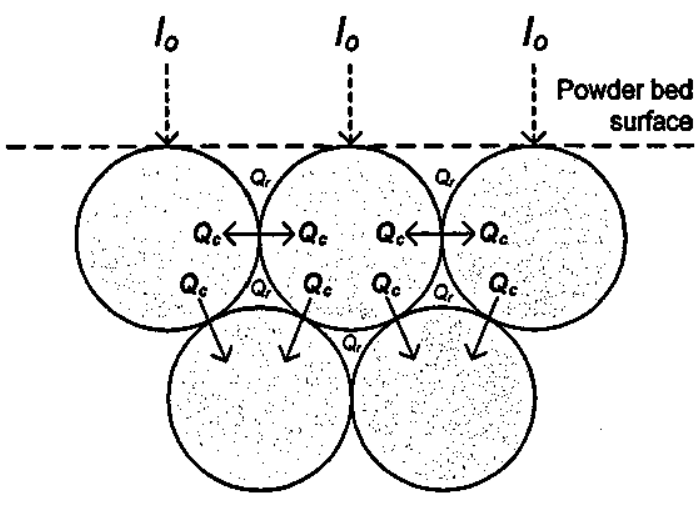

(a)

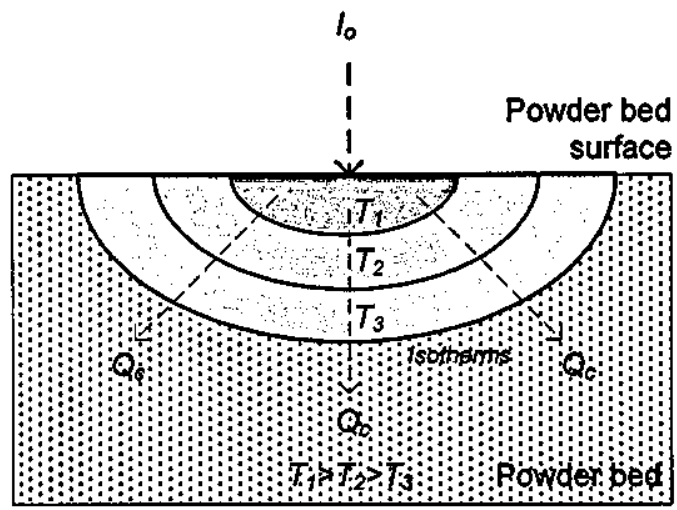

(b)

Figure 3-5 Transfer of heat between powder particles via conduction (a) \& heat distribution within powder bed (b)

The energy required to heat and melt a particle of radius $r_{p}$, volume $v_{p}$ and density $\rho$ can be given by the melt energy required to melt material $\Delta H_{m}$ (heat balance):

$$
\Delta H_{m}=\rho v_{p}\left(C_{p} \Delta T_{m}+L_{f}\right)=\frac{4}{3} \pi \rho r_{p}^{3} E_{m} \quad \text { [Karapatis 2002] }
$$

$C_{p}$ specific heat capacity, $\Delta T_{m}$ difference between ambient temperature and material melting temperature, $L_{f}$ latent heat of fusion and $E_{m}$ melt energy. According to the heat balance preheating of a powder bed will reduce the difference between ambient temperature and melt temperature and subsequently reduce required heat to melt material. As mentioned in 3.1.3 there were differences in conclusions regarding the merits of powder bed preheating. It had been reported that a powder bed could only be heated to $200-250^{\circ} \mathrm{C}$ (depending on material and powder size) before "caking" or solid state sintering would occur [Das 1998; Elsen 2007]. The reduction in $\Delta H_{m}$ when employing powder bed heating can be calculated using equation (3-6). Elsen [2007] calculated that the reduction in energy required to melt stainless steel powder with a powder bed preheating temperature of $500^{\circ} \mathrm{C}$ was only $22 \%$ lower as compared to the use of no preheating. 


\subsubsection{Energy Loss}

It has already been mentioned that the incident laser radiation can either be absorbed or lost through reflectance and transmittance. The absorbed energy can also be lost in the stages after a melt pool formation. Heat can be lost through thermal diffusion, vaporisation loss (discussed further in section 3.4) and radiation loss as shown in Figure $3-6$.

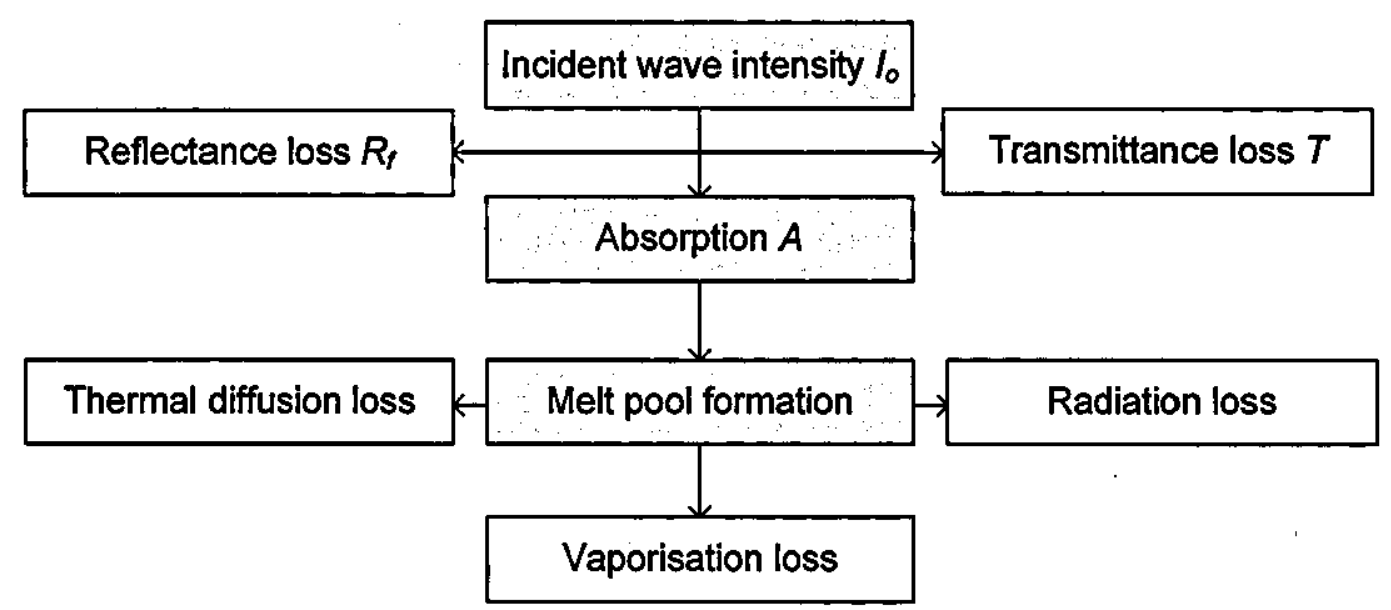

Figure 3-6 Energy loss leading up to and after melt pool formation

\subsection{Melt Pool Dynamics}

Melt pool dynamics studies the fluid behaviour of a melt pool. The fluid behaviour of the melt pool heavily influences heat distribution, movement of molten material, surface tension, part morphology and the internal structure of a solidified part. Understanding its behaviour is critical in controlling SLM part properties. Molten material should spread well over any underlying solid forming a consistent structure along the direction of the scanned laser beam. However various physical phenomena can prevent this from occurring including the generation of instabilities within the melt pool. This section discusses melt pool capillary instabilities and thermocapillary effects within a melt pool including influential properties such as wettability, surface tension and liquid viscosity. 


\subsubsection{Wetting}

Wettability determines the spreading behaviour of a liquid on a solid. It affects interlayer connections, porosity and strength of SLM parts [Singheiser et al. 2001; Steen 2003]. It also affects the tendency of the melt pool to break up into smaller entities in order to reduce surface tension variations (capillary instabilities see section 3.3.2) of the melt pool. Figure 3-7 shows a non reactive liquid in contact with a solid in the presence of a vapour phase and their respective surface tension at each interface. The liquid intersects the solid at a contact angle $\theta$ and is governed by the equation of Young:

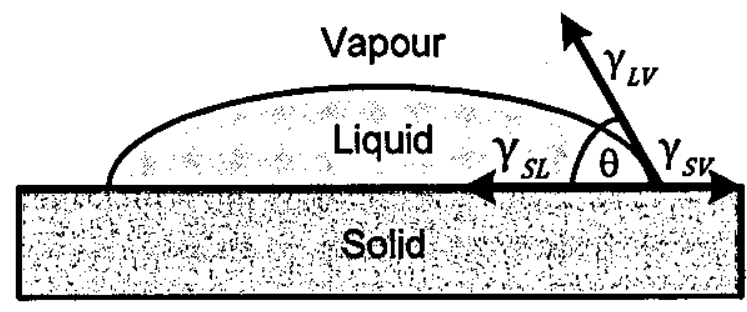

$$
\cos \theta=\frac{\gamma_{S V}-\gamma_{L S}}{\gamma_{L V}}
$$

Figure 3-7 Three phase equilibrium for wetting of a liquid on a solid (adapted from [Eustathopoulos et al. 1999])

$\gamma_{L V}, \gamma_{S V}$ and $\gamma_{L S}$ represent the surface free energy at the liquid-vapour, solid-vapour and liquid-solid interfaces respectively. The liquid will wet if $\gamma_{L V} \leq \gamma_{S V}-\gamma_{L S}$ as long as $\gamma_{S V}>$ $\gamma_{L S}\left(\right.$ i.e $\gamma_{S V} \gamma_{L S}>0$ ), with improved wetting at contact angles smaller than $90^{\circ}$ [Agarwala et al. 1995; Extrand 2003]. Homologous wetting is when a liquid spreads over a solid with the same chemical composition and commonly occurs within SLM/SLS. High solubility also improves wetting behaviour and assists the rearrangement of material compositions within the melt pool [Kruth 1991]. The surface energy of metal oxides are lower than the corresponding liquid-vapour surface free energy. Therefore liquid metals exhibit poor wetting on a solid with an oxide film [Das 1998] causing higher contact angles and increases the liquid's tendency to undergo the balling mechanism (balling is discussed in section 3.3.2). Figure 3-8 shows an example of a liquid with poor and complete wetting. 

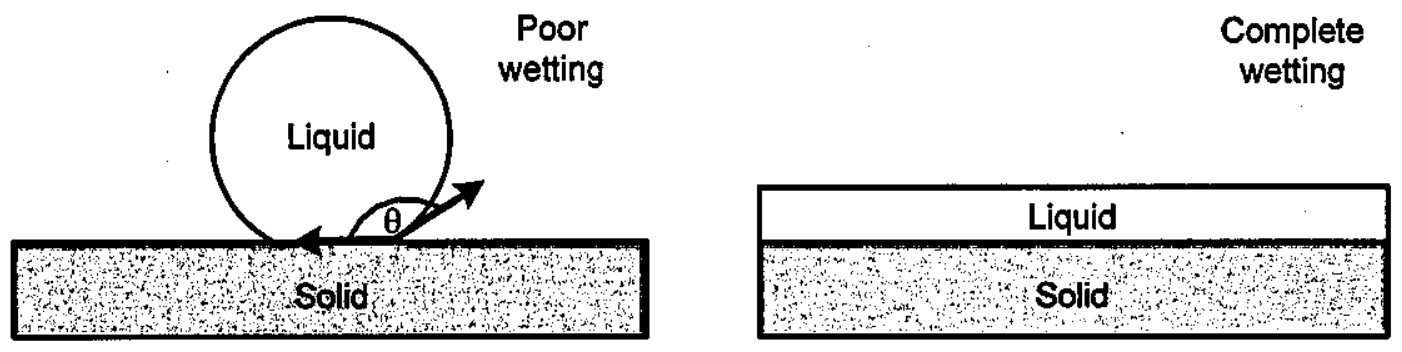

Figure 3-8 Poor wetting behaviour \& complete wetting of liquid on a solid

Due to the rapid melt pool solidification during SLM it is important that a melt pool is given sufficient time to spread and improve its wetting. It has been reported that as temperature increases $\gamma_{L V}$ reduces improving the wetting behaviour [Kruth et al. 2003]. At higher temperatures a liquid's viscosity $\eta$ decreases and improves its spreading behaviour:

$$
\eta=\eta_{0} \exp \left|\frac{Q}{R T}\right|
$$

$\eta_{0}$ is a constant, $Q$ activation energy for flow, $R$ universal gas constant and $T$ absolute temperature. Wetting properties can be improved by introducing small quantities of insitu deoxidiser or fluxing agent into the powder. This can improve liquid spreading by lowering the wetting angle between the liquid and solid phase [Agarwala et al. 1995]. Preheating a powder bed improves the wetting angle of the melt onto the substrate [Agarwala et al. 1995; Morgan et al. 2001]. It has been reported that the increased recoil pressures generated by higher laser peak powers exert a pressure on the melt pool reshaping the spherical melt pool to a flatter profile [Fischer et al. 2003; Morgan et al. 2004], this causes the contact angle made between liquid and substrate to reduce improving the spreading and subsequent wettability of the melt pool [Mumtaz et al. 2008].

\subsubsection{Capillary Instabilities}

Capillary instabilities relate to the breakup of a liquid into smaller entities in an effort to reduce surface free energy, this mechanism within SLM is known as balling and is commonly related to Plateau-Rayleigh instabilities. These smaller entities resemble molten spheres due to a natural tendency of the surface free energy to minimise its area 
[Morgan et al. 2004]. Surface free energy/surface tension variations across the melt pool can cause the melt pool to break up into these smaller entities and reduce surface tension variations across the melt pool. Surface tension is a function of temperature, due to non uniform heating distribution across the melt pool will inevitably induce surface tension variations, this is discussed further in section 3.3.3. The breakup of molten spheres can attach and solidify to the external part of the melt pool or scatter onto the surface of the powder bed. Balling often occurs due to the molten material not wetting to the underlying substrate [ONeill et al. 1998]. It can hinder interlayer connections [Fuh et al. 1995], increase part porosity [Hauser et al. 2005], obstruct deposition of further powder layers [Kruth et al. 2003] and increase the surface roughness of parts. Surface roughness developed during powder processing is still a dominant issue and needs to be better controlled [Glardon et al. 2001; Ramos et al. 2006].

Kruth et al. [2004] states that when the total surface of a molten pool becomes larger than that of a sphere with the same volume the balling effect takes place, shown in Figure 3-9. This schematic represents one scenario in which a melt pool may become unstable affecting its spreading behaviour and increasing its tendency to undergo the balling mechanism. In order to reduce this instability of the melt pool the length to diameter ratio of the melt pool should be as small as possible [Karapatis 2002]. This requires that the melt pool re-solidifies rapidly behind the spot (short melt length). With the use of pulsed lasers, the melt pool solidifies very quickly and therefore large length to diameter ratios are less likely to occur (unless high scan speed in combination with high repetition are used).

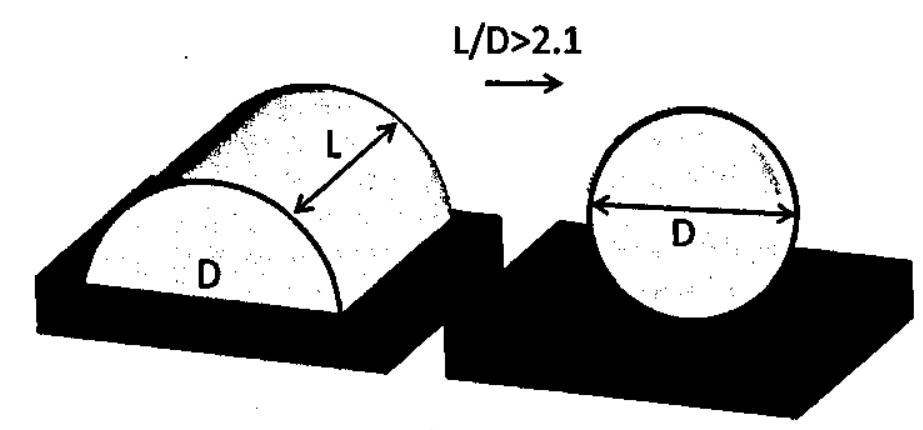

Figure 3-9 Transition from half cylinder to sphere, adapted from Kruth et al. [2004]

Excessive molten material or very low viscosity within a melt can promote balling [Agarwala et al. 1995]. However if the time it takes for a melt pool to break up into 
spheres exceeds the solidification time for the melt pool, then no balling will occur. The break up time is delayed when the liquid within a melt pool has a higher level of viscosity $\eta$, but will subsequently form larger molten spheres if break up does occur [Donnelly \& Glaberson 1965; Rombouts et al. 2006]. A liquid on a substrate is stable if the contact angle $\theta$ made between the liquid solid interfaces is less than $\pi / 2$, improving its wetting behaviour and reducing its tendency to undergo balling [Schiaffino \& Sonin 1997].

\subsubsection{Thermocapillary Flows}

The heating over the region exposed to the laser beam is not homogenous causing thermal variation along the generated melt pool. This thermal variation generates surface tension forces that produce convective fluid flows known as Marangoni flow. Marangoni flows dominate the shape of the solidified melt pool and stability of the melt pool. Fluid flow within the melt pool can also affect part porosity which can form under two main circumstances, voids can form when gas within part is not given enough time to escape or when powder particles have not fully melted [Choi \& Chang 2005]. A quick melt pool solidification time will reduce time for which molten material can flow and reduces the chances of trapped gas to escaping the melt. Material that is not given enough energy to fully melt or enable thermocapillary flows to redistribute material will also form porosity. Porosity that is spherical in shape tends to be gas occluded porosity whereas irregular shaped porosity tends to be due to lack of melting [Haboudou et al. 2003].

Buoyancy forces can also affect fluid flow and are caused by variations in density of fluid due to a temperature gradient. Gravity is the driving force for buoyancy forces but its influence is generally regarded as negligible for a melt pool within SLM/SLS due to small melt pool sizes (1mm or less in width) [Kruth et al. 2007]. Buoyancy forces are more relevant in welding processes in which the melt pool is larger [Mills et al. 1998]. 


\subsubsection{Marangoni convection}

Surface free energy $\gamma$ is a function of temperature, therefore a non uniform temperature distribution along the melt pool induces surface tension variations resulting in shear stress and induces fluid motion known as Marangoni convection. The forces generated under Marangoni convection are generally larger than natural convection and gravity and tend to dominate flow. Other external factors such as recoil pressure and shielding gas also affect flow and are discussed in section 3.4. Areas central to the incident laser beam are at temperatures higher than those at the edge of the melt pool. A steep thermal gradient induces motion of liquid flow from regions of low surface free energy to regions of high surface free energy. The strength of the Marangoni effect can be estimated:

$$
M_{\mathrm{a}}=\frac{d \gamma_{L V}}{d T} \frac{d T}{d s} \frac{L}{2 \eta \delta}
$$

$\frac{d V_{L V}}{d T}$ is the surface tension gradient, $\frac{d T}{d s}$ is thermal gradient ( $s$ is position), $L$ is the length of the melt pool, $\eta$ is viscosity and $\delta$ is the thermal diffusivity. The Marangoni number $M_{a}$ is the ratio of the rate of convection and the rate of conduction. Molten steel has a higher $M_{a}$ than molten aluminium and will therefore be more greatly affected by Marangoni convection. This will aid homogenisation by convection resulting in a shallow, wider melt pool [Ion 2005]. Figure 3-10 illustrates Marangoni convection within the cross-section of a melt pool. Surface tension $\gamma$ decreases with increasing temperature in pure liquid metals. In Figure 3-10, $T_{1}>T_{2}$ producing a negative thermal gradient $\frac{d T}{d s}$ causing $\gamma_{1}<\gamma_{2}$ inducing an outwardly directed flow from the centre of the melt pool [Beer \& Ring 1972]. The flow descends towards the edges of the melt pool to the bottom where it rises again to the melt pool centre generating shallow wide melt pools [Hegge \& Hosson 1991]. The surface tension gradient $\frac{d \gamma_{L V}}{d T}$ of pure metals and many alloys is negative, however positive values are reported for alloys that contain sufficiently high content of surface active elements such as sulphur and oxygen [Lu et al. 2004]. This would cause surface tension $\gamma$ to increase with increasing temperature, 
reversing the fluid flow and generating narrow deep melt pools. Marangoni flow in the melt pool is proportional to the square of the melt pool size and has significant implications for the overall melt pool shape and stability [Pinkerton et al. 2006]. Larger melt pools with a stronger Marangoni flow have been shown to increase a melt pool instability and therefore enhance the balling effect during laser processing of metal powders [Niu \& Chang 1999; Gu \& Shen 2007].

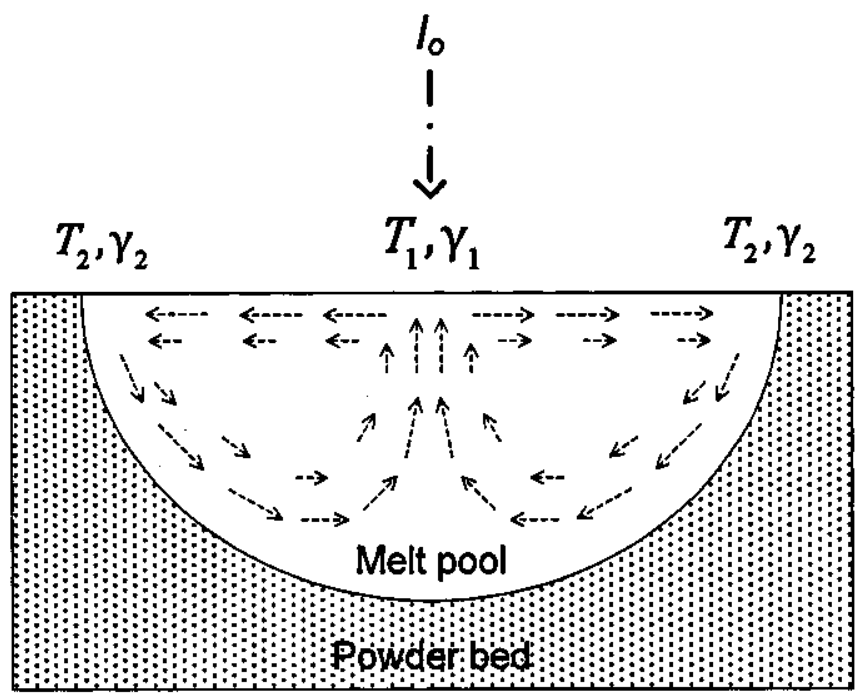

Figure 3-10 Marangoni convection within the cross-section of a melt pool

\subsubsection{Melt pool topology}

As mentioned earlier the flow of movement of fluid heavily influences the shape of the solidified melt pool. Thermal gradients also exist perpendicular to the surface of the melt pool in the direction of the traversing laser. These thermal gradients have been shown to contribute to surface ripple effect [Anthony \& Cline 1977; Ramos et al. 2003]. Figure 3-11 shows a model of fluid flow driven by Marangoni convection caused by temperature differences between a moving laser beam and solidifying zone. The surface tension differences cause liquid movement towards the solidifying front producing a ripple. Gravity and surface curvature effects counteract the surface tension forces and restore the surface height to a free level (relaxation) once thermal gradients have vanished [Ramos et al. 2003]. The restoration of the liquid surface height to a free level is limited by solidification time and is delayed due to liquid viscosity. 
Figure 3-12 shows in more detail the periodic structure formed, including direction of surface tension, gravitational forces and relief height $\Delta h$. Once the laser has moved a distance greater than $\lambda 2$ away from the ripple restoring forces of gravity, surface curvature commence to reduce $\Delta \mathrm{h}$. Lower scan speed increase the temperature directly below the laser spot, increasing the amplitude of the periodic structure and surface roughness $R_{a}$.

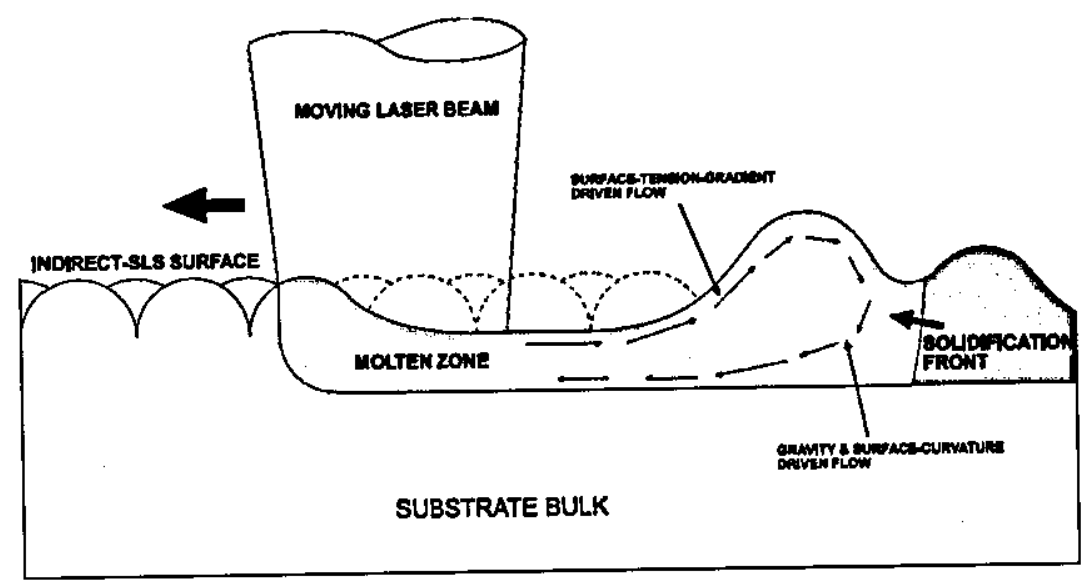

Figure 3-11 Surface rippling along laser scan direction [Ramos et al. 2003]

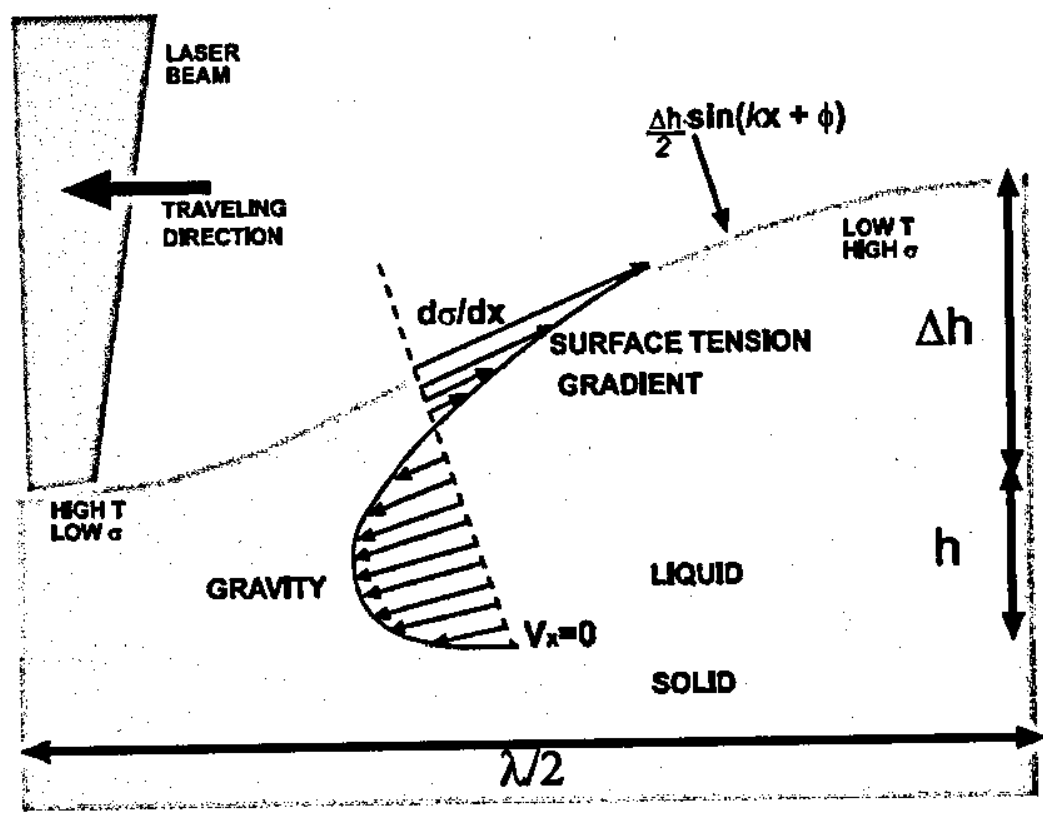

Figure 3-12 Relaxation mechanism restoring liquid to surface free level [Ramos et al. 2003] 
This thermo-physical modelling for the generation of surface ripples is based on the use of a constant power output (continuous wave laser) without any breaks in laser output and the movement of the laser beam relative to the solidifying front. Melt pools generated by pulsed wave lasers emit a more complex heat distribution than that of a continuous wave and are prone to very rapid solidification of melt pool due to use of short pulse duration and reduced HAZ. Short laser-on times are not heavily influenced by the movement of the laser. For example a laser spot of size $0.8 \mathrm{~mm}$ is moving with a scan speed $V$ of $7 \mathrm{~mm} / \mathrm{s}$ along a powder bed. If the laser operates at a pulse duration of $5 \mathrm{~ms}$, the laser spot will move $0.035 \mathrm{~mm}$ during the pulses duration. This distance is just under $4 \%$ of the laser spot size. Melt pool formation and dynamics will therefore be more heavily influenced by pulse duration and spot overlap.

The melt pools produced by a pulsed laser solidify and overlap each other according to the rate, duration and traversing speed of each emitted pulse (spot overlap). The variation in temperature profiles of each melt pool induces Marangoni convection shaping the melt pool as shown in Figure 3-13. A sinusoidal wave profile similar to the surface rippling effect can contribute to top surface roughness. This wave profile exist as peaks and troughs on the melt pool surface produced by pulsed lasers, these correspond to contacts made between solidifying melt pool as shown in Figure 3-14. Relaxation forces described in Figure 3-11 and Figure 3-12 can reduce the peak-trough height variation once thermal gradients have reduced, however melt pool solidification time is very short and gravity my not have enough time to effectively reduce this variation. 


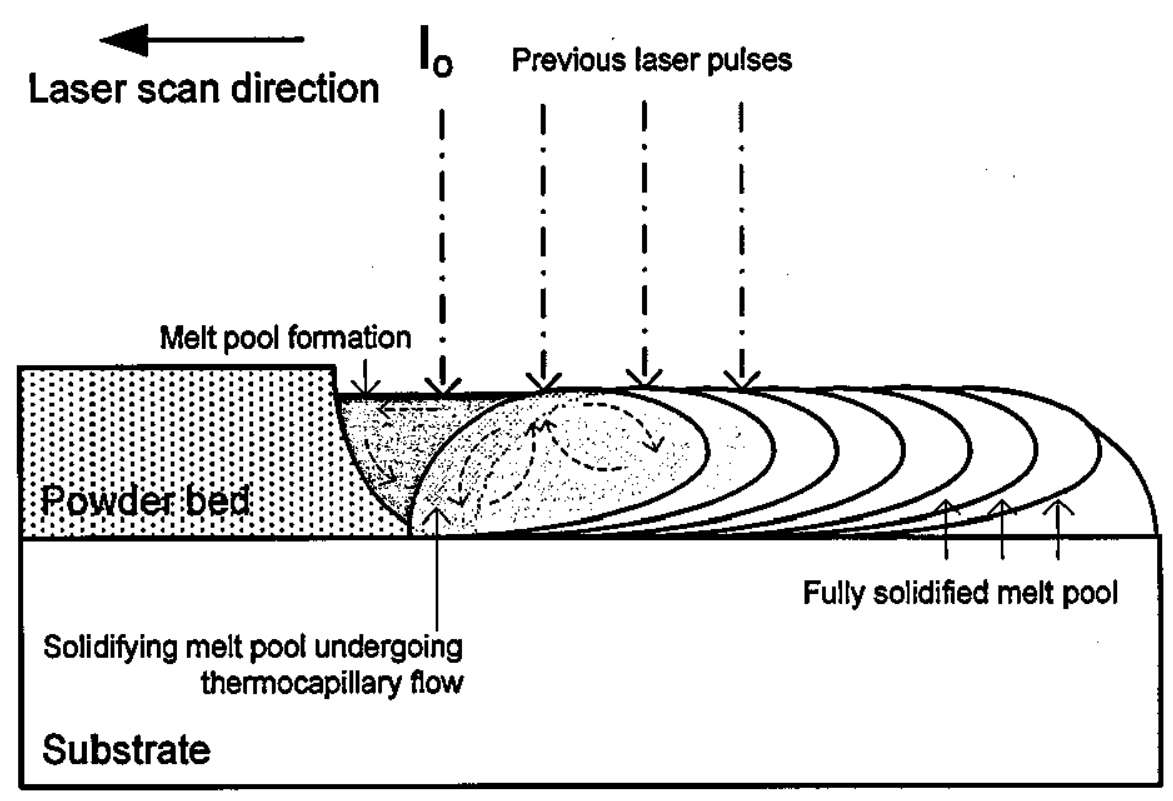

Figure 3-13 Melt pool formation and solidification by a pulsed laser

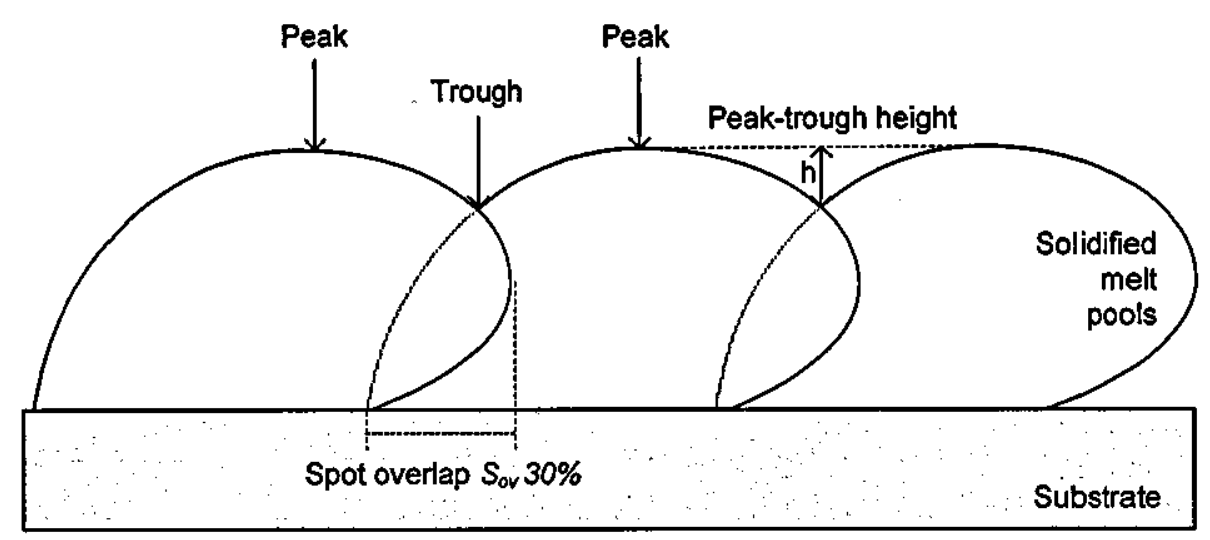

Figure 3-14 Peaks and troughs between solidified melt pools

Ramos et al. [2003] has described that relaxation of the melt pool reduces the surface rippling effect and therefore top surface roughness. Research has shown that a number of other factors can contribute to improving top surface roughness of a solidified melt pool produced using a pulsed laser. Increasing laser spot overlap can reduce peaktrough height variation and reducing overall top roughness as well as regulating a steadier melt pool [Niu \& Chang 1998; Mazumder et al. 2000; Childs 2005; Syed et al. 2006]. Fisher et al.[2003] found that increasing the repetition rate when processing titanium from a powder bed, generated a sufficient volume of molten material allowing surface tension forces to flatten out the melt pool surface. It has been reported that the 
generation of high peak powers with the use of pulsed lasers applies a mechanical component to material consolidation as high recoil pressures exert a force on the melt pool. This has been shown to reduce top surface roughness as well as side surface roughness, this is discussed further in section 3.4 .

\subsection{Vaporisation and Recoil Pressures}

SLM changes the state of metal powder from solid to liquid by heating it above its melting temperature $T_{m}$. However if material temperature exceeds the vaporisation temperature $T_{v a p}$ of the material it will subsequently transform into a gas. This metal vapour can interact with the incident laser beam causing it to ionise. This ionisation generates a plasma plume above the surface of the processed material. A plasma plume can absorb incident laser radiation and dissipate a significant fraction of the laser power before it reaches the target [Duley 1999]. The plasma plume continuues to absorb laser energy, generating a heat sustained shock wave that moves radially outward from the interaction site. The high pressure region immediately behind the shockwave exerts a recoil pressure on the melt pool [Morgan et al. 2004]. This increase in recoil pressure has been described as an "enforced wetting" of a melt pool to a substrate [ONeill et al. 1998]. Recoil pressure adds a mechanical element to material consolidation and increases in intensity with increasing peak power and surface temperature [Semak \& Matsunawa 1997]. Recoil pressure has been shown to improve the density of parts [ONeill et al. 1998; Kruth et al. 2004], increase molten material ejection from the melt pool [Fujinaga et al. 2000] (see section 3.4.1) and modify the shape of melt pool (see section 3.4.2). Too high a recoil pressure can also blow powder away from laser material interaction zone.

\subsubsection{Melt Pool Ejection}

High recoil pressures can eject material from the edge of the melt pool as shown in Figure 3-15. Park et al. [1999] examined the effects of plasma plume and spatter generation during the laser processing of metals. It was found that an increase in plasma plume generation is accompanied by an increase in ejected material from the melt pool known as spatter due to increases in recoil pressure. An increase in melt pool ejection is 
directly linked with excessive heat input, which increases the amount of plasma plume generated. The force generated by recoil pressure acts like a piston on the melt pool and forces the liquid out [Almen 1987]. Therefore suppression of plasma plume should also reduce spatter potential. The threshold hold value for melt ejection is determined by the surface tension force, which must be overcome by the radial pressure gradient [Rombouts 2006]. The bulk density of powder particles also affects melt pool ejection with particles of a lower density and thus lower mass being expelled more easily, melt thickness and the viscosity also influence ejection potential from the melt pool [Semak \& Matsunawa 1997].
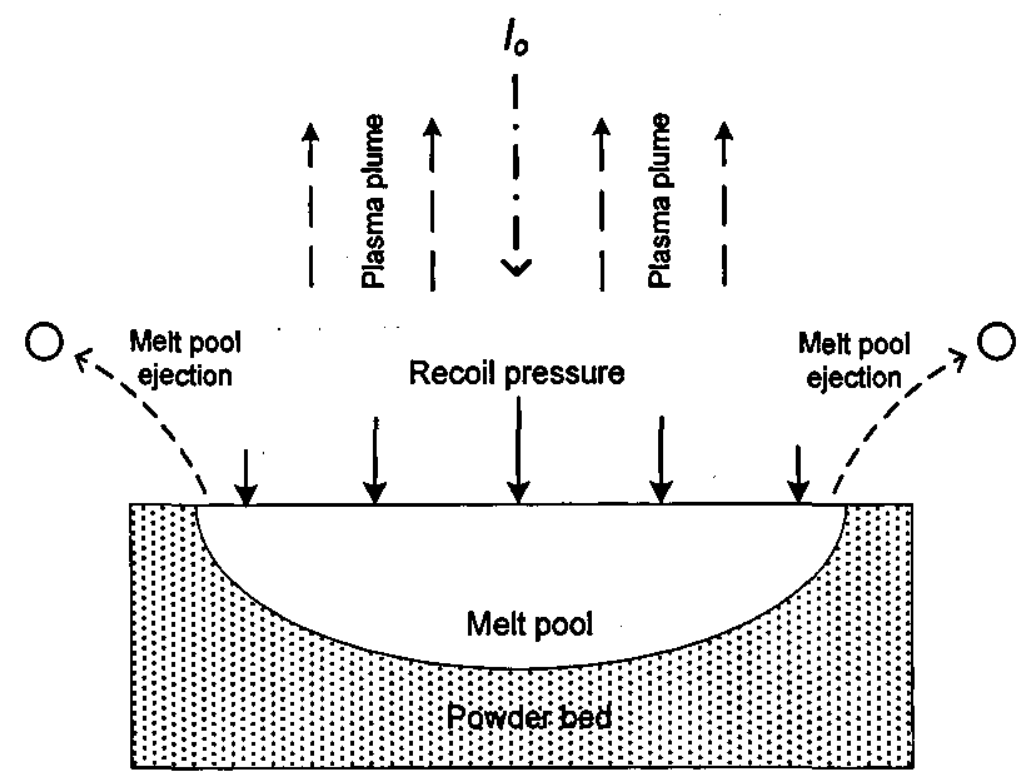

Figure 3-15 Melt pool ejection as a result of high recoil pressure on melt pool surface

Spatter has negative effects upon the SLM process. Vaporised and ejected material lowers the material yield available for processing [Kruth et al. 2004] and may cause part geometric inaccuracies to develop (possibly causing porosity). Spatter can form as contamination on a powder bed or increase surface roughness if impinged upon the outer surface of a part. Solidified spatter can also cause problems with powder deposition if it were to collide with a roller/scraper/slot feeder deposition system when flattening the powder bed and can cause failure of the entire fabrication. Spatter can also solidify directly below the laser beam trajectory (on protective glass or lens) interfering with laser beam delivery. Another explanation for melt pool expulsion may be related to the superheating of the gas trapped in the powder bed, which leads to a rapid gas expansion and finally in a removal of the liquid metal above it [Rombouts 2006]. 
Commercial laser melting powder bed processes (EOS M270, MTT Realizer, Concept LaserCUSING) have a degree of plasma plume formation and subsequently emit spatter during processing. Some of these systems employ an air knife located below the laser lens to displace any spatter from solidifying in and around the laser's optics system.

\subsubsection{Melt Pool Shape Modification}

The high peak powers generated with pulsed lasers can generate a recoil pressure that can exert an external force on the melt pool. This external force can overcome surface tension and convective stirring motions within the melt pool, modifying the melt pool from a circular shape to a flatter profile [Ohmura et al. 1997; Morgan et al. 2004; Fischer et al. 2005]. In studies conducted by Kruth et al. [2004] it was found that use of high peak powers suppressed the balling mechanism, improved interlayer connection and improved surface quality of SLM iron powder. The reduction in the balling mechanism may have occurred due to improvement of melt pool wetting onto the substrate. Mumtaz et al.[2008] used SLM to process waspaloy powder using an Nd:YAG pulsed laser. It was found that the use of higher peak powers (generated with the use of higher pulse energies at a fixed pulse duration) reduced the contact angle $\theta$ made between liquid-solid interface by flattening the melt pool and hence improving wetting characteristics as shown in Figure 3-16. A balance should be found between beneficial levels of recoil pressure leading to melt pool shape modification and excessive recoil pressures that can eventually lead to material expulsion [ONeill et al. 1998].
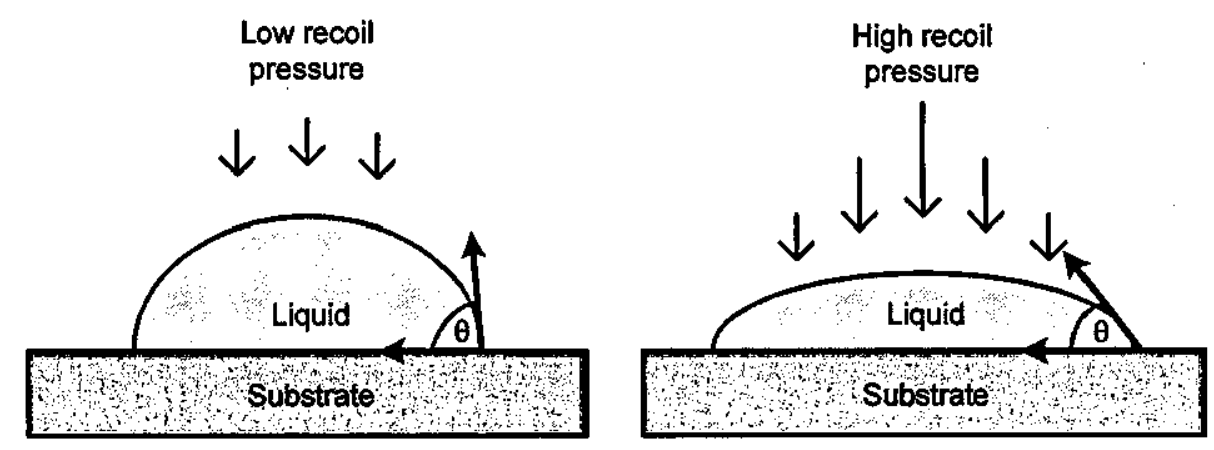

Figure 3-16 Melt pool modification influenced by external recoil pressure 


\subsection{Oxidation}

Oxidisation is the combining of an element with $\mathrm{O}_{2}$. A metal's surface is continually oxidising when exposed to the environment. Metal powder has a large surface area and therefore has an increased oxidisation potential compared to a flat surface. The extent to which oxidation occurs dramatically increases with a rise in temperature, especially when a metal is in a molten state:

$$
\Delta G=R T \ln p_{0_{2}}
$$

[Rombouts 2006] (3-10)

With $\Delta G$ being the free energy of the reaction (increasing values indicate increasing oxidation), $R$ is the universal gas constant, $T$ is the absolute temperature and $p_{02}$ being the partial pressure of $\mathrm{O}_{2}$. Oxidation can have a number of influences on SLM processing and part properties. As the laser energy absorption coefficient is greater for oxides than for pure metals, a sharp increase in absorption coefficient provides likely conditions for material overheating, which in turn intensifies the oxidation process [Xie \& Kar 1999; Yakovlev et al. 2005]. Oxidisation of a substrate or previously processed layer results in a lower surface tension $\gamma_{s v}$ and thus according to Equation (3-7) results in worse wetting by the melt pool [Rombouts 2006]. This can lead to reduced mechanical properties and increased part porosity [Kou 2003; Morgan et al. 2004; Gower et al. 2005].

\subsubsection{Inert Environment}

Protecting the molten metal from oxidisation can be achieved in one of two ways, material can either be processed within an inert gas chamber or an inert gas flow can be fed directly onto the molten material (shown in Figure 3-17). 


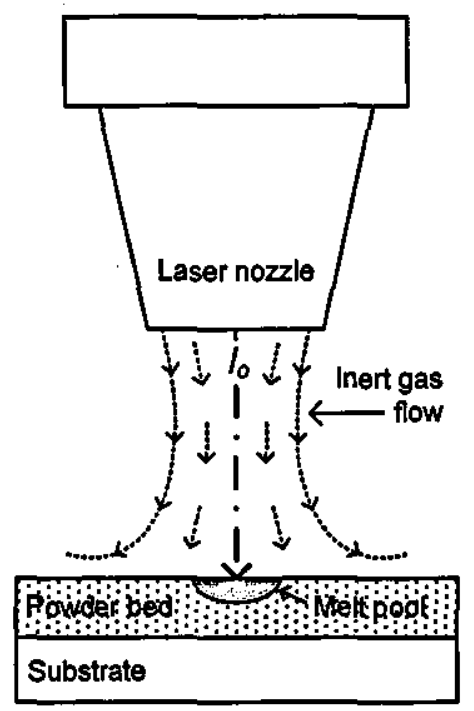

Figure 3-17 Inert gas shield fed from laser nozzle onto laser material interaction zone

Maintaining the material within an inert gas chamber is a secure method of reducing the $\mathrm{O}_{2}$ content within a processing environment. However it should be noted that inert gas chambers still can experience issues related to $\mathrm{O}_{2}$ being trapped within loose powder. This is because $\mathrm{O}_{2}$ partial pressure is relatively low and can still yield oxide formation [German 1994]. Inert gas chambers are more costly to run than use of a shielding gas, it also incurs a longer setup time and requires constant chamber pumping to ensure a movement of gas. The use of shielding gases within the laser processing of metals has many benefits. Apart from providing protection against oxidisation it also suppresses plasma plume formation, a reduction in spatter generation and protects the focusing optic [Duley 1999]. The inert gas can also cool the melt pool surface and influence melt pool shape if gas flow pressures are high enough to overcome thermocapillary flows.

\subsubsection{Inert Gases}

There are a variety of gases that can be used as a shielding gas, these include $\mathrm{H}_{e}, \mathrm{~N}_{2}$ and $\mathrm{A}_{\mathrm{r}}$. $\mathrm{N}_{2}$ can react and form nitrides with metals such as $\mathrm{Ti}, \mathrm{Mn}$ and $\mathrm{Cr}$ (elements within Inconel 625) significantly affecting the mechanical properties of a part [Kou 2003]. $\mathrm{H}_{e}$ is one of the more expensive inert gases and has high ionisation potential, therefore generating larger plasma plumes [Duley 1999]. $\mathrm{H}_{e}$ is lighter than $\mathrm{O}_{2}$ making it difficult to use as a shielding gas to protect the molten material. $A_{r}$ is a relatively inexpensive gas and is heavier than $\mathrm{O}_{2}$ enabling a more effective shielding and greater 
resistance to cross-draft [Messler 1999]. $A_{r}$ is used in inert gas chambers for commercial laser based processes such MTT Realizer, EOS M270 and is used within POM DMD as a gas shielding.

\subsection{Thermal Stresses}

SLM rapidly generates large heat intensities within a small area, due to non uniform heating, variable cooling, and repetitive heating cycles, a steep thermal gradient can develop across the powder bed. These thermal gradients induces stresses that cause dimensional inaccuracies due to shrinkage, solidification cracks and layer delamination [Karapatis 2002]. Cooled parts fabricated using SLM can contain residual stresses reducing its overall fatigue strength [Osakada \& Shiomi 2006]. Often parts are processed while employing powder bed preheating and may undergo further postprocessing (furnace cycles) to alleviate these stresses [Santos et al. 2006].

\subsubsection{Shrinkage}

Shrinkage and warping occurs as a material cools due to contraction, often leading to unacceptable dimensional tolerance losses and stress build up. Material shrinkage can affect part microstructure and may present many important stability problems because of internal stresses induced by the shrinkage of a new layer bonded to the cooler material below it [Karapatis et al. 1998]. The level of shrinkage can vary according to the material, laser processing parameters, build strategy and melting conditions [Jeng \& Lin 2003]. The effects of shrinkage can be reduced by decreasing laser spot size, decreasing pulse duration and increasing scan speed [Pohl et al. 2001]. Other ways of reducing the effects of shrinkage includes preheating the powder bed [Sexton et al. 2002] use a near zero shrinkage powder or use a powder presenting a highly controlled uniform shrinkage such as EOS Direct Steel 50 [Karapatis et al. 1998; Over 2003; Shellabear \& Nirhylä 2004]. CAD software can incorporate geometric adjustments to compensate and reduce the effect of part shrinkage. Niu et al.[1998] investigated the surface of laser melted steel powder and found that the size of formed agglomerates increases with increasing laser power. As a result the dimensional accuracy decreases with increasing laser power when used above a certain laser power threshold [Song 
1997; Niu \& Chang 1998]. Smaller layer thicknesses require less heat input to fully melt powder and will therefore be less prone to shrinkage.

\subsubsection{Solidification Cracking}

Solidification cracking (also known as hot cracking) result from the inability of the semi-solid material to accommodate the thermal shrinkage strains associated with solidification and cooling [Cieslak 2002]. It can be formed from thermal residual stresses that occur due to an over exposure to heat or rapid melt pool solidification time [Kruth et al. 2004; Shannon \& Li 2004; Zhong et al. 2005]. Cracks usually propagate along grain boundaries or interfaces between different materials. Cold cracks can also appear as a result of the contamination of the material by hydrogen. This can appear weeks or months after processing [Cieslak 2002]. Powder bed preheating can help reduce thermal gradients and therefore a materials susceptibility to undergo cracking [Sexton et al. 2002]. 


\section{Chapter 4 Principal Hypothesis \& Research \\ Objectives}

The fabrication of fully functional metal parts using laser based SFF is a difficult process to control. A number of processing issues can arise from the formation of a fully molten melt pool. The use of pulse shaping control within the laser welding of metal sheets offered an additional element of control over processing and was able to reduce the size of weld profiles, improve surface roughness etc. This research proposes that the additional degree of process control available by employing pulse shaping, can also improve the properties of SLM parts, especially part top/side surface roughness and part resolution.

A quantitative understanding of the relationship between independent process parameters (laser power, scan speed, repetition rate, etc.) and part properties should be undertaken to identify a usable processing window for Inconel 625. It is suggested that the use of high repetition rates and low scan speeds can improve top surface finish. However high repetition rates and low scan speeds have been shown to promote melt pool breakup and potentially increase surface roughness due to increases in melt pool width producing larger surface tension variations. A suitable balance between low top and side surface roughness can be achieved through varying scan speeds and repetition rates. Process parameters can be monitored and optimised continuously to obtain the desired part properties. It is suggested that the use of short pulse durations will produce less distorted parts due to the production of smaller melt pools.

It has been shown that use of pulse shaping can alleviate the problems associated with standard Rectangular pulses and improve the quality of parts. Controlling the heat input to the material can produce a specified volume of liquid which maintains its liquid form for a desired period of time without overheating or significantly increasing the width of the melt pool. Different pulse shapes can gradually heat a material such that the spatter generated during processing is also reduced. A prolonged melt pool solidification period 
may allow more time for molten material to spread, improving its top surface roughness and wetting characteristics.

\subsection{Research Novelty}

The majority of academic work processing Inconel 625 powder using lasers has been conducted using powder feed processes [Nakao et al. 1993; Griffith et al. 2000; Xue et al. 2000; Sexton et al. 2002; Xue 2006; Paul et al. 2007]. Limited academic work has focused on processing Inconel 625 directly from a powder bed. Das et al.[1998] processed Inconel 625 from a powder bed using indirect SLS. This research post processed parts using Hot Isostatic Pressing (HIP) to reduce part porosity from 1.5 to $0.5 \%$. Yadroitsev et al.[2007] used Phenix Systems SLM process to directly fabricate Inconel 625 test parts, which were tested for mechanical properties. Little research has produced systematic works on the relationship between the process parameters and the formation of laser processed supernickel parts [Wu et al. 2004].

Research has shown that different laser process parameters affect melt pool instabilities (i.e "balling") [Karapatis 2002; Hauser 2003; Hauser et al. 2003; Kruth et al. 2004; Hauser et al. 2005; Rombouts et al. 2006] that can contribute to SLM part surface roughness. Limited in depth research has simultaneously investigated the relationship between top and side roughness of SLM parts. These results would further increase understanding of laser material processing within SLM as well as strengthening the optimisation procedure.

Pulse shaping has been researched within the field of laser welding of metal sheets. Numerous benefits have been found, including a reduction in spatter generation, reduction in surface roughness, minimal crack propagation and minimal porosity inclusion within welds [Katayama et al. 1993; Fujinaga et al. 2000; Gower et al. 2005]. The majority of this work had focused on the use of steels and aluminium. Sparks et al. [2006] is the only researcher to employ pulse shape control during laser processing of metal powders. Sparks et al. [2006] preliminary research examined the effect pulse shaping had on the microstructure of laser processed tool steel parts processed using 
powder injection deposition. No research has been conducted using pulse shaping control to process material with a laser using a powder bed deposition approach.

\subsection{Aims and Objectives}

This research focused on producing thin walled Inconel 625 parts using the SLM process while applying pulse shaping and non pulse shaping control techniques. The SLM equipment used (GSI Lumonics JK701H Nd:YAG laser) had been previously used to process a variety of materials such as tool steel, copper, waspaloy and zirconia powders [Su et al. 2003; Mumtaz et al. 2007; Mumtaz et al. 2008]. The effects various pulse shape energy distributions had on the property of thin wall single tracks (top/side surface roughness and width) were examined. A number of objectives were set:

1. Establish a useable laser processing parameter window for successful melting of Inconel 625 without generating excessive spatter during processing.

2. Observe effects of laser pulse energy, pulse duration, repetition rate and scan speed on part top/side surface roughness and width.

3. Optimise processing parameters and produce thin wall part with low top and side surface roughness while maintaining a low wall width.

4. Employ pulse shaping control working primarily with Ramp Up and Ramp Down energy distributions and examine effects on part top/side surface roughness and width.

5. Identify pulse shapes most effective in improving part properties and validate by producing larger test parts with different geometries.

6. Develop a new in depth understanding into laser/material interactions and the development of top/side surface roughness and width of parts.

7. Publish and present work. 


\section{Chapter 5 System Characterisation}

This chapter details the SLM apparatus used within this investigation including its capabilities, beam size and processing adjustments. Details are also given about the material processed within this investigation. The remainder of the chapter focuses on adjustments made to the existing system in an effort to improve the SLM processing standard before major experimentation took place.

\subsection{System Setup and Processing}

The SLM system used within this investigation includes a GSI Lumonics JK701H solid state Nd:YAG laser, this can be seen in Figure 5-1. The laser has a TEM 00 Gaussian profile, a maximum power output of $550 \mathrm{~W}$. The laser head remains static during processing but can move along the z-axis prior to processing. A processing table within the systems housing (see Figure 5-2) can move along the $x-y$ axis during processing and is driven by a 2-axis CNC controlled machine. The laser pulses are generated by electrically pumping flash lamps that are located above the system housing. The system control centre regulates and monitors the laser parameters and movement of the CNC controlled machine. The system also displays a plan view of the processing bed through a charger coupling device camera to ensure that focusing and alignment of the laser is accurate.

Figure 5-2 shows the main SLM apparatus within the processing area of the system. The pulses of laser light generated by the flash lamps are fed through a $\emptyset 1 \mathrm{~mm}$ silica optical fibre delivery cable to the laser head and are deflected through $90^{\circ}$ by a reflecting mirror onto a focusing optic. The light is focused and passes through the laser nozzle onto the work piece. The inert gas flow rate of argon is regulated using a gas flow meter. The inert gas is fed through pipes into the laser nozzle where it shrouds the processing area. 


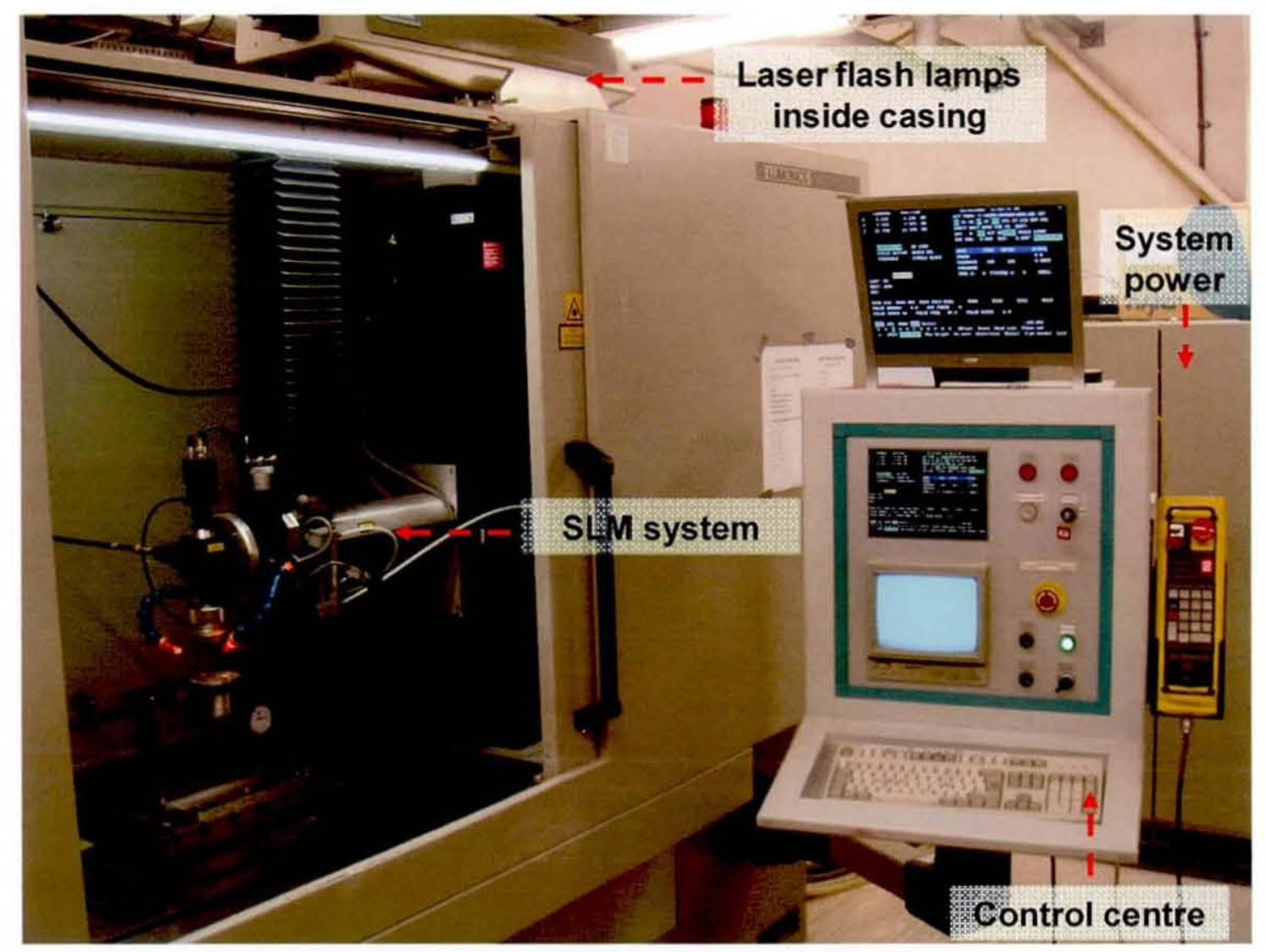

Figure 5-1 GSI Lumonics JK701H Nd:YAG laser system

The processing table shown in Figure 5-2 and Figure 5-3 was modified to facilitate lower layer thickness deposition. The previous processing table consisted of a scissor lift that raised the processing table up and down. This apparatus produced large inaccuracies in deposited powder layer thicknesses not allowing thin layers to be reliably deposited. The new piston based processing table allows powder layers as thin as $50 \mu \mathrm{m}$ in thickness to be accurately and consistently deposited. A lower layer thickness reduces the required heat input to melt material as well as reducing the surface roughness of parts due to the stair stepping effect [Shellabear \& Nirhylä 2004]. 


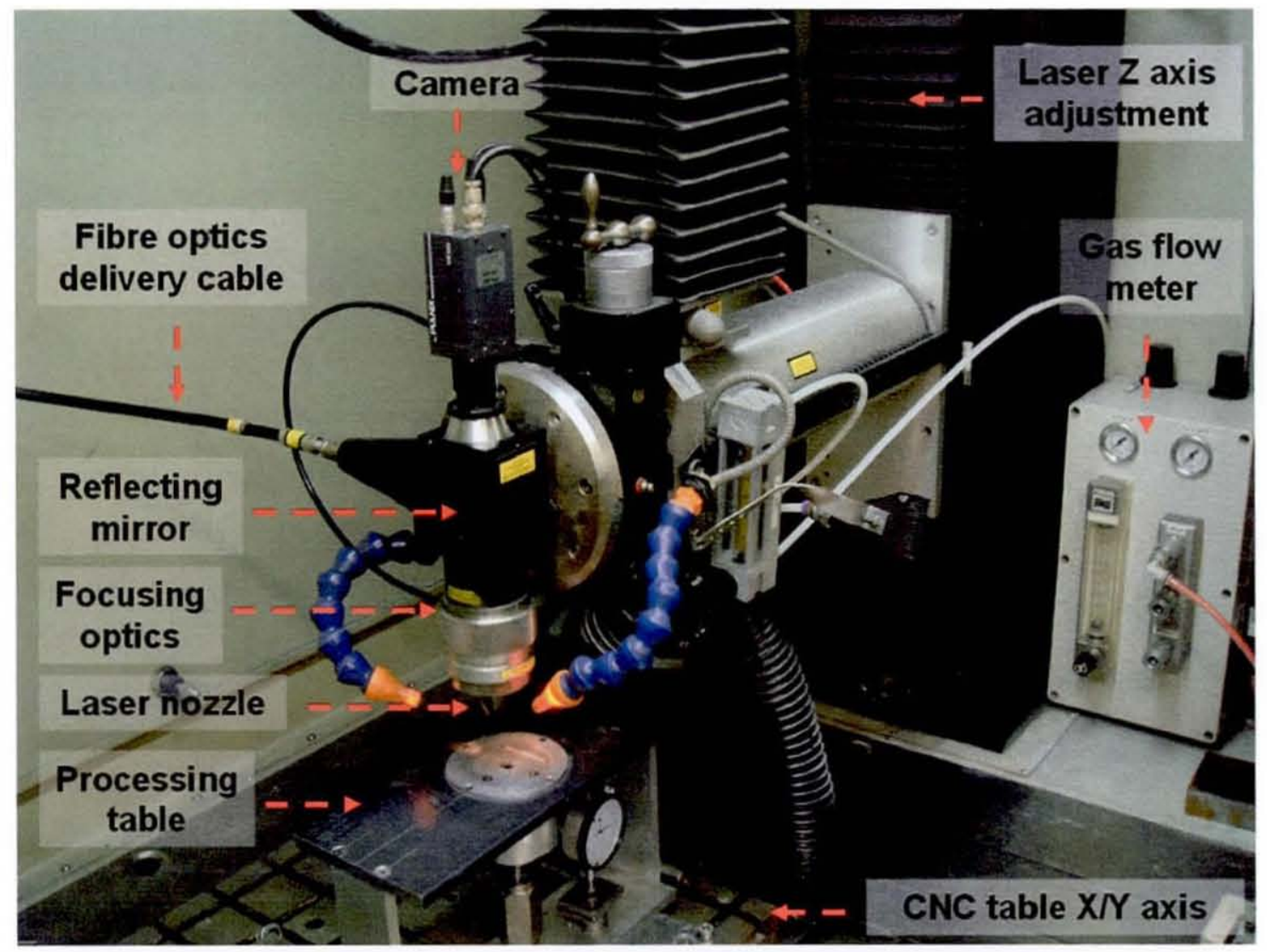

Figure 5-2 Main selective laser melting apparatus

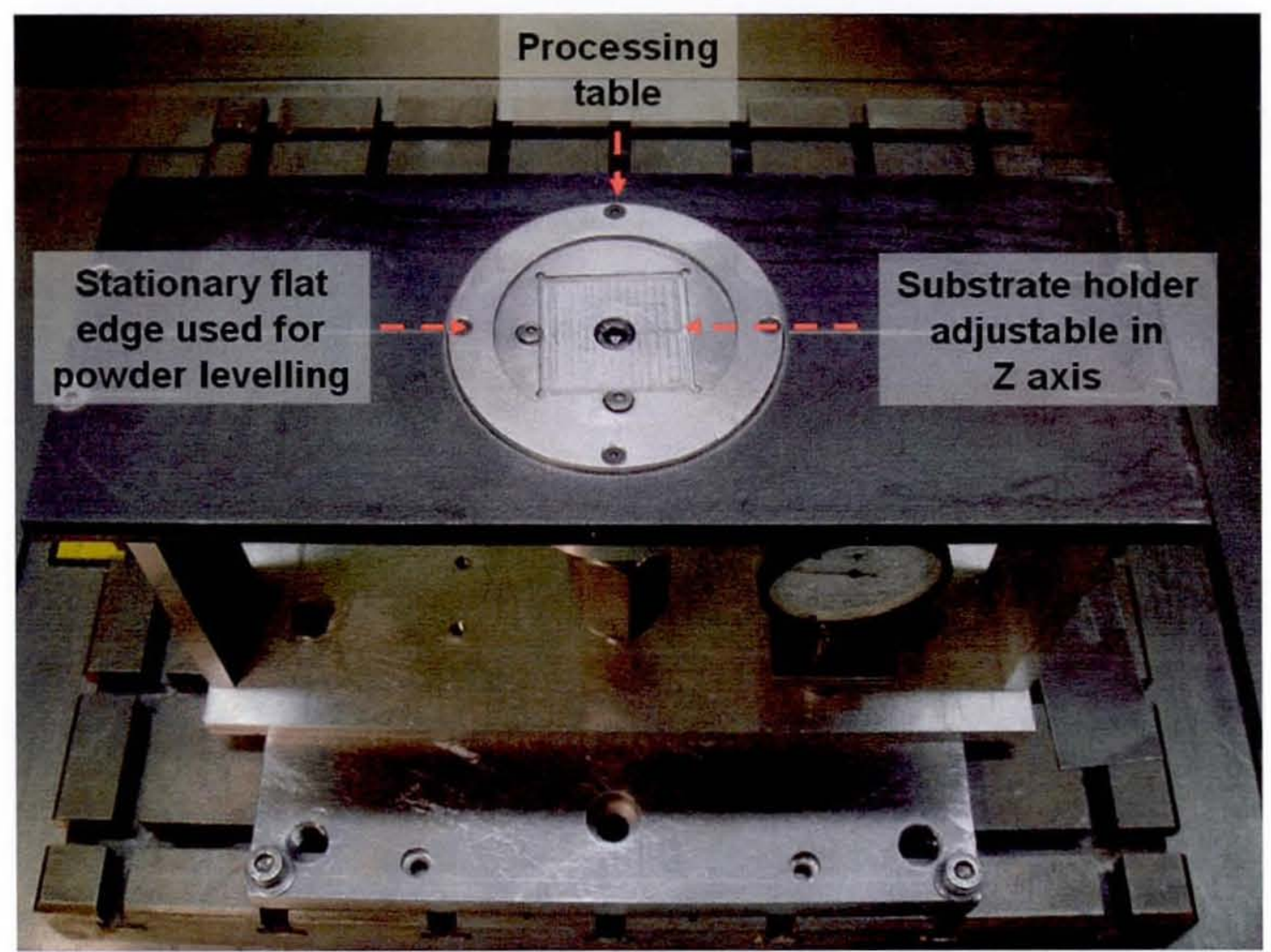

Figure 5-3 Plan view of processing table 


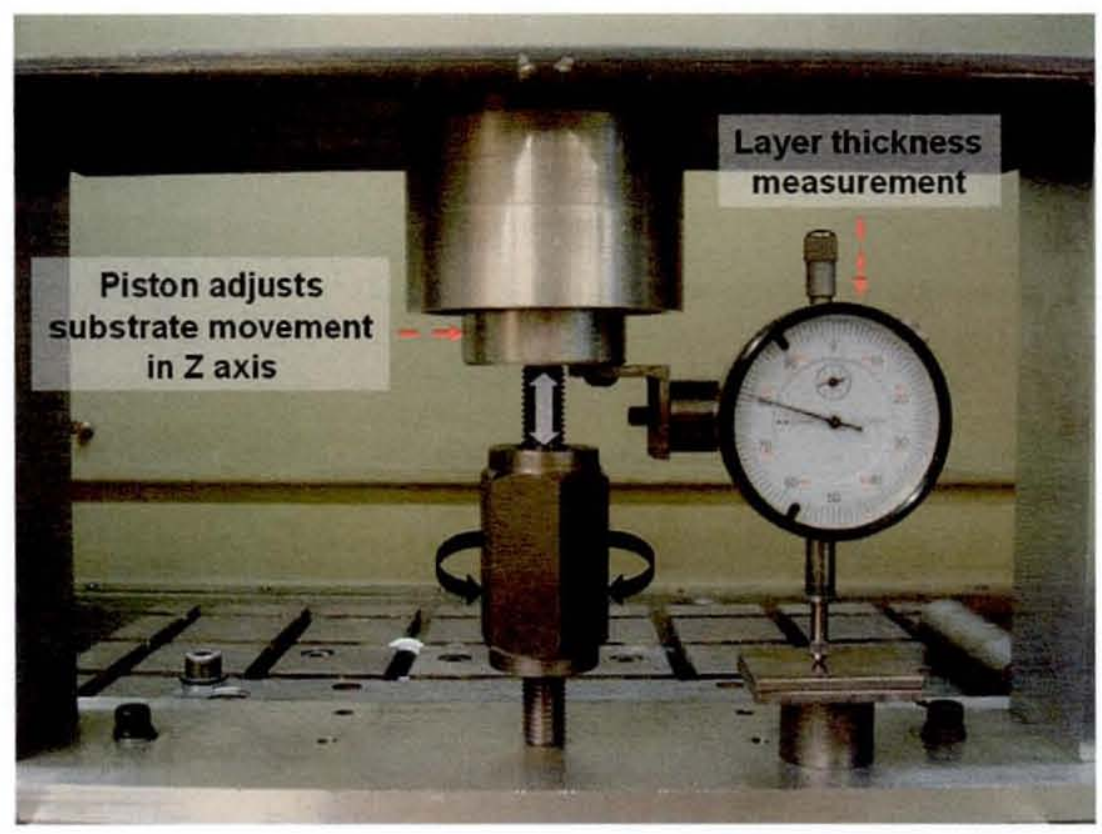

Figure 5-4 Side view of processing table

Figure 5-5 illustrates the powder deposition methodology. The adjustable piston was lowered a set distance and a funnel was used to manually deposit powder onto the substrate. Levelling of powder is achieved using a steel cylinder that is manually moved across the powder bed while counter rotating.

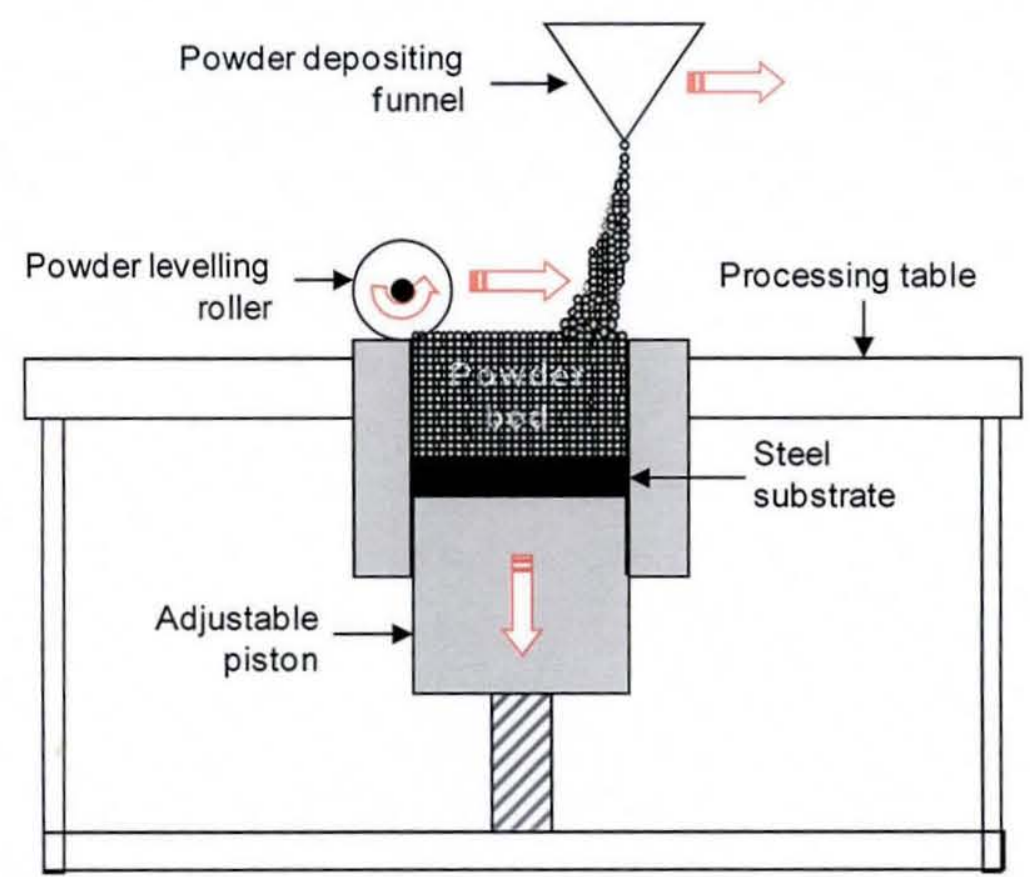

Figure 5-5 Side view of processing table with powder deposition \& levelling methodology 
The actual placement of a substrate onto the processing table and deposition of $0.1 \mathrm{~mm}$ Inconel 625 is shown in Figure 5-6. The substrate was secured in place by two lock nuts. Figure 5-7 shows the alignment of the laser nozzle with the powder bed prior to processing.
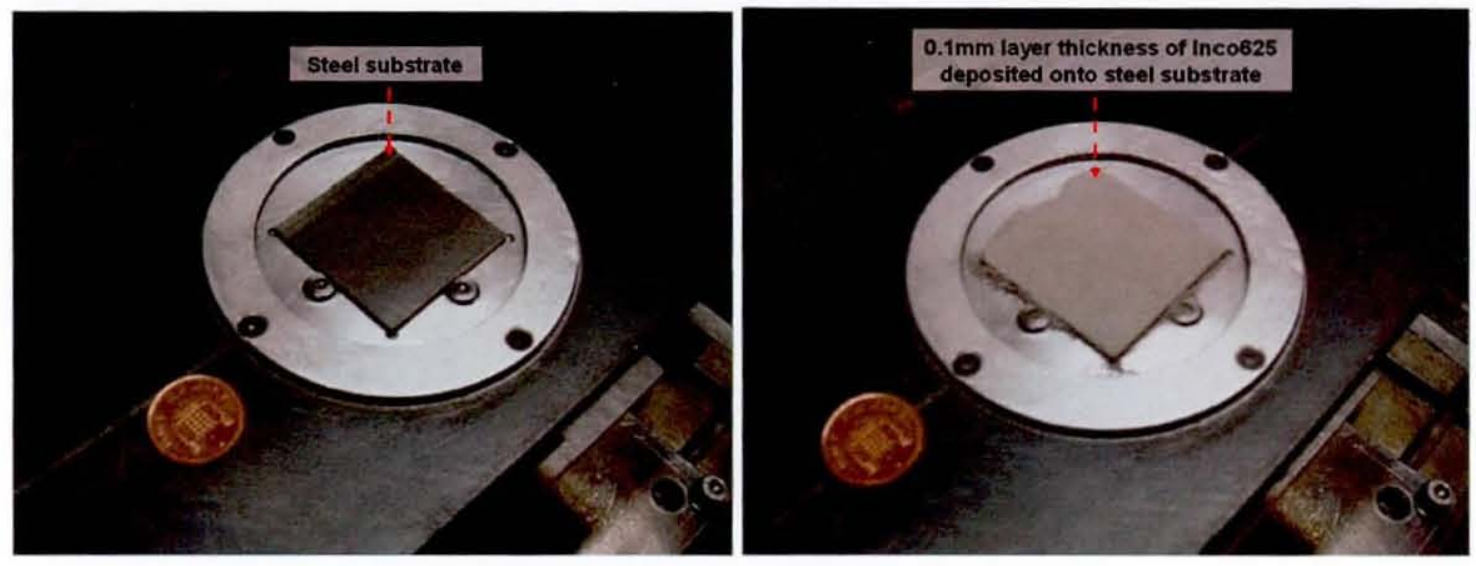

Figure 5-6 Substrate placement on processing table \& $0.1 \mathrm{~mm}$ powder deposition

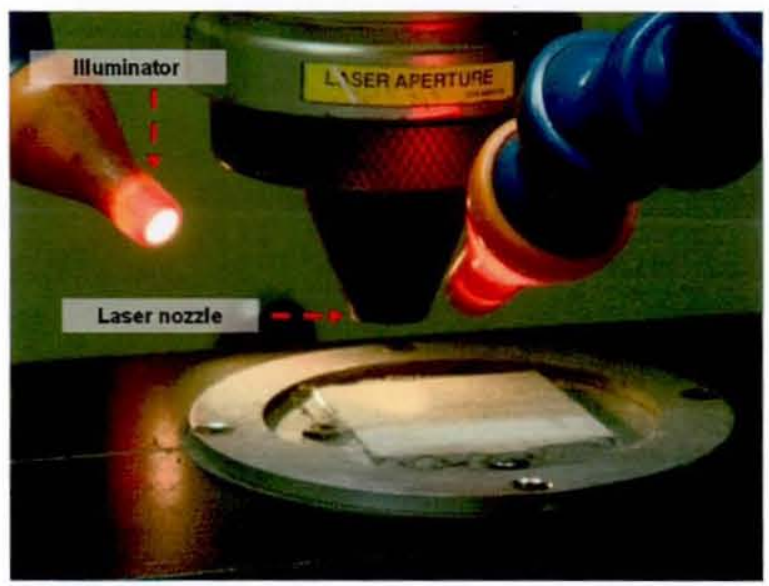

Figure 5-7 Laser nozzle alignment with powder bed prior to processing

\subsubsection{System Capabilities and Working Principle}

The Nd:YAG laser emits laser energy in pulses. Each pulse has a set duration of exposure known as pulse duration (ms) and contains a defined pulse energy (J). This pulse can be repeatedly emitted at a set repetition rate $(\mathrm{Hz})$. The speed at which the processing table moves relative to the laser is known as the scan speed ( $\mathrm{mm} / \mathrm{min})$. From these control parameters a number of processing factors can be derived, these include spot overlap (\%), peak power (W), average power (W) and specific energy density 
$\left(\mathrm{J} / \mathrm{mm}^{2}\right)$. The full range of the SLM system's control parameters are shown in Table 5-1.

\begin{tabular}{ll}
\hline Variable & Range/Value \\
\hline Pulsed Laser & \\
\hline Pulse Duration & $0.5-20 \mathrm{~ms}$ \\
Pulse Energy & $0.1-70 \mathrm{~J}$ \\
Pulse Repetition Rate & $0.2-500 \mathrm{~Hz}$ \\
Average Power & $0-550 \mathrm{~W}$ \\
\hline Processing Table and laser head range of movement & \\
\hline Powder Bed Thickness & $0.01-100 \mathrm{~mm}$ (z-axis) \\
X-axis & $0.1-600 \mathrm{~mm}$ \\
Y-axis & $0.1-450 \mathrm{~mm}$ \\
Laser Head Z-axis & $0.1-450 \mathrm{~mm}$ \\
Table Scan Speed & $1-20000 \mathrm{~mm} / \mathrm{min}$ \\
\hline Other & \\
\hline Spot Size (theoretical) & $0.8 \mathrm{~mm}$ \\
Spot Overlap & $0-100 \%$ \\
Gas Flow Rate & $0-50 \mathrm{ml} / \mathrm{s}$ \\
\hline
\end{tabular}

Table 5-1 Full range of GSI Lumonics JK701H Nd:YAG laser control parameters

\subsubsection{Beam Quality and Spot Size}

This section describes an experiment used to improve beam quality by determining the optimum focal distance. The laser spot size was also determined experimentally.

\subsubsection{Focal distance adjustment}

The Lumonics JK701 Nd:YAG used within this investigation underwent a full maintenance service by Lumonics engineers prior to experimentation. Maintenance included the full adjustment of the beam expander telescope collimator to reduce laser spot size and improve the quality of delivered beam. This adjustment caused there to be a change in the previously set optimum focal distance (achieved at a laser height of $382.5 \mathrm{~mm}$ from the powder surface [Su 2002]). Figure 5-8 shows that a minimal beam diameter is achieved at an optimal focal distance. 


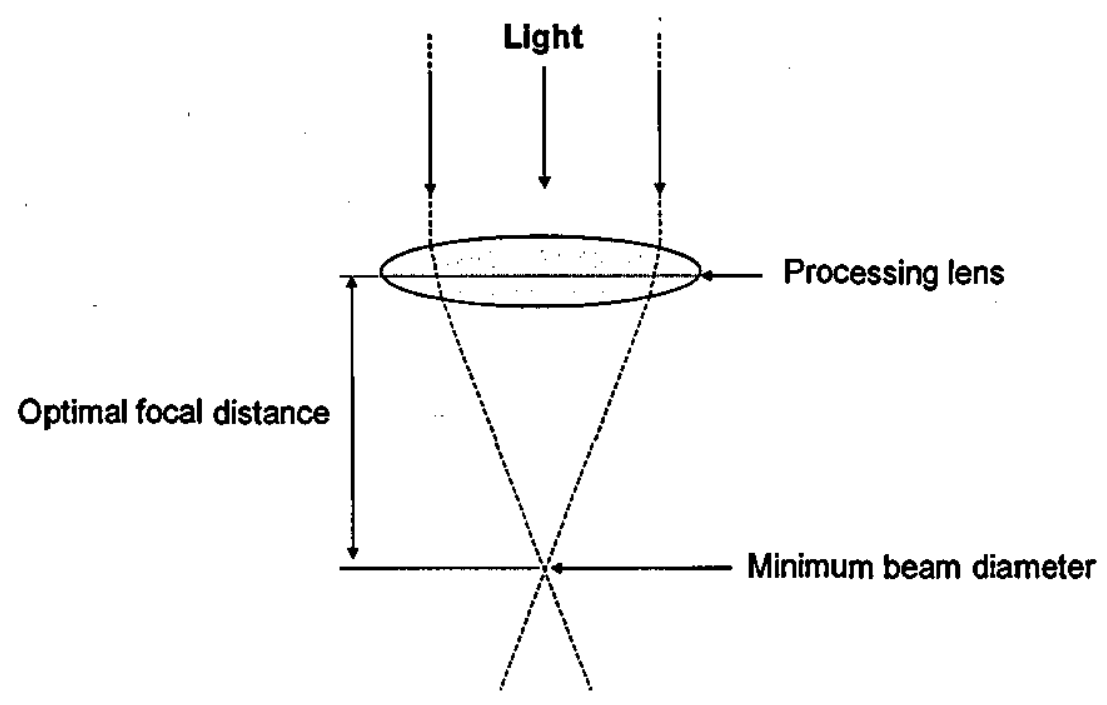

Figure 5-8 Lens focal distance

\section{Methodology}

A focal distance optimisation methodology recommended by GSI Lumonics [Lumonics 1995] was used to re-establish the correct focal distance and maintain a good quality beam with minimal spot size. A pulse duration of $0.5 \mathrm{~ms}$ and a pulse energy of $0.5 \mathrm{~J}$ was used to fire single pulsed shots at a stainless steel substrate (melting temperature $1400^{\circ} \mathrm{C}$, measuring $3 \mathrm{~mm}$ in thickness). Stainless steel was used due to it being readily available and cheap to purchase. This generated a peak power of $1000 \mathrm{~W}$, this laser energy was not sufficient to generate enough heat to penetrate the $3 \mathrm{~mm}$ substrate, however it would enable melting at the surface of the substrate forming a melted circular profile resembling the laser's TEM profile. This would serve as a reference point as the focal distance was adjusted (circular melted profile proportional to focused diameter of laser at workpiece). The laser height was adjusted in the $\mathrm{z}$ axis $2.5 \mathrm{~mm}$ above and below the previously set optimal height $(382.5 \mathrm{~mm})$, this gave a range of $5 \mathrm{~mm}$ to work within. The spot size would vary as laser height increased and decreased due to the adjustment of focal distance. The laser height that produced a minimum spot size would produce the optimal focal distance. Spot sizes were measured optically using a Nikon microscope.

\section{Results}

Figure 5-9(a) shows the spot size of single laser pulses as the laser head is moved at variable distances from the steel substrate. Note the distance in Figure 5-9 is the 
distance the laser head was from the moveable platform as given by computer control system. As the laser approached a height of $383-384 \mathrm{~mm}$ the spot size was at its lowest value. A more detailed examination of this $383-384 \mathrm{~mm}$ region was measured and results are shown in Figure 5-9(b). Within this $383-384 \mathrm{~mm}$ region single pulsed shots were fired at stainless steel and Kapton film (melting temperature $400^{\circ} \mathrm{C}, 1 \mathrm{~mm}$ thickness) to test result consistency when processing different materials. Note, both materials top surface was brought to the same level, flush with the top of the processing table. A laser height of $383.6 \mathrm{~mm}$ produced a minimum spot size in both steel and Kapton film, with spot sizes of $0.46 \mathrm{~mm}$ and $0.56 \mathrm{~mm}$ respectively. The reason for variation in spot size generated on steel and Kapton film is explained in section 5.1.2.2. It can be concluded that a laser height of $383.6 \mathrm{~mm}$ consistently produced the smallest spot size for this laser system due to the distance between the processing lens and substrate being correctly focused (matching the focal length of the processing lens). This is a $1.1 \mathrm{~mm}$ increase from the previously set laser height. The focusing optic in the charger coupling device camera was adjusted such that the camera would focus clearly on the work piece when optimal focal distance was achieved.
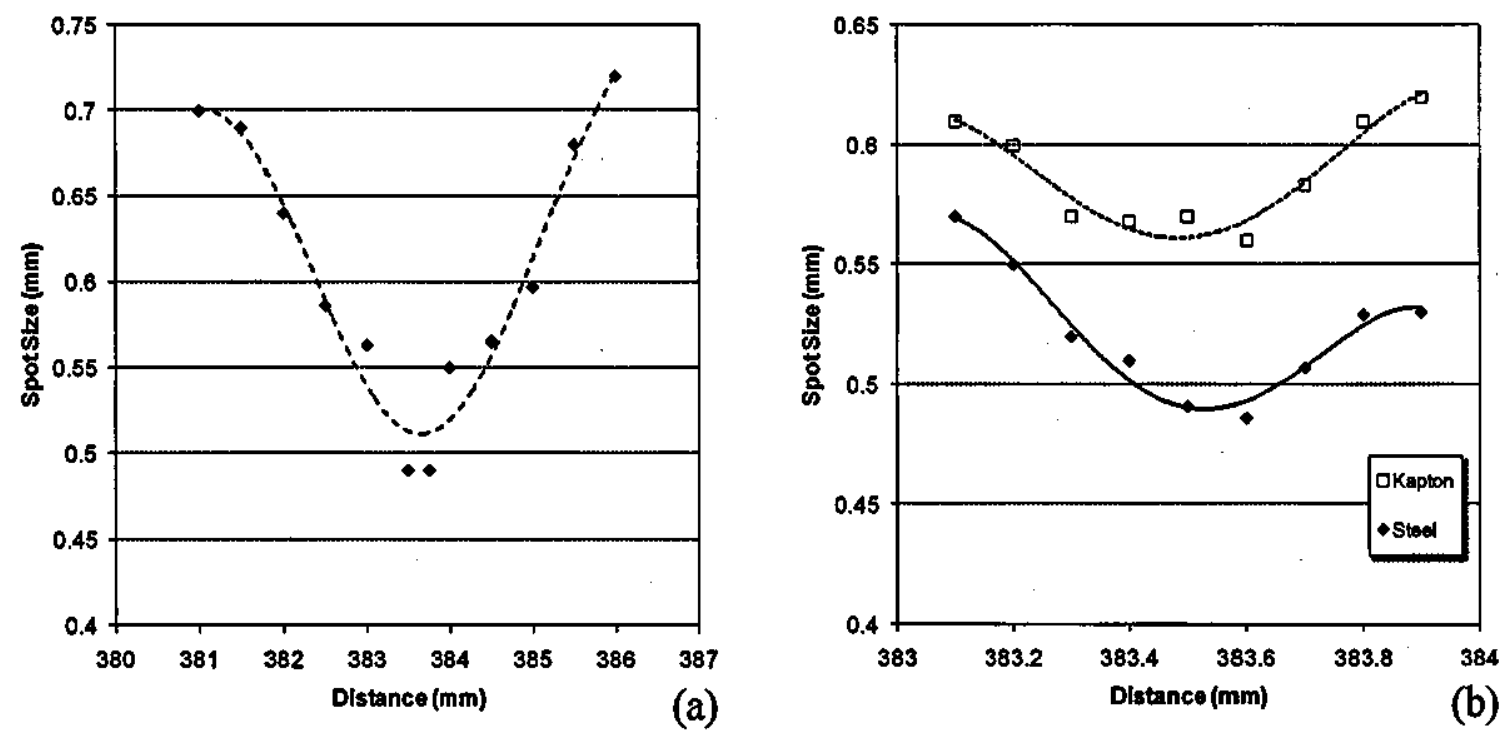

Figure 5-9 Spot size against focal distance for steel (a) \& Kapton/Steel at refined focal distance region (b) 


\subsubsection{Laser spot size}

It is important to determine the experimental laser spot size in order to evaluate capabilities of the system. There are generally two interpretations for the spot size of a laser beam. This can be the calculated diameter of the beam at the surface of the work piece, based on knowledge of laser beam characteristics and parameters of optical focusing system. Alternatively this can be the actual diameter of the weld, cut or hole made on a specific material. The theoretical spot size $(s)$ can be calculated by knowing the laser's focal length, beam diameter at front mirror/plate, beam divergence and using equation (3-11).

$$
s=d f\left(\frac{\Theta}{D}\right)
$$

With $d$ representing the beam diameter at front mirror $(10 \mathrm{~mm}), f$ focal length of process lens $(80 \mathrm{~mm}), D$ beam diameter at laser head front plate $(16 \mathrm{~mm})$ and $\Theta$ beam divergence of the laser $(0.016 \mathrm{rad} / \mathrm{s})$. The theoretical spot size for the $\mathrm{Nd}: \mathrm{YAG}$ laser was calculated to be $0.8 \mathrm{~mm}$.

This spot size does not dictate the size to which features can be melted as this depends on laser powers and material properties. Figure 5-9 in section 5.1.2.1 illustrated that there was a variation between the attained spot size when firing single laser pulses at stainless steel and Kapton film with an optimal focus and when similar laser powers were used. A fixed energy input will have more of a melting effect on Kapton film than stainless steel due to absolute melting temperature variation, thermal conductivity, specific heat capacity, reflectivity and material thickness. The heat generated by a single laser pulse on Kapton film continues to melt the film after the pulse has been fired. This continual radial movement of heat via conduction would not necessarily represent an accurate value for spot size of the laser beam due to excessive melting. The phenomenon of continual radial melting of material is less of an issue within metals processing due to its higher melting temperatures and higher latent heat of fusion. The variation in attained spot sizes is not solely restricted to the type of material used. It can vary according to laser power parameters due to the laser's Gaussian distribution [Lumonics 1995]. 


\section{Methodology}

Tests were conducted using the full range of pulse energies and pulse durations available to the system to fire single pulses onto steel substrates and Kapton film. The spot sizes were measured optically using a Nikon microscope.

\section{Results}

The variation in spot size produced on steel substrates and Kapton film are shown in Figure 5-10. Low pulse energies produced spot sizes of $0.3-0.45 \mathrm{~mm}$ on steel. Short pulse durations combined with low pulse energies did not generate enough heat to melt the surface of steel. Increased pulse energies and pulse durations produced larger melted areas or spot sizes as more heat was delivered to the material and more time was given for heat to conduct and spread out away from the centre of the laser spot. The spot sizes produced on the Kapton film greatly surpassed the theoretical spot size of $0.8 \mathrm{~mm}$ with the use of long pulse duration and increased pulse energy. Using a pulse energy of $10 \mathrm{~J}$ and $20 \mathrm{~ms}$ pulse duration the laser melted a circular profile on the Kapton film that measured $1.9 \mathrm{~mm}$ in diameter. This is over double the theoretical spot size of the laser, this in itself proves that the theoretical spot size cannot be used to directly determine the accuracy or resolution to which parts can be made. Thermal gradients can induce the thermal lensing effect causing further variations in attained spot size [Rombouts 2006].
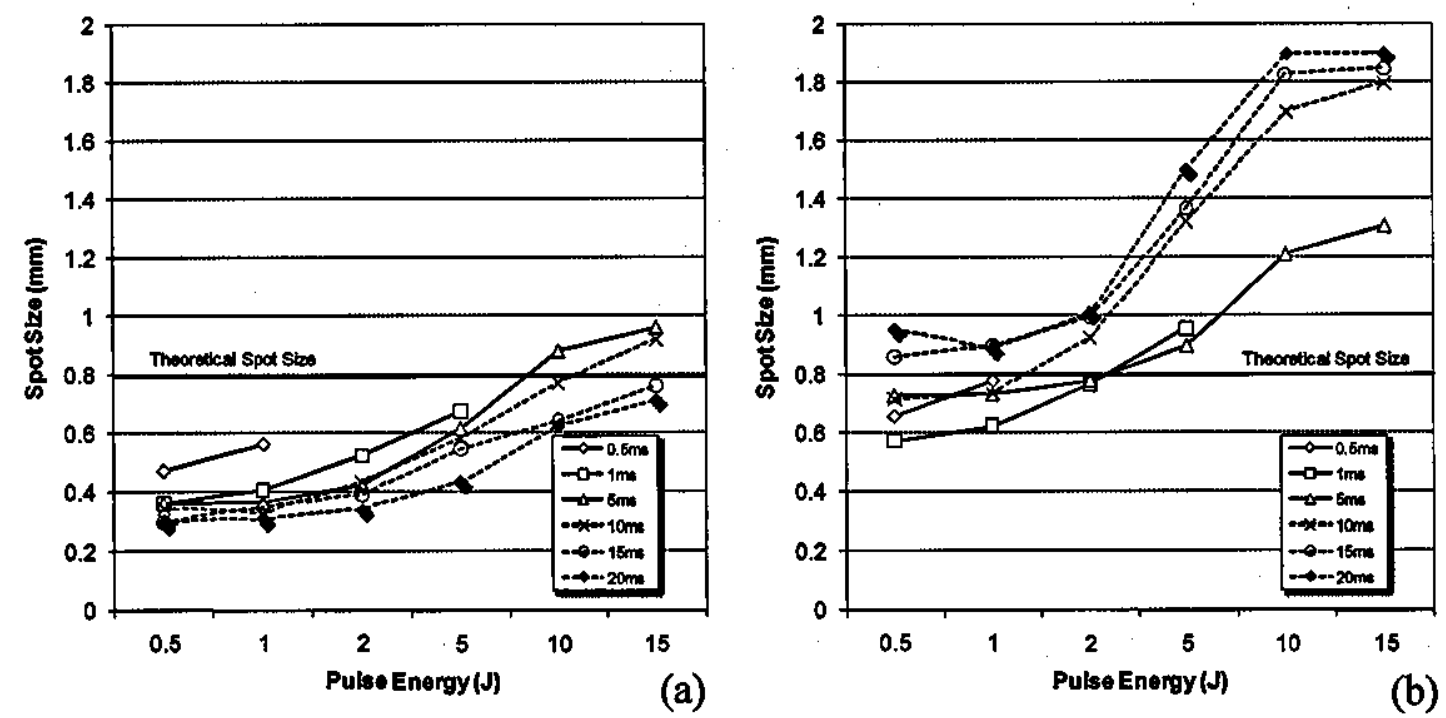

Figure 5-10 Spot size against pulse energy on steel (a) \& Kapton film (b) 
Figure 5-11(a) shows the results of a single laser shot on steel using a high pulse duration and high energy. A number of concentric circles can be seen radiating from the centre of the spot. A central area approximately $0.25 \mathrm{~mm}$ in diameter appears to produce the greatest melting effect. The pattern consists of a bright central spot surrounded by rings of rapidly diminishing intensity. This is a result of the Airy disk phenomenon, with the majority of the laser's energy being distributed within the centre of the laser spot [Dragesco 1995]. The heat intensity reduces as the distance from the centre of the spot is increased. In the absence of any lens imperfection, $84 \%$ of the energy is contained within the central spot and the remainder is distributed within the outer rings [Miller 1994]. Figure 5-11(b) shows a schematic of the central spot that contains the majority of the laser energy.
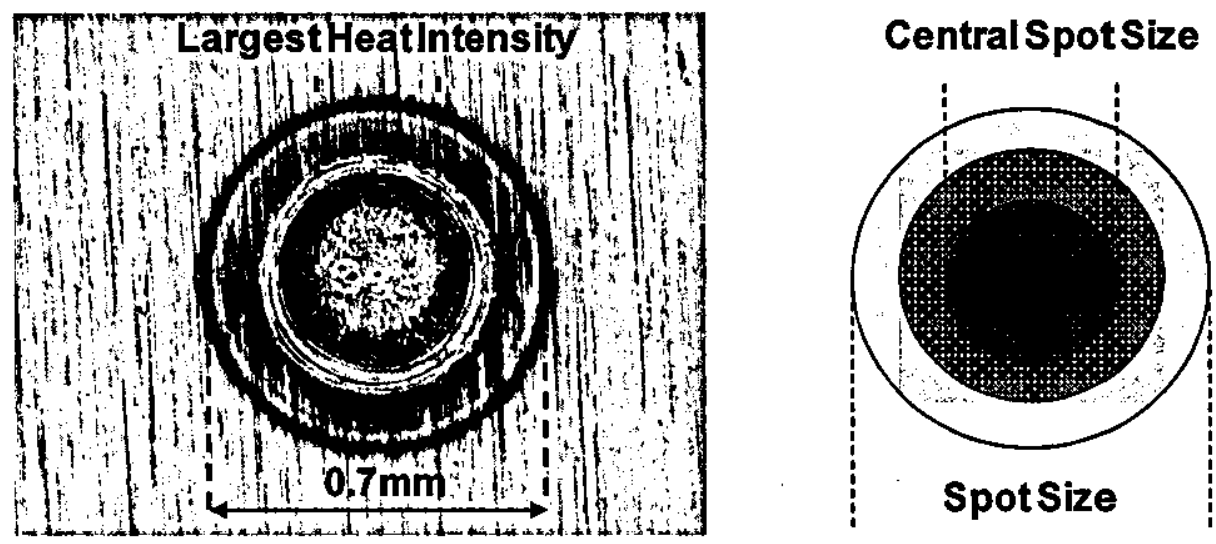

(a)

Figure 5-11 Single laser shot on stainless steel at $20 \mathrm{~ms} 15 \mathrm{~J}(750 \mathrm{~W}$ ) (a) \& laser beam intensity (b)

\subsubsection{Summary}

A smaller theoretical spot size will generally produce a smaller melt area and smaller parts, however smaller spot sizes can lead to increased material vaporisation (if energy density is too high) and slower part fabrication times. Regardless of theoretical spot size or material, an increase in pulse energy and pulse duration tends to increase the size of melted areas with the majority of heat being delivered from within the central portion of the laser beam. 


\subsection{Material Characterisation}

Inconel 625 is a nickel based superalloy that has a melting temperature of approximately $1290-1350^{\circ} \mathrm{C}$. Inconel 625 inherently has a high tensile, rupture and thermal fatigue strength [Metals 2006]. This material typically suits applications that require high strength at elevated temperatures or a degree of oxidation/corrosive resistance. It has been used in high temperature applications such as heat exchangers and gas turbine components with extensive usage within the aerospace industry. It has been used in aircraft ducting systems, engine exhaust systems, fuel and hydraulic line tubing, turbine shroud rings, and heat-exchanger tubing in environmental control systems [Das et al. 1998; Shankar et al. 2001; Arafin et al. 2007]. The high alloy content of Inconel 625 also gives it good corrosive properties, making it an ideal choice for sea-water applications and some acid process equipment [Metals 2006]. Inconel 625 can withstand many corrosive environments such as alkaline, salt and fresh water. It is used as wire rope for mooring cables, propeller blades for motor patrol gunboats, submarine auxiliary propulsion motors, submarine quick-disconnect fittings and exhaust ducts for Navy utility boats.

\subsubsection{Inconel 625 Powder Properties}

This section describes the properties of Inconel 625 powder used within this investigation. This includes material chemical composition determined by Energy Dispersive Spectroscopy (EDS) using a Stereoscan 360. The EDS analysis presents a variety of information regarding a samples chemical composition. The materials particle size distribution is also shown, this was determined using a Malvern Mastersizer.

\subsubsection{Chemical composition}

The Inconel 625 powder used within this research was acquired from Special Metals Corporation based within the UK. The Inconel 625 powder was fabricated using argon gas atomisation. This assures that the oxygen content introduced into the manufacturing procedure was minimised. Figure 5-12 shows the chemical composition of Inconel 625 measured using EDS. The material contains twelve different material elements, approximately $58 \%$ nickel, $20-23 \%$ chromium, and $8-10 \%$ molybdenum. Chromium 
provides resistance to oxidation at high temperatures, molybdenum provides resistance to pitting and crevice corrosion. Phosphorus improves the wetting behaviour of iron and reduces the effects of oxidisation. Because of the different elements contained within Inconel 625 , it is envisioned that some of the low melting temperature elements will vaporise prior to full melting. The EDS analysis revealed that the powder contained traces of $\mathrm{O}_{2}(2 \%)$. The powder was originally received from Special Metals Corporation in sealed vacuum containers. It is assumed because of powder handling namely exposing powder to the environment $\left(\mathrm{O}_{2}\right)$ caused the surface of the powder to oxidise.

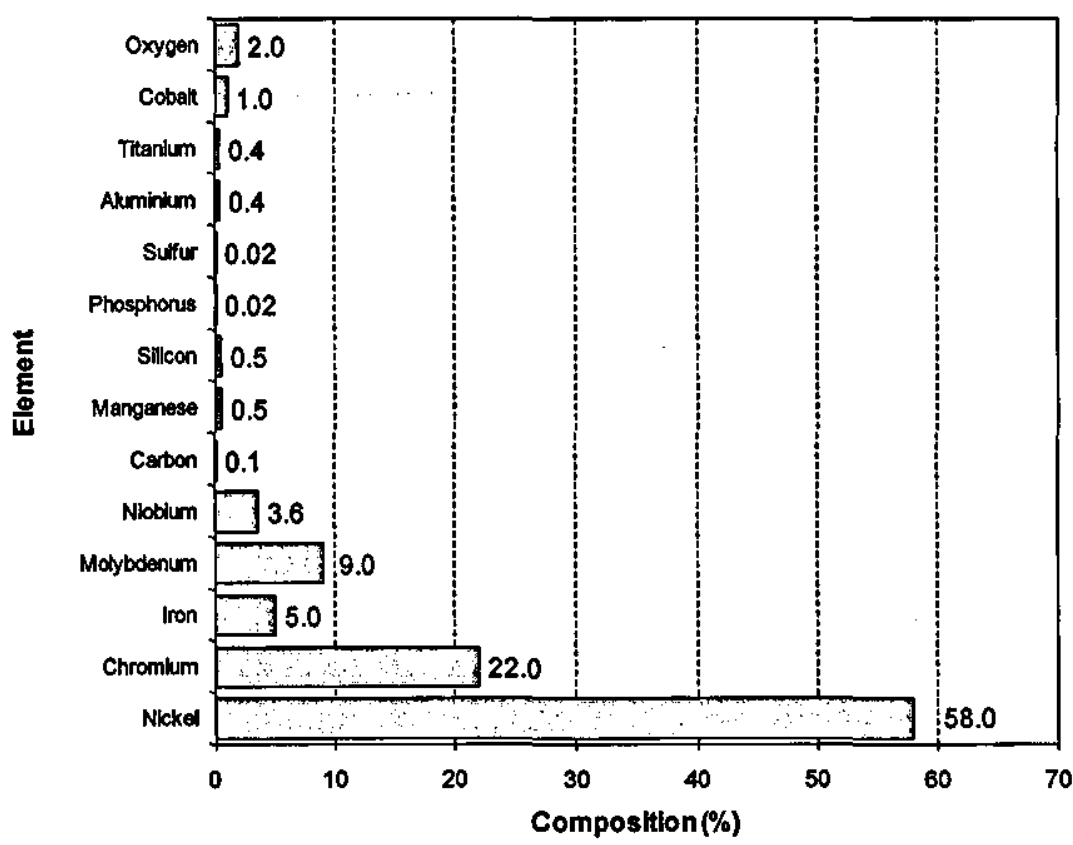

Figure 5-12 Inconel 625 chemical composition obtained from EDS

\subsubsection{Particle size distribution}

The Inconel 625 powder was supplied sized to $-53 \pm 25 \mu \mathrm{m}$. The choice of powder size was selected after considering the powder sizes used by other researchers and the quality of parts they were able to produce using powder bed processes. Consultation from Special Metals Corporation also played a role in the selection of material size. The exact PSD was measured to be $\mathrm{D}(\mathrm{v}, 0.1)$ of $22.51 \mu \mathrm{m}, \mathrm{D}(\mathrm{v}, 0.5)$ of $45.48 \mu \mathrm{m}$ and $\mathrm{D}(\mathrm{v}, 0.9)$ of $58.59 \mu \mathrm{m}$. This data was acquired using a Malvern Mastersizer, this distribution is shown in Figure 5-13. 


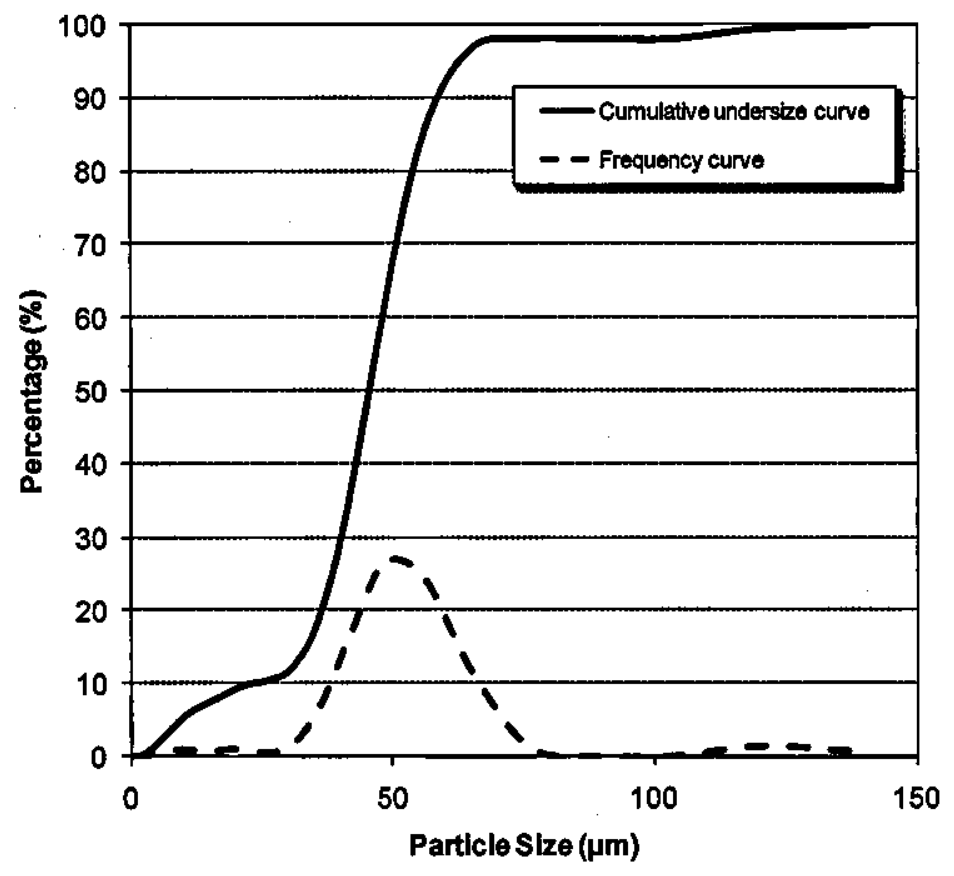

Figure 5-13 Inconel 625 particle size distribution

Figure 5-14 (a) and (b) show SEM images of the Inconel 625 powder at magnification $\mathrm{X} 200$ and X1300 respectively. There appear to be no pore inclusions within the powder particles. The absence of pore inclusions is an important factor in reducing the overall surface area per unit volume of the powder and hence susceptibility to absorb contaminants [Das 1998]. The powder was highly spherical and should increase powder packing density and improve flow characteristics when deposited onto a SLM processing bed [Das 1998]. It was extremely important that the powder could be easily deposited onto a processing bed without agglomerating. Other batches of powder with smaller particle sizes were trialed but were rendered unusable due to agglomerations. Figure 5-15(a) shows an example of a failed batch of Inconel 625 that suffered from powder agglomeration. This batch had an average particle size of $14 \mu \mathrm{m}$ and a particle size distribution of $\mathrm{D}(\mathrm{v}, 0.1)$ of $2.8 \mu \mathrm{m}, \mathrm{D}(\mathrm{v}, 0.5)$ of $9.88 \mu \mathrm{m}$ and $\mathrm{D}(\mathrm{v}, 0.9)$ of $25.9 \mu \mathrm{m}$. Due to the smaller size of particles the powder would stick and agglomerate together due to surface tension forces. The powder could not be consistently spread onto a processing bed and would pull away previously spread layers as top surface layers were flattened with a roller (or flat edge scraper). Figure 5-15(b) shows a comparison of the unusable batch of Inconel 625 that had agglomerated with the successful batch showing signs of improved flowability. 


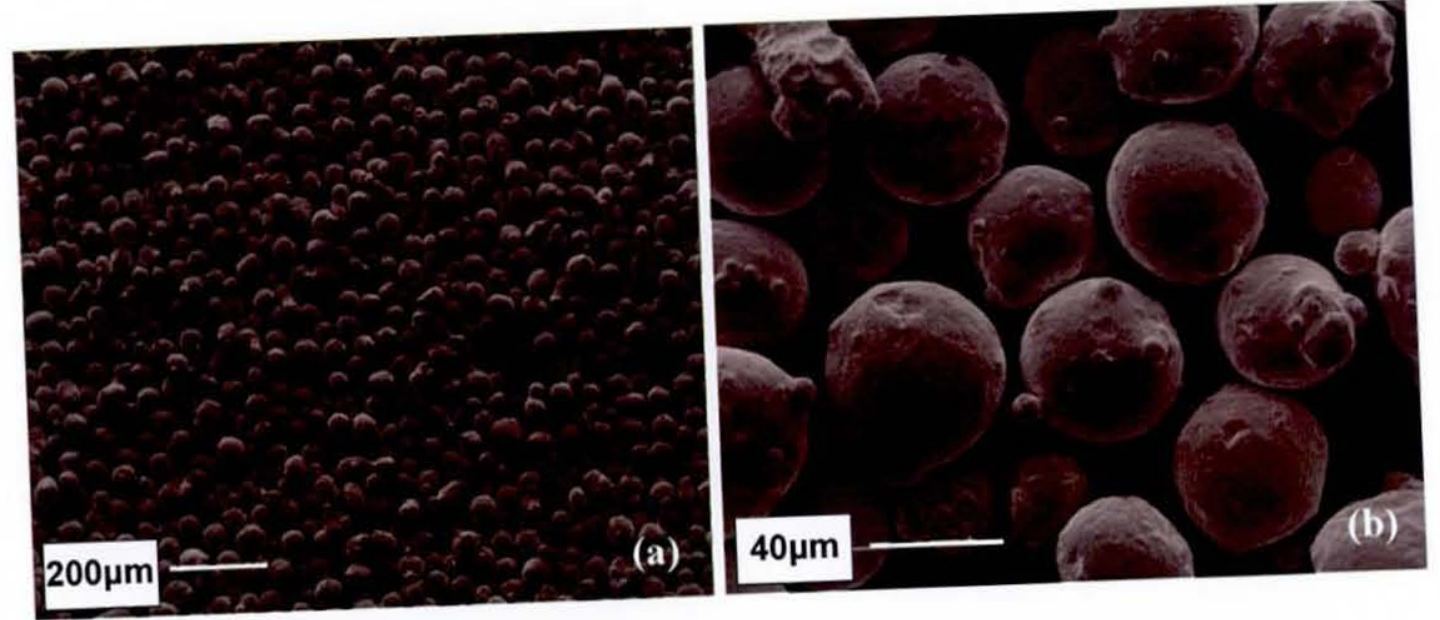

Figure 5-14 SEM image of Inconel 625 powder at X200 (a) \& X1300 (b)
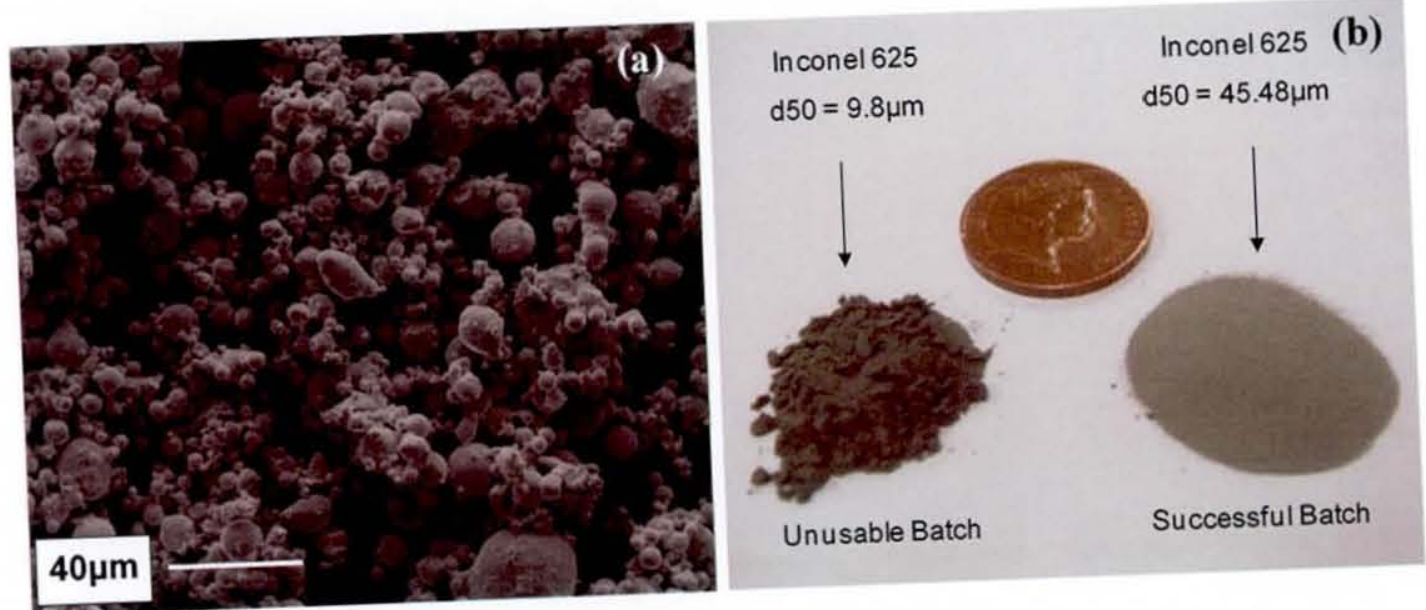

Figure 5-15 SEM image of failed batch of Inconel 625 powder at X1300 (a) \& comparison between different powder sizes (b) 


\subsection{System Adjustments}

This section summarises processing improvements made to the SLM system prior to main experiments. The inert gas flow that shields the processing region from the effects of oxidisation was optimised to provide improved protection. An electrostatic effect that was observed to displace powder away from the laser material interaction zone was also neutralised.

\subsubsection{Inert Gas Optimisation}

Tests were conducted to optimise inert gas shielding of Inconel 625 powder during processing. The optimisation of gas flow rate would minimise any potential defects produced as a result of insufficient gas shielding. Experimental results indicated that an $A_{r}$ gas flow rate of $10 \mathrm{ml} / \mathrm{s}$ directly above the laser material interaction zone minimised the melt pool oxidisation potential. Full experimental methodology and results for inert gas optimisation are covered in Appendix 12.1.

\subsubsection{Electrostatic effect}

During experimentation it was observed that un-melted powder was being displaced in and around the laser material interaction zone. This even occurred at low energy densities and when the inert gas flow was set to zero. This displacement of loose powder was attributed to an electrostatic effect that built up during processing. Appendix 12.2 details the identification of the electrostatic effect and the method employed to remove this during processing. 


\section{Chapter 6 Rectangular Pulse Optimisation Experiments}

Chapter 2.3 demonstrated that SLM is a complex process and that a variety of processing issues can arise and affect the formation of metal parts. With the use of a new material it is critical to have information on how the material will behave when processed using certain laser process parameters. This knowledge can be most readily attained through practical experience and will subsequently minimise and reduce any processing setbacks. The operator will be able to quickly identify any problems within part fabrication and make suitable decisions to improve the process. This will allow for successful, reliable and repeatable metal fabrication [Griffith et al. 1999].

The use of a new material presents a new set of uncertainties. At this early stage it was important to identify a precise mapping of the processing window/region in which Inconel 625 successfully melts. The identification of an operating window allowed further experiments to focus within this defined range and eventually optimise parameters such that thin walled parts with low top and side surface roughness were produced. This was achieved in two stages, the first identified an operating window for which Inconel 625 would successfully melt without under melting the material or delivering excessive heat input, causing material to vaporise and produce excess spatter during processing. The second part investigated key parameters identified from the literature search by qualitative and quantitative methods to develop an optimal set of process parameters that produced thin wall parts with minimum top and side surface roughness. Table 6-1 shows the main experimental stages used to optimise processing parameters required to produce thin walled Inconel 625 parts with low top and side roughness. Pulse shape control techniques were at this stage not used and therefore the laser's standard Rectangular pulse energy distribution was used throughout this Chapter. 


\begin{tabular}{lll}
\hline Experimental Stage & Objective & Deliverables \\
\hline Inconel 625 Operating & Identify Usable processing & Process single layers of \\
Window & parameter region in which Inconel. Identify usable pulse \\
Melting region & melts without under/over process regions. Select pulse \\
(section 6.1.1) & melting or generating durations that minimise melt \\
& excessive spatter. Observe pool width in order to produce \\
& pulse durations that generate parts to a high resolution \\
& low melt pool width & \\
Spot overlap region & Using the identified pulse Identify useable repetition \\
(section 6.1.2) & energy/duration operating rates and scan speed within \\
& window, vary repetition rate pulse energy/duration region \\
& and scan speeds. Identify already identified \\
& useable repetition rate and & \\
scan speed operating window & \\
in which excessive energy \\
densities are not generated.
\end{tabular}

Table 6-1 Chapter 6 optimisation experiments

\subsection{Inconel 625 Operating Window}

The methods employed for the complete melting of metal varies from process to process and material to material. Within SLM a large variety of processing parameters can dramatically affect the level heat delivered to a powder bed. Within early testing it is important to identify a usable processing parameter region and diagnose as early as possible any problems that may arise due to the use of certain laser parameters. These problems included insufficient heating, material vaporisation and excessive spatter generation during processing. 


\subsubsection{Methodology}

Experiments were undertaken to identify the laser operating window in which Inconel 625 would fully melt, over/under heat and emit excessive spatter during processing (for spatter testing see section 6.1.1.1). The widths of the specimens were also examined with the aim of selecting processing parameters capable of minimising melt pool width and facilitate the future production of high resolution parts.

A $0.1 \mathrm{~mm}$ layer thickness of Inconel 625 was deposited onto a steel substrate (as described in Chapter 5) and a single laser scan measuring $25 \mathrm{~mm}$ in length was performed (note, steel substrates were used instead of Inconel 625 substrates due to the lower purchasing cost). At this stage solely the pulse duration and pulse energy were varied as only a general operating window in which to work within was required, the full range of processing parameters used is shown in Table 6-2. These process parameters represent the full range of pulse energies and pulse durations available on the Nd:YAG laser (generating energy densities of $7.6-18.8 \mathrm{~J} / \mathrm{mm}^{2}$ ). The repetition rate was maintained at $10 \mathrm{~Hz}$ with a scan speed of $168 \mathrm{~mm} / \mathrm{min}$ generating a spot overlap of $65 \%$. These repetition rates and scans speed parameters were initially chosen due to their successful use in previous research when producing high density, crack free waspaloy parts using the same SLM apparatus [Mumtaz et al. 2008].

\begin{tabular}{llll}
\hline Pulse Energy $(\mathrm{J})$ & Pulse Duration $(\mathrm{ms})$ & $\begin{array}{l}\text { Repetition Rate } \\
(\mathrm{Hz})\end{array}$ & $\begin{array}{l}\text { Scan Speed } \\
(\mathrm{mm} / \mathrm{min})\end{array}$ \\
\hline $0.1-15$ (varied) & $0.5-20($ varied $)$ & $10($ fixed $)$ & 168 (fixed) \\
\hline
\end{tabular}

Table 6-2 Laser processing parameters used within melting region experiments

Design of Experiments (DOE) methods such as Taguchi, factorial analysis and doptimal are statistical techniques used to study the effects of multiple variables simultaneously. DOE methods assist in the reduction of experimental runs when many processing parameters exist. However, by employing a DOE methodology such as Taguchi or d-optimal the strength of derived parameters relationships may not be as strong as full factorial experimental runs (running the full number of experimental runs). Therefore all experiments conducted within this research were completed using a full factorial approach. Only one sample for each set of test conditions was produced. 


\subsubsection{Spatter characterisation}

Section 3.4 discussed that increases in plasma plume directly related to the amount of spatter ejected from the laser material interaction zone due to excessive recoil pressure [Park \& Rhee 1999]. A reduction in plasma plume is therefore beneficial in reducing melt pool expulsion and all issues that arise from it (section 3.4). Monitoring the relationship between levels of plasma plume generated and the amount of spatter solidified on top of the powder bed led to the identification of an acceptable level of plasma plume height before spatter generation became excessive.

A Sony digital video camera (Sony DSC-T30) was mounted on a fixed tripod 32 inches directly in front of the processing area. In all tests the camera's position remained fixed (including elevation and processing table start position) relative to the processing area. The digital camera recorded the laser processing throughout each experimental run at 25 frames per second and recorded for approximately 4 seconds. The acquired video was split into individual frames using a video editing software (Adobe Premier Elements®) and visually monitored for maximum plasma plume formation, this was generally observed as an increase in vertical height reached by plasma from processing area. The individual frames that emitted the largest plasma plume during processing were selected for further examination using ImageTool analysis software. Using the software's spatial calibration function the height of the plasma plume could be accurately measured. An example of an individual frame is shown in Figure 6-1(a), a vertical scale and crosshairs have been superimposed onto the centre of the laser processing area. This illustrates the area from which the plasma plume was measured including the maximum height that could be measured for a plasma plume $(14 \mathrm{~mm}$, reaching laser nozzle head). Tests were conducted observing the level of plasma plume and the amount of spatter contaminants solidified on the powder bed. Figure 6-1(b) shows a bright plasma plume (characteristic of an ion plasma) reaching approximately $5 \mathrm{~mm}$ in height when Inconel $625(0.5 \mathrm{~ms} \&$ $0.6 \mathrm{~J})$. This level of plasma produced little if no contamination on top of the powder bed due to low spatter ejection. Figure 6-1(c) shows an increased plasma plume intensity reaching halfway to the laser nozzle at $7 \mathrm{~mm}$ in height $(0.5 \mathrm{~ms} \& 1 \mathrm{~J})$. Increasing the pulse energy increased plasma plume intensity and therefore spatter ejection from the laser material interaction zone. Plasma plumes that reached $7 \mathrm{~mm}$ and above in height visibly increased the amount spatter generated and solidified contaminants on the 
processing bed. Figure 6-1(d) shows the powder bed contamination produced as a result of the $7 \mathrm{~mm}$ in height plasma plume. The black solidified material scattered around the powder bed surface is ejected spatter that has oxidised. As discussed earlier, the spatter contaminates the powder bed and can also impinge on external surfaces increasing surface roughness and potentially reducing the accuracy of parts. After examining the direct relationship between the height of a plasma plume and amount of powder bed contamination produced, a plasma plume height of $7 \mathrm{~mm}$ and above was identified to cause an "undesirable level in material spatter" with the processing parameters that produced this deemed unusable for further use. Plasma plumes below $7 \mathrm{~mm}$ in height produced no notable signs of powder bed contamination. Author acknowledges that definition of "undesirable level in material spatter" is open to interpretation. However it was based upon experience and allowed a qualified figure ( $7 \mathrm{~mm}$ plasma plume height) to be identified as a quality benchmark for further experiments.
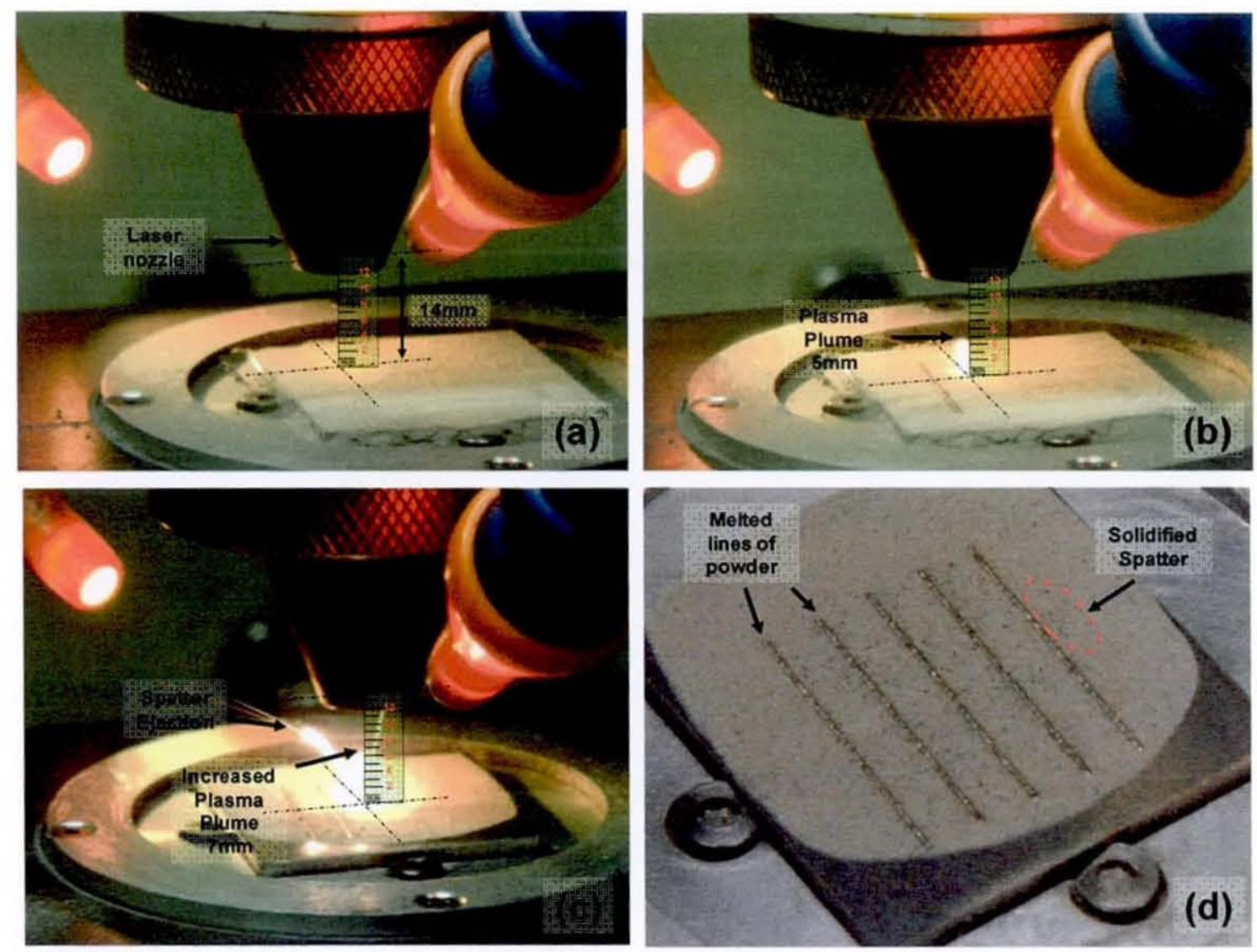

Figure 6-1 Plasma plume measurement $(\mathrm{a}, \mathrm{b}, \mathrm{c})$ \& powder bed contamination due to undesirable level of material spatter (d) 


\subsubsection{Width measurements}

Due to the samples being processing from only a $0.1 \mathrm{~mm}$ powder bed thickness, there were difficulties in physically measuring the sample widths using digital callipers. The widths were therefore measured using a Flash optical measurement device. This equipment automatically measured the distance between multiple points along the entire length of the samples outer profile when viewed from above. The same operating conditions were applied to all samples during Flash optical measurement $(47 \mathrm{x}$ magnification, 22 surface light, 3 ring light, 73 backlight) A screenshot of the software used to capture data points and measure average sample width is shown in Figure 6-2.

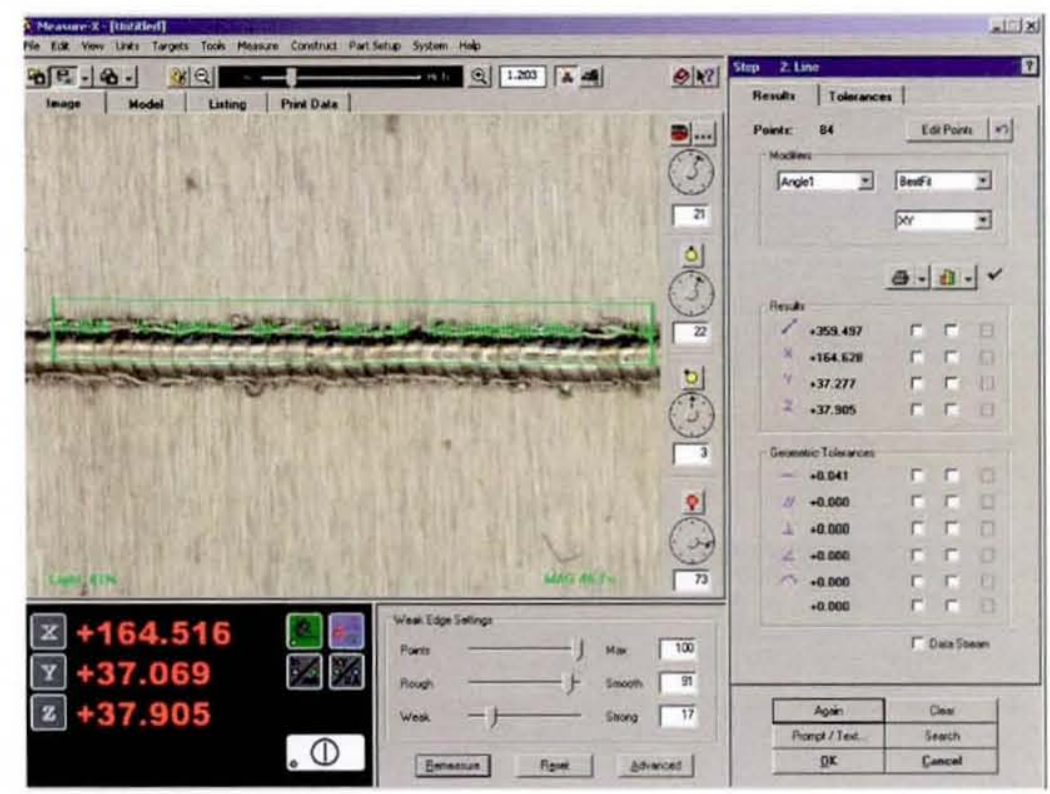

Figure 6-2 Screenshot of Flash optical measurement software measuring width of a single scan sample

\subsubsection{Laser Melting Region}

Figure 6-3 show a range of samples produced at varying pulse energies displaying signs of insufficient melting, sufficient melting and excessive melting leading to large amounts of material vaporisation. The samples shown in Figure 6-3 were produced using a pulse duration of $0.5 \mathrm{~ms}$ and pulse energies ranging from $0.1-2.5 \mathrm{~J}$. Each sample is shown embedded within the powder bed (top) and with the surrounding powder removed (bottom), this assists visualisation of loose powder consolidated into a melted scan track. Pulse energies of $0.5 \mathrm{~J}$ (1000W peak power) and below did not generate 
enough heat to fully melt the powder. Either this or a melt pool may have formed but the temperature may not have been high enough to catch or absorb a sufficient amount of powder into the melt pool [Liu \& Li 2004], resulting in an uneven and inconsistent solidified melt geometry. Insufficient heat input also caused a lack of fusion between the processed material and the underlying layer such that Inconel melted tracks would easily detach from the substrate. Other consequences of lack of heat input include porosity inclusion and reduced wettability [Singheiser et al. 2001; Liu \& Dupont 2003; Choi \& Chang 2005]. Complete melting of Inconel 625 in bulk form would require heating to approximately $1290-1350^{\circ} \mathrm{C}$. Powder has a higher absorbance than dense material due to the voids in interstitial spaces within the powder packing acting as a more effective absorber. Therefore less energy would be required to melt material in powder form compared to it being in bulk form. Pulse energies between 0.7-1J (1000$2000 \mathrm{~W}$ peak power, when using a fixed pulse duration of $0.5 \mathrm{~ms}$ ) produced well formed samples that fully melted to the steel substrate.

As pulse energies increased above $1 \mathrm{~J}$ (2000W peak power) the samples showed signs of excessive laser heat input and produced large amounts of spatter during processing. Samples increased in width due to the extra heat input increasing the volume of liquid produced forcing the melt pool to spread out and expand. These samples also possessed a brownish/yellow surface colour, indicating possible oxidisation as a result of the melt pool surface interacting with traces of $\mathrm{O}_{2}$ present within the processing environment (see appendix 12.1). A similar discoloration was observed when using SLS to process layers of stainless steel powder in an air atmosphere. Due to its colour, it was believed to be slag rich in chromium $\left(\mathrm{Cr}_{2} \mathrm{O}_{3}\right)$ [Hauser 2003]. 


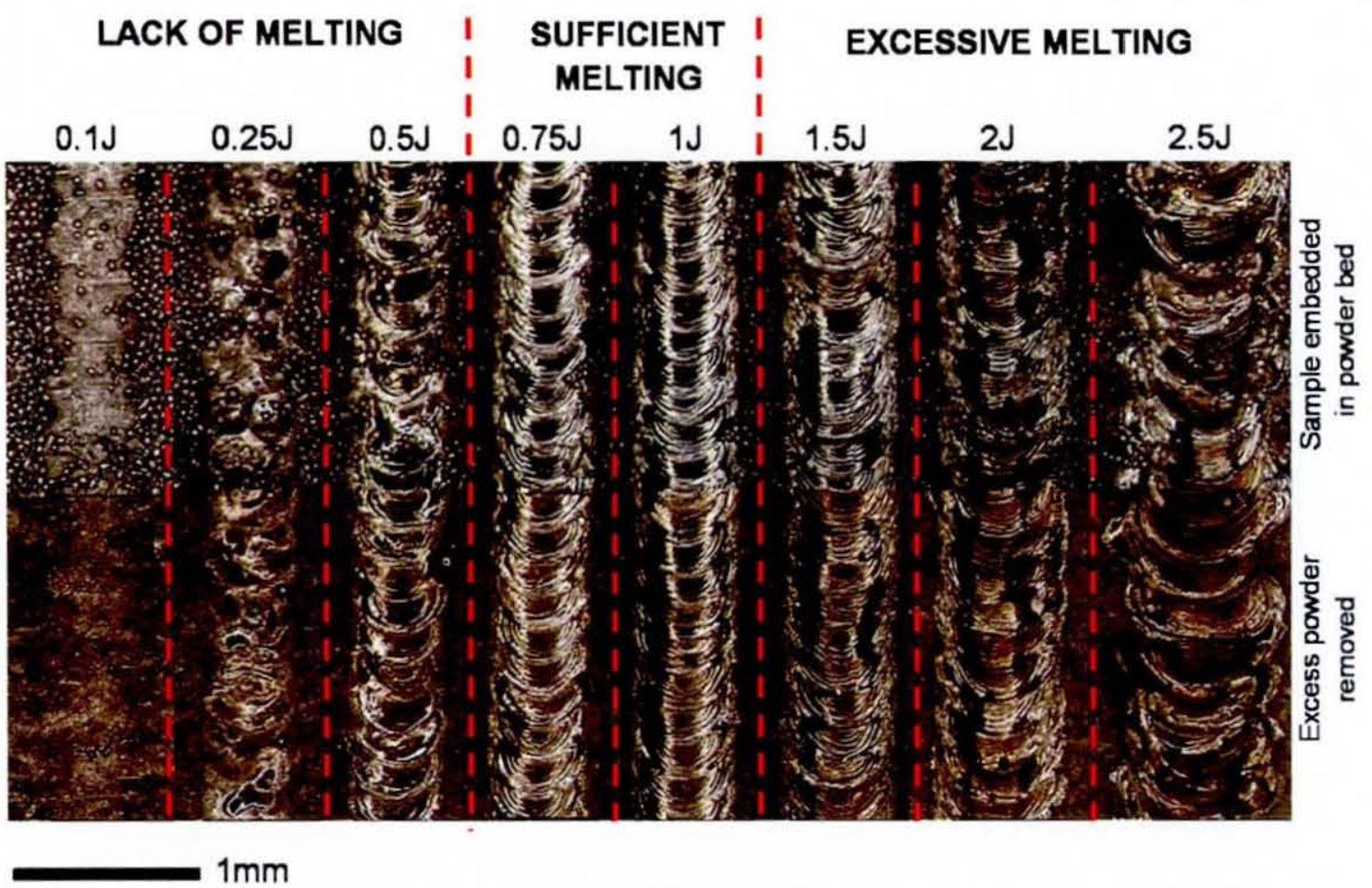

Figure 6-3 Inconel 625 single scans at $0.5 \mathrm{~ms}, 10 \mathrm{~Hz}, 168 \mathrm{~mm} / \mathrm{m} \& 0.1-2.5 \mathrm{~J}$

Figure 6-4 shows an Inconel 625 melting region using variable pulse energies/durations. As pulse duration increased, the amount of energy required within each pulse to melt the powder increased. This is because increased pulse duration delivers the pulses energy over a longer period and subsequently reduces the average peak power. Increased pulse durations have the potential to increase the length of time that a melt pool remains molten, this gives more time for heat to be lost from the melt pool to the surrounding environment (thermal diffusion and radiation loss). Longer pulse durations also tend to melt powder over a larger area as heat travels through the powder bed via conduction. The increased melting area requires more energy to generate and maintain a melt pool than that of a smaller area produced at a shorter pulse duration due to a reduced volume of material melted. In order to compensate for the reduction in peak power and potentially increased heat loss to the environment, the pulse requires more energy to maintain/generate a melt pool. The undesirable increase in material spatter boundary falls $1-2 \mathrm{~J}$ below the excessive heat input region. As mentioned earlier an increase in material spatter/plasma plume is an early indicator that too much heat input is being delivered to the material, the results reinstate this fact. It should be noted that material vaporisation will always occur to a certain degree within SLM when attempting to completely melt a metal alloy. Different material compositions within the 
alloy will have variable melting temperatures causing metals with lower melting temperatures to vaporise more easily.

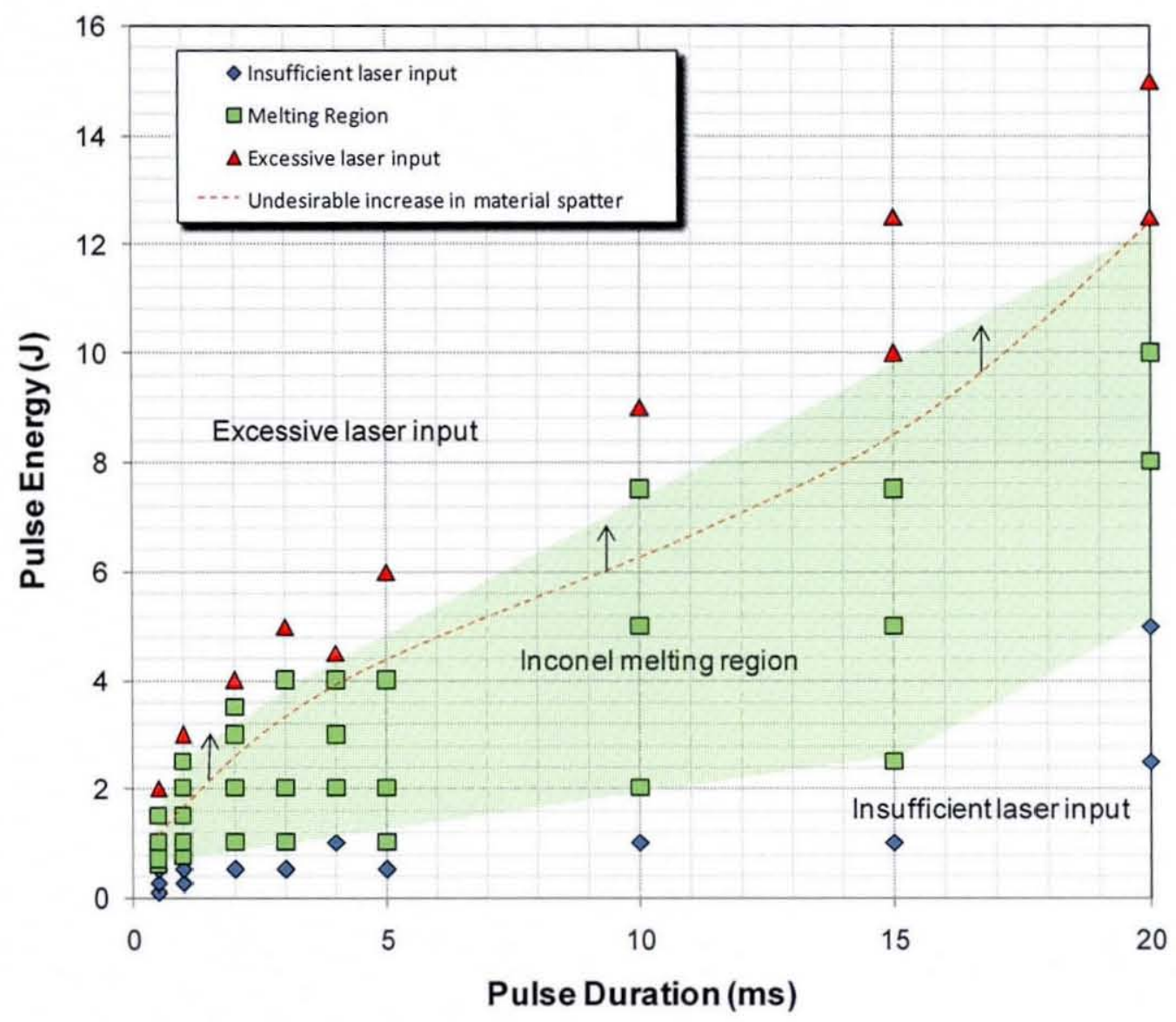

Figure 6-4 Inconel 625 melting region $(10 \mathrm{~Hz} \& 168 \mathrm{~mm} / \mathrm{min})$

Figure 6-5 shows the usable processing parameter region in the form of average peak powers. As mentioned earlier the average peak power is a key factor determining the level of heating within a specified time duration. Inconel 625 can be successfully melted without generating excessive spatter using peak powers of between 1000-1500W using a $0.5 \mathrm{~ms}$ pulse duration, repetition rate of $10 \mathrm{~Hz}$ and a scan speed of $168 \mathrm{~mm} / \mathrm{min}$. A further increase in peak power will cause an undesirable increase in spatter/plasma generation. Using a pulse duration of $2 \mathrm{~ms}$ Inconel 625 can be successfully melted without excessive spatter using peak powers between $500-1000 \mathrm{~W}$. Results indicate that successful melting at shorter pulse durations occur at higher average peak powers, this is due to the material requiring larger heat intensities when the laser-on time and overall pulse energy is reduced. As the laser-on time is increased the peak power intensity is reduced, the pulse then requires more energy within the pulse to melt the material. 


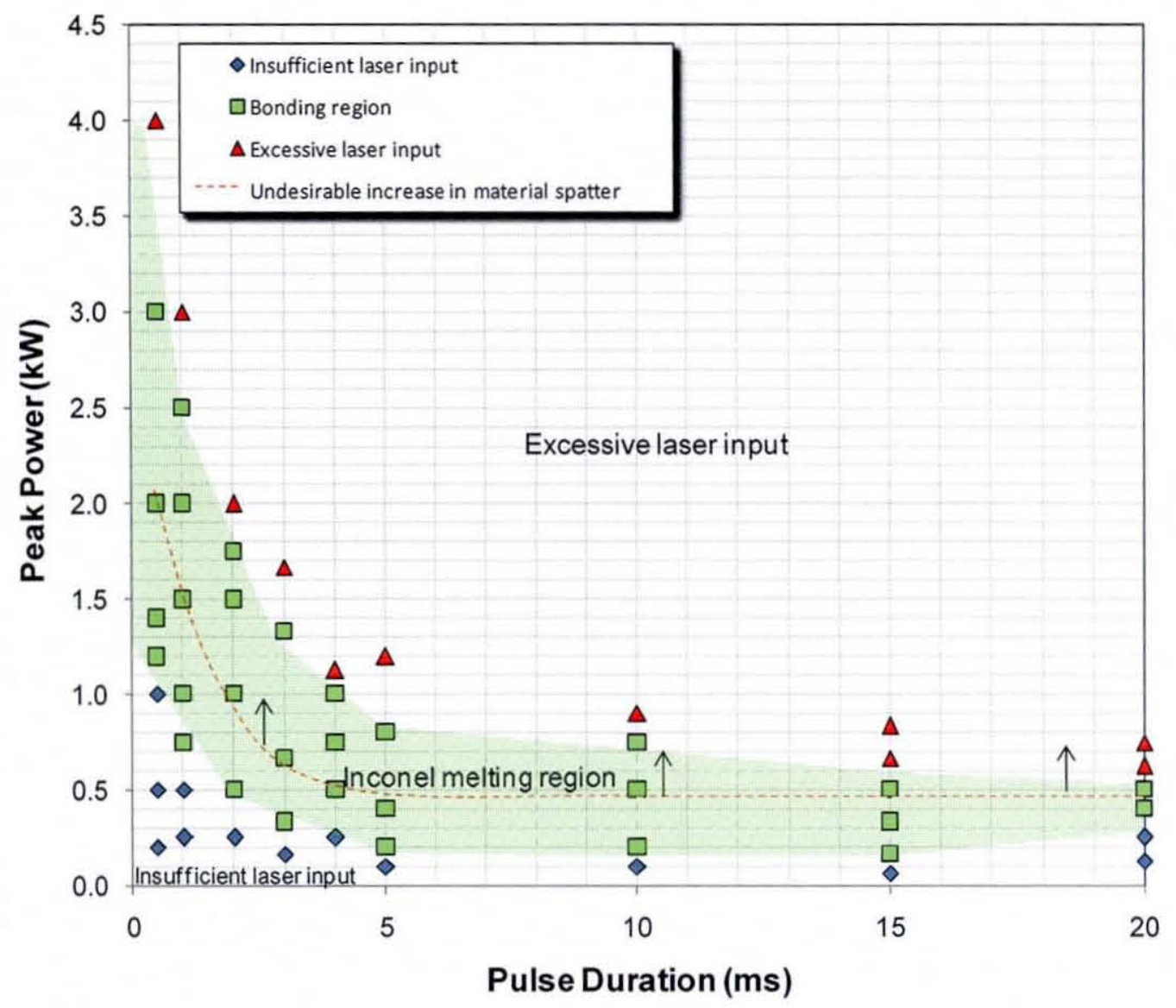

Figure 6-5 Peak power against pulse duration

The widths of samples produced at different pulse duration and pulse energies were measured using a Flash optical microscope. Figure 6-6 displays the widths of samples that successfully melted without generating excessive spatter during processing. It can be seen that shorter pulse duration produced the smallest melt pool and therefore have the greater potential to facilitate the production of high resolution parts. Longer pulse durations melted powder over a larger area due to an extended laser-on time and therefore produced thicker melted scan tracks. The smallest melt pool width was created using the shortest pulse duration of $0.5 \mathrm{~ms}(0.48-0.52 \mathrm{~mm})$. One of the objectives of the experiment was to identify pulse durations that were capable of producing the smallest melt pools such to enable the production of high resolution parts. Pulse durations of $0.5 \mathrm{~ms}$ were therefore selected for use in further experimentation as it held the most potential in producing thin walled parts. The generation of larger melt pool with the use of longer pulse durations has also been seen within research processing waspaloy powder [Mumtaz et al. 2008]. Figure 6-4 indicates that Inconel 625 can be successfully melted using pulse energies of $0.6-1 \mathrm{~J}$ when using a pulse duration of $0.5 \mathrm{~ms}$. 


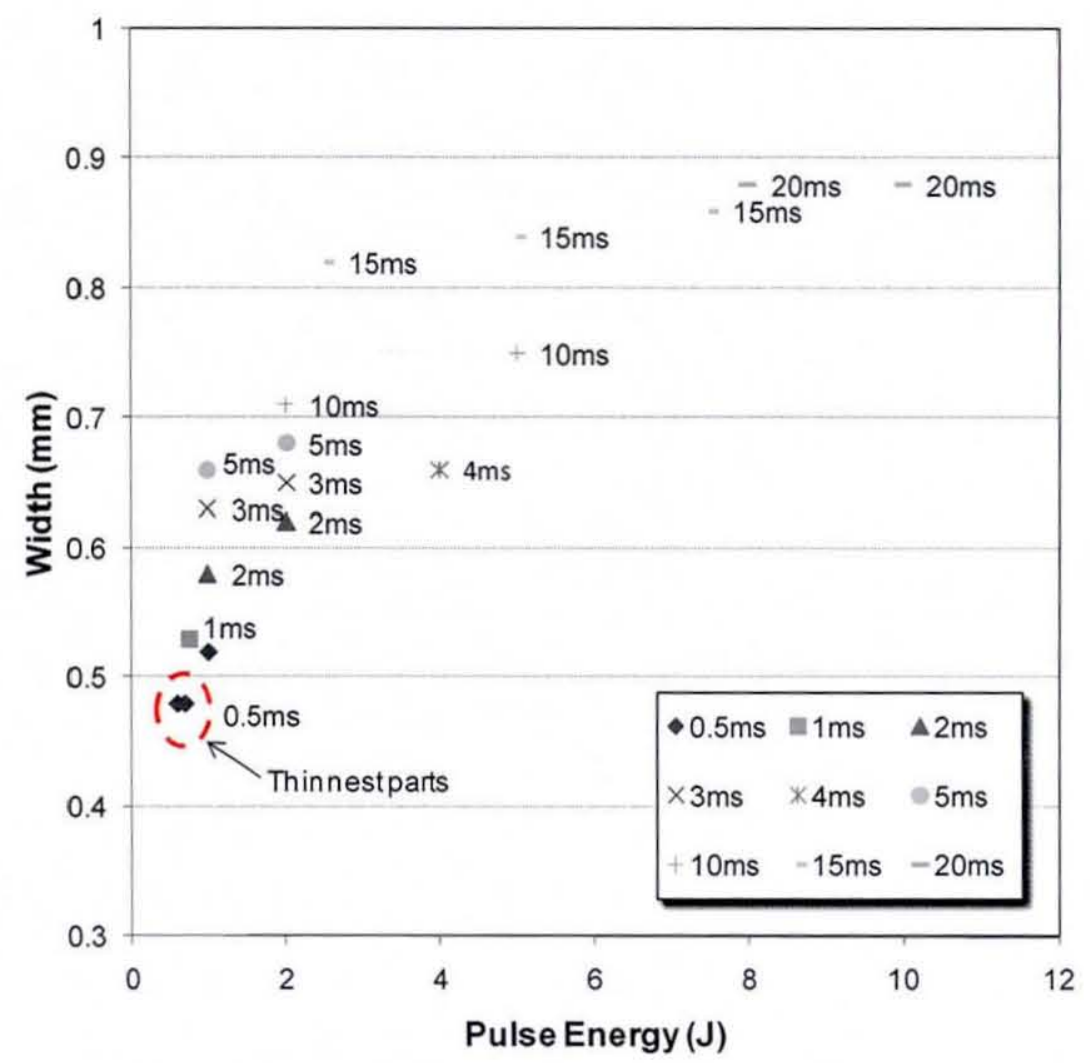

Figure 6-6 Width of samples produced using variable pulse durations and energy

\subsubsection{Laser Spot Overlap Region}

A general melting region in which Inconel 625 would successfully melt using variable pulse durations and pulse energies were found. A pulse duration of $0.5 \mathrm{~ms}$ and pulse energies of between $0.6-1 \mathrm{~J}$ were shown to produce thin parts without overheating the material or generating excessive material spatter. However these laser parameters were found using a fixed laser repetition rate and scan speed. In order to fully optimise process parameters and explore effects of other key parameters on the production of thin walled parts it was critical to identify a laser spot overlap operating window and experiment within this range. The laser spot overlap dictates the energy density delivered to a material, the formation of the melt pool and eventual part formation, including surface roughness [Mazumder et al. 2000; Fischer et al. 2003; Kruth et al. 2004; Syed et al. 2006]. Laser spot overlap is a combination of the pulsed laser's repetition rate, scan speed and spot size, Equation (3-4). The laser system is capable of producing high spot overlaps, however these high overlaps can cause an increase in material vaporisation due to an increase in energy density, Equation (3-3). In an attempt 
to identify a useable spot overlap region the plasma plume height was again monitored and visual observations of samples were made.

\subsubsection{Methodology}

As with the laser melting region experimentation (section 6.1.2), 25mm single scans were processed from a $0.1 \mathrm{~mm}$ powder bed. A pulse duration of $0.5 \mathrm{~ms}$ was used throughout all experimentation as it had been defined in section 6.1.2 to be the most suitable parameter in minimising melt pool width and facilitate the further production of thin walled parts. Repetition rates and scan speeds were varied between $30-200 \mathrm{~Hz}$ and $100-400 \mathrm{~mm} / \mathrm{min}$ respectively. These parameters would represent a wide range of generated spot overlaps and fall within the laser systems power limitations and processing speed capabilities. Section 6.1.2 had identified that pulse energies of $0.6-1 \mathrm{~J}$ successfully melted Inconel 625 when using a pulse duration of $0.5 \mathrm{~ms}$ and fixed repetition rates and scan speeds producing a spot overlap of $65 \%$. However due to the generation of higher spot overlaps (72-99\%) within this experimentation it was envisioned that larger energy densities would be produced, resulting in more material vaporisation and spatter generation. In order to readdress this balance laser spot overlap experiments operated within a lower pulse energy range of $0.5-0.7 \mathrm{~J}$ as shown in Table 6-3. Only one sample for each set of test conditions was produced. Solely plasma plume height was monitored during this experimentation.

\begin{tabular}{cccc}
\hline Pulse Width (ms) & Pulse Energy $(\mathbf{J})$ & Repetition Rate $(\mathbf{H z})$ & Scan Speed $(\mathbf{m m} / \mathbf{m i n})$ \\
\hline 0.5 & 0.5 & $30-200$ & $100-400$ \\
0.5 & 0.6 & $30-200$ & $100-400$ \\
0.5 & 0.7 & $30-200$ & $100-400$ \\
\hline
\end{tabular}

Table 6-3 Processing parameters used to identify useable laser spot overlap region

\subsubsection{Results}

Figure 6-7 displays useable repetitions and scan speeds during processing using pulse energies of $0.5-0.7 \mathrm{~J}$. Lower pulse energies are capable of operating at higher repetition rates before undesirable increases in material spatter occur. Figure 6-7 also represents the same data in the form of laser spot overlaps. Higher scan speeds increase part processing times, but incur a reduction in the spot overlap range available due to spatter 
generation. This is because faster scan speeds require a higher repetition rate to maintain the same level of laser spot overlap and degree of melting. This in turn puts a greater working load on the laser's flash lamps reducing its lifetime. Maintaining a high spot overlap at faster scan speed requires an aggressive increase in the rate of melting, inducing higher pressures on melt pool and subsequently larger spatter generation. Using higher pulse energies the range of useable repetition rates becomes limited, as spatter generation becomes more dominant. This is because the use of a higher pulse energy at a fixed pulse duration, generate larger peak powers which can increase the maximum temperature attained by the melt. A melt pool maintained at a higher temperature is more prone to increased material vaporisation, plasma plume generation and spatter ejection from melt pool.
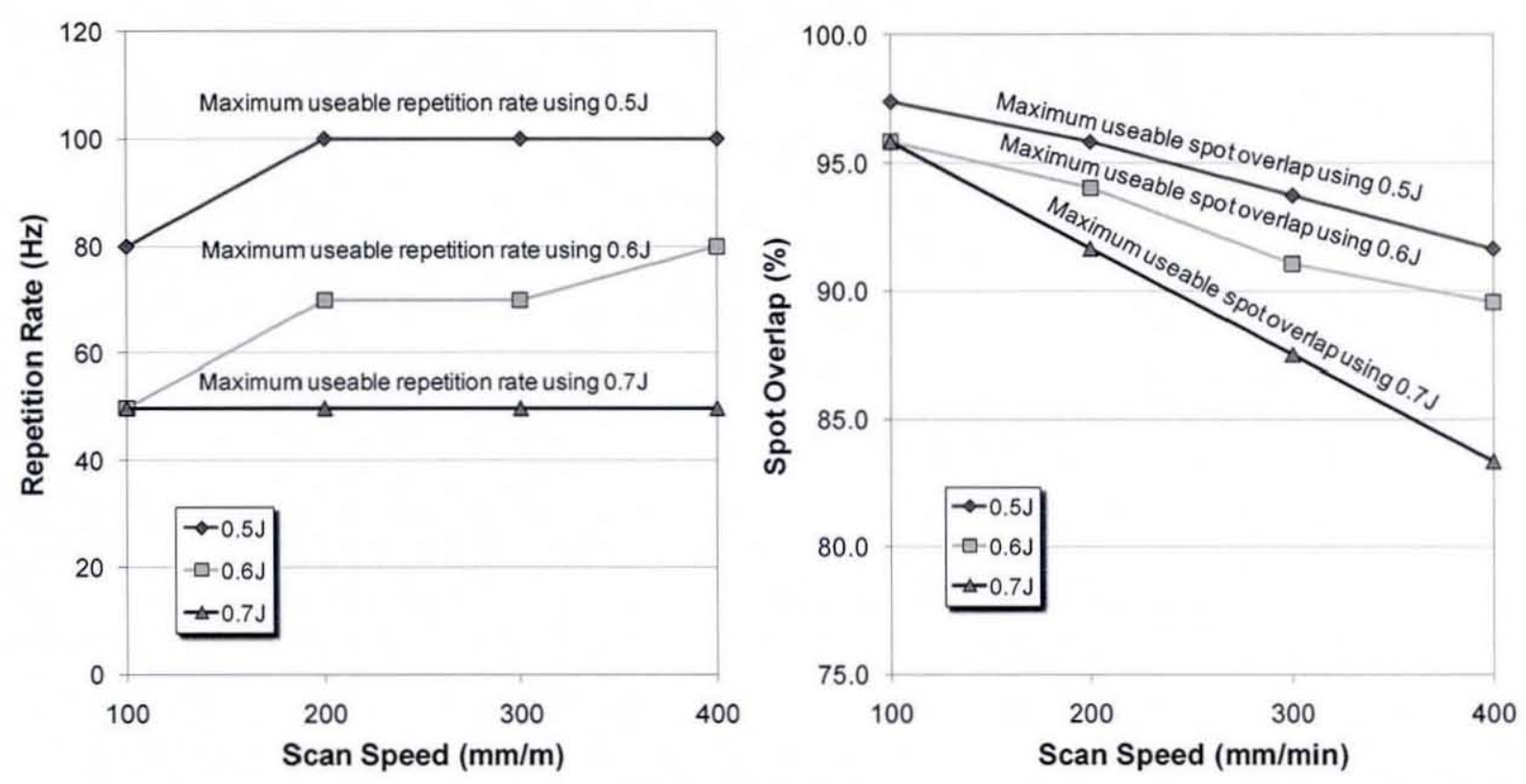

Figure 6-7 Repetition rate/spot overlap against scan speed useable region

\subsubsection{Summary}

A laser operating window in which Inconel 625 can fully melt without producing excessive spatter during processing has been identified. This region specifies the pulse energy required to successfully melt a $0.1 \mathrm{~mm}$ layer of Inconel 625 at different pulse duration using a fixed overlap. It was found that the use of shorter pulse durations produced the smallest melt pool width and would best facilitate the production of high resolution parts. Using a pulse duration of $0.5 \mathrm{~ms}$ a useable spot overlap range was also 
identified. The identification of an operating window for Inconel 625 formed the foundation for further experimentation. 


\subsection{Thin Walled Samples}

As discussed in Chapter 3, part qualities such as surface roughness and melt width are heavily influenced by laser processing parameters used to generate a melt pool. Capillary/thermocapillary flows and external shear forces from recoil pressures and gas flow rate make control of the melt pool a complex process to fully control. Research reported in this section used the laser processing parameters discussed in section 6.1 to build thin wall Inconel 625 parts. The effects of various laser parameters had on top/side surface roughness and wall thickness were investigated.

\subsubsection{Methodology}

Four layer $0.1 \mathrm{~mm}$ powder layers were processed using single scans measuring $25 \mathrm{~mm}$ in length (shown in Figure 6-8). The processing parameters used are shown in Table 6-4 (defined in section 4.1). Only one sample for each set of test conditions was produced. Sample top and side surface roughness and width were measured.

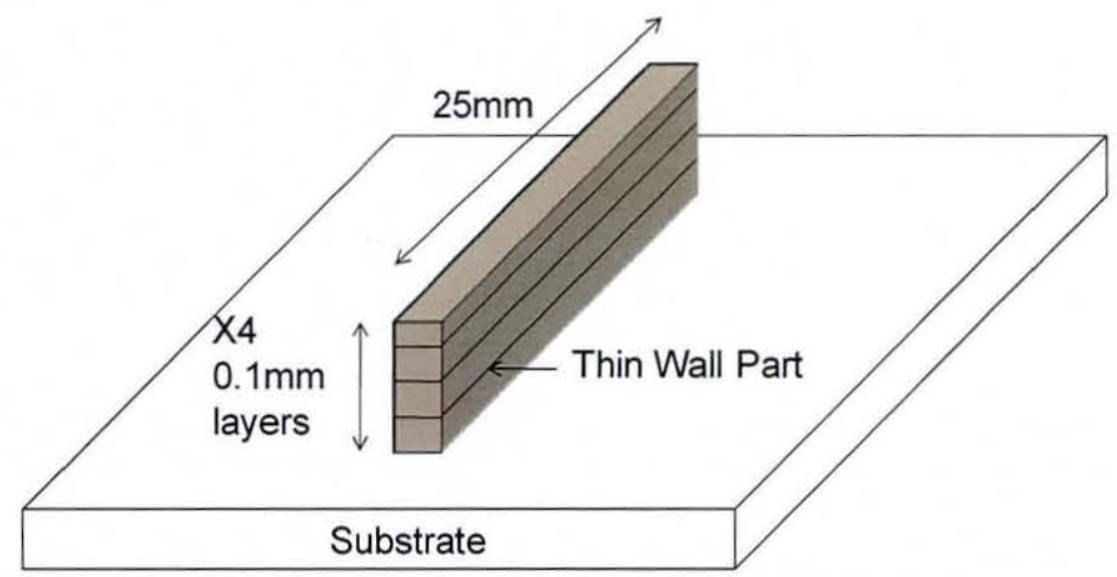

Figure 6-8 Thin wall part

\begin{tabular}{cccc}
\hline Pulse Width $(\mathrm{ms})$ & Pulse Energy $(\mathrm{J})$ & Repetition Rate $(\mathrm{Hz})$ & Scan Speed $(\mathrm{mm} / \mathrm{m})$ \\
\hline 0.5 & 0.5 & $30-100$ & $100-400$ \\
0.5 & 0.6 & $30-70$ & $100-400$ \\
0.5 & 0.7 & $30-50$ & $100-400$ \\
\hline
\end{tabular}

Table 6-4 Process parameters used for the fabrication of thin wall parts 


\subsubsection{Width measurement}

Due to the increased height of samples, width of the samples could be measured physically using digital callipers. It is believed that this contact method for measurement is more accurate than visual measurement. Measurements were taken at three points along each samples length. This measurement did not include the extreme ends of samples due to a larger volume of material being present at the start and end of each scan. This is caused by the presence of additional laser energy at the beginning of the laser scan due to the laser being turned on (laser overshoot and delay in processing table movement) and the extra energy present at the end of the scan (laser off delay and the stopping of processing table movement).

\subsubsection{Surface roughness measurement}

The top and side surface roughness of samples was measured using a Talysurf CLI 2000 contact method (shown in Figure 6-9). This apparatus measures surface roughness by dragging a stylus across the surface of the sample. The arithmetic average for surface roughness $\left(\mathrm{R}_{\mathrm{a}}\right)$ was measured using a $12.5 \mathrm{~mm}$ traverse length and $2.5 \mathrm{~mm}$ cut-off length. The arithmetic average is the most common measurement taken for examining roughness and is directly proportional to an increase/decrease in roughness on a surface. The Top $R_{a}$ was measured three times from the central portion of the samples length, the side $R_{a}$ was also measured three times while alternating the side being measured (note, one side would be measured twice and the other measured once) Figure 6-10 shows the surfaces of the thin wall parts which were measured. Sample were removed from the steel substrate using a circular saw prior to side $\mathrm{R}_{\mathrm{a}}$ measurements, in order for the Talysurf's stylus to make correct contact with the side surface of the sample without obstruction. Top $\mathrm{R}_{\mathrm{a}}$ was measured along the direction of laser scanning and side $\mathrm{R}_{\mathrm{a}}$ was only measured in one direction along the $y$-axis. The sample could not be measured for side $R_{a}$ along the $z$-axis because the sample was not tall enough (currently less than $0.4 \mathrm{~mm}$ in height). The production of taller specimens involving at least 50 layers each, would incur a long production and testing time. However samples produced in later experiments (Chapter 8) were tested for side $\mathrm{R}_{\mathrm{a}}$ in both horizontal and vertical directions. 


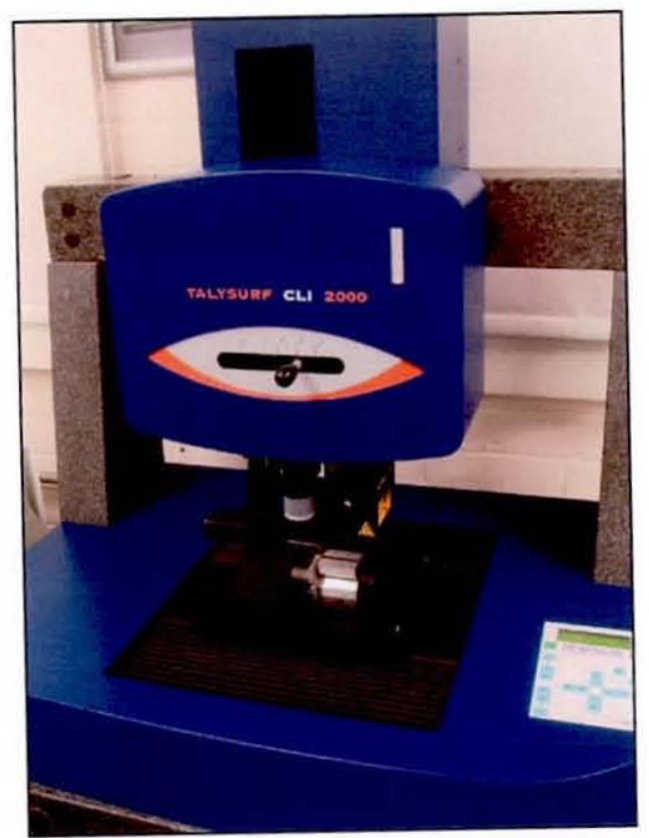

Figure 6-9 Talysurf CLI 2000 apparatus used to measure surface roughness

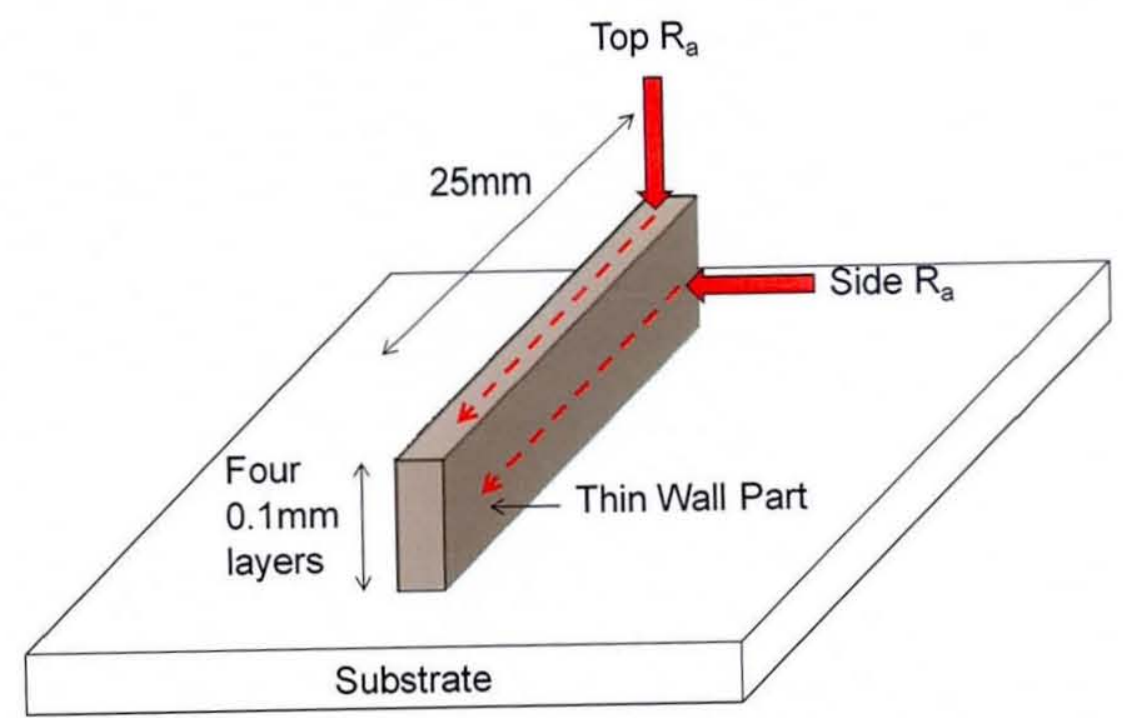

Figure 6-10 Top/Side $\mathrm{R}_{\mathrm{a}}$ measurement of thin wall part

\subsubsection{Top Surface Roughness}

The top $R_{a}$ of thin wall samples is discussed in this section. The effects of laser parameters on increasing/reducing top $\mathrm{R}_{\mathrm{a}}$ are examined with particular attention focused on spot overlap, energy density and pulse energies. 


\subsubsection{Spot overlap}

Table 6-5 shows the top $R_{a}$ results for thin wall samples produced using a pulse energy of $0.5 \mathrm{~J}$ and a scan speed of $400 \mathrm{~mm} / \mathrm{min}$, at repetition rates of $30-100 \mathrm{~Hz}$. The top surface of a melt pool is directly in contact with the incident laser during processing and the laser's pulsed imprint can be most easily recognised from viewing the top surface. Results indicate that top $R_{a}$ of parts reduced as repetition rate was increased this reduction in top $R_{a}$ is clearly linked to a reduced variation in top surface profile as spherical masses generated by each laser pulse come closer together as shown in Figure 6-11. Figure 6-12 shows the plan view of the samples produced from laser parameters shown in Table 6-5. The lowest $\mathrm{R}_{\mathrm{a}}$ was achieved using a repetition rate of $100 \mathrm{~Hz}$ generating a laser spot overlap of $91.7 \%$.

\begin{tabular}{|c|c|c|c|c|c|c|}
\hline $\begin{array}{l}\text { Rep. Rate } \\
(\mathrm{Hz})\end{array}$ & $\begin{array}{l}\text { Spot Overlap } \\
(\%)\end{array}$ & $\begin{array}{l}\text { Av. Power } \\
\text { (W) }\end{array}$ & $\begin{array}{l}\text { Energy Density } \\
\left(\mathrm{J} / \mathrm{mm}^{2}\right)\end{array}$ & $\begin{array}{l}\text { Top } R_{a} \\
(\mu \mathrm{m})\end{array}$ & $\begin{array}{l}\text { Std } \\
\pm(\%)\end{array}$ & Deviation \\
\hline 30 & 72.2 & 15 & 2.8 & 16 & & 1.2 \\
\hline 40 & 79.2 & 20 & 3.8 & 14 & & 2.3 \\
\hline 50 & 83.3 & 25 & 4.7 & 12 & & 1.2 \\
\hline 60 & 86.1 & 30 & 5.6 & 11 & & 2.9 \\
\hline 70 & 88.1 & 35 & 6.6 & 11 & & 2.3 \\
\hline 80 & 89.6 & 40 & 7.5 & 10 & & 1.2 \\
\hline 90 & 90.7 & 45 & 8.4 & 9 & & 1.2 \\
\hline 100 & 91.7 & 50 & 9.4 & 9 & & 2.3 \\
\hline
\end{tabular}

Table 6-5 Top $R_{a}$ for a selection of samples produced using $0.5 \mathrm{~J} \& 400 \mathrm{~mm} / \mathrm{min}$

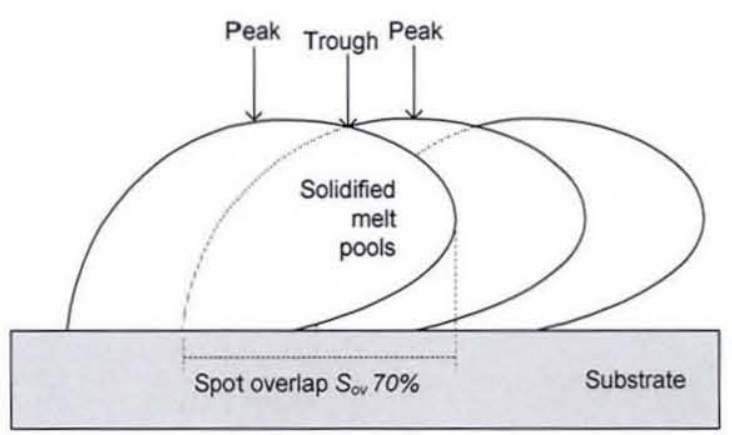

(a)

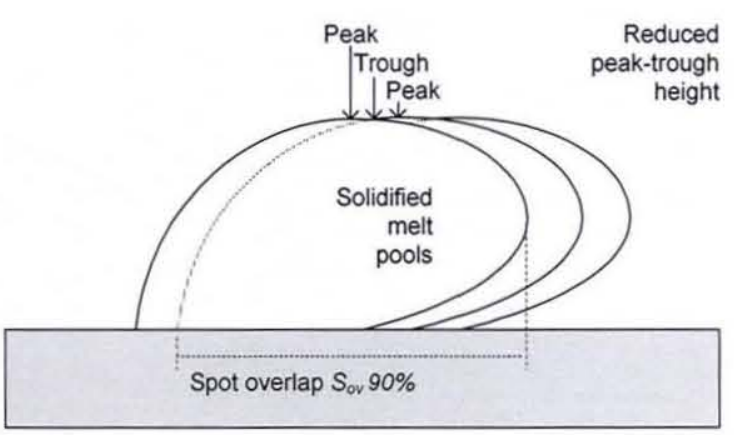

(b)

Figure 6-11 Solidified melt pool produced with a 70\% laser spot overlap (a) \& 90\% spot overlap (b) 

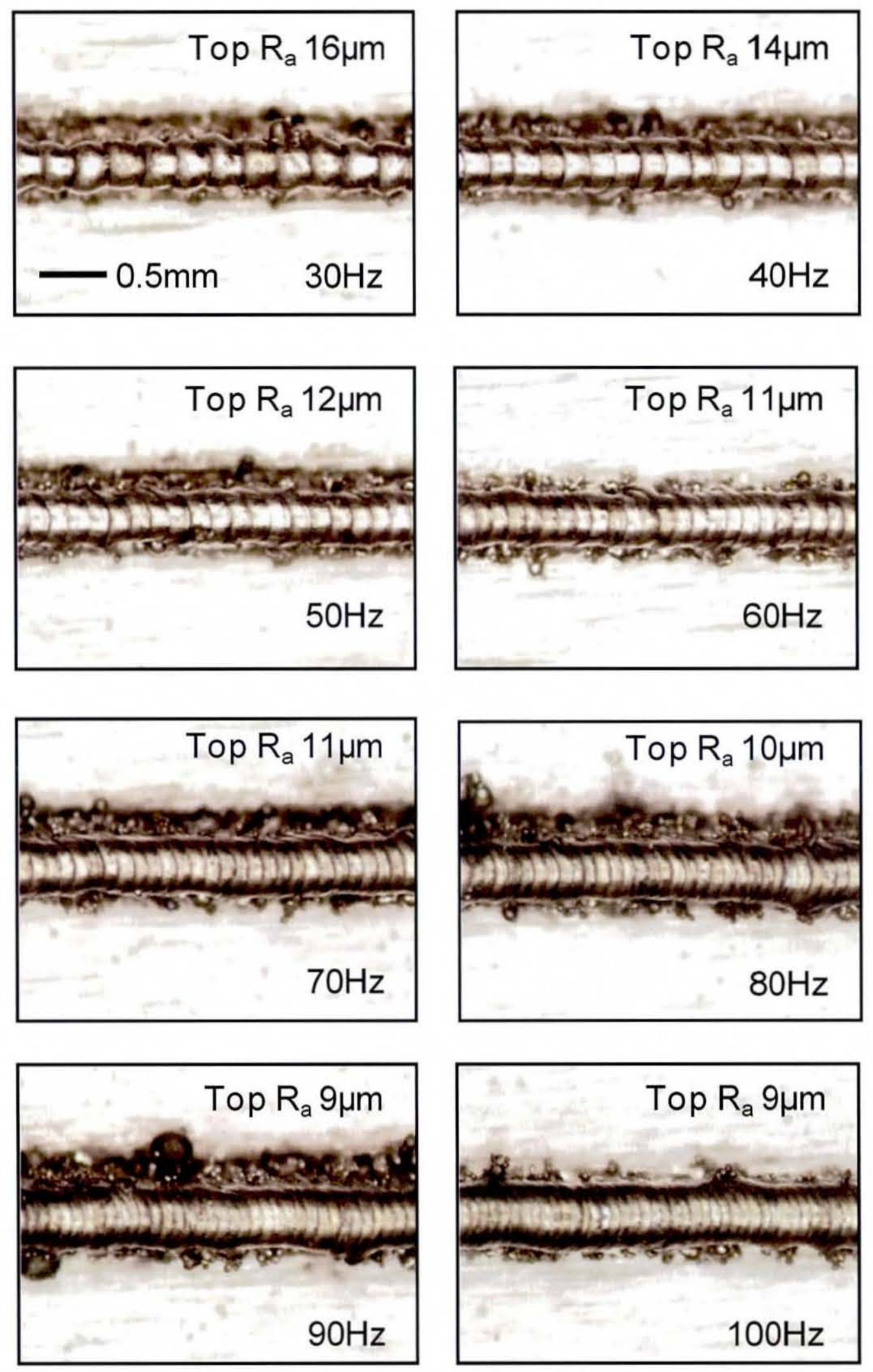

Figure 6-12 Top $\mathrm{R}_{\mathrm{a}}$ of 4 layered samples processed using $0.5 \mathrm{~J}, 400 \mathrm{~mm} / \mathrm{min}$ at variable repetition rates 
Figure 6-13 represents the top $\mathrm{R}_{\mathrm{a}}$ of samples when processed at variable repetition rates and scan speeds, combining to form spot overlap. High repetition rates and low scan speeds reduce top $R_{a}$ due to the generation of high laser spot overlap. A percentage overlap above $90 \%$ tended to produce the lowest top $\mathrm{R}_{\mathrm{a}}$ for samples. This is related to the spherical masses produced by the laser coming nearer together reducing the variation in surface profile. It is believed that the use of slower scan speeds gives more time for molten material to flow and smoothen out the top surface profile [Ramos et al. 2003]. This movement of molten material is influenced by Marangoni convection. The material that is processed nearest to the incident laser will have the highest temperature and lowest surface tension. Areas processed behind the traversing laser are cooler and therefore have a higher surface tension. The use of a slower scan speed in effect steadies the melt pool as more time is given for molten material to flow and reduce the ripple effect generated by pulsed laser. However, this reduction in top $\mathrm{R}_{\mathrm{a}}$ may also be related to the fact that higher spot overlaps are more easily generated at lower scan speeds, therefore reducing variation in surface profile.

A scan speed of $100 \mathrm{~mm} / \mathrm{min}$ produces a sample with the lowest top $R_{a}$ of $4 \mu \mathrm{m}$, at a repetition rate of $50 \mathrm{~Hz}$ and scan speed of $100 \mathrm{~mm} / \mathrm{min}$ ( $96 \%$ overlap). As spot overlaps increased above $96 \%$ the top $\mathrm{R}_{\mathrm{a}}$ would begin to increase. It is believed that as spot overlap approaches $96 \%$, the top $\mathrm{R}_{\mathrm{a}}$ would have already reached its optimum value (for set laser parameters and system setting) and that further increases would begin to degrade top $\mathrm{R}_{\mathrm{a}}$. This could be due to high energy density disrupting melt pool stability which can increase top $\mathrm{R}_{\mathrm{a}}$ [Ramos et al. 2003]. It is believed that high spot overlaps (above 96\%) can be used, however either pulse energy and/or pulse duration should be adjusted in order to reduce heat input. 


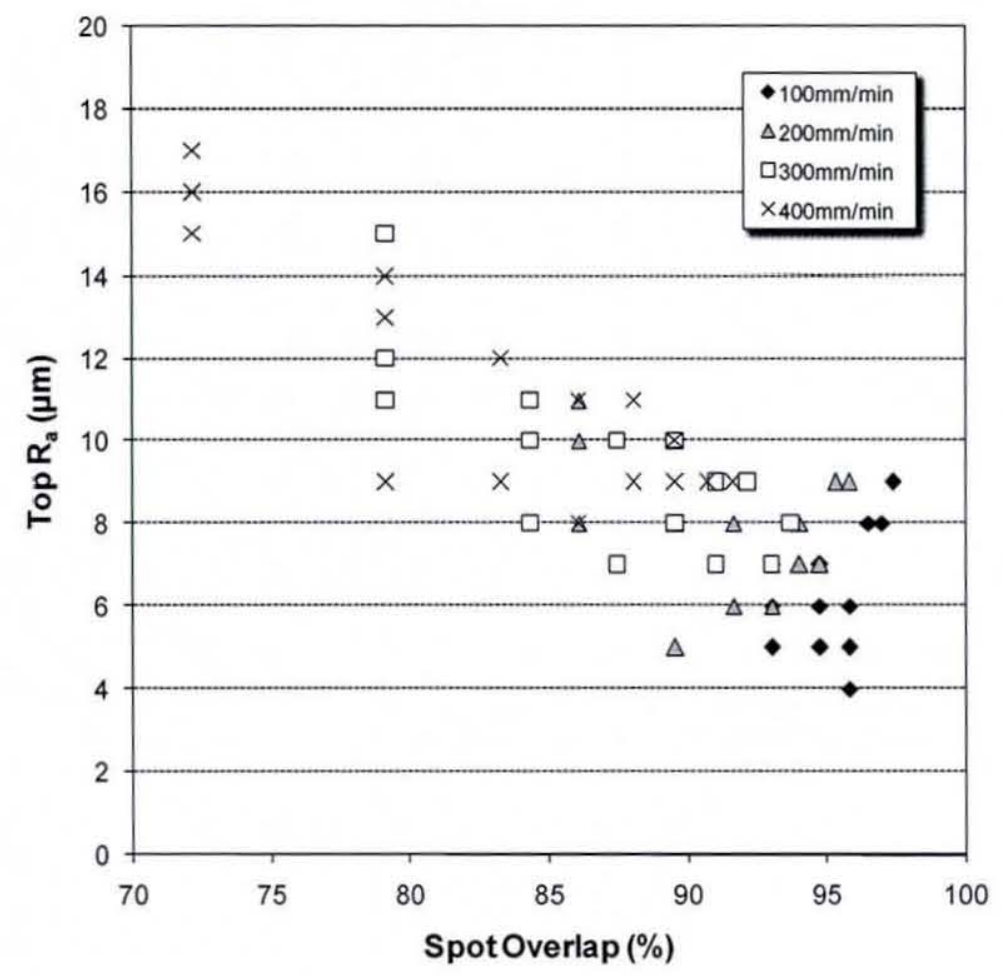

Figure 6-13 Top $\mathrm{R}_{\mathrm{a}}$ against spot overlap

\subsubsection{Energy density}

Figure 6-14 shows results of top $\mathrm{R}_{\mathrm{a}}$ against energy densities. As expected, higher energy densities reduce the top $\mathrm{R}_{\mathrm{a}}$ of samples. Higher energy densities are produced at high pulse energies, high repetition rates and low scan speed. Reductions in top Ra with increases in energy density are related to a combination of factors. As already discussed the use of higher repetition rates reduces top $R_{a}$ due to increases in spot overlap. Higher pulse energies increase the volume of liquid produced. Melt pools containing a higher volume of liquid will take a longer time to solidify, allowing more time for relaxation forces to reshape and reduce variation between overlapping melt pools. According to Equation (3-2), the use of high pulse energies at a fixed pulse duration will automatically generate a larger average peak power and subsequently induce higher recoil pressures on the melt pool. This will assist the flattening and reshaping of the melt pool and reduce top $\mathrm{R}_{\mathrm{a}}$. However if energy density became too high (above 26 $\mathrm{J} / \mathrm{mm}^{2}$ ), the $R_{a}$ began to degrade. Again this is could be related to a disruption of the melt pool. 


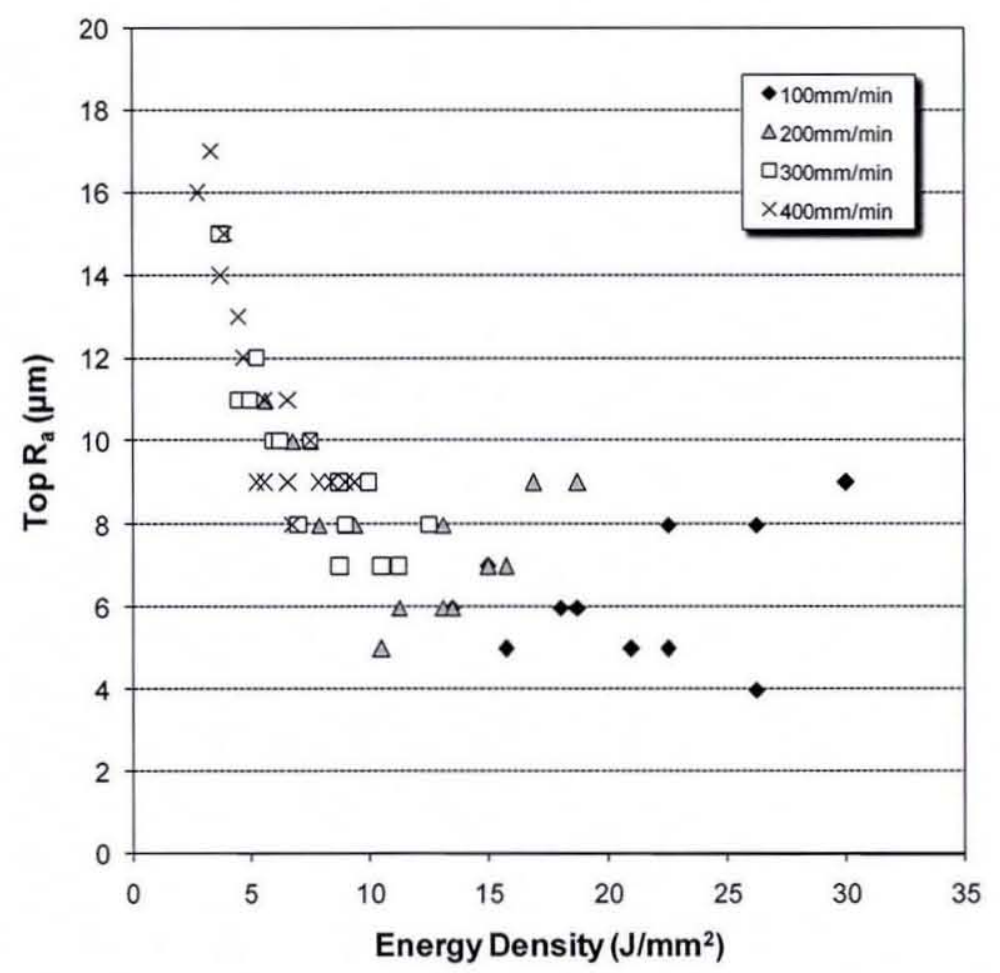

Figure 6-14 Top $\mathrm{R}_{\mathrm{a}}$ against energy density

\subsubsection{Pulse energy}

Results have clearly indicated that lower scan speeds and higher repetition rates reduce top $\mathrm{R}_{\mathrm{a}}$. Figure 6-15 shows top $\mathrm{R}_{\mathrm{a}}$ against repetition rate at a low scan speed of $100 \mathrm{~mm} / \mathrm{min}$ and variable pulse energy. Samples produced at higher pulse energies tended to possess lower top $\mathrm{R}_{\mathrm{a}}$. This collaborates with data shown in Figure 6-14 indicating higher energy densities reduce top $\mathrm{R}_{\mathrm{a}}$ As explained in section 6.2.2.2, the use of higher pulse energies at a fixed pulse duration would increase the average peak power. This would generate larger recoil pressures flattening out the melt pool [Morgan et al. 2001; Mumtaz et al. 2008]. A minimum top $R_{a}$ of $4 \mu \mathrm{m}$ is achieved using a pulse energy of $0.7 \mathrm{~J}$ and a repetition rate of $50 \mathrm{~Hz}$ when employing a scan speed of $100 \mathrm{~mm} / \mathrm{min}$, the sample produced is shown in Figure 6-16.

As repetition rates progress past $50 \mathrm{~Hz}$, the top $\mathrm{R}_{\mathrm{a}}$ increases due to what is believed to be an unsteadying and disruption of the melt pool. This was shown in Figure 6-13 with spot overlaps increasing above $96 \%$. 
The use of higher pulse energies increases the melt pool temperature. Higher temperatures can cause the melt pool viscosity to reduce and will subsequently increase the strength of any Marangoni convection (Equation (3-9)) present within the melt pool. This can assist fluid flow and melt pool reshaping before complete solidification, possibly reducing any melt pool top surface variation developed due to overlapping pulses. Higher pulse energies also generate larger peak powers at a fixed duration, causing larger recoil pressures to be generated. These larger recoil pressures flatten out the melt pool producing a smoother top surface profile.

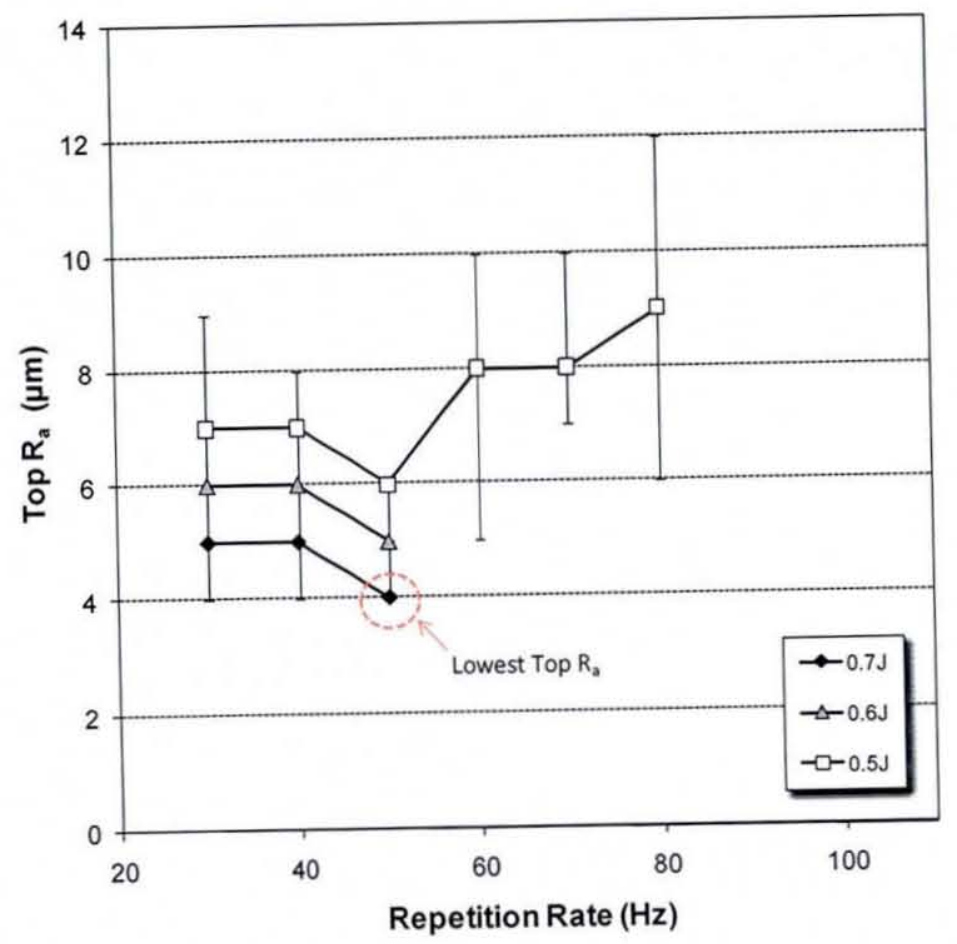

Figure 6-15 Top $\mathrm{R}_{\mathrm{a}}$ against repetition rate at $100 \mathrm{~mm} / \mathrm{min}$

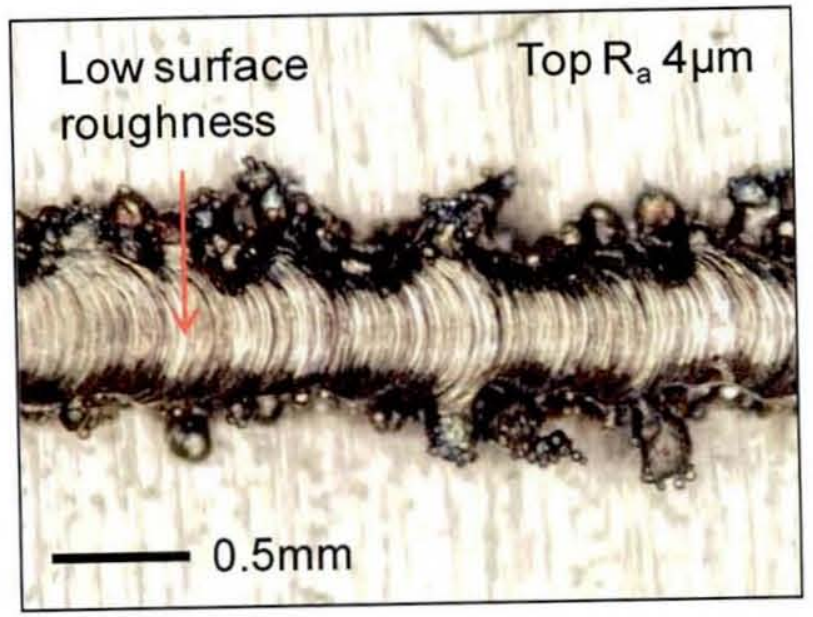

Figure 6-16 Lowest top $\mathrm{R}_{\mathrm{a}} 4 \mu \mathrm{m}$ achieved using $0.5 \mathrm{~ms}, 0.7 \mathrm{~J}, 50 \mathrm{~Hz}$ and $100 \mathrm{~mm} / \mathrm{min}$ 


\subsubsection{Summary}

It has been found that increasing the repetition rates up to a certain value will reduce top $\mathrm{R}_{\mathrm{a}}$ due to the centres of individual spherical masses becoming nearer together reducing the variation in top $R_{a}$. Lower scan speeds will produce parts with lower top $R_{a}$ due to the generation of a steadier melt pool as well as the molten material been given more time to flow freely. It was found that a scan overlap of between $91.1-95.8 \%$ produced the lowest top $\mathrm{R}_{\mathrm{a}}$ on samples. Higher energy densities increase the volume of liquid within the melt pool. A melt pool with a higher volume may be more influenced as a bulk mass by the forces of gravity as it flattens the melt pool causing it to spread out. This flattening may reduce top $\mathrm{R}_{\mathrm{a}}$ due to there being less variation in height between the individual spherical masses formed by the pulsed laser. Higher pulse energies at a fixed duration increase the overall peak power that subsequently produces larger recoil pressures. This pressure flattens out the melt pool and produced a smoother top surface profile. Repetition rate and scan speed combinations that produce spot overlaps of $96 \%$ and above caused increases in top $\mathrm{R}_{\mathrm{a}}$ due to a possible unsteadying or disruption of the melt pool. 


\subsubsection{Side Surface Roughness}

During processing during SLM, the breakup of a melt pool along its scan direction into small spherical spheres (known as balling) has been shown to be the main contributor to side $R_{a}$ of SLM parts [Rombouts 2006]. Other contributors include partially melted, entrained powder particles that stick to the outer edge of the solidified melt pool known as "satellite formation" or "hillocks" [Tolochko et al. 2004; Elsen 2007]. Satellite formation mainly occurs when powder particles are not given enough time to penetrate the melt pool due to melt pool solidification. Side $R_{a}$ contributors are often more difficult to control and eliminate compared to factors related to top $R_{a}$ of parts. The final layer on the top surface of a part can often be re-melted using a lower power and higher repetition rate to reduce top surface roughness [Su 2002; Morgan et al. 2004], however this option is not practical for side surfaces as laser direction is perpendicular to the side facing surface.

\subsubsection{Spot overlap}

Table 6-6 shows the results of a selection of thin wall samples processed using a pulse energy of 0.7J, Figure 6-17 displays the plan view of these samples. At the edges of the samples there appear to be small spherical structures approximately the same size as that of the powder particles used during processing. It is believed that these are partially melted powder particles from the surrounding powder bed that attach to the edge of the solidified melt pool, known as satellite formation. The degree of satellite formation at the edge of samples can be seen to reduce as scan speeds are increased and repetition rate is reduced, thus reducing side $R_{a}$. However when repetition rates were increased and scan speeds reduced (generating high energy densities) these spherical structures increased in size. Due to the increased size of these structures, they are believed to be a result of molten material that may have broken up from the melt pool itself. This can be described as "lateral balling" in which melt pool breakup is perpendicular to scan direction and triggered due to surface tension variations. The appearance of lateral balling could occur when large volumes of liquid are produced (due to high energy densities) subsequently inducing strong Marangoni flow. This has been shown to directly affect the degree of melt pool breakup during conventional balling along the scan track [Niu \& Chang 1999; Gu \& Shen 2007; Xiao \& Zhang 2007]. These large spherical structures may also be a result of melt pool ejection due to high recoil 
pressures exerting large pressures on the melt pool, forcing molten material out of the melt pool and cause it to solidify on the edge of the melt pool. Alternatively these large spherical structures may simply be a result of satellite formation and appear larger in size due to more heat being given to smaller powder particles melting outside the melt pool to agglomerate and form large structures. Multiple theories can be used to explain the appearance of these structures. The only way this can be ascertained is through further research (high speed imaging of melt pool during processing). This further research is outside the remit of this investigation.

The width of samples appeared to increase with use of lower scan speeds, higher repetition rates generating high energy densities. This increase in energy density widens the melt pool as surrounding powder enters the already formed melt pool (this is discussed in more detail in section 6.3). The reduced scan speed also allowed more time for heat to conduct through the material producing a larger volume of melt.

\begin{tabular}{rrrrrrr}
\hline $\begin{array}{l}\text { Rep. Rate } \\
(\mathrm{Hz})\end{array}$ & $\begin{array}{l}\text { Scan Speed } \\
(\mathrm{mm} / \mathrm{m})\end{array}$ & $\begin{array}{l}\text { Spot Overlap } \\
(\%)\end{array}$ & $\begin{array}{l}\text { Av. Power } \\
(\mathrm{W})\end{array}$ & $\begin{array}{l}\text { Energy Density } \\
\left(\mathrm{J} / \mathrm{mm}^{2}\right)\end{array}$ & $\begin{array}{l}\text { Side } \mathrm{R}_{\mathrm{a}} \\
(\mu \mathrm{m})\end{array}$ & $\begin{array}{l}\text { Std Dev. } \\
\pm(\%)\end{array}$ \\
\hline 30 & 100 & 93.1 & 21 & 15.7 & 30 & 2.0 \\
40 & 100 & 94.8 & 28 & 21 & 30 & 3.2 \\
50 & 100 & 95.8 & 35 & 26.2 & 32 & 0.6 \\
30 & 200 & 86.1 & 21 & 7.8 & 15 & 2.6 \\
40 & 200 & 89.6 & 28 & 10.5 & 16 & 0.0 \\
50 & 200 & 91.7 & 35 & 13.1 & 25 & 2.5 \\
30 & 300 & 79.2 & 21 & 5.2 & 11 & 0.6 \\
40 & 300 & 84.4 & 28 & 7 & 13 & 2.5 \\
50 & 300 & 87.5 & 35 & 8.7 & 16 & 2.9 \\
30 & 400 & 72.2 & 21 & 3.9 & 10 & 2.0 \\
40 & 400 & 79.2 & 28 & 5.2 & 10 & 1.5 \\
50 & 400 & 83.3 & 35 & 6.5 & 12 & 2.6 \\
\hline
\end{tabular}

Table 6-6 Thin wall samples processed using $0.7 \mathrm{~J}$ pulse energy 

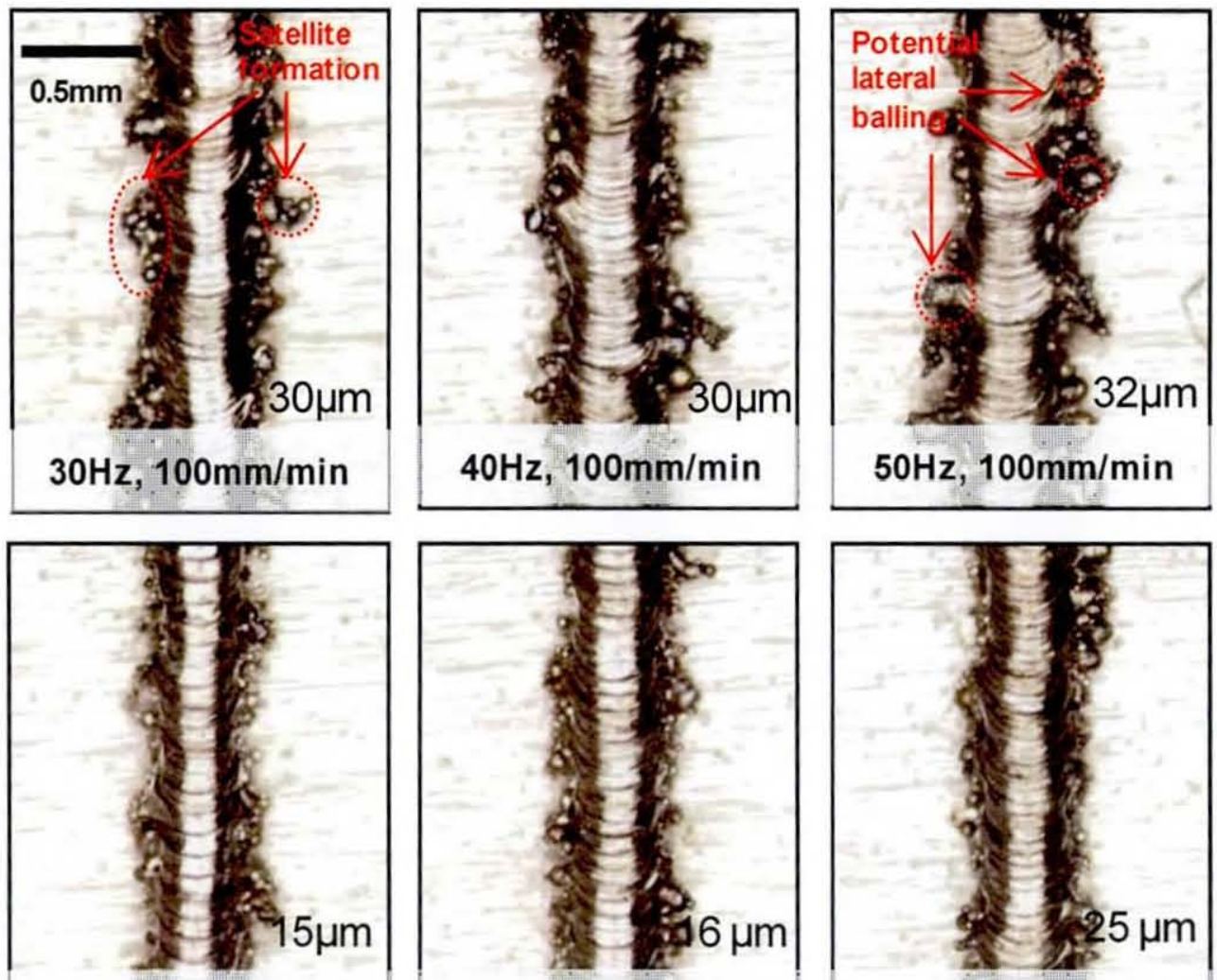

$50 \mathrm{~Hz}, 100 \mathrm{~mm} / \mathrm{min}$

$30 \mathrm{~Hz}, 200 \mathrm{~mm} / \mathrm{min}$
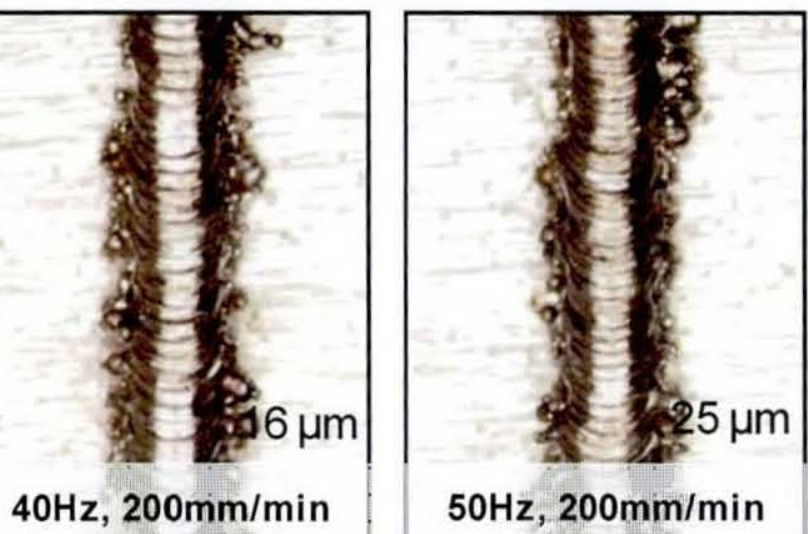

$50 \mathrm{~Hz}, 200 \mathrm{~mm} / \mathrm{min}$
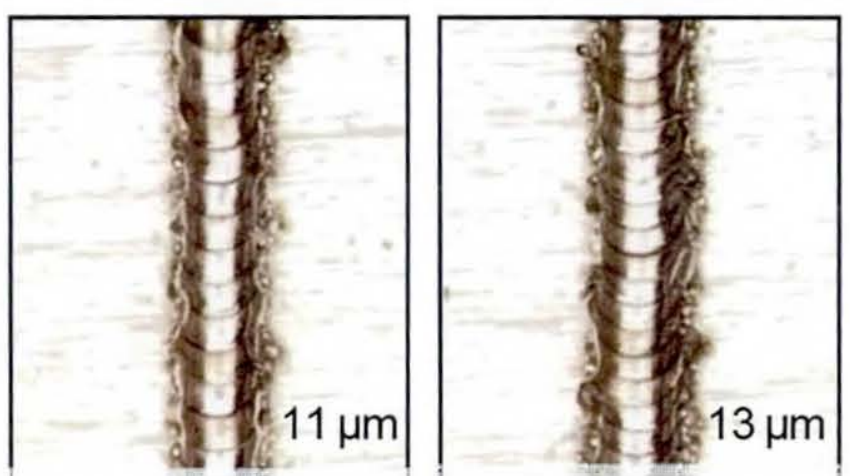

$30 \mathrm{~Hz}, 300 \mathrm{~mm} / \mathrm{min}$
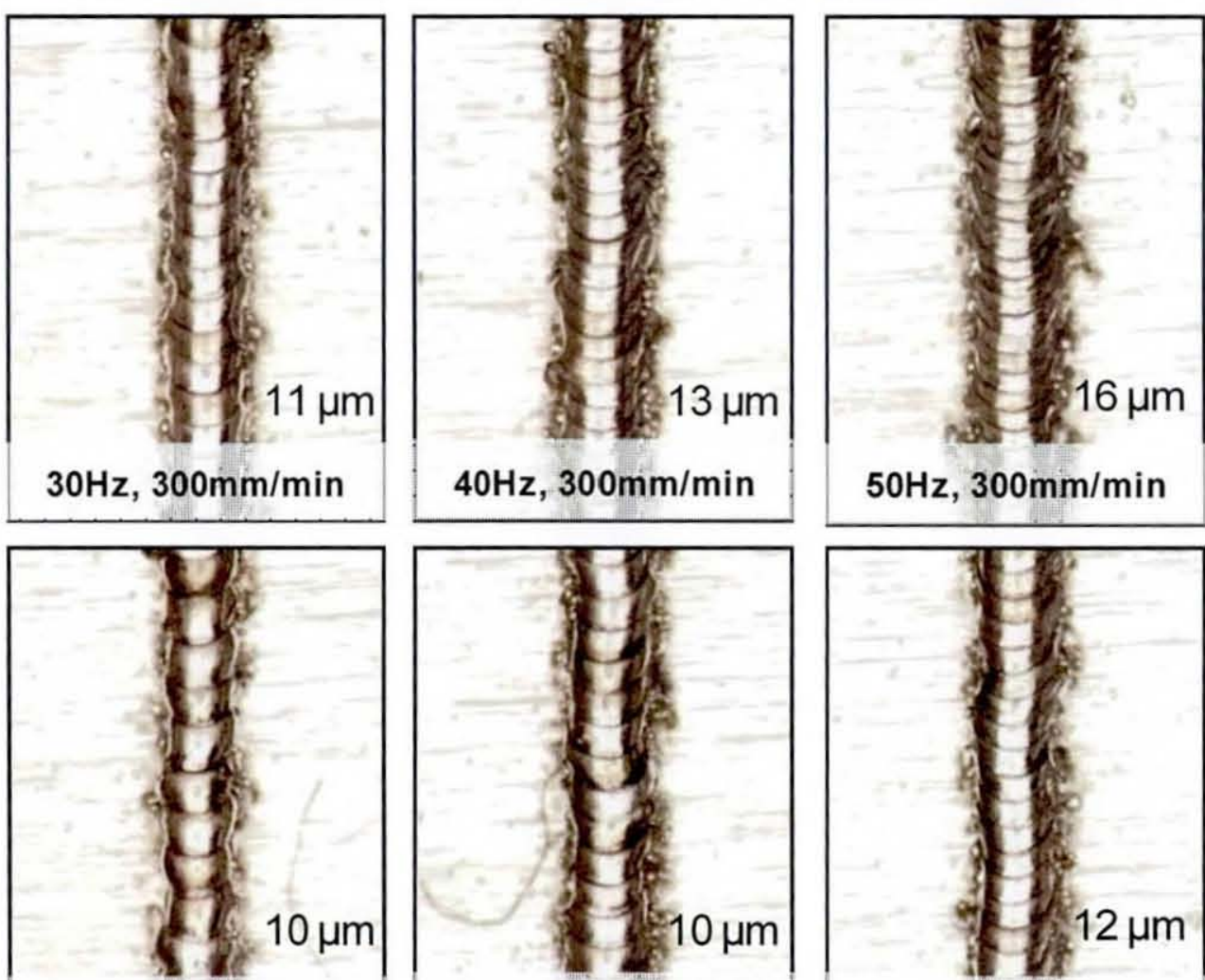

$50 \mathrm{~Hz}, 300 \mathrm{~mm} / \mathrm{min}$
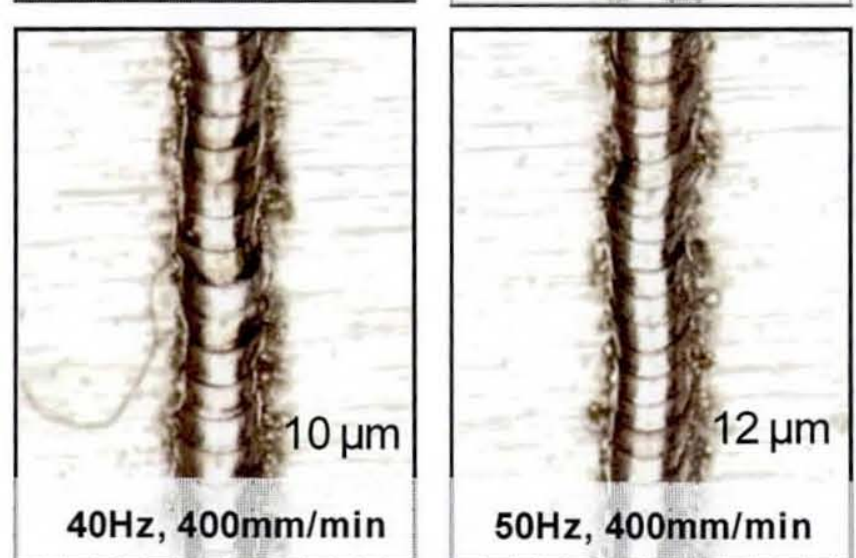

Figure 6-17 Thin walled parts processed at $0.7 \mathrm{~J}$ at varied repetition rates \& scan speeds 
Figure 6-18 shows the plan views taken from the central areas of a sample when processed using $0.5 \mathrm{~J}$ and a fixed repetition rate of $40 \mathrm{~Hz}$. It can be seen that there are many spherical structures (satellite formation/balling) attached to the edges of samples processed using a low scan speed of $100 \mathrm{~mm} / \mathrm{min}$ (a). These structures appeared to be less predominant in the sample produced at a higher scan speed of $400 \mathrm{~mm} / \mathrm{min}(\mathrm{d})$. At a fixed repetition rate lower scan speeds produce more liquid within the melt pool due to an increase in energy density (see section 6.2.3.2). The widened melt pool may subsequently reduce its surface tension variation by breaking up into spherical molten balls (lateral balling) that tend to solidify on the outer edge of the melt pool. Increasing the scan speed reduced the degree of satellite formation/lateral balling and subsequently causes side $R_{a}$ to reduce from $29 \mu \mathrm{m}$ to $14 \mu \mathrm{m}$. Results confirm that the amount of spherical structures at the edge of the samples directly affected part side $R_{a}$.

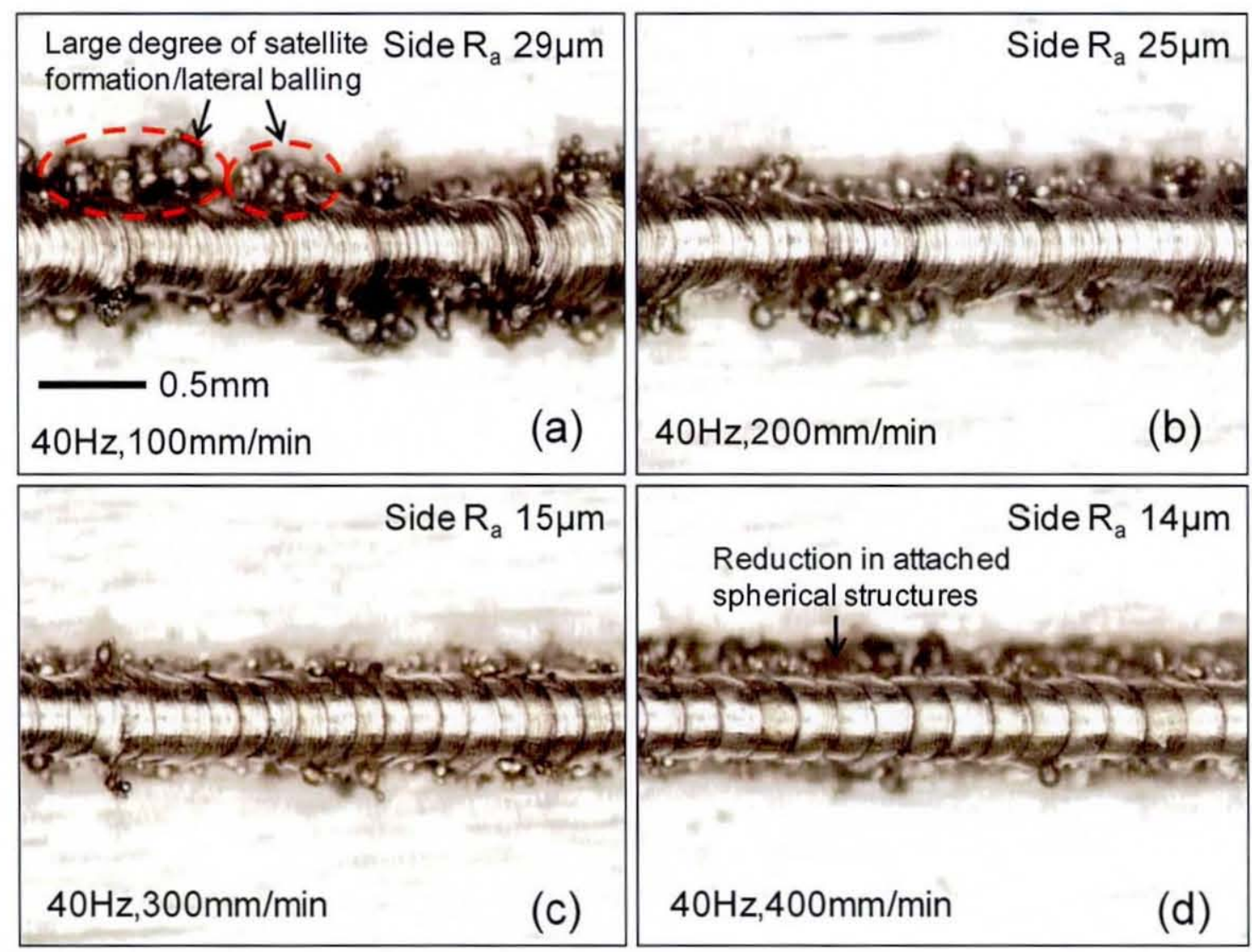

Figure 6-18 Four layer processed using $0.5 \mathrm{~J}, 40 \mathrm{~Hz} \&$ varying scan speeds 
Figure 6-19 shows a graph of side $\mathrm{R}_{\mathrm{a}}$ against spot overlap. Samples produced at overlaps below $85 \%$ tended to have a side $R_{a}$ of between $10-15 \mu \mathrm{m}$. Further increasing the overlap increased side $\mathrm{R}_{\mathrm{a}}$. The satellite formation/lateral balling effect is promoted, as increases in spot overlap form too much liquid that exists for too long.

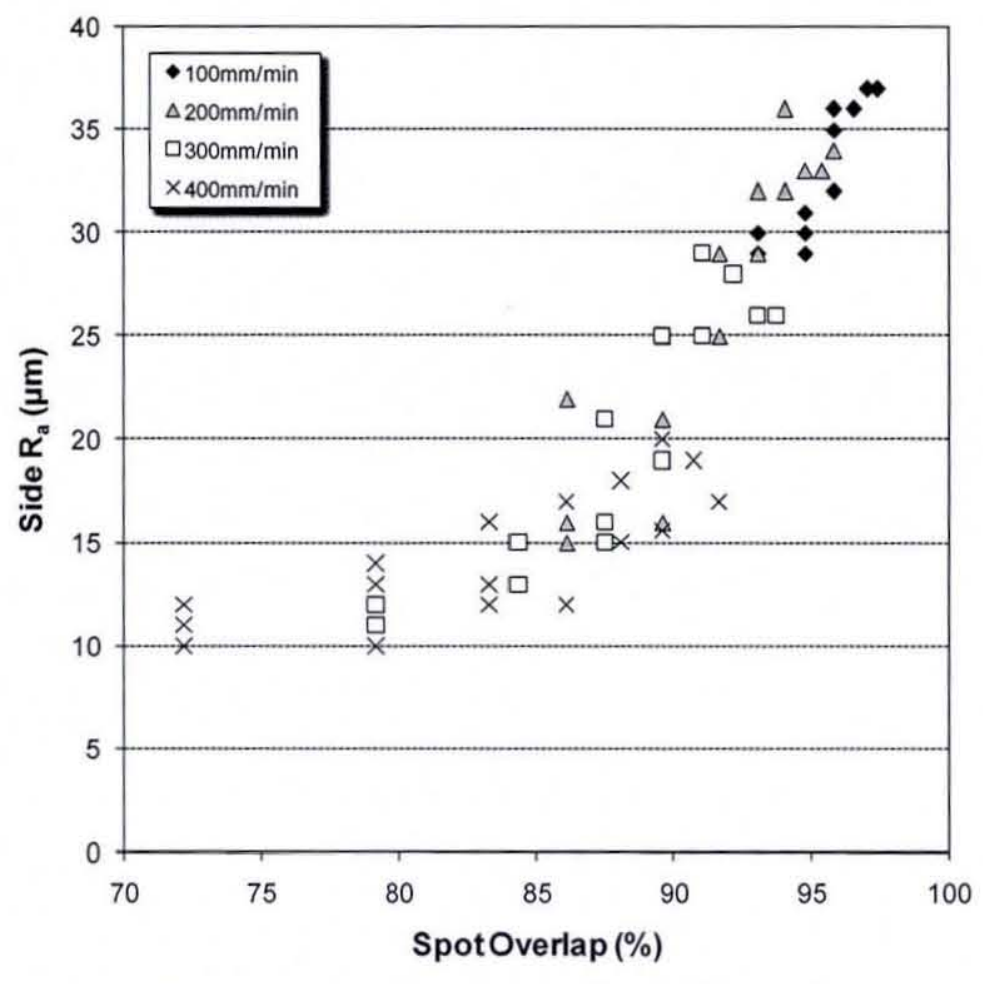

Figure 6-19 Overlap against side roughness

\subsubsection{Energy density}

Figure 6-20 shows results of side $R_{a}$ against energy density at different scan speeds. Clearly increasing energy density increases the side $R_{a}$ of samples. This is related to a widening of the molten component as heat conducts through the powder bed causing more powder particles to stick (agglomerate) to the side of the melt pool and/or cause melt pool break up. Higher scan speeds produce lower energy densities due to less energy per unit area being delivered to the powder bed. When similar energy densities are generated at varying scan speeds, it is the higher scan speed that produces the lower side $\mathrm{R}_{\mathrm{a}}$. This is contrary to the effect decreasing energy density has on top $\mathrm{R}_{\mathrm{a}}$, as discussed in section 6.2.2.2 (Figure 6-14). A maximum side $\mathrm{R}_{\mathrm{a}}$ occurred using a pulse energy of $0.5 \mathrm{~J}$, scan speed of $100 \mathrm{~mm} / \mathrm{min}$ and a repetition rate of $80 \mathrm{~Hz}$. Producing a 
side $R_{a}$ of $37 \mu \mathrm{m}$. This maximum side $R_{a}$ occurs when the energy density is at its highest.

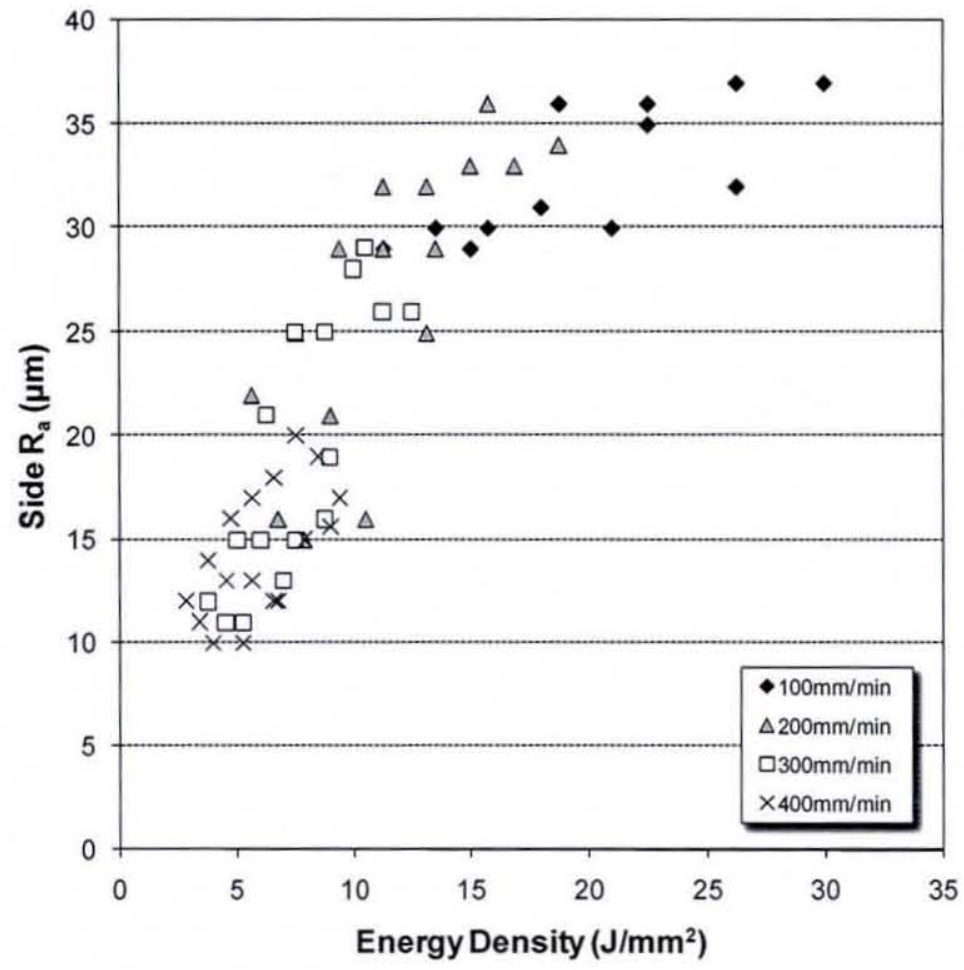

Figure 6-20 Side $\mathrm{R}_{\mathrm{a}}$ against energy density

\subsubsection{Pulse energy}

Results so far have indicated that faster scan speeds and low repetition rates reduce side $\mathrm{R}_{\mathrm{a}}$. However the effects of pulse energy on side $\mathrm{R}_{\mathrm{a}}$ have not been examined comparatively. Figure 6-21 shows the graph of side $\mathrm{R}_{\mathrm{a}}$ against repetition rate when a high scan speed of $400 \mathrm{~mm} / \mathrm{min}$ was used. It can be seen that samples produced at higher pulse energies possess a lower side $\mathrm{R}_{\mathrm{a}}$. At first this may appear to contradict earlier results which state that an increase in energy density increases side $R_{a}$ as increases in pulse energy contribute to increases in energy density. However in this case it may not be the extra energy within the pulse that reduces the side $R_{a}$ but rather the increase in peak power generated as a consequence of the larger pulse energy. At higher pulse energies larger peak powers are generated at a fixed pulse duration, this improves the interlayer connection [Kruth et al. 2004] and lowers side $\mathrm{R}_{\mathrm{a}}$ possibly due to improved wetting of melt. This is also related to the larger recoil pressures generated on the melt pool, causing any satellite formation/lateral balling to completely detach from 
solidifying melt pool (onto the rest of the powder bed) or completely suppress its initial formation by forced melt pool wetting onto the substrate. A minimum side $R_{a}$ of $10 \mu \mathrm{m}$ is achieved using a pulse energy of $0.7 \mathrm{~J}$ and repetition rates between $30-40 \mathrm{~Hz}$, scan speed of $400 \mathrm{~mm} / \mathrm{min}$ producing an overlap between $72.2-79.2 \%$. The samples with the lowest side $\mathrm{R}_{\mathrm{a}}$ are shown in Figure 6-22.

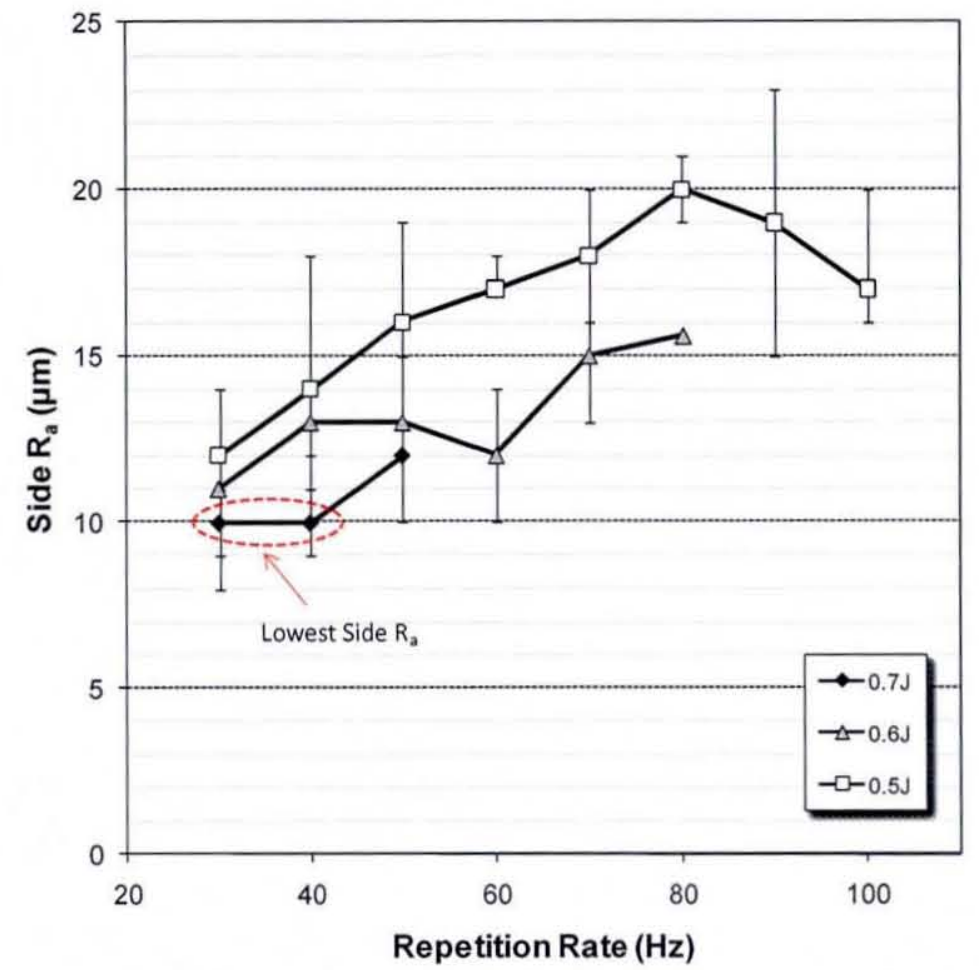

Figure 6-21 Side roughness against repetition rate at $400 \mathrm{~mm} / \mathrm{min}$

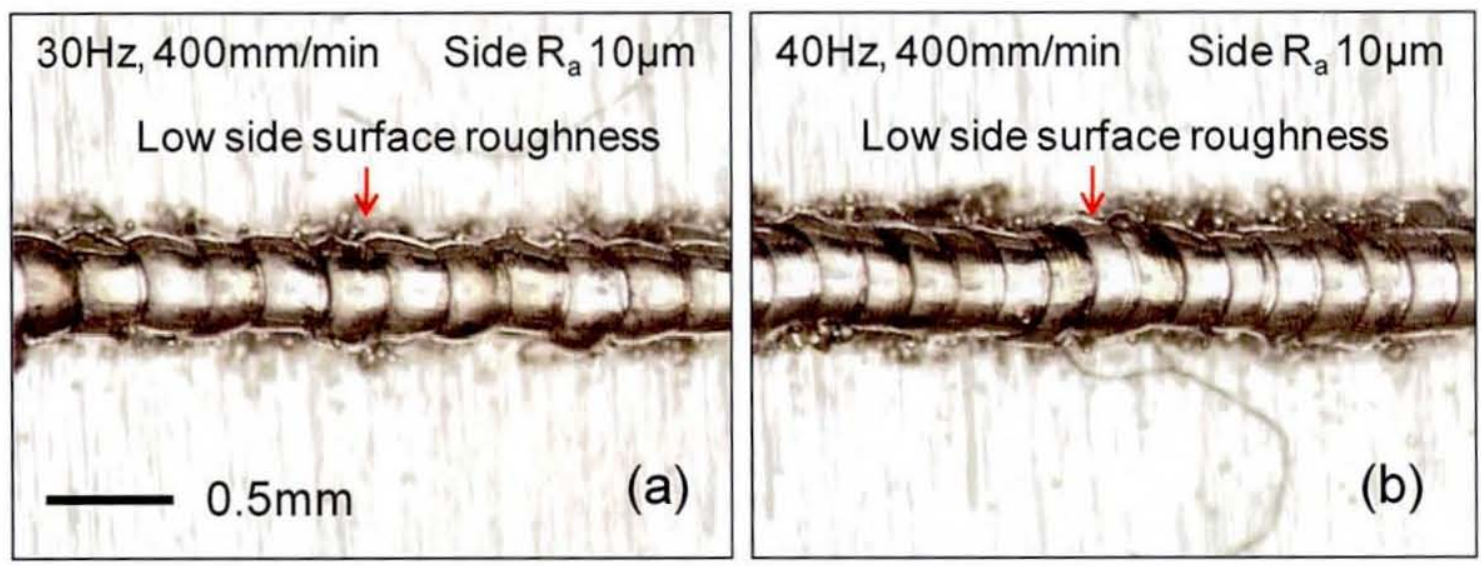

Figure 6-22 Lowest side roughness of $10 \mu \mathrm{m}$ produced using $30 \mathrm{~Hz}$ (a) \& 40Hz (b) at $0.5 \mathrm{~ms}, 0.7 \mathrm{~J} \& 400 \mathrm{~mm} / \mathrm{min}$. 


\subsubsection{Summary}

Spherical structures attached to the edge of the solidified melt pool have been shown to directly affect the side $R_{a}$ of samples. Multiple theories exist to why these structures form, these include satellite formation due to partially melted powder particles, lateral balling due to surface tension and melt pool ejection due to high recoil pressures. It has been demonstrated that the use of certain processing parameters within SLM promote satellite/balling formation. Increasing scan speeds and reducing repetition rate reduce side $R_{a}$. Processing at higher scan speeds not only reduces side $R_{a}$ but increases part production time.

\subsubsection{Width}

The fabrication of thin wall parts to a high resolution complements the SLM technology's main advantage of producing highly complex geometries. Thinner parts will improve the resolution and potentially the accuracy to which parts can be made. The minimum melt pool width can be limited to laser wavelength and spot size (as discussed in section 5.1). Laser parameters used to process the material also heavily influence the width of a melt pool. This section examines the effects spot overlap, energy density and pulse energy on the width of thin walled samples.

\subsubsection{Spot overlap}

Figure 6-23 shows the trend of increasing sample width with increasing laser spot overlap. Increased spot overlaps will increase the energy density and subsequently widen the melt pool as the volume of liquid produced increases. Lower scan speeds are capable of generating high spot overlaps than that of higher scan speeds. Scan speeds of $100 \mathrm{~mm} / \mathrm{min}$ produced spot overlaps of $93-97 \%$ depending on repetition rate. Figure 6-23 shows that the sample width varied considerable between $0.47-0.65 \mathrm{~mm}$, when operating within this spot overlap range. The large range in sample width is as a result of using different pulse énergies when processing the samples (see section 6.2.4.3). 


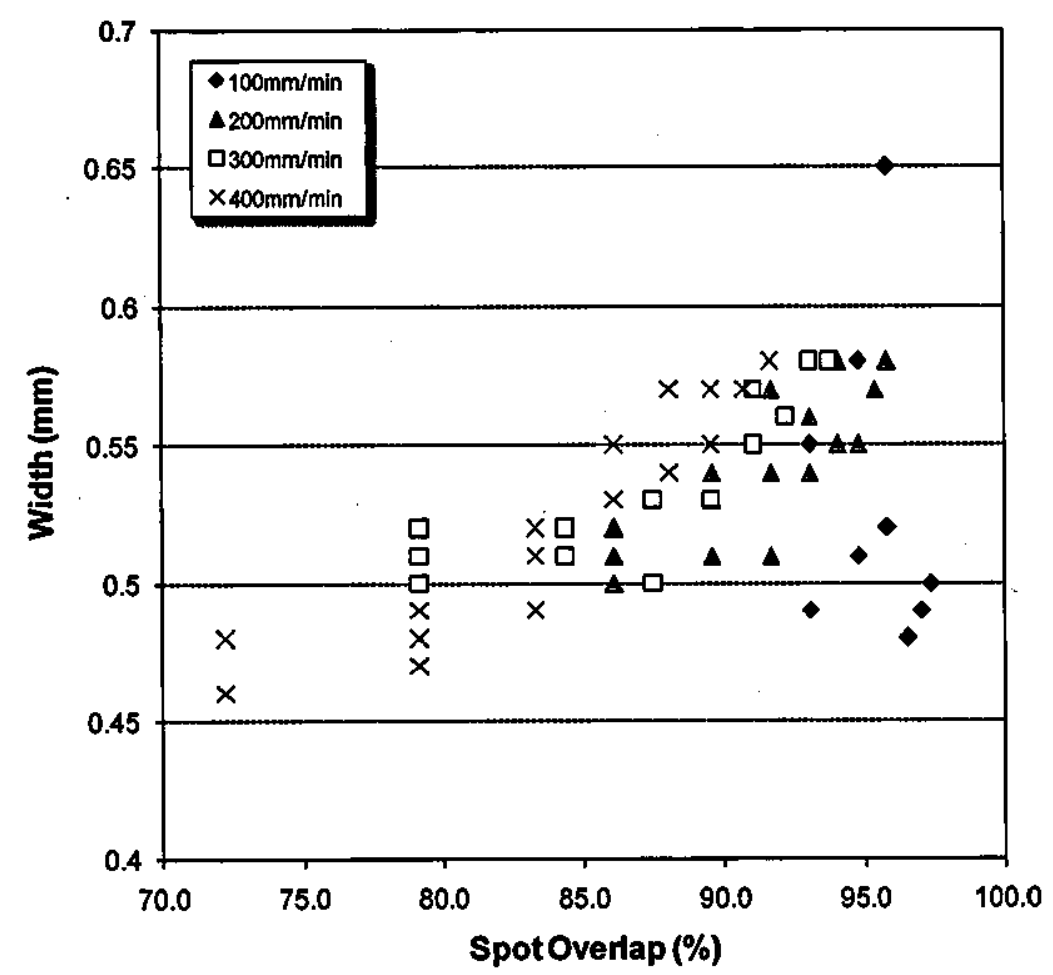

Figure 6-23 Width against spot overlap

\subsubsection{Energy density}

Figure 6-24 shows the width of the samples produced at variable scan speeds and energy densities. An increase in energy density increases the width of samples as additional heat spreads out via conduction regardless of processing scan speed. The volume of liquid produced increases and the melt pool subsequently widens. The lowest sample widths were produced using a scan speed of $400 \mathrm{~mm} / \mathrm{min}$ and energy densities below $5 \mathrm{~J} / \mathrm{mm}^{2}$. 


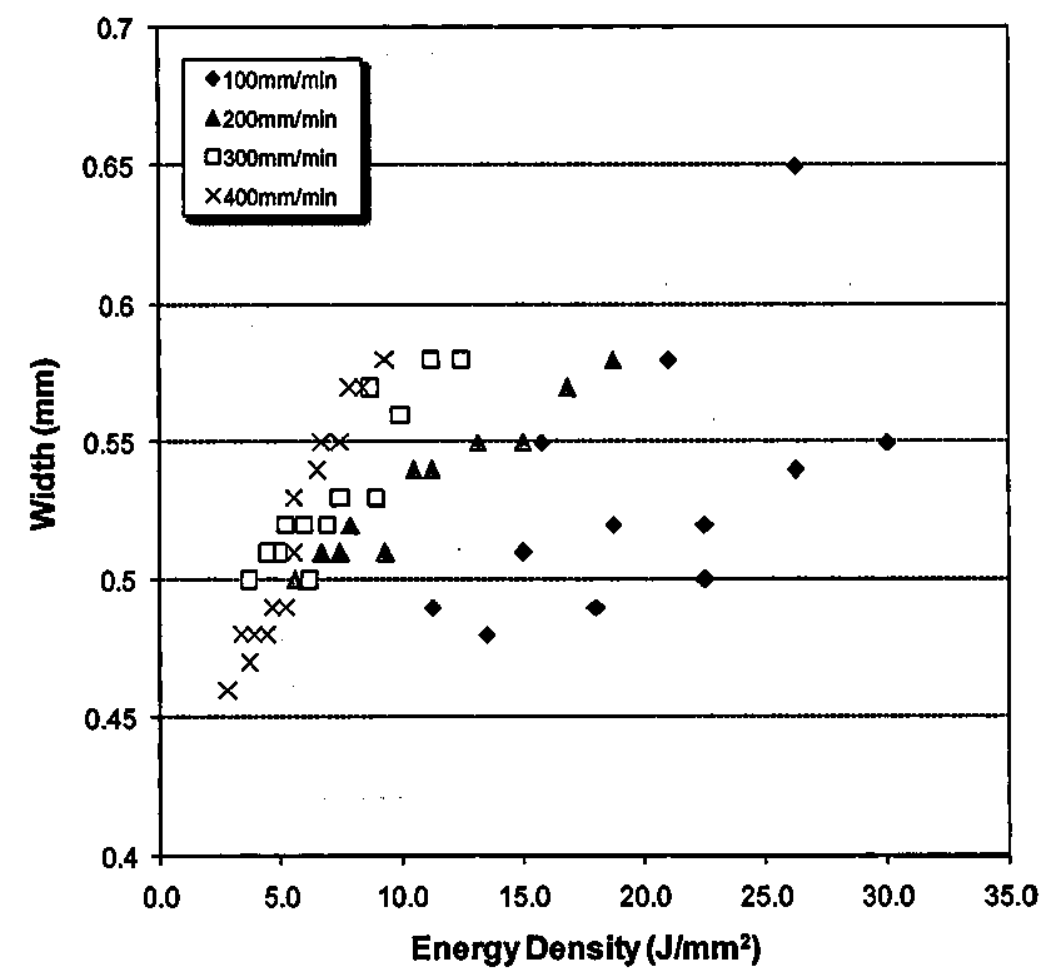

Figure 6-24 Sample width against energy density

\subsubsection{Pulse energy}

Results have indicated that minimum melt width is generated at high scan speeds and low repetition rates (low spot overlap and energy density). Figure 6-25 shows that the use of low pulse energies generates thinner melt pools. Less energy reduces the volume of liquid produced and limits that spread and widening of the melt pool. The use of lower pulse energies at a fixed pulse duration do not promote the generation of high recoil pressures that flatten out and widen the melt pool. A minimum melt pool width of $0.47 \mathrm{~mm}$ is created using a pulse energy of $0.5 \mathrm{~J}$, scan speed $400 \mathrm{~mm} / \mathrm{min}$ and repetition rate of $30 \mathrm{~Hz}$. 


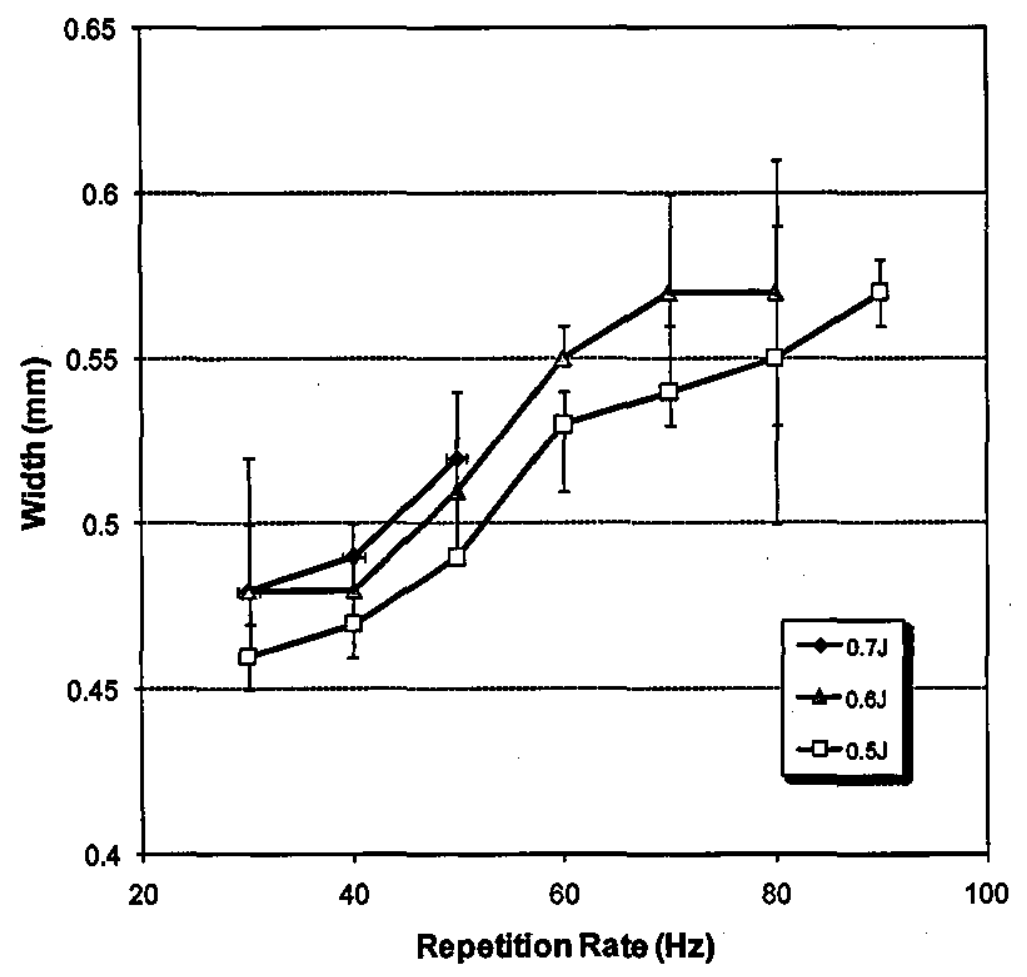

Figure 6-25 Width against repetition rate for samples produced at $400 \mathrm{~mm} / \mathrm{min} \&$ variable pulse energies

\subsubsection{Summary}

Low pulse energies, high scan speed and low repetition rate produce smaller melt pools.

Lower energy densities therefore facilitate the production of thin wall parts. 


\subsection{Optimisation of Surface Roughness and Wall Thickness}

Section 6.2 has shown that different factors affect top/side $R_{a}$, and wall thickness of samples. High pulse energies reduced the top $R_{a}$ of samples due to improved fluidity and movement of molten material within melt pool. This allows for the top surface profile to smoothen out under the forces of gravity reducing variation between overlapping melt pools. The use of larger pulse energies (at a fixed pulse duration) generate larger peak powers that increase recoil pressures, flattening and smoothing out the top surface profile of the melt pool. However the amount of pulse energy delivered to the material has conflicting results in regards to side $R_{a}$. Increases in pulse energy increase the amount of molten liquid produced, widening the melt pool and therefore increasing the temperature differences as outer regions of the melt pool become cooler due to their distance from the centre of the incident laser beam, as seen in Figure 6-26. This results in a melt pool with a higher surface tension variation than a melt pool with a smaller width. Figure 6-27 shows that a melt pool with a larger melt pool width $(W)$ and volume $(V)$ have larger surface tension variations across the melt pool as a result of the greater temperature gradients. In order to reduce this large variation in surface tension across the melt pool the melt pool may breaks up laterally into small spherical balls, subsequently increasing side $R_{a}$ as shown in Figure 6-28. The pressures generated by recoil pressures may assist the break up of molten material and cause it to eject to either side pool rather than settle on top. Larger melt pools have more heat to conduct into the surrounding powder bed than that of smaller melt pools. This causes more powder particles to attach to the outer edge of the solidifying melt pool (satellite formation) subsequently increasing side $R_{a}$. 

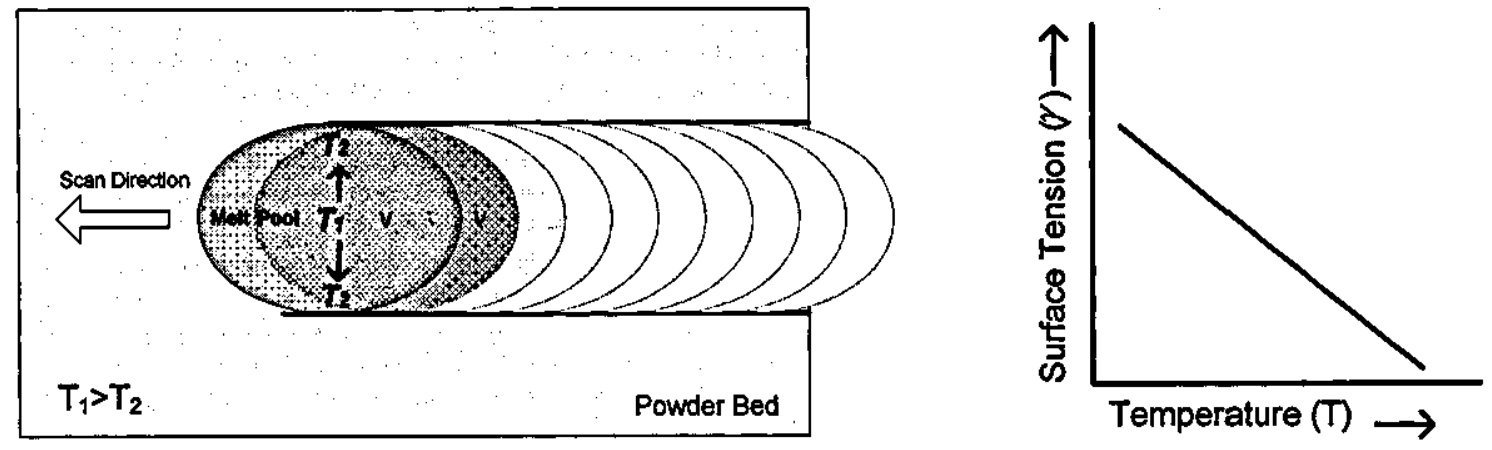

Figure 6-26 Plan view of melt pool formed by pulsed laser \& variations in surface tension due to temperature gradient
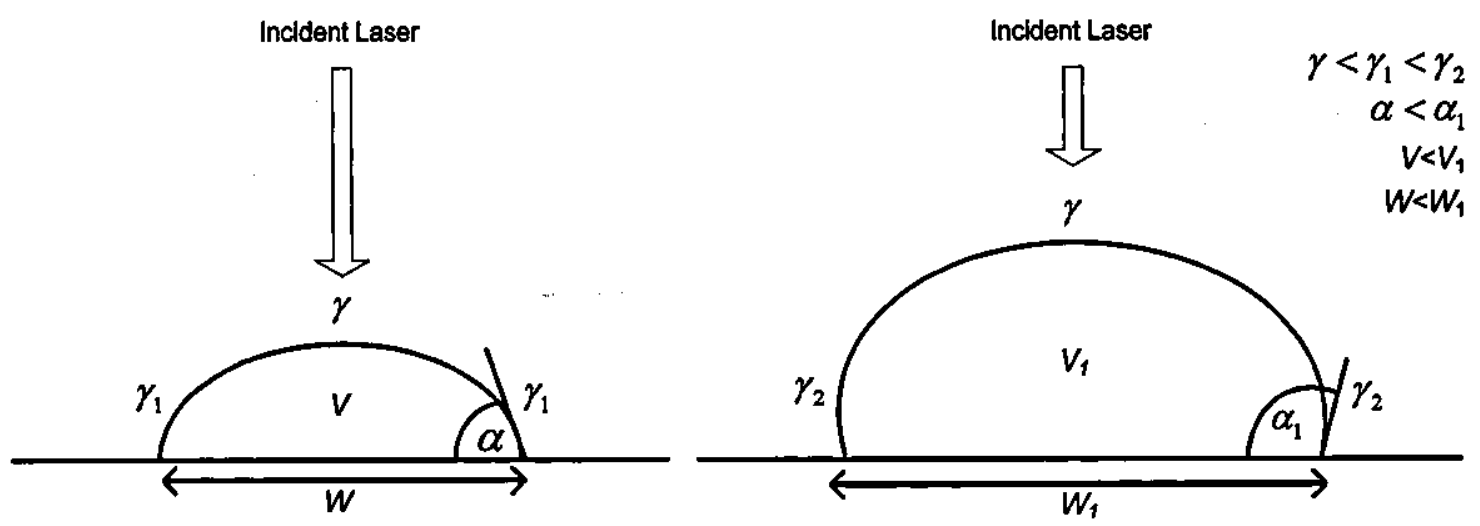

Figure 6-27 Variations in surface tension as a results of melt pool volume and width

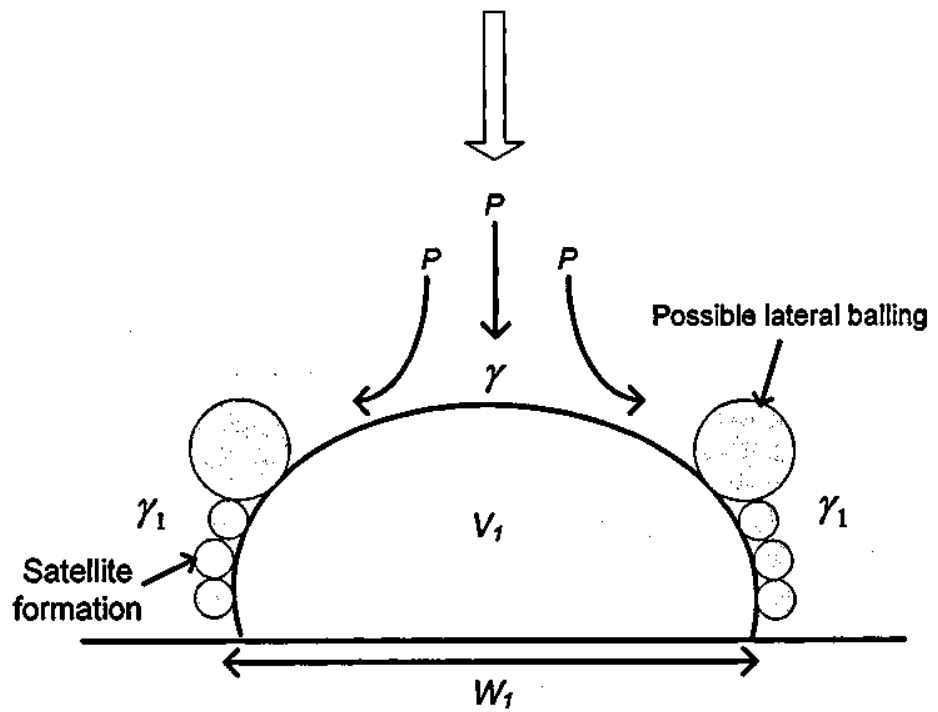

Figure 6-28 Reduction in surface tension via the theory of lateral balling

From first impressions it appears that increases in pulse energy increase side $R_{a}$, however larger pulse energies inherently produce larger peak powers (at a fixed pulse 
duration) due to the nature of a pulsed laser when using a standard Rectangular pulse shape. This increased peak power may reduce part side $R_{a}$ in two ways. The higher peak power improves the wetting (forced wetting) of the melt onto a substrate and therefore reduces a melt pools tendency to undergo possible lateral balling. The larger pressure generated by the increased peak power may also displace any satellite formation from solidifying on the outer edge of the melt pool.

It has been shown that processing with high processing peak powers is beneficial in simultaneously improving top and side surface finish. However other factors have a tendency to pull part properties in opposite directions by improving one part property to the detriment of the other, this is illustrated in Figure 6-29, Figure 6-30 and Table 6-7. A high repetition rate improves the top $R_{a}$ by reducing the variation in overlapping pulses thus causing there to be less variation on the top profile of the melt. This subsequently reduces top surface $R_{a}$. Increasing the repetition rate increased energy density, which may have contributed to an increase in material vaporisation causing an increase in recoil pressures. Subsequently flattening out the melt pool and producing a smoother top surface. However this increase in energy density consolidated more material causing the width of the melt pool to increase. Larger temperature differences build-up across the melt pool, causing an increase in melt pool surface tension variation and hence possible lateral balling. Also larger melt pools will have more heat to conduct into the surrounding powder leading to more satellite formation and higher side $\mathrm{R}_{\mathrm{a}}$. 


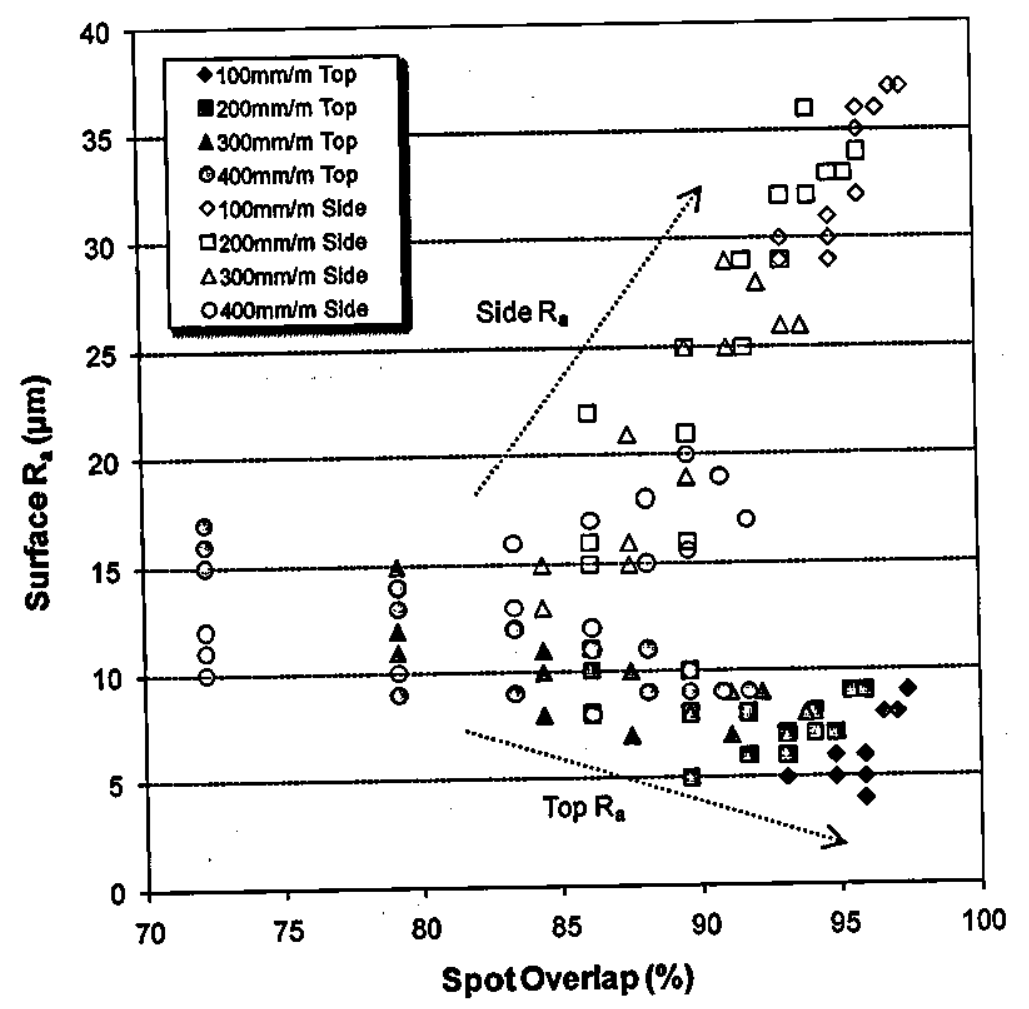

Figure 6-29 Surface $\mathrm{R}_{\mathrm{a}}$ at variable laser spot overlaps

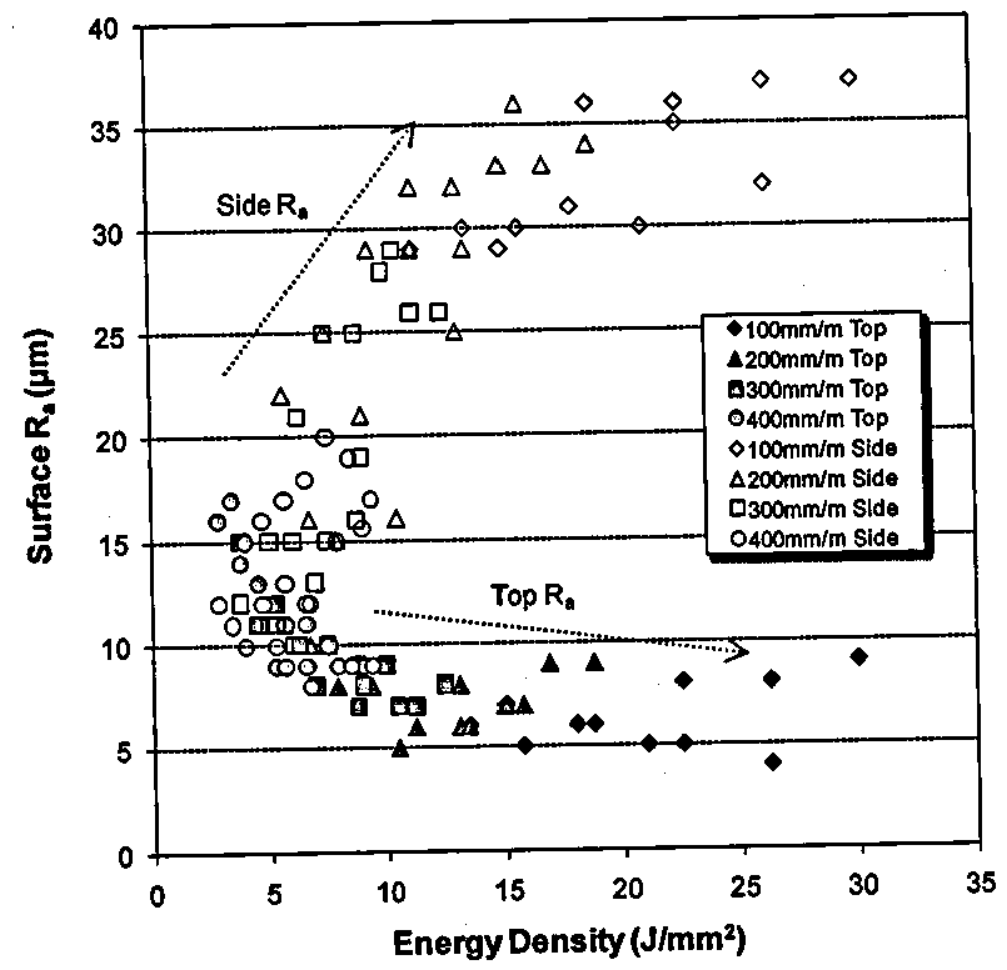

Figure 6-30 Surface $R_{a}$ at variable energy densities

Table 6-7 shows the part properties that are improved with increasing/decreasing laser pulse energy, repetition rate etc. For example the table shows that high repetition rates 
are required to improve top $R_{a}$. A low repetition rate is required to improve side $R_{a}$ and a low repetition rate is required to reduce sample wall width. It shows that all three part properties (top/side $R_{a}$ and width) cannot be simultaneously improved due to each part property requiring different processing parameters inputs (high/low values).

\section{Low Top $\mathbf{R}_{\mathbf{a}}$}

Low Side $\mathbf{R}_{\text {. }}$

Low wall width

Pulse Energy (J)

HIGH

HIGH/LOW

LOW

Repetition Rate (Hz)

HIGH

LOW

LOW

Scan Speed (mm/min)

LOW

HIGH

HIGH

Peak Power (W)

$\mathrm{HIGH}$

HIGH

LOW

SED (J/mm $\left.{ }^{2}\right)$

$\mathrm{HIGH}$

LOW

LOW

Spot Overlap (\%)

HIGH

LOW

LOW

Table 6-7 Process parameters that reduce part top/side $R_{\mathbb{a}} \&$ wall width

\subsubsection{Optimum Parameter Selection}

Figure 6-31 plots side $\mathrm{R}_{\mathrm{a}}$ data acquired from the thin walled parts built in section 6.2 and their respective top $R_{a}$ values. The graph clearly indicates that a sample cannot simultaneously attain an optimum top $R_{a}(4 \mu \mathrm{m})$ and side $R_{a}(10 \mu \mathrm{m})$. As top surface $R_{a}$ improves side $R_{a}$ worsens, as side $R_{a}$ improves top $R_{a}$ worsens. Figure 6-16 showed a sample with a minimum top surface $R_{a}$ of $4 \mu \mathrm{m}$ produced at a high energy density of 26 $\mathrm{J} / \mathrm{mm}^{2}$ and spot overlap of $96 \%$. However because this sample possessed a large wall width of $0.65 \mathrm{~mm}$ it suffered from a high degree of balling, causing it to have a side $R_{a}$ of $33 \mu \mathrm{m}$. A minimum side $R_{\mathrm{a}}$ of $10 \mu \mathrm{m}$ was achieved in two samples, one sample with a wall thickness of $0.45 \mathrm{~mm}$ and the other $0.49 \mathrm{~mm}$ (Figure 6-22). The first sample Figure 6-22(a) was produced using a relatively low overlap (72\%) and energy density (4 $\mathrm{J} / \mathrm{mm}^{2}$ ) causing the top $R_{\mathrm{a}}$ to be $13 \mu \mathrm{m}$. The second sample Figure 6-22(b) was produced at a higher overlap and therefore had a lower top $R_{a}$ of $9 \mu \mathrm{m}$. 


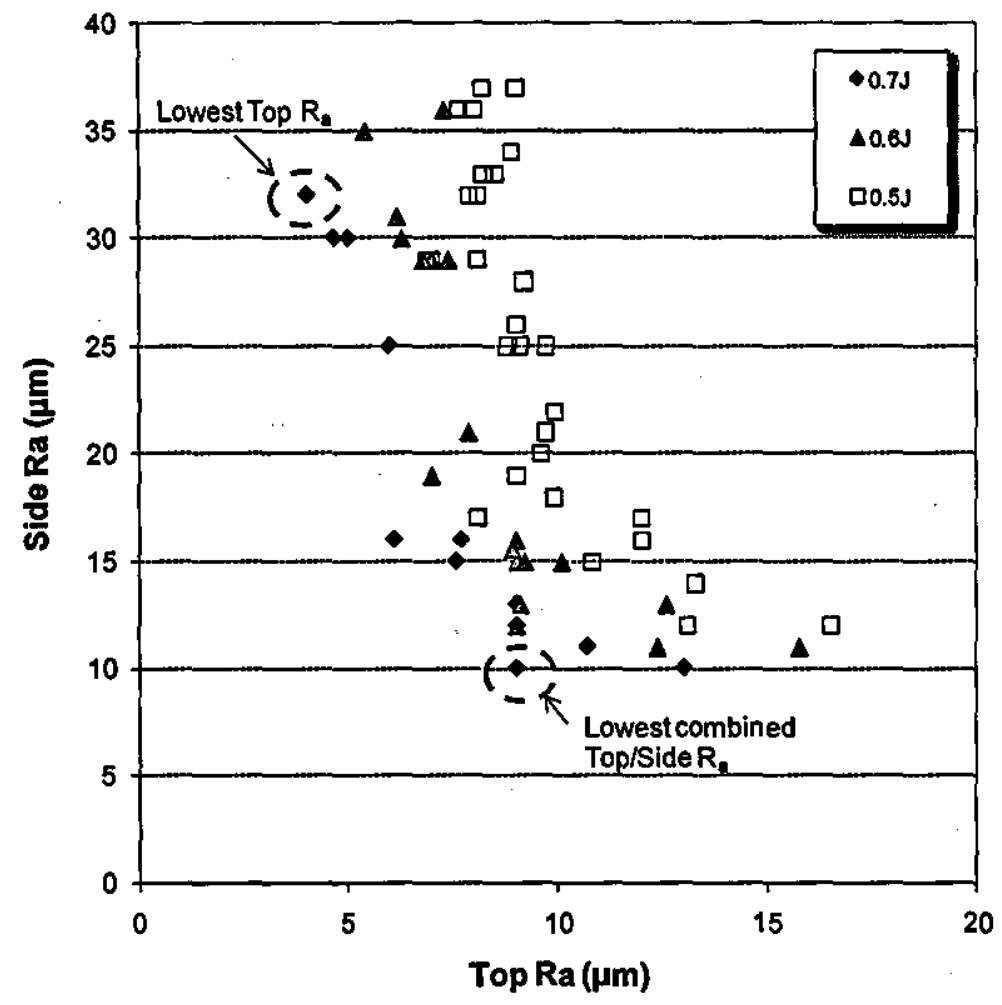

Figure 6-31 Side $R_{a}$ against top $R_{a}$ for thin wall parts

Having seen that minimum top and side $\mathbf{R}_{\mathbf{a}}$ cannot be simultaneously achieved the best solution would be to compromise on optimum top/side $R_{a}$ values and settle for values which when combined produced the lowest value for top/side $R_{a}$. This is illustrated in Figure 6-31 with the actual sample shown in Figure 6-32. This was produced using a pulse energy of $0.7 \mathrm{~J}$, repetition rate $40 \mathrm{~Hz}$ and a scan speed of $400 \mathrm{~mm} / \mathrm{min}$. This produced a top surface $R_{a}$ of $9 \mu \mathrm{m}$, side $R_{a}$ of $10 \mu \mathrm{m}$ and wall thickness of $0.49 \mathrm{~mm}$. These were the optimum processing parameters selected within the initially set range of processing conditions, these parameters may therefore not represent the optimum value for the system. The sample produced was $0.04 \mathrm{~mm}$ thicker then the sample with equal side $R_{a}$ shown in Figure 6-22(a) but had a top $R_{a}$ that was $4 \mu \mathrm{m}$ lower. The simultaneously low top and side $\mathrm{R}_{\mathrm{a}}$ combined with a relatively low wall thickness was a valid reason to select these process parameters as the optimum in producing thin walled parts with minimum top and side $R_{\mathrm{a}}$. At this stage of research more emphasis is put on the reduction of side $R_{a}$ rather than top $R_{a}$. This is because top $R_{a}$ only affects the final layer of parts and can easily be reduced using surface re-melting techniques, whereas the sides of parts are more difficult to re-melt and treat with a laser. 


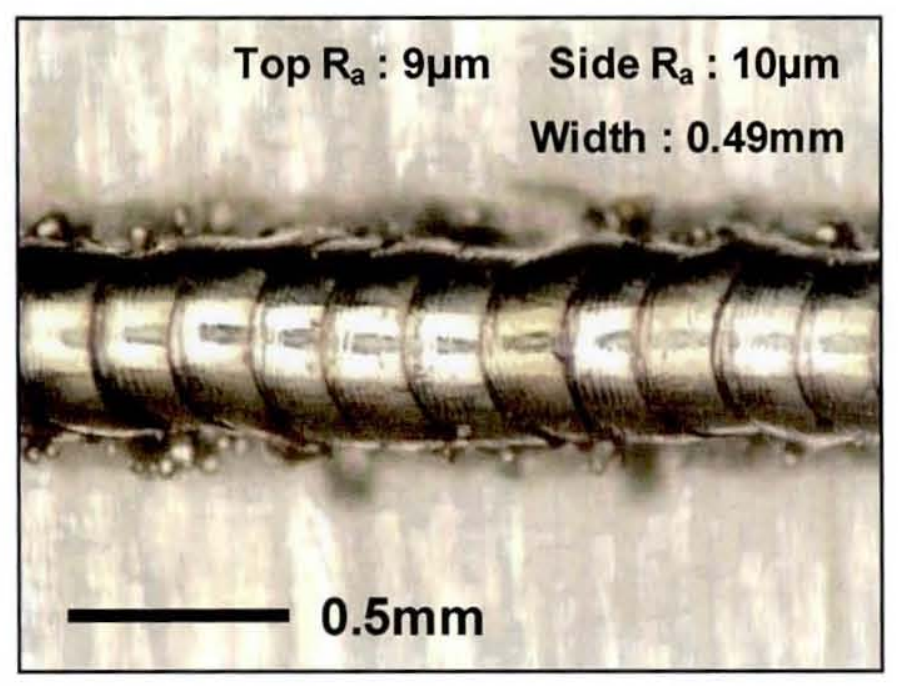

Figure 6-32 Lowest combined top \& side $\mathrm{R}_{\mathrm{a}}$ thin walled part $(0.7 \mathrm{~J}, 40 \mathrm{~Hz}$ at $400 \mathrm{~mm} / \mathrm{min})$

\subsubsection{Summary}

It was found that trying to simultaneously attaining a minimum top/side $R_{a}$ with minimal wall thickness is difficult to achieve. Reducing scan speed and increasing repetition rate reduced top $\mathrm{R}_{\mathrm{a}}$ (as it increased spot overlap) reducing variation in the top surface profile, it also allowed the melt pool to cool at a slower rate. The prolonged cooling gave more time for Marangoni convection to generate stirring forces within melt pool flattening out the top surface profile generated by the pulsed laser. However these factors had an opposite effect in reducing side $\mathrm{R}_{\mathrm{a}}$, as slower scan speeds and high repetition rates cause for the melt pool to extend in width. This extension increases surface tension variation as temperature differences between the central and outer portions of the melt pool increase. The molten material may possibly break up into small spherical balls in order to break up this surface tension causing side $R_{a}$ to increase. The larger volume of liquid present within the melt pool will emit more heat into the surrounding powder bed via conduction causing increases in satellite formation producing higher side $\mathrm{R}_{\mathrm{a}}$. The pulse energy was also shown to hold a conflicting effect on part surface properties. This shows that simultaneously achieving a minimum surface and side $R_{a}$ is difficult to achieve with the current method of SLM. The key to generating parts with low surface roughness and low wall width is carefully controlled 
heat input. Too little or too much pulse energy, peak power or energy will result in the detriment of either top or side $\mathrm{R}_{\mathrm{a}}$.

Therefore future success in minimising top and side $\mathrm{R}_{\mathrm{a}}$ requires careful control such that the melt pool width remains short and remains molten for a shorter period of time. Finally recoil pressures should be sufficiently high to reshape and flatten the melt pool, improving its wetting characteristics. 


\section{Chapter 7 Pulse Shaping Experiments}

Section 2.3 demonstrated that research using pulse shaping in the laser welding of metal sheets revealed certain advantages over non pulse shaped pulses. These advantages included the production of smoother welds, reduced crack propagation and reduced spatter generation during processing. The extended degree of control over energy distribution within a pulse allowed these advantages to be realised.

Chapter 6 discovered that controlling certain laser parameters allowed SLM part properties such as surface roughness and width to be minimised. Using the four main processing parameters pulse energy, pulse duration, repetition rate and scan speed an optimum set of processing parameters were found, such that a thin walled part with low top and side surface roughness was attained. However the optimum value for either top or side roughness could not be simultaneously attained due to limited melt pool control, as certain factors improved one part characteristic while deteriorating the other. The SLM of parts is a complex process that imparts large heat intensities within a very short space of time. This rapid heating requires a greater degree of control such that the correct volume of liquid is present within the melt pool and possesses stable qualities (high wettability, low thermal variation across melt pool and low tendency to undergo balling). The four main processing parameters allow the user to have four degrees of control over the heat input delivered to the material. However if the user is given further control over process parameters, a more precise and tailored control over the heat input could allow a refining and improvement over current part properties. Control of the pulse shape may allow a pulse to possess an ideal energy distribution identified in section 6.3 (i.e. high peak power, low pulse energy and short pulse duration) that would produce thin wall parts with improved surface roughness properties. With the use of non pulse shaping methods, the user is restricted to the degree of control over the pulse's energy distribution (i.e. difficult to achieve high peak power, low pulse energy combination). 
This chapter will investigate the effects of pulse shaping on the formation of SLM thin wall Inconel 625 parts. The pulse shaping experimentation focused on the use of traditional pulse shapes such as Ramp Up and Ramp Down and examined what effect they had on top $R_{a}$, side $R_{a}$ and wall width. An added benefit of pulse shaping highlighted in the literature review, was the potential to extend the processing range of the laser by reducing the spatter generation during processing (achieved using the Ramp Up pulse shape).

\subsection{Pulse Shaping Characterisation}

As mentioned in section 2.3 pulse shaping is achieved by varying the pulse current used to excite a laser's flash lamp into as many as 20 individual sectors and specifying the duration and peak power of each sector. The splitting up of the pulse into sectors are in essence the building blocks of the pulse shape. The current supplied by the electric power supply causes the flashlamps to emit light that is absorbed and amplified by the Nd:YAG crystal. This amplified light is emitted in short bursts/pulses through various focusing lenses and eventually onto a work piece.

Figure 7-1 shows the SLM control screen used to adjust laser parameters including pulse shape control. The number of sectors within the pulse is specified, pulse shapes must always contain at least one sector with a minimum width of $0.5 \mathrm{~ms}$ for the main sector and $0.3 \mathrm{~ms}$ for all other sectors. The power for each sector within a pulse is set using a Height $(\mathrm{Hgt} \%)$ control that proportionately supplies a current demand profile to the laser flashlamps. A Hgt of $100 \%$ equates to $100 \%$ lamp power (or 9V) and a Hgt of $0 \%$ equates to $0 \%$ lamp power (or $0 \mathrm{~V}$ ). Once the Hgt and duration of each sector within a pulse has been set, the profile of the power demand input to the laser can be visualised. However the actual laser output shape was not known until an external digital storage oscilloscope is attached to the system. 


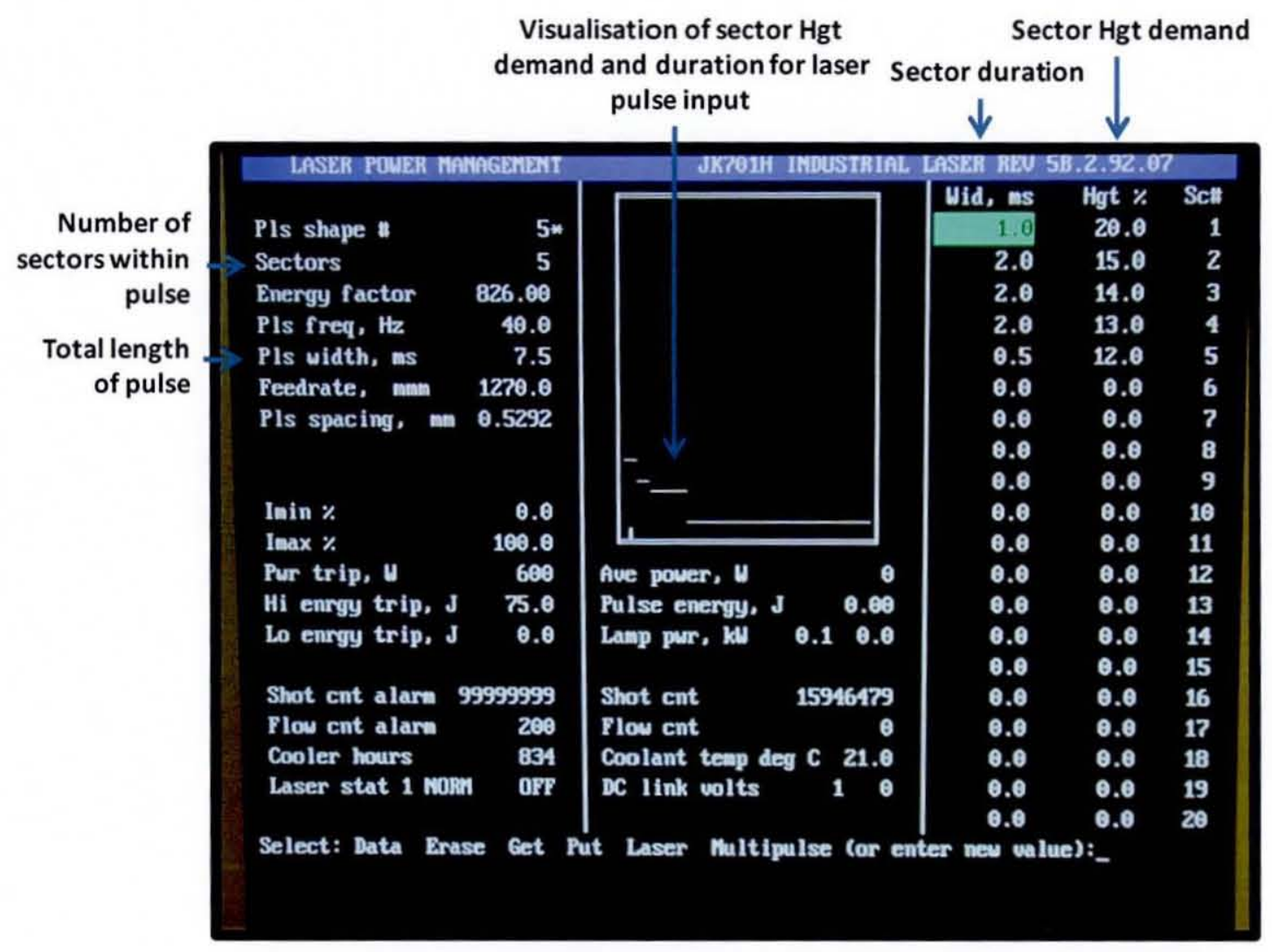

Figure 7-1 SLM laser control screen with pulse shaping control

\subsubsection{Pulse Shape Monitoring}

The true laser output pulse shape will indicate the energy distribution within a single pulse. Accurate representation of an output pulse shape will allow the user to expound on results and relate the pulse shape to the heat distribution throughout the pulse. Simply observing the laser input (as seen in Figure 7-1) is not enough to effectively understand the effects of the laser output pulse. There are signal amplification, noise, power overshoot, system resistance, and other pulsed laser characteristics that affect the true shape of the output pulse shape. A few authors have conducted pulse shaping experimentation without monitoring the true output shape of pulses [Gower et al. 2005; Pan et al. 2005; Sparks et al. 2006]. Failure to monitor the true output of the laser can lead to errors in results and derived relationships.

The actual monitoring of the laser's output was observed using a PicoScope 3205 PC digital storage oscilloscope to display voltage waveforms as shown Figure 7-2. This has all the benefits of a standard digital storage oscilloscope but with the advantage of auto 
ranging, real time recording filters and measurement analysis. The PicoScope was connected to the laser system via three BNC connectors. These plugged into current input, laser output and trigger. The current input would send data to the PicoScope relating to the input to the laser, the laser output represent the laser's true pulse shape. The trigger specifies a point in time in which the PicoScope software would begin collecting data.

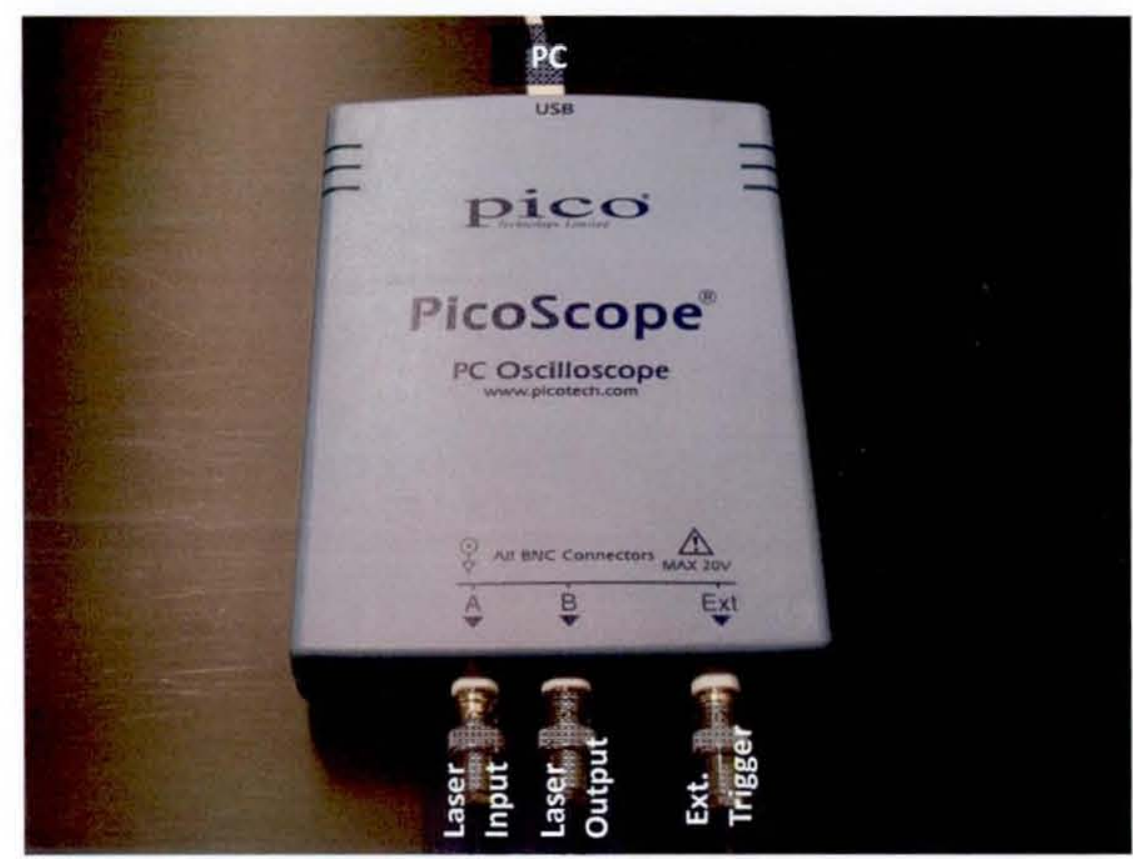

Figure 7-2 PicoScope 3205 PC oscilloscope

Figure 7-3 shows the oscilloscope window in the PicoScope PC software. The oscilloscope displays the laser input and output for a standard Rectangular pulse shape. The laser input pulse (represented in blue) consisted of one sector with a duration of approximately $0.5 \mathrm{~ms}$ and a $\mathrm{Hgt}$ of $44 \%$, this gave a voltage reading of approximately $4 \mathrm{~V}$. The laser output (represented in red) displays the energy distribution throughout the pulse's duration. Section 7.1.2 discusses how peak power and energy levels were interpreted by analysing the laser output. 


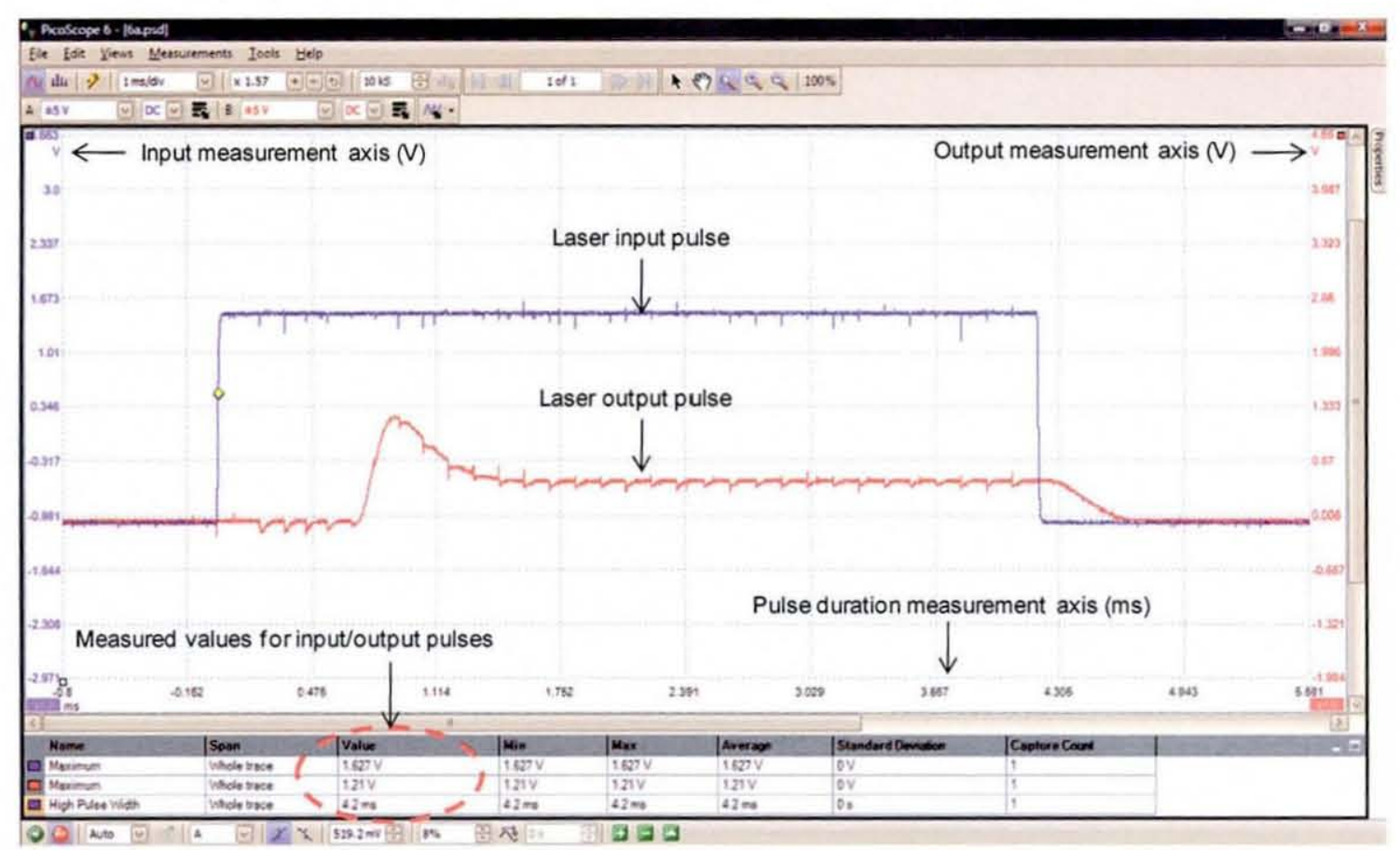

Figure 7-3 Oscilloscope window in PicoScope PC software

\subsubsection{Peak Power and Energy Levels}

Changing the energy distribution within a pulse can completely change the melting behaviour of a material. Peak powers and pulse energies once deemed useable within a standard Rectangular pulse shape may become redundant due to suboptimal melting of material (excessive molten material produced, temperature too low to melt material etc.). Observing the shape of a pulse shape (Rectangular, Ramp up, Ramp Down, spiking etc.) is not enough to completely understand the full characteristics of the pulse. The energy distribution and peak power intensities throughout the pulse need to be determined.

\subsubsection{Pulse energy}

Figure 7-4 shows the input and output of a Rectangular pulse shape that has a $4 \mathrm{~ms}$ pulse duration and contains $2 \mathrm{~J}$ of energy. The integrated area under the laser output curve is known as the pulse energy. The pulse energy contained within a pulse is automatically determined by the laser system using a silicon photodiode power monitor within the laser head unit and displays the pulse energy on the control panel shown in Figure 7-1. It can be seen that a large amount of energy is delivered throughout the first $1 \mathrm{~ms}$ of the output pulse (overshoot or spiking) due to non-linear flashlamp effects during warm up 
(note output pulse is generated approximately $1 \mathrm{~ms}$ after input to the flashlamps). The remaining 1-3ms contains a lower energy density that tapers off quickly towards the end of the pulse.

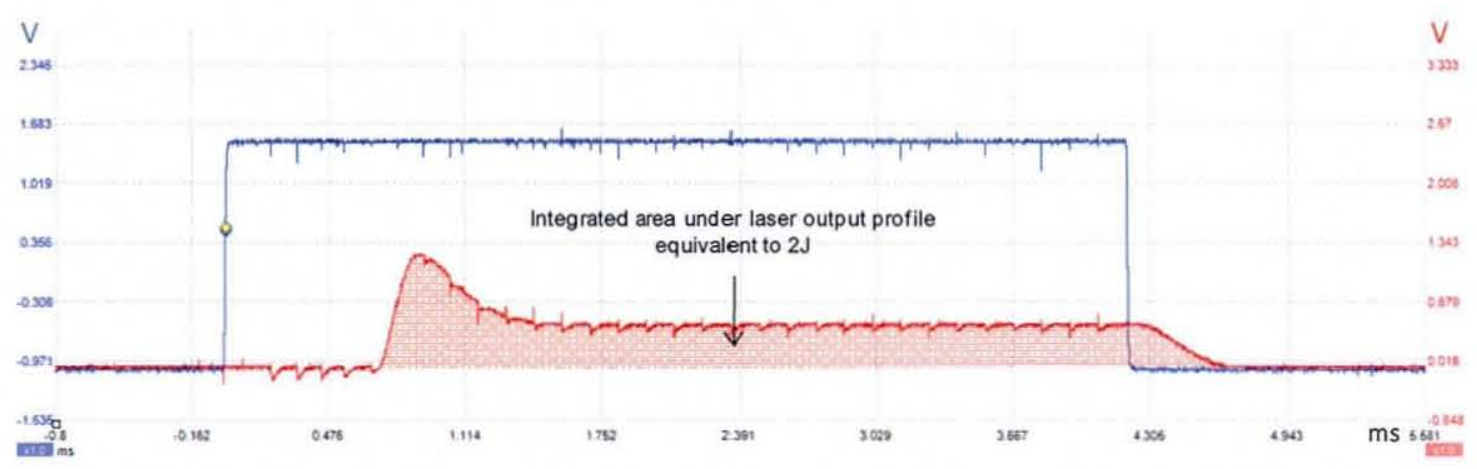

Figure 7-4 Rectangular pulse shape with a $4 \mathrm{~ms}$ durations \& $2 \mathrm{~J}$ energy

\subsubsection{Peak power}

The peak power is the maximum output of the laser. The method commonly used to determine average peak power is to divide the pulse energy by pulse duration as shown in Equation(3-2) [Tam et al. 1993; Fujinaga et al. 2000; Tzeng \& Chen 2000; Osakada $\&$ Shiomi 2006]. However this formula cannot be used to determine the maximum peak power. Therefore a method to accurately determine the pulse's peak power was required. Figure 7-5 shows a $4 \mathrm{~ms}$ Rectangular pulse shape with $1 \mathrm{~J}$ of energy (produced at $40 \mathrm{~Hz}$ ) as displayed using the PC oscilloscope. By measuring the voltage reading along the $y$-axis it is possible to calculate the pulse's power (W) at any point along its duration. The highest point on the laser output curve is equivalent to the maximum peak power of the laser. Lumonics state that for the JK701 Nd:YAG laser every volt displayed on an oscilloscope is approximately equivalent to $1000 \mathrm{~W}$ peak power [Lumonics 1995]. For the pulse shown in Figure 7-5 the maximum peak on the laser output registers as $1.2 \mathrm{~V}$, according to Lumonics this is equivalent to $1.2 \mathrm{~kW}$ peak power. For this Rectangular pulse shape the average peak power can be calculated by dividing the pulse energy by pulse duration. This results in an average peak power of $500 \mathrm{~W}$ $(0.5 \mathrm{~V})$. 


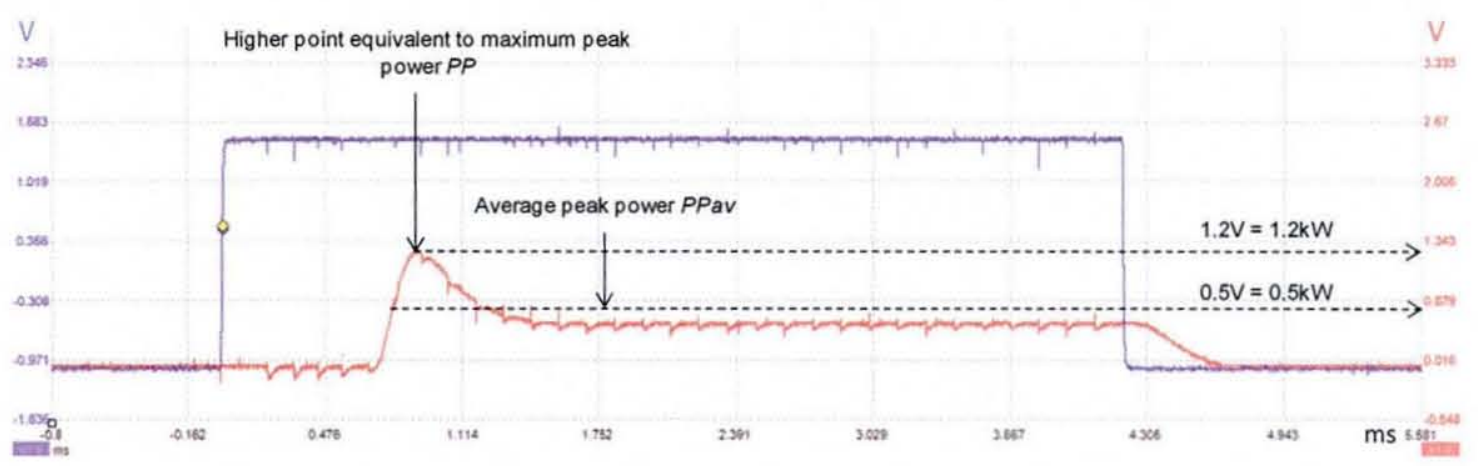

Figure 7-5 Maximum/average peak power of a $5 \mathrm{~ms}$, $1 \mathrm{~J}$ Rectangular pulse shape

\subsubsection{Characteristics of the Optimised Non Shaped Rectangular Pulse}

Having identified the key parameters involved in pulse shape measurement and analysis, the pulse shape of the optimised laser parameters developed in section 6.3 for producing thin wall parts with low top/side $\mathrm{R}_{\mathrm{a}}$ can be examined. Figure 7-6 displays this optimised non shaped standard Rectangular pulse and an image of the part produced. The laser input pulse was set to $0.5 \mathrm{~ms}$ and consisting of one sector set at $35 \mathrm{Hgt}$ powering the flash lamps to give a laser output pulse of $0.7 \mathrm{~J}$. The laser output initiates approximately $0.5 \mathrm{~ms}$ after the laser input and takes approximately $0.2 \mathrm{~ms}$ to reach maximum peak power. After the peak power has been reached the power quickly tapers off to zero. The rapid increase and decrease in laser power equates to very rapid heating and cooling of material.

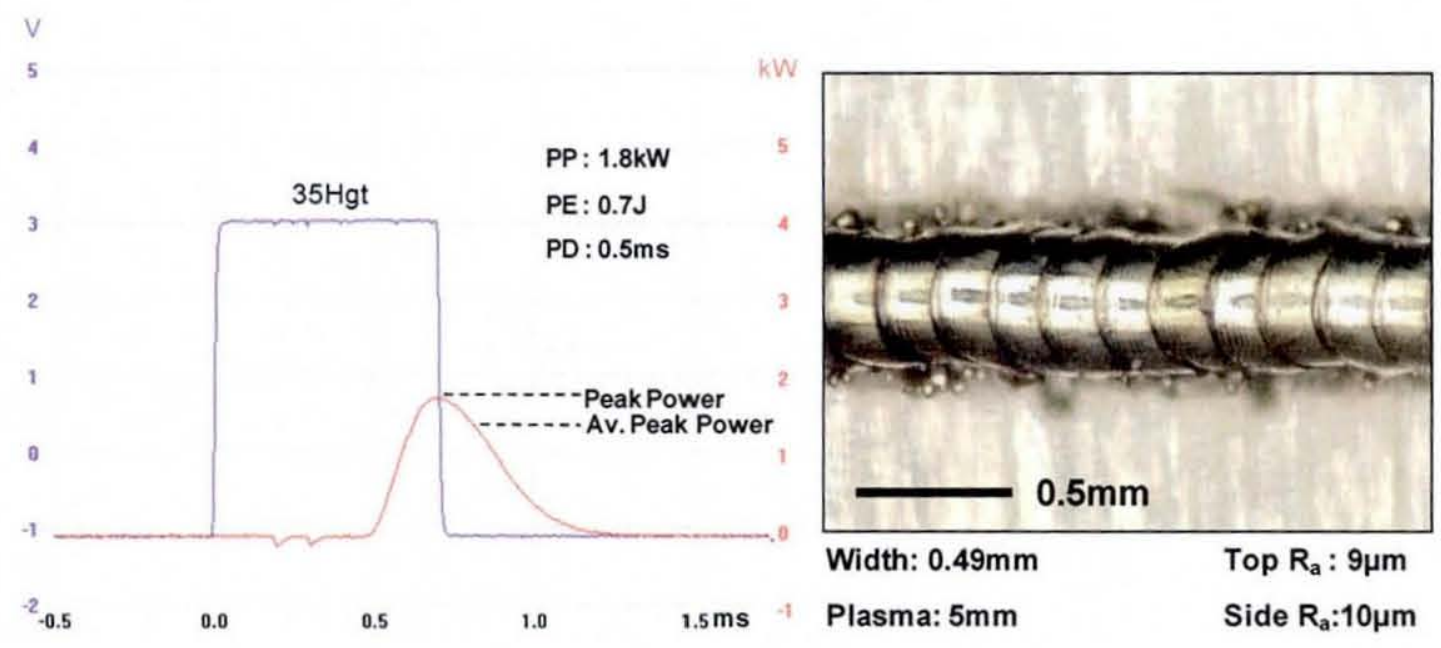

Figure 7-6 Optimised Rectangular pulse shape 
Unexpectedly, rather than the pulse being $0.5 \mathrm{~ms}$ in duration it became $0.7 \mathrm{~ms}$. Therefore in reality, the laser system's minimum pulse duration was in fact $0.7 \mathrm{~ms}$ (as opposed to $0.5 \mathrm{~ms}$ ). It is known for the power in pulsed lasers to overshoot, however there has been no information regarding pulse duration overshoot. This pulse duration overshoot extends the time for which the material is heated, possibly increasing the volume of liquid within the melt pool and/or allowing more time for a melt pool to solidify.

\subsection{Ramp Up Pulse Shapes}

A Ramp Up pulse shape is intended to gradually increase the power output from zero at the beginning of the pulse to a maximum peak power at the end of the pulse. Within this experiment a Ramp Up pulse will be defined as a pulse that has a consistent and a gradual power build up, with no significant drops or loss in power.

\subsubsection{Methodology}

Pulse shaping requires a pulse to be divided into sectors. The more sectors the more complex the pulse shape. Pulse shaping began with the splitting of the pulse shape into very few sectors with short durations and low powers. The pulse shaping experimentation progressively increased the overall duration and power within the pulse when producing individual samples (as shown in Figure 7-7). Extra sectors were introduced where needed such that a smooth Ramp Up power delivery would be generated. Parts were built consisting of four multi-layer single scans measuring $25 \mathrm{~mm}$ in length which were processed from a $0.1 \mathrm{~mm}$ powder bed. All pulse shaping experiments maintained the same repetition rate $(40 \mathrm{~Hz})$ and scan speed $(400 \mathrm{~mm} / \mathrm{min})$ using the optimised processing parameters found in section 6.3. The pulse duration and energies of the Ramp Up pulses varied between $1-10 \mathrm{~ms}$ and $0.6-3.5 \mathrm{~J}$ respectively (as shown in Table 7-1). Three samples were built for each set of processing parameters. Samples were measured for top/side $\mathrm{R}_{\mathrm{a}}$ and width using the same methods described in section 6.2.1. Due to other work reporting a reduction in spatter generation using Ramp Up pulses during laser welding [Katayama et al. 1993; Fujinaga et al. 2000; Gower et al. 2005; Pan et al. 2005] the level of plasma plume/spatter generation was also 
monitored during all pulse shaping experiments in order to verify whether spatter reduction could also be achieved during processing using SLM.

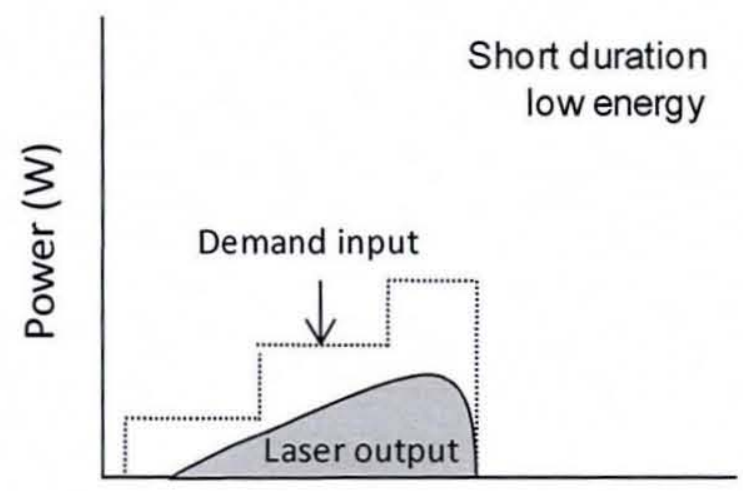

Pulse Duration (ms)

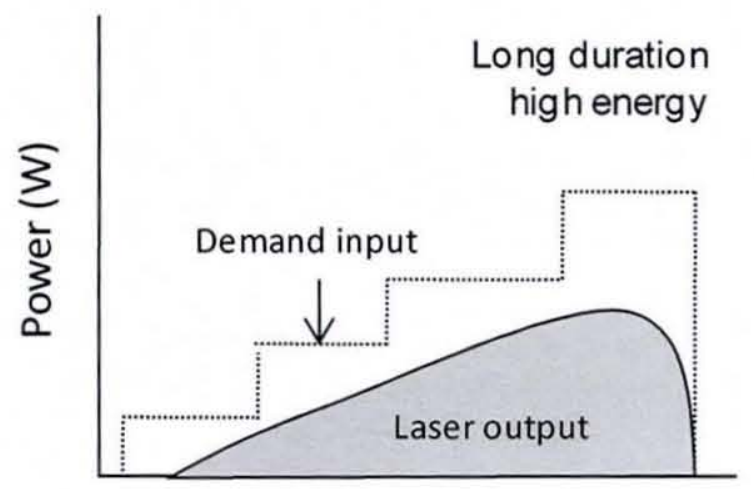

Pulse Duration (ms)

Figure 7-7 Ramp Up pulse shape methodology

\begin{tabular}{cccc}
\hline Pulse Duration $(\mathrm{ms})$ & Pulse Energy $(\mathrm{J})$ & Rep. Rate $(\mathrm{Hz})$ & Scan Speed $(\mathrm{mm} / \mathrm{min})$ \\
\hline $1-10$ & $0.6-3.5$ & 40 & 400 \\
\hline
\end{tabular}

Table 7-1 Ramp Up pulse generation parameter range

\subsubsection{Ramp Up issues}

Initially a pulse was divided into two sectors, the minimum required for pulse shaping. The first sector was assigned at a low $10 \mathrm{Hgt}$ and $0.5 \mathrm{~ms}$ duration, the second sector was assigned a $35 \mathrm{Hgt}$ and $0.5 \mathrm{~ms}$ duration (optimised). This produced a pulse that was overall $1 \mathrm{~ms}$ in duration as shown in Figure 7-8. From literature it was expected that the laser output pulse would gradually Ramp Up to maximum peak power, however this was found not to be the case. Instead of the gradual Ramp Up to an optimised $1.8 \mathrm{~kW}$ peak power, the additional sector at the beginning of the pulse increased the initial spike of the pulse. Figure 7-8 also displays the original standard Rectangular pulse shape for comparison (abbreviated with R PS). The modified pulse shape not only had a larger peak power and pulse energy than the Rectangular pulse shape, but also marginally increased the rate of heating. Further trials were undertaken to explore and understand how a Ramp Up pulse shape could be effectively developed. 


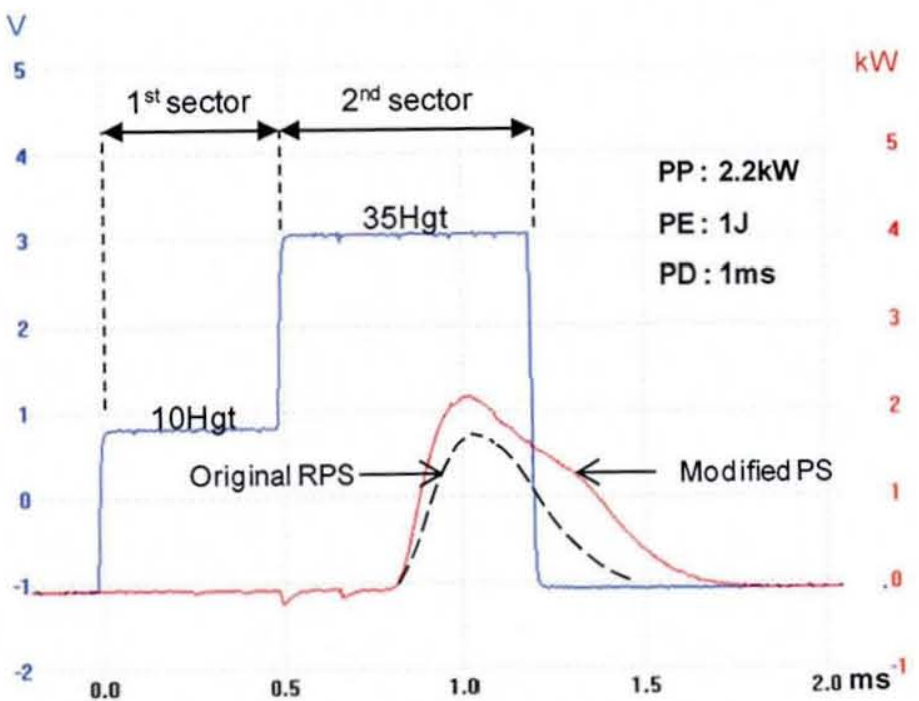

Figure 7-8 Issues with Ramp Up pulses, $1 \mathrm{~ms} \& 1 \mathrm{~J}$

At variable sector heights the same problem occurred with Ramping Up the pulse as shown in Figure 7-9. The two short sectors tended to merge and form an amplified Rectangular pulse shape. An increase in sector Hgt within the first sector increases the peak power, pulse energy and reduces the rate at which power drops off towards the end of the pulse, producing a Ramp Down effect. If first sector Hgt is too low (i.e 5\% Hgt) the sector will have little or no effect on the pulse's output and will subsequently behave as a single sector $0.5 \mathrm{~ms}$ Rectangular pulse shape. Pulsed lasers characteristically produce large overshoots at the beginning of a pulse (as mentioned in section 2.3) and because of this there was difficulty in changing the nature of a pulsed laser when attempting to produce a Ramp Up pulse shape.

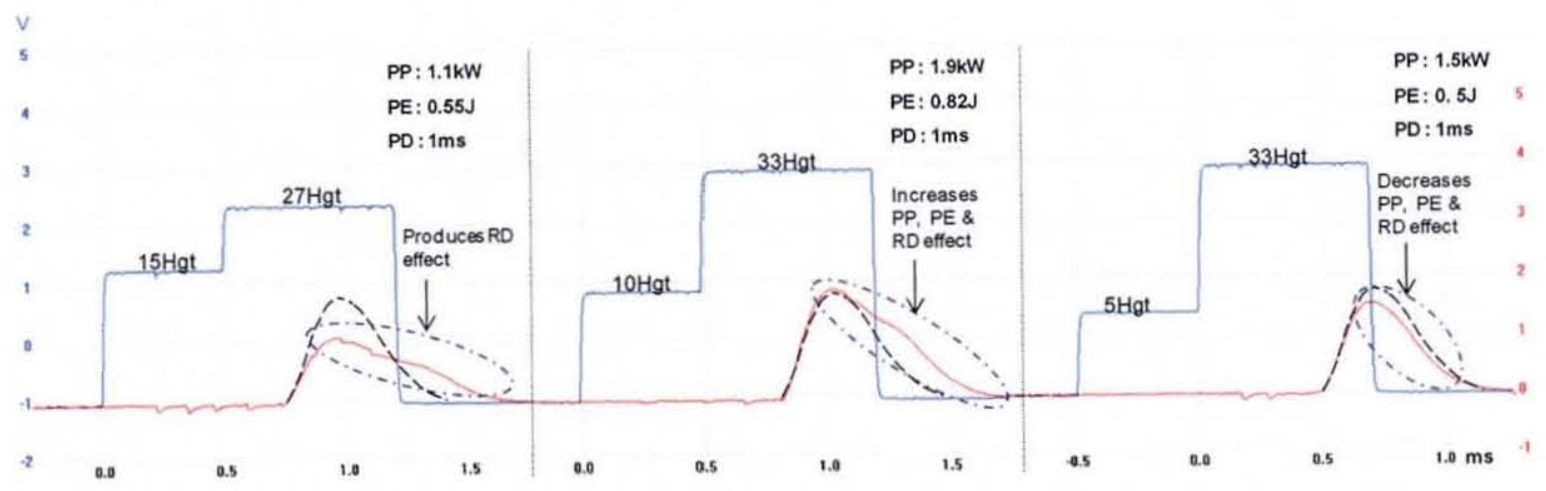

Figure 7-9 Issues with Ramp Up pulses, effects of different sector heights 
It was envisioned that prolonging the duration of first sector would aid the separation of the amplified peak generated by the two sector inputs thus producing a gradual increase in laser power.

Figure 7-10 shows a pulse produced with a prolonged first sector $(2 \mathrm{~ms})$ and shorter second sector $(0.5 \mathrm{~ms})$. At the beginning of the pulse a small peak is generated producing only $100 \mathrm{~W}$ power. This laser power may only gently warm the surface of the material it is processing. A low heat input at the beginning of a Ramp Up pulse is precisely what is required to introduce a gradual heating effect. However $0.5 \mathrm{~ms}$ into the pulse the power completely drops off to zero. The laser input's second sector then generates a second peak power. This has a peak power of $850 \mathrm{~W}$ and then drops off to zero after just over $0.5 \mathrm{~ms}$. Because the power dropped off to zero halfway through the pulses duration, the pulse shape cannot be considered Ramped Up and resembles more a pre-pulse used to condition the material before main processing. A constant heating effect should be in place when building up to the main peak power otherwise multiple heating and cooling effects will take place.

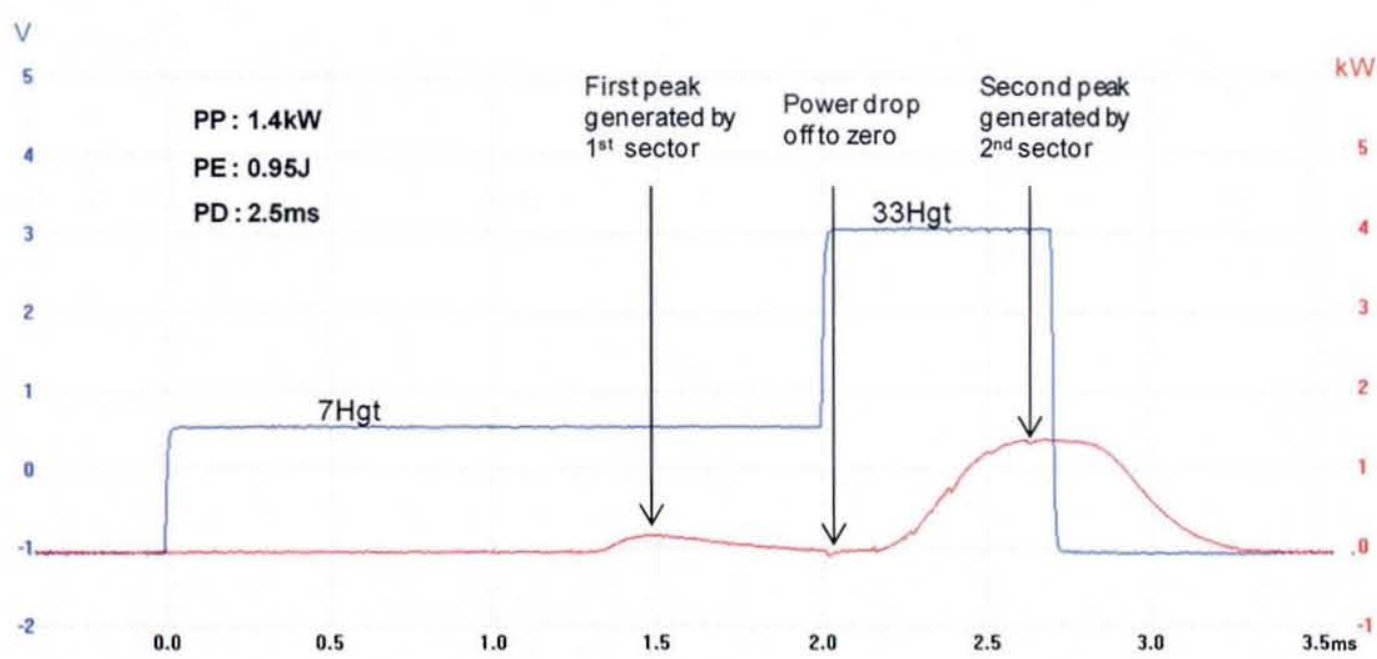

Figure 7-10 Ramp Up issues, prolonged $1^{\text {st }}$ sector causes power drop off

\subsubsection{Ramp Up solution}

As a possible remedy for the power drop off during the middle of the pulse the first sector pulse was varied between $0.5 \mathrm{~ms}$ and $2 \mathrm{~ms}$. It was found that a pulse duration of exactly $1.2 \mathrm{~ms}$ would suppress the first peak and allow a gradual Ramp Up of power 
deliver onto the second $0.5 \mathrm{~ms}$ sector as shown in Figure 7-11. This produced an overall pulse duration of $1.7 \mathrm{~ms}$ if the second sector was $0.5 \mathrm{~ms}$ as shown in Figure $7-11$. First sectors shorter than $1.2 \mathrm{~ms}$ would cause an overshoot at the beginning of the pulse and first sectors longer than $1.2 \mathrm{~ms}$ would suffer from a power drop immediately before the second sector input had initiated. Ramp Up pulse durations longer than $1.7 \mathrm{~ms}$ needed to be investigated in order to gain a fuller understanding into the effects of pulse shaping. Producing longer pulse duration required the extension of either the first or second sector within the pulse. It was found that the addition of a third sector within longer pulses would counteract the power drop off experienced as a result of the first sector. The middle sector would maintain power such that the power produced by the first sector would be continued onto the third and final sector. Figure 7-12 shows the successful generation of a Ramp Up pulse shape for a longer pulse using this methodology. The $1.2 \mathrm{~ms}$ first sector suppressed the formation of any power peak formed at the beginning of the pulse allowing a smoother and consistent power transition. The power generated by the second sector $(0.3 \mathrm{~ms})$ is consistent at $300 \mathrm{~W}$ for approximately $0.5 \mathrm{~ms}$. The power then gradually increases and reaches a peak power of $1000 \mathrm{~W}$, approximately $1 \mathrm{~ms}$ after the pulse started. A method that incorporated three sectors had been successfully developed to produce a Ramp Up energy distribution.

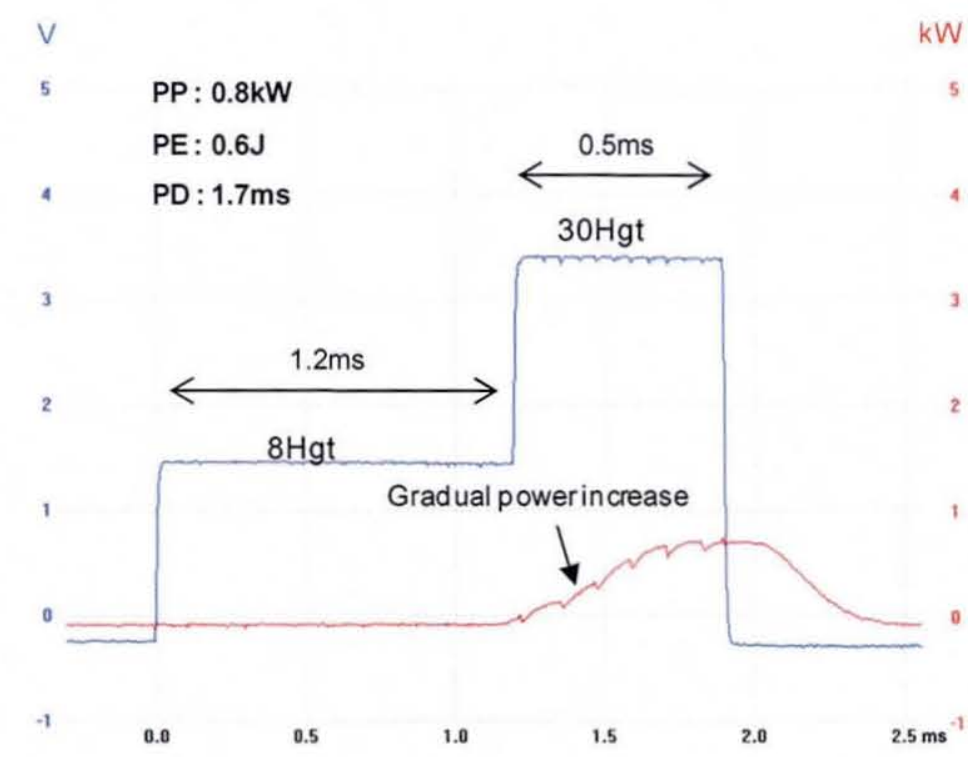

Figure 7-11 Successful Ramp Up pulse generation for pulses $1.7 \mathrm{~ms}$ in duration 


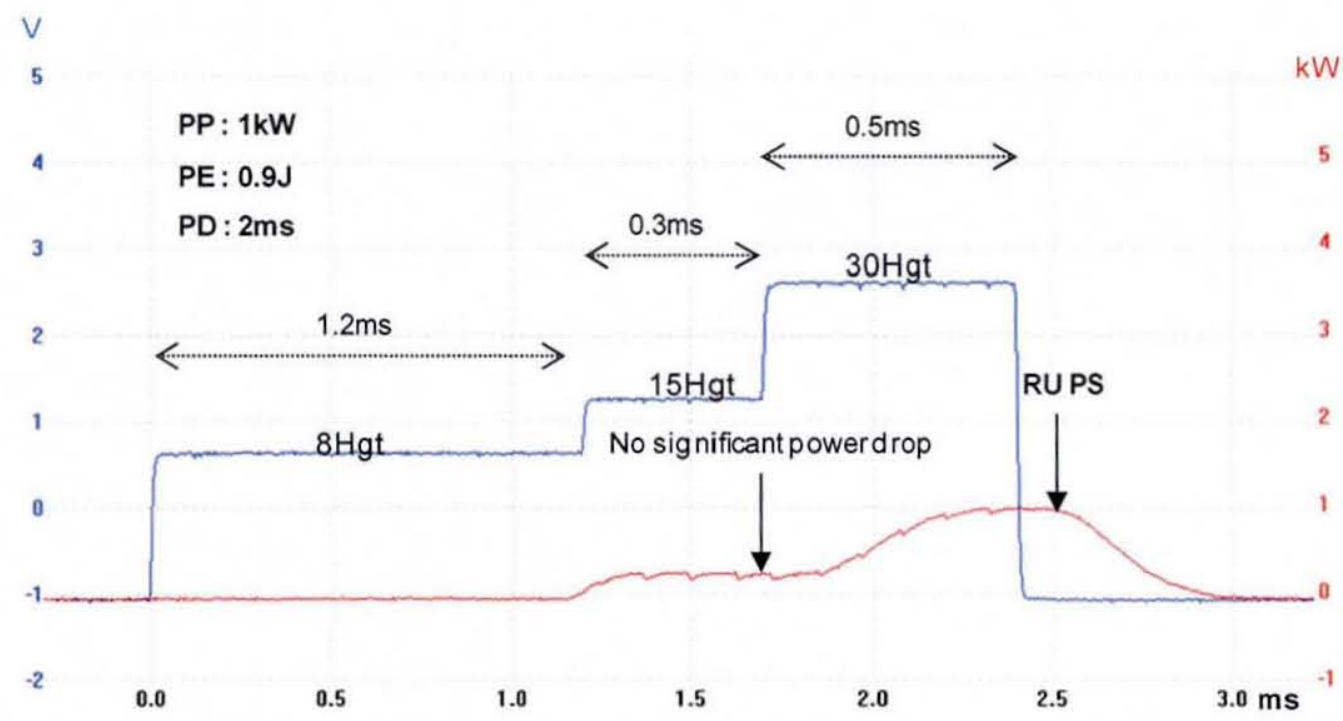

Figure 7-12 Successful Ramp Up pulse generation employing three sectors

As pulse durations were increased the power drop problem became more of an issue as shown in Figure 7-13. The central sector serves as a transition point between the start and the end of the pulse. In order to reduce the power drop-off the input pulse was split into four sectors rather than three as shown in Figure 7-14. The additional sector reduced the power drop-off by increasing or boosting the power in the middle of the pulse. The author acknowledges that for longer pulse durations some power drop off may occur (as shown in Figure 7-14). However measures had been taken to reduce the extent of this.

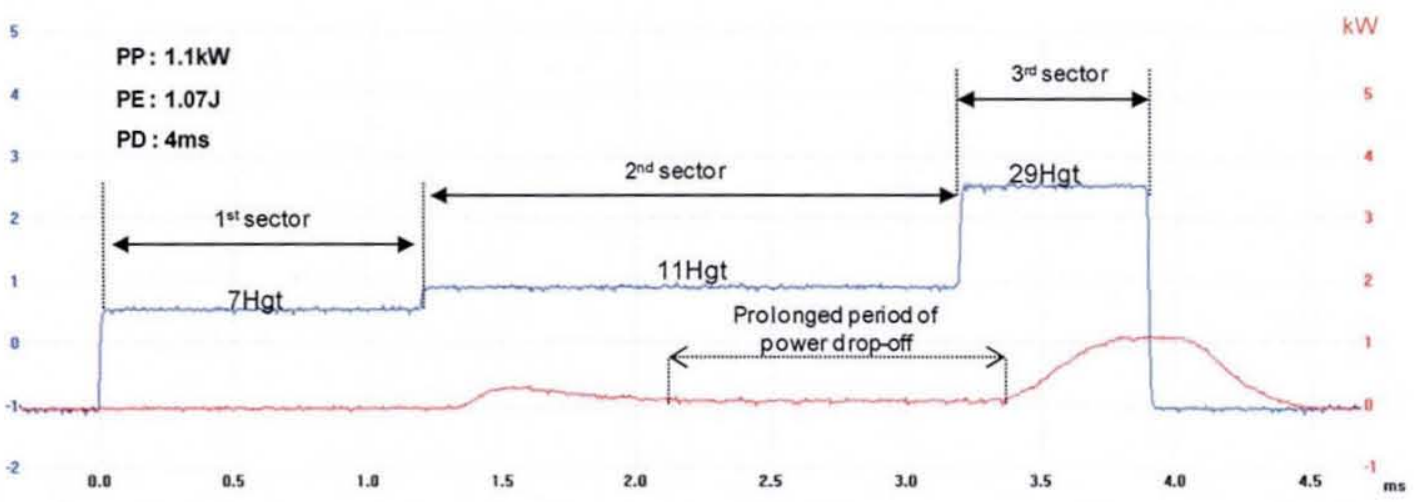

Figure 7-13 Increased pulse duration \& power drop-off using three sectors 


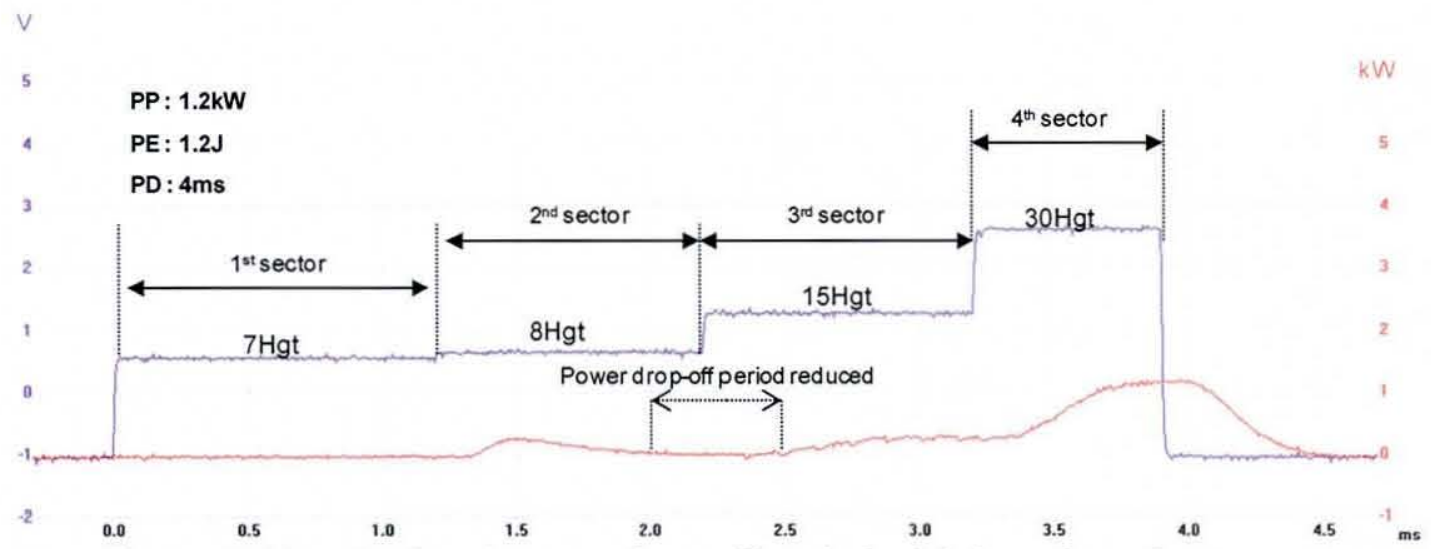

Figure 7-14 Reduced power drop-off period with insertion of extra sector

\subsubsection{Ramp Up Results}

The experiments initially processed Inconel 625 using short Ramp Up pulse durations with low powers. Experiments progressively increased the duration and power of each of these Ramp Up pulses. Section 7.2.2.1 to 7.2.2.3 shows a selection of samples produced using Ramp Up pulse shapes with increasing pulse durations and investigated what effect it has on the production of thin walled parts. Section 7.4 discusses all the Ramp Up results collectively and examines its potential merits and downfalls for use within SLM.

\subsubsection{Pulse duration $1.7 \mathrm{~ms}$ to $2 \mathrm{~ms}$}

Figure 7-15 to Figure 7-19 show 1.7ms Ramp Up pulses used to process samples from an Inconel powder bed, each figure displays the plan view of the thin walled samples produced and the actual power input/output demand profile of the pulse shape used to process them. All figures have the Rectangular pulse shape (from section 6.3) superimposed onto the modified pulse shape profile for comparison (abbreviated with RPS). The figures also include information regarding the samples width, top/side $\mathrm{R}_{\mathrm{a}}$ and height plasma plume generated during processing. Figure 7-15 shows a sample produced with a pulse energy of $0.6 \mathrm{~J}$ and low peak power of $0.8 \mathrm{~kW}$. This produced a relatively thin sample measuring $0.42 \mathrm{~mm}$ in width. It was expected that the side $R_{a}$ would remain low between $10-15 \mu \mathrm{m}$ when the melt pool width was small (as discussed in section 6.3). However due to the low peak power intensity there was not sufficient recoil pressure to improve the melt wetting to the substrate and retard any possible lateral balling formation. Alternatively the low recoil pressures may have not been high 
enough to remove partially melted powder particles from attaching to the edge of the melt solidified pool. Figure 7-16 shows a pulse with the same amount of energy used within the Rectangular pulse shape (0.7J). Even though the pulses have the same amount of energy, the samples produced are distinctly different. The Rectangular pulse produced a sample that was $0.49 \mathrm{~mm}$ in width while generating a plasma plume measuring $5 \mathrm{~mm}$ in height. The Ramp Up pulse produced a thinner sample of $0.43 \mathrm{~mm}$ with only $1 \mathrm{~mm}$ of plasma plume produced. This shows that even if two pulses contain the same amount of energy, the properties of a generated part will depend heavily on how this energy is distributed within the pulse. As the peak power and energy within the pulse increased (as shown in Figure 7-17 to Figure 7-19) the top $R_{a}$ reduced and the sample side $\mathrm{R}_{\mathrm{a}}$ and width increased. However as the peak power approached $1 \mathrm{~kW}$, the plasma plume increased producing higher levels of material spatter.

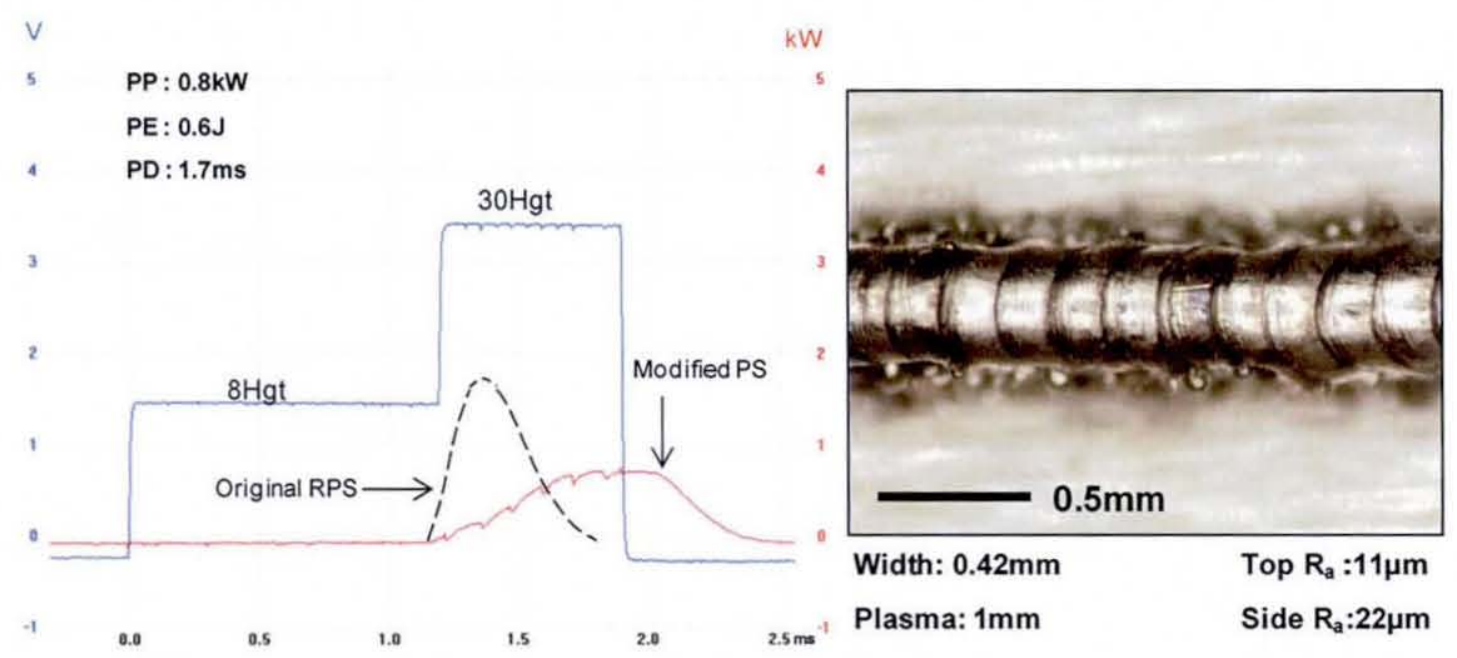

Figure 7-15 Ramp Up pulse 1.7ms \& 0.6J

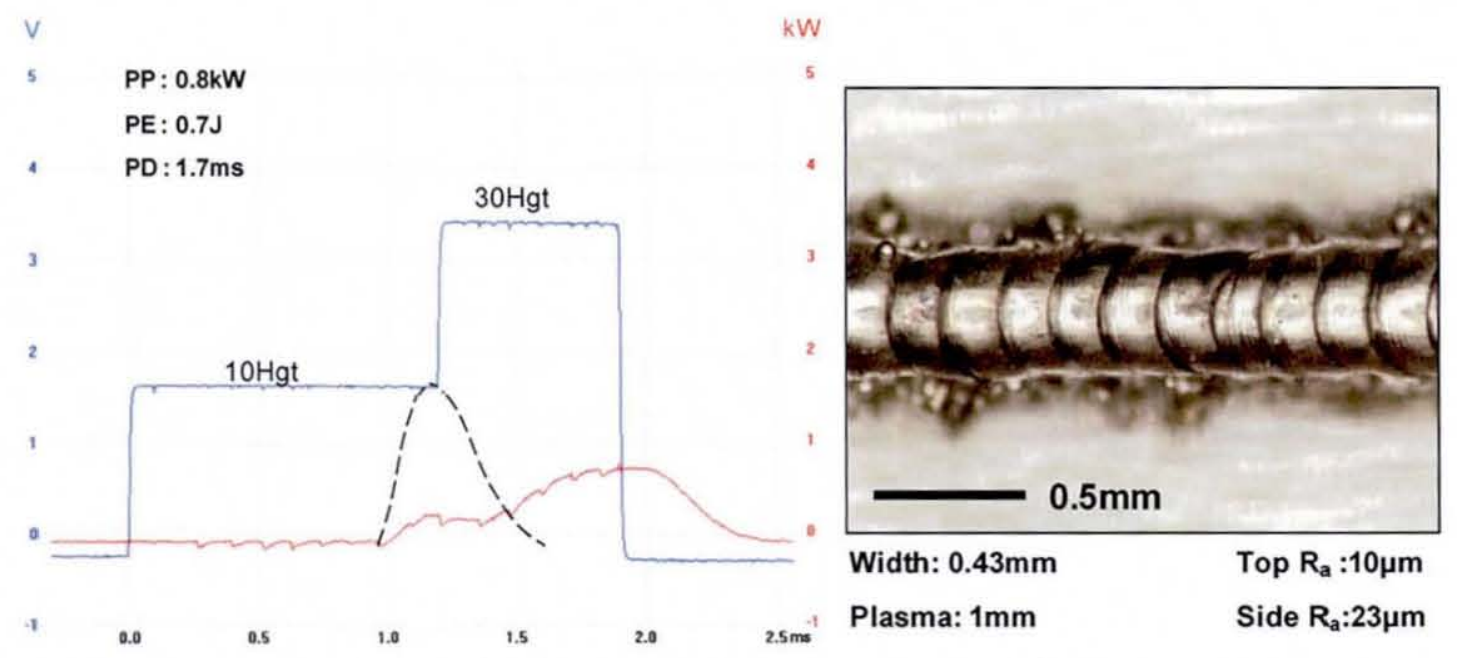

Figure 7-16 Ramp Up pulse 1.7ms \& 0.7J 


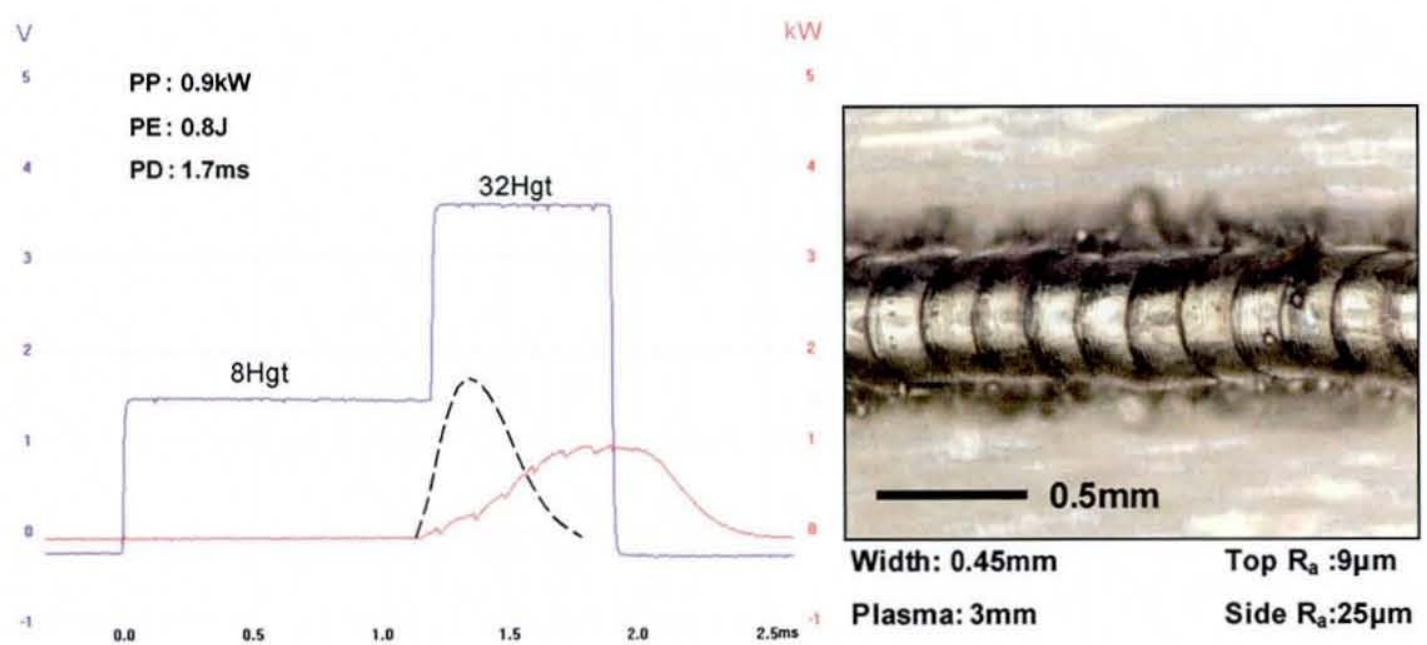

Figure 7-17 Ramp Up pulse 1.7ms \& 0.8J

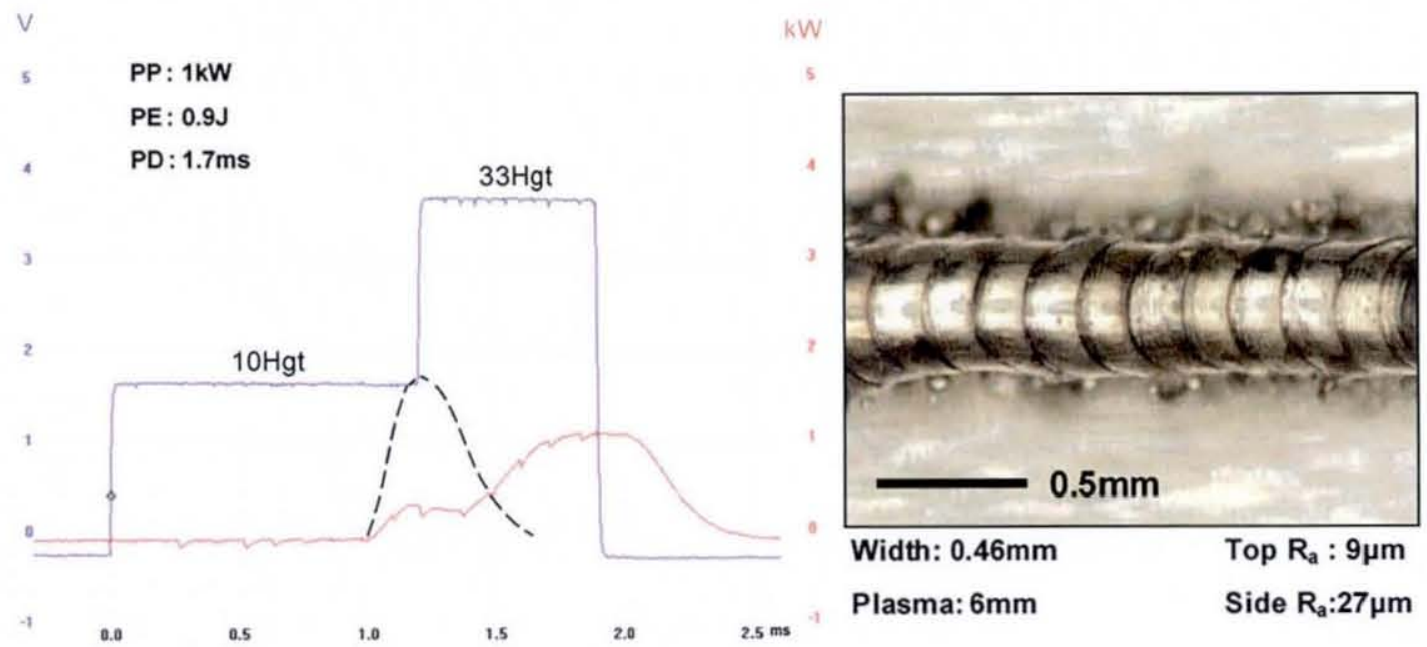

Figure 7-18 Ramp Up pulse 1.7ms \& 0.9J

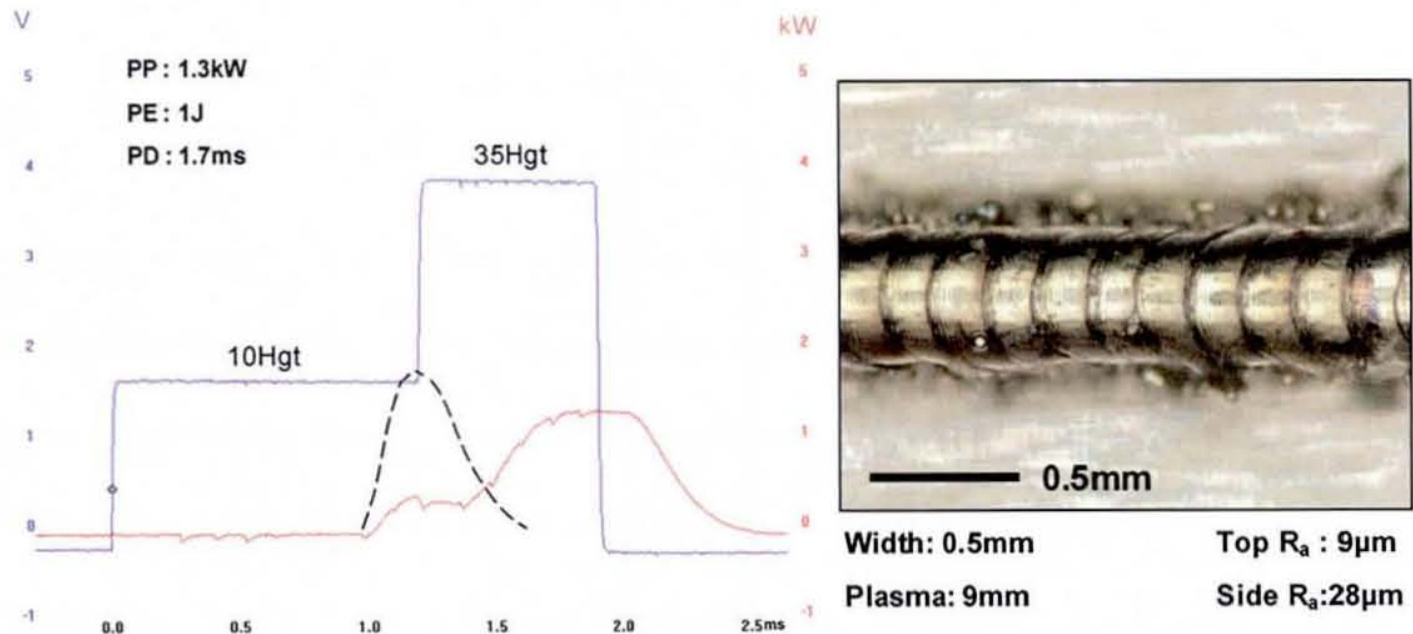

Figure 7-19 Ramp Up pulse 1.7ms \& 1J

Figure 7-20 to Figure 7-22 shows Ramp Up pulse shapes with durations of 2ms. Figure $7-20$ shows a sample processed using $0.6 \mathrm{~J}$ and $0.7 \mathrm{~kW}$ peak power. The sample 
produced was thin measuring only $0.4 \mathrm{~mm}$ with minimal plasma plume height during processing. The sample's edge profile does not appear to be flat and consistent and displays a wavy profile. Low peak power causes melt pools to form a spherical shape due to surface tension, as opposed to a wider flatter profile formed as a result of greater recoil pressures generated using larger peak powers. Low heat input and short pulse durations can cause a melt pool to remain relatively small and solidify quickly. This may restrict the flow of molten material causing an inconsistent edge profile. Figure 7-21 shows a similar Ramp Up pulse but with an increased power input demand for the third sector. The height of the third sector was increased from $25 \mathrm{Hgt}$ to $30 \mathrm{Hgt}$. This increased the peak power from $0.7 \mathrm{~kW}$ to $1 \mathrm{~kW}$ and produced a well formed sample with a consistent flat edge profile. This increase in peak power would increase the maximum temperature attained by the melt pool increasing the volume of liquid produced as well as the amount of material vaporisation, causing the level of spatter to increase. Figure 7-22 shows a Ramp Up pulse similar to the one shown in Figure 7-21, however the middle sector's height was increased from $15 \mathrm{Hgt}$ to $17 \mathrm{Hgt}$. This did not affect the overall peak power of the pulse but rather increased the pulse energy by $0.1 \mathrm{~J}$. This increase in the middle sector appears to marginally reduce the gradient of the Ramp Up pulse, this would result in a more gradual heating effect. The samples produced had a width of $0.5 \mathrm{~mm}$, this increase in width is due to more pulse energy being delivered to the material causing an increase in the volume of liquid produced. The edges of the samples had consistent and smooth profiles. This is attributed to the gradual heating of the material allowing molten material to flow more freely and easily as melt pool viscosity reduces. However due to the increased melt pool width there was a larger variation in the thermal properties across the melt pool causing thermal differences from the centre and outer edges of the melt. This would have increased side $R_{a}$ due to satellite formation or as the molten pool broke up into smaller entities in an attempt to reduce surface tension variations across the melt pool (lateral balling). The surface $R_{a}$ of the samples drops to $8 \mu \mathrm{m}$, this may be as a result of a more steady melt pool being produced due to gradual increase in heat input. 


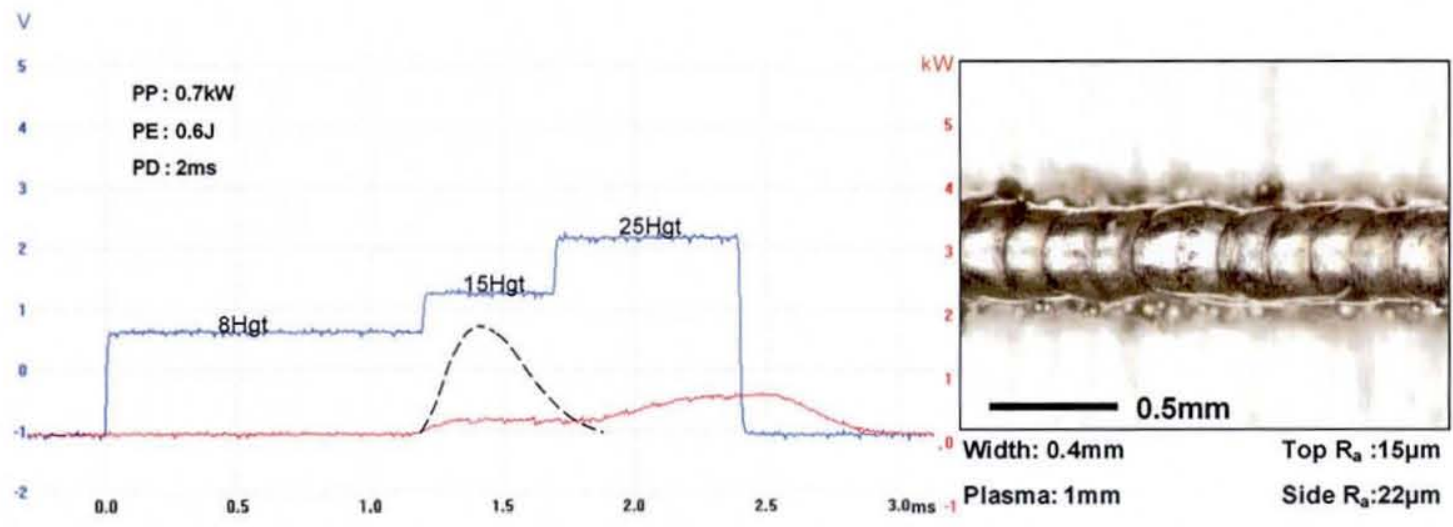

Figure 7-20 Ramp Up pulse $2 \mathrm{~ms} \& 0.6 \mathrm{~J}$

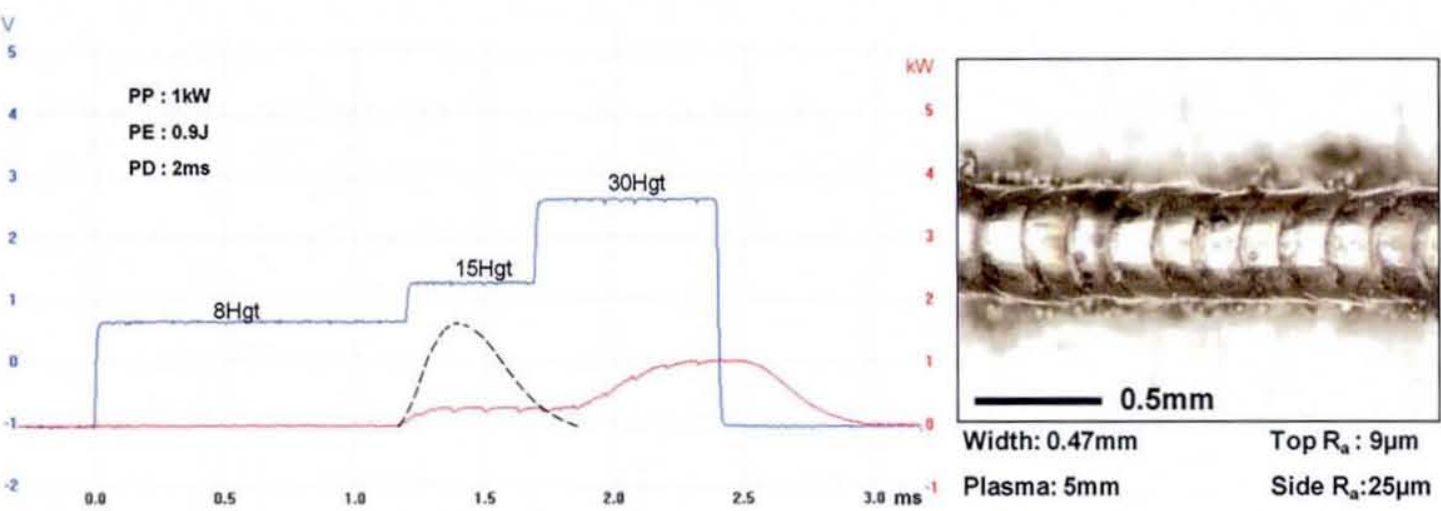

Figure 7-21 Ramp Up pulse 2ms \& 0.9J

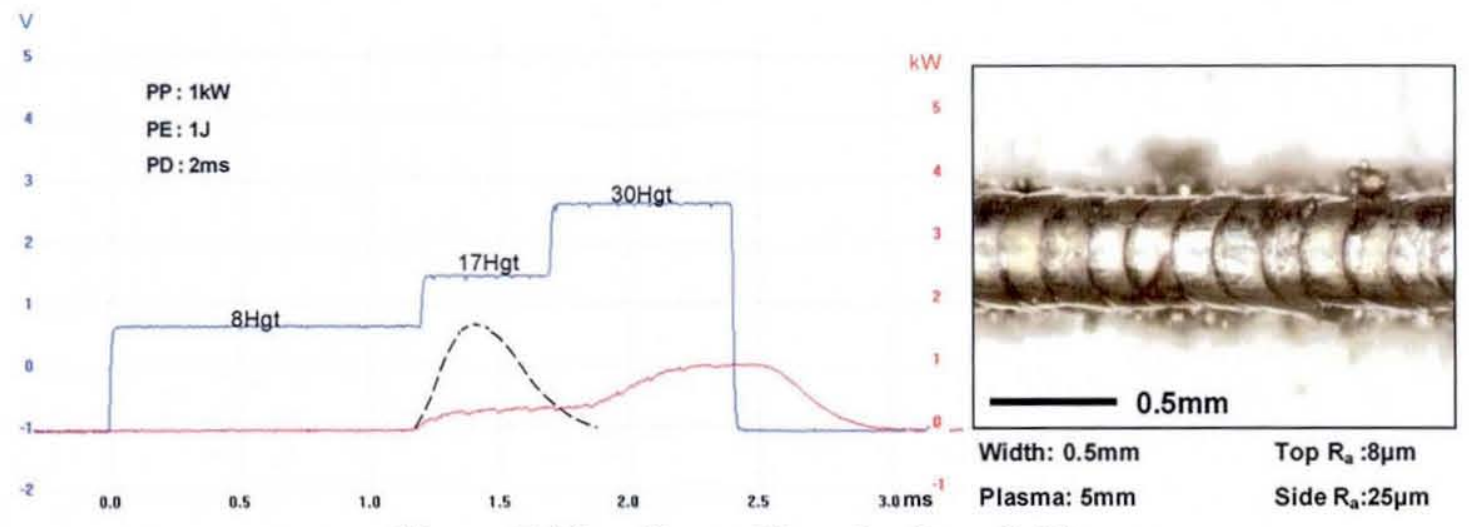

Figure 7-22 Ramp Up pulse 2ms \& $1 \mathrm{~J}$

Figure 7-23 shows a comparison of a standard Rectangular pulse ( $0.5 \mathrm{~ms}$ duration) and a Ramp Up pulse (2ms duration, abbreviated with RU PS). The Rectangular pulse had a higher peak and very short heating time, the peak power $1.8 \mathrm{~kW}$ was sufficient enough to produce a melt pool which would solidify relatively quickly. The edge definition of the sample produced using the Rectangular pulse shape is not consistent and tends to be wavy in formation. The rapid laser on/off time may not allow enough time for the powder particles to form a sufficient liquid volume. If the particles are molten for a very short time this does not allow the melt pool to spread or relax under the forces of 
gravity. Surface tension and Marangoni forces heavily influence the flow of the melt pool and the subsequent solidified geometry of the part. If sufficient time is not given for these forces to fully affect the molten flow then the geometry may become more dominantly effected by other factors. These include recoil pressures and an inconsistent powder bed density. It is believed that a larger volume of molten material produced for a longer period of time will form a more consistent part geometry. However larger melt pools have a greater tendency to undergo the balling mechanism due to surface tension variations. The Ramp Up pulse shown in Figure 7-23, has a lower peak power than the Rectangular pulse yet has a higher pulse energy, this is because the energy is spread over a period four times greater than that of the Rectangular pulse. Having a more consistent edge profile aids a reduction in geometric variation along the samples length. However this reduction in edge profile waviness does not indicate that side $R_{a}$ is lower. Images can also be deceptive and not reveal the full extent of any satellite/balling formation due to images being focused solely on the top surface of a sample. Therefore satellite/balling formation at lower layers along the samples side may not be visually apparent given a false indication of improved side $\mathrm{R}_{\mathrm{a}}$. The only accurate method to determine side $\mathrm{R}_{\mathrm{a}}$ is from a measured value (as has been conducted).

The top $\mathrm{R}_{\mathrm{a}}$ of the sample produced using the Ramp Up pulse was marginally better than that of the Rectangular pulse. This could be a result of a steadier melt pool formation due to gradual heat input, reducing ripple formation along its surface. The Ramp Up pulse required less peak power to form a melt pool of similar width compared with that formed by the Rectangular pulse. This could be due to the gradual heating introduced at the beginning of the pulse as a result of the Ramp Up pulse. The temperature of the powder particles have already been raised by the time the main peak power is initiated. The powder particles therefore would have a higher energy absorbance subsequently requiring less heat input to become fully molten. Even though the period of time is short ( $2 \mathrm{~ms}$ ) metals can convert phonon excitation from laser radiation into thermal energy within 1ps [Chichkov et al. 1996]. Therefore certain Ramp Up pulse energy distributions may produce the same effect as that produced by conventional power bed preheating. Section 7.2.2.4 displays the full set of results for 1.7-2ms Ramp Up pulses as well as other Ramped Up pulse durations. 


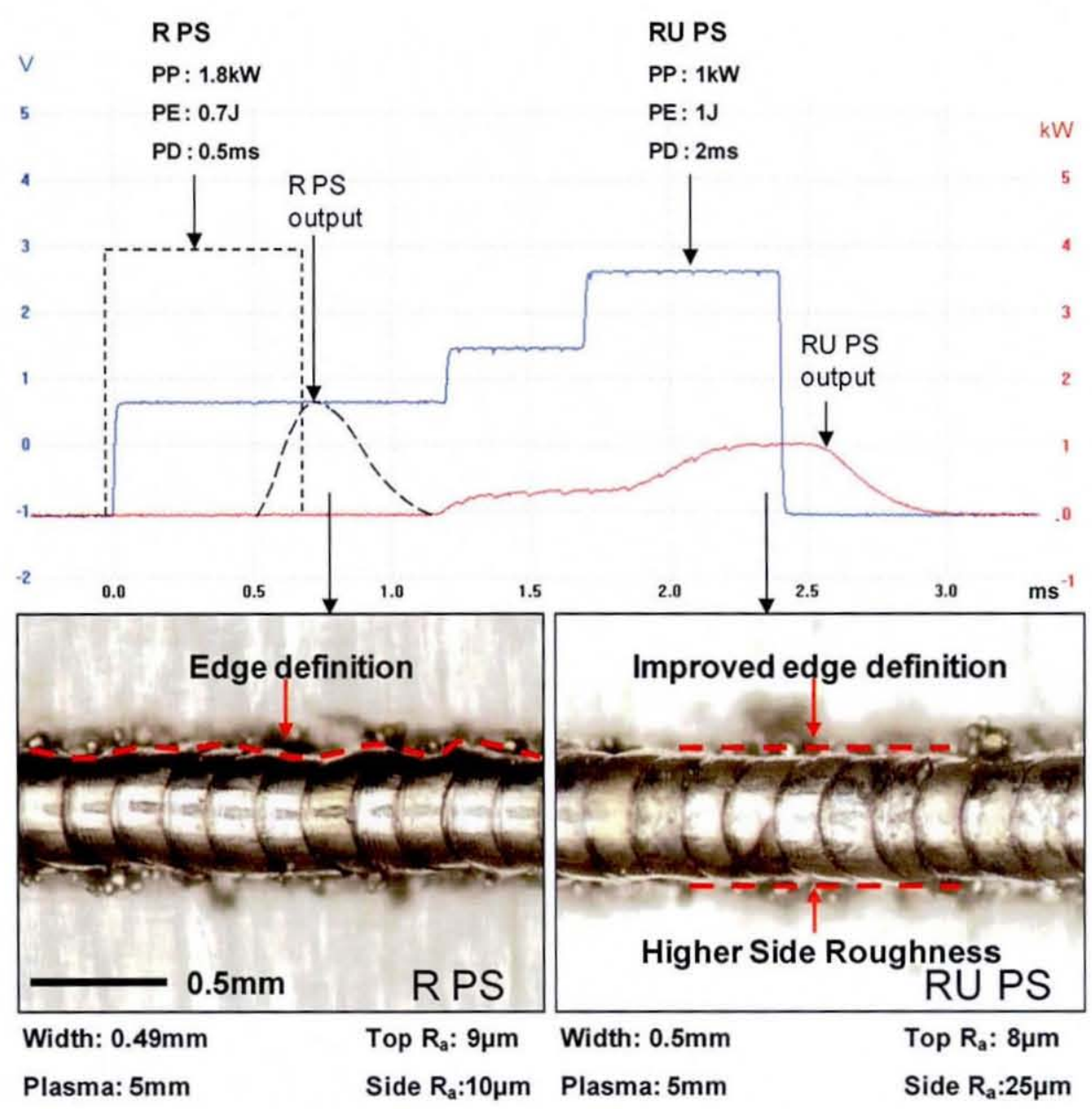

Figure 7-23 Comparison of the Rectangular \& Ramped Up pulse shape (2ms)

\subsubsection{Pulse durations $4 \mathrm{~ms}$ to $6 \mathrm{~ms}$}

Figure 7-24 shows a Ramp Up pulse with a $4 \mathrm{~ms}$ pulse duration. The pulse initiates with a small peak, something difficult to regulate and completely remove within pulsed lasers. The power then drops off at $0.5 \mathrm{~ms}$ and the slowly ramps up to $0.4 \mathrm{~kW}$ for another $0.5 \mathrm{~ms}$. Towards the end of the pulse the power ramps up to a peak power of $0.9 \mathrm{~kW}$. The sample produced using this pulse shape appears to be fully melted and has a relatively flat edge profile. However the side $\mathrm{R}_{\mathrm{a}}$ was higher than other samples produced using shorter pulse durations with a similar melt pool width. The pulse duration was approximately $4 \mathrm{~ms}$, there was a possibility that the melt pool was initiated early within the pulse as opposed to the latter part. This may result in the melt pool remaining molten for a longer period of time producing an excessive volume of liquid which would trigger the lateral balling mechanism. Alternatively more time would have been 
given for heat to conduct into the powder bed causing more powder particles to partially melt and agglomerate to the side of the solidifying melt pool.

Figure 7-25 shows a similar Ramp Up pulse with a duration of $4 \mathrm{~ms}$. The fourth sector input of this pulse was increased from $27 \mathrm{Hgt}$ to $28 \mathrm{Hgt}$. The overall shape of the pulse remained the same, however the increase in sector height caused the pulse's peak power to increase from $0.9 \mathrm{~kW}$ to $1 \mathrm{~kW}$ and increased the pulse energy to $1.1 \mathrm{~J}$. This increase in peak power and pulse energy increased the plasma plume height to $8 \mathrm{~mm}$ resulting in an undesirable increase in material spatter. The width of the sample increased to $0.53 \mathrm{~mm}$ as the volume of liquid within the melt pool increased.

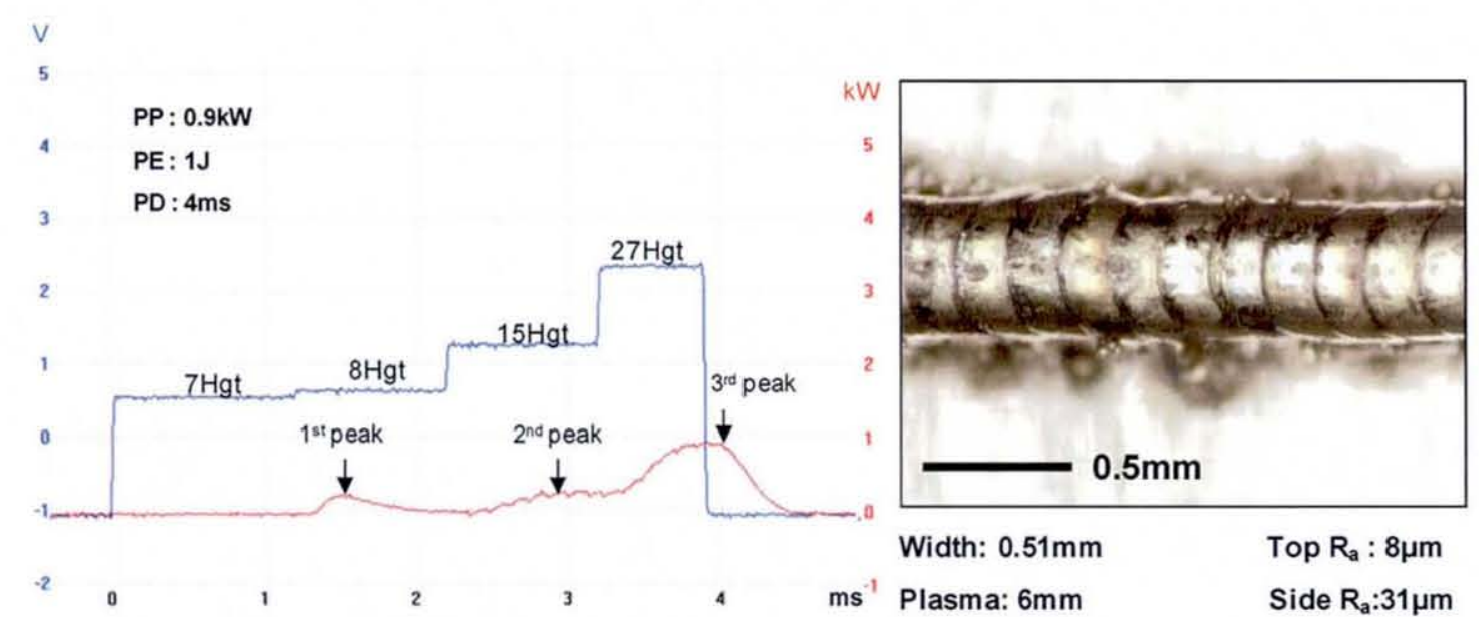

Figure 7-24 Ramp Up pulse 4ms \& 1J

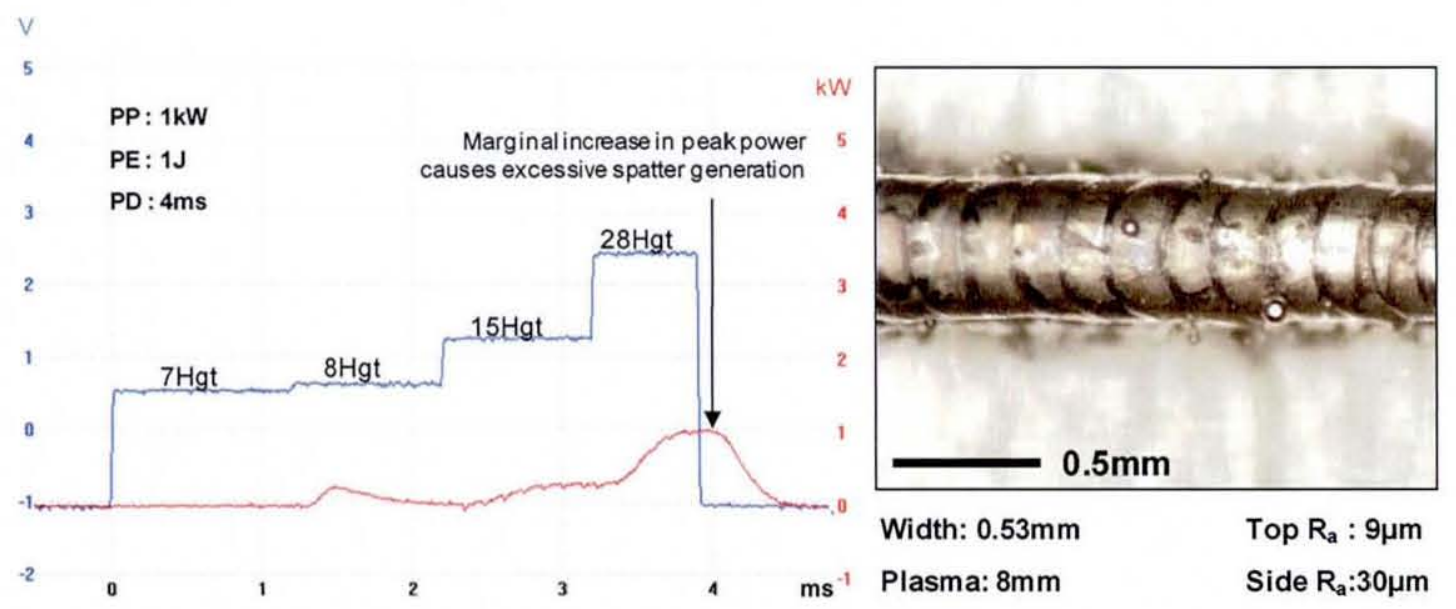

Figure 7-25 Ramp Up pulse $4 \mathrm{~ms} \& 1.1 \mathrm{~J}$

Samples produced using $6 \mathrm{~ms}$ in duration showed similar trends to the $4 \mathrm{~ms}$ Ramp Up pulse with low top $\mathrm{R}_{\mathrm{a}}$ and high side $\mathrm{R}_{\mathrm{a}}$. The pulses required less peak power to produce 
a melt pool as more energy was distributed earlier on within the pulse. Section 7.2.2.4 displays the full set of results for 4-6ms Ramp Up pulses.

\subsubsection{Pulse duration $8 \mathrm{~ms}$ and $10 \mathrm{~ms}$}

Figure 7-26 shows a Ramp Up pulse with a $8 \mathrm{~ms}$ pulse duration. The pulse begins with a small peak measuring $0.4 \mathrm{~kW}$ power and then a period of power drop-off. This is followed by an increase in power up to $0.2 \mathrm{~kW}$, this power is maintained for approximately $3.5 \mathrm{~ms}$ and then the power is Ramped Up to a peak power of $0.7 \mathrm{~kW}$. This Ramp Up pulse produced a sample width measuring $0.51 \mathrm{~mm}$ with a top $\mathrm{R}_{\mathrm{a}}$ of $9 \mu \mathrm{m}$. The edges of the sample are consistently formed similar to the samples produced in Figure 7-22. However the plasma plume height was reduced. The peak power used to melt the Inconel with the $8 \mathrm{~ms}$ Ramp Up pulse was only $0.7 \mathrm{~kW}$, this is a significant factor aiding the reduction in plasma plume generation. The initial peak generated by the pulse would have heated the powder particles, $3.5 \mathrm{~ms}$ at $0.2 \mathrm{~W}$ would have preheated the material prior to the main pulse. Therefore less heat was required to completely liquefy the powder particles and hence a lower peak power was required. It is possible that the initial peak of the pulse and $3.5 \mathrm{~ms}$ of preheating would have at least sintered or liquefied smaller powder particles before the main peak power. The surface of the sample shows signs of discoloration possibly a result of oxidisation. When a metal becomes molten the oxidisation potential rapidly increases. It is believed that the use of longer pulse duration increased the melt pool's surface susceptibility to oxidise due to it remaining molten for a longer period of time.

Figure 7-27 shows a similar Ramp Up pulse with a similar same shape, however the fourth sector was increased from $20 \mathrm{Hgt}$ to $23 \mathrm{Hgt}$, this increased the peak power by $0.2 \mathrm{~kW}$ and pulse energy by $0.1 \mathrm{~J}$. This slight adjustment in pulse shape input demands caused the plasma plume height generated to increase from $2 \mathrm{~mm}$ to $5 \mathrm{~mm}$. This shows that the small adjustments made to a pulse's energy distribution can significantly affect part processing and formation. Again there is top surface blackening or discoloration indicating possible oxidisation. Figure 7-28 shows a Ramp Up pulse that has had the presence of the initial spike/peak removed by increasing the sector height of the second, third and forth sectors. This increase caused the power level of the pulse to remain at approximately $0.4 \mathrm{~kW}$ for the majority of the pulse $(7.4 \mathrm{~ms})$, this was then followed by a 
peak power of $1 \mathrm{~kW}$. The sample produced shows clearly that too much heat was delivered to the material over the period of the pulse. The sample measured $0.68 \mathrm{~mm}$ in width, clearly too much powder was drawn into the melt pool and liquidised. The plasma plume height generated was also very high. This sample also showed evidence of possible oxidisation.

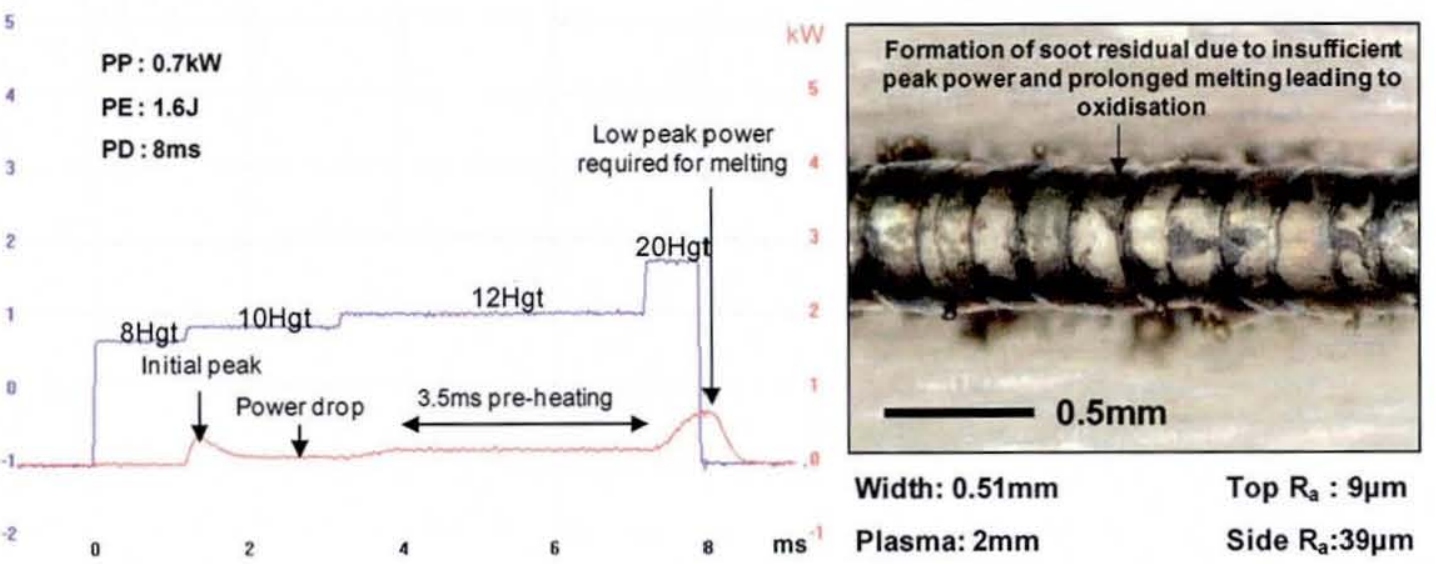

Figure 7-26 Ramp Up pulse $8 \mathrm{~ms} \& 1.6 \mathrm{~J}$

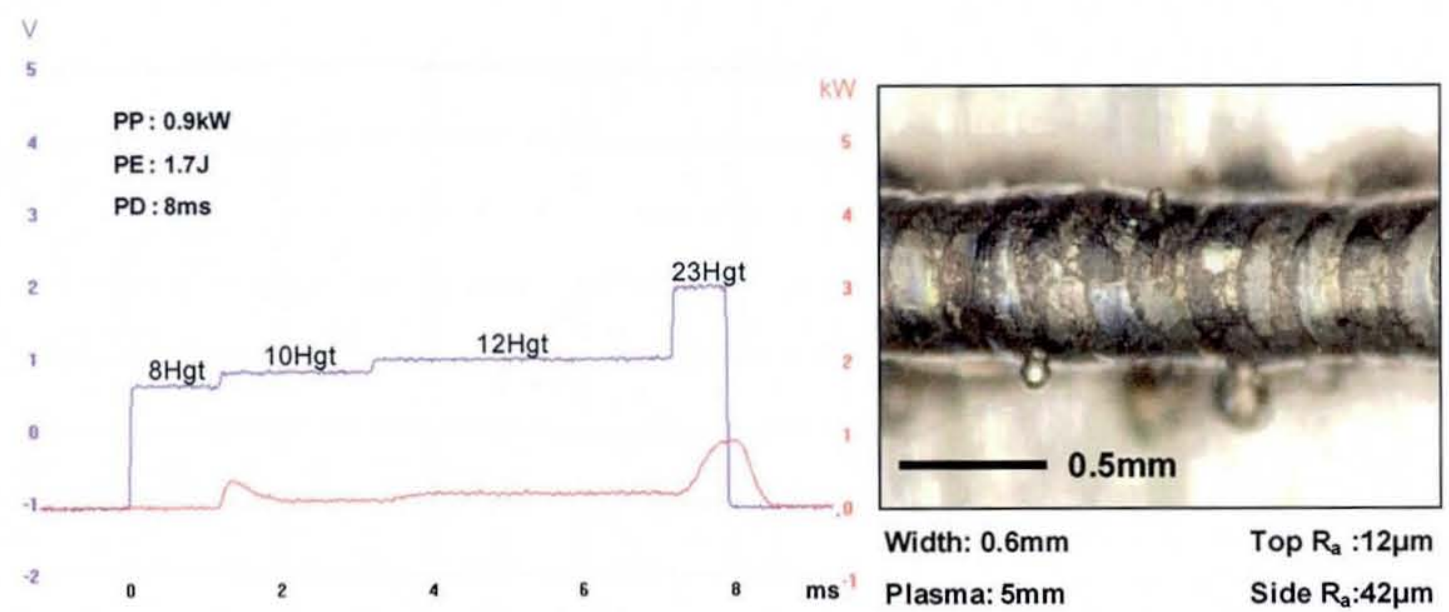

Figure 7-27 Ramp Up pulse $8 \mathrm{~ms} \& 1.72 \mathrm{~J}$ 
V

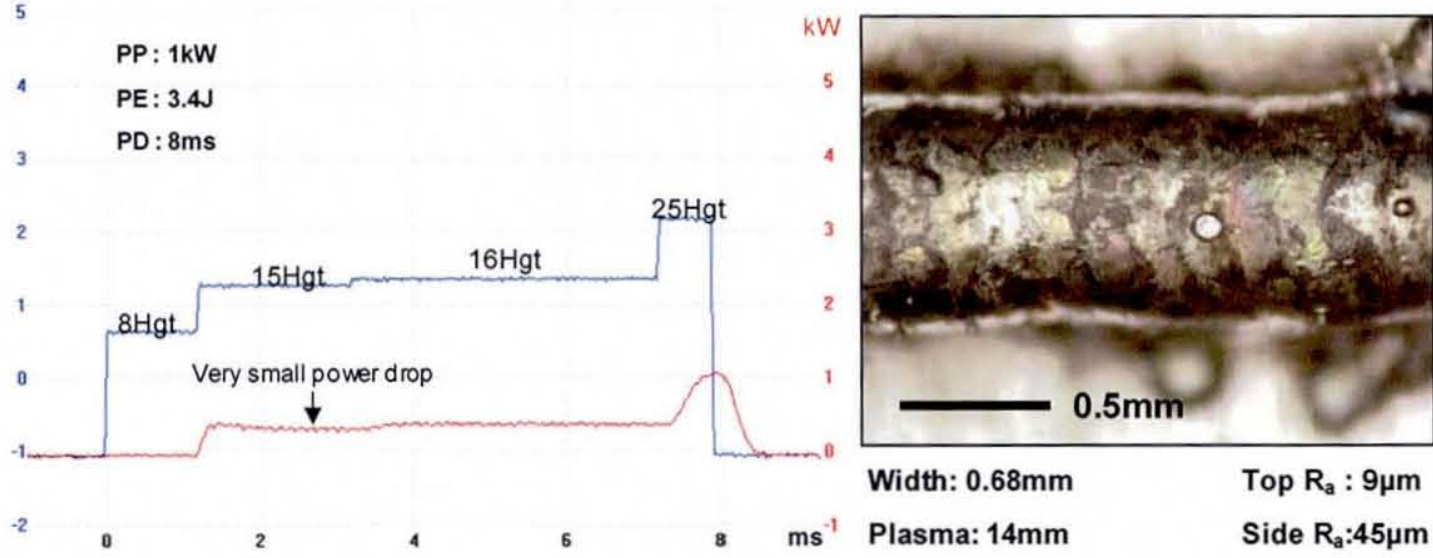

Figure 7-28 Ramp Up pulse 8ms \& 3.4J

Figure 7-29 shows a comparison of a standard Rectangular pulse and a Ramp Up pulse with an $8 \mathrm{~ms}$ pulse duration. The Ramp Up pulse is just over fifteen times longer than the Rectangular pulse shape, it requires $0.7 \mathrm{~kW}$ less peak power but $0.9 \mathrm{~J}$ more to melt the Inconel. This reduced the plasma plume height during processing and subsequently reduces the risk of powder bed contamination due to spatter ejection. The edge profile of the Ramp Up pulse samples is more consistently defined due to the melt pool existing for a longer period of time allowing more time for it to flow under the force of gravity. However due to the melt pool remaining molten for longer period of time it became more susceptible to oxidisation as well as allowing more time for melt pool instabilities to develop. 


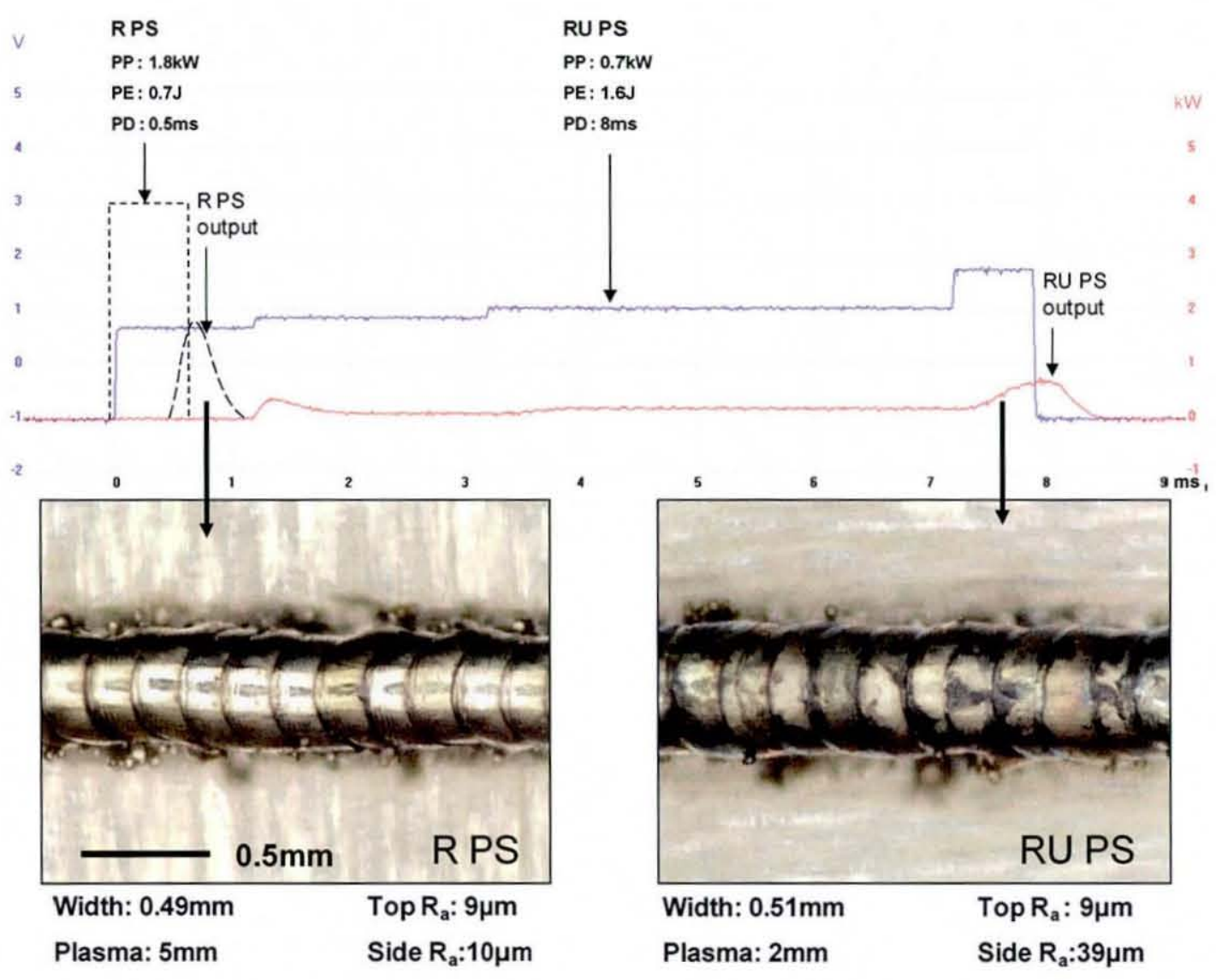

Figure 7-29 Comparison of a Rectangular pulse \& a Ramp Up pulse (8ms)

Samples produced using 10ms Ramp Up pulse showed similar results to those obtained using 8ms Ramp Up pulse. They produced large melt pool widths (larger than $0.57 \mathrm{~mm}$ ), side $\mathrm{R}_{\mathrm{a}}$ was high (above $38 \mu \mathrm{m}$ ) and there were indications of surface oxidisation. It was difficult to create Ramp Up pulses longer than $6 \mathrm{~ms}$, rather a constant heat input was delivered for some time before main pulse as shown in Figure 7-28. With longer pulse durations, by the time the power has Ramped Up to the main peak power already too much heat had been delivered to the material producing a large melt pool, high side roughness, spatter and excessive oxidisation on sample surface. None of these part qualities would benefit the SLM process in producing functional metal parts with a high resolution and low side $\mathrm{R}_{\mathrm{a}}$. Section 7.2.2.4 displays the full set of results for $8-10 \mathrm{~ms}$ Ramp Up pulses.

\subsubsection{Overview of ramp up pulse shapes}

Figure 7-30 shows the full range of top/side $\mathrm{R}_{\mathrm{a}}$ and width results produced as a result of different Ramp Up pulses (plasma plume height has also been included). For 
comparison the data obtained from the standard optimised Rectangular pulse shape parameters and results developed in section 6.3 has been included on the graph (abbreviated with R PS). Each pulse's energy has been shown including energy and durations that caused an excessive amount of spatter to be generated during processing.

Throughout all the pulse durations the top $\mathrm{R}_{\mathrm{a}}$ did not vary significantly remaining between $6-15 \mu \mathrm{m}$ as shown in Figure 7-30. However the side $\mathrm{R}_{\mathrm{a}}$ was more difficult to control and varied between $10-45 \mu \mathrm{m}$. Side $R_{a}$ was heavily influenced by the pulse energy and pulse duration. As pulse energy and duration increased the side $\mathrm{R}_{\mathrm{a}}$ increased. Increased pulse energy causes a larger volume of liquid to be produced causing the melt pool to expand in width. A melt pool that is larger in width has more thermal variation across the melt pool and therefore larger variation in its surface tension. The melt pool may then breaks up into small molten spheres (lateral balling) to reduce surface tension variation. These molten spheres are also a result of satellite formation that tend to solidify to the outer surface of the melt pool and increase its roughness (side surface is more predominately affected due to scattering of molten spheres to the side of the melt pool as a result of recoil pressure generated by plasma plume). Even though side $R_{a}$ has a trend of increasing roughness with increasing pulse duration and pulse energy there appear to be slight variations in the measured values. This is a result of the different peak powers used within the pulse and the order in which the data is presented. High/low peak powers have the potential to dramatically change sample properties including top/side $\mathrm{R}_{\mathrm{a}}$ and sample width. Data in the graphs are first presented in order of low pulse duration and low pulse energy, with samples produced at variable peak powers being randomly presented. This would explain the slight variation in some of the trends. On both graphs it can be seen that the optimised parameters developed in section 6.3 (Rectangular pulse shape) produces a sample with a side $R_{a}(10 \mu \mathrm{m})$ far superior than any other of the samples produced using a Ramp Up pulse shape. Ramp Up samples that had a side $R_{a}$ lower than $20 \mu$ m were produced when plasma plume height had already risen to an unacceptable level. The larger plasma plumes that arise causing more spatter ejection would also exert a larger pressure on the melt pool. This would work in favour of reducing side $R_{a}$ by reshaping the melt from a spherical structure to a flatter wider profile, thus improving the melt wetting and reducing a melt pools tendency to undergo balling. The larger recoil pressure may have also removed 
any satellite/balling formation from solidifying along the edges of the melt pool and dispersed it along the powder bed. It should be noted that if recoil pressures become too high, side $\mathrm{R}_{\mathrm{a}}$ can also increase. This may be a result of melt pool ejection to either side of the melt pool. There was one sample that was produced with relatively low top/side $R_{a}$ without excessive spatter generation. This sample was produced using a $2 \mathrm{~ms}$ Ramp Up pulse with $0.9 \mathrm{~J}$ pulse energy and $1 \mathrm{~kW}$ peak power. This sample had a top and side $R_{a}$ of $9 \mu \mathrm{m}$ and $25 \mu \mathrm{m}$ respectively and width of $0.47 \mathrm{~mm}$ (as previously shown in Figure 7-21). Even though this sample was produced using very a different energy distribution to that of the Rectangular pulse, the sample part properties were similar (including the same level of plasma plume). There appeared to be no obvious advantage in the use of this Ramp Up pulse over that of the non pulse shaped Rectangular pulse. However any advantages of one particular pulse shape over another may have been discovered through further testing (hardness, tensile, microstructure etc.), however this is beyond the remit of this research.

Figure 7-30 also displays the height of plasma plumes generated during the use of the Ramp Up pulses. As expected, the plasma plume generation is more predominant with use of high pulse energies. Marked on the graph is a $1.7 \mathrm{~ms}$ pulse that contains the same amount of energy as that of a Rectangular pulse $(0.7 \mathrm{~J})$, but a lower peak power of only $0.8 \mathrm{~kW}$ (shown in Figure 7-16). Due to this the width of the sample is small $(0.43 \mathrm{~mm}$ ), top $R_{a}$ was low $(10 \mu \mathrm{m})$ but the side $R_{a}$ is higher $(23 \mu \mathrm{m})$. The Ramp Up nature of pulses is the completely opposite to that of a standard Rectangular pulse or that of a Ramp down pulse. The main advantage of this pulse over that of a Rectangular is the reduced maximum peak power required to initiate full melting. As a result of the redistribution of energy (gradual heating) and low peak power, the plasma plume height reduced and ejected less spatter during processing. This would reduce any processing issues related to spatter generation during processing (spatter issues discussed in section 3.4.1) 


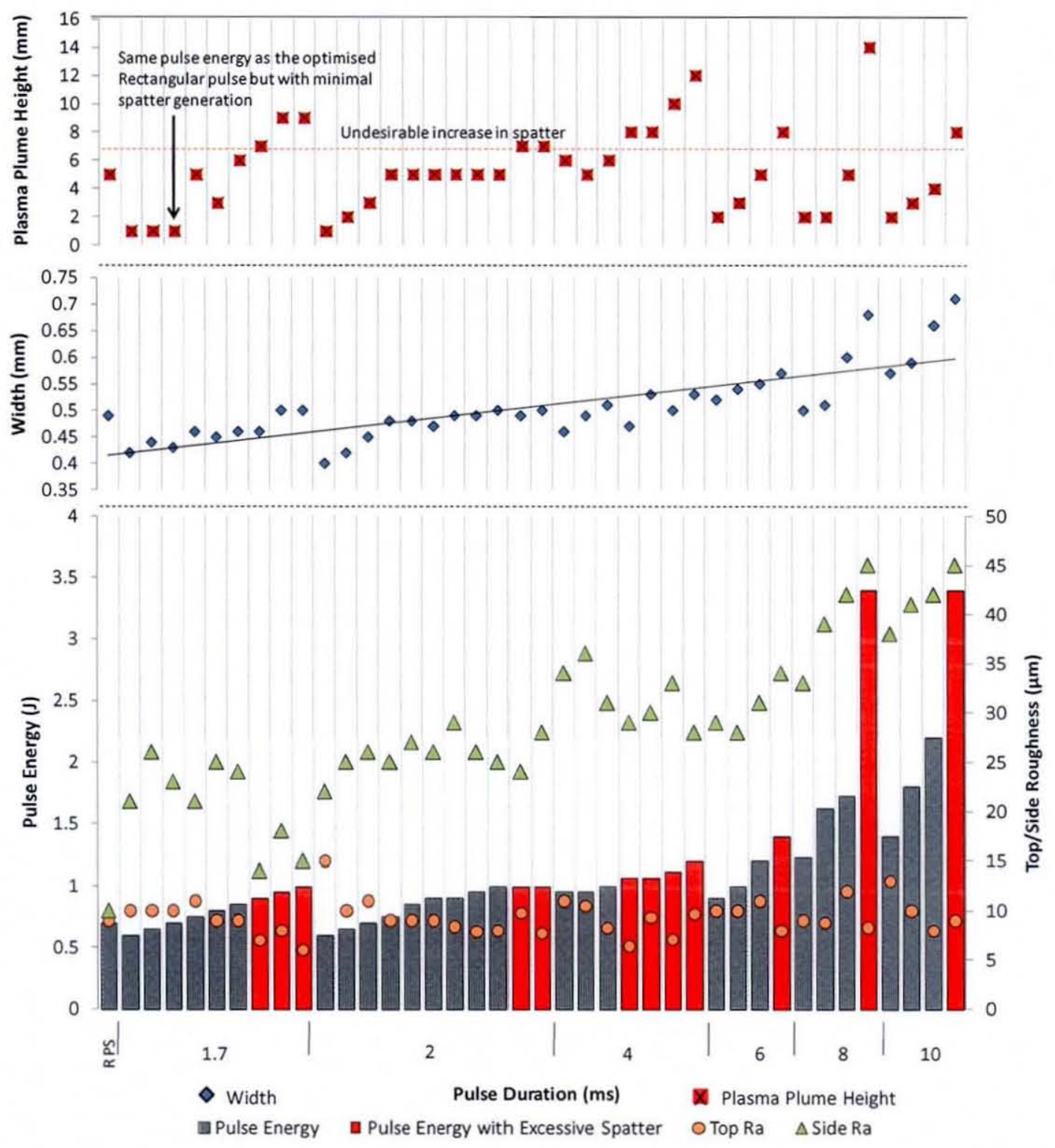

Figure 7-30 Ramp Up top/side $\mathrm{R}_{\mathrm{a}}$ 


\subsection{Ramp Down Pulse Shapes}

Ramp Down pulse shapes are essentially opposite in their nature than that of a Ramp Up pulse. Figure 7-31 shows a standard Rectangular pulse developed in section 6.3. This pulse takes approximately $0.2 \mathrm{~ms}$ to reach a peak power and $0.5 \mathrm{~ms}$ for its power to tail off to zero. A Ramp Down pulse will therefore be defined as a pulse that emits the majority of its power within the first few millisecond and takes more than $0.5 \mathrm{~ms}$ to completely power down.

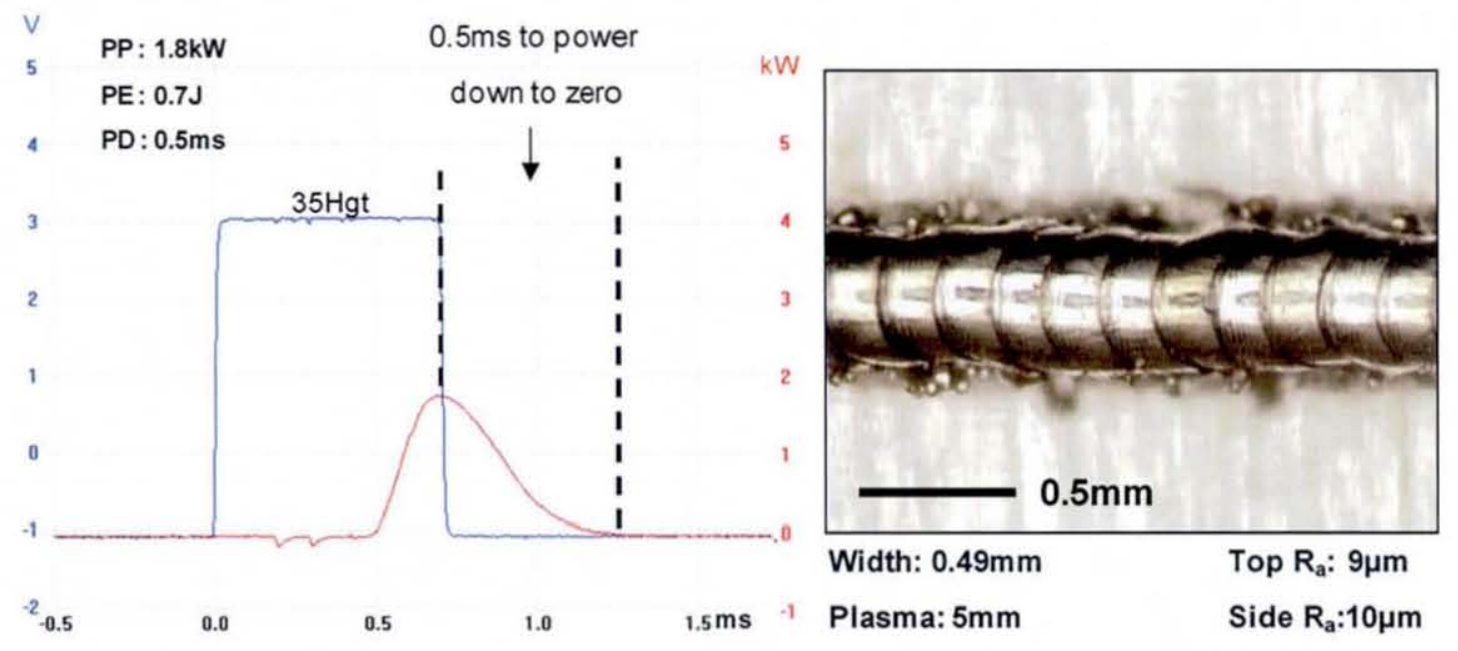

Figure 7-31 Standard Rectangular pulse shape, $0.5 \mathrm{~ms} \& 0.7 \mathrm{~J}$

\subsubsection{Methodology}

The same methodology used in section 7.2.1 of inserting sectors and adjusting current demand profiles was used to generate Ramp Down pulses. However the problems experienced within Ramp Up pulses such as power drop-off, inconsistent power Ramp Up were not experienced within the generation of Ramp Down pulses. This is because the nature of a Ramp Down pulse is essentially the same as a standard Rectangular pulse, both have a maximum peak power early within the pulse with a reduction in energy immediately after. The only differences are the rate at which the energy drops off and the potential to tailor high peak power, low energy combination or low peak power, high energy combinations (something not possible with a non pulse shaped pulse). 
As with Ramp Up pulses the Ramp Down pulse shaping experimentation progressively increased the overall duration and power within the pulse (as shown in Figure 7-32). Extra sectors were introduced into the pulse such that a consistent Ramp Down power delivery was generated. Parts were built consisting of four multi-layer single scans measuring $25 \mathrm{~mm}$ in length which were processed from a $0.1 \mathrm{~mm}$ powder bed. All pulse shaping experiments maintained the same repetition rate $(40 \mathrm{~Hz})$ and scan speed $(400 \mathrm{~mm} / \mathrm{m})$ using the optimised processing parameters developed in section 6.3. Ramp Down pulse duration and energies varied between $1-10 \mathrm{~ms}$ and $0.4-3 \mathrm{~J}$ respectively (as shown in Table 7-2). Again three samples were made for each set of processing parameters. Samples were measured for top/side $\mathrm{R}_{\mathrm{a}}$, width and observed for plasma plume height during processing.

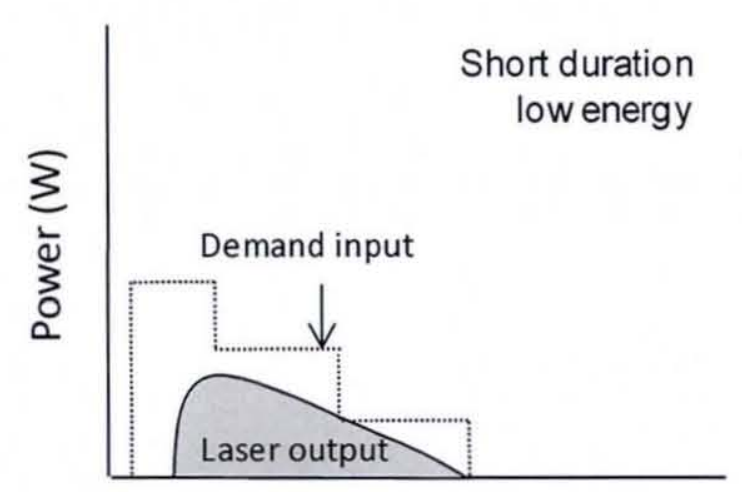

Pulse Duration (ms)

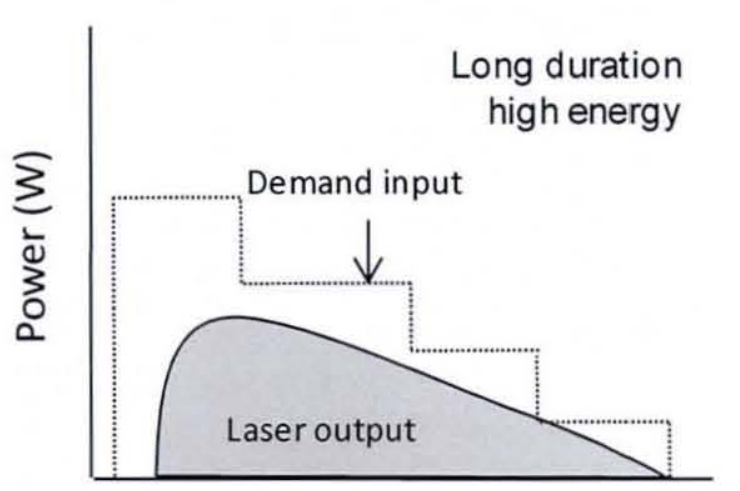

Pulse Duration (ms)

Figure 7-32 Ramp Down pulse shape methodology

\begin{tabular}{cccc}
\hline Pulse Duration (ms) & Pulse Energy $(J)$ & Rep. Rate $(\mathrm{Hz})$ & Scan Speed $(\mathrm{mm} / \mathrm{min})$ \\
\hline $1-10$ & $0.4-3$ & 40 & 400 \\
\hline
\end{tabular}

Table 7-2 Ramp Down pulse generation parameter range

\subsubsection{Ramp Down Results}

The experiments initially processed Inconel 625 using short Ramp Down pulses with low powers and progressively increased the power and length of these pulse shapes. Section 7.3.2.1 to 7.3.2.3 shows a selection of samples produced using these pulses with increasing pulse durations and investigated the effect it has on the production of thin 
wall parts. Section 7.3.2.5 discusses all the Ramp Down results collectively and examines its potential merits and downfalls for use within SLM.

\subsubsection{Pulse duration $1 \mathrm{~ms}$ to $2 \mathrm{~ms}$}

A $1 \mathrm{~ms}$ pulse was split into two sectors, the first sector was set to $35 \mathrm{Hgt}$ (equal to the Rectangular pulse's sector Hgt) and the second sector was set to $20 \mathrm{Hgt}$ as shown in Figure 7-33. The peak power of the pulse was approximately $1.9 \mathrm{~kW}$ with a pulse energy of $0.78 \mathrm{~J}$. This Ramp Down pulse caused the melt pool to remain molten for a longer period of time as the energy imparted by the laser gradually reduced. Gravity was given more time to flatten the melt pool and reduce top $\mathrm{R}_{\mathrm{a}}$. The use of a higher pulse energy may have also contributed to lower top $R_{a}$, as higher melt temperatures reduce melt pool viscosity allowing molten material to flow more freely under Marangoni convection. Lower top $\mathrm{R}_{\mathrm{a}}$ could also be due to an increase in recoil pressure, modifying the shape of the melt pool. This energy distribution caused the top $R_{a}$ of the sample to reduce to $7 \mu \mathrm{m}$. However prolonged heating and melt pool volume expansion probably caused an increase in side $\mathrm{R}_{\mathrm{a}}(20 \mu \mathrm{m})$. Figure 7-34 shows the pulse with its first sector increased to $37 \mathrm{Hgt}$, this caused the peak power to increase to $2.3 \mathrm{~kW}$ causing large plasma plume generation. The pulse had an energy of $0.92 \mathrm{~J}$ and side $\mathrm{R}_{\mathrm{a}}$ of $16 \mu \mathrm{m}$. This is a reasonable side surface finish considering the laser on time and increased melt pool width. This is possibly due to increased recoil pressures on the melt pool The recoil pressures reshaped the melt pool making it flatter inducing enforced wetting onto the substrate. Improved wettability relieves the melt pool surface tension variations and suppresses the balling mechanism. Increased recoil pressure may also have removed any satellite formation from attaching to the edge of the solidifying melt pool.

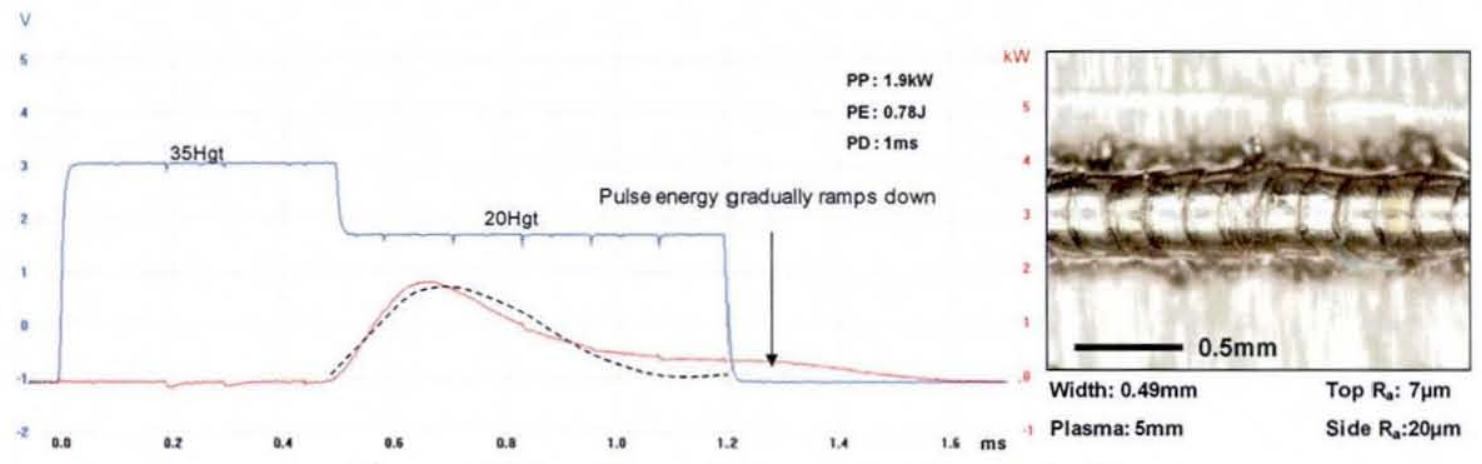

Figure 7-33 Ramp Down pulse $1 \mathrm{~ms} \&$ 0.78J 


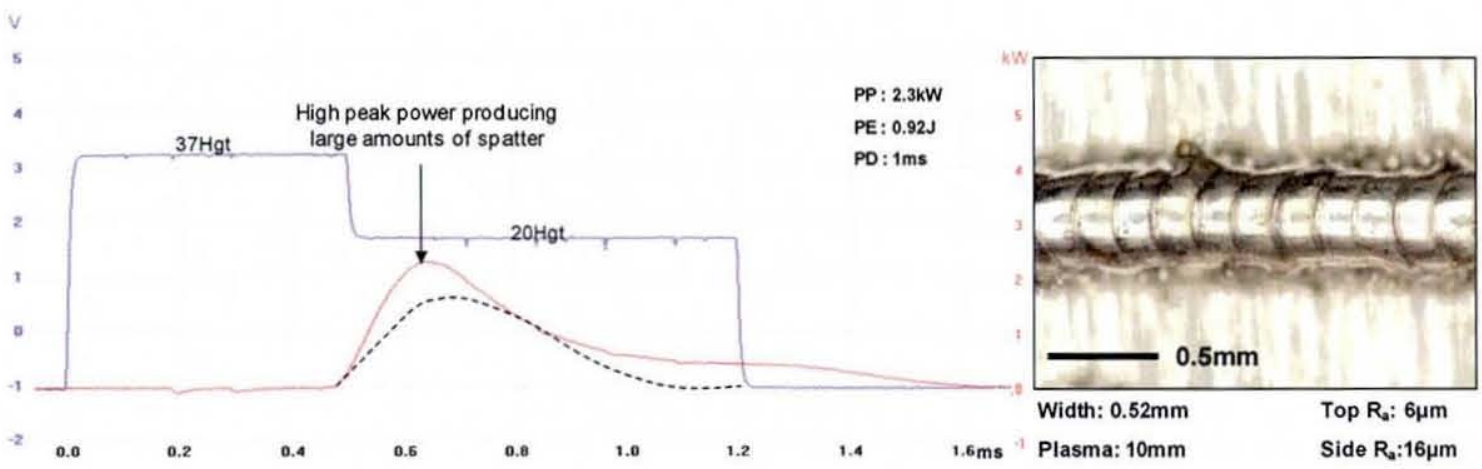

Figure 7-34 Ramp Down pulse $1 \mathrm{~ms} \&$ 0.92J

The Ramp Down pulse duration was extended such that the second sector was $1 \mathrm{~ms}$ in duration and the first sector remained constant at $0.5 \mathrm{~ms}$. This produced a pulse with a duration of $1.5 \mathrm{~ms}$. Figure $7-35$ shows that after the main peak power had been generated it took $1.4 \mathrm{~ms}$ for the power to reach zero. This prolonged melt pool solidification allowed the top $R_{a}$ to remain low, however the side $R_{a}$ suffered as a result of more time being given for melt pool instabilities to develop. The peak power of $2 \mathrm{~kW}$ caused the generation of a larger plasma plume height of $6 \mathrm{~mm}$. Figure 7-36 shows that the peak power of the pulse was reduced while the energy distribution within the latter part of the pulse was increased. The reduction in peak power assisted the reduction in plasma plume generation while the increase in pulse energy helped to reduce top $R_{a}$ to $6 \mu \mathrm{m}$. This was one the lowest top $\mathrm{R}_{\mathrm{a}}$ value achieved while plasma plume generation remained low. The combination of melt pool heating time and melt temperature produced a steady melt pool allowing molten material to redistribute within the extended relaxation time. However because of this the side $R_{a}$ was high, measuring $28 \mu \mathrm{m}$. 


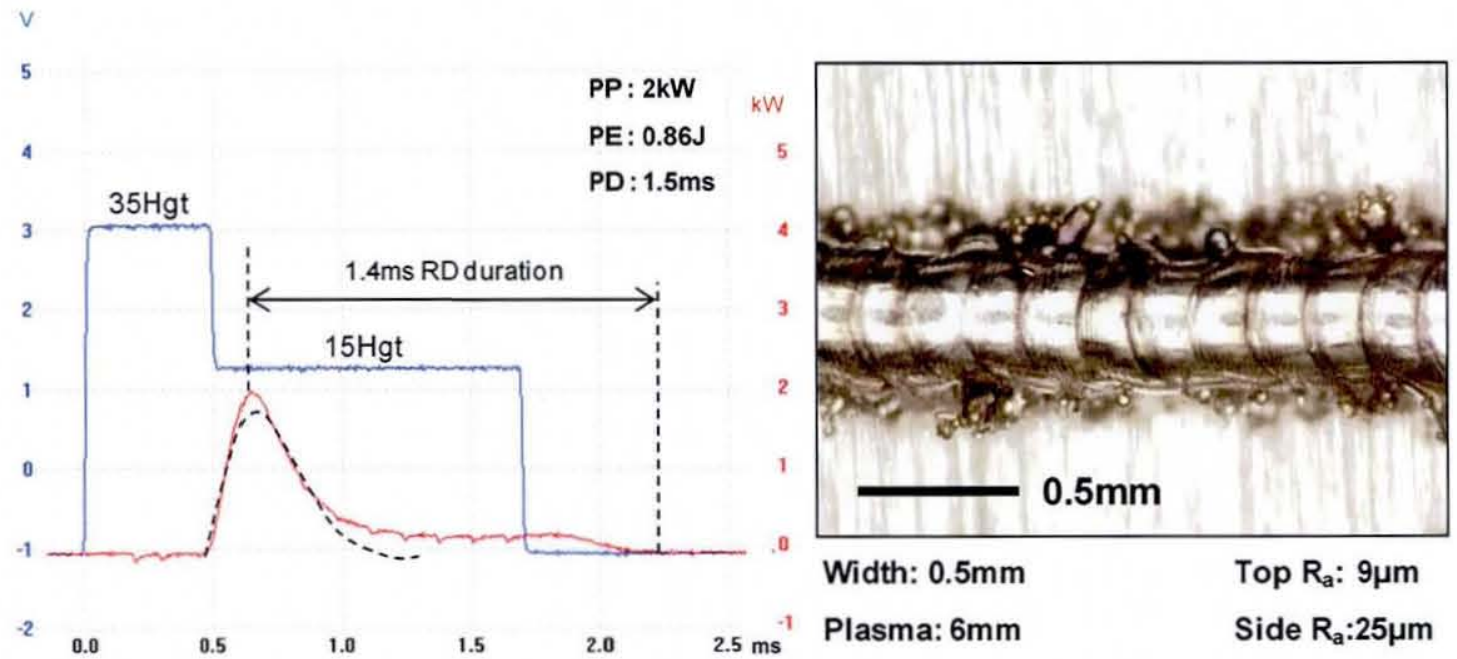

Figure 7-35 Ramp Down pulse $1.5 \mathrm{~ms} \& 0.86 \mathrm{~J}$
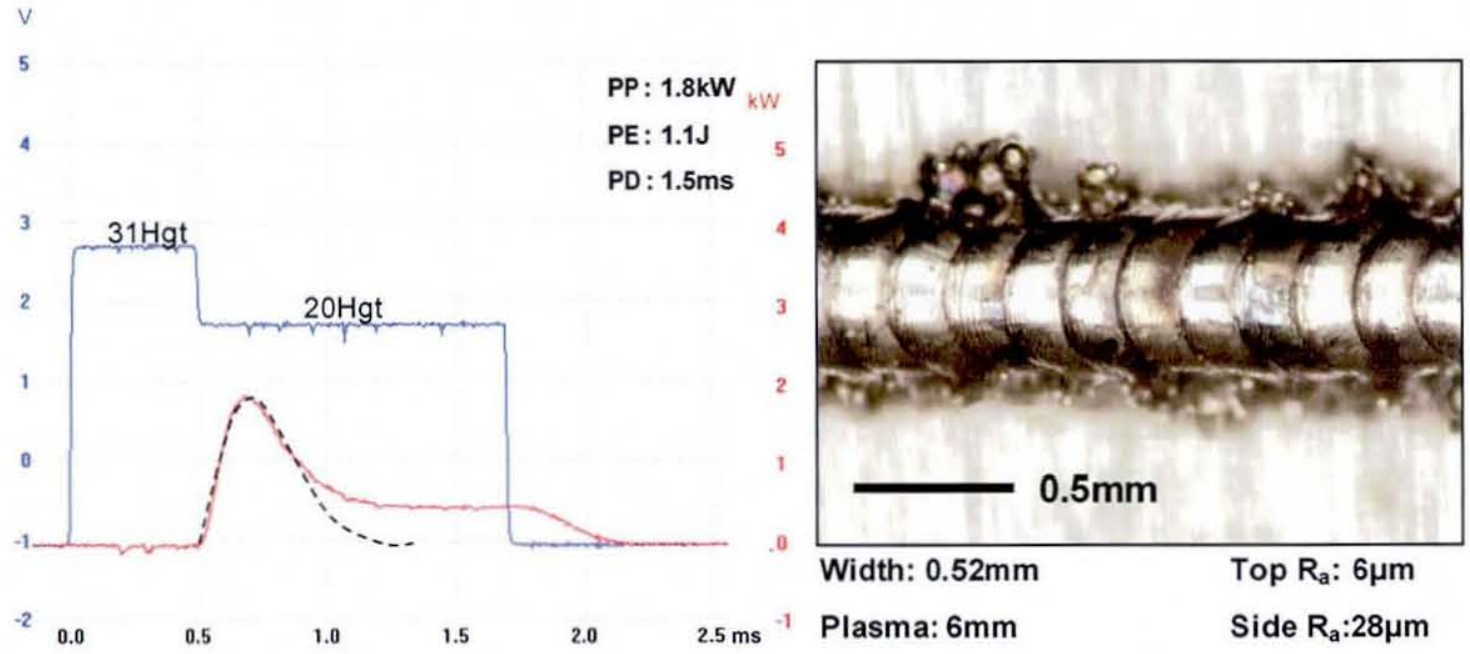

Figure 7-36 Ramp Down pulse $1.5 \mathrm{~ms} \& 1.08 \mathrm{~J}$

Pulse durations were extended to $2 \mathrm{~ms}$ by increasing the second sector length to $1.5 \mathrm{~ms}$. The sample showed similar trends to those produced by a $1.5 \mathrm{~ms}$ Ramp Down pulse. However the side $\mathrm{R}_{\mathrm{a}}$ progressively worsened as melt pool width increased. Section 7.3.2.5 displays the full set of results for 1-2ms Ramp Down pulse along with other pulse durations.

\subsubsection{Pulse duration $4 \mathrm{~ms}$ to $6 \mathrm{~ms}$}

Extending the Ramp Down pulse duration to $4-6 \mathrm{~ms}$ required an additional third sector in order to maintain a steady Ramp Down power transition. Figure 7-37 shows a $4 \mathrm{~ms}$ Ramp Down pulse with the first, second and third sectors measuring $0.5 \mathrm{~ms}, 1.5 \mathrm{~ms}$ and $2 \mathrm{~ms}$ in duration respectively. The sample produced is most notable for its high side $R_{a}$ $(45 \mu \mathrm{m})$. This is probably a result of the larger melt pool width remaining molten for too 
long, further to this the surface of the sample shows sign of oxidisation due to a discoloration and blackening of the surface. Figure 7-38 shows a similar pulse with the main peak power increased to $1.9 \mathrm{~kW}$. This increase improved the top and side $\mathrm{R}_{\mathrm{a}}$ of the sample due to recoil pressures generated. This also caused plasma plume/spatter generation to increase. Figure 7-39 shows a Ramp Down pulse with a peak power of $1.8 \mathrm{~kW}$. The current demand profile for sectors two and three were increased such that more energy was distributed within the central portion of the pulse. This caused part width to greatly increase to $0.59 \mathrm{~mm}$. The initial peak power generates a melt pool, but this does not have a large influence on extending the width of melt due to its very short duration. The energy immediately after the main peak power has more influence due to its longer duration. There was no improvement to the top $\mathrm{R}_{\mathrm{a}}$, nor was there any further deterioration of side $R_{a}$ past the $45 \mu \mathrm{m}$ recorded maximum.
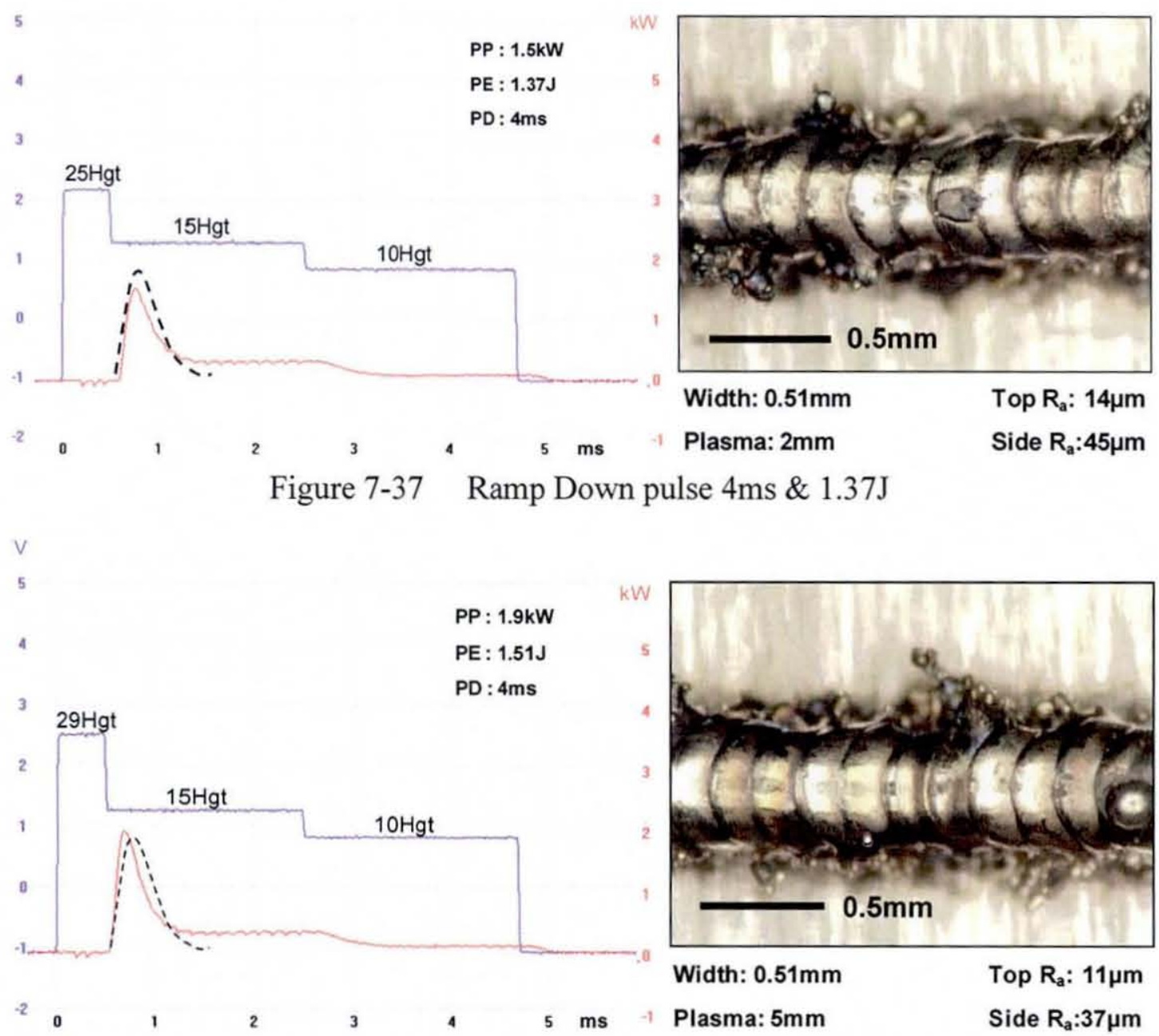

Figure 7-38 Ramp Down pulse $4 \mathrm{~ms} \& 1.51 \mathrm{~J}$ 


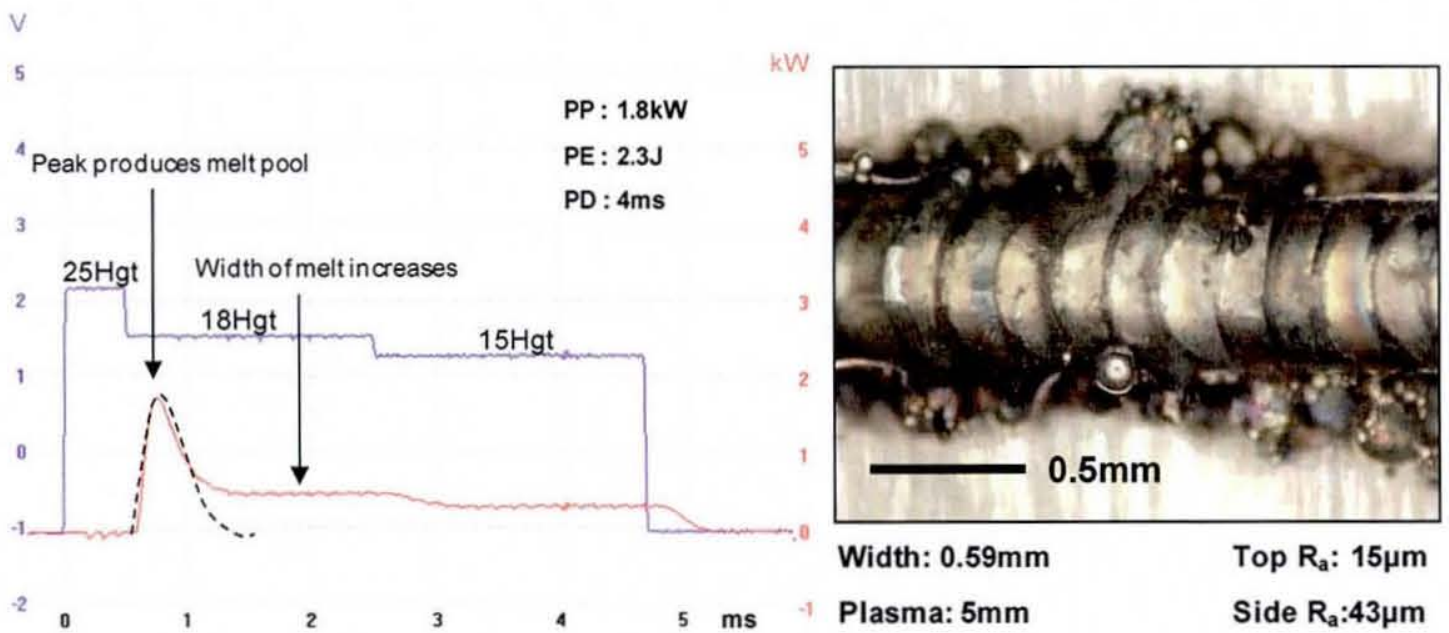

Figure 7-39 Ramp Down pulse $4 \mathrm{~ms} \& 2.3 \mathrm{~J}$

Ramp Down pulses measuring 6ms in duration were produced by extending the second and third sector to $0.5 \mathrm{~ms}$ and $2.5 \mathrm{~ms}$ respectively. These were found to produce samples that increased in width and produced side surface $R_{a}$ above $34 \mu \mathrm{m}$. Samples also continued to suffer from what appeared to be oxidisation. Section 7.3.2.5 displays the full set of results for 4-6ms Ramp Down pulses.

\subsubsection{Pulse duration $8 \mathrm{~ms}$ to $10 \mathrm{~ms}$}

Ramp Down pulses of $8 \mathrm{~ms}$ and $10 \mathrm{~ms}$ required only three sectors to deliver gradual power Ramp Down. They were produced using 3.5ms (second sector), 4ms(third sector) and $4.5 \mathrm{~ms}$ (second sector) and $5 \mathrm{~ms}$ (third sector) respectively. There were no unexpected results. The use of longer pulse duration extended the time for which the melt pool remained molten and subsequently increased its susceptibility to oxidise Samples progressively increased in width, darkened in colour and possessed high side $\mathrm{R}_{\mathrm{a}}$ values above $34 \mu \mathrm{m}$. None of these part qualities/characteristics would benefit the SLM process in producing functional metal parts with a high resolution and low surface roughness. Section 7.3.2.5 displays the full set of results for 8-10ms Ramp Down pulses.

\subsubsection{Unexpected "Suppressed Pulse Shape" generation}

Within Ramp Down pulse shape generation an unexpected pulse shape was generated within early experimentation. A $1 \mathrm{~ms}$ pulse was originally split into two sectors, the first sector was set to $39 \mathrm{Hgt}$ and the second sector to $0 \mathrm{Hgt}$. It was expected that the pulse would follow the same energy distribution as that of the optimised $0.5 \mathrm{~ms}$ Rectangular 
pulse (reaching $0.7 \mathrm{~ms}$ in duration), as the power would essentially only be present within the first $0.5 \mathrm{~ms}$ sector. However the result was most unexpected with the pulse's total duration being suppressed down to $0.5 \mathrm{~ms}$, as shown in Figure 7-40. By inserting a second sector with a demand of $0 \mathrm{Hgt}$ the pulse duration is effectively reduced in order to achieve zero power between $0.5-1 \mathrm{~ms}$ (second sector). The pulse had the same peak power as the standard optimised Rectangular pulse $(1.8 \mathrm{~kW})$ but with $0.3 \mathrm{~J}$ less energy and a shorter duration $(0.2 \mathrm{~ms}$ shorter). This resulted in producing a sample measuring $0.42 \mathrm{~mm}$ in width. The width of the sample was reduced compared to the Rectangular pulsed sample, due to reduced pulse duration and pulse energy. Shorter laser-on time restricts the melt pool width as heat has less time to conduct radially from the centre of the melt pool. Less pulse energy reduces the volume of liquid produced within the melt pool. The high peak power at the beginning of the pulse is sufficient to generate a melt pool that very rapidly solidified. The low pulse energy caused plasma plume to be restricted to $3 \mathrm{~mm}$. The combination of low melt pool width and sufficiently high peak power allowed the sample to possess a low top/side $R_{a}$ of $10 \mu m$. So in summary this Suppressed pulse was able to attain very similar top/side $R_{a}$ as that of a standard Rectangular pulse, but with a reduced part width and lower plasma plume during processing. Figure 7-41 shows a similar Ramp Down pulse with an increased first sector Hgt. This caused the peak power of the pulse to increase to $1.9 \mathrm{~kW}$ and the pulse energy to $0.5 \mathrm{~J}$. As expected, this caused both the width and plasma plume height to increase. This had no effect on top/side $\mathrm{R}_{\mathrm{a}}$. Figure 7-42 shows a pulse with increased peak power reaching $2.3 \mathrm{~kW}$. This energy distribution further increased the width of the melt but caused an excessive increase in spatter generation. Even though the pulse energy was only $0.6 \mathrm{~J}$ the short laser-on time and high peak power generated large amounts of heat within a very short space of time causing material to rapidly vaporise. 

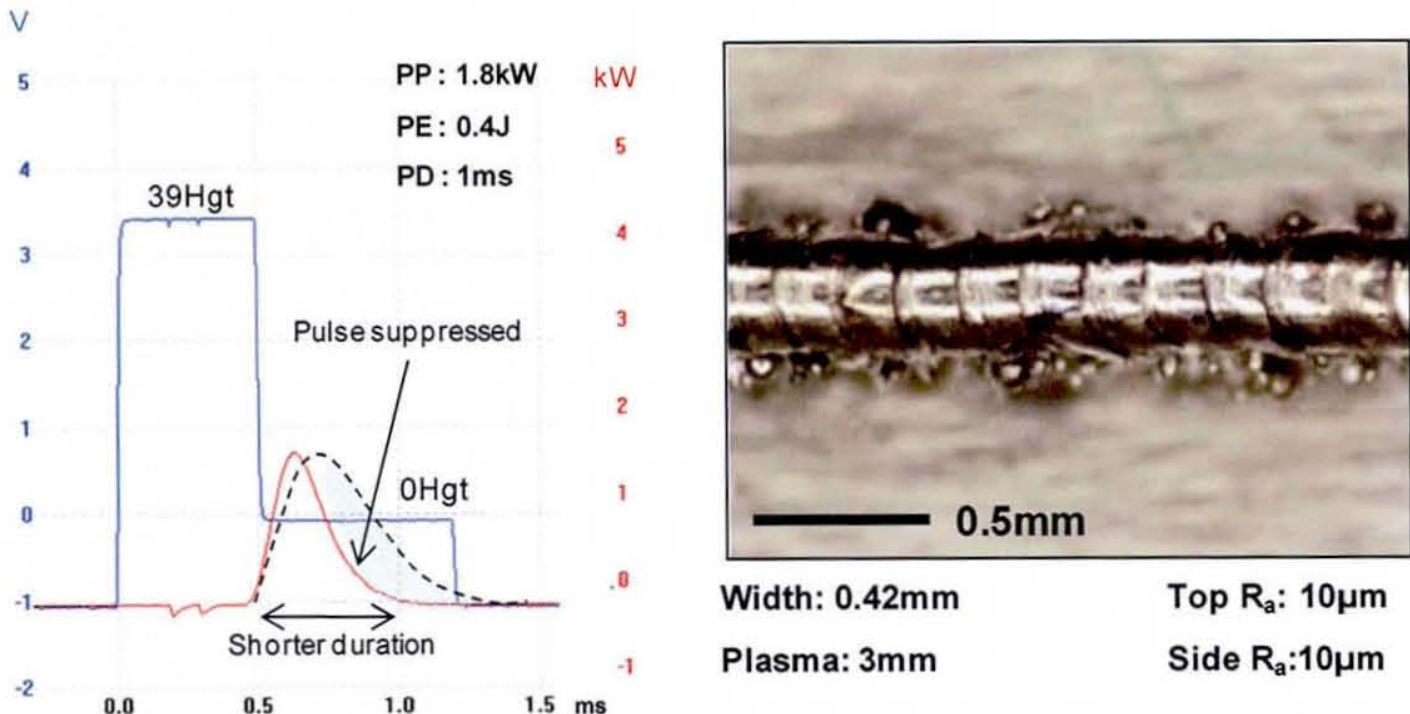

Width: $0.42 \mathrm{~mm}$

Plasma: $3 \mathrm{~mm}$
Top $R_{\mathrm{a}}: 10 \mu \mathrm{m}$

Side $R_{a}: 10 \mu m$

Figure 7-40 Suppressed pulse shape $1 \mathrm{~ms} \& 0.4 \mathrm{~J}$
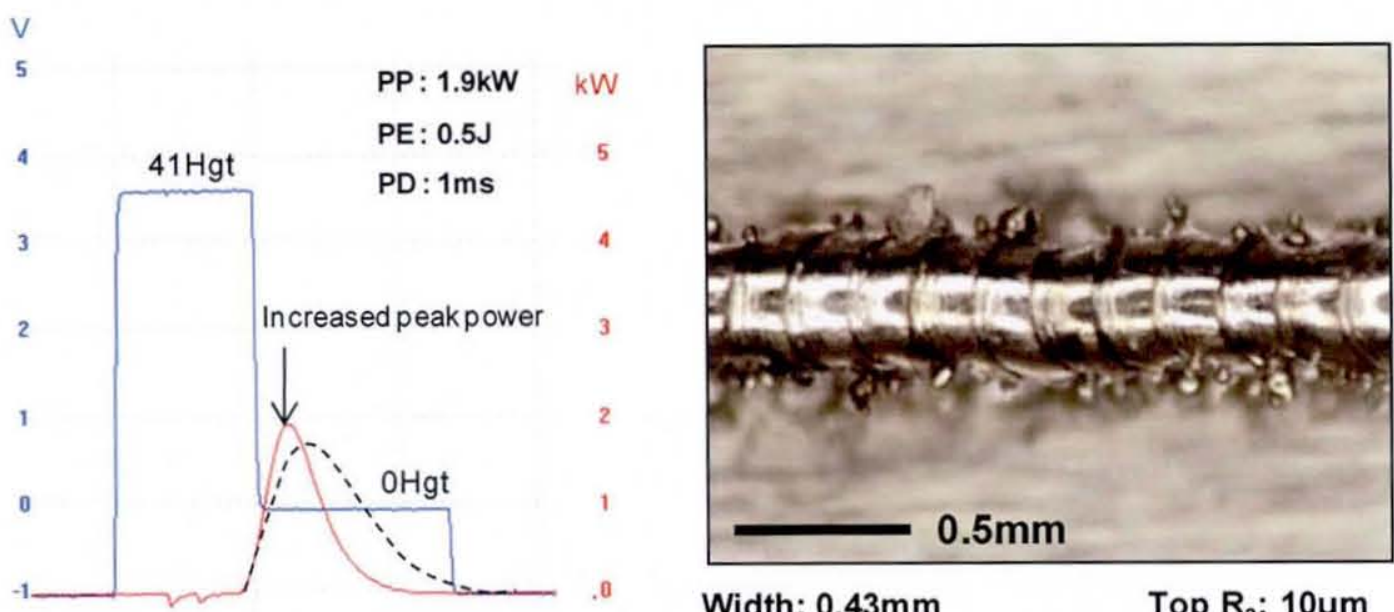

Width: $0.43 \mathrm{~mm}$

Top $R_{\mathrm{a}}: 10 \mu \mathrm{m}$

Plasma: $5 \mathrm{~mm}$

Side $R_{\mathrm{a}}: 10 \mu \mathrm{m}$

Figure 7-41 Suppressed Pulse shape $1 \mathrm{~ms} \& 0.5 \mathrm{~J}$

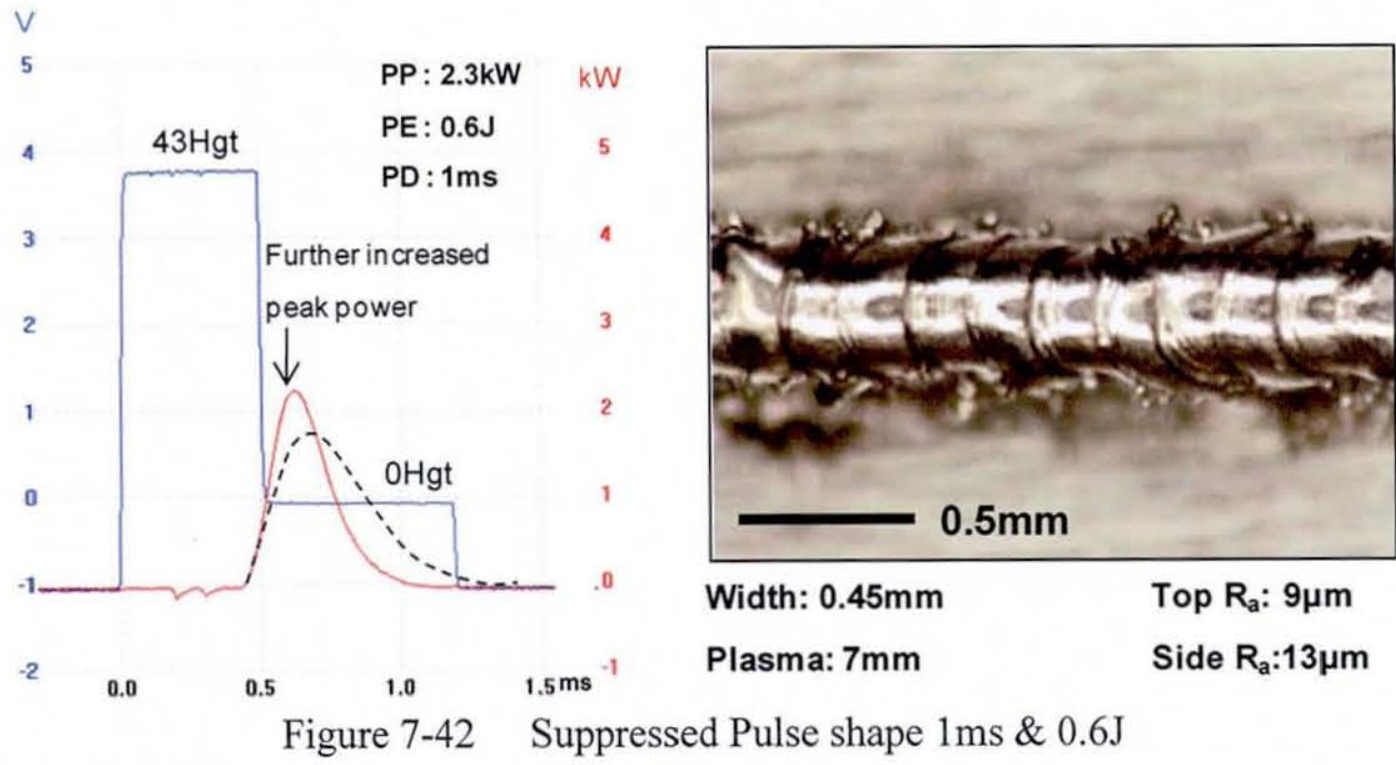




\subsubsection{Overview of Ramp Down/Suppressed Pulse Shapes}

Figure 7-43 shows the full range of top/side $R_{a}$ results produced as a result of different Ramp Down pulse shapes including Suppressed pulses. For comparison the data obtained from the standard optimised Rectangular pulse parameters developed in Chapter 6 has been included on the graph. Each pulse's energy has been shown including the energies that caused an excessive amount of spatter to be generated during processing. Figure 7-43 shows that the side $\mathrm{R}_{\mathrm{a}}$ of samples degrades as both pulse duration and pulse energy of the pulse is increased. This is likely to be due to a greater degree of satellite formation the melt pool becomes larger and is held in a molten state for longer. The larger melt pool volume can cause a larger thermal variation across the melt pool which increases surface tension variations and subsequently may induce lateral balling of the melt pool. Top $\mathrm{R}_{\mathrm{a}}$ improves when pulses between $1.5 \mathrm{~ms}$ and $2 \mathrm{~ms}$ are used. This could be the result of improved melt pool relaxation as molten material is given more time to flow under the force of gravity, thus reducing the variation between overlapping melt pools. Another possible explanation for the reduction of Top $\mathrm{R}_{\mathrm{a}}$ could be associated with the total vaporisation of surface oxides. Gower et al. [2005] found that the use of Ramp Down pulse shapes to laser weld steel sheets produced a smoother weld surface because of surface oxide vaporisation as a result of the Ramp Down energy distribution. Surface oxidisation can contribute to increases in top $R_{a}$ as shown in section 5.3.1. A sample produced using a 1.5ms Ramp Up pulse and 1.08J produced the lowest top $R_{a}$ of $6 \mu \mathrm{m}$. However prolonged melt pool cooling with increases in pulse duration appeared only to be beneficial for a certain period. At pulse durations above $4 \mathrm{~ms}$ the top $R_{a}$ would degrade. This could be a result of too much volume of liquid being produced as a result of a high energy per unit length. As heat is delivered to the melt pool for an increased period of time the volume of the melt pool will expand. This expansion will increase the surface area of the melt pool. More surface area equates to a stronger Marangoni flow and greater rate of heat loss from the surface of the melt pool into the environment. This heat loss produces a larger variation in the temperature profile across the melt pool and greater tendency to undergo lateral balling. To add to this samples produced using Ramp Down pulses of $4 \mathrm{~ms}$ or higher were visually observed to undergo surface discoloration that was presumed to be oxidisation (as mentioned in 6.1). Increases in heat input can also lead to part distortion due to variations in cooling rates and material shrinkage. 
Figure 7-43 shows that samples produced with the Suppressed pulse shape held low top and side $\mathrm{R}_{\mathrm{a}}(10 \mu \mathrm{m}$ minimum) and a low melt pool width ( $0.42 \mathrm{~mm}$ minimum). This was produced using the pulse shape that suppressed the pulse's energy and pulse duration down to $0.42 \mathrm{~J}$ and $0.5 \mathrm{~ms}$ respectively, as previously shown in Figure 7-40. This Suppressed pulse can be seen to have an advantage over the Rectangular pulse due to the reduced melt pool width, allowing parts to be built to a higher resolution.

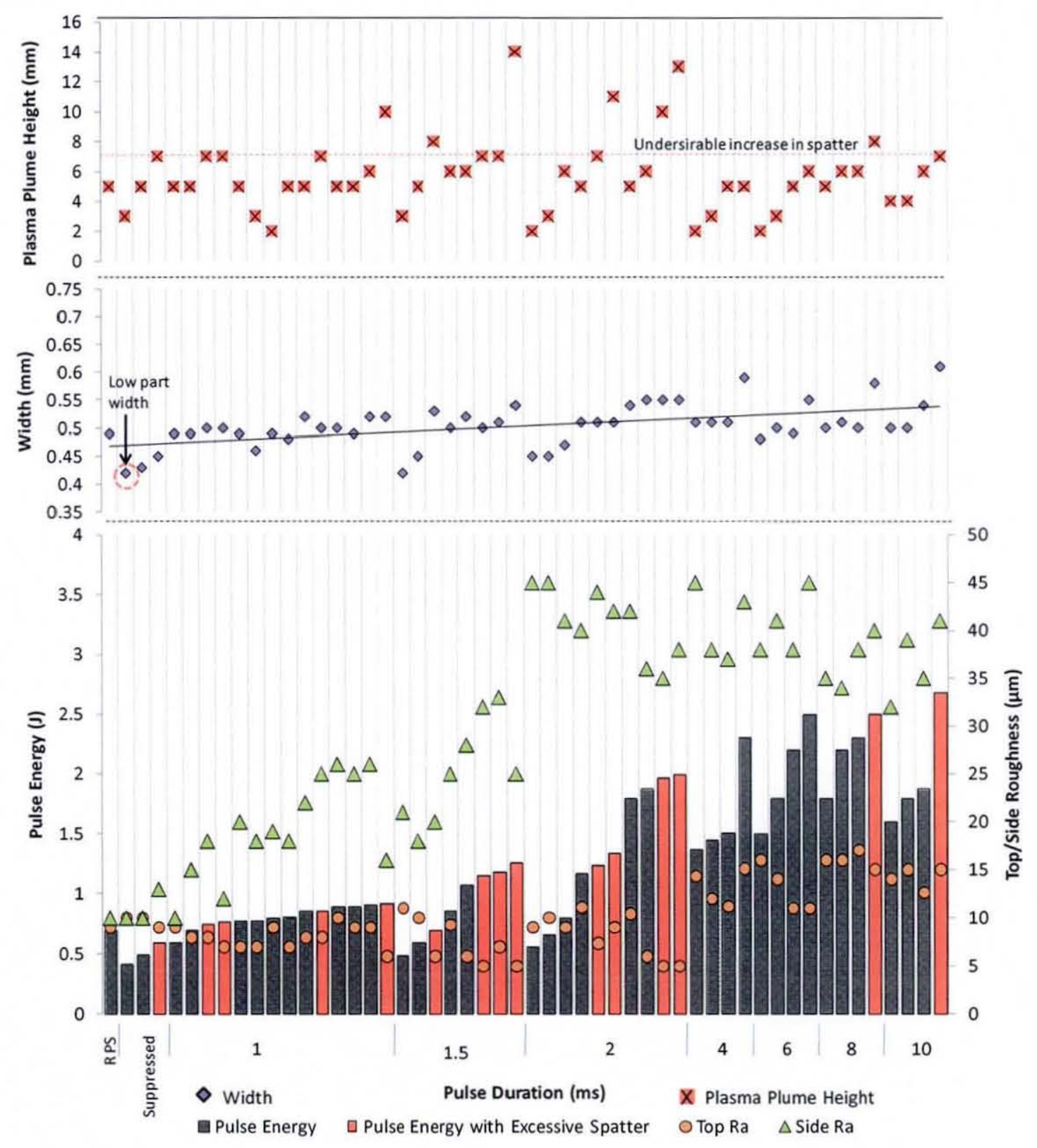

Figure 7-43 Ramp Down/Suppressed pulse shape top/side $R_{a}$ \& width 
Figure 7-43 also shows the plasma plume height generated throughout the use of different Ramp Down/Suppressed pulse shapes. As expected Ramp Down pulses did not have advantages over Rectangular pulses in regards to plasma plume height reduction during processing. Plasma plume height is sporadic not reaching below $2 \mathrm{~mm}$ in height. However due to the low pulse energies in some of the Suppressed pulses the height of plasma plume generated during processing was comparatively lower than a Rectangular or Ramp Down pulse employing the same peak power. 


\subsection{Pulse Shape Trends and Effectiveness}

The individual effects of Ramp Up and Ramp Down/Suppressed pulse shapes have been examined. This section briefly examines the general trends experienced with the use of these pulse shapes. Pulse shapes that were effective in improving any aspect of SLM processing or sample properties will be identified in this section and used for further testing in section Chapter 8. Figure 7-44 shows top/side $R_{a}$, width results of samples produced utilising useable pulse shapes. The graph also displays the heights of plasma plume generated (pulse shapes that generated undesirable increases in material spatter have been excluded).

Trend lines show that top $\mathrm{R}_{\mathrm{a}}$ of samples do not vary considerably when different pulse shapes are employed. The lowest top $R_{a}(6 \mu m)$ was achieved in two samples generated using Ramp Down pulse shapes. One of these (containing a lower side $R_{a}$ ) is indicated on Figure 7-44 with $\mathrm{RD}_{1}$. At higher Ramp Down pulse durations (above $4 \mathrm{~ms}$ ) top $\mathrm{R}_{\mathrm{a}}$ is generally higher compared to those produced using Ramp Up pulses. This is possibly a result of a larger volume of liquid being produced by Ramp Down pulses due to a stronger heating effect [Tzeng 2000]. Ramp Down pulses cause an early melt pool generation with its initial peak power, the remainder of energy increasing the volume of liquid present within the melt pool. Within laser processing of metal powders a lot of energy is required to initiate a melt pool due to initial high reflectivity. Once the melt pool has been initiated the material's reflectivity drops and subsequently requires less energy to initiate full melting or remain molten. The increased melt pool expansion generated with Ramp Down pulses produces larger surface tension variations that increases the melt pools tendency to undergo possible lateral balling which subsequently increasing side $\mathrm{R}_{\mathrm{a}}$. Another factor that could contribute to higher side $\mathrm{R}_{\mathrm{a}}$ with the use of Ramp Down pulses is the prolonged melt pool cooling. The longer a melt pool remains molten the more time there is for instabilities such as balling to develop as well as encouraging satellite formation. 

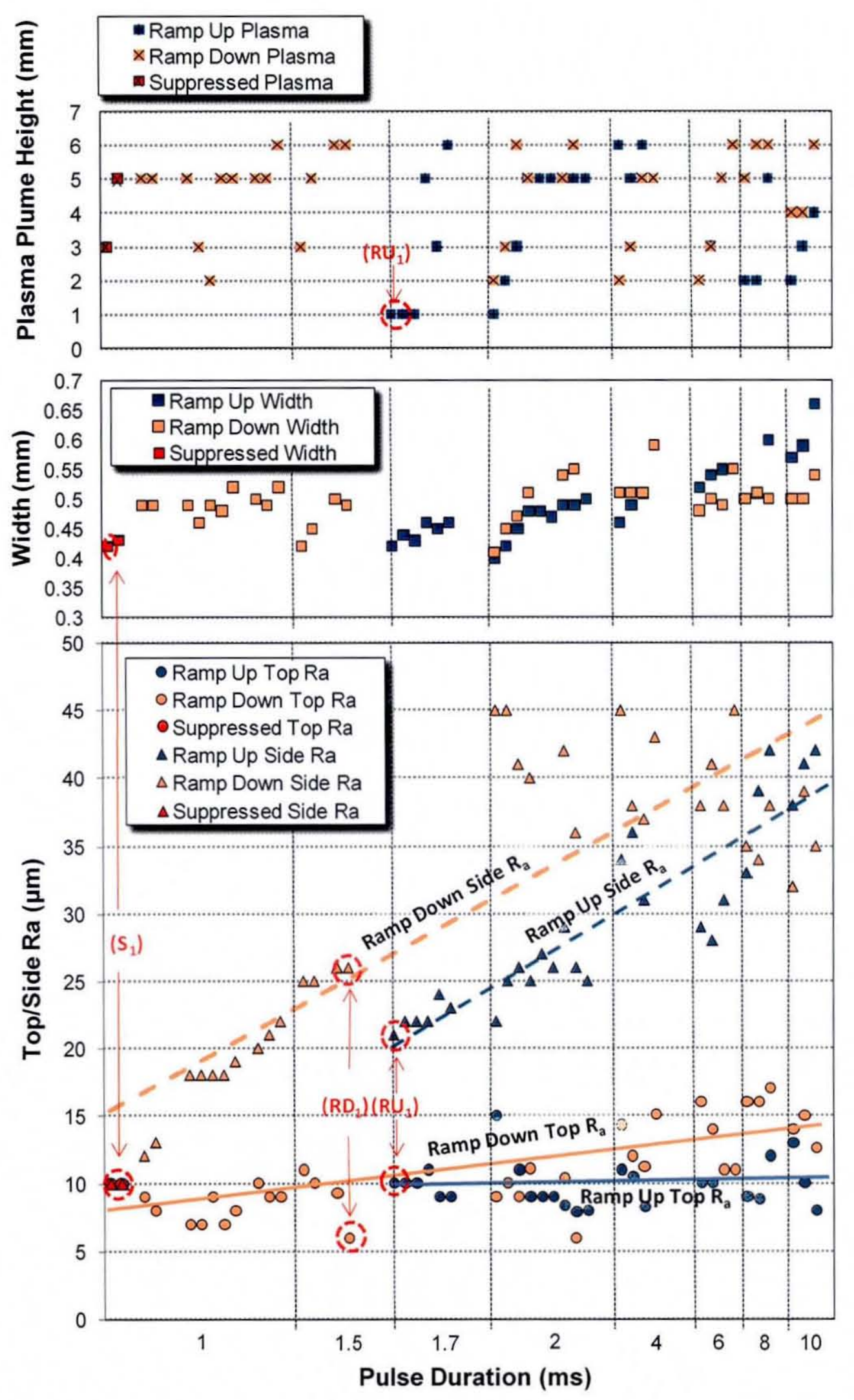

Figure 7-44 Top/Side $\mathrm{R}_{\mathrm{a}}$, width of samples and plasma plume height produced using Ramp Up, Ramp Down/Suppressed pulse shapes 
The Ramp Up pulse would possibly have generated a melt pool at the end of the pulse, once the melt pool has been generated the energy within the pulse abruptly ends and does not facilitate the expansion of melt pool volume (only the case when energy density is low early within pulse). This abrupt loss in power using Ramp Up pulses has been observed to inhibit the flow of molten material with the laser welding of metal sheets [Bransch et al. 1994]. The Ramp Up pulses generally were not successful in reducing top/side $\mathrm{R}_{\mathrm{a}}$ due to low peak power, the lowest combined top and side for all Ramp Up pulses are indicated on Figure 7-44 with $\mathrm{RU}_{1}$. This Ramp Up pulse was also effective in reducing the level of plasma height during processing due to gradual heating of material (indicated with $\mathrm{RU}_{1}$ ). This pulse produced one of the lowest plasma plume heights out of all pulse shapes.

The results for two Suppressed pulses are shown in Figure 7-44. Due to the pulses high peak power, short duration and low pulse energy the samples produced contained the lowest combined top/side $\mathrm{Ra}$ out of all samples produced using the pulse shaping technique as well as a low sample width. Both Suppressed pulses produced a top/side $\mathrm{R}_{\mathrm{a}}$ of $10 \mu \mathrm{m}$, the thinner out of the two samples measured $0.42 \mathrm{~mm}$ and is indicated on Figure 7-44 with $\mathrm{S}_{1}$.

Figure 7-45 shows the top and side $\mathrm{R}_{\mathrm{a}}$ values for the standard optimised Rectangular pulse and useable Ramp Up, Ramp Down and Suppressed pulses. The Rectangular pulse was developed in Chapter 6 such that simultaneously low top/side $\mathrm{R}_{\mathrm{a}}$ values were attained. All pulse shaping experimentation used the same repetition rate and scan speed as the Rectangular pulse and attempted to improve part properties by employing variable energy distributions. Figure 7-45 shows that Ramp Up and Ramp Down energy distribution tended to degrade side $\mathrm{R}_{\mathrm{a}}$ values. Ramping Down tended to be more successful in regards to reducing Top $R_{a}$. However as Top $R_{a}$ reduced below $9 \mu m$ the side $\mathrm{R}_{\mathrm{a}}$ would increase, displaying similar trends to that shown in Figure 6-31. Only two useable suppressed pulses were used to produce parts, both these exhibited low top and side $\mathrm{R}_{\mathrm{a}}$ (both $10 \mu \mathrm{m}$ ). 


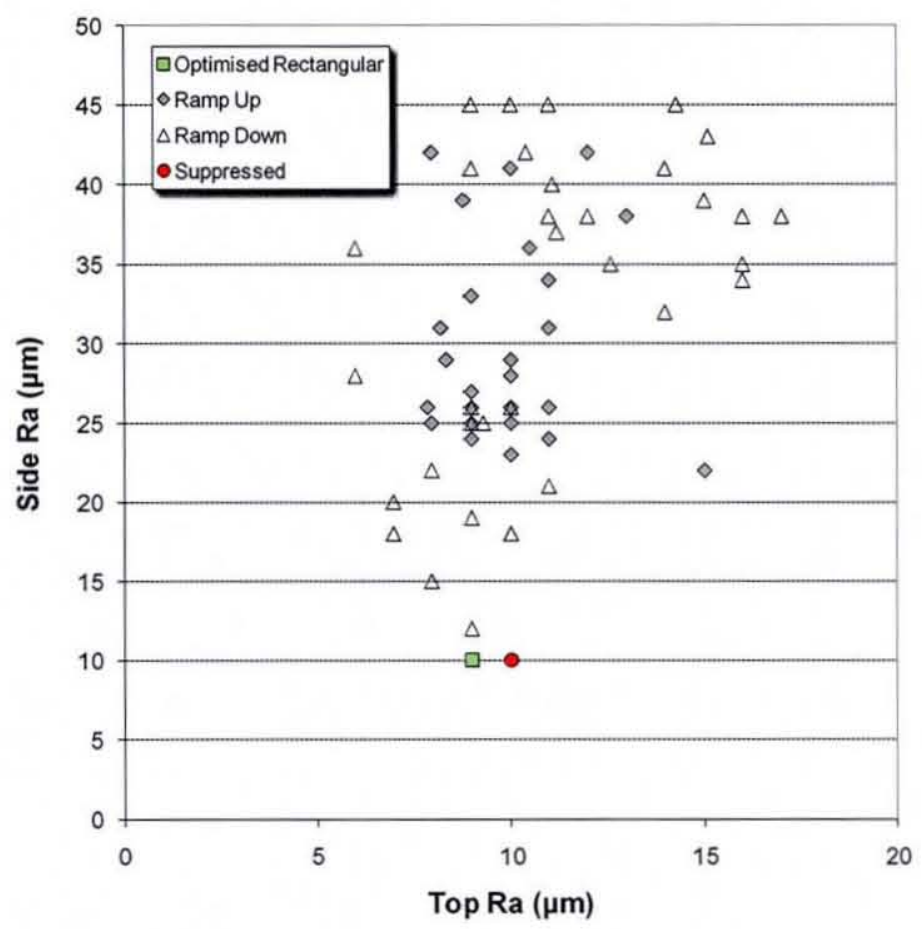

Figure 7-45 Side $R_{a}$ against Top $R_{a}$ for different pulse shapes

Figure 7-46(a-b) shows the top and side $R_{a}$ of samples produced using different pulse shapes at variable peak powers. Both graphs indicate a lower top $R_{a}$ compared to side $\mathrm{R}_{\mathrm{a}}$. However another main difference between the pulse shapes are the peak power which they employ. Ramp Up pulse required lower peak powers than Ramp down pulses in order to melt Inconel power. This is directly related to the distribution of energy within each pulse. With Ramp Down pulses the high peak powers are required $(1.3-2 \mathrm{~W})$ to melt the powder at room temperature. At room temperature metal powder's reflectivity is high and requires large amounts of heat to change the state of the powder from solid to liquid. With Ramp Up pulses the powder is exposed to lower amounts of heat before main peak power is initiated. The gradual increase in power Ramp Up causes the powder particles temperature to increase. This also causes the materials reflectivity to drop and even sinter the powder. By the time the main pulse is reached less heat is required to change the powder from solid or semi-molten to completely molten. Even though the period of time is short (milliseconds) the energy distribution provided by Ramp Up pulses can be viewed as similar to that of powder bed preheating. As a result of this a more gradual and steady melt pool is formed with less spatter generation. The Ramp Up pulses required between $0.7-1.1 \mathrm{~kW}$ to melt the Inconel. On average the Ramp Up pulses required $0.8 \mathrm{~kW}$ less peak power than those required by Ramp Down pulses to successfully process the material. Suppressed pulses had a 
similar peak power demand to that of the Ramp Down pulses due to the requirement for early melt pool generation.
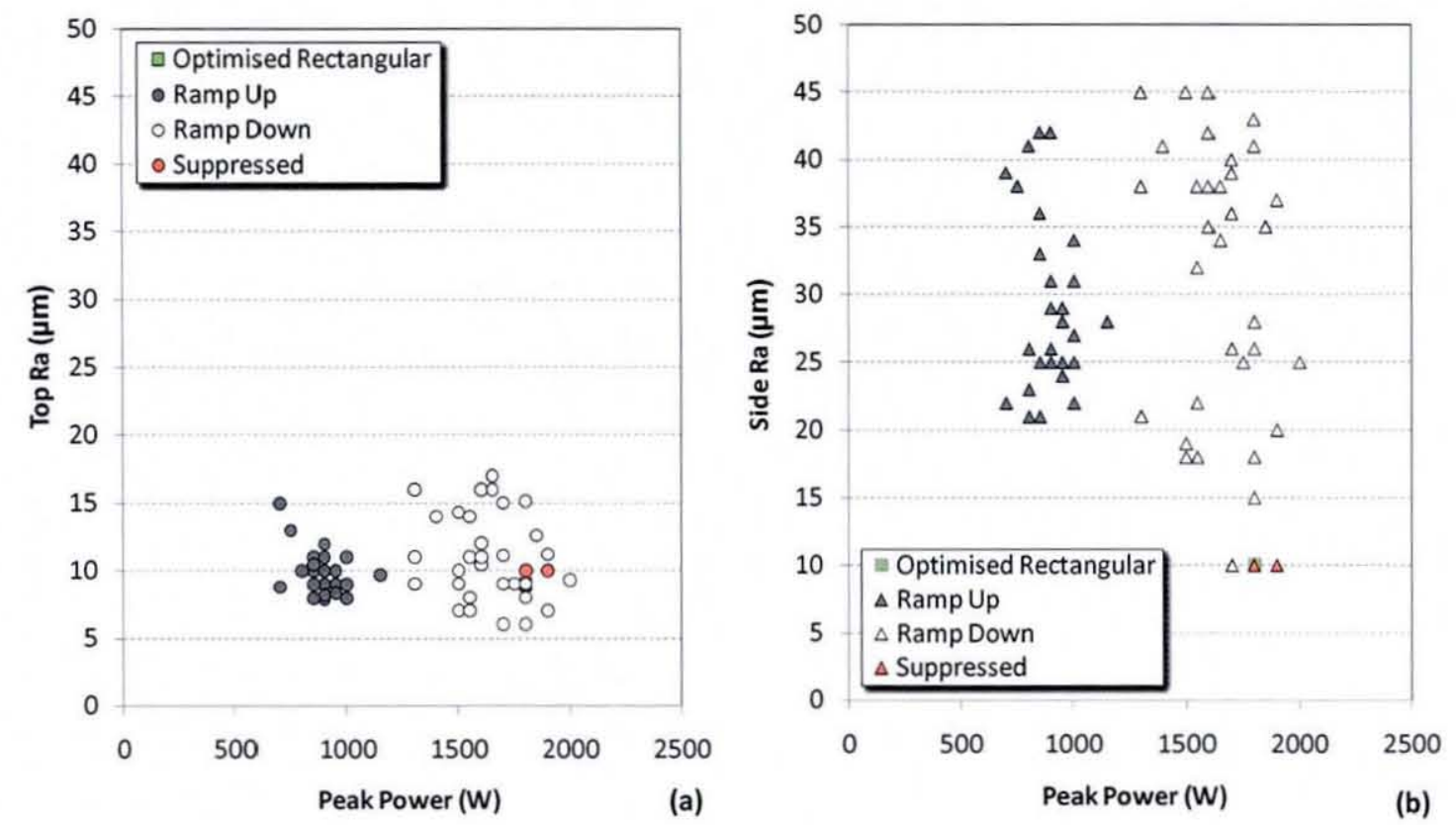

Figure 7-46 Ramp Up\Ramp Down $\backslash$ Suppressed pulse Top $\mathrm{R}_{\mathrm{a}}$ (a) \& Side $\mathrm{R}_{\mathrm{a}}$ (b) at different peak powers

\subsubsection{Pulse Shaping Effectiveness}

Pulse shaping experiments in section 7.2.2 and 7.3.2 generated a variety of different samples at pulse duration between $1-10 \mathrm{~ms}$. The part properties were observed for top/side $R_{a}$, width and spatter generation during processing. Pulse shapes that could offer advantages over the non shaped pulses developed in section 6.3 were identified.

\subsubsection{Ramp Up pulse shape}

Ramp Up pulse shapes have the potential to produce samples with top $R_{a}$ equal to those produced using non pulse shaping techniques. As the duration of the Ramp Up pulse increased side $R_{a}$ progressively worsened reaching a maximum $42 \mu \mathrm{m}$. The use of low peak powers within Ramp Up pulses generally contributed to the poor side $R_{a}$. As mentioned in section 6.3 , high peak powers are beneficial in reducing top and side $R_{a}$ due to the increased recoil pressure reshaping the melt pool. However due to the use of low peak powers samples could be produced with minimal spatter generation. A Ramp Up pulse with the same amount of pulse energy $(0.7 \mathrm{~J})$ contained within the Rectangular 
pulse parameter developed in section 6.3 was able to produce a sample with very low plasma plume formation. This pulse shape and sample is shown in Figure 7-47 and has already been indicated on Figure 7-44 with $\mathrm{RU}_{1}$. Due to the gradual rise in energy density and low peak power the sample was produced with only $1 \mathrm{~mm}$ plasma plume height. This is compared to the $5 \mathrm{~mm}$ generated by the Rectangular pulse. As mentioned in section 3.4.1 a reduction in spatter generation is beneficial for SLM processes. It reduces powder bed contamination and potential damage to the powder leveller. If spatter solidifies on the external surface of a part it can also increase surface $\mathrm{R}_{\mathrm{a}}$. Due to the low peak and late melt pool generation the width of the sample remained relatively low at $0.43 \mathrm{~mm}$. Other potential benefits stemming from the low heat input and reduced HAZ within this Ramp Up pulse include the possibility of alleviating issues related to shrinkage and residual stress build up within a part. From the Ramp Up pulse experimentation the pulse displayed in Figure 7-47 was the only pulse that offered some potential for the SLM process and will further investigated in Chapter 8 .

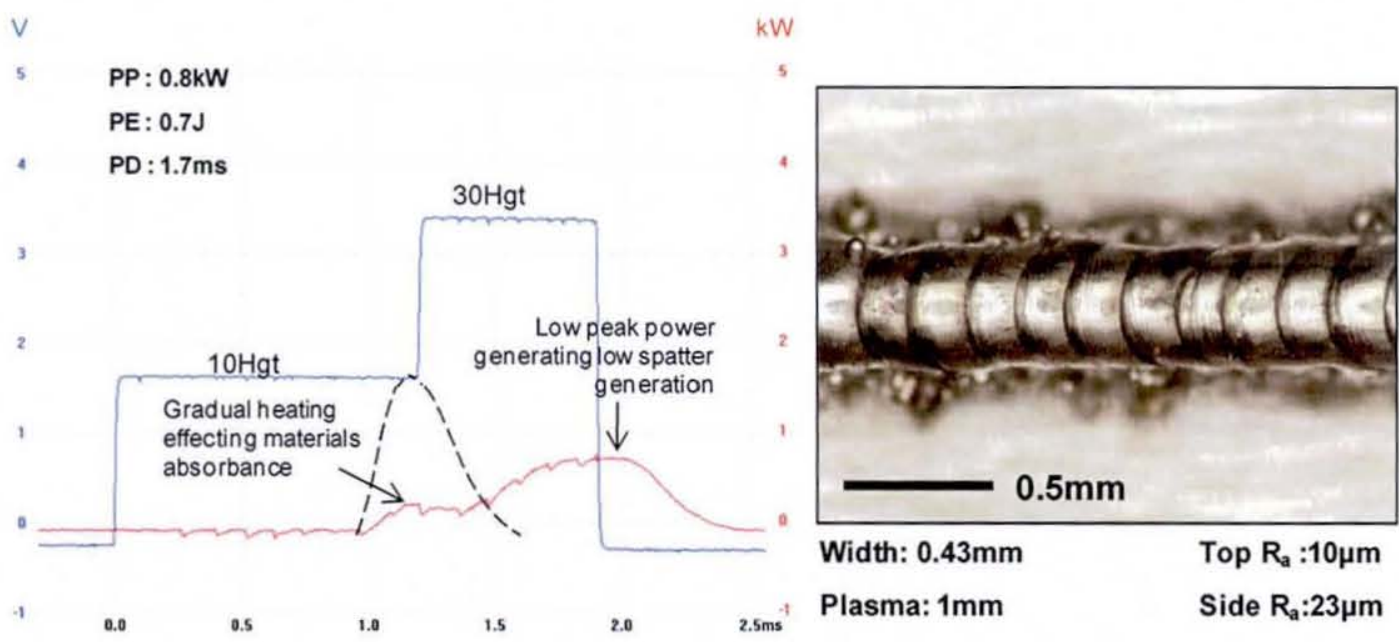

Figure 7-47 Ramp Up pulse producing low wall width \& generating minimal spatter generation during processing

\subsubsection{Ramp Down pulse shape}

Due to the extended melt pool width and prolonged time for which a melt pool remained molten, the Ramp Down pulses did not provide any benefit in regards to improvement to side surface quality or reducing sample width. However a Ramp Down pulse with a duration of $1.5 \mathrm{~ms}$ produced the lowest sample top $\mathrm{R}_{\mathrm{a}}$ measuring $6 \mu \mathrm{m}$. This 
pulse and sample are displayed in Figure 7-48 and has already been indicated on Figure 7-44 with $\mathrm{RD}_{1}$. However as a result of the pulse's increased pulse energy and duration the side $R_{a}$ was high at $28 \mu \mathrm{m}$. This pulse shape overall may not be practical for use over a Rectangular pulse (due to high side $R_{a}$ ), however the samples top $R_{a}$ property had been improved and was therefore further investigated in Chapter 8 .

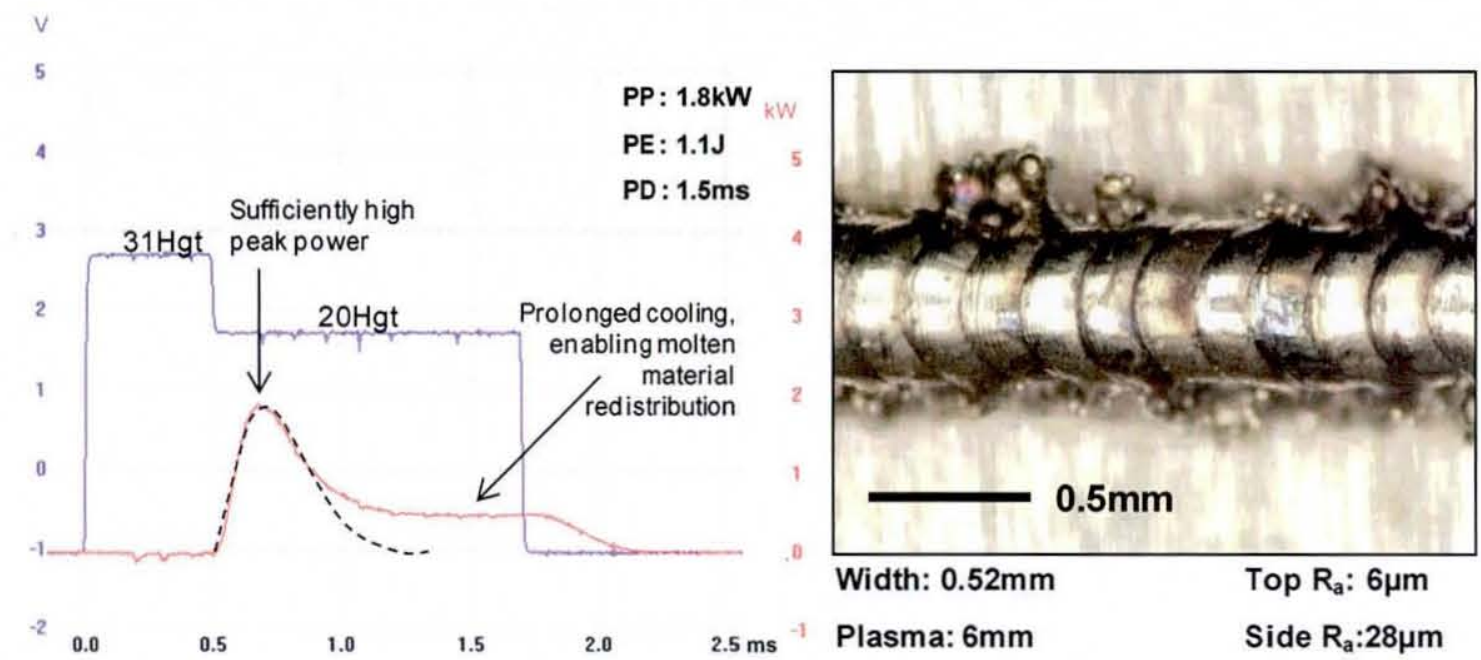

Figure 7-48 Ramp Down pulse with lowest top $\mathrm{R}_{\mathrm{a}}$

\subsubsection{Suppressed pulse shape}

Within Ramp Down pulse experimentation a Suppressed pulse was unexpectedly created when the second sector demand for the laser input was set to zero. This caused the $1 \mathrm{~ms}$ pulse duration to be reduced to a duration lower than that possible with the non pulse shaped optimised Rectangular pulse. One of the Suppressed pulses generated (shown in Figure 7-49 and already indicated in Figure 7-44 with $\mathrm{S}_{1}$ ) had an energy of $0.4 \mathrm{~J}$, duration of $0.5 \mathrm{~ms}$ and the same peak power as that used with the Rectangular pulse $(1.8 \mathrm{~kW})$. This high peak power, low pulse energy and low duration pulse was able to produce a sample with low top/side $\mathrm{R}_{\mathrm{a}}$ (each $\left.10 \mu \mathrm{m}\right)$ and low sample width of $0.42 \mathrm{~mm}$ (compared to the $0.49 \mathrm{~mm}$ generated by the Rectangular pulse). This Suppressed pulse was further investigated in Chapter 8. 

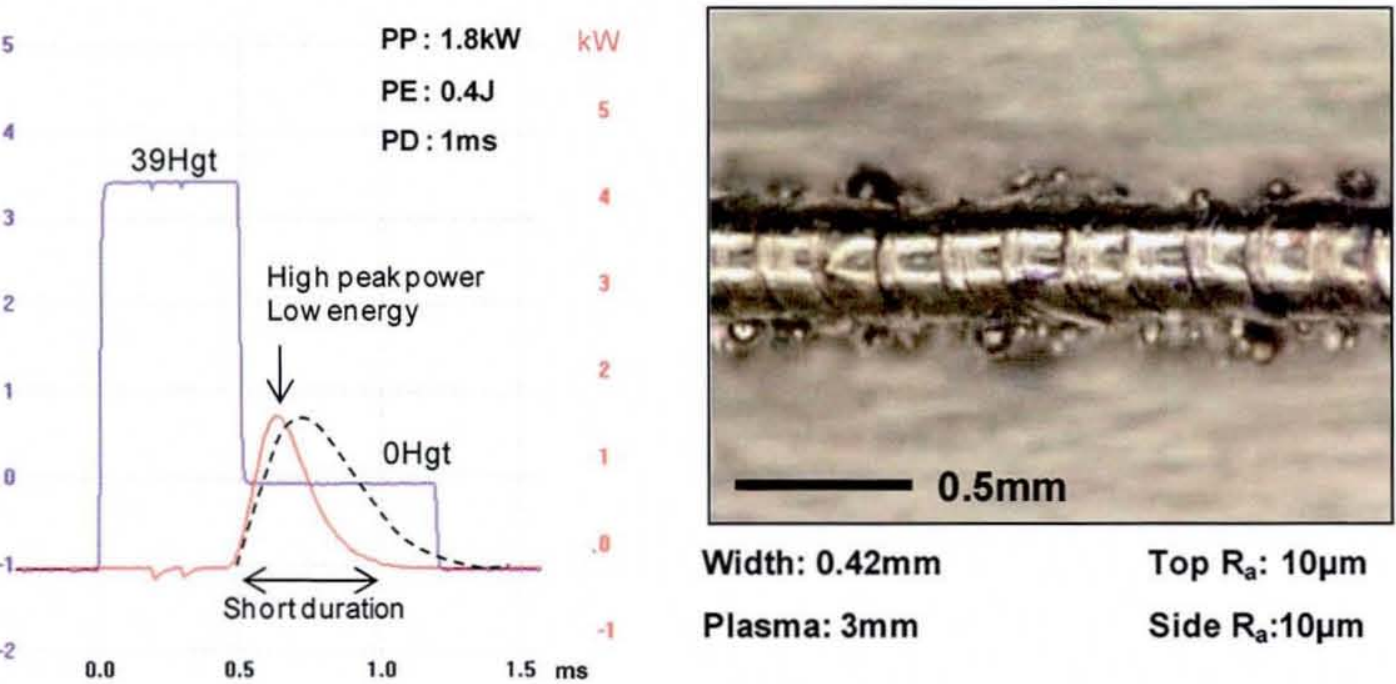

Width: $0.42 \mathrm{~mm}$

Top $R_{\mathrm{a}}: 10 \mu \mathrm{m}$

Plasma: $3 \mathrm{~mm}$

Side $R_{\mathrm{a}}: 10 \mu \mathrm{m}$

Figure 7-49 Suppressed pulse with low top/side $\mathrm{R}_{\mathrm{a}}$ \& low width

\subsubsection{Summary}

Table 7-3 displays details of the optimised Rectangular pulse developed in Chapter 6 and the three most effective pulse shapes identified in Chapter 7. Black ticks indicate the part property that the respective pulse shapes were effective in improving. Red ticks indicate the part property in which the respective pulse shape was highly effective in improving. The table indicated that the optimised non shaped Rectangular pulse was effective in producing samples with low top/side $R_{a}$ and low width. The selected Ramp Up pulse was effective in reducing sample Top $R_{a}$, width and highly effective in reducing spatter generation during processing. The selected Ramp Down pulse was highly effective in reducing top $\mathrm{R}_{\mathrm{a}}$. The Suppressed pulse was effective in reducing top/side $R_{a}$, spatter generation during processing and highly effective in reducing sample width.

\begin{tabular}{c|c|c|c|c}
\hline & Rectangular & Ramp Up & Ramp Down & Suppressed \\
\hline Top $\mathrm{R}_{\mathrm{a}}(\mu \mathrm{m})$ & $\checkmark$ & $\checkmark$ & $\checkmark$ & $\checkmark$ \\
Side $\mathrm{R}_{\mathrm{a}}(\mu \mathrm{m})$ & $\checkmark$ & & & $\checkmark$ \\
Width $(\mathrm{mm})$ & $\checkmark$ & $\checkmark$ & & $\checkmark$ \\
Spatter & & $\checkmark$ & & $\checkmark$ \\
\hline
\end{tabular}

Table 7-3 Benefits of selected pulse shapes to be used within test part validation 
Chapter 8 produces further test parts using the standard non shaped Rectangular pulse (developed in Chapter 6) and the three pulse shapes recently identified. The test parts will allow for validation of initial findings and examine the repeatability and effectiveness of each pulse when used to produce larger parts with variable geometries. 


\section{Chapter 8 Test Part Validation}

The pulse shape experimentation in Chapter 7 had identified three pulse shapes that potentially held advantages over the use of a standard optimised Rectangular pulse developed in Chapter 6. These advantages include improvements to part properties such as surface roughness, part width and reduction in spatter generation during processing. In order to validate and test the effectiveness of these pulse shapes multilayer test parts were built. Parts were built using the non shaped Rectangular pulse developed in Chapter 6 and the three pulse shaped pulses identified at the end of Chapter 7. Parts were tested for surface $R_{a}$ in a variety of directions in different locations along the test part. The width of parts was measured as well as monitoring of plasma plume height during processing.

\subsection{Methodology}

A total of four test parts with different geometries were designed for fabrication using laser parameters developed in Chapter 6 and Chapter 7. The test parts were designed such that not only thin straight walls were produced. Section 8.1.1 details the test part design and fabrication method.

\subsubsection{Test Part Design}

The test part design centred around the extrapolation of previous straight thin wall structures. Other geometries included a thin wall cylinder and solid block structure. The structures were built on steel substrates measuring $40 \times 40 \times 3 \mathrm{~mm}$. All structures were built from 80 powder layers, each with a thickness of $0.1 \mathrm{~mm}$. The first structure was a cylinder measuring $1 \mathrm{~mm}$ in diameter, this could only be measured for side $\mathrm{R}_{\mathrm{a}}$ along the $\mathrm{Z}$-axis. The second structure was a thin wall measuring $10 \mathrm{~mm}$ in length. This could be 
tested for top $\mathrm{R}_{\mathrm{a}}$ and side $\mathrm{R}_{\mathrm{a}}$ in both horizontal and vertical directions. The third structure was another thin wall part scanned perpendicular to the previous wall and measured $7 \mathrm{~mm}$ in length. This structure again could be measured for top $R_{a}$ and side $R_{a}$ in both horizontal and vertical directions. The fourth and final structure was a $7 \times 7 \mathrm{~mm}$ cube. All four side surfaces of the cube were measured for horizontal and vertical $\mathrm{R}_{\mathrm{a}}$. This cube structure was included to test whether there was any variation in side wall $R_{a}$ as the thicker structures were produced due to heat build up. There was also the option for the measurement of top $\mathrm{R}_{\mathrm{a}}$ in a variety of different directions (along scan path, across scan path etc.) however this would have been outside the remit of this investigation due to the requirement for producing parts using variable hatch distances. Each of these four structures were produced a total of three times (see Figure 8-1) using one specific pulse shape on the same substrate so that repeatability of results could be tested.

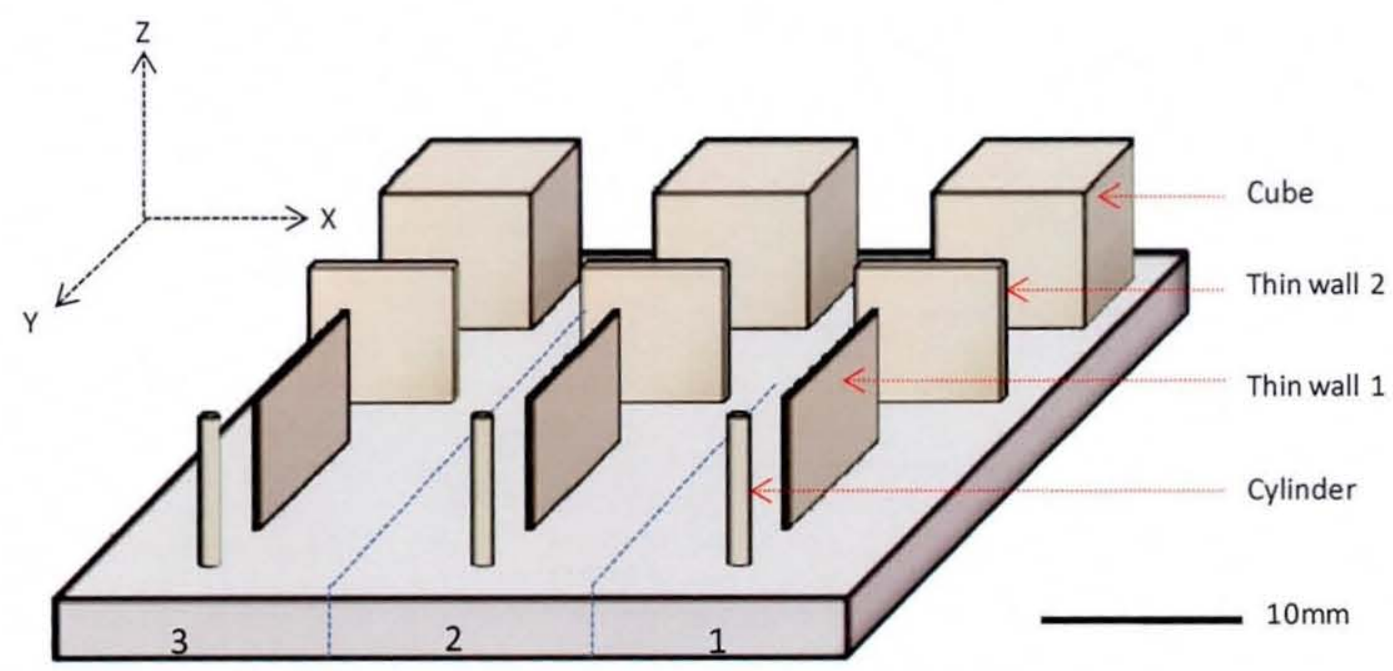

Figure 8-1 Test part design

Figure 8-2 shows the final stages of test part fabrication. Image 1 shows the top layer of the powder bed after the $80^{\text {th }}$ powder layer had been processed. Image 2-3 shows the powder bed level being raised and excess powder removal. Image 4 shows the test part on the processing table with all excess powder removed. 
The width of the thin wall parts were measured using digital callipers. Due to the restricted height and length of parts, surface $R_{a}$ measurements were made using a $5.6 \mathrm{~mm}$ traverse length and $0.8 \mathrm{~mm}$ cut-off.

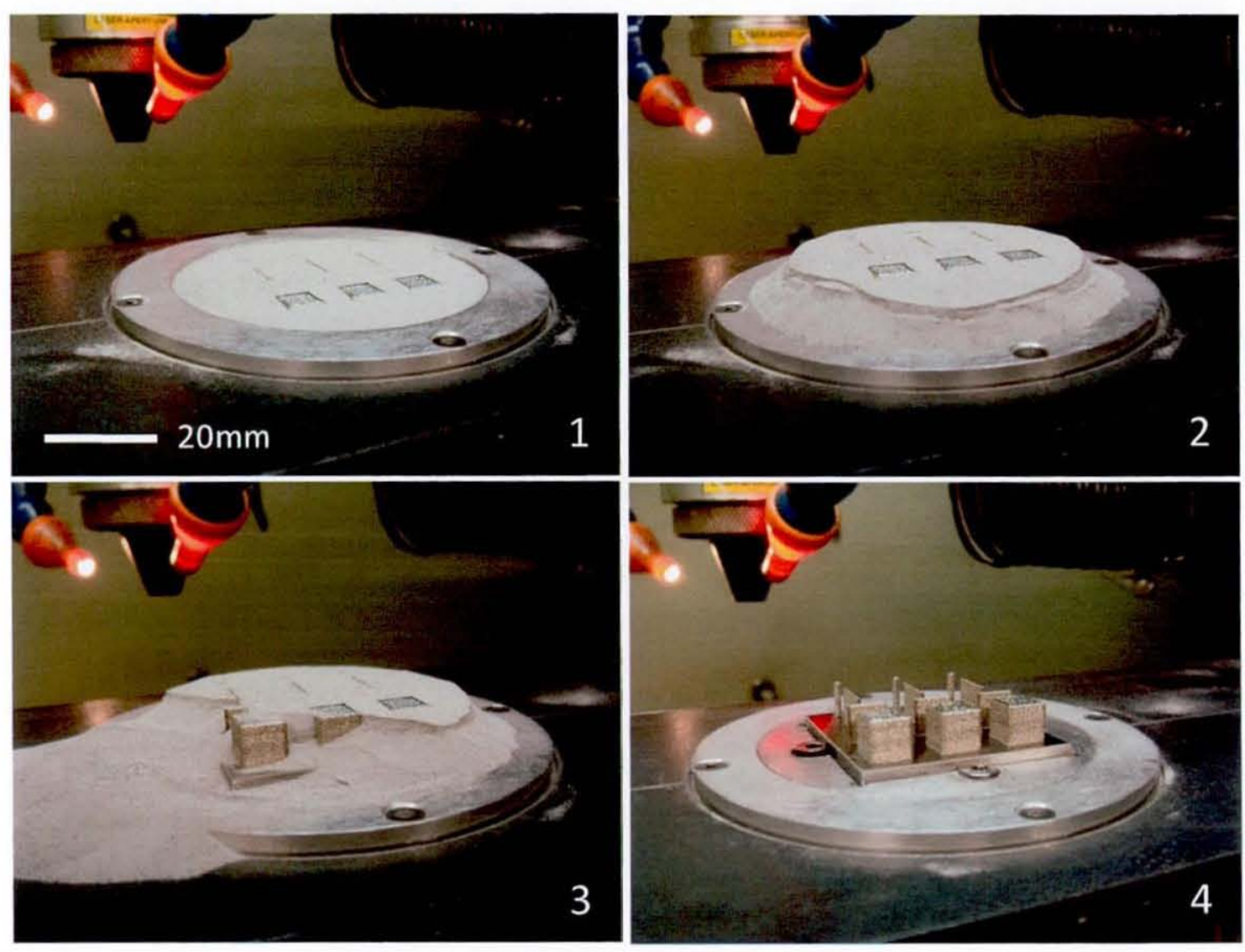

Figure 8-2 Final stages of test part fabrication 


\subsection{Test Part Results}

Four test parts were built using the laser parameters shown in Table 8-1. Each part was built using a repetition rate of $40 \mathrm{~Hz}$ and a scan speed of $400 \mathrm{~mm} / \mathrm{m}$ (optimised parameters developed in Chapter 6). Pulse duration highlighted in italic indicates the actual measured pulse duration. The four test parts are shown in Figure 8-3. Figure 8-4 shows the pulse shapes used to produce the test parts.

\begin{tabular}{llllll}
\hline $\begin{array}{l}\text { Pulse } \\
\text { Description }\end{array}$ & $\begin{array}{l}\text { Pulse Energy } \\
(\mathrm{J})\end{array}$ & $\begin{array}{l}\text { Pulse Duration } \\
(\mathrm{ms})\end{array}$ & $\begin{array}{l}\text { Peak } \\
\text { Power }(\mathrm{W})\end{array}$ & $\begin{array}{l}\text { No. of sectors } \\
\text { within pulse }\end{array}$ & $\begin{array}{l}\text { Hgt demand } \\
\text { of sectors }\end{array}$ \\
\hline Rectangular & 0.7 & $0.5(0.7)$ & 1800 & 1 & 35 \\
Ramp Up & 0.7 & $1.7(1.5)$ & 800 & 2 & $10 \& 30$ \\
Ramp Down & 1.08 & $1.5(1.6)$ & 1800 & 2 & $31 \& 20$ \\
Suppressed & 0.42 & $1(0.5)$ & 1800 & 2 & $39 \& 0$ \\
\hline
\end{tabular}

Table 8-1 Laser parameters used to produced test parts

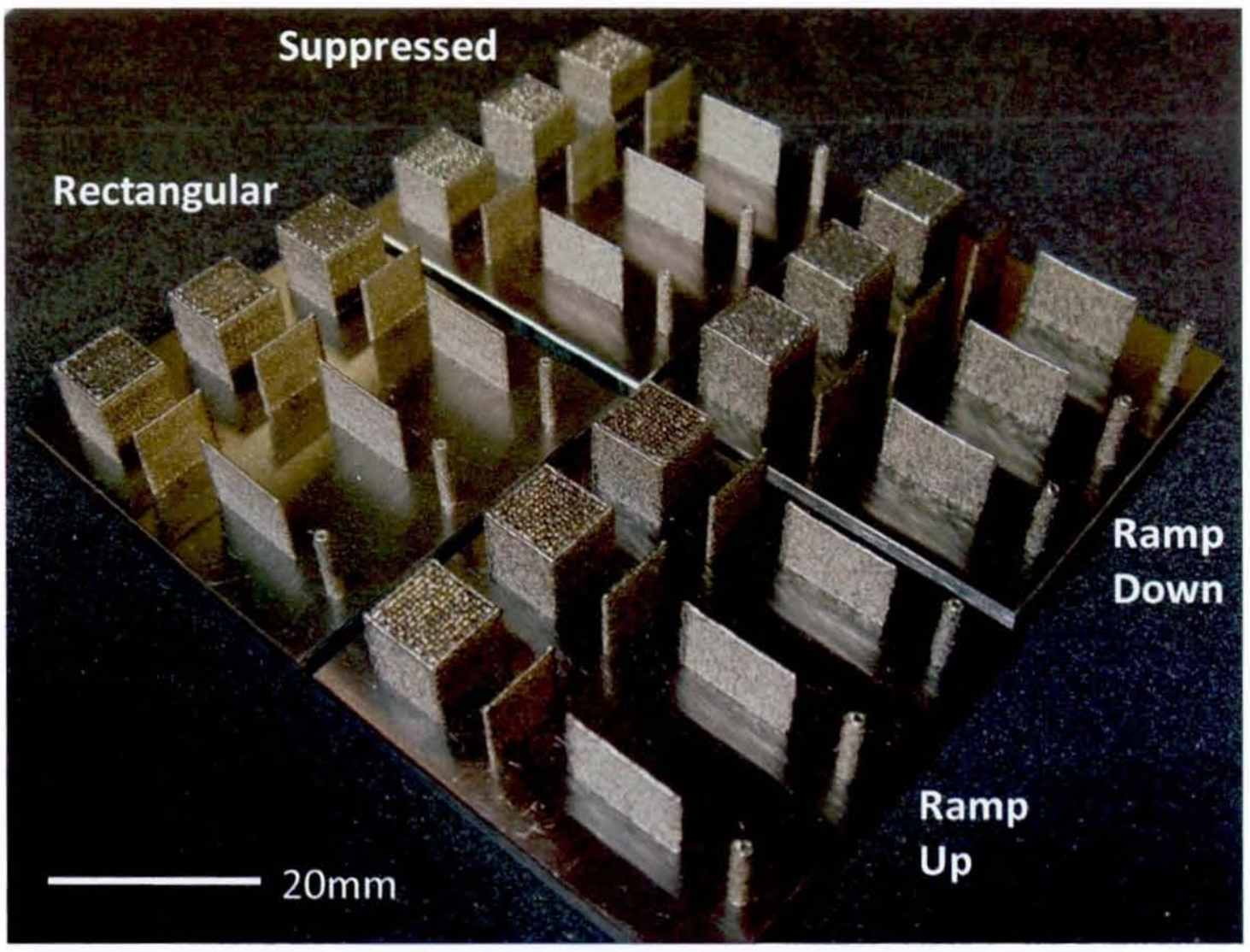

Figure 8-3 Test parts made with different pulse shapes 

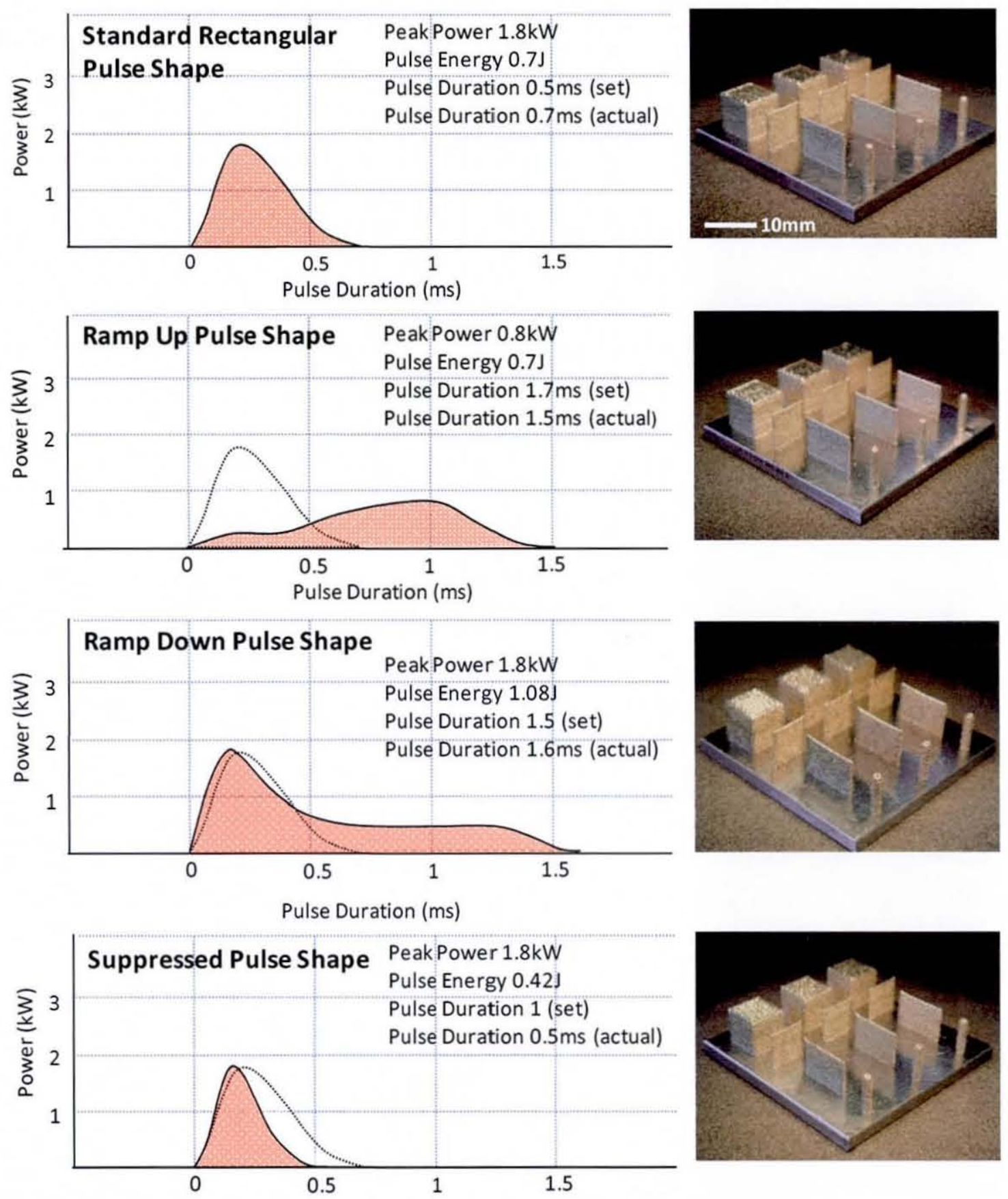

Figure 8-4 Pulse shapes used to produce test parts

\subsubsection{Thin Wall Properties}

This section will focus exclusively on the properties of the thin wall structures and examine the effects various pulse shapes had on their surface $R_{a}$, width and plasma plume generation during processing. Figure 8-5 shows the results of surface $R_{a}$ and width of the thin wall structures made using the different pulse shapes. Figure 8-6 shows the side view of some of the thin wall parts produced. 


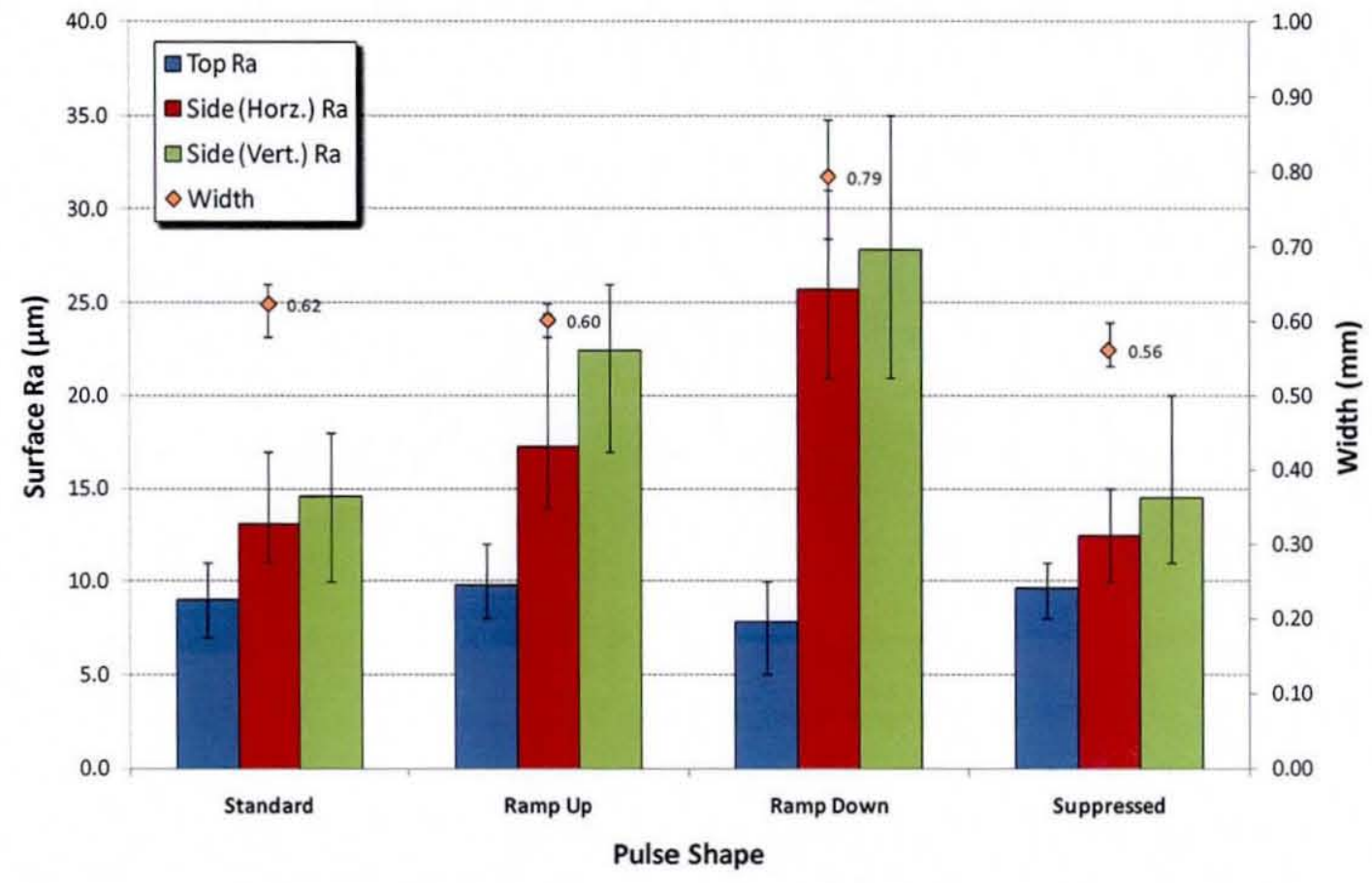

Figure 8-5 Thin wall part surface roughness and width
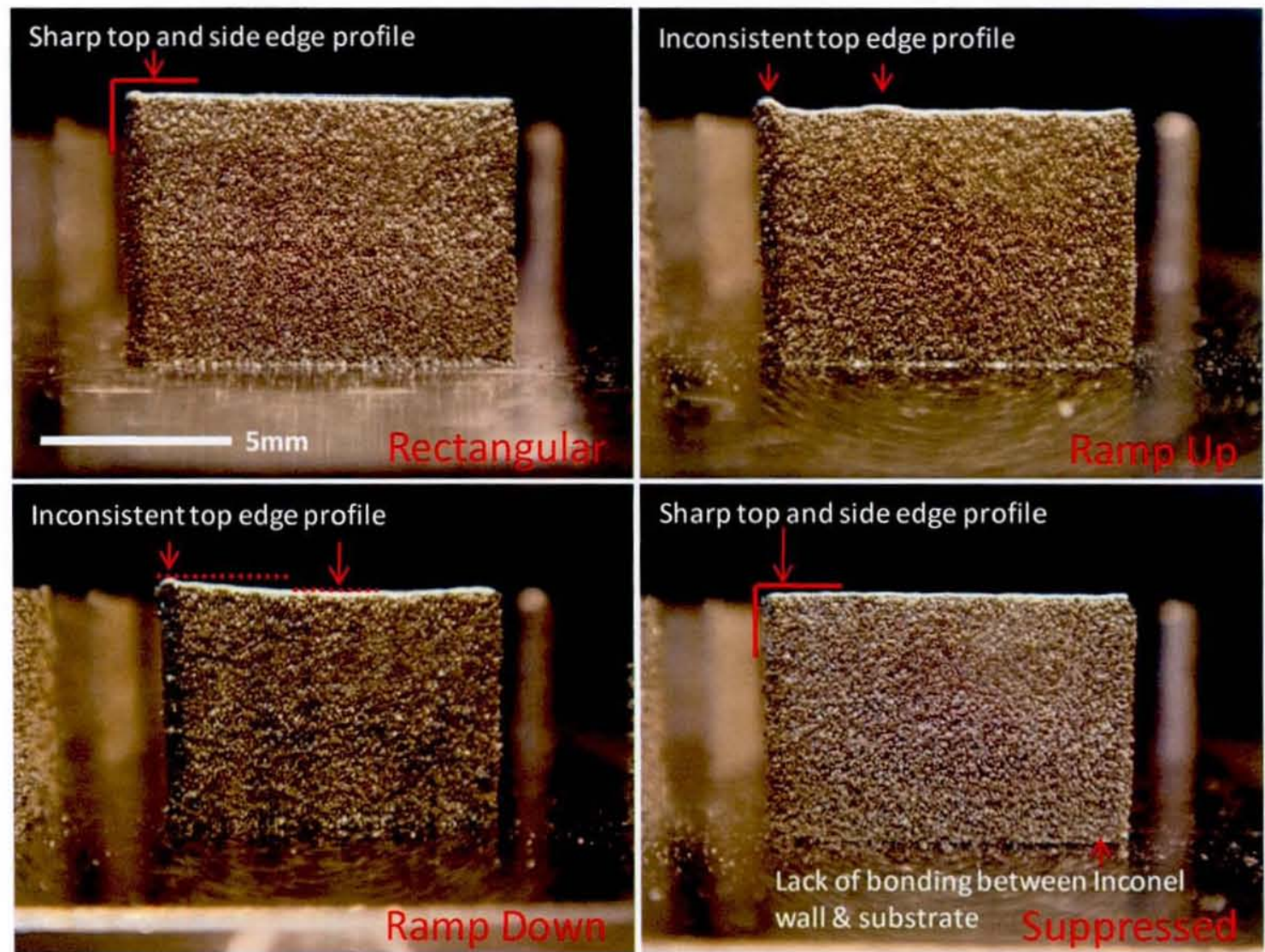

Figure 8-6 Side view of thin wall parts 


\subsubsection{Rectangular pulse}

The Rectangular pulse as expected produced thin walls $(0.62 \pm 0.04 \mathrm{~mm}$ in width $)$ with a top $R_{a}$ values $(9 \pm 2 \mu \mathrm{m})$ lower than that of side $R_{a}(13 \pm 4 \mu \mathrm{m}$ horizontal \& $15 \pm 5 \mu \mathrm{m}$ vertical). It was found that the side $\mathrm{R}_{\mathrm{a}}$ in the vertical direction was higher than that of the side $\mathrm{R}_{\mathrm{a}}$ in the horizontal axis. This could be a result of interlayer connections between melted layers of powder increasing $R_{a}$ in this direction. Measuring side $R_{a}$ along the horizontal axis will only take into consideration one layer. Measuring in the vertical axis measures across multiple layers, a melted interlayer connection has the potential to increase side $\mathrm{R}_{\mathrm{a}}$. The thickness of the thin walled structure measured $0.62 \pm 0.04 \mathrm{~mm}$. Using the same laser parameters a thin walled part produced from four layers measured lower at $0.49 \mathrm{~mm}$ (section 6.3). This variation in measured wall thickness could be related to the multiple reheating cycles and heat build-up when producing tall parts. Four $0.1 \mathrm{~mm}$ layers will produce a thin wall not more than $0.4 \mathrm{~mm}$ in height, there would not be a lot of heat build up as the substrate would very quickly conduct heat away from the thin wall structure [Morgan et al. 2001]. The processing of further layers ( 80 layers in this case) will cause the average width of the wall to increase due to multiple re-heating cycles and build up of heat within the bulk of the processed Inconel structure. More heat build up will cause the melt pool to expand as more heat is available for powder particles to melt. This expansion of the melt pool with taller specimens may also be a reason why the side $\mathrm{R}_{\mathrm{a}}$ in the horizontal direction for the test parts are higher than those produced from four 0.1 mm layers. Figure 8-6 shows the side view of the thin wall structure produced using a Rectangular pulse. The top and edge profile for the structure is flat and consistent. This indicates that the heat input delivered to the material was sufficient in creating flat consistent melt pool that did not suffer from part distortion as a result of excessive heat input. The height of plasma plume generated during processing using the Rectangular pulse was approximately $5 \mathrm{~mm}$, the same result measured in early experiments employing the same pulse shape.

\subsubsection{Ramp Up pulse}

Chapter 7 had identified that the a Ramp Up pulse had been particularly useful in minimising spatter generation during processing. This pulse used the same pulse energy as that of the Rectangular pulse but instead spread the energy over a period of $1.5 \mathrm{~ms}$ (as opposed to $0.7 \mathrm{~ms}$ ) and operated at a lower peak power of just $0.4 \mathrm{~kW}$ (as shown in 
Figure 8-4). Compared to the other three pulse shapes, the Ramp Up pulse was the most successful in its reduction of spatter generation producing only $1 \mathrm{~mm}$ of plasma plume. This observation of lower spatter ejection during processing is consistent with other researches employed Ramp Up pulse shapes to laser weld metal sheets [Katayama et al. 1993; Fujinaga et al. 2000; Gower et al. 2005; Pan et al. 2005]. As expected the side $\mathrm{R}_{\mathrm{a}}$ $(17 \pm 8 \mu \mathrm{m}$ horizontal \& $22 \pm 5 \mu \mathrm{m}$ vertical) of parts processed using this pulse degraded as a result of reducing peak power and increasing pulse duration (as shown in Figure 8-5). Late melt pool generation and rapid melt pool solidification occurred as a result of the Ramp Up energy distribution, this produced a lower volume of liquid present within the melt pool and subsequently a smaller part width $(0.6 \pm 0.02 \mathrm{~mm})$. Figure 8-6 shows the side view of the thin wall structure produced using the Ramp Up pulse. The top edge profile of the structure it not completely flat and consistent. It is possible that the use of low peak powers caused bulges on the top surface to appear. Low peak powers exert lower recoil pressures on a melt pool. The recoil pressure contributes to reshaping of the melt pool from a spherical structure to a flatter wider profile. Generally the shape of the melt pool is dominated by surface tension forces which are affected by melt pool temperature variations. If inconsistencies exist within the melt pool and powder bed, recoil pressures can then assist the melt pool in acquiring a more uniform consistent shape (due to a mechanical force compacting the surface of the melting pool). If lower recoil pressures are present then the inconsistencies that form during melt pool solidification will be more heavily influenced by thermocapillary flows and surface tension caused by thermal temperature variations. The low heat input and low HAZ produced as a result of the Ramp Up energy distribution may alleviate processing issues associated with shrinkage, residual stress build up and cracking.

\subsubsection{Ramp Down pulse}

The Ramp Down pulse was identified in Chapter 7 as the pulse shape that would produce parts with the lowest top $\mathrm{R}_{\mathrm{a}}$. The pulse had the same peak power as the Rectangular pulse but contained a higher pulse energy of $1.08 \mathrm{~J}$ spread over a longer duration. Figure 8-5 shows the results of this pulse. The top $\mathrm{R}_{\mathrm{a}}$ and side $\mathrm{R}_{\mathrm{a}}$ (horizontal $\&$ vertical) were measured to be $8 \pm 3 \mu \mathrm{m}, 26 \pm 5 \mu \mathrm{m}$ and $28 \pm 7 \mu \mathrm{m}$ respectively. The top $\mathrm{R}_{\mathrm{a}}$ of the thin walls was lower than any of the other parts produced with other pulse shapes. The high temperature generated using this pulse increased the melt pools viscosity, this combined with the increased melt pool solidification time assisted the movement and 
spreading of molten material reducing variations in overlapping melt pools. As expected the side $R_{a}$ values were the highest out of all the pulse shapes. This was because the pulse contained the largest pulse energy of $1.08 \mathrm{~J}$. This combined with longer pulse durations (1.6ms actual) causes a larger volume of liquid to be produced and a subsequent widening of the melt pool. The majority of the heat from within the melt pool is drawn away from the edge of the melt pool into surrounding material and environment, causing large thermal gradients between the centre and extremities of the melt pool. This generates large surface tension variations which may subsequently induce lateral balling. The melt pool may have had too low a viscosity, this has been shown to promote conventional balling formation [Agarwala et al. 1995]. Larger melt pools have more heat to conduct in the surrounding powder bed than smaller melt pools, this encourages satellite formation and increases side $R_{a}$. The wall thickness was $0.79 \mathrm{~mm} \pm 0.08$, this was the largest wall thickness out of all the samples and as expected would therefore possess the largest side $\mathrm{R}_{\mathrm{a}}$. Figure 8-6 shows the side view of the thin wall part produced using the Ramp Down pulse. The top edge profile is not flat and dips towards the centre. This is possibly a result of too large a heat input causing large amounts of powder to enter the melt pool. As more powder enters the melt pool there is less material available for the laser to process in the direction of the scan causing a reduction in melt pool widths and height. During the first series of pulses, molten pools are created, which attract powder particles lying next to it under capillary action [Song 1997]. This leaves areas deficient in powder at the position of the second set of pulses and after solidification a depression is formed there. Due to the Ramp Down pulses higher energy and stronger heating effect the spatter generation during processing was higher as a result of $6 \mathrm{~mm}$ plasma plumes. The larger spatter generation with the use of Ramp Down pulses has also been observed during the laser welding of metal sheets [Tzeng 2000; Gower et al. 2005].

\subsubsection{Suppressed Pulse}

The Suppressed pulse identified in Chapter 7 was able to minimise the actual pulse duration of the laser down to $0.5 \mathrm{~ms}$. The pulse was also designed to contain a high peak power, low energy and short duration. This ideal combination had previously been identified in section 6.3 as the best combination for producing parts with top/side low $R_{a}$ and minimal width. However due to limited control using non pulse shape methods this 
energy distribution was originally not possible. The results shown in Figure 8-5 for the Suppressed pulse show that thin wall structures had low top and side $R_{a}(10 \pm 2 \mu \mathrm{m}$ top, $12 \pm 3 \mu \mathrm{m}$ horizontal \& $15 \pm 6 \mu \mathrm{m}$ vertical) values that were very similar to those produced with the Rectangular pulse. As expected the melt pool width remained low producing an average wall width of $0.56 \pm 0.04 \mathrm{~mm}$. This was $0.06 \mathrm{~mm}$ smaller then the thin wall part produced using the Rectangular pulse. This would allow parts to be built to a higher resolution. In addition the part was processed using a lower pulse energy of $0.42 \mathrm{~J}$, this could reduce part distortion due to a reduced heat build-up. Due to the melt pool width remaining relatively small, the melt pool would solidify at a faster rate than that of a larger melt pool and restrict partially melted powder particles from agglomerating to the side of the solidifying melt pool. This reduced solidification time also restricts the time available for melt pool instabilities to develop. The low energy within the pulse reduced the plasma plume height to $3 \mathrm{~mm}$ such that spatter generation during processing was minimal. It should be noted that a reduction in plasma plume height may indicate a reduction in the magnitude of recoil pressure exerted on the melt pool. Figure 8-6 shows the side view of the thin wall part created using the Suppressed pulse. This structure has sharp edge profiles and a level top surface, similar to that produced using the Rectangular pulse. This indicates that a correct level of material heating occurred such that Inconel would successfully melt without causing too much part distortion or slumping of parts due to insufficient powder. However the bottom right corner of the thin wall part produced with the Suppressed pulse in Figure 8-6 did not fully bond/attach to the steel substrate. Warping caused the Inconel 625 wall moved/curled away from the substrate. The low energy and short pulse duration may not have provided a sufficient enough bond between the Inconel and steel substrate. However this lack of bonding was only observed in one of the six thin wall parts with the other five appearing to have fully bonded. This sharp edge profile is possibly due to the thin melt pools produced and relatively quick melting and cooling of the melt pools. Commercial systems such as MTT SLM process emit their laser output in short burst in order to minimise the HAZ and reduce part distortion. However this method is not able to fully simulate that of a Suppressed pulse due to the continuous wave output not generating as high peak powers as those achieved with a pulsed wave laser. 


\subsubsection{Other Geometries}

\subsubsection{Thin wall cylinder}

In order to further test the validity of results and examine whether alternative vertical geometries affect side surface $R_{a}$ values, thin wall cylinders (diameter $1 \mathrm{~mm}$ ) were measured for Side $R_{a}$ in the vertical direction (shown in Figure 8-7). Results indicate that side $\mathrm{R}_{\mathrm{a}}$ vertical trends match up closely with those previously attained producing straight thin wall parts. The largest variation in results occur with the use of Ramp Down pulses, however this larger variation may simple be a result of scaled proportions as $R_{a}$ values produced using the Ramp Down pulse were higher compared to the those produced using other pulse shapes.

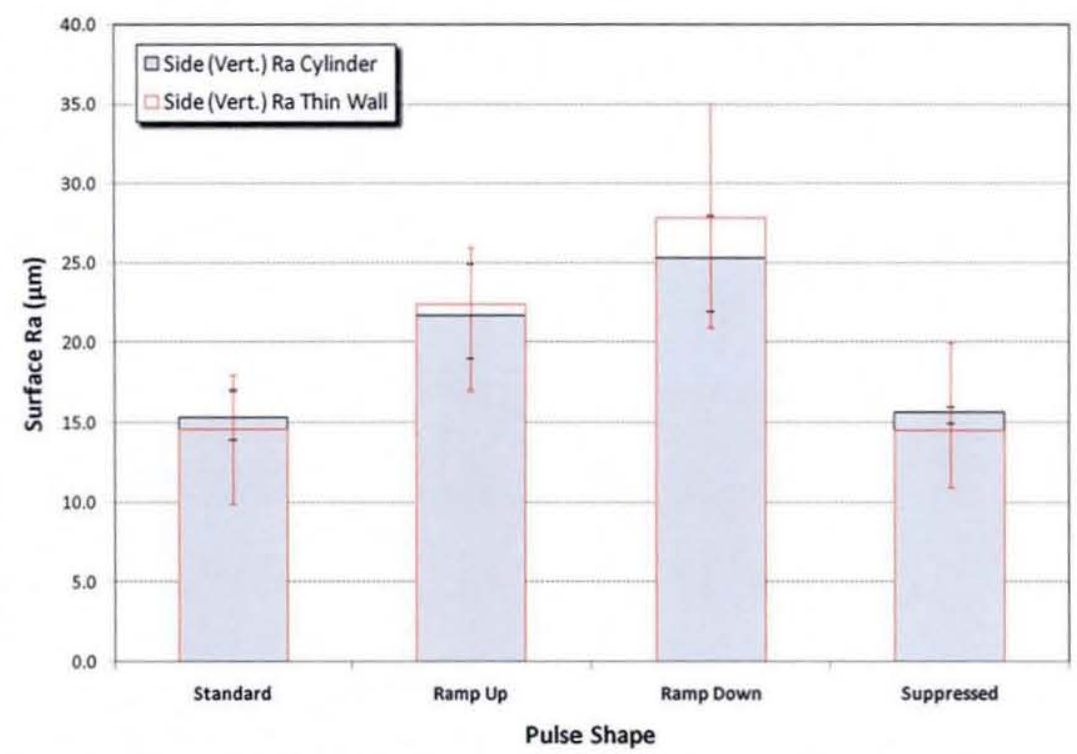

Figure 8-7 Side $\mathrm{R}_{\mathrm{a}}$ vertical for thin wall cylinder (including thin wall part results for comparison)

\subsubsection{Block parts}

The side $\mathrm{R}_{\mathrm{a}}$ in the horizontal and vertical directions were measured for all four sides of the block parts, the results are shown in Figure 8-8. Again results indicate the side $R_{a}$ in both horizontal and vertical values match up closely with those previously attained producing straight thin wall parts. The largest variation in results again occur with the use of Ramp Down pulses. However this larger variation may simply be a result of scaled proportions as $R_{a}$ values produced using the Ramp Down pulse were higher compared to the those produced using other pulse shapes. Because the block parts were 
built from multiple thin walls overlapping each other, there was a potential for a variation in side $R_{a}$ results due to a variation in heat distribution/build up. However results indicate that the production of thicker structures had little impact on the value of side $R_{a}$ for vertical structures.

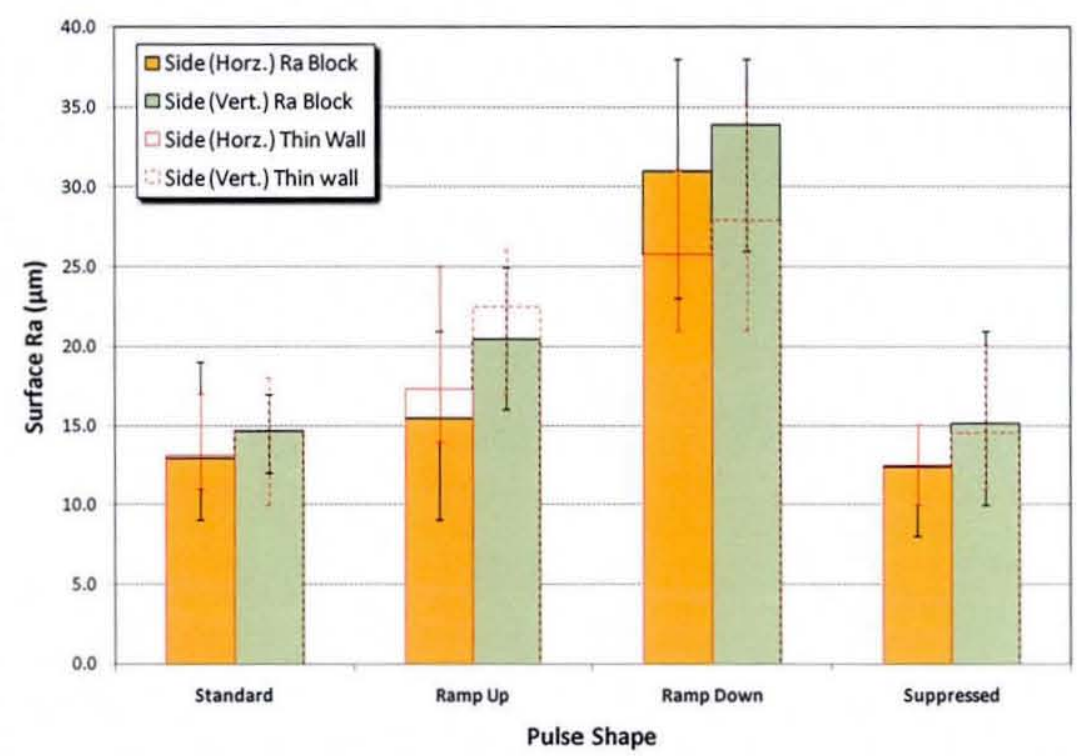

Figure 8-8 Side $\mathrm{R}_{\mathrm{a}}$ horizontal \& vertical average for all four sides of block parts (including thin wall part results for comparison)

\subsubsection{Summary}

The use of different pulse shapes has revealed a number of differences in part properties. A Rectangular pulse developed within section 6.3 represented the best non pulse shaped laser parameter that produced thin wall parts with low top/side surface $\mathrm{R}_{\mathrm{a}}$. This formed a benchmark to which all other parts were compared. Within the testing of different Ramp Up/Down and Suppressed pulse shapes identified in Chapter 7, only three were identified as pulse shapes that offered advantages over the non shaped Rectangular pulse.

The first pulse shape was a Ramp Up pulse that gradually melted powder over a period of $1.5 \mathrm{~ms}$ and only required a peak power of $0.4 \mathrm{~kW}$ to successfully melt the Inconel powder. The only benefit of this Ramp Up power distribution was the lower spatter generation during processing due to the more gradual heating and use of lower peak power. As expected a consequence of this modified energy distribution would be higher side $R_{a}$ due to lower peak powers. 
The second pulse used was a Ramp Down pulse shape that allowed the melt pool more time to cool after melt pool generation. This pulse was able to reduce the top $R_{a}$ of the test part due to extra energy flattening out the melt pool over a larger area. Due to the prolonged cooling of the melt pool more time was giving for gravity to redistribute liquid within the melt pool and reducing the variation between overlapping melt pool subsequently reducing top $\mathrm{R}_{\mathrm{a}}$. However due to larger melt pool and extended time for which the melt pool remained molten the part suffered from high side $\mathrm{R}_{\mathrm{a}}$ due to satellite formation and possible lateral balling.

A Suppressed pulse unexpectedly developed during Ramp Down pulse generation revealed the best potential from shaping. The pulse's energy distribution resembled the characteristics of an ideal pulse identified in section 6.3 when producing thin wall parts with low surface $\mathrm{R}_{\mathrm{a}}$ and low melt pool width. These ideal pulse qualities included a high peak power, low energy and low pulse duration. With the use of standard non shaped Rectangular pulses the amount of control the operator had on these pulse qualities was restricted. The Rectangular pulse duration had been set to a minimum of $0.5 \mathrm{~ms}$ but in reality produced a pulse measuring $0.7 \mathrm{~ms}$ in duration due to a natural overshoot present within pulsed lasers. To add to this a high peak power often resulted in more pulse energy spread throughout the duration of the pulse, a factor that can prove detrimental to the side $\mathrm{R}_{\mathrm{a}}$. The use of pulse shaping allowed the user to extend the control over the pulse's energy distribution. It was tailored such that pulse duration was minimised, peak power was high while energy remained low. The Ramp Down pulse (shown in Figure 8-4) as expected produced parts with a surface $R_{a}$ comparable to those of a Rectangular pulse but surpassed the non shaped pulse by reducing part width. This reduction in melt pool width will allow part to be produced to a higher resolution. Due to the lower energy density and subsequently lower heat input parts would be less prone to distortion. As expected the lower energy within the pulse caused less spatter to be produced reducing powder bed contamination and other problems associated with this.

Table 8-2 summarises the properties of test parts for all geometries and Table 8-3 reconfirms the effectiveness of each pulse shape (black ticks indicate property pulse shape was effective in improving with red ticks indicating the property the pulse shape was highly effective in improving) 


\begin{tabular}{l|c|cc|ccc|c}
\cline { 2 - 8 } & Top $\mathrm{R}_{\mathrm{a}}(\mu \mathrm{m})$ & \multicolumn{2}{|c|}{ Side $\mathrm{R}_{\mathrm{a}}$ Horizontal $(\mu \mathrm{m})$} & \multicolumn{2}{|c|}{ Side $\mathrm{R}_{\mathrm{a}}$ Vertical $(\mu \mathrm{m})$} & Width $(\mathrm{mm})$ \\
\cline { 2 - 8 } & Wall & Wall & Block & Wall & Cylinder & Block & Wall \\
\hline Rectangular & 9 & 13 & 13 & 15 & 15 & 15 & 0.62 \\
Ramp Up & 10 & 17 & 15 & 22 & 22 & 21 & 0.6 \\
Ramp Down & 8 & 26 & 31 & 28 & 25 & 34 & 0.79 \\
Suppressed & 10 & 12 & 12 & 15 & 16 & 15 & 0.56 \\
\hline
\end{tabular}

Table 8-2 Test part properties

\begin{tabular}{c|c|c|c|c}
\hline & Rectangular & Ramp Up & Ramp Down & Suppressed \\
\hline Top $\mathrm{R}_{\mathrm{a}}(\mu \mathrm{m})$ & $\checkmark$ & $\checkmark$ & $\checkmark$ & $\checkmark$ \\
Side $\mathrm{R}_{\mathrm{a}}(\mu \mathrm{m})$ & $\checkmark$ & & & $\checkmark$ \\
Width $(\mathrm{mm})$ & $\checkmark$ & $\checkmark$ & & $\checkmark$ \\
Spatter & & $\checkmark$ & & $\checkmark$ \\
\hline
\end{tabular}

Table 8-3 Measured effectiveness of various pulse shapes when employed within SLM
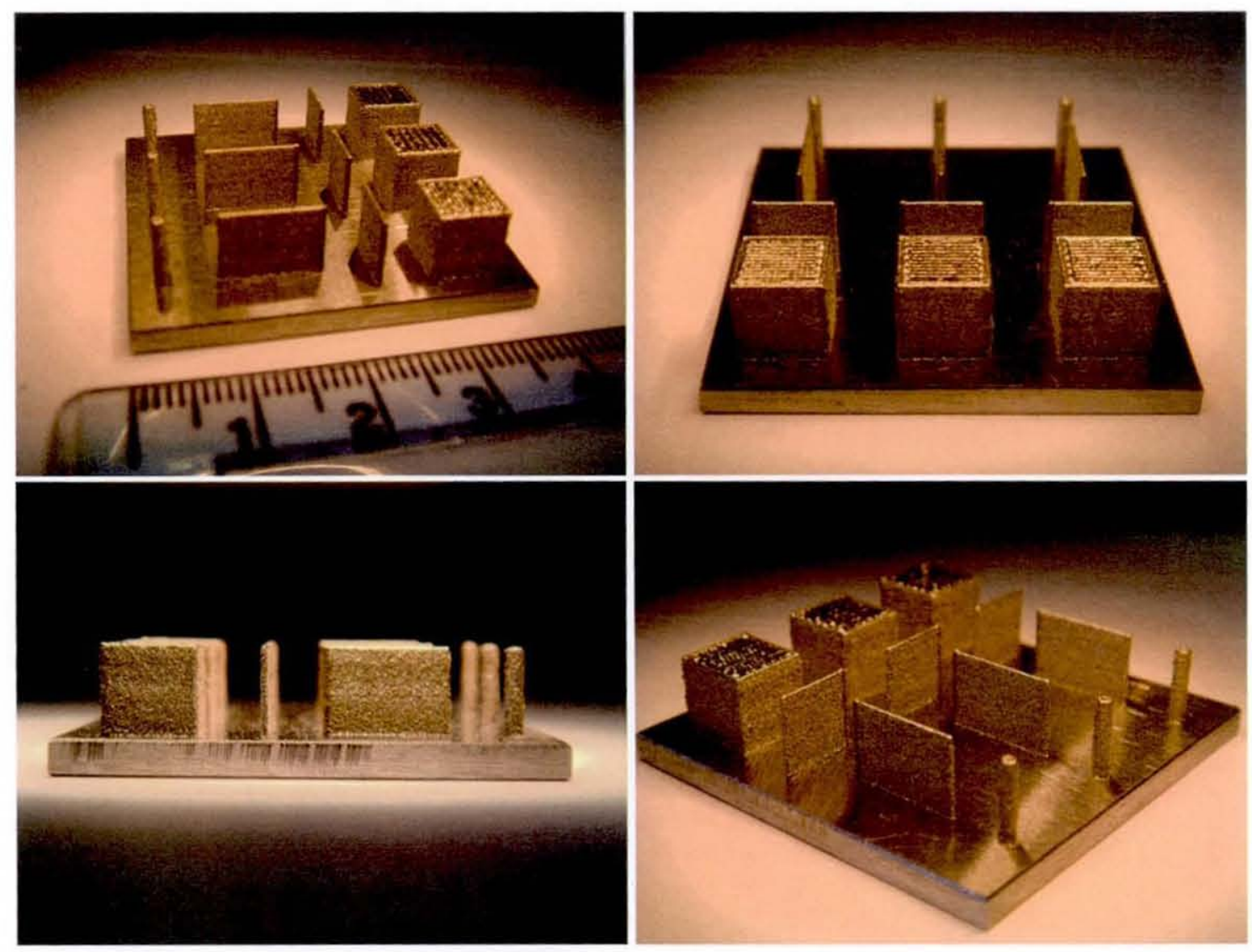

Figure 8-9 Test part produced using the Suppressed pulse shape. 


\section{Chapter 9 Conclusions}

\subsection{Summary of Conclusions}

Pulsed lasers are extensively used throughout industry and academic institutions for use within metal processing. The use of pulsed wave lasers over that of continuous wave lasers within processes such as SLM has become even more inviting as its many advantages are realised. This research will benefit users of laser metal processing technologies that wish to further understand the extended degree of process control capable using pulse shaping. This work is a comprehensive study examining the effects laser pulse shaping has on the formation of metal parts processed from a powder bed. It details information on how pulse shaping can be practically implemented including limitations that must be considered. New knowledge is presented relating to the control of part properties (top and side roughness and wall thickness) through pulse shape control.

Early experimentation found that simultaneous low top/side part surface roughness was difficult to achieve due to fluid dynamics and melt pool instabilities. Some key characteristics required by a laser to produce thin wall samples with low top/side $R_{a}$ were identified (high peak power and low energy/duration). However due to the limited degree of laser output control a compromise between optimal part properties had to be made. Pulse shaping control was employed to investigate whether the additional degree of control over laser parameters allowed the pulsed energy distribution to be refined and tailored such that part properties could be varied and improved without the need for such compromise.

Experiments were conducted using a variety of Ramp Up and Ramp Down pulse shapes and investigated the effects these had on part properties. Measurement of power delivered by the laser using different pulse shapes showed significant discrepancies between input profile and actual energy output delivered. Consequently, the power 
delivered by the laser using different input pulse profiles was monitored closely during the development of new pulse shapes. Ramp Up pulses gradually increased the power distribution during its duration and facilitated a gradual heating of material. This energy distribution was shown to be beneficial in reducing spatter generation during processing. This was because Ramp Up pulses required overall less peak power due to a progressive material preheating. This preheating also caused the material's energy absorbance to increase prior to main pulse peak power at the end of the pulse. The late melt pool generation and rapid solidification of the melt pool did not result in melt pool expansion allowing parts to be produced to a higher resolution (parts were thinner than those produced using a Rectangular pulse). The low heat input and reduced HAZ generated by this Ramp Up pulse may have alleviated part build issues associated with shrinkage, residual stress build up and cracking.

Ramp Down pulses gradual taper off their energy distribution allowing more time for a melt pool to cool after melt pool generation. The extended time for which the melt pool remained molten enabled more time for molten material to redistribute under the force of gravity and reduced the variation in top surface profile (reducing top $R_{a}$ ). The additional energy supplied after melt pool generation may have increased material vaporisation, causing larger recoil pressures to flatten the top surface of the melt, contributing to a lower top $\mathrm{R}_{\mathrm{a}}$. Due to the additional energy supplied to the melt pool its temperature would have risen, this reduces the melt pool viscosity allowing a more free movement of molten material under the forces of Marangoni convection and gravity. This movement assisted the reduction in top $\mathrm{Ra}$ as the variation in peak-trough height between melt pools reduced. However the additional energy and extended time for which the melt pool remained molten increased melt pool width. Both these would have actively encouraged powder particles to partially melt and attach to the edge of the solidifying melt pool (satellite formation). Larger melt pools also have a larger variation in temperature across the melt pool (hotter at the centre and cooler at the edges) which subsequently increases surface tension variations. These variations in surface tension and possible too low melt pool viscosity promoted melt pool instabilities and increased side surface roughness due to a possible lateral balling.

Ramp Up and Ramp Down energy distributions were useful in improving certain individual part properties and aspects of SLM processing (low top $R_{a}$, low width, low 
spatter generation during processing) however these pulse shapes were overall not useful in achieving the main aim of this research, which was to produce thin wall parts with combined low top and side surface roughness. This aim however was achieved using a Suppressed pulse shape that consisted of a high peak power, low energy and short time duration. This enabled an aggressive rapid melting and rapid cooling of material including the generation of high recoil pressures exerting an external force on the melt pool (it should be noted that high recoil pressure may also increase side $R_{a}$ by promoting melt pool expulsion to either side of the melt pool). Often the development of melt pool instabilities can be inhibited through increased melt wetting to a substrate. Conventionally, prolonged melt pool solidification and high energies are required for a melt pool to spread under the force of gravity and improve this wetting characteristic. However this extended duration also gives more time for melt pool instabilities to develop and encourage satellite formation. A possible remedy for this simultaneous favourable/unfavourable scenario was through the generation of a high recoil pressure (generated with the Suppressed pulse). This would improve the wetting of the melt pool onto the substrate within a short space of time due to the external force it exerts on the melt pool. As a result of the short laser-on time and low pulse energy the width of the melt pool was reduced due to less energy and less time for heat to diffuse sideways causing a narrowing of the melt region This caused any thermallsurface tension variation across the melt pool to be reduced, this contributed to a reduction in the development of melt pool instabilities and reduced part side $\mathrm{R}_{\mathrm{q}}$. These combined factors and added degree of control available through pulse shaping improved part properties producing superior high resolution parts with combined low top and side surface roughness.

\subsection{Specific Conclusions}

- High laser spot overlaps reduce the variations in a melt pools top surface profile improving top $R_{\mathrm{a}}$.

- High laser spot overlaps produce high energy densities that can increase the volume of liquid present within a melt pool subsequently increasing its width. 
- Large melt pools that are held molten for a longer period of time will conduct more heat into the surrounded powder bed than that of a smaller melt pool and subsequently increase satellite formation around the solidified melt pool

- A wider melt pool has larger thermal variation across the melt pool, these induce surface tension variations that may increase the melt pools tendency to undergo lateral balling and increase side $\mathrm{R}_{\mathrm{a}}$.

- Low laser scan speeds allow more time for gravity and recoil pressure to flatten a melt pool reducing the variations in top surface profile (low top $R_{a}$ ).

- High laser scan speeds reduce the melt pool width and solidification time, limiting time available for satellite/balling formation to take place (reducing side $\mathrm{R}_{\mathrm{a}}$ ).

- Attaining a simultaneously low top/side $\mathrm{R}_{\mathrm{a}}$ with minimal wall width is difficult to further optimise using non pulse shaping control.

- High recoil pressures were beneficial in reducing top/side $R_{a}$, however high recoil pressures also contribute to increases in spatter generation during processing

- Pulsed lasers not only experience power overshoots but also pulse duration overshoots.

- Ramp Up pulses generally melted the material at lower peak powers as compared to Rectangular or Ramp Down pulses. This is because a gradual heating of material induced by the Ramp Up pulse causes powder particles to heat up prior to the main pulse peak power. Also as the powder heats up its reflectivity drops requiring less energy to completely melt.

- Due to the use of low peak powers, Ramp Up pulses generated less plasma plume/spatter during processing.

- Even though Ramp Up pulses heat a material within a matter of milliseconds it can be viewed as similar to powder bed preheating.

- Ramp Up pulses may reduce part distortion, build up of residual stress and susceptibility to crack due to a lower heat input. 
- Ramp Down pulses increased the energy delivered to the material, it extended the time for which the melt pool remained molten allowing more time for gravity and recoil pressures to reshape the melt pool and improve top $R_{\mathrm{a}}$.

- Ramp Down pulses increased melt pool solidification times enabling more time for satellite formation and melt pool instabilities to develop.

- Ramp Down pulses contained higher energy densities at the beginning of the pulse and encouraged early melt pool generation. Subsequent energy within the pulse increased the volume of liquid within the melt pool, increasing the temperature variation across the melt pool and the melt pool's tendency to undergo possible lateral balling increasing side $R_{a}$

- Extended Ramp Down pulse durations may increase the oxidisation potential of a melt pool

- Of the pulse shapes investigated, a Suppressed pulse's high peak power, low energy/duration contained the best energy distribution to produce thin wall parts with low top/side $\mathrm{R}_{\mathrm{a}}$

- The Suppressed pulse's high peak power generates a large recoil pressure that exerts an external force on the melt pool improving the melt pool's wetting to a substrate and reduces its tendency to undergo lateral balling balling. The high recoil pressure may remove any satellite formation from attaching to the edge of the solidifying melt pool

- The Suppressed pulse's low energy reduced the volume of molten material within the melt pool and reduced its width. This limits satellite formation, thermal/surface tension variations across the melt pool and subsequent lateral balling formation. Low energy also reduced the extent of material vaporisation and spatter ejection during processing. The low energy may reduce part distortion due to low heat input

- The Suppressed pulse's short pulse duration gave less time for heat to conduct radially from the centre of the melt pool also reducing the volume/width of the melt pool. The melt pool remained molten for a short period of time restricting the development of satellite formation and melt pool instabilities such as balling.

- Controlling melt pool properties is fundamental to the production of high quality parts. 
- Pulse shaping control extended the degree of control over a melt pool and was shown to improve the overall properties of thin wall parts compared to non pulse shaped Rectangular pulses. 


\section{Chapter 10 Recommendations for further \\ study}

A number of further research areas have been identified:

- The way in which heat is delivered too and conducted within a material affects a part's microstructure. Essentially it is the thermal history of a melt pool that determines the microstructure and thus mechanical properties of resulting parts. Since the microstructure determines the material properties, it is essential to understand, and control its formation. Part microstructure is heavily influenced by processing conditions, namely cooling rates and heat flow. It can affect mechanical strength, hardness, brittleness and dictates the performance of a part. Further research could investigate effects of pulse shaping on part hardness, tensile strength and microstructure. By doing this other unknown pulse shaping advantages may be discovered. Short pulse durations may increase the hardness of parts due to rapid cooling of melt.

- Powder bed preheating using conventional techniques can lead to solid state sintering of smaller powder particles. Arcam EBM process rapidly scans the surface of a powder bed prior to melting in order to preheat the material and alleviate any issues related solid state sintering. Further studies should examine in more detail laser Ramp Up pulses and its potential use as a powder bed pre heating technique.

- Investigate pulse shaping within the use of nanosecond pulses. Use of nanosecond pulse may improve part properties as these pulses operate at durations shorter than millisecond pulses.

- The range of processing parameters used in initial optimisation experiments (Chapter 6) could be extended with particular attention paid to scan speed. An increase in scan speed would further reduce the time for which a melt pool remains molten and reduce melt pool width.

- Examine the variations in part width and side $R_{a}$ with increases in part height due to variation in heat build up.

- Examine the surface roughness of angled surfaces and overlapping flat surfaces. 
- Ascertain through high speed imagery whether large solidified spherical structures attached to edge of melt pool throughout this research were a result of lateral balling due to surface tension, high recoil pressure or simply satellite formation. 


\section{Chapter 11 References}

Abe, F., K. Osakada, M. Shiomi, K. Uematsu and M. Matsumoto (2001). The manufacturing of hard tools from metallic powders by selective laser melting. Journal of Materials Processing Technology 111(1): 210-213.

Abe, F., E. C. Santos, Y. Kitamura, K. Osakada and M. Shiomi (2003). Influence of forming conditions on the titanium model in rapid prototyping with the selective laser melting process. Proceedings of the I MECH E Part C Journal of Mechanical Engineering Science 217: 119-126.

Agarwala, M., D. Bourell, J. Beaman, H. Marcus and J. Barlow (1995). Direct selective laser sintering of metals. Rapid Prototyping Journal 1(1): 26-36.

Aggarangsi, P. and J. L. Beuth (2006). Localized preheating approaches for reducing residual stress in additive manufacturing. Solid Freeform Fabrication Symposium, Austin, TX.709-720

Allen, J. (2007). Powder stream and powder bed systems. AILU Technology Workshop, Rotherham, UK

Almen, M. V. (1987). Laser - beam interactions with materials. New York, Springer Verslag.3540594019

Anthony, T. R. and H. E. Cline (1977). Surface rippling induced by surface tension gradient during laser surface melting alloying. Journal of Applied Physics 46(9): 3888-3894.

Arafin, M. A., M. Medraj, D. P. Turner and P. Bocher (2007). Transient liquid phase bonding of Inconel 718 and Inconel 625 with BNi-2: Modeling and experimental investigations. Materials Science and Engineering A 447(1-2): 125-133.

Ashley, S. (1995). Rapid prototyping is coming of age. Mechanical Engineering 117(7): 62.

Badawi, K., M. Paindavoine and G. Jacrot (1989). Effects of spatial and temporal pulse energy distribution of a laser beam on welding Revue de Métallurgie 86: 162170.

Bagger, C., K. Neilsen and F. Olsen (1993). Hot Cracking of AISI 316 in Pulsed C02 and Pulsed Nd:YAG Laser Welding. ICALEO.693-703

Beer, R. T. and E. M. Ring (1972). Liquid metals: chemistry and physics. New York, Marcel Dekker.0824710320

Blackwell, P. L. (2005). The mechanical and microstructural characteristics of laserdeposited IN718. Journal of Materials Processing Tech. 170(1): 240-246.

Boivie, K. (2001). Limits of Loose Metal Powder Density in the Sinterstation. Solid Freeform Fabrication Symposium, Austin, TX.264-275

Bourell, D. L., M. Wohlert, N. Harlan, S. Das and J. J. Beaman (2002). Powder densification maps in selective laser sintering. Engineering Materials 9(4): 663669.

Bransch, H. N., D. C. Weckman and H. W. Kerr (1994). Effects of Pulse Shaping on Nd:YAG Spot Welds in Austenitic Stainless Steel. Welding Research Supplement(June): 141-151.

Carroll, P. (2007). Information on laser deposition at TWI. AILU Technology Workshop, Rotherham, UK 
Castillo, L. (2005). Study about the rapid manufacturing of complex parts of stainless steel and titanium. TNO Report, Instituto Technologico Metalmecanico.

Cheng, J., F. Kahlen and A. Kar (2000). Effects of Intra-Pulse Structure on Hole Geometry In Laser Drilling. ICALEO.58-67

Chichkov, B. N., C. Momma and S. Nolte (1996). Femtosecond, picosecond and nanosecond laser ablation of solids. Appl Phys A(63): 109-115.

Childs, T. H. C. (2005). Raster scan selective laser melting of the surface layer of a tool steel powder bed. Proc. IMechE 219(B): 379-384.

Childs, T. H. C., C. Hauser and M. Badrossamay (2005). Selective laser sintering (melting) of stainless and tool steel powders: experiments and modelling. Proceedings of the I MECH E Part B Journal of Engineering Manufacture 219: 339.

Choi, J. and Y. Chang (2005). Characteristics of laser aided direct metal/material deposition process for tool steel. International Journal of Machine Tools and Manufacture 45(4-5): 597-607.

Choi, J. and Y. Chang (2005). Characteristics of laser aided direct metal/material deposition process for tool steel. International Journal of Machine Tools and Manufacture 45(4-5): 597.

Cieslak, M. J. (2002). Fundamentals of welding - Cracking phenomena associated with welding, ASM Handbook 6: Welding, brazing and soldering

Cormier, D., West H., Harrysson O. and Knowlson K. (2004). Characterization of Thin Walled Ti-6Al-4V Components Reduced via Electron Beam Welding. Solid Freeform Fabrication Symposium, Austin, TX

Crane, N. B., J. Wilkes, E. Sachs and S. M. Allen (2005). Improving Accuracy of Powder Sintering-based SFF Processes by Metal Deposition from Nanoparticle Dispersion. Solid Freeform Fabrication Symposium, Austin, TX.261-272

Cubberly, W. and R. Bakerjian (1989). Tool and Manufacturing Engineers Handbook, SME. 0872633519

Das, S. (1998). Direct Selective Laser Sintering of High Performance Metals - Machine Design, Process Developement and Process Control., Austin, Texas. Phd.

Das, S., J. J. Beaman, M. Wohlert and D. L. Bourell (1998). Direct laser freeform fabrication of high performance metal components. Rapid Prototyping Journal 4(3): $112-117$.

De Lima, M. S. F. (2005). Laser beam welding of titanium nitride coated titanium using pulse-shaping. Materials Research 8(3): 323-328.

Donnelly, R. J. and W. Glaberson (1965). Experiments on the capillary instability of a liquid jet. Proceedings of the Royal Society of London A290: 547-556.

Dragesco, J. (1995). High Resolution Astrophotography, Cambridge University Press. 0521415888

Duley, W. (1999). Laser Welding, Wiley - Interscience.0-471-246679-4

Dumitru, G., B. Luscher, M. Krack, S. Bruneau, J. Hermann and Y. Gerbig (2005). Laser processing of hardmetals: Physical basics and applications. Int. Journal of Refractory Metals and Hard Materials 23(4): 278-286.

Elsen, M. V. (2007). Complexity of Selective Laser Melting: a new optimisation approach. Department of Mechanical Engineering. Leuven, Katholieke Universiteit Leuven. Phd.

Eos. (2006). "http://www.eos-gmbh.de/." March 2006.

Eustathopoulos, N., M. G. Nicholas and B. Drevet (1999). Wettability at High Temperatures, Published by Pergamon,.0080421466 
Extrand, C. W. (2003). A thermodynamic model for wetting free energies from contact angles. Langmuir 19(3): 646-649.

Fankhauser, F. and S. Kwasniewska (2003). Lasers in Ophthalmology: Basic, Diagnostic, and Surgical Aspects : a Review, Kugler Publications. 9062991890

Fcubic. (2008). "www.fcubic.com." August 2008.

Feenstra, F., Holmer B., Pohl H., G. Tromans, M. N. and Mieritz B. (2003). RP, RT, RM Trends and Developments/Research Survey. 4th National Conference of Rapid \& Virtual Prototyping and Applications, Buckinghamshire, UK.95-138.

Fischer, P., N. Karapatis, V. Romano, R. Glardon and H. P. Weber (2002). A model for the interaction of near-infrared laser pulses with metal powders in selective laser sintering. Applied Physics A 74: 467-474.

Fischer, P., V. Romano, A. Blatter and H. P. Weber (2005). Highly precise pulsed selective laser sintering of metallic powders. Laser Physics Letters 2(1): 48-55.

Fischer, P., V. Romano, H. P. Weber, N. P. Karapatis, E. Boillat and R. Glardon (2003). Sintering of commercially pure titanium powder with a Nd:YAG laser source. Acta Materialia 51(6): 1651-1662.

Flemmer, R. I. C., J. Pickett and N. N. Clark (1993). Experimental study on the effect of particle shape on fluidization behavior. Powder Technology(77): 123-133.

Fuh, J. Y. H., Y. S. Choo, A. Y. C. Nee, L. Lu and K. Lee (1995). Improvement of the V curing process for the laser lithography technique. Materials \& Design 16(1): 23-32.

Fujinaga, S., H. Takenaka, T. Narikiyo, S. Katayama and A. Matsunawa (2000). Direct observation of keyhole behaviour during pulse modulated high-power Nd:YAG laser irradiation. Journal of Physics D: Applied Physics 33(5): 492-497.

German, R. M. (1994). Powder Metallurgy Science, Metal powder industries federation.1-878954-42-3

Glardon, R., N. Karapatis, V. Romano and G. N. Levy (2001). Influence of Nd:YAG Parameters on the Selective Laser Sintering of Metallic Powders. CIRP Annals Manufacturing Technology 50(1): 133-136.

Gower, H. L., R. R. G. M. Pieters and I. M. Richardson (2005). Pulsed laser welding of metal-polymer sandwich materials using pulse shaping. Journal of laser applications 18(1): 35-41.

Griffith ML, Harwell LD, Romero JT, Schlienger E, Atwood CL and S. JE. (1997). Multi-Material Processing by LENS. Solid Freeform Fabrication Symposium, Austin.TX.387-392

Griffith, M. L., M. E. Schlienger, L. D. Harwell, M. S. Oliver, M. D. Baldwin, M. T. Ensz, M. Essien, J. Brooks, C. V. Robino and J. E. Smugeresky (1999). Understanding thermal behavior in the LENS process. Materials and Design 20(2): 107-113.

$\mathrm{Gu}, \mathrm{D}$. and Y. Shen (2007). Balling phenomena during direct laser sintering of multicomponent $\mathrm{Cu}$-based metal powder. Journal of Alloys and Compounds 432(1-2): 163-166.

Haboudou, A., P. Peyre, A. B. Vannes and G. Peix (2003). Reduction of porosity content generated during Nd:YAG laser welding of A356 and AA5083 aluminium alloys. Materials Science and Engineering: $A$ 363(1): 40-52.

Hague, R., S. Mansour and N. Saleh (2004). Material and design considerations for Rapid Manufacturing. International Journal of Production Research 42(22): 4691.

Hauser, C. (2003). Selective Laser Sintering of a Stainless Steel Powder, University of Leeds. 
Hauser, C. (2003). Selective Laser Sintering of Stainless Steel Powder. School of Mechanical Engineering. Leeds, Leeds University. $\mathrm{PhD}$.

Hauser, C., T. H. C. Childs, C. M. Taylor and M. Badrossamay (2003). Direct selective laser sintering of tool steel powders to high density. Part A. Effect of laser beam width and scan strategy,. Solid Freeform Fabrication Symposium, Austin, Tx.644-655

Hauser, C., C. Sutcliffe, M. Egan and P. Fox (2005). Spiral Growth Manufacturing (SGM) - A Continuous Additive Manufacturing Technology For Processing Metal Powder by Selective Laser Melting. Solid Freeform Fabrication Symposium, Austin, Tx.1-12

Hegge, H. J. and J. Hosson (1991). The influence of convection on the homogeneity of laser-applied coatings. Journal of Material Science(26): 711-714.

Howard, P. and M. J. Koczak (1981). How porosity and atmosphere effect the thermal conductivity of P/M parts. International Journal of Powder Technology(17): 2535.

$\mathrm{Hu}$, D. and R. Kovacevic (2003). Sensing, modelling and control for laser-based additive manufacturing. International Journal of Machine Tools and Manufacture 43(1): 51-60.

Ion, J. C. (2005). Laser Processing of Engineering Materials, ButterworthHeinemann.0750660791

Jeng, J. Y. and M. C. Lin (2003). Mold fabrication and modification using hybrid processes of selective laser cladding and milling. Journal of Materials Processing Technology 1(110): 98-103.

Kanzler, K. (2006 ). How much energy are you throwing away? Photonics Spectra. July.

Karapatis, N. P. (2001). A sub-process approach of selective laser sintering, Ecole Polytechnique Federal de Lausanne. Phd Thesis.

Karapatis, N. P. (2002). A sub-process approach of selective laser sintering. Switzerland, Ecole Polytechnique Federal de Lausanne. Thesis no.2506.

Karapatis, N. P., J. P. S. van Griethuysen and R. Glardon (1998). Direct rapid tooling: A review of current research. Rapid Prototyping Journal 4(2): 77-89.

Katayama, S., S. Kohsaka, M. Mizutani, K. Nishizawa and A. Matsunawa (1993). Pulse Shape Optimization for Defect Prevention in Pulsed Laser Welding of Stainless Steels. ICALEO Orlando, Flo.

Khaing, M. W., J. Y. H. Fuh and L. Lu (2001). Direct metal laser sintering for rapid tooling: processing and characterisation of EOS parts. Journal of Materials Processing Technology 113(1-3): 269.

Kou, S. (2003). Welding Metallurgy, Wiley Interscience.0-471-43491-4

Kruth, J. P. (1991). Material incress manufacturing by rapid prototyping techniques. CiRP Annals 40(2): 603-614.

Kruth, J. P., L. Froyen, M. Rombouts, J. V. Vaerenbergh and P. Mercelis (2003). New ferro powder for selective laser sintering of dense parts. CIRP Annals Manufacturing Technology 1(52): 139-142.

Kruth, J. P., L. Froyen, J. Van Vaerenbergh, P. Mercelis, M. Rombouts and B. Lauwers (2004). Selective laser melting of iron-based powder. Journal of Materials Processing Technology 149(1-3): 616-622.

Kruth, J. P., G. Levy, F. Klocke and T. H. C. Childs (2007). Consolidation phenomena in laser and powder-bed based layered manufacturing. CIRP Annals Manufacturing Technology 56(2): 730-759. 
Kruth, J. P., P. Mercelis, J. V. Vaerenbergh, L. Froyen and M. Rombouts (2005). Binding mechanisms in selective laser sintering and selective laser melting. Rapid Prototyping Journal 11(1): 26-36.

Kruth, J. P., X. Wang, T. Laoui and L. Froyen (2003). Lasers and materials in selective laser sintering. Assembly Automation 23: 357-371.

L. Costa, T. R. R. Vilar, R. Colaço, A. M. Deus and I. Felde (2005). Simulation of phase transformations in steel parts produced by laser powder deposition,. Material Science Forum 473-474: 315-320.

Laeng, J., J. G. Stewart and F. W. Liou (2000). Laser metal forming processes for rapid prototyping - a review. International Journal of Production Research 38: 3973.

Laoui, T., E. Santos, K. Osakada, M. Shiomi, M. Morita, S. K. Shaik, N. K. Tolochko, F. Abe and M. Takahashi (2006). Properties of titanium dental implant models made by laser processing. Proceedings of the Institution of Mechanical Engineers, Part C: Journal of Mechanical Engineering Science 220(6): 857863.

Laser, C. (2006). "http://www.concept-laser.de/." March 2006.

Lewis, G. K. and E. Schlienger (2000). Practical considerations and capabilities for laser assisted direct metal deposition. Materials and Design 21(4): 417-423.

Liu, J. and L. Li (2004). In-time motion adjustment in laser cladding manufacturing process for improving dimensional accuracy and surface finish of the formed part. Optics \& Laser Technology 36(6): 477.

Liu, W. and J. N. Dupont (2003). In-situ reactive processing of nickel aluminides by laser-engineered net shaping. Metallurgical and Materials Transactions A: Physical Metallurgy and Materials Science 34 A(11): 2633.

Low, D. K. Y., L. Li and P. J. Byrd (2001). The influence of temporal pulse train modulation during laser percussion drilling. Optics and Lasers in Engineering 35(3): 149-164.

Lu, L., J. Fuh and W. Y.S (2001). Laser-Induced Materials and Processes for Rapid Prototyping, Kluwer Academic Publishers.0-7923-7400-2

$\mathrm{Lu}, \mathrm{S} ., \mathrm{H}$. Fujii and K. Nogi (2004). Marangoni convection and weld shape variations in Ar-O2 and Ar-CO2 shielded GTA welding. Materials Science and Engineering A 380(1-2): 290-297.

Lu.L, J. Fuh and W. Y.S (2001). Laser-Induced Materials and Processes for Rapid Prototyping, Kluwer Academic Publishers.0-7923-7400-2

Lumonics (1995). JK701-702 Nd:YAG Laser manual and programming

Marlow, F. B. and W. L. Galvery (2001). Welding Essentials: Questions and Answers, Industrial Press Inc.0831131519

Mazumder, J., D. Dutta, N. Kikuchi and A. Ghosh (2000). Closed loop direct metal deposition: art to part. Optics and Lasers in Engineering 34(4-6): 397.

Mercelis, P. and J.-P. Kruth (2006). Residual stresses in selective laser sintering and selective laser melting. Rapid Prototyping Journal 12(5): 254-265.

Messler, R. W. (1999). Principles of Welding, processes, physics, chemistry and metallurgy, Wiley - Interscience.0-471-25376-6

Metals, S. (2006). "http://www.specialmetals.com/products/inconelalloy625.htm." June 2006.

Michaud, E. J., D. C. Weckman and H. W. Kerr (1994). Effects of Pulse Shape on Predicted Thermomechanicl Strains in Nd:YAG Laser Welded Aluminium. ICALEO, Orlando, Flo.461-470

Milewski, J. O., P. G. Dickerson, R. B. Nemec, G. K. Lewis and J. C. Fonseca (1999). Application of a manufacturing model for the optimization of additive 
processing of Inconel alloy 690. Journal of Materials Processing Technology 91(1): 18-28.

Milewski, J. O., G. K. Lewis, D. J. Thoma, G. I. Keel, R. B. Nemec and R. A. Reinert (1998). Directed light fabrication of a solid metal hemisphere using 5-axis powder deposition. Journal of Materials Processing Technology 75(1): 165-172.

Miller, J. (1994). Principles of Infrared Technology: A Practical Guide to the State of the Art, Van Nostrand Reinhold.0442012101

Mills, C., B. J. Keene and K. Brooks (1998). Marangoni effects in welding. Philosophical Transactions of the Royal Society A: Mathematical, Physical and Engineering Sciences 356(1739): 911-925.

Morgan, R., A. Papworth, C. Sutcliffe, P. Fox and B. O'Neill. (2001). Direct Metal Laser Re-Melting (DMLR) of 316L Stainless Steel Powder. Part 1: Analysis of Thin Wall Structures. Solid Freeform Fabrication Symposium, Austin, TX.276282

Morgan, R., C. J. Sutcliffe and W. ONeill (2001). Experimental investigation of nanosecond pulsed Nd:YAG laser re-melted pre-placed powder beds. Rapid Prototyping Journal 7(3): 159-172.

Morgan, R., C. J. Sutcliffe and W. ONeill (2004). Density analysis of direct metal laser re-melted 316L stainless steel cubic primitives. Journal of Materials Science 39(4): 1195-1205.

Mumtaz, K., N. Hopkinson and P. Erasenthiran (2006). High Density Selective Laser Melting of Waspaloy. Solid Freeform Fabrication Symposium, Austin, TX.220232

Mumtaz, K., N. Hopkinson and P. Erasenthiran (2007). Selective Laser Melting Functionally Graded Compositions of Waspaloy and Zirconia. Journal of Material Science 42(18): 7647-7656.

Mumtaz, K., N. Hopkinson and P. Erasenthiran (2008). High Density Selective Laser Melting of Waspaloy. Journal of Material Processing Technology 195: 77-87.

Nickel, A., D. Barnett, G. Link and Prinz. F. (1999). Residual stresses in layered manufacturing. Proc. Solid Freeform Fabrication symposium, Austin. TX

$\mathrm{Niu}, \mathrm{H}$. J. and I. T. H. Chang (1998). Liquid phase sintering of M3/2 high speed steel by selective laser sintering Scripta Materialia 30(1): 67-72.

Niu, H. J. and I. T. H. Chang (1999). Instability of scan tracks of selective laser sintering of high speed steel powder. Scripta Materialia 41(11): 1229-1234.

O'Neill, W., C. J. Sutcliffe, R. Morgan and K. K. B. Hon (1998). Investigation of Short Pulse Nd:YAG Laser Interaction with Stainless Steel Powder Beds. Solid Freeform Fabrication Symposium, Austin, TX.147-160

Ohmura, E., H. Hayashi, S. Fujimori and I. Miyamoto (1997). Thermohydrodynamics analysis of fusion phenomena accompanied with evaporation due to laser irradiation. Proceedings of the Laser Institute of America ICALEO, CA, USA.pp 9-15

Onuh, S. O. and B. Hon (1998). Application of the Taguchi method and new hatch styles for quality improvement in stereolithography. Journal of Engineering Manufacture B6(212): 461-472.

Osakada, K. and M. Shiomi (2006). Flexible manufacturing of metallic products by selective laser melting of powder. International Journal of Machine Tools and Manufacture 46(11): 1188-1193.

Over, C. (2003). Generative Fertigung von Bauteilen aus Werkzeugstahl X38CrMoV51 und Titan TiAl6V4 mit Selective Laser Melting. RWTH Aachen. Germany, RWTH Aachen. PhD. 
P. Fischer, V. R. A. B. H. P. W. (2005). Highly precise pulsed selective laser sintering of metallic powders. Laser Physics Letters 2(1): 48-55.

Pan, L. K., C. C. Wang, Y. C. Hsiao and K. C. Ho (2005). Optimization of Nd:YAG laser welding onto magnesium alloy via Taguchi analysis. Optics \& Laser Technology 37(1): 33-42.

Park, H. and S. Rhee (1999). Analysis of mechanism of plasma and spatter in CO2 laser welding of galvanized steel. Optics and Laser Technology 31(2): 119-126.

Peng, L., Y. Taiping, L. Sheng, L. Dongsheng, H. Qianwu, X. Weihao and Z. Xiaoyan (2005). Direct laser fabrication of nickel alloy samples. International Journal of Machine Tools and Manufacture 45(11): 1288-1294.

Petrov, A. L., A. A. Gusev and K. S.V (1992). New advances in industry applications of YAG pulse lasers. Proc. LAMP 92.993-997

Photonics, R. (2008). "http://www.rp-photonics.com/fiber lasers.html." December 2008.

Pinkerton, A. J. (2007). Matching the additive process and parameters to the purpose. AILU Technology Workshop, Rotherham, UK

Pinkerton, A. J., M. Karadge, W. Syed and L. Li (2006). Thermal and microstructural aspects of the laser direct metal deposition of waspaloy. JOURNAL OF LASER APPLICATIONS 18(3): 216-226.

Pogson, S., P. Fox, W. ONeill and C. J. Sutcliffe (2004). The direct metal laser remelting of copper and tool steel powders. Materials Science and Engineering A 386(1-2): 453.

Pohl, H., A. Simchi, M. Issa and H. C. Dias (2001). Thermal Stresses in Direct Metal Laser Sintering. Solid Freeform Fabrication Symposium, Austin Texas.366-372

Ramos, J. A., D. L. Bourell and J. J. Beaman (2003). Surface over-melt during laser polishing of indirect-SLS metal parts. Materials Research Society, Boston, MA, United States.53-61

Ramos, J. A., J. Guerrero, T. Larraín and Ramírez A. (2006). Direct Metal Laser Fabrication of $\mathrm{Cu}$ slabs from powder precursor: surface depth of melt and furnace temperature issues. Proc. Solid Freeform Fabrication Symposium, Austin. Tx.246-260

Rangaswamy, P., T. M. Holden, R. B. Rogge and M. L. Griffith (2003). Residual stresses in components formed by the laser-engineered net shaping (LENS) process. The Journal of Strain Analysis for Engineering Design 38: 519-528.

Rehme, O. and C. Emmelmann (2005). Reproducibility for properties of Selective Laser Melting products. Proceedings of the Third International WLT-Conference on Lasers in Manufacturing, Munich

Rombouts, M. (2006). Selective laser sintering/melting of iron based powders. Faculteit Ingenieurswetenschappen. Leuven, Katholieke Universiteit Leuven. Phd.

Rombouts, M., J. P. Kruth, L. Froyen and P. Mercelis (2006). Fundamentals of Selective Laser Melting of alloyed steel powders. CIRP Annals - Manufacturing Technology 55(1): 187-192.

Rossi, S., F. Deflorian and F. Venturini (2004). Improvement of surface finishing and corrosion resistance of prototypes produced by direct metal laser sintering. Journal of Materials Processing Technology 148(3): 301-309.

Santos, E. C., K. Osakada, M. Shiomi, Y. Kitamura and F. Abe (2004). Microstructure and mechanical properties of pure titanium models fabricated by selective laser melting. Proceedings of the I MECH E Part C Journal of Mechanical Engineering Science 218(7): 711-719. 
Santos, E. C., M. Shiomi, K. Osakada and T. Laoui (2006). Rapid manufacturing of metal components by laser forming. International Journal of Machine Tools and Manufacture 46(12-13): 1459-1468.

Schiaffino, S. and A. A. Sonin (1997). Formation and stability of liquid and molten beads on a solid surface. Journal of Fluid Mechanics 343: 95-110.

Schlienger, E., D. Dimos, M. Griffith, J. Michael, M. Oliver, T. Romero and J. Smugeresky (1998). Near Net Shape production of metal components using LENS. 3rd Pacific Rim international conference on advanced materials and processing, , Honolulu, HI (United States)

Schneider, M. F. (1998). Laser cladding with powder, effect of some machining parameters on clad properties. U. o. Twente. The Netherlands, Phd Thesis, University of Twente. Phd.

Schwendner, K. I., R. Banerjee, P. C. Collins, C. A. Brice and H. L. Fraser (2001). Direct laser deposition of alloys from elemental powder blends. Scripta Materialia 45(10): 1123-1129.

Sears, J. W. (1999). Direct laser powder deposition - 'State of the Art'. ASTM/TMS Materials Week, Cincinnati, $\mathrm{OH}$ (US).16 pages

Semak, V. and A. Matsunawa (1997). The role of recoil pressure in energy balance during laser materials processing. Journal of Physics D: Applied Physics 30(18): 2541-2552.

Sexton, L., S. Lavin, G. Byrne and A. Kennedy (2002). Laser cladding of aerospace materials. Journal of Materials Processing Technology 122(1): 63.

Shankar, V., K. Rao and S. L. Mannan (2001). Microstructure and mechanical properties of Inconel 625 superalloy. Journal of Nuclear Materials(288): 222232.

Shannon, G. J. and B. Q. Li (2004). Investigation of weld cracking in Nd:yttrium-aluminum--garnet keyhole spot welding of materials used in optoelectronic packaging. Journal of Laser Applications 16(2): 73-78.

Shellabear, M. (2007). EOS GmbH. Personal communication

Shellabear, M. and O. Nirhylä (2004). DMLS-Development history and state of the art. Proceedings of the 4th LANE 2004, Germany.393-404

Simchi, A., F. Petzoldt and H. Pohl (2003). On the development of direct metal laser sintering for rapid tooling. Journal of Materials Processing Technology 141(3): 319-328.

Singheiser, L., R. Steinbrech, W. J. Quadakkers and R. Herzog (2001). Failure aspects of thermal barrier coatings. Materials at High Temperatures 18(4): 249-259.

Song, Y.-A. (1997). Experimental study of the basic process mechanism for direct selective laser sintering of low-melting metallic powder. CIRP Annals Manufacturing Technology 46(1): 127.

Sparks, T., J. Ruan, Z. Fan, Y. Bap and F. Liou (2006). Effect of Structured Laser Pulses on Grain Growth in H13 Tool Steel Solid Freeform Fabrication Symposium, Austin, TX.261-267

Steen, W. M. (2003). Laser Material Processing. Verlag, Springer 1-85233-698-6

$\mathrm{Su}, \mathrm{W}$. N. (2002). Layered Fabrication of Tool Steel and Functionally Graded Materal with a Nd:YAG Pulsed Laser. Rapid Manufacturing Research Group, Loughborough University. Phd.

Su, W. N., P. Erasenthiran and P. M. Dickens (2003). Investigation of fully dense laser sintering of tool steel powder using a pulsed Nd: YAG (neodymium-doped yttrium aluminium garnet) laser. Proceedings of the Institution of Mechanical 
Engineers, Part C: Journal of Mechanical Engineering Science 217(1): 127138.

Sun, S., Y. Durandet and M. Brandt (2005). Parametric investigation of pulsed Nd: YAG laser cladding of stellite 6 on stainless steel. Surface and Coatings Technology 194(2-3): 225-231.

Syed, W. U. H., A. J. Pinkerton and L. Li (2005). A comparative study of wire feeding and powder feeding in direct diode laser deposition for rapid prototyping. Applied Surface Science 247(1): 268.

Syed, W. U. H., A. J. Pinkerton and L. Li (2006). Simultaneous wire and powder feed direct metal deposition. Journal of Laser Applications 18(1): 65-72.

Systems, P. (2008). "http://www.phenix-systems.com." March 2008.

Syvanen, T., J. Nyrhila, J. Kotila and J. E. Lind (2000). Direct Metal Laser Sintering of Very Fine Metal Powder. ICALEO, Orlando, Flo.21-29

Tam, S. C., C. Y. Yeo, S. Jana, M. W. S. Lau, L. E. N. Lim, L. J. Yang and Y. M. Noor (1993). Optimization of laser deep-hole drilling of Inconel 718 using the Taguchi method. Journal of Materials Processing Technology 37(1): 741-757.

Tolochko, N. K., S. Mozzharov, I. A. Yadroitsev, T. Laoui, L. Froyen, V. I. Titov and M. B. Ignatiev (2004). Balling process during selective laser treatment of powders. Rapid Prototyping Journal 10(2): 78-87.

Toyserkani, E., A. Khajepour and S. Corbin (2004). 3-D finite element modeling of laser cladding by powder injection: Effects of laser pulse shaping on the process. Optics and Lasers in Engineering 41(6): 849-867.

Tzeng, Y. F. (2000). Process Characterisation of Pulsed Nd:YAG Laser Seam Welding. Int J Advanced Manufacturing Technology 16: 10-18.

Tzeng, Y. F. and F. C. Chen (2000). Effects of Operating Parameters on the Static Properties of Pulsed Laser Welded Zinc-Coated Steel. The International Journal of Advanced Manufacturing Technology 18: 641-647.

Tzeng, Y. F. and F. C. Chen (2001). Effects of Operating Parameters on the Static Properties of Pulsed Laser Welded Zinc-Coated Steel. The International Journal of Advanced Manufacturing Technology 18: 641-647.

Von, A. M. (1987). Laser-beam interactions with materials. Berlin, Springer-Verlag

Wang, H. and X. Chen (2003). Three-dimensional modelling of the laser-induced plasma plume characteristics in laser welding. Journal of Physics D: Applied Physics(36): 628-639.

Watanabe, T., S. Ichijyo and J. S. Kim (1998). Suppression Of Cracks By Means Of Added Ultrasonic Vibration And/Or Controlled Pulse-Train In Laser Spot Welding Of Aluminium Alloys. ICALEO, Orlando, Flo.1-9

Watkins, K. G. (2001). Achieving the Potential of Direct Fabrication with Lasers Proc 3rd International. LANE Erlangen.25 - 38

Webb, C. E. and J. D. C. Jones (2004). Handbook of Laser Technology and Applications, Taylor \& Francis.0750309660

Weedon, T. M. (1987). Nd:YAG lasers with controlled pulse shape. Proc. LAMP.75-80

Williams C.B, Mistree F. and Rosen D.W (2005). Towards the design of layer-based additive manufacturing process for the realization of metal parts of designed microstructure. Proceedings of the Solid Freeform Fabrication Symposium, Austin, Tx.217-230

Williams, C. B., F. Mistree and D. W. Rosen (2005). Towards the design of layer-based additive manufacturing process for the realization of metal parts of designed microstructure. Solid Freeform Fabrication Symposium, Austin, Tx.217-230 
Wohler, T. (2006). Wohlers Report 2006, Rapid Prototyping and Manufacturing State of the Industry. $0-9754429-2-9$

Wohlers, T. (2008). Wohlers Report 2008, Rapid Prototyping and Manufacturing State of the Industry.0-9754429-3-7

Woldman, N. E. (1948). Metal Process Engineering, Reinhold Pub

Wu, X., J. Liang, J. Mei, C. Mitchell, P. S. Goodwin and W. Voice (2004). Microstructures of laser-deposited Ti-6Al-4V. Materials and Design 25(2): 137144.

Xiao, B. and Y. Zhang (2007). Laser sintering of metal powders on top of sintered layers under multiple-line laser scanning. Journal of Physics D: Applied Physics 40(21): 6725-6734.

Xie, J. and A. Kar (1999). Laser Welding of Thin Sheet Steel with Surface Oxidation. Welding Research Supplement: 343-348.

Xue, L. (2006). Laser Consolidation - A Rapid Manufacturing Process for Making NetShape Functional Components. Rapid Manufacturing 1st Internation Conference, Loughborough, UK.143-152

Xue, L. and M. U. Islam (2000). Free-form laser consolidation for producing metallurgically sound and functional components. Journal of Laser Applications 12(4): $160-165$.

Yadroitsev, I., P. Bertrand and I. Smurov (2007). Parametric analysis of the selective laser melting process. Applied Surface Science 253(19): 8064-8069.

Yadroitsev, I., L. Thivillon, P. Bertrand and I. Smurov (2007). Strategy of manufacturing components with designed internal structure by selective laser melting of metallic powder. Applied Surface Science 254(4): 980-983.

Yakovlev, A., E. Trunova, D. Grevey, M. Pilloz and I. Smurov (2005). Laser-assisted direct manufacturing of functionally graded 3D objects. Surface and Coatings Technology 190(1): 15-24.

Yevko, V., C. B. Park, G. Zak, T. W. Coyle and B. Benhabib (1998). Cladding formation in laser-beam fusion of metal powder. Rapid Prototyping Journal 4(4): 168-184.

Zhong, M., H. Sun, W. Liu, X. Zhu and J. He (2005). Boundary liquation and interface cracking characterization in laser deposition of Inconel 738 on directionally solidified Ni-based superalloy. Scripta Materialia 53(2): 159-164. 


\section{Chapter 12 Appendices}

\subsection{Inert Gas Optimisation}

An inert gas is defined as a gas that is not reactive under normal circumstances [Kou 2003]. It plays a fundamental role in metal welding/melting processes such as gas metal arc, gas tungsten arc, EBM, LENS, DMLS, SLM etc. An inert gas can be used to protect or screen solidifying metal from the adverse reaction of gases (especially oxygen) within the surrounding atmosphere.

The rate at which the inert gas is jetted/delivered onto molten material to protect it against the effects of oxidisation can be adjusted. No studies had been conducted on the Lumonics JK701 laser apparatus detailing the optimum gas flow required to provide sufficient protection against oxidisation. Previous work had used a fixed gas flow rate of $15 \mathrm{ml} / \mathrm{s}$ for processing $\mathrm{H} 13$ tool steel with a particle size of $136 \mu \mathrm{m}$ [Su 2002]. However there was no evidence to suggest this was the optimum flow rate. It may seem obvious that a greater gas flow rate would equate to improved protection. However if the gas flow rate is too high it may cause powder to be blown away from the interaction zone or it may induce turbulence and deliver more oxygen to interaction zone [Marlow $\&$ Galvery 2001]. If the gas flow rate is too low the melt area may oxidise due to insufficient shielding. It is therefore important to study the effects of gas flow rate on part formation in order to obtain an optimum flow rate setting and produce improved parts.

\subsubsection{Methodology}

The gas flow rate was adjusted at varying scan speeds. It is important to observe whether scan speed is a factor affecting oxidisation, this is because faster scan speeds may move the solidifying molten material out from the protection of the shielding gas before it cools below oxidisation temperature. Plasma plume generation was monitored during processing. Parts were examined for signs of oxidisation by observing 
discoloration, surface roughness (measured using a Talysurf surface contact method) and oxygen content (measured using EDS).

The gas flow rate was varied between $0-25 \mathrm{ml} / \mathrm{s}$ when processing three $0.1 \mathrm{~mm}$ layers of Inconel 625 . Low pulse durations of $0.5 \mathrm{~ms}$ were used so that the material would remain molten for a minimum time duration (reducing oxidising potential). The scan speed was varied between $100-400 \mathrm{~mm} / \mathrm{min}$. A constant spot overlap of $75 \%$ was used throughout these experiments to maintain consistency, repetition rates were varied between 10 $40 \mathrm{~Hz}$ to maintain the $75 \%$ spot overlap. The processing parameters used within the experiment are shown in Table 12-1.

\begin{tabular}{|lllll|}
\hline $\begin{array}{l}\text { Pulse Energy } \\
(\mathbf{J})\end{array}$ & $\begin{array}{l}\text { Pulse Width } \\
(\mathbf{m s})\end{array}$ & $\begin{array}{l}\text { Repetition Rate } \\
(\mathbf{H z})\end{array}$ & $\begin{array}{l}\text { Scan Speed } \\
(\mathbf{m m} / \mathbf{m i n})\end{array}$ & $\begin{array}{l}\text { Argon flow rate } \\
(\mathbf{m l} / \mathbf{s})\end{array}$ \\
\hline 1 & 0.5 & $10-40$ & $100-400$ & $0-25$ \\
\hline
\end{tabular}

Table 12-1 Processing parameters used for gas flow rate optimisation study

\subsubsection{Results}

It was found that increasing the gas flow rate above $10 \mathrm{ml} / \mathrm{s}$ caused some of the Inconel 625 powder to be displaced from the powder bed. This value is lower than the $15 \mathrm{ml} / \mathrm{s}$ gas flow rate previously used for processing $136 \mu \mathrm{m}$ tool steel [Su 2002]. Due to this powder displacement gas flow rates above $10 \mathrm{ml} / \mathrm{s}$ were no longer considered.

\subsubsection{Oxygen content within operating window}

A Hitech Z210 oxygen analysing probe was placed within the region shielded by the inert gas flow. Figure 12-1 shows the oxygen content within the laser material interaction zone at different gas flow rates. It can be seen that the use of $5-10 \mathrm{ml} / \mathrm{s}$ flow rate dramatically reduces the oxygen content to a minimum of $0.26 \%$. Elsen [2007] states that a processing environment should have an oxygen level below $0.3 \%$ for laser the processing of metal powders. The experimental apparatus used within this research 
does not use an inert gas chamber but is still capable of reaching the minimum oxygen content requirements.

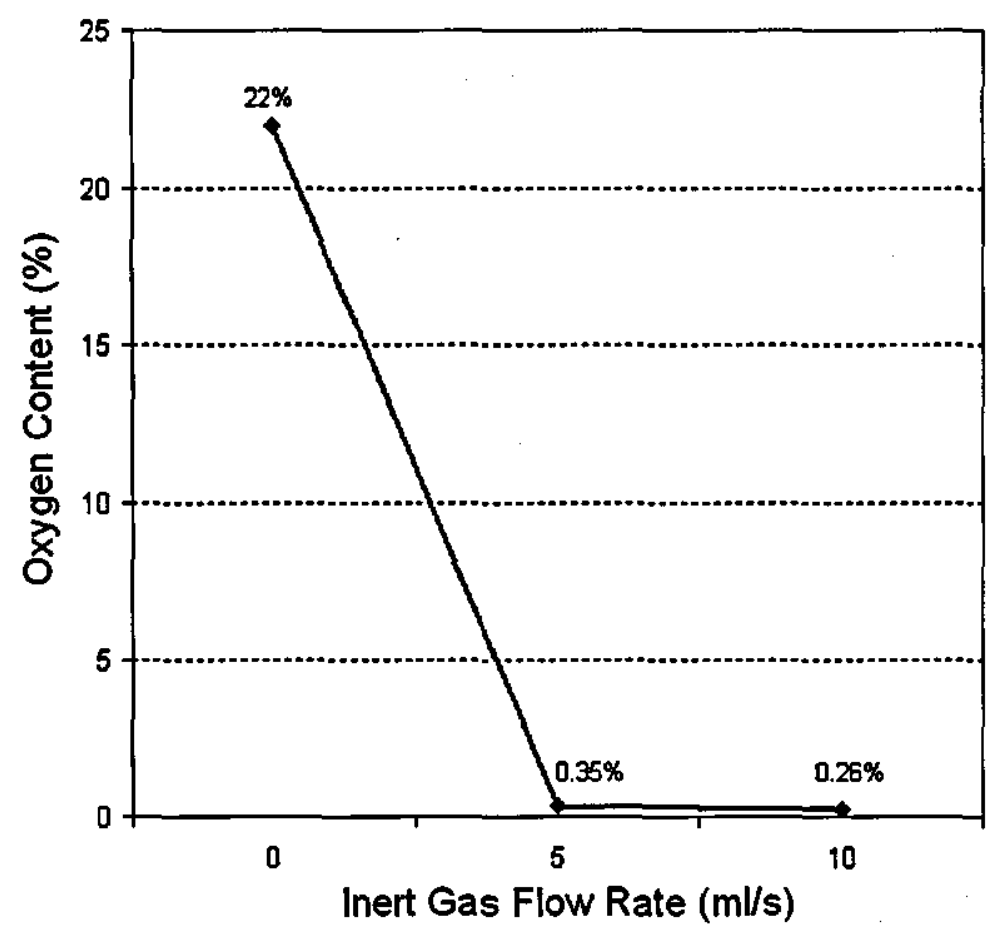

Figure 12-1 Oxygen content within processing region at different inert gas flow rates

\subsubsection{Plasma plume and spatter}

Increases in plasma plume generation and spatter can negatively affect SLM processing. Park et al. [1999] examined the effects of plasma plume and spatter generation within laser processing of metals. It was found that an increase in plasma plume generation is accompanied by an increase in material spatter. Therefore suppression of plasma plume should also reduce spatter potential. Figure 12-2 shows the level of plasma and spatter generated at different processing conditions. Large amounts of plasma and spatter were generated without the use of a shielding gas. An increase in scan speed and repetition rate generally increased the amount of plasma and spatter generated due to an increase in energy density. The use of $10 \mathrm{~m} / \mathrm{s}$ gas flow rate significantly reduced both plasma and spatter generation. This gas flow rate suppressed the plasma plume at all scan speed. This reduction in plasma and spatter generation may have been associated with the absorbance of the material [Xie \& Kar 1999]. Presence of oxygen can increase the exothermic reaction when a laser melts powder, an increase in gas flow rate reduces the oxidisation potential and subsequently reduces the intensity of melting generating less 
plasma and spatter [Syed et al. 2006]. The shielding gas may have also provided a cooling effect suppressing the plasma plumes intensity and modifying its geometry[Wang \& Chen 2003].
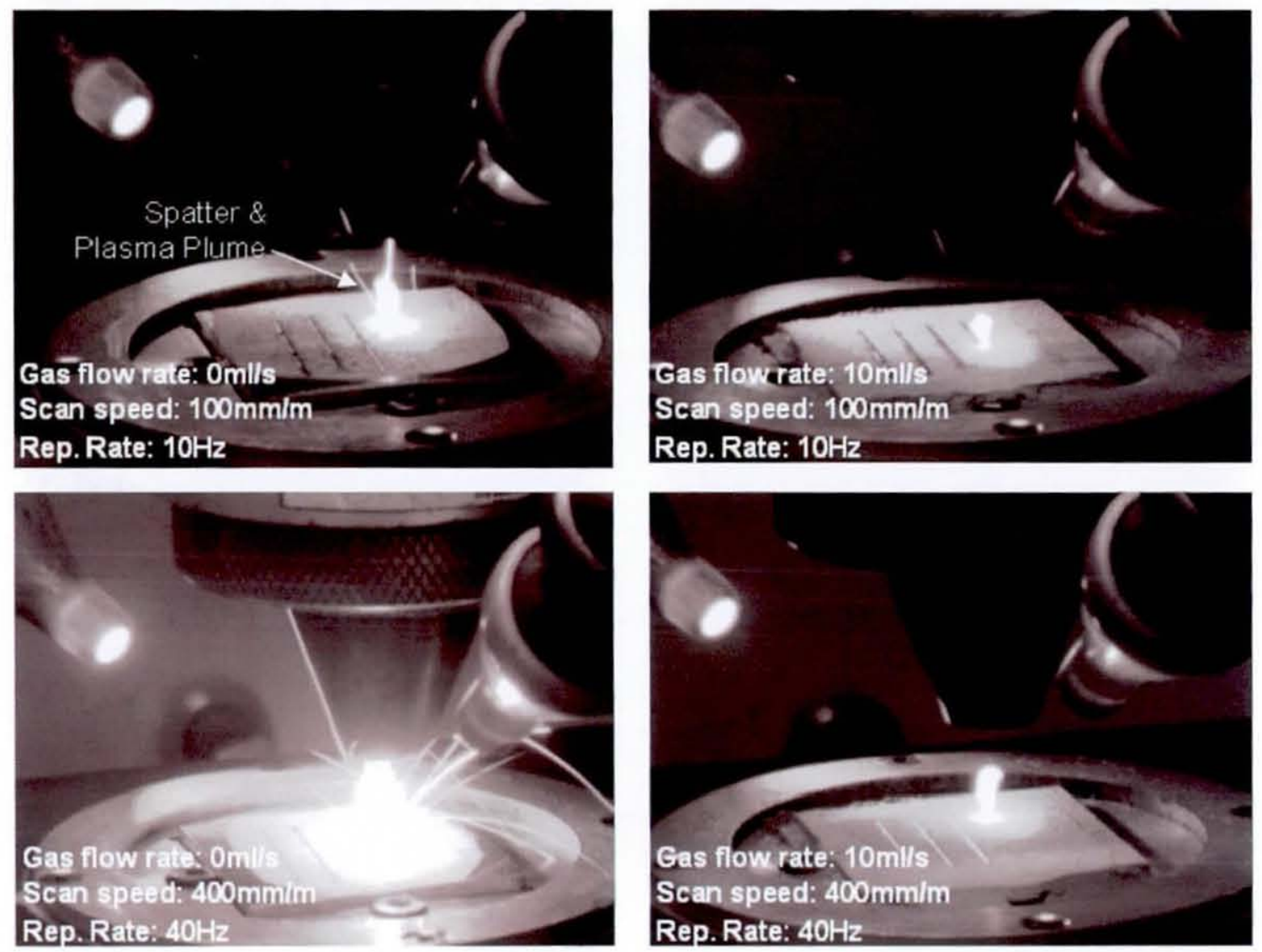

Figure 12-2 Plasma Plume/spatter generated with \& without the assistance of an inert shielding gas

\subsubsection{Surface discoloration}

Specimens processed without a shielding gas emitted a black smoke from the laser material interaction zone during processing (at all scan speed). These specimens were surrounded by small traces of black soot (possibly carbon, manganese or aluminium). Other powder bed systems such as EOS M270 and MTT Realizer also experience a small amount of soot residual during processing of powders (within an inert gas chamber). Black soot are nanoparticles produced with the laser interacts with the powder bed. These nanoparticles have a high aspect ratio and therefore appear black [Shellabear 2007]. The soot was gently brushed off the surface of the sample to reveal a 
grey/yellow surface, shown in Figure 12-3(a). This type of surface discoloration is characteristic of surface oxidisation [Woldman 1948]. As the flow rate of gas was increased between $5-10 \mathrm{ml} / \mathrm{s}$ the amount of black smoke/soot reduced. A gas flow rate of $10 \mathrm{ml} / \mathrm{m}$ produced no smoke or soot leaving a bright shiny silver surface on the sample, shown in Figure 12-3(b). This indicated that the amount of oxidisation reduced as gas flow rate was increased.
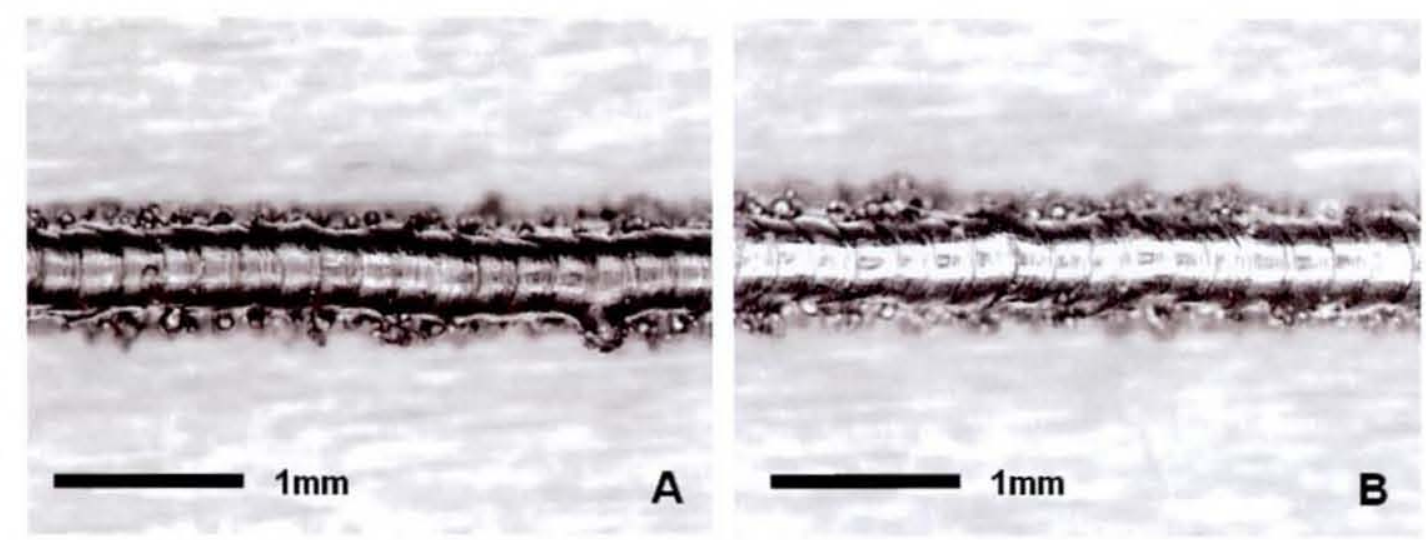

Figure 12-3 Three layers single scan processed with no shielding gas (a) \& $10 \mathrm{ml} / \mathrm{s}$ shielding gas (b) (both processed at $0.5 \mathrm{~ms}, 1 \mathrm{~J}, 10 \mathrm{~Hz} \& 100 \mathrm{~mm} / \mathrm{m}$ )

\subsubsection{Oxygen content on sample surface}

Discoloration of a part's surface is indicative of oxidisation but not proof that a reaction has occurred. Therefore an oxygen content analysis was performed on the surface of parts by EDS. An SEM image of the samples surface is shown in Figure 12-4. Spectrum 1 is a region on the samples surface that was measured for it's chemical composition. 


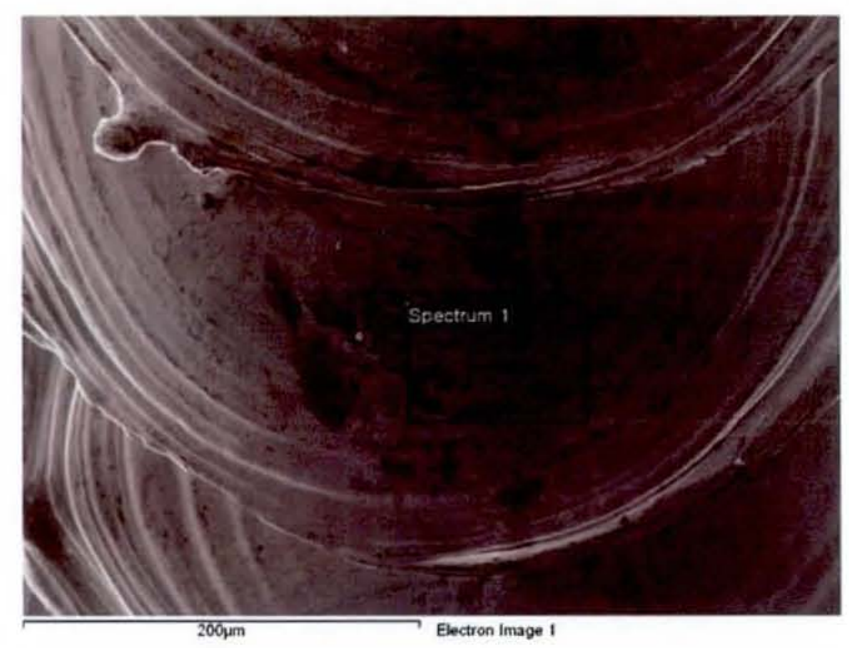

Figure 12-4 SEM image of parts surface measured for oxygen content

Figure 12-5 shows the oxygen content of the samples when processed under varying gas flow rates and scan speed. Oxygen content level peaked around $9.7 \%$ when processed without a shielding gas. A gas flow rate of $5 \mathrm{ml} / \mathrm{s}$ reduced the oxygen content to 2.7$3.1 \%$. A gas flow rate of $10 \mathrm{ml} / \mathrm{s}$ caused the oxygen content to drop just below $2 \%$. Varying the scan speed between $100-400 \mathrm{~mm} / \mathrm{m}$ did not have a significant effect on oxygen content.

Even though samples processed under a gas flow rate of $10 \mathrm{ml} / \mathrm{s}$ held an average oxygen level of $2 \%$ it should taken into consideration that the EDS analysis was conducted approximately 30 minutes after processing. After processing and cooling of molten the component the surface will continue to oxidise (due to exposure to air). Powder has more surface area to oxidise then solid, it is interesting to note that the Inconel 625 powder itself was analyzed using EDS (chapter 5.2.1) and contained an average oxygen content of $2 \%$ after being exposed to the environment over a period of time. Therefore it can be assumed that the surface of an Inconel 625 sample produced using SLM will also oxidise over time when exposed to the environment. The $10 \mathrm{ml} / \mathrm{s}$ gas flow rate may have completely removed the oxidising potential within processing with the laser ablating any oxides already present on the powders surface. This is then followed by reoxidisation on the surface of the solidified melt. 


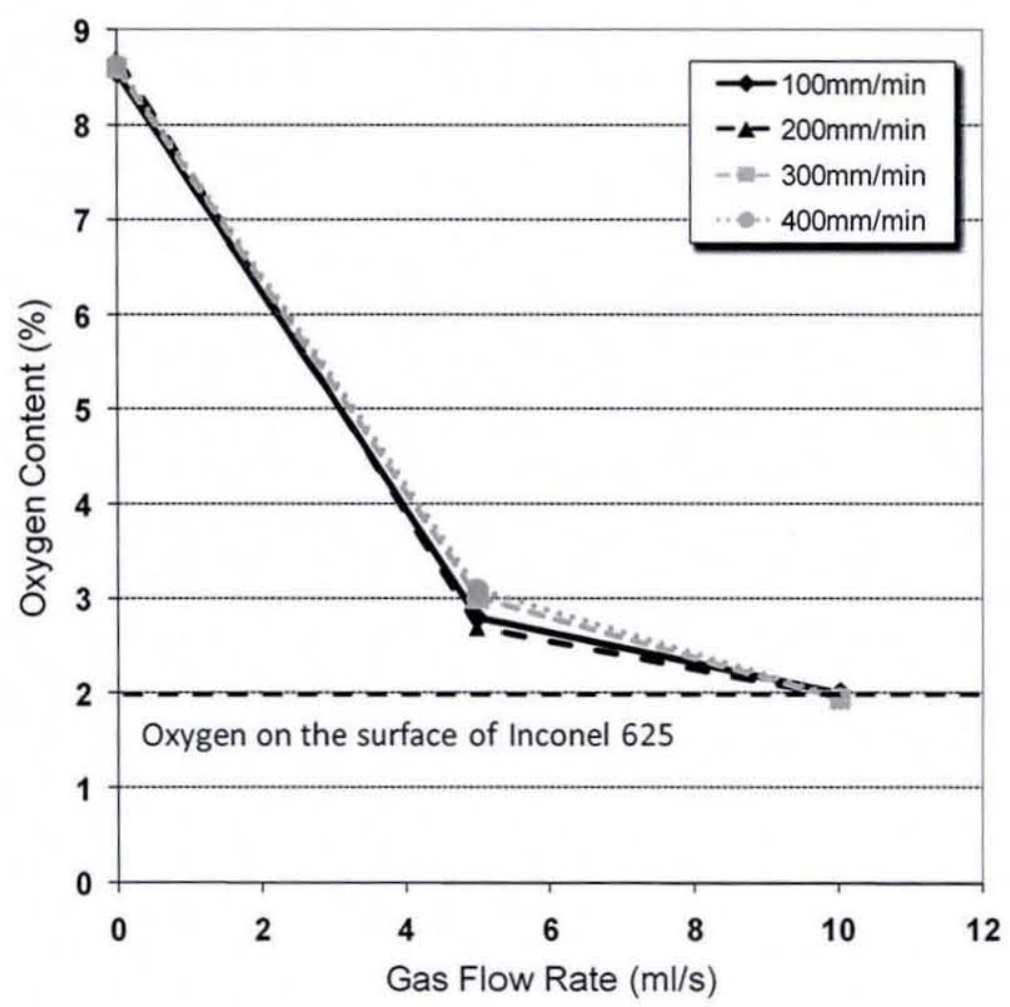

Figure 12-5 Oxygen content on samples at different gas flow rates and processing scan speeds

\subsubsection{Surface roughness}

Figure 12-6 shows the measured surface roughness of samples produced at varied scan speeds, repetition rates and gas flow rates. There is a marginal reduction in the surface roughness of the specimens as the gas flow rate is increased to $10 \mathrm{ml} / \mathrm{s}$. Gower et al.[2005] noticed that a reduction in the oxide layers formed on the surface of laser welded steel produced smoother welds. This suggests that a reduction in surface oxides improves the surface finish of parts. This information corroborates with the EDS results suggesting that a $10 \mathrm{ml} / \mathrm{s}$ gas flow rate produces minimal oxidisation. The degree of overlap remained the same throughout the experiment. 


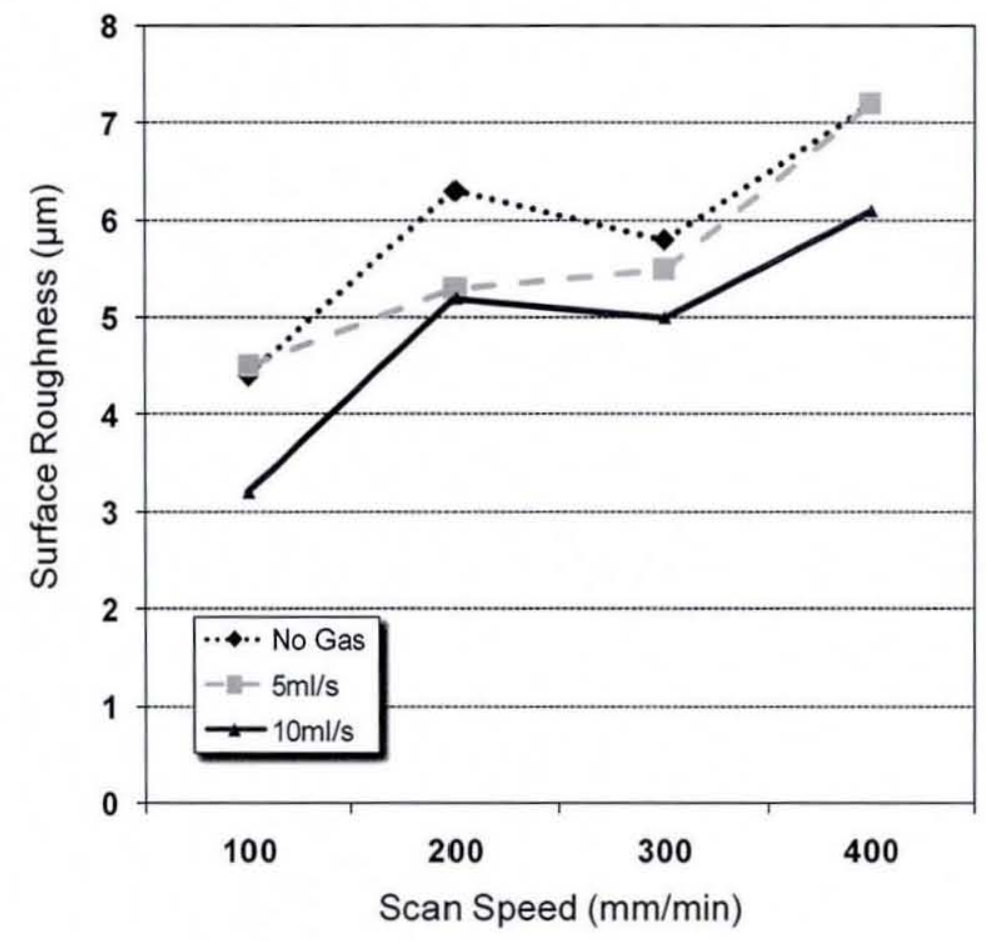

Figure 12-6 Surface roughness against repetition rate for specimens produced at varied shielding gas flow rates

\subsubsection{Summary}

It has been demonstrated that an inert gas shielding is an extremely important factor within laser metal processing. The shielding gas helped to reduce part contamination (excessive spatter, black soot residual), reduce oxygen content within a part and improves surface finish (reduction in oxides). A gas flow rate of $10 \mathrm{ml} / \mathrm{s}$ was selected as the optimum gas flow rate for shielding the molten metal during processing using SLM.

\subsection{Electrostatic effect}

Within powder handling and processing there is a potential for electrostatic charge generation and retention. The magnitude of resulting electrostatic charge depends on factors such as material properties powder surface area, resistivity and handing/processing methods. A laser beam interacting with the surface of a material generates an intense electric field, localised under the irradiated surface [Dumitru et al. 2005]. 
During the inert gas optimisation experiments it was found that an optimised gas flow rate of $10 \mathrm{ml} / \mathrm{m}$ did not move or scatter material away from the laser interaction zone. However it was noticed that after processing the powder not consolidated into melt was located a distance away from the laser material interaction zone. There was a void between processed material and loose material. This effect occurred with and without the use of a shielding gas. The size of this void increased as higher repetition rates were used. Figure 12-7 shows the plan view of a single laser scan from a $0.1 \mathrm{~mm}$ powder bed using $0.5 \mathrm{~ms}, 1 \mathrm{~J}, 50 \mathrm{~Hz}$ and $100 \mathrm{~mm} / \mathrm{min}$ laser processing parameters. There is approximately a $0.25 \mathrm{~mm}$ gap between consolidated material and loose powder. This is a most unfavorable scenario when building parts from a powder bed. Subsequent scans to the left or right of this initial scan will be devoid of material, producing parts with possible increased porosity and geometric distortions.

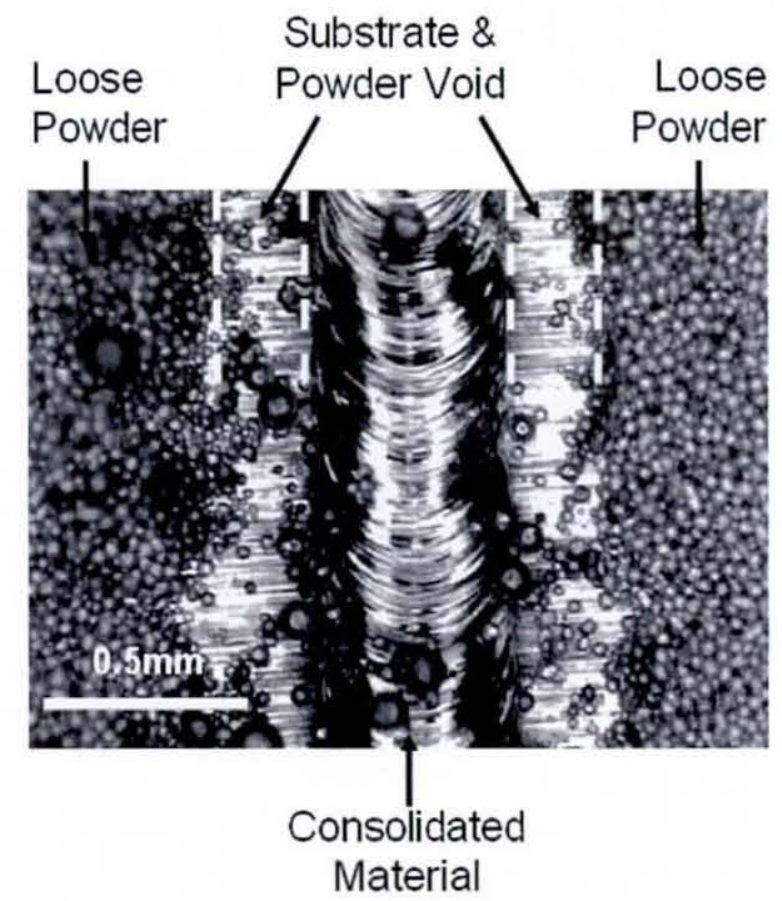

Figure 12-7 Plan view of single scan of Inconel 625 from powder bed

It was suspected that recoil pressure generated from the plasma plume may have dislodged powder, however there did not visually appear to be substantial differences in the gap generated with larger or smaller plasma plume. A very small plasma plume (using low pulse energy) still produced a gap. It was believed that an electrostatic charge built up as the laser interacted with the material. This charge build up may have 
caused material to scatter away from laser material interaction zone. Figure 12-8 illustrates the theory of the electrostatic effect.

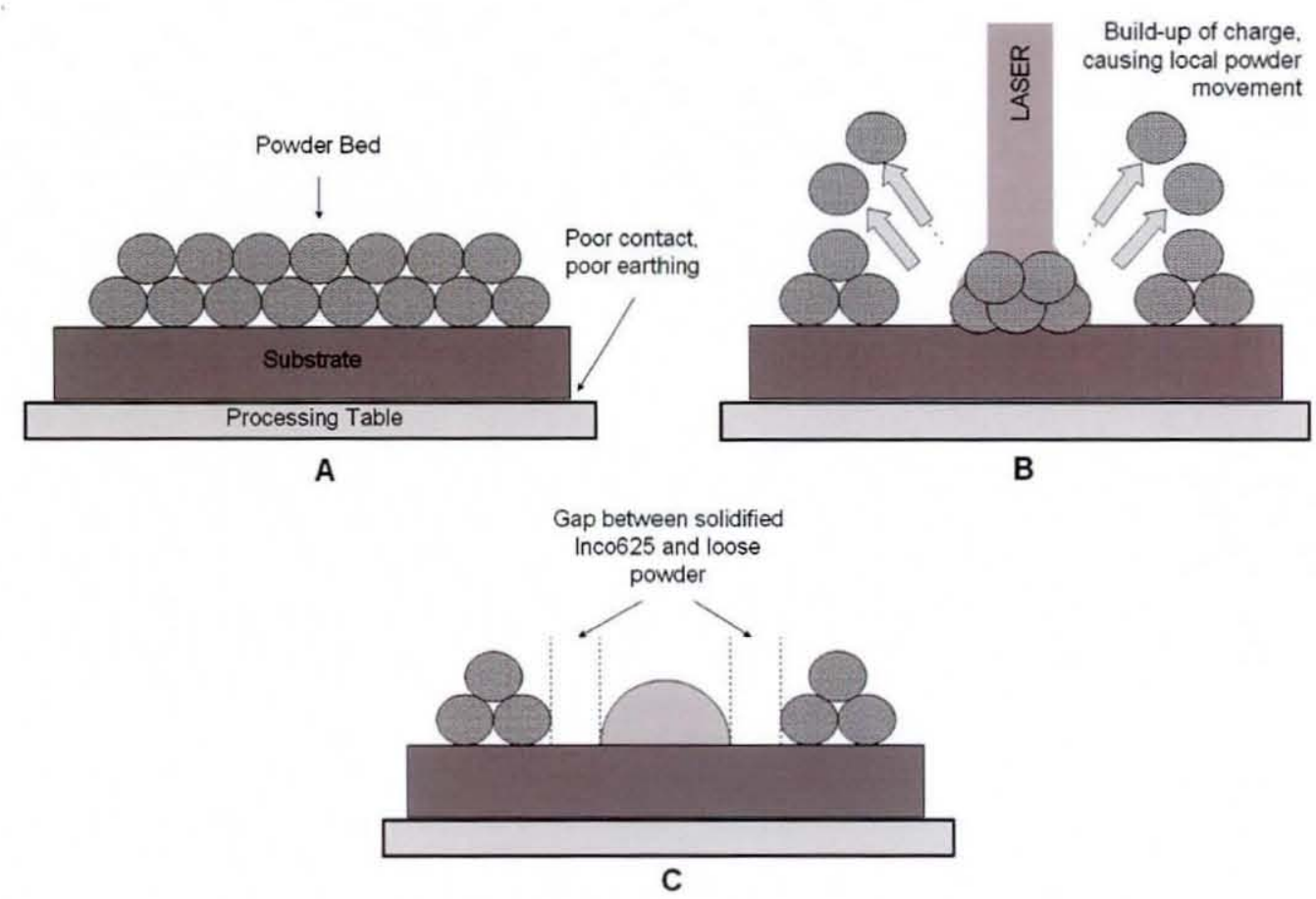

Figure 12-8 Possible electrostatic effect generated by laser material interaction

\subsubsection{Removal of the Electrostatic Effect}

As a possible solution to the electrostatic effect it was envisioned that a discharge of any static charge through the substrate would prevent movement of powder. This is conventionally known as "earthing". The steel substrate sits on top of an aluminium processing table that is connected to a $\mathrm{CNC}$ adjustable table, the table is then earthed. It was believed that a poor contact was being made between substrate and processing table. A revised earthing arrangement was made, the substrate was directly connected to a designated earthing point shown in Figure 12-9. 

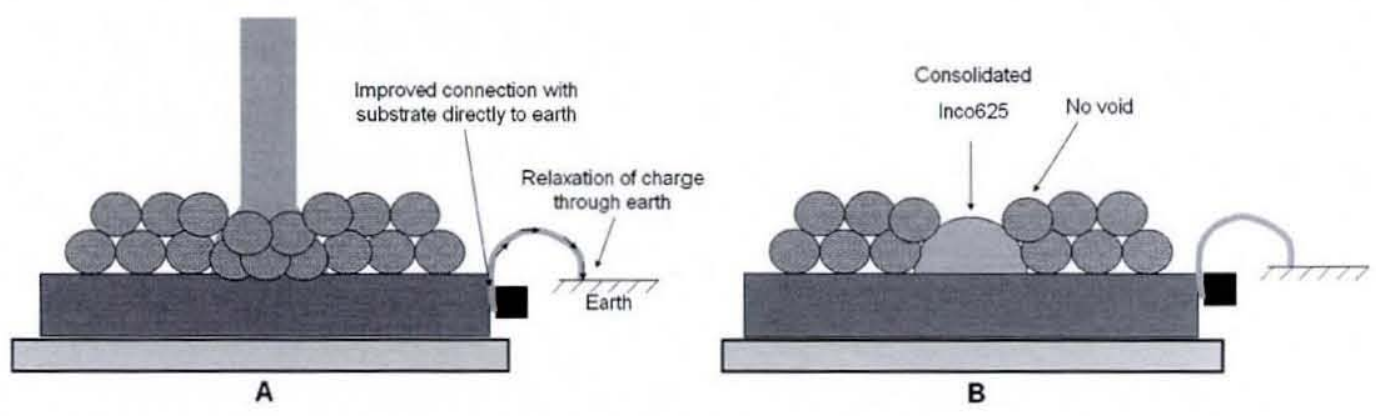

Figure 12-9 Revised earthing arrangement

This revised earthing arrangement was fitted to the existing SLM system. It was found that this modification significantly reduced effects of electrostatic charge. A plan view comparison of improved earthing is shown in Figure 12-10. The loose powder engulfs the side of the consolidated material, indicating that there is no undesirable movement of powder due to the electrostatic effect.
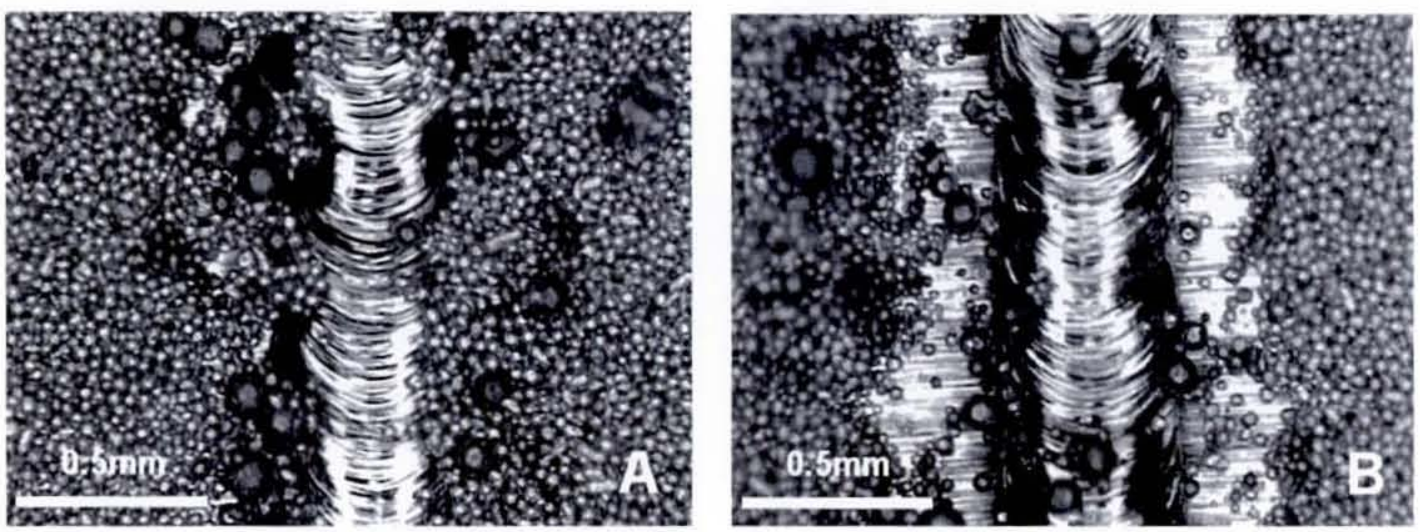

Figure 12-10 Plan view of powder bed processed with improved earthing (a) \& without (b) (processed using $0.5 \mathrm{~ms}, 1 \mathrm{~J}, 60 \mathrm{~Hz} \& 100 \mathrm{~mm} / \mathrm{min}$ )

The generation of any static charge is discharged through substrate into earthing points. This system modification is a key refinement in the processing using SLM. The quality of parts should improve due to a sufficient amount of material being available for subsequent laser scans. 


\title{
12.3 Publications by Author
}

\subsubsection{Laser melting functionally graded composition of Waspaloy and Zirconia powders}

\section{Laser melting functionally graded composition of Waspaloy ${ }^{(}$ and Zirconia powders}

\author{
Kamran Aamir Mumtaz · Neil Hopkinson
}

Received: 13 November 2006/Accepted: 5 March 2007/Published online: 3 June 2007

(8) Springer Science+Business Media, LLC 2007

\begin{abstract}
An approach for fabricating functionally graded specimens of supernickel alloy and ceramic compositions via Selective Laser Melting (SLM) is presented. The focus aimed at using the functionally graded material (FGM) concept to gradually grade powdered compositions of Zirconia within a base material of Waspaloy ${ }^{8}$. A high power Nd:YAG laser was used to process the material compositions to a high density with gradual but discrete changes between layered compositions. The graded specimens initially consisted of $100 \%$ Waspaloy ${ }^{\circ}$ with subsequent layers containing increased volume compositions of Zirconia $(0-10 \%)$. Specimens were examined for porosity and microstructure. It was found that specimens contained an average porosity of $0.34 \%$ with a gradual change between layers without any major interface defects.
\end{abstract}

\section{Introduction}

Functionally graded materials

Functionally graded materials (FGMs) are composites of two or more materials with continuously varying volume fractions. Used as coatings and interfacial zones, they help to reduce mechanical and themally induced stresses caused by a material property mismatch and can improve bonding strength. Functionally graded materials can be

K. A. Mumtaz (四) - N. Hopkinson

Rapid Manufacturing Research Group, School of Mechanical

and Manufacturing Engineering, Loughborough University.

Wolfson Building, Loughborough LEII 3TU. UK

e-mail: k.a.mumtaz@lboro.ac.uk designed at varied material gradients to suit a specific application and achieve levels of performance superior to that of homogeneous materials. They can be applied to metals, ceramics and organic composites to generate improved components, they are increasingly being considered in industry for various applications to maximize strengths and integrities of many engineered structures [1]. Functionally graded materials are ideal candidates for applications that experience severe thermal gradients, ranging from thermal structures in aerospace engines to computer circuit boards [2].

An area in which FGMs could play an important role in is the protection of aerospace, automobile and heavy duty industrial utility applications from high temperature environments. Studies related to the improvement in performance of materials for high temperature applications have mainly focused upon the thermally insulating systems of Thermal Barrier Coatings (TBC) [3]. This heat resistant coating is made from materials that have low thermal conductivities such as the ceramic Zirconia. They are applied to the surface of parts or substrates by metallurgical methods of diffusion saturation, electroplating. plasma spraying and Electron Beam Physical Vapor Deposition (EB-PVD) [4]. Initially a bond coat (conventionally MCrALY [5]) is applied to the surface of the application followed by the TBC, this is shown in Fig. 1 .

Nozzle guide vanes (NGV) found within jet engines are a typical application that uses a TBC. These applications experience poor bonding strength between TBC and component when subjected to mechanical and themal stresses after long-term usage. Applications that employ a TBC are typically used within an oxidizing environment and undergo repetitive thermal cycles $[6,7]$. Thermal stresses are induced by a thermal expansion mismatch between coatings, leading to cracking, delamination and eventual 


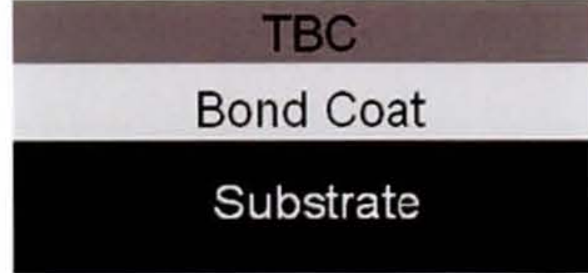

Fig. 1 Cross-sectional view of a TBC. bond coat and application substrate

part failure [8-10]. Significant oxidization problems occur due to the presence of the bond coat resulting in a large coating expansion and generation of high residual stresses at interfaces [11, 12].

In an effort to release the residual stresses and improve the properties of conventionally formed heat protective coatings, the concept of FGM has recently been used to alleviate these problems [13]. Functionally grading the protective heat resistant coating within the substrate of the application can be used to alleviate the defects associated with multiple material interfaces [14, 15]. A gradual transition in properties through the coating thickness can be established [16, 17] and will assist the reduction in residual stresses $[18,19]$ associated with thermal expansion mismatch $[20,21]$. The external part of the specimens resistance to thermal shock cracking can be improved [22. 23] as well as a superior bonding between compositions of material allowing for it to become more resistant to delamination $[24,25]$. It has been demonstrated that functionally graded heat resistant coatings also have better resistance to oxidization and a longer lifetime during test conditions than those formed without any type of functional grading [26].

\section{Solid freeform fabrication}

A great interest has been expressed by industry for the production of FGM components via solid freeform fabrication (SFF) [27]. Solid freeform fabrication is family of processes that involve the layer-wise shaping and consolidation of material (e.g. powder, wire). Due to the additive nature of SFF it inherently holds many manufacturing advantages with the potential to produce parts with a high geometric freedom directly from a CAD model. Solid freeform fabrication also reduce the time period between the initial conceptual design of a part and its actual fabrication. The benefits of using SFF to produce functionally graded parts have led to world-wide research activities [28].

Research has been conducted by Domack et al. to test the suitability of different SFF processes in the production of functionally graded Nickel and Titanium parts. Functionally graded parts were formed using Laser Engineered Net Shaping (LENS). flat wire welding and Ultrasonic Consolidation (UC). LENS builds parts by melting metal powder injected into the trajectory of a high power laser beam. Flat wire welding also uses a laser. however it melts wire without the need to create a molten pool of metal on to a substrate. UC is a process in which metal foil under an applied load is fused together using ultrasonic vibration. It was found that functionally graded parts processed using LENS contained macroscopic cracking and significant elemental segregation. Flat wire welding of materials exhibited good mixing between the wire layers but also exhibited cracking. UC parts demonstrated good metallurgical bonding between Nickel and Titanium interfaces. The researchers concluded that all three manufacturing methods require further development before functionally graded parts can be reliably produced [29]. Liu et al. also used the LENS process to fabricate functionally graded Titanium Carbide and Titanium ( $\mathrm{TiC} / \mathrm{Ti}$ ) parts. The researchers successfully processed these compositions of material to produce functionally graded TiC/Ti crack free parts [30]. Another laser based SFF process known as Selective Laser Sintering (SLS) also uses a laser to melt powders. however the material is processed from a prelayered powder bed rather then by powder injection. Jepson et al. used SLS to produce functionally graded Cobalt and Tungsten Carbide ( $\mathrm{Co} / \mathrm{WC}$ ) parts. The grading of these materials would suit applications that require hardness and wear resistance while maintaining a degree of ductility [3I].

Selective Laser Melting (SLM) is a tool-less manufacturing process capable of building 3D parts by a consecutive layer by layer production of thin cross-sections. SLM produces parts by selectively melting powder from a powder bed using a scanning focused laser beam. this makes the process extremely similar to SLS. The high power laser beam used within SLM can generate heat capable of fully melting powders. This allows for the direct production of high-density parts without any breaks in the build cycle. The SLM process has great potential within the manufacturing of specialized functional applications [32].

Limited research has been conducted using SLM to produce functionally graded parts. Research has included functionally graded Tungsten Carbide/Tool Steel parts [33] and Tool Steel/Copper parts [34]. Literature suggests that no research has used SLM to produce functionally graded nickel alloy and ceramic parts.

Benefits of SLM functionally graded parts

The use of SLM to fabricate functionally graded parts would allow for the control in composition of material 
within each layer and precise regulation of the FGM gradient. Gradually changing the composition of material from metal to ceramic could allow for a TBC to be applied to a component without the requirement of a bond coat. This in turn should reduce the residual stress build up between TBC and application. Combining these technologies could extend the scope and capabilities of laser-assisted manufacturing by reducing production costs improving the performance and durability of functionally graded parts.

Waspaloy ${ }^{\infty}$ is a high temperature supernickel alloy that is typically used within jet engine components. Zirconia is a ceramic that is most commonly used to provide a TBC for these components. This investigation focused on using an Nd:YAG laser to fabricate by SLM functionally graded compositions of these materials. The laser processing parameters used had been specifically optimized for the production of high-density crack free Waspaloy ${ }^{\otimes}$ parts [35].

\section{Experimental methodology}

Setup

A high power $550 \mathrm{~W}$ Nd:YAG pulsed laser (GSI Lumonics $\mathrm{JK} 701 \mathrm{H}$ ) operating at a wavelength of $1.06 \mu \mathrm{m}$ was used. The laser beam had a spot diameter of $0.8 \mathrm{~mm}$. The beam was carried through a fibre optics delivery system and was installed on a 4-axis CNC controlled machine. Powder layers were deposited in one direction by means of a hopper that traversed across the powder bed. Argon was used as a shield gas to prevent parts from oxidization. Parts were built on $43 \times 30 \times 4 \mathrm{~mm}$ steel substrates. The experimental setup is shown in Fig. 2 .

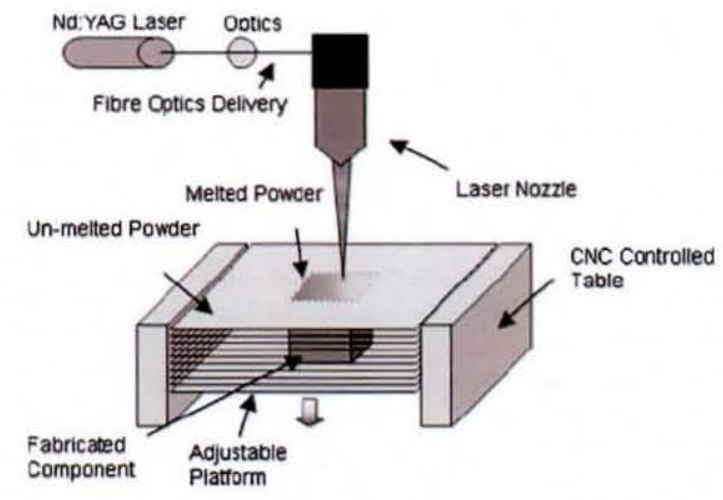

Fig. 2 Experimental setup

\section{Powder properties}

Two powders were used within this investigation. a supemickel alloy Waspaloy ${ }^{8}$ shown in Fig. $3 \mathrm{a}$ and a partially stabilized ceramic Zirconia with $7-9 \%$ yttria (PSZ) shown in Fig. 3b.

The Waspaloy ${ }^{*}$ powder had a mean particle size of $63 \mu \mathrm{m}$. In its solid form it has excellent strength at high temperature and good resistance to corrosion and oxidization. This material has a melt temperature of around 1.330$1,360^{\circ} \mathrm{C}$. Waspaloy ${ }^{\circ}$ has been used for aerospace and gas turbine engine components as well as other miscellaneous engine hardware [36]. The chemical composition of Waspaloy ${ }^{*}$ is shown in Table 1.

The PSZ powder had a mean particle size of $0.5 \mu \mathrm{m}$. The yttria content stabilizes the ceramic at higher temperatures, avoiding phase transition and increasing the spallation life of the ceramic. The material's melting temperature is around $1.350-1.500^{\circ} \mathrm{C}$. its chemical composition is shown in Table 2 . Figure 3 b shows regions of the PSZ powder that has agglomerated. these agglomerates are comparable in size to that of the Waspaloy ${ }^{8}$ powder.

\section{Specimen preparation and processing}

Four composition s of material were used. the first consisted of $100 \%$ Waspaloy ${ }^{*}$ followed by increasing additions of PSZ. Each powder blend was mixed by volume fraction. placed in a ceramic container and blended for six hours. The volume fractions used are shown in Table 3. the SEM images of these mixed composition are shown in Fig. 4.

The placement of these powered compositions is shown in Fig. 5. The layers were graded in the $z$-axis and comprised a total of eight $0.4 \mathrm{~mm}$ thick layers with the PSZ content changed stepwise from 0 to $10 \%$ volume. The first two layers consisted of $100 \%$ Waspaloy ${ }^{*}$. The third and the fourth layers contained volume compositions of $99 \%$ Waspaloy ${ }^{*}$ and 1 \% PSZ. Layers 5 and 6 consisted of $95 \%$ Waspaloy ${ }^{*}$ and $5 \%$ PSZ. Layers 7 and 8 consisted of $90 \%$ Waspaloy ${ }^{*}$ and $10 \%$ PSZ.

\section{Fabricating functionally graded $25 \times 5 \mathrm{~mm}$ specimens}

Initial specimens were $25 \mathrm{~mm}$ in length. $5 \mathrm{~mm}$ in width and consisted of eight $0.4 \mathrm{~mm}$ layers with the functionally graded compositions shown in Fig. 5 .

Five test specimens were made and analyzed under Scanning Electron Microscopy (SEM) and Energy Dispersive X-ray Spectroscopy (EDS) analysis. These test specimens were observed for grading of layers and material segregation. 
Fig. 3 Micrograph of

Waspaloy ${ }^{*}$ (a) and PSZ

particles (b)
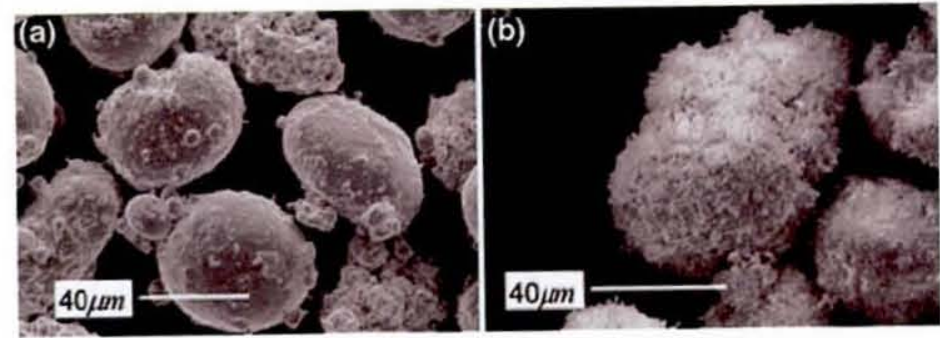

Table I Waspaloy ${ }^{*}$ nominal weight composition in percent [36]

\begin{tabular}{llllllllll}
\hline $\mathrm{Ni}$ & $\mathrm{Co}$ & $\mathrm{Cr}$ & $\mathrm{Mo}$ & $\mathrm{Fe}$ & $\mathrm{Si}$ & $\mathrm{Mn}$ & $\mathrm{Al}$ & $\mathrm{Ti}$ & $\mathrm{Cu}$ \\
\hline 54.0 & 13.5 & 19.5 & 4.2 & 2.0 & 0.7 & 1.0 & 1.4 & 3.0 & 0.5 \\
\hline
\end{tabular}

Table 2 Partially stabilized ceramic Zirconia with 7-9\% yttria weight composition [37]

\begin{tabular}{lllll}
\hline $\mathrm{Y}_{2} \mathrm{O}_{3}$ & $\mathrm{SiO}_{2}$ & $\mathrm{TiO}_{2}$ & $\mathrm{Fe}_{2} \mathrm{O}_{3}$ & $\mathrm{SO}_{3}$ \\
\hline 8 & 0.01 & 0.01 & 0.005 & 0.05 \\
\hline
\end{tabular}

Table 3 Volume compositions of material

\begin{tabular}{llc}
\hline Layer Composition & Waspaloy ${ }^{*} \%$ by volume & PSZ \% by volume \\
\hline Ist & 100 & 0 \\
2nd & 99 & 1 \\
3rd & 95 & 5 \\
4 th & 90 & 10 \\
\hline
\end{tabular}

In previous work a refill scanning strategy that had been successfully developed to produce high-density steel parts using SLM was used for processing these specimens. This scanning and refill strategy is shown in Fig. 6 [33]. Scans 1a. 2a. 3 a etc. were initially performed followed by a refill strategy Ib. 2b, 2c to complete the layer.

Parts were fabricated using optimized laser processing parameters developed in previous work to fabricate high density Waspaloy ${ }^{*}$ specimens [35]. these are shown in Table 4.

\section{Fabricating functionally graded $10 \times 10 \mathrm{~mm}$ specimens}

The $10 \times 10 \mathrm{~mm}$ blocks were created using a varied scanning strategy. The scanning strategy used was similar to that employed for processing the $25 \times 5 \mathrm{~mm}$ (Sect. "Fabricating functionally graded $25 \times 5 \mathrm{~mm}$ specimens"). However. each successive layer was scanned perpendicular to the previous. It was proposed that the use of this seanning strategy would improve the grading of layers and remove any periodic wave structure within the part. This should help avoid the formation of interconnected porosity

Fig. 4 Micrograph images of (a) $99 \%$ Waspaloy $1 \%$ PSZ (b) $95 \%$ Waspaloy $^{3}$ 5\% PSZ (c) $90 \%$ Waspaloy* $10 \%$ PSZ

Fig. 5 Layers of functionally graded composition of Waspaloy ${ }^{*}$ and PSZ graded in the $z$-axis
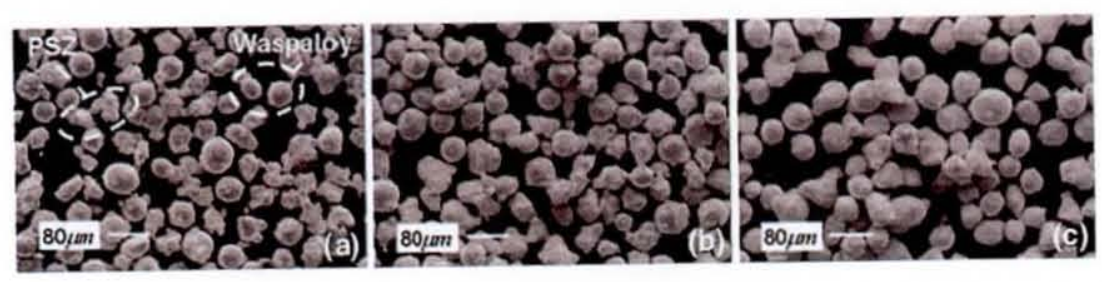

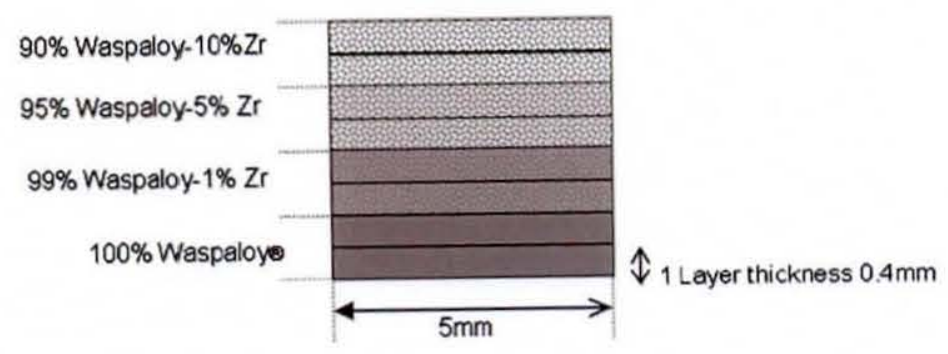




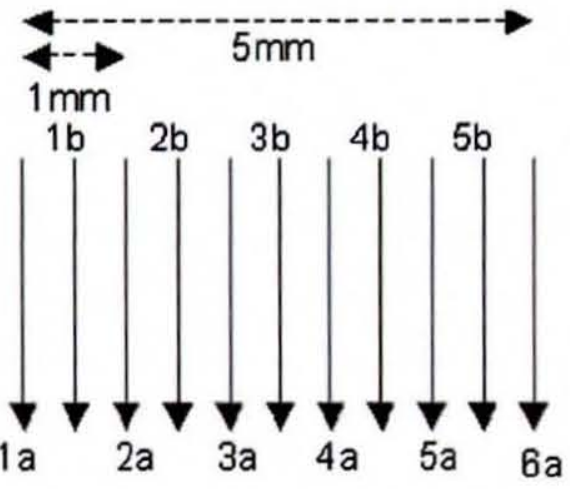

Fig. 6 Plan view of optimized scan and refill strategy

Table 4 Optimized processing parameters for processing Waspaloy ${ }^{2}$

\begin{tabular}{llll}
\hline $\begin{array}{l}\text { Pulse width } \\
(\mathrm{ms})\end{array}$ & $\begin{array}{l}\text { Pulse energy } \\
\text { (J) }\end{array}$ & $\begin{array}{l}\text { Repetition rate } \\
(\mathrm{Hz})\end{array}$ & $\begin{array}{l}\text { Scan speed } \\
(\mathrm{mm} / \mathrm{min})\end{array}$ \\
\hline 5 & 9 & 10 & 168 \\
\hline
\end{tabular}

$[38]$ and prevent layer delamination. The cross-hatching technique and specimen dimensions are shown in Fig. 7.

The same laser processing parameters used for fabricating the $25 \times 5 \mathrm{~mm}$ specimens were also used in the construction of the $10 \times 10 \mathrm{~mm}$ specimens. Once again five test specimens were produced, these specimens were observed for geometrical distortions, the occurrence of porosity and general microstructure including grading of layers. Standard metallographic techniques were used for the preparation of specimens by SEM analysis. An etching solution of $\mathrm{CuSO}_{4}$ was used to reveal the microstructure. The porosity of the specimen was measured using Image Tool [39], a PC software which visually analyses a specimen's cross section and determines its porosity.
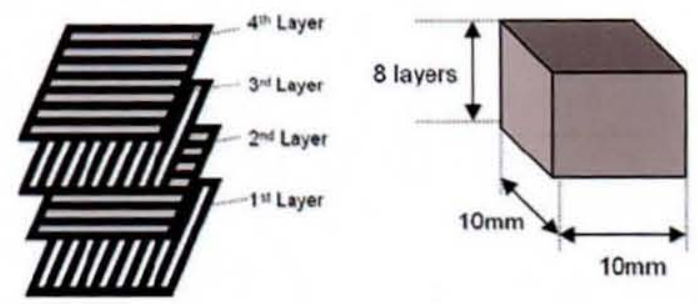

Fig. 7 Illustration of layer cross hatching technique and specimen dimensions

\section{Results}

Functionally graded $25 \times 5 \mathrm{~mm}$ specimen

The $25 \times 5 \mathrm{~mm}$ functionally graded specimen was crosssectioned and viewed using SEM. shown in Fig. 8 . This specimen contained an average porosity of $0.45 \%$. The variation in material composition between the layers is shown with a change in colour. The layers lighten in colour as the PSZ content is increased. Layers with $5-10 \%$ PSZ contain dark segments or bands that could be a result of alloy or ceramic segregation. The locations of each dark segment appear at interlayer boundaries or where the laser initially strikes the materials.

As the volume percentage of Waspaloy ${ }^{*}$ decreased and PSZ increased. there was less likelihood of full melting occurring due to PSZ having a higher melting temperature than Waspaloy ${ }^{8}$. Further to this the laser processing parameters used were specifically optimized for high density processing of $100 \%$ Waspaloy ${ }^{8}$ and not for mixed composition of high temperature ceramic.

The composition within the final layers may not have been fully homogenized within the mixture of materials. In order to determine the content of darker or lighter patches specific regions were subjected to EDS.

\section{Energy Dispersive X-ray Spectroscopy analysis}

A further magnified image of the $25 \times 5 \mathrm{~mm}$ specimen within the region containing 1-5\% PSZ is shown in Fig. 9. A number of dark patehes were observed with one highlighted in region 1. A EDS analysis of the dark area and lighter area within this region was analyzed, the results are shown in Fig. 10.

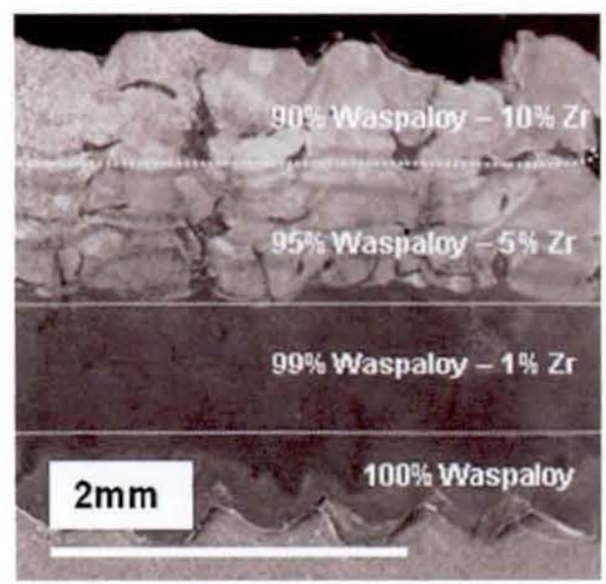

Fig. 8 Cross-sectional image of $25 \times 5 \mathrm{~mm}$ layered FGM specimen 
Fig. 9 Micrograph

characteristics of layers of $1 \%$ and $5 \%$ PSZ layers

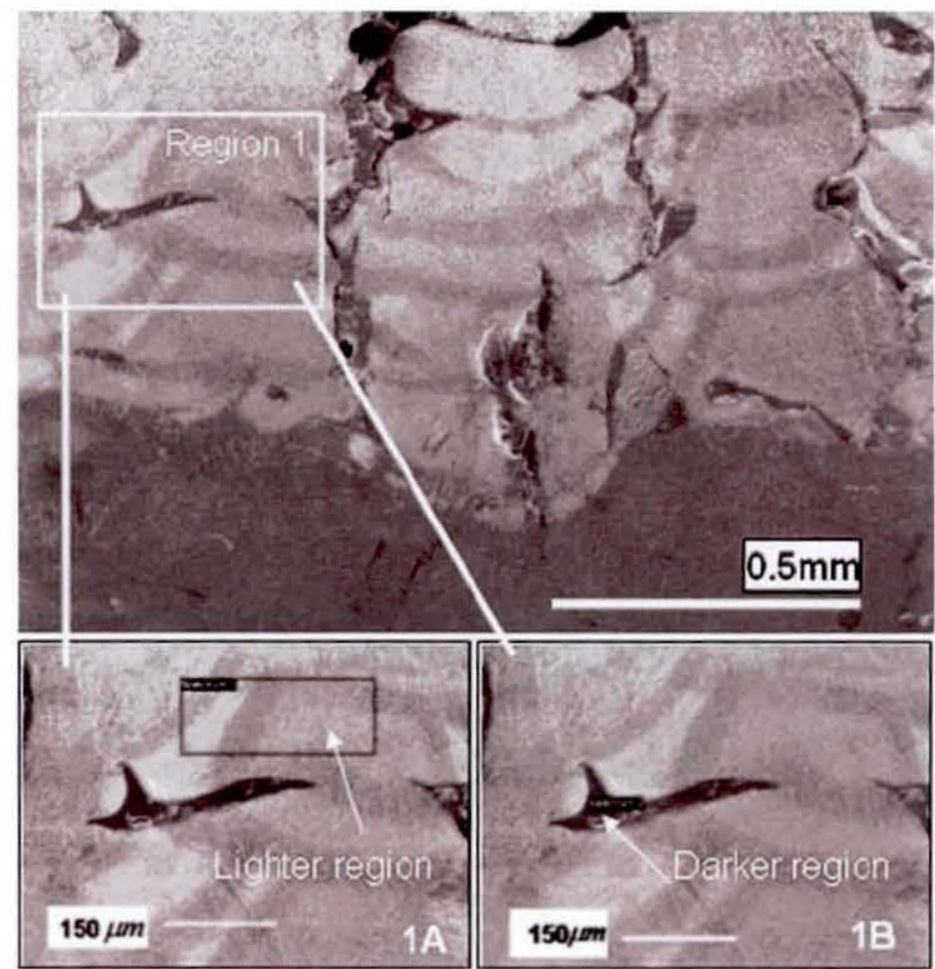

Fig. 10 EDS analysis results

(a) for region $\mathbf{a}$ and $\mathbf{b}$
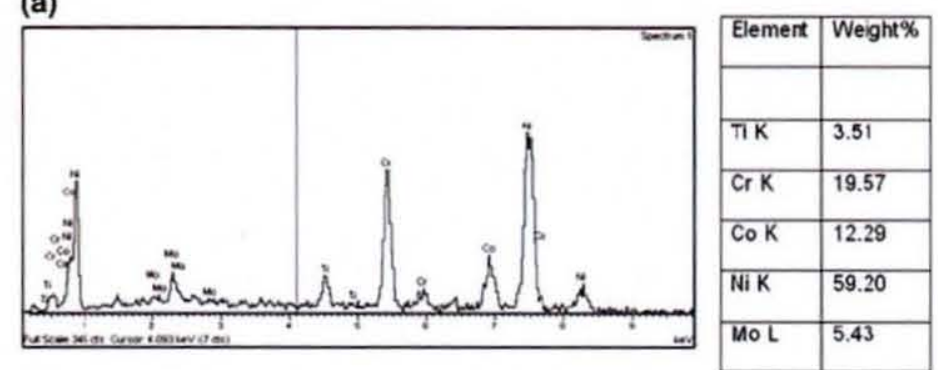

(b)
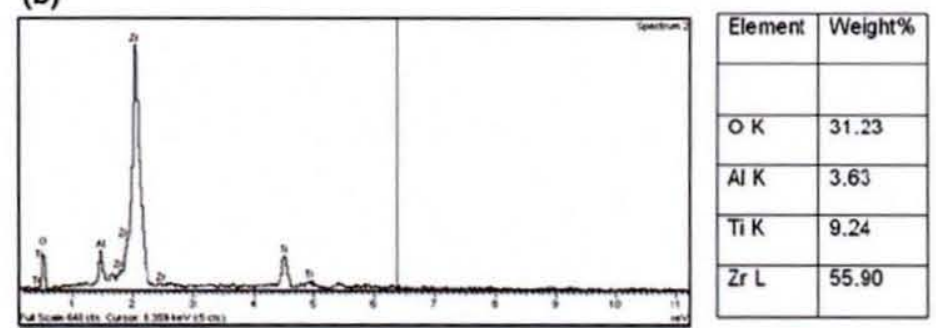

The lighter region in Fig. 9(1A) was analyzed and revealed it contained the same elements present within Waspaloy shown in Fig 10a. The darker area in

Fig. 9(1B) consisted mostly of PSZ with small elements of titanium and aluminium. shown in Fig. 10b. This shows that the PSZ particles accumulated in one particular area

\section{Q Springer}


with only small amounts of Waspaloy ${ }^{8}$ elements (titanium and aluminium). This could be a result of the original agglomeration of PSZ observed in Fig. 3b. The small particle size of PSZ may make it difficult to break up agglomeration, separate and distribute particles within a Waspaloy ${ }^{\otimes}$ mixture causing isolated patches of PSZ to form.

The difference in material properties of Waspaloy ${ }^{\otimes}$ and PSZ may cause these materials to react differently within the melt pool. The heat generated from the centre of the laser interaction zone lowers the density of heated powders. Cooler powders at the edge of the Heat Affected Zone (HAZ) will have higher densities. Therefore gravity will cause molten material at the edge of the HAZ to sink within the melt pool due to gravity (Buoyancy force). Different material densities will cause variations in the movement of material within the melt. This may explain why there is segregation of materials within layers containing higher PSZ content (Fig. 8), Another factor that may contribute to material segregation is surface tension. The surface tension of a materia reduces with increasing temperature, cooler material at the edge of a HAZ will pull material from the centre of the HAZ to the edge (Marangoni convection). Waspaloy ${ }^{\$}$ conducts heat better than PSZ (subsequently melting over a larger HAZ) due to it having a higher thermal conductivity. There will therefore be a variation in movement of materials within melt due to surface tension forces.
Despite the segregation of materials, the solidification structure around the embedded PSZ shows good wettability, resulting in a dense structure. This scenario is certainly preferable to experiencing defined voids around the agglomerated PSZ due to poor wetting.

High density $10 \times 10 \mathrm{~mm}$ functionally graded specimen

A fabricated $10 \times 10 \mathrm{~mm}$ specimen can be seen in Fig. 11. The cross-sectioned specimen viewed under an SEM microscope is shown in Fig. 12 .

The top layer of the specimens is not completely flat, it is assumed that the scanning strategy as well as the differences between material properties of Waspaloy ${ }^{8}$ and PSZ contributed to the unevenness of the top layer. As mentioned in Sect. "Functionally graded $25 \times 5 \mathrm{~mm}$ specimen" the differences in material properties may have caused an alloy ceramic segregation. The placement and movement of material within the melt pool could have affected the formation of the top layer. The extreme ends of the block suffer from bulges or elevated edges that rise above the rest of the specimen. This is attributed to the scanning strategy initially processing the outer profile of the specimen. A large amount of material will initially be drawn into the melt pool, this leaves less material for subsequent scans within the same layer. Another factor connected to the availability of loose powder is the presence of existing solidified material.
Fig. 11 Functionally graded $10 \times 10 \mathrm{~mm}$ specimen before and after cross-sectioning
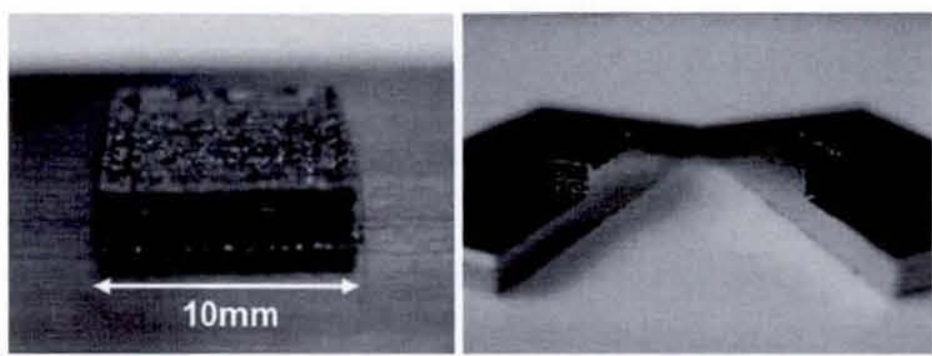

Fig. 12 Cross-sectional view of $10 \times 10$ specimen

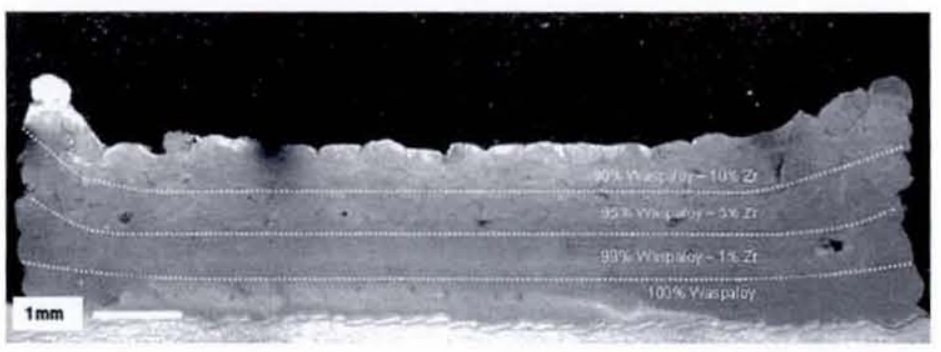


The functionally graded specimens were crack free and contained an average porosity of $0.34 \%$. The porosity was more prevalent within layers containing higher compositions of PSZ.

The layers of different material composition appear to grade more evenly than that seen in Fig. 8, producing less discrete changes in layer boundaries. The dark bands that were predominate in the previous specimen are far less recognizable, indicating an improved and more even distribution of Waspaloy ${ }^{\alpha}$ and PSZ particles. The presence of PSZ is noticeable with increasing volume of PSZ that appear to lighten the contrast of layers. The four compositions of material result in four varying contrasts throughout the layer, occurring at $0.5 \mathrm{~mm}$ intervals along the $z$-axis.

The improved grading of $\mathrm{W}_{\text {aspaloy }}{ }^{*}$ and PSZ may have been a result of the cross-hatching strategy breaking up any unevenly distributed and repetitive heat build-up along the layer causing segregation of materials due to differences in material properties. Research has shown that porosity can propagate through subsequent layers forming an interconnected void. Cross-hatching can eliminate interconnected porosity and maintain a level of uniformity throughout the layer [36]. This is demonstrated by the fact that the $25 \times 5 \mathrm{~mm}$ functionally graded specimen was produced without cross-hatching and contained porosity $0.45 \%$. whereas the $10 \times 10 \mathrm{~mm}$ sample was fabricated using cross-hatching and held a lower porosity of $0.34 \%$.

Figure 13 shows the cross-sectioned specimen at a higher magnification, little transition between the different material compositions was observed. The exact location and morphology of PSZ was not visible due to low magnification. However initial observation and comparison of specimen cross-sections generally indicates an improvement within the grading and distribution of materials. This is a promising feature for high temperature applications as it may alleviate the problems associated with defined interfaces between two different materials. These problems include thermal expansion mismatch. high residual stresses, cracks and possible delamination.

Region one in Fig. 13 shows very fine precipitates of gamma prime with angles between the grain boundary. This very fine or amorphous microstructure may be a consequence of the rapid cooling rate near the top of the specimen.
Fig. 13 Micrographs characteristics of specimen of FGM
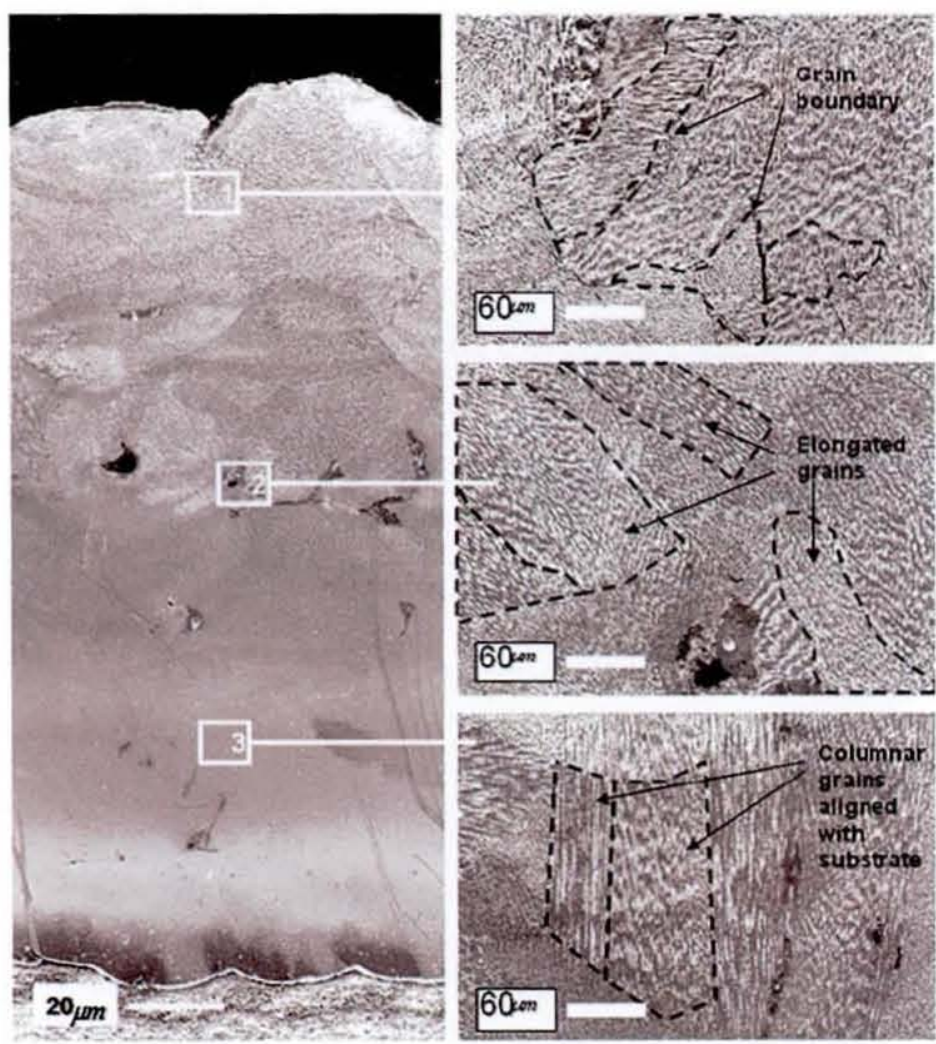
Region two in Fig. 13 shows an area where a small amount of porosity is present. The grain sizes appear to increase in size possibly due to a longer cooling period as result of re-melting and slow processing speed.

Region three in Fig. 13 shows a columnar grain structure that aligns along the direction of the substrate due to directionally cooling through the steel substrate.

It has been shown that despite stepwise stacking, the microstructure and distribution of metal and ceramic elements gradually change without distinct observable boundaries throughout the layers.

\section{Conclusion}

Using SLM to produce functionally graded parts has proven successful when grading small amounts of ceramic within a nickel alloy. It has been demonstrated that despite stepwise stacking, the microstructure and the distribution of material elements are gradually changed without distinct boundaries between layers when a cross-hatching scan strategy is used.

Increasing compositions of $1 \%, 5 \%$ and $10 \%$ PSZ were functionally graded with Waspaloy ${ }^{10}$ and processed using optimized laser parameters. Initially a $25 \times 5 \mathrm{~mm}$ functionally graded specimen was fabricated to a density of $99.55 \%$. This contained dark bands or regions that may have been caused by an alloy or ceramic segregation. EDS analysis found that small areas or patches of agglomerated material were compositions of PSZ. This segregation of material may have been due to the difference in Waspaloy ${ }^{*}$ and PSZ material properties. This could have caused a variation in movement of material within the melt pool due to the Buoyancy and Marangoni affects. The PSZ compositions within the layers showed good wettability when embedded amongst solidified Waspaloy ${ }^{\infty}$ and therefore good bonding.

The $10 \times 10 \mathrm{~mm}$ specimen fabricated using a crosshatching scanning technique had an average density of $99.66 \%$. The specimen experienced geometric unevenness and tended to bulge at its edges due to large amounts of material being pulled into the melt of initial scans. However the cross-hatching scanning technique was found to improve the grading of material compositions making interlayer boundaries less discrete. Initial analysis suggests that the segregation and agglomeration of PSZ experienced within earlier specimens had been significantly reduced as a result of the modified scanning strategy.

The laser's heat distribution when processing compositions of material from a powder bed is a significant factor. It affects the part porosity, location of material within a layer and has the potential to generate uniformity within functionally graded parts.
Early tests suggest that functionally graded compositions of Waspaloy ${ }^{\circ}$ and PSZ can be processed using SLM to produce high-density crack free parts. The functionally graded layers are less likely to experience severe thermal expansion mismatch and delamination. Whether fabrication using the SLM process improves the performance of the component and avoids premature failure can only be determined after further advanced testing and analysis.

\section{References}

1. Gu Y, Nakamura T, Prchlik L, Sampath S. Wallace J (2003) Mater Sci Eng A 345(1-2):223

2. Aboudia J. Pinderab MJ. Arnold SM (1999) Composites Part B 30:777

3. Polat A, Sarikaya O, Celik E (2002) Mater Des 23:641

4. Movchan BA, Yu K (2004) Surf Coat Technol 188-189:85

5. Quadakkers WJ, Shemet V. Sebold D, Anton R. Wessel E. Singheiser L (2005) Surf Coat Technol 199(1):77

6. Miller RA. Lowell CE (1982) Thin Solid Films 95(3):265

7. Miller RA, Berndt CC (1984) Thin Solid Films 119(2):195

8. Gill SC, Clyne TW (1990) Metall Trans 21 B:377

9. Ferrari M. Lutterotti L (1992) J Eng Mech 118(9):1928

10. Singheiser L Steinbrech L. Quadakkers WJ. Herzog R (2001) Mater High Temp 18(4):249

11. Nomura N, Gasik M. Kawasaki A. Watanabe R (2000) Ceram Trans 114:233

12. Limarga AM, Widjaja S, Yip LK (2002) Int J Mod Phys B 16:233

13. Choules BD. Kolini K (1996) J Eng Mater Technol Trans ASME 118(4):522

14. Sasaki M. Hirirai T (1991) Cent Mem Iss Ceram Soc Jpn 99:1002

15. Watanabe R. Takahashi H. Tamura M. Shiota I. Yoshida T. Kurino T (1993) Functionally gradient materials. FGM Forum Japan. p 1-10

16. Khor KA, Dong ZL_, Gu YW (1999) Mater Lett 38:437

17. Dong ZL, Khor KA, Gu YW (1999) Surf Coat Technol V 113:181

18. Jung $\mathrm{YH}$, Choi SC (1997) J Mater Sci 32:3841, DOI: 10.1023/A 1018640126751

19. Demirkiran AS, Elik E, Avci E (2001) Proc Fifth Int Fract Conf 10:1341

20. Demirkiran AS, Avci E (1999) Surf Coat Technol 116-119:292

21. Demirkiran AS, Elik E. Avci E (1997) Oxidation of functionally gradient materials. Proceedings of the Fifth Combustion Sym posium, Bursa (Turkey), July 21-23, p 543

22. Kawasaki A. Watanabe R. Yuki M. Nakanishi Y, Onabe H (1996) Mater Trans JIM 37(4):788

23. Khor KA, Gu YW (2000) Thin Solid Films 372:104

24. Kokini K, Dejonge J. Rangaraj SV. Beardesly BM (2002) Surf Coat Technol 154:223

25. Yonushonis T (1995) NASA Conf Publ 3312:235

26. Gasik M. Kawasaki A. Kang YS (2005) Mater Sci Forum 492 $49: 9$

27. Wohlers T (2003) Wohlers Report 2003 Rapid prototyping and tooling state of the industry. Annual Worldwide Progress Repor

28. Cherradi N. Kawasaki A. Gasik M (1999) Comp Eng 4:883

29. Domack MS, Baughman JM (2005) Rapid Prototyping J I(11):41

30. Liu W, Dupont JN (2003) Scripta Mater 9(48):1337

31. Jepson L, Beaman JJ, Bourell DL, Wood KL, (1997) SLS processing of functionally graded materials. SFF Symposium Proceedings, p 67

32. Kruth P, Froyen L, Vaerenbergh JV, Mercelis P, Rombouts M. Lauwers B (2004) J Mater Process Technol 149(1-3):616 
33. Su WN (2002) Layered fabrication of tool steel and functionally graded material with a Nd:YAG pulsed laser. PhD Thesis, Loughborough University

34. Beal VE, Erasenthiran P. Hopkinson N, Dickens P, Ahrens CH (2006) Int J Adv Manuf Technol 30:844

35. Mumtaz K. Hopkinson N, Erasenthiran P (2006) High density selective laser melting of Waspaloy ${ }^{*}$. SFF Symposium Proceedings, p 220
36. Special Metals, "Waspaloy". www.SpecialMetals.com, as on Ist August 2006

37. Mel chemical, "Zirconia", www.mel-chemical.com, as on Ist August 2006

38. Morgan RH. Papworth AJ. Sutcliffe C. Fox P. O'neill W (2002) J Mater Sci 37:3093, DOI: 10.1023/A: 1016185606642

39. Uthsea. The University of Texas Health Science Center at San Antonio, ImageTool Version 3.0 


\title{
12.3.2 High density selective laser melting of Waspaloy
}

JOURNAL OF MATERTALS PROCESSING TECHNOLOGY 195 (2008) 77-87

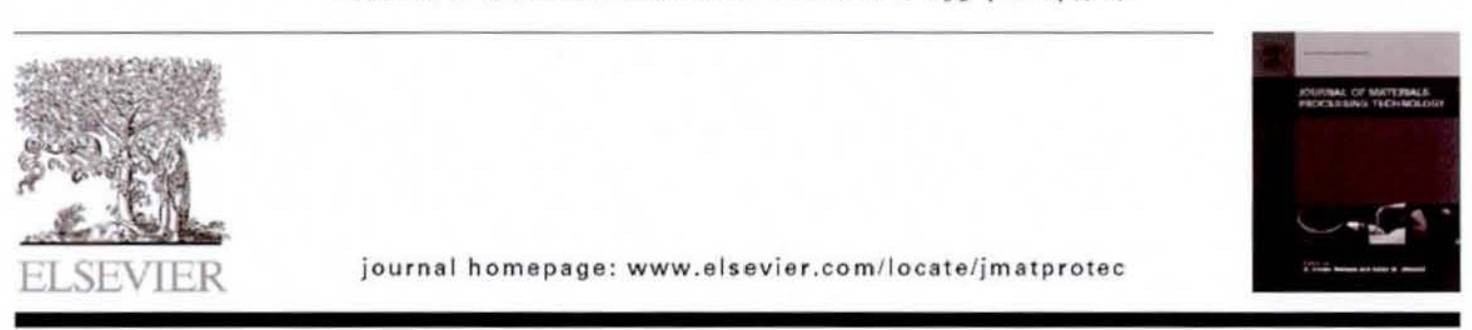

\section{High density selective laser melting of Waspaloy ${ }^{\circledR}$}

\author{
Kamran Aamir Mumtaz*, Poonjolai Erasenthiran, Neil Hopkinson \\ Loughborough University, Rapid Manufacturing Research Group, Loughborough LE11 3TU, United Kingdom
}

\section{A R I C LE IN FO}

\section{Articie history}

Received 31 August 2006

Received in revised form

17 April 2007

Accepted 18 April 2007

Keywords:

Selective laser melting

Laser melting

Solid freeform fabrication

Direct fabrication

Waspaloy ${ }^{\circ}$

\begin{abstract}
A B S T R A C T
In this work, high density Waspaloy" specimens were produced using selective laser melting (SLM). SLM of Waspaloy* powder was performed using a high power pulsed Nd:YAG laser. The laser parameters pulse energy $(\mathrm{J})$, pulse width (ms), repetition rate $(\mathrm{Hz})$ and scan speed $(\mathrm{mm} / \mathrm{min})$ were varied. Process parameter optimization was achieved using factorial analysis to investigate the relationship between specific processing parameters and the formation of Waspaloy ${ }^{5}$ specimens. The optimized processing parameters produced Waspaloy ${ }^{3}$ specimens that were $99.7 \%$ dense. The resultant laser melted specimen's height, width and contact angles were measured. Specimens were also tested for the occurrence of porosity and observed for microstructure.
\end{abstract}

๑ 2007 Elsevier B.V. All rights reserved.

\section{Introduction}

Due to an increased demand for shortened product development cycles, there exists a need to rapidly fabricate fully functional high density metal parts without the requirement for hard tooling (Yevko et al., 1998). Theses demands can be met using solid free fabrication (SFF), a family of processes that involve a layer-wise shaping and consolidation of material (e.g. powder, wire) with the potential to produce fully dense parts with a high geometric freedom directly from a CAD model. SFF processes have the potential to drastically reduce the time period between the initial conceptual design of a part and its actual fabrication (Wohlers, 1996).

Laser-based SFF processes use the heat generated by a focused laser beam to partially or completely melt deposited material. Research efforts have demonstrated the great potential available within laser-based SFF to manufacture metal components with microstructures and mechanical properties equivalent or superior to conventionally processed materials (e.g. casting, hot isostatic pressing) (Bourell et al., 2002).
Xue et al. used an Nd:YAG laser to fully melt Inconel 625 and $316 \mathrm{~L}$ stainless steel powders. This produced fully functional Inconel parts to a full density having higher yield and ultimate tensile strengths than cast material and comparable to wrought material (Xue and Islam, 2000). The 316 stainless steel parts had a higher yield strength compared to cast or wrought material, while the ultimate strength and elongation were comparable. Zhang et al. used laser processing techniques to melt 663 copper alloy and 316L stainless steel powders (Zhanget al., 2003). The parts were fabricated to a full density, having mechanical properties reported to be equivalent to conventionally processed metal parts.

Several laser-based SFF processes have been developed and commercialized for processing metal. These include laser engineered net shaping (LENS ${ }^{\mathrm{TM}}$ ), direct light fabrication (DLF), direct metal laser sintering (DMLS) and selective laser melting (SLM) (Keicher et al., 1998; Lewis et al., 1994; Childs et al., 2005). These processes are capable of completely melting material from a powder bed or deposited/injected material by means of heat generated from a high powered focused laser

\footnotetext{
- Corresponding author. Tel: +44 7830128 444; fax: +44 1509227549

E-mail address: K.a.mumtazelboro.ac.uk (K.A. Mumtaz).

$0924-0136 / \$$ - see front matter $\odot 2007$ Elsevler B.V. All rights reserved. dot:10.1016/).jmatprotec.2007.04.117
} 
beam. LENS and DLF melt powder injected into the trajectory of the laser, this method of powder deposition is ideal for metal component repair operations. However this deposition technique always requires a solid substrate and can suffer from large material wastage due scattering of powder during processing. It also has the added complexity of powder flow rate, a precise flow rate must be maintained at all times during part fabrication to ensure the deposition of an accurate layer thickness. DMLS and SLM melt material in a pre-laid powder bed. Accurate powder layer thicknesses can be deposited with the remaining un-melted powder serving as a supporting structure for the processed material. However in some instances, such as the production of large flat down facing surfaces a support structure is required. The DMLS and SLM processes also allow for recycling and re-use of un-melted powder.

The SLM process is currently the subject of major research studies (Childs et al., 2005; Agarwala et al., 1995), it has been shown to be a viable option for the production of fully functional metallic parts, including titanium dental implants (Tolochko et al., 2002). SLM uses a high power Nd:YAG laser to melt thin layers of metal powder. Nd:YAG lasers operate at lower wavelengths compared to those used by $\mathrm{CO}_{2}$ lasers. This makes processing of metals with high melting temperatures or high reflectivity easier due to the material's increased absorbance to the laser's energy (Santos et al., 2006). SLM research has focused on metals such as aluminium, chromium, nickel (Abe et al., 2001), copper (Pogson et al., 2003), iron (Kruth et al., 2004), tool steel (Su, 2002) and titanium (Santos et al., 2004). Currently no research has been conducted using SLM or any other SFF process to produce fully dense components from powdered compositions of the supernickel alloy Waspaloy ${ }^{2}$. This advanced material can operate at the high temperatures required for high performance aerospace vehicles and propulsive systems. Waspaloy has been processed using casting and forging techniques (Jones and Jackman, 1999) for fabricating high strength/temperature components.

The use of SLM to process Waspaloy ${ }^{6}$ powder gives the user the ability to fabricate fully functional (Lu et al., 2001) high density parts (Over et al., 2002) with high geometric freedom in a single step. There is the potential to produce metal parts with microstructural properties superior to those conventionally processed. These benefits have motivated a considerable amount of exploration into metal processing using SLM (German, 1994; Das, 2003).

Using lasers to fabricate full density components from powders can be difficult due to the formation of part porosity (Jeng and Lin, 2001). The processed material can suffer from the effects of balling, vaporization and reduced wetting between layers. Maintaining part accuracy is another factor that further complicates matters when using high powered lasers. In order to reduce these problems a processing window for each type of material needs to be determined experimentally. Therefore the range of commercially used powders for laser metal processing is limited today (Kruth et al., 2005).

This investigation used SLM to process Waspaloy ${ }^{\circ}$ pow. der from a powder bed. Factorial analysis was used to identify the statistically significant parameters and how it affected the formation of parts. A laser processing region was identified for full bonding of the material and a set of optimized laser processing parameters were developed for the fabrication of fully dense Waspaloy's specimens. These specimens were tested for the occurrence of porosity and observed for microstructure.

\section{Experimental methodology}

\subsection{Set-up}

A high power 550W Nd:YAG pulsed laser (GSI Lumonics $J \mathrm{~K} 701 \mathrm{H}$ ) operating at a wavelength of $1.06 \mu \mathrm{m}$ and spot size of $0.8 \mathrm{~mm}$ was used. The set-up for this system is shown in Fig. 1 . Powder layers were deposited in one direction by means of a hopper that traversed across a $43 \mathrm{~mm} \times 30 \mathrm{~mm} \times 4 \mathrm{~mm}$ steel substrate. Each substrate was secured onto an adjustable platform that was part of a 4-axis CNC controlled processing table. The adjustable platform was lowered in the $z$-axis and allowed for the deposition of further powder layers. The pulsed laser beam generated by the Nd:YAG laser was carried through a fibre optics delivery system, the beam then passed through a laser nozzle onto the powder bed. The heat generated by the laser melted regions of the powder bed that corresponded to the cross-section of the part at that particular layer. Once the layer had been melted the process was repeated until the part had been fully fabricated. An argon gas flow rate of $15 \mathrm{ml} / \mathrm{s}$ was fed through the laser nozzle onto the melt area to prevent parts from the effects of oxidization.

\subsection{Powder properties}

Waspaloy ${ }^{\oplus}$ is a nickel-based, age-hardenable superalloy with excellent high-temperature strength and good resistance to corrosion, notably to oxidation. It is used for aerospace and gas turbine engine components. Current applications include compressors, rotor discs, shafts, spacers, seals, rings and casings, fasteners and other miscellaneous engine hardware, airframe assemblies and missile systems (Special Metals, 2006). The Waspaloy ${ }^{8}$ powder used within this investigation had an average particle size of $63 \mu \mathrm{m}$, this was determined using a Malvern Mastersizer. The chemical composition of Waspaloy ${ }^{2}$ is shown in Table 1.

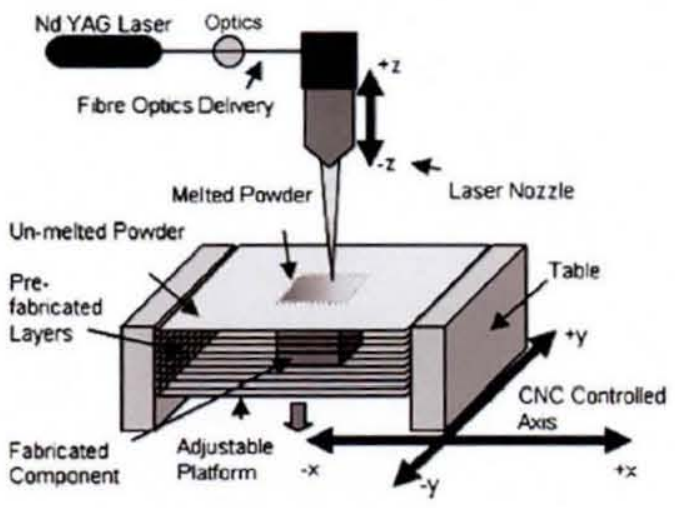

Fig. 1 - Experimental set-up. 


\begin{tabular}{|c|c|}
\hline $\mathrm{Ni}$ & 54.0 \\
\hline Co & 13.5 \\
\hline $\mathrm{Cr}$ & 19.5 \\
\hline Mo & 4.2 \\
\hline $\mathrm{Fe}$ & 2.0 \\
\hline $\mathrm{Si}$ & 0.7 \\
\hline $\mathrm{Mn}$ & 1.0 \\
\hline Al & 14 \\
\hline $\mathrm{T} \mathrm{I}$ & 3.0 \\
\hline $\mathrm{Cu}$ & 0.5 \\
\hline
\end{tabular}

\subsection{Optimization of laser processing parameters}

Processing parameters pulse width (ms), pulse energy (D), repetition rate $(\mathrm{Hz})$ and scan speed $(\mathrm{mm} / \mathrm{min})$ all hold an impact upon the formation of material processed using SLM. Optimization of laser processing parameters for the production of high density Waspaloy parts would involve a number of steps.

Multi-layered parts are built from multi-single layers, therefore it is critical to fully understand and resolve any problems within single layer fabrication before progressing onto multi-layer part fabrication. Initially single scans or beads of Waspaloy were produced in order to gain a quantitative understanding of laser material interactions and the formation of parts.

A bonding region for which the material would fully fuse to the substrate was established. Factorial analysis was employed using the software Statgraphics ${ }^{\mathrm{TM}}$ (Statgraphics, in press) for the test set-up and initial analysis of results. Statgraphics ${ }^{\mathrm{TA}}$ produced a vast array of information analyzed from experimental results and suggested which processing parameters held the greatest influence upon the formation of single layers of Waspaloy ${ }^{s}$. This allowed for an accurate interpretation of results and optimization of pulse width, repetition rate and scan speed values. Multi-layers of Waspaloy ${ }^{\circ}$ were then fabricated using the previously determined optimized parameters, laser pulse energies were adjusted and it's effect on part porosity examined.

\subsubsection{Single scan optimization}

Single scans were processed from a powder bed thickness of $0.9 \mathrm{~mm}$. The scanned tracks were $25 \mathrm{~mm}$ in length and processed within the range of process parameters shown in Table 2. The processing region in which the material would fully fuse, not bond and vaporize was initially investigated. The influence these processing parameters had on the response variables width, height and contact angle was examined. The percentage beam spot overlap shown in Fig. 2 was varied between 60 and $85 \%$. Its impact upon the response

\section{Table 2 - Processing parameters}

Pulse width (ms)

2-8

Pulse energy (t)

$5-15$

Repetition rate $(\mathrm{Hz})$

5-15

Scan speed ( $\mathrm{mm} / \mathrm{min})$

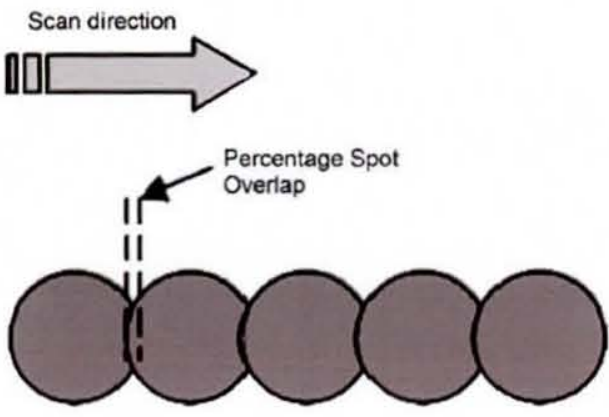

Fig. 2 - Percentage spot overlap.

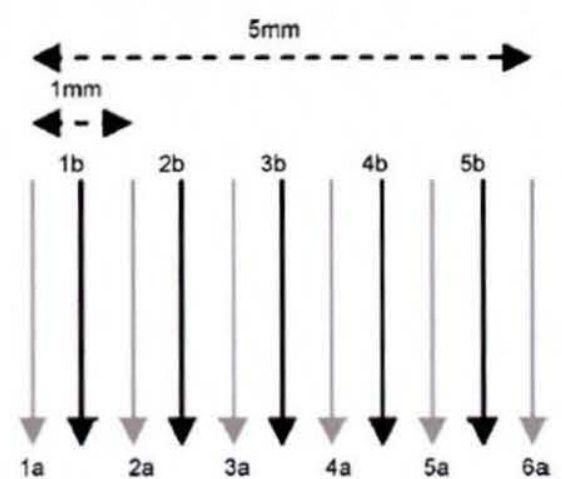

Fig. 3 - Plan view of optimized scan and refill strategy.

variables was also examined. The single scan investigation allowed for the development and identification of optimized processing parameter for pulse width, repetition rate and scan speed.

\subsubsection{Multi-layer optimization}

Further experiments were conducted to identify the influence of pulse energies on multiple layer fabrication. Optimized process parameters for pulse width, repetition rate and scan speed had already been determined from single scan testing. Pulse energies were varied between 6 and $10 \mathrm{~J}$ and were processed from a powder bed thickness of $0.4 \mathrm{~mm}$. The layered specimens were $25 \mathrm{~mm}$ in length, $5 \mathrm{~mm}$ in width and consisted of three $0.4 \mathrm{~mm}$ layers.

A scanning and refill stage strategy that had been successfully developed (Su, 2002) to produce high density steel parts using SLM was used for processing the multi-layered specimens. This scanning and refill strategy is shown in Fig. 3. Scans 1a, 2a, 3a, 4a, 5a and 6a were initially performed, each of these scans were spaced $1 \mathrm{~mm}$ apart from each other. The layer was then refilled to the same layer thickness and the laser continued to scans $1 b, 2 b, 3 b, 4 b, 5 b$ and $6 \mathrm{~b}$ directly in-between the previous scans to complete the layer. This refill strategy helped prevent unnecessary distortion as powder lost to either side of the initial scan area was replaced, allowing for a plentiful supply of powder (Morgan et al., 2002). This scanning strategy also reduced part porosity as powder packing density of the layer was increased. 


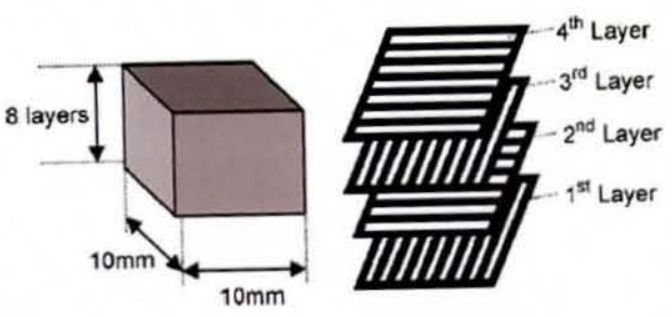

Fig. 4 - Illustration of layer cross-hatching technique and spec.

The effects pulse energies had on the occurrence of porosity within the specimen was observed using optical analysis.

\subsection{Fabricating high density Waspaloy specimen}

A $10 \mathrm{~mm} \times 10 \mathrm{~mm}$ square that consisted of eight $0.4 \mathrm{~mm}$ powdered layers was produced. A refill strategy and cross-hatched scanning technique was used to produce the specimen. A
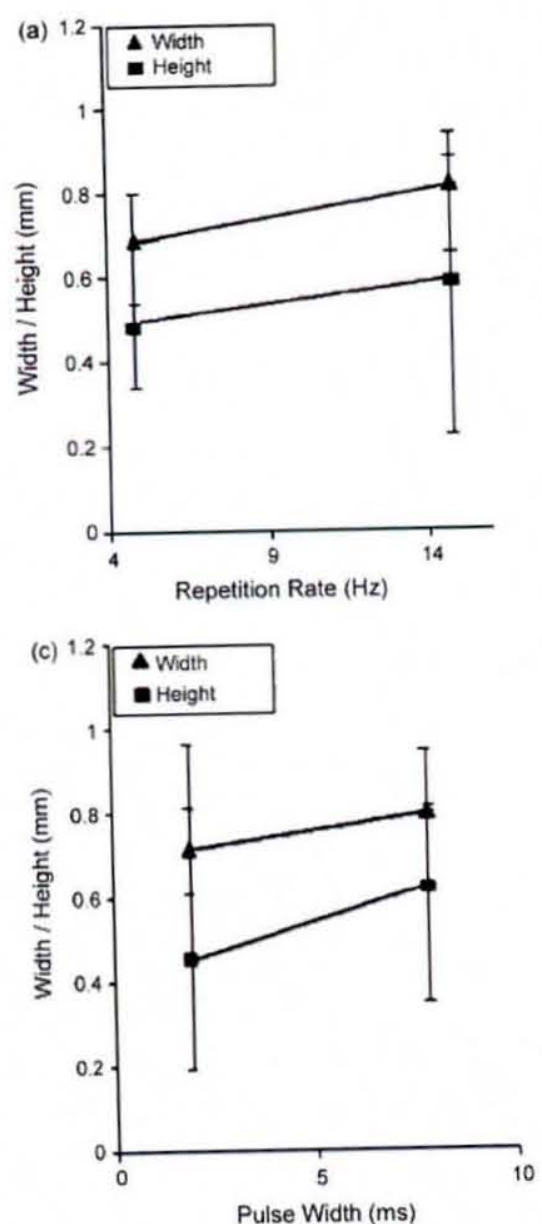

Fig. 6 - (a-d) Single bead, processing parameters against width/height.
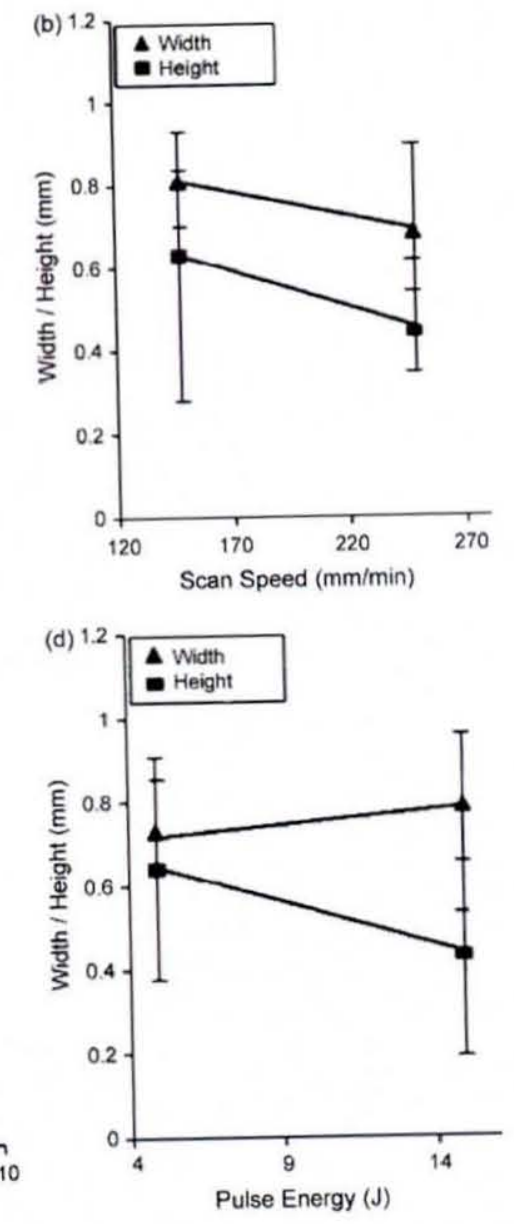

cross-hatching technique scans successive layers perpendicular to the previous, this technique and specimen dimensions are shown in Fig. 4. Cross-hatching would aid the removal of any periodic wave structure by breaking up any predominant or repetitive heat build up in areas along each layer. Research 
has shown that once a pore has formed, it's presence can propagate through subsequent layers forming an interconnected void. Thus cross-hatching helps to avoid the formation of interconnected porosity (Morgan et al., 2002).

The specimen was cross-sectioned and examined for porosity and microstructure.

\subsection{Testing procedure}

2.5.1. Measurement of bead geometry

All samples were cross-sectioned with an abrasive cut-off wheel. Fig. 5 shows the bead height, width and contact angle that were measured using UTHSCA Image Tool Version 3.0 (UTHSCA, 2006).

\subsubsection{Scanning electron microscopy}

Standard metallographic techniques were used in the preparation of specimens for optical scanning electron microscopy (SEM) analysis. An etching solution of $\mathrm{CuSO}_{4}$ was used to reveal the microstructures within the specimen.
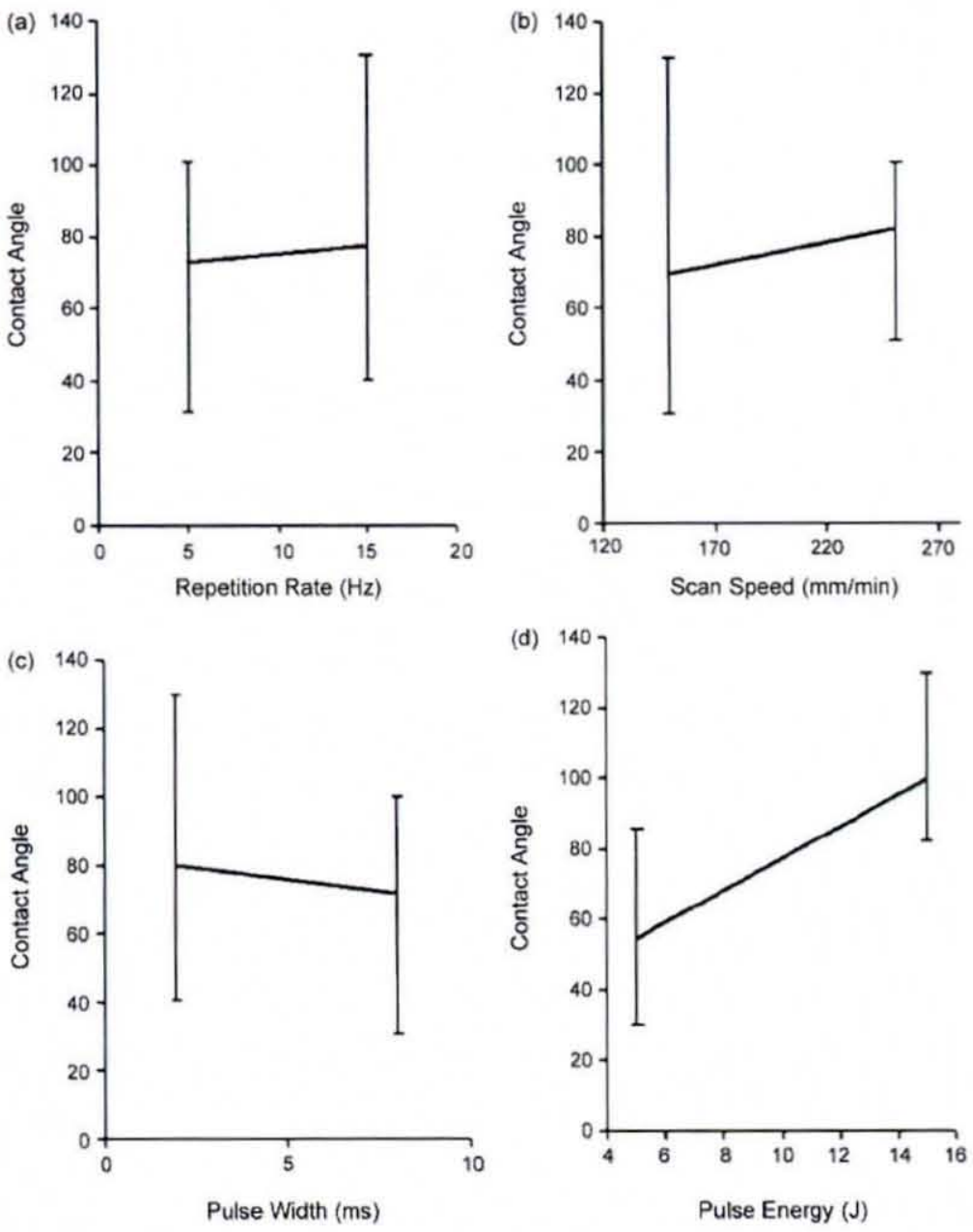

Fig. 7 - (a-d) Single bead processing, processing parameters against contact angle.

\subsection{Optimized processing parameters}

\subsubsection{Single scan testing}

Fig. 6 shows the results of bead width and height dimen sions as processing parameters were varied within the definec ranges shown in Table 2 using factorial analysis. Fig. $6 \mathrm{a}$ show that increasing the repetition rate from 5 to $15 \mathrm{~Hz}$ increase both the width and height of the bead. This shows that th. repetition rate directly affects the melt pool as the surfac tension force draws in local material into the melt. As repeti tion rate increases the total energy delivered to the interactior site is increased and hence more powder melting occurs. Thi subsequently increases the size of the bead width and height Fig. $6 \mathrm{~b}$ shows that an increase in scan speed between 150 an $250 \mathrm{~mm} / \mathrm{min}$ decreases the size of the bead width and height This decrease is a result of the energy input per unit lengtl being reduced causing less powder melting to occur. Fig. 6 
shows that at higher pulse widths or pulse durations more material is consolidated into the melt pool and hence larger bead widths and heights were produced. This is due to a prolonged laser on time, allowing more time for heat to conduct throughout the material. Fig. $6 \mathrm{~d}$ shows that increasing pulse energy between 5 and $15 \mathrm{~J}$ produced larger bead widths and smaller bead heights due increased heat intensity. Increasing heat intensity aided full melting of particles, but will induce vaporization if heat intensity is too high. Beads flatten out as more energy is introduced into the melt pool as well as more material being consolidated.

The change in repetition rate does not have great impact upon the size of the bead contact angle, this is shown in Fig. 7a. Fig. $7 \mathrm{~b}$ shows that an increase in scan speed produced smaller flatter beads due to an increase in surface tension creating larger contact angles. Fig. 7c shows that increases in pulse width reduced the contact angle. Increasing the pulse width reduces the peak power and therefore the heat intensity generated. This could allow more porosity within the bead to develop reducing the width to height ratio of the bead, this subsequently reduces the contact angle. Fig. $7 \mathrm{~d}$ shows that the pulse energy also has a substantial effect upon the bead contact angle. An increase in pulse energy promotes melting, the molten component has enough energy for capillary forces to spread the material out. This increases the contact angle and therefore improves the wettability. As SLM is a layered technology, reduced wetting is a severe impediment and can cause defects such as porosity and delamination (Steen, 1991). Another disadvantageous phenomenon arising during SLM is balling. It occurs when the molten material does not wet the underlying substrate due to the surface tension, which tends to spheroidise the liquid. This results in a rough, bead-shaped surface, obstructing a smooth layer deposition and decreasing the density of the part (Kruth et al., 2004).

The scan speed used for producing beads was varied at a fixed repetition rate of $10 \mathrm{~Hz}$, producing percentage spot overlaps of between 60 and $85 \%$. The correlation between bead width, height and contact angle at varying spot overlaps is shown in Fig. 8. Fig. 8 a shows that the bead width and height varied as spot overlap was increased. Within the overlap range of $60-85 \%$ the bead width did not vary a considerable amount. At overlaps between 70 and $80 \%$, a trend in increasing and decreasing bead height was observed. Bead height is it's lowest at $65 \%$ overlap and highest at $75 \%$. There is a similar trend between the increase and decrease in bead width and height at specific overlaps. Fig. $8 \mathrm{~b}$ shows the contact angle steadily drops and then rises between 70 and $80 \%$. The trend shown in this graph is inversely proportional to the trends of the bead height shown in Fig. 8a. When the bead height increases, the contact angle reduces. As the bead height decreases the contact angle increases. The bead contact angle is highest $\left(103^{\circ}\right)$ at an overlap of $65 \%$, achieved at a scan speed of $168 \mathrm{~mm} / \mathrm{min}$. A higher contact angle is preferable as it improves the wettability of the melt.

Examples of cross-sectioned beads that have undergone the effects of vaporization, full and partial bonding are shown in Fig. 9. Fig. 9a shows that the heat intensity generated by the laser was too high, causing the Waspaloy ${ }^{2}$ powder and part of the steel substrate to vaporize. When this phase transformation occurs, the rapidly moving evaporated powder particles
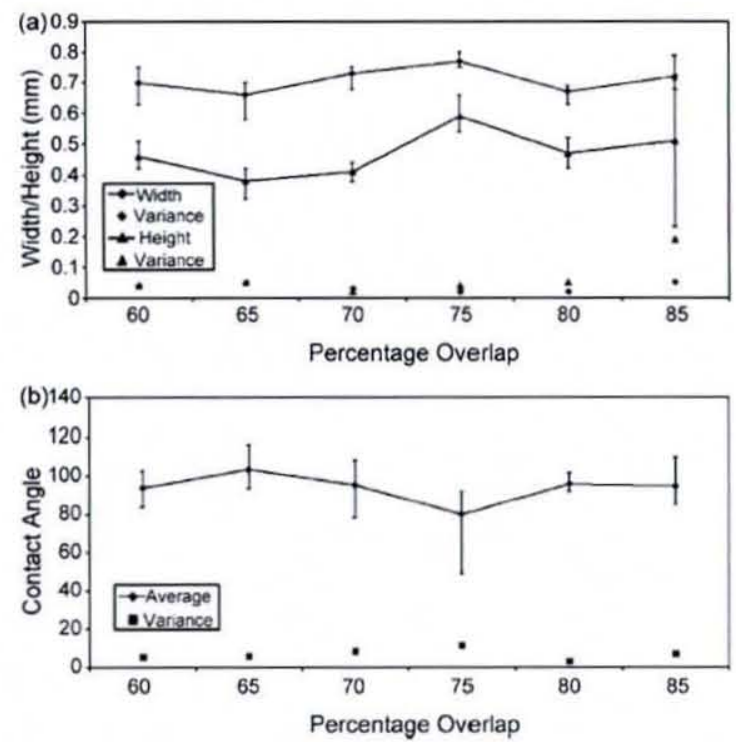

Fig. 8 - (a) Single bead, percentage overlap against width/height and (b) contact angle.

expand and generate a recoil pressure on the molten pool (Kruth et al., 2004). In this case the recoil pressure was sufficient to move some of the powder away from the interaction zone, causing the laser to penetrate the steel substrate causing little or no specimen formation. Fig. $9 \mathrm{~b}$ shows that the heat generated allowed for full bonding of Waspaloy to the substrate with good wetting characteristics. Fig. $9 \mathrm{c}$ shows a low contact angle is made between melted Waspaloy ${ }^{2}$ and substrate, this is a sign of insufficient bonding due to insufficient supply of heat energy.

From the single scan experiments a process operating window was obtained and is plotted in Fig. 10. Fig. 10 shows that at a lower pulse width the material is more susceptible to vaporization at lower specific energy densities compared to those at higher pulse widths. This is a result of greater heat intensities being generated at lower pulse widths. Vaporization of material is more likely to occur at lower pulse widths and therefore requires less specific energy to fully fuse the powder. As the pulse width increases the specific energy required for complete bonding of Waspaloy" increases. A lack of bonding occurred at specific energies below $5 \mathrm{~J} / \mathrm{mm}^{2}$ due to an insufficient heat input. It has been shown that heat generated by the laser and its intensity over a defined area had a considerable effect upon the fusion of the Waspaloy ${ }^{6}$ powder.

From the single scan investigation the optimized parameters were identified. Pulse width $(5 \mathrm{~ms})$, repetition rate $(10 \mathrm{~Hz})$ and scan speed $(168 \mathrm{~mm} / \mathrm{min})$ were found to be the best parameters to produce well formed fully fused specimens with high contact angles. In the subsequent experiment producing multi-layer specimens, the previously mentioned parameters were used. However, the effects of varied pulse energies were further investigated as described in Section 3.1.2. 

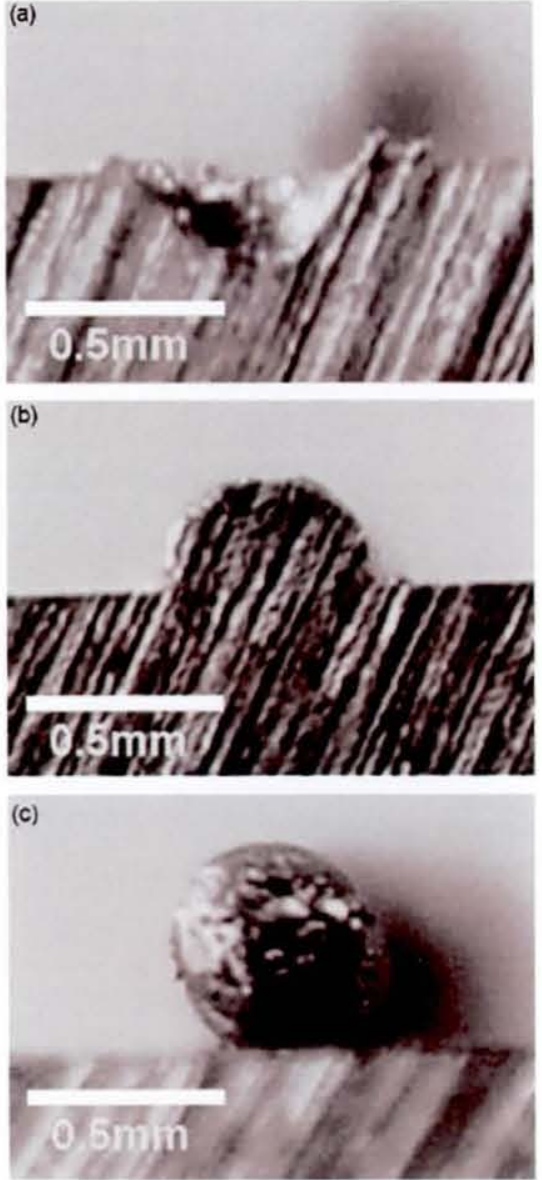

Fig. 9 - Cross-sectioned beads, affect of vaporization (a), full melting (b); (c) lack of fusion.

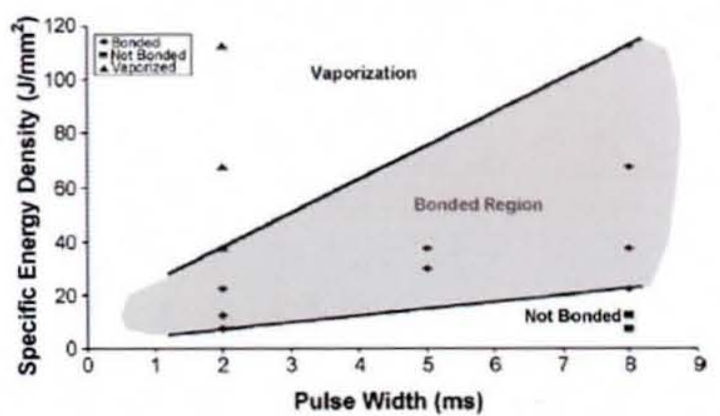

Fig. 10 - Specific energy $0 / \mathrm{mm}$ ) against pulse width ( $\mathrm{ms}$ ).

3.1.2. Multi-layers of Waspaloy

Fig. 11 shows the amount of porosity within layered specimens produced at a pulse width of $5 \mathrm{~ms}$, repetition rate $10 \mathrm{~Hz}$, scan speed $168 \mathrm{~mm} / \mathrm{min}$ and pulse energies between 6 and $9 \mathrm{~J}$.

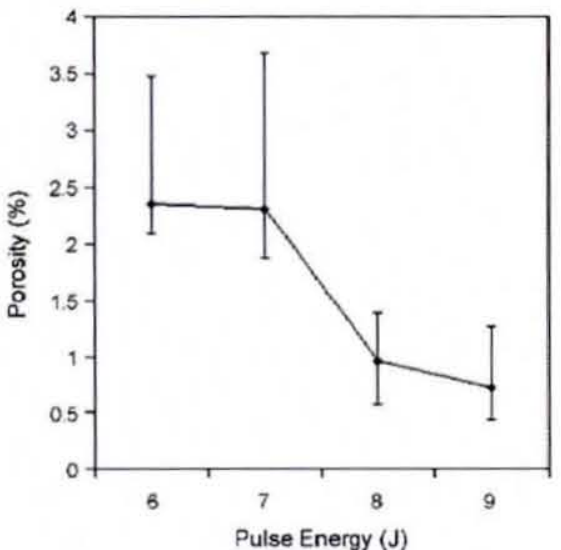

Fig. 11 - Layered specimen, porosity against pulse energy 0).

A reduction in porosity is observed with increasing pulse energy, low pulse energies do not generate enough heat to fully melt powder and hence a higher porosity is present. An increase in pulse energy results in a higher attained temperature of melt and therefore promotes an improved interlayer connection (Shiomi et al., 1999). A pulse energy of 9 J produced a part that contained an average porosity of only $0.7 \%$. The scanning laser beam delivered a sufficient amount of heat to each layer in order to sufficiently re-melt the previous layer without over melting the current layer. At a pulse energy of $10 \mathrm{~J}$ a peak power of $2 \mathrm{~kW}$ was generated causing the plasma plume from vaporized material to crack the protective glass lens in the Nd:YAG nozzle. Cross-sectioned layers produced at the four different pulse energies are shown in Fig. 12. The darker areas or spots represent the porous parts of the structure. The pores are irregular shaped and are predominant between adjacent scans and layer boundaries. It can be seen that as the processing pulse energy is increased to $9 \mathrm{~J}$ there is a reduction in the amount of porosity within the layered specimen due to increased melting.

The optimized parameters developed from the single scan and layered Waspaloy ${ }^{\oplus}$ investigations are listed in Table 3.

\subsection{High density Waspaloy ${ }^{*}$ specimen}

Fig. 13a shows a specimen that was fabricated using the optimized laser processing parameters (shown in Table 3) that were optimized specifically for the fabrication of high density

Table 3 - Optimized laser processing parameters for processing Waspaloy ${ }^{*}$

Pulse width (ms)

Pulse energy (I)

Average power $(W)$

Peak power $(W)$

Repetition rate $(\mathrm{Hz})$

Scan speed $(\mathrm{mm} / \mathrm{min})$

5
9
90
1800
10
168



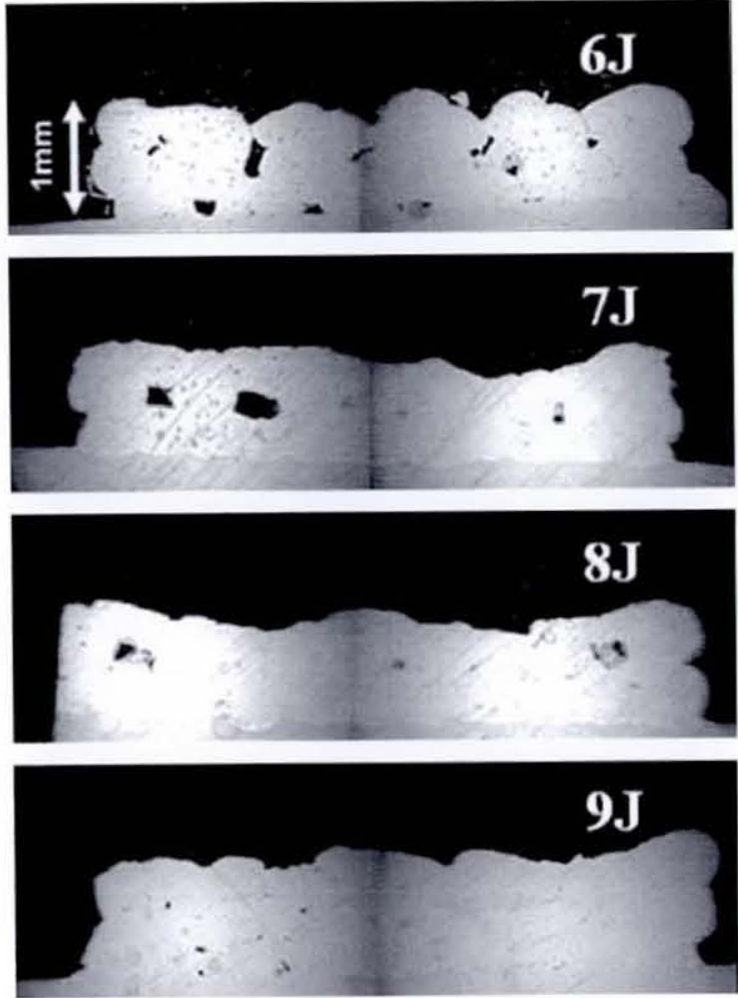

Fig. 12 - Layered specimen cross-sections $(2.5 \mathrm{x})$.
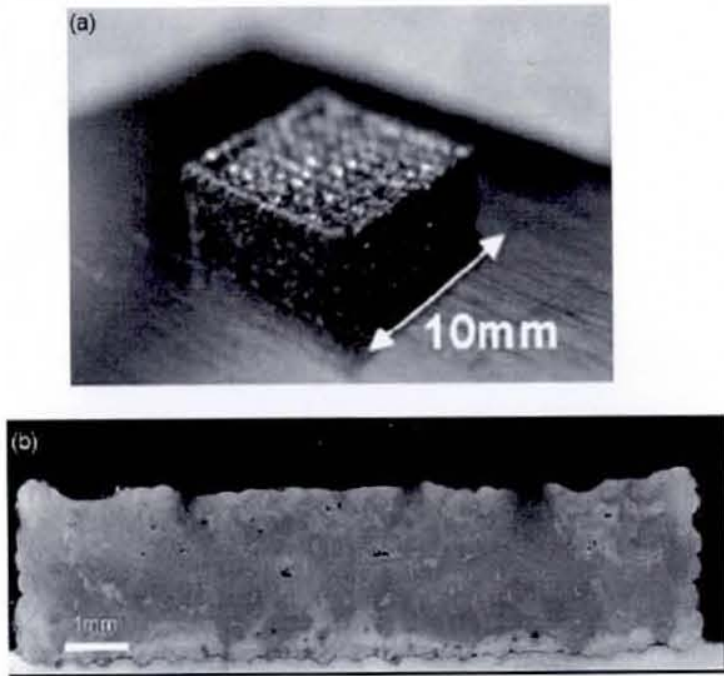

Fig. 13 - (a) Waspaloy ${ }^{2}$ specimen; (b) cross-sectioned SEM image.
Waspaloy ${ }^{\odot}$ specimens. This $10 \mathrm{~mm} \times 10 \mathrm{~mm}$ specimen was approximately $2.5 \mathrm{~mm}$ in height and consisted of eight $0.4 \mathrm{~mm}$ layers. From observing the specimen's geometric accuracy and after applying suitable shrink factors, it is possible to produce parts with accuracies of $X Y \pm 0.2 \mathrm{~mm}$ and $Z \pm 0.3 \mathrm{~mm}$.

\subsubsection{Density}

Fig. 13b shows the cross-section of the specimen magnified under SEM. Image tool analysis indicates that the specimen held a low porosity of $0.3 \%$ (this implies that the structure itself was $99.7 \%$ dense). There was a small amount of porosity present, this may have developed as a consequence of ball formation and entrapment of gas in melting powder mass. The outer top surface of the structure is not completely flat, it experiences elevated edges of solidified material. This is a consequence of the rate at which material is consolidated within the melt pool. The material can be exposed to a high temperature for long periods of time depending on scan spacing and bead overlap. Initial scans consume powder leaving a finite amount of powder for the subsequent scans to consolidate within the layer. The longer a bead is maintained at an elevated temperature the more powder it consumes leaving less material remaining for subsequent scans within the same layer. An inconsistent amount of material available for subsequent scans would produce an uneven top surface. A refill and cross-hatch scanning strategy was initially employed to resolve these issues. However this scanning strategy in combination with a reduction in layer thickness and further optimization of other laser parameters are required to fully deal with geometric unevenness.

A comparison between processing specimens at optimized parameters and lower pulse energies was made. Pulse width, repetition rates and scan speed were maintained at $5 \mathrm{~ms}$, $10 \mathrm{~Hz}$ and $168 \mathrm{~mm} / \mathrm{min}$, respectively. Pulse energy processing parameters were varied between $9 \mathrm{~J}$ and a lower $8 \mathrm{~J}$. Fig. 14(a) shows that the specimen processed at an optimized $9 \mathrm{~J}$ pulse energy held a porosity of $0.3 \%$. Fig. 14 (b) shows that the specimen produced at $8 \mathrm{~J}$ pulse energy contained $3.4 \%$ porosity, 10 times more porosity then the specimen produced at an optimized $9 \mathrm{~J}$ pulse energy.

Fig. 15 shows the specimens produced at a lower pulse energy contained large dimensional inaccuracies, porosity inclusion and crack propagation within the structure. This is due to the insufficient heat input used to melt interconnected layers.

\subsubsection{Microstructure}

The specimen produced using the optimized parameters (shown in Fig. 13) was magnified and examined for its micrograph characteristics, this is shown in Fig. 16(a). The microstructure was free from major defects such as high porosity and cracks. There was little if no contamination from the SLM process. Fig. 16 (b) shows the dendrite formation near the top surface of the specimen (region 1) contained small coarse equiaxed dendrites known as primary dendrites. Primary dendrites are the first series of branches that form from the nucleus of the grain. The spacing between these dendrite arms are equivalent to the grain size of the material. The small sizes of these dendrites indicate that cooling occurred very quickly after laser processing. This phenomenon of rapid 


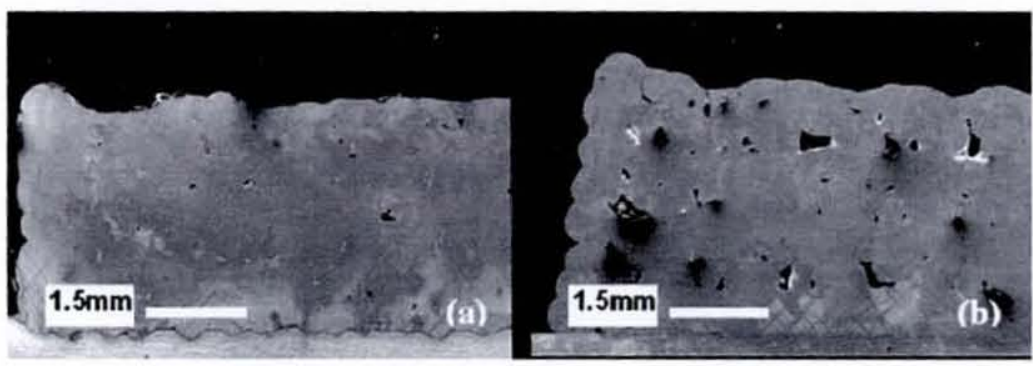

Fig. 14 - (a and b) Processed at 9 and 8 J.

or quick cooling at the top surface of the structure is due to the lack of subsequent re-heating caused due to no extra layers being processed above these layers. The grain boundaries shown in Fig. 16(b) act as reaction fronts that sweep out the dislocations in their path and leave strain-free grains behind (ASM, 1973). The grain boundaries in region one are at high angles to each other, these contain large areas of poor fit. High angle grain boundaries have an open structure with lots of free volume. These can cause problems as they can lead to defects and high strain at the interface between grain boundaries. The cube like dendrites shown are typical of nickel superalloys (Donachie and Donachie, 2002) and are likely to be cuboid $\gamma^{\prime}$ (gamma prime) precipitates. Nickel-base superalloys have a matrix $\gamma$ with a face-centred cubic structure. These $\gamma^{\prime}$ are precipitates that help the superalloy derive it's strength and are formed from aluminium and titanium which react with nickel. These $\gamma$ can also appear as spheres as well as cuboids when properly formed. The formation of large grain boundaries and small dendrites are only formed at the top surface of the structure.

Fig. 16(c) shows regions nearer the substrate (region 2), the dendrite formation was more favourable from a structural point of view. Dendrites exhibit secondary dendrite arms that are larger in size and longer then those in region one. This indicates that the region was allowed more time to cool after laser processing. This is due to subsequent layers being processed above this region causing a multiple reheating effect. This would cause the processed material within this region to $\mathrm{cool}$ at a slower rate. The substrate would heat up and reduced the temperature gradient experienced by the lower parts of the specimen compared to that at the top. The dendrites exhibit columnar dendrite forms that are aligned along the direction of the substrate. This alignment is characteristic of solidifcation that is dominant due to heat dissipation through the substrate. The angle between the grain boundaries are relatively low, this is a more favourable scenario compared to the large grain angle boundaries seen in region one. Low grain angle boundaries contain large areas of good fit separated by misfit dislocations, they contain little free volume (Charre, 1997).

Fig. 16(d) shows the region nearest to the substrate (region $3)$, this does not reveal a great deal about the specimen's dendrite formation, this could be due to a new alloy formation of Waspaloy ${ }^{2}$ and steel from the substrate. Fig. 17 shows region 3 (of Fig. 16(d)) at a higher magnification, the larger white areas could be an oxide film layer. In laser welding oxide or soot from metal vapour from the previous spot can enter the weld pool on the next. It is therefore necessary that gas shielding is correctly used to prevent contamination and maintain weld quality. The smaller white segments on the image appear to be precipitation of carbide. This is a hard substance that is revealed once the specimen is etched (Charre, 1997). Carbides are major strengtheners and are found mostly at grain boundaries and hold a beneficial effect on rapture strength by reducing grain boundary sliding.

The period of time for which a material is held at a high temperature directly effects the formation of grain structure. Material that is molten for longer periods of time will allow for any oxides to disassociate (Pogson et al., 2003). The disadvantage of holding the material at a high temperature for a longer period of time is that greater thermal stresses may be induced.

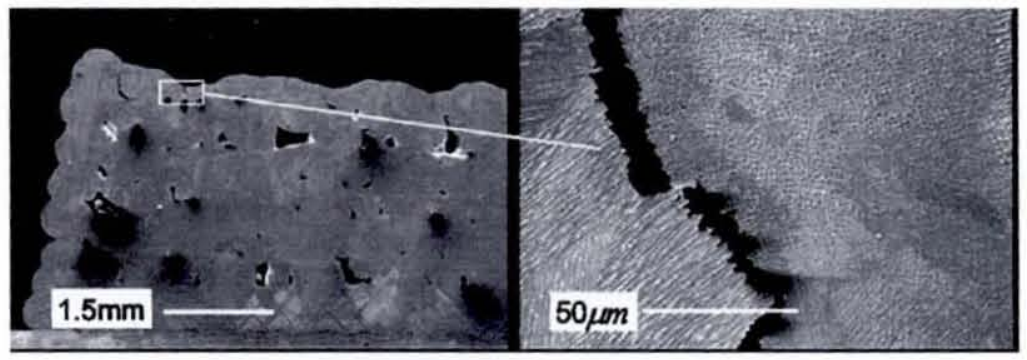

Fig. 15 - Waspaloy specimen processed at a deficient $8 \mathrm{~J}$ pulse. 


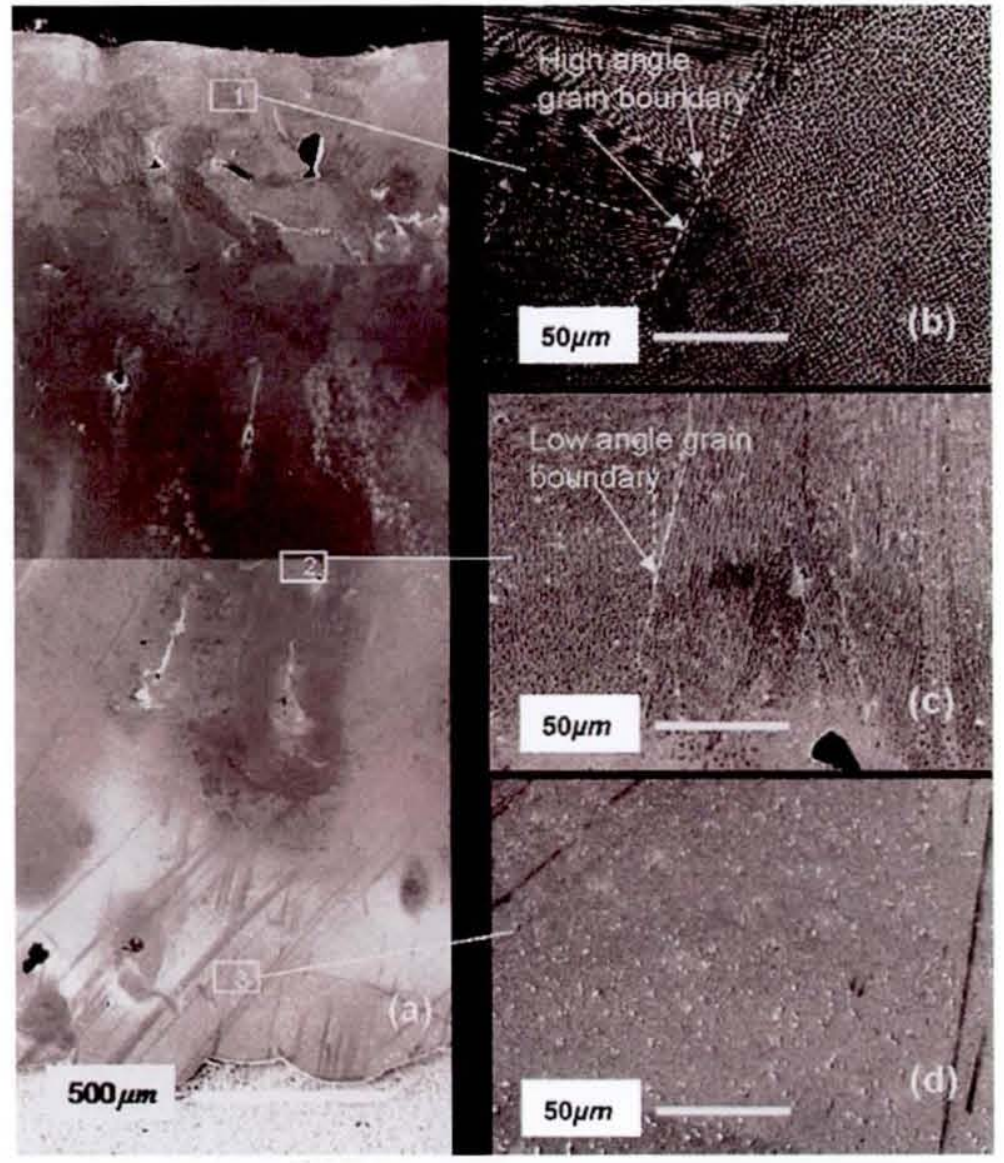

Fig. 16 - (a-d) Micrograph characteristics of cross-sectioned high density specimen.

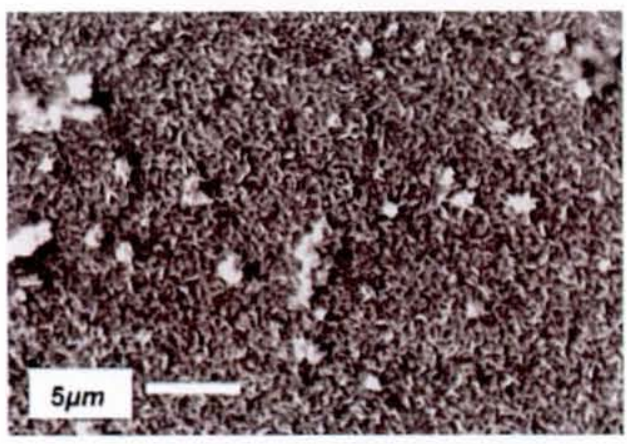

Fig. 17 - Microstructure of region 3.

\section{Conclusion}

A set of experiments using an Nd:YAG laser to SLM Waspaloy ${ }^{6}$ powder was conducted with the aim of obtaining optimized processing parameters for the fabrication of high density specimens.
Single beads were produced at varying pulse width $(\mathrm{ms})$, pulse energy $(\mathrm{J})$, repetition rate $(\mathrm{Hz})$ and scan speeds $(\mathrm{mm} / \mathrm{min})$. Bead height, width and contact angles were measured and a processing bonding region was identified. To further develop and optimize these process parameters layered specimens were produced and examined for part porosity. Porosity within the layered specimen reduced as the pulse energy increased to 9 J. However a further increase in pulse energy would cause large amounts of material vaporization to occur.

The optimized process parameters pulse width $5 \mathrm{~ms}$, pulse energy 9J, repetition rate $10 \mathrm{~Hz}$ and scan speed $168 \mathrm{~mm} / \mathrm{min}$ were used to fabricate a high density $(99.7 \%$ dense) $10 \mathrm{~mm} \times 10 \mathrm{~mm}$ specimen consisting of eight $0.4 \mathrm{~mm}$ layers. The microstructure was examined for dendrite formation, cracks and porosity. A comparison was made between specimens produced at optimized parameters and those fabricated at a lower pulse energy. There appeared to be less crack formation within the specimen using optimized process parameters compared to the specimen produced at insufficient lower pulse energies. 
It has been shown that the formation of Waspaloy" parts can be controlled by the adjustments of laser processing parameters. Defining an operating processing window and developing processing parameters allowed for the fabrica. tion of high density Waspaloy specimens by means of SLM. The SLM process holds high potential for the production of fully functional high density components in a single step as well as the possibility of fabricating functionally graded parts.

\section{Acknowledgements}

The authors acknowledge the support of technical staff Peter Wileman, Rod Springthorpe and Andy Sandavor.

\section{REFERENCES}

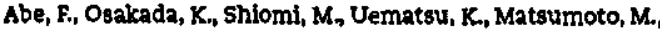
2001. The manufacture of hard tools from metallic powders by selective laser melting. J. Mater. Process. Technol. 111 (1), 210-213.

Agarwala, M. Bourell, D., Beaman, J., Marcus, H., Barlow, J., 1995. Direct selectve laser sintering of metals. Rapid Prototyping J. 1 (1), 26-36.

ASM Handbook Committee, Metallographic, structures and Phase Diagrams, Metals Handbook, 8th ed., vol. 9, USA, 1973.

Bourell, D.L., Wohlert, M., Harlan, N., Das, S., Beaman, J.J., 2002. powder densification maps in selective laser sintering. Eng. Mater. 4 (9), 663-669.

Charre, M.D., 1997. The Microstructure of Superalloys. Gordon and Breach Sclence Publ.

Childs, T.H.C., Hauser, C., Badrossamay, M., 2005. Selective laser sintering (meiting) of stainless and tool steel powders: experiments and modelling. In: Proc. IMechE 219 part B: l. Eng. Manuf., pp. 999-357.

Das, S., 2003. Physical aspects of process control in direct selective laser sintering of metals. Adv. Eng. Mater. J. $5(10)$, 701-711.

Donachte, M.J., Donachte, S.J., 2002 Superalloys, a technical gulde. ASM int..

German, R.M., 1994. Powder Metallurgy sclence 2nd Edition Metal Powder industries Federation, Princeton, $N$.

Jeng, J.Y., Lin, M.C., 2001. Mold fabrication and modilfcation using hybrld processes of selective laser cladding and milling. J. Mater, Process. Technol. 110 (1) 98-109.

Jones, R.M.F., Jackman, L. A, 1999. The structural evolution of superalloy ingots during hot woritng. JOM 51 (1), 27-31.

Kelcher, D.M., Mlller, W.D., smugeresky, J.E., Romero, JA, 1998. Laser engineered net shaping (LENS), beyond rapid prototyping to direct fabrication. In: Proceedings of the 1998 TMS Annual Meeting, San Antonio, TX, pp. 369-\$77.

Kruth, J.R., Froyen, L., Veerenbergh, J.V., Mercelis, R. Rombouts, M., Lauwers, B., 2004. Selective laser melting of iron-based powder. J. Matex. Process, Technol 149 (1-9), 616-622.
Kruth J.P., Mercelis, P., Vherenbergh, J.V., 2005. Binding mechanisms in selective laser sintering and selective laser melting. Rapid Prototyping I. 11 \{1\}, 26-36.

Lewis, G.K., Nemec, R., Milewsld, J., Thoma, D.j., Cremers, D, Barbe, M., 1994. Directed light fabrication. In: Proceedings of the Intemational congress on Applications of Lasers and Electro-Optics, Orlando, Florida, USA, pp. 17-26.

Lu, L., Fuh, J.Y.H., Wong, Y.S., 2001. Laser-Induced Materials and Processes for Rapid Prototyping. Kluwer Academic Publishers, USA.

Morgan, R.J., Papworth, A.J., Sutcliffe, C., Fox, P., O'Neill, W., 2002. High density net shape components by direct laser re-melting of single-phase powders. J. Mater. Scl. 37 (15), 3093-3100.

Over, C., Meiners, W., Wissenbach, K., Lindemann, M., Huthess, J. 2002. Laser melting a new approach for the direct manufacturing of metal parts and tools. In: Proceedings of the Euro-Rapid 2002 International User's Conference, Frankfurt. Germany, p. 5.

Pogson, S.R., Fox, R., Sutcliffe, C.j., O'Neill, W., 2003. The production of copper parts using DMLR. Rapid Prototyping J. 9 (5) $334-343$.

Santos, E.C., Osakada, K., Shiomi, M., Kitamura, Y., Abe, F., 2004 Microstructure and mechanical properties of pure titanium models fabricated by selective laser melting. Proc. I MECH E Part C: J. Mech. Eng Scl. 218 (7), 711-719.

Santos, E.C., Shiomi, S., Osakada, X., Laoui, T., 2006. Rapid manufacturing of metal components by laser forming. int. J. Mach. Tools Manuf. Des. Res. Appl. 46, 1459-1468.

Shlomi, M., Yoshidome, A., Abe, F., Osakada, K., 1999. Finite element analysis of melting and solldifying processes in laser rapid prototyping of metallic powders. Int. J. Mach. Tools Manuf. 39 \{2\}, 237-252.

Speclal Metals, Waspaloy, November 2006, http:/hwww.SpecialMetals.com.

statgraphics, Stat Point inc, http://www,statgraphics.com, Virginia, USA

Steen. W.M., 1991. Laser Material Processing. Springer Verlag.

W. Su, Lryered Fabrication of Tool Steel and Functionally Graded Materials with a Nd:YAG Pulsed Laser, PhD Thesis, Loughborough University, Loughborough, Leicestershire, United KIngdom, 2002.

Tolochko, N.K., Savich, V.V., Laoul, T., Froyen, L., 2002. Dental root implants produced by the combined selective laser sintering/meltung of titanium powders, Proc. I MECH E Part L: J. Mater.: Des. Appl. 216, 267-270.

UTHSCA, The University of Texas Health Science Center at San Antonto, ImageTool Version 3.0. January 2006, http://ddsdr.uthscsa.edu/digfitdesc.html.

Wohlers, T., 1996. Proceedings of the Rapid Prototyping \& Manufacturing Conference, Dearborn, MI, USA.

Xue, L., Islam, M.U., 2000. Free-from laser consolication for producing metallurglcally sound and functional components. f. Laser Appl. 12 (4), 160-165.

Yevko, V., Park, C.B, Zak, G., Benhablb, B., 1998 . Cladding formation in laser-beam fusion of metal powder. Rapid Prototyping ]. 4 (4), 168-184

Zhang, Y. XI, M., Gao, S, ShI, L., 2009. Characterization of laser direct deposited metallic parts. J. Mater. Process. Technol. 142 (2), 582-585. 


\title{
12.3.3 Top surface and side roughness of inconel 625 parts produced using pulse shaping
}

\section{Top surface and side roughness of Inconel 625 parts processed using selective laser melting}

\author{
Kamran Mumtaz and Neil Hopkinson \\ Rapid Manufacturing Research Group, Wolfson School of Mechanical and Manufacturing Engineering, \\ Loughiborough University, Loughborough, UK
}

\begin{abstract}
Purpose - Obtaining the required part top surface roughness and side roughness is critical in some applications. Each of these part properties can often be improved to the detriment of the other during selextive laser metting (S.MA. The purpose of this paper is to investigate the selective laser meithng of laconel 625 using an Nd;YAG pulsed laser to produce thin wall parts with an emphasis on attaining parts with minimum top surface and side surface roughness. Design/method ologylapproach - A full factorial approach was used to vary process parameters and identify a usable inconel 625 processing region. The effects laser process parameters had on the formation of part surface roughness for multi-layer parts were examined. Processing parameters that specifically affected top surface and side roughness were identified.

Findings - Higher peak powers tended to reduce top surface roughness and reduce side roughness as recoil pressures flatten out the melt pool and reduce balling formation by increasing wettability of the melt. Increased repetition rate and reduced scan speed reduxed top surface roughness but increased side roughness. A compromise between attaining a relatively low surface roughness and side roughness can be attained by comparing part surface roughness values and understanding the factors that affect them. A sample with $9 \mu \mathrm{m}$ top surface roughness and $10 \mu \mathrm{m}$ side roughness was produced.

Originalityivalue - The research is the first of its kind directly processing Inconel 625 using SLM and investigating processing parameters that affect top surface and side roughness simultaneousty. it is a useful aid in unvelting a relationship between processparameters and top/side roughness of thin walled parts.
\end{abstract}

Keywords Surface texture, Surface-roughness measurement

Paper type Research paper

\section{Introduction}

Solid freeform fabrication (SFF) is family of processes that involve the layer-wise shaping and consolidation of material (e.g. powder, wire). Due to the additive nature of SFF it can produce parts with a high geometric freedom directly from a CAD model. SFF also has the potential to reduce the time period between the initial conceptual design of a part and its actual fabrication. In recent years laset based SFF processea such as selective laser melting (SLM) has been shown to be a viable and promising technology for the production of fully functional metal parts.

\subsection{Selective laser melting}

SLM is a powder bed process that begins with the deposition of a thin layer of powder onto a substrate held in place by an adjustable platform. A laser then raster scans the surface of the powder, the heat generated by the laser causes powder particles to melt and form a melt pool (Santos et al, 2004).

The current lssue and full text archive of this journal is available at www.emeraldinsight.com/1355-25 46 , htm

Rapid Procotypteng Joumal

$13 / 2(2009) 96-103$

O Emanid Cooup Publiching timitad [LSSN 1358-2546]

[DOt 10.110813552540010943397]
As the laser beam moves away from the melt pool, the molten material is solidified forming a structure. Once the layer has been scanned the adjustable platform lowers in the 2-axis, another layer of powder is deposited onto the previous layer and is again melted by the laser. Figure 1 shows a schematic of this process. Powder particles that are not melted remain loose and are removed once the component is complete.

Research efforts have demonstrated the great potential available within SLM to manufacture metal components with microstructures and mechanical properties equivalent or superior to bulk material and conventionally processed materials (e.g. processed by casting or hot isostatic pressing) (Bourell et al, 2002, Kruth et at, 2005), this makes the SLM process the subject of major research activities (Childs et al., 2005, Agarwala et at. 1995). Schwendner et al. (2001) believe DMLF processes such as SLM are the most viable technologies for the direct fabrication of complex metallic components. This is due to the versatility, accuracy and small spot size of a leser beam. The small spot size reduces the melt width enabling the production of high resolution parts.

Direct processing using SLM is not without its difficulties and constraints. SLM issues mainly revolve around the use of high powered lasers to produce high ternperatures capable of

Received: 6 September 2007

Revised: 11 January 2008, 21 June 2008

Accepred: 10 October 2008 
Figure 1 Selective laser melting process

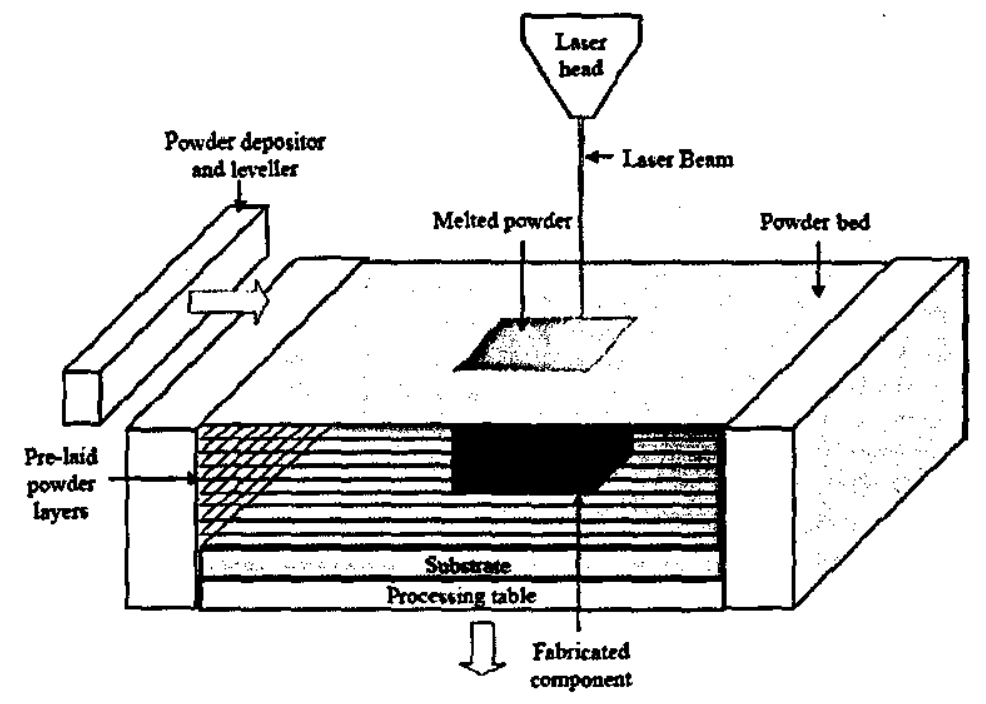

completely melting the powder. High heat input often causes an increase in spatter generation and material vaporization during processing. However, the main topic of discussion centre around part top and side surface finish due to particle melting and re-solidifying mechanisms (Connolly, 2001, Ramos et al., 2001).

\subsection{Surface roughness}

The surface roughness of a part is critical in many applications with some applications requiring a surface roughness of $0.8 \mu \mathrm{m}$ or better to avoid premature failure from surface initiated cracking (Dalgamo, 2007). Commercial powder bed machines such as MCP's Realizer, EOS M270, etc. often require post processing operations such as surface machining, polishing and shor peening to attain final part surface finish. These unwanted post processing operations incur further time and delay part completion.

The top surface roughness of a melt pool can be affected by a rippling effect that occurs due to surface tension forces exerting a shear force on the liquid surface. This is primarily due to a surface temperature difference between the laser beam and the solidifying zone caused by the motion of the laser beam. As the thermal gradients reduce, gravity and melt pool surface curveture counter this external shear force, eventually restoring the surface height of the melt pool to the free level (Ramos a al, 2003). However, viscous forces delay this relaxation process, quick melt pool solidification time often ensures that complete relaxation is not fully achieved.

Investigations using pulsed lasers have reported that top surface roughness can be reduced using a low scan speed and high repetition rate due to melt pool stabilization and reduced surface profile variation on top of the solidified melt pool (Niu and Chang, 1998, Fischer et al, 2003). However, low scan speeds and high repetition rates can significantly increase the volume of melt pool produced and promote a phenomenon known as balling. Balling is the breakup of the melt pool into small spheres. It occurs when molten material does not wet well to the underlying substrate or material due to high surface tension differences generated as a result of variations in thermal properties within the melt pool (Kruth a al, 2004, Morgan a ah, 2004, Fuh et al, 1995). These thermal gradients cause a thermocapillary flow of a fluid within the melt pool from regions with low surface tension to regions with high surface tension, known as Marangoni convection (Rombouts, 2006). The breaking up of the melt pool into smaller entities reduces the variation in melt pool surface tension. Balling can increase with the generation of excessive molten material or if too bw viscosity exists within a melt pool (Agarwala et al, 1995). Kruth et al (2004) stated that when the total surface of a molten pool becomes larger than that of a sphere with the same volume, the balling effect takes place. Balling is a severe impediment on interlayer connection, it decreases part density and increases top surface roughness and side roughness. However, the balling effect more dominantly affects the side roughness of parts due to the direction of balling scattering to either side of the melt pool rather than setting on the top surface.

Due to the complexity of SLM there is still a requirement to understand the issues that affect top surface and side roughness and a demand to further improve the surface finish of parts produced using SLM. This would reduce the requirement for furcher post processing operations.

\section{Selective laser melting system and powder properties}

The SLM system used within this investigation included a GSI Lumonics JK701H Nd:YAG laser. The laser has a $T E M_{\infty}$ Gaussian profile, a spot size of $0.8 \mathrm{~mm}$ and a maximum power output of $550 \mathrm{~W}$. The pulses of laser energy generated by the flash lamps are fed through a $1 \mathrm{~mm} \sigma$ silica optical fibre delivery cable to the laser head and are deflected through $90^{\circ}$ by a reflecting mirror onto a focusing optic. The light is focused and passes through the laser nozzle onto 
Top surface and slde roughness of inconel 625 parts

Kamnan Mumtaz and Nail Hopkiscon

the powder bed. Powder is deposited onto a steel substrate that is itself placed on a processing table that is driven by a 4-axis CNC controlled machine. Argon gas is fed through the laser nozzle onto the processing area at a rate of $10 \mathrm{ml} / \mathrm{s}$ to protect the parts from the effects of oxidization.

The Inconel 625 powder (Sandvik Osprey, UK) was supplied sized to $53 \pm 25 \mu \mathrm{m}$. The exact particle size distribution was measured to be $\mathrm{D}(\mathrm{v}, 0.1)$ of $22.51 \mu \mathrm{m}$ ( 10 per cent of the volume distribution is below this value), $\mathrm{D}(\mathrm{v}, 0.5)$ of $45.48 \mu \mathrm{m}$ (volume median diameter, 50 per cent of the distribution is above and 50 per cent is below this value) and $D(v, 0.9)$ of $58.59 \mu \mathrm{m}$ (90 per cent of the volume distribution is below this value). Particle size distribution data were acquired using a Malvern Mastersizer.

\section{Methodology}

Within SLM of metal powders there exists a level of uncertainty related to the required laser process parameters needed for successful part fabrication. Achieving a fine balance with processing parameters is something that is fundamental for the production of fully functional metal parts. To add to this the processing of a new and relatively under researched material within SLM brings about many unknowns that require optimization of parameters.

In order to produce thin walled parts with low top surface and side roughness usable melting region for Inconel 625 was first established. Once the processing region had been identified multi-layer thin walled parts were then bult using varied processing parameters. Parts were examined for top and side surface roughness.

Images of the top surface of samples were acquired using Optical Gaging Product's Flash 200 SmartScope, this equipment had a scale resolution of $0.5 \mu \mathrm{m}$. Sample top surface and side roughness was measured using a Talysurf CLI 2000. The Talysurf evaluated $12.5 \mathrm{~mm}$ of the samples surface. This was taken about the middle portion of the sample as the first portion showed a higher roughness due to the first line scan balling phenomenon (Das, 2003). For measurement of side surface roughness the stylus tip was positioned around the centre of these four layers and traversed along the side of the sample in the same direction used to measure top surface roughness. Side roughness was measured along the layers as opposed to across the layers ( $z$-axis) due to the requirement of taller samples measuring at least $12.5 \mathrm{~mm}$ in height. A cut-off length of $2.5 \mathrm{~mm}$ was used to determine the arithmetic average roughness $\left(R_{2}\right)$ for both top and side surfaces. This cut-off would best represent the overall surface coughness allowing the high-frequency roughness oscillations to be distinguished from the low-frequency waveforms.

\section{Results}

4.1 Inconel 625 melting region

Thble I shows the range of laser processing parameters used to process single scuns measuring $25 \mathrm{~mm}$ in length from

Table I laser processing parameters used to determine inconel 625 meking region

\begin{tabular}{lccc}
\hline $\begin{array}{l}\text { Pulse energy } \\
\text { (J) }\end{array}$ & $\begin{array}{c}\text { Pulse duration } \\
\text { (ms) }\end{array}$ & $\begin{array}{c}\text { Repetition rate } \\
\text { (H2) }\end{array}$ & $\begin{array}{c}\text { Scan speed } \\
\text { (mm/min) }\end{array}$ \\
\hline $0.1-15$ (varled) & $0.5-20$ (varied) & 10 (fixed) & 168 (fixed) \\
\hline
\end{tabular}

Rapld Prototyping Journal

Wbium 15 - Numbor $2 \cdot 2009 \cdot 96-103$

$0.1 \mathrm{~mm}$ powder bed thickness. The Inconel 625 powder was placed on a stainless steel substrate measuring $60 \times 60 \times 3 \mathrm{~mm}$. A fixed repetition rate of $10 \mathrm{~Hz}$ and scan speed of $168 \mathrm{~mm} / \mathrm{min}$ were initially used to determine a melting region. These process parameters were successfully used in research conducted by Mumtaz a al. (2007) when processing Waspaloy ${ }^{\oplus}$ using SLM.

Figure 2 shows a range of samples that were visually observed for signs of a lack of melting, sufficient melting and excessive melting leading to overheating/oxidization and widening of melt pool. These samples were produced using a pulse duration of $0.5 \mathrm{~ms}$ and pulse energies ranging from 0.1 to $2.5 \mathrm{~J}$. Pulse energies below $0.5 \mathrm{~J}$ did not produce enough heat to fully melt the powder. Pulse energies between 0.75 and $1 \mathrm{~J}$ produced well formed samples that fully melted to the substrate. As pulse energies increased above $1 \mathrm{~J}$ the samples show signs of excessive heat input. They increased in width due to the heat conduction increasing the volume of liquid produced and subsequently the width of the melt pool. The samples produced using high pulse energies possessed a brownish surface colour (indicating possible oxidizarion), material vaporization and spatter generation increased. The samples processed at increased pulse durations increased in width due to more time being given for heat to conduct out radially from the centre of the melt pool.

Figure 3 shows the regions in which Inconel 625 fully melted using variable pulse energies and pulse durations. The graph also indicates insufficient/excessive heat input regions as well as a boundary indicating an undesirable increase in material spatter in which powder bed contamination became visually apparent. As pulse duration increased the amount of energy within the pulse required to melt the powder increased. This is because increased pulse duration will effectively melt powder over a larger area due to the spread of heat through the powder bed via conduction. This prolonged beating effect over a larger area requires more energy to maintain the same degree of melting than that of a shorter pulse duration. The undesirable increase in material spatter boundary falls 1-2 J below the excessive heat input region. The increase in material spatter is therefore an eady indicator that too much heat input is being delivered to the material.

4.2 Top and side surface roughness

Multi-layer parts were produced using the laser parameter shown in shown in Table II, these were measured for top/side surface $R_{\text {. }}$. Muldi-layer parts were $25 \mathrm{~mm}$ in length and consisted of four $0.1 \mathrm{~mm}$ powder layers. During part fabrication the laser's pulse duration was fixed at $0.5 \mathrm{~ms}$ as it minimized the laser on time and produced the smallest melt pool width. This would allow parts to be produced to a higher resolution. The pulse energy values used for multi-layer part fabrication are within the useable melting regions shown in Figure 3. The repetition rates used are within the laser's operational limit for specife pulse duration and power. The scan speeds used would facilitate the production of a spot overlap between 72 and 98 per cent. The maximum useable repetition rate used within processing was restricted with the use of higher pulse energies. This was done in order to reduce excessive spatter generation due to an increase in energy density. 
Figure 2 Inconel 625 single scans using $0.5 \mathrm{~ms}$ and $0.1-2.5$.

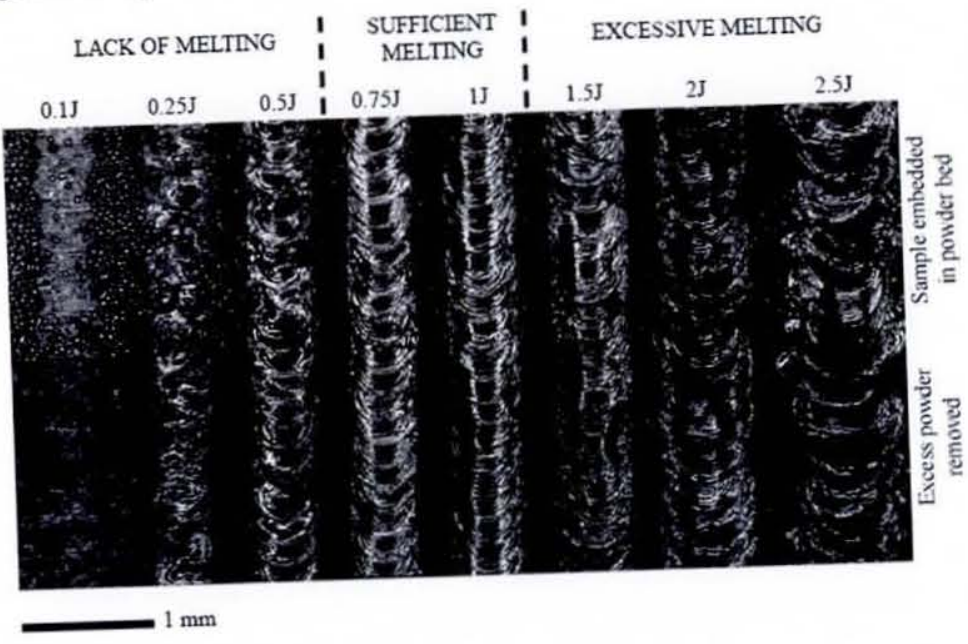

Figure 3 Inconel 625 melting region

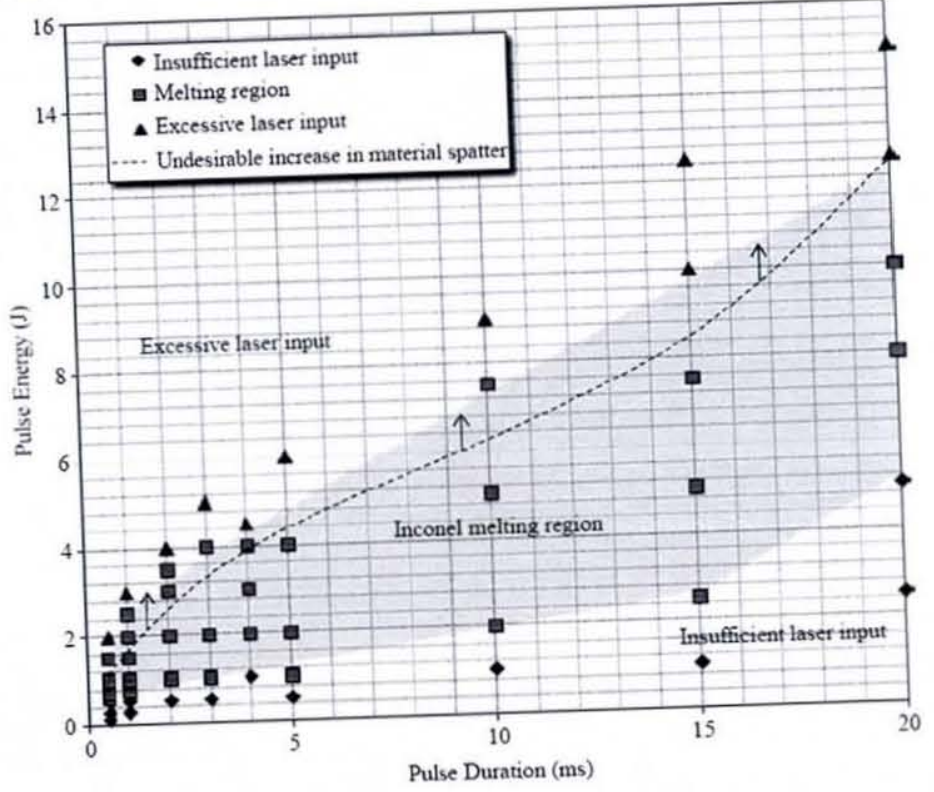

Table II Laser parameters used within single layer processing

\begin{tabular}{lccc}
\hline $\begin{array}{l}\text { Pulse width } \\
(\mathrm{ms})\end{array}$ & $\begin{array}{c}\text { Pulse energy } \\
(\mathrm{J})\end{array}$ & $\begin{array}{c}\text { Repetition rate } \\
(\mathrm{Hz})\end{array}$ & $\begin{array}{c}\text { Scan speed } \\
(\mathrm{mm} / \mathrm{m})\end{array}$ \\
\hline 0.5 & 0.5 & $30-100$ & $100-400$ \\
0.5 & 0.6 & $30-70$ & $100-400$ \\
0.5 & 0.7 & $30-50$ & $100-400$ \\
\hline
\end{tabular}

Pulsed lasers produce melt pools that are kept molten by laser pulses that are repeatedly emitted at an adjustable repetition pulses that are reper molten rate $(f)$. As the laser pulses move away from the molten material it cools to form overlapped solidified material, the level of overlap is directly related to the laser spot overlap level of overlap is directs raises the temperature profile of the generated as each pulses raises the temperature profle of the melt pool. Calculation of spot overlap $\left(S_{o v}\right)$ is made using
equation 1 with $V$ and $S_{d}$ representing the laser scan speed and laser spot size, respectively: 


$$
s_{o v}=\left|1-\frac{V}{S_{d f}}\right| 100
$$

Figure 4 shows the top/side $R_{\mathrm{a}}$ of parts produced at variable scan speeds and spot overlaps. The top $R_{a}$ of parts reduced as the spot overlap increased. At a fixed scan speed spot overlap is increased with increasing repetition rates causing the overlapping spots to become closer together and reduce the variation in surface profile, thus producing a smoother surface finish. Higher spot overlaps were achieved with the use of high repetition rates and low scan speeds. Figure 5 shows the top view of a selection of thin walled parts produced using a pulse energy of $0.5 \mathrm{~J}, 40 \mathrm{~Hz}$ and variable scan speeds. It can be seen that the use of lower scan speeds improves top $R_{a}$ at a fixed repetition rate due to increased spot overlap.

Figure 4 shows that side $R_{4}$ of parts are at their highest with the use of lower scan speeds and highrepetition rates. This is a result of the high overlaps and a higher energy density causing the liquid lifetime to increase and promote balling formation. Figure 5 shows that there is a high-degree of degree of balling in samples processed using slower scan speed of $100 \mathrm{~mm} / \mathrm{min}$. Balling appeared to be less predominant in samples produced at a higher scan speed of $400 \mathrm{~mm} / \mathrm{min}$ due to a reduced spot overlap at a fixed repetition rate. The reduction in balling caused the side $R$. to fall from $29 \mu \mathrm{m}$ to $14 \mu \mathrm{m}$. The width of samples also reduced with higher scan speeds due to less time being given for heat to conduct radially from the centre of the melt pool.

Results have so far indicated that low scan speeds and high repetition rates (high spot overlap) reduce top $R_{3}$, high scan speeds and low repetition rate (low spot overlap) reduce side $R_{2}$.

Figure 6(a) shows the lowest values for top $R_{a}$ when processed using a low scan speed of $100 \mathrm{~mm} / \mathrm{min}$ at variable repetition rates and pulse energies. Samples produced at higher pulse energies tended to have a lower top $R_{a}$. A minimum top surface $R_{a}$ of $4 \mu \mathrm{m}$ is achieved using a pulse energy of $0.7 \mathrm{~J}$ and a repetition rate of $50 \mathrm{~Hz}$ when using a scan speed of $100 \mathrm{~mm} / \mathrm{min}$ (spot overiap 95.8 per cent)

Figure 6(b) shows the lowest values for side $R_{a}$ when processed using a high scan speed of $400 \mathrm{~mm} / \mathrm{min}$ at variable repetition rates and pulse energies. As expected, lower repetition rates produced parts with lower side $R_{a}$. However, it was found that parts produced at higher pulse energies also reduced side $R_{\alpha}$. A minimum side $R_{\alpha}$ of $10 \mu \mathrm{m}$ was achieved in two samples using a pulse energy of $0.7 \mathrm{~J}$, repetition rate of $30-40 \mathrm{~Hz}$ and scan speed of $400 \mathrm{~mm} / \mathrm{min}$ (spot overlap 72.2-79.2 per cent).

Figure 7 displays images of parts with minimum top $R_{\mathrm{a}}$ (a) and side $R_{a}$ (b). Part (a) was produced with a high overlap such that there was little variation between overlapping melt pools. However, the high energy density caused large amounts of balling to occur increasing the side $\mathrm{R}_{\mathrm{a}} 32 \mu \mathrm{m}$. Part (b) was produced using a lower spot overlap causing less balling to occur. However, due to increased variation between overlapping melt pools the top $\mathrm{R}_{\mathrm{a}}$ increased by $5 \mu \mathrm{m}$ to $9 \mu \mathrm{m}$.

\section{Discussion}

Results clearly indicate that a sample cannot simultaneously attain an optimum top $\mathrm{R}_{\mathrm{a}}(4 \mu \mathrm{m})$ and side $\mathrm{R}_{\mathrm{a}}(10 \mu \mathrm{m})$ as shown in Figure 8. As top $R_{a}$ improves side $R_{a}$ worsens, as

Figure 4 Top/Side $R_{a}$ at variable scan speeds and spot overlaps

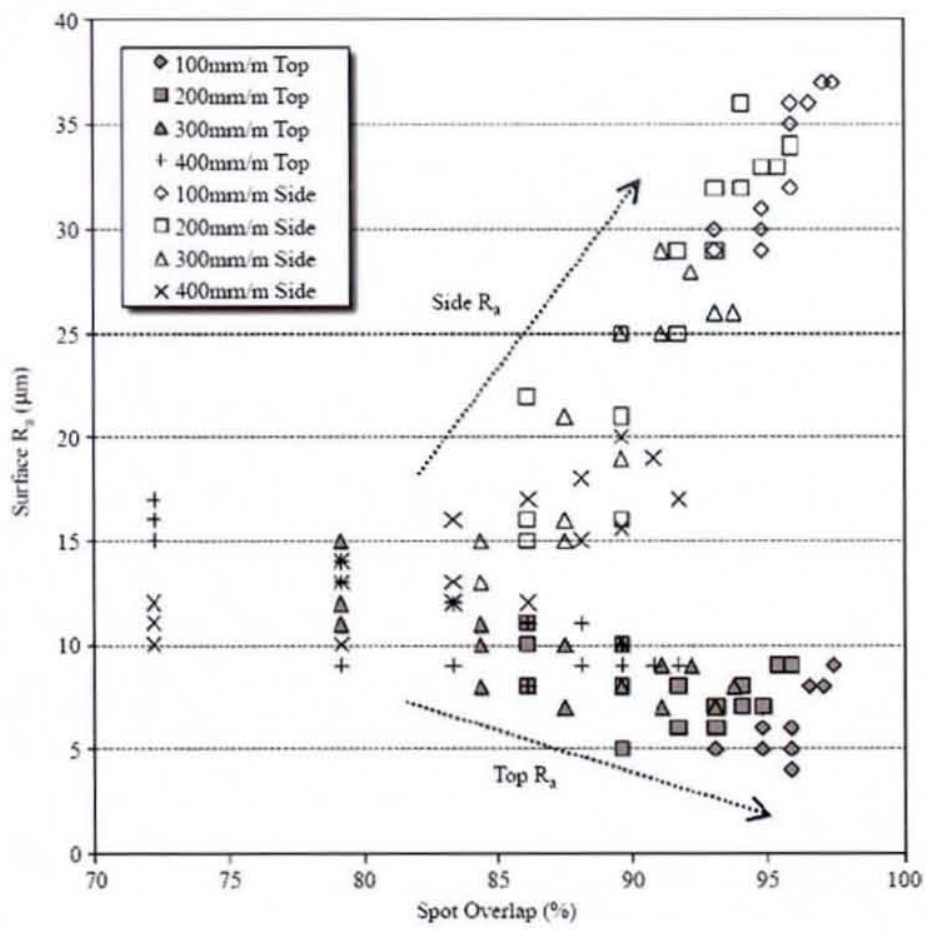


Figure 5 Thin wall parts processed using $0.5 \mathrm{~J}, 40 \mathrm{~Hz}$ and variable scan speeds

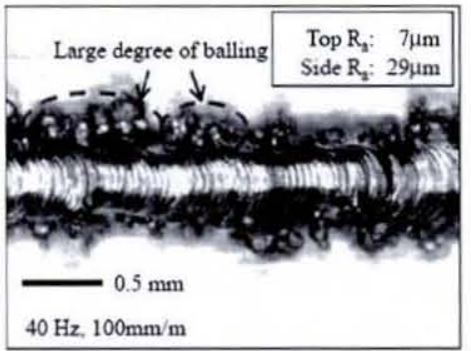

(a)

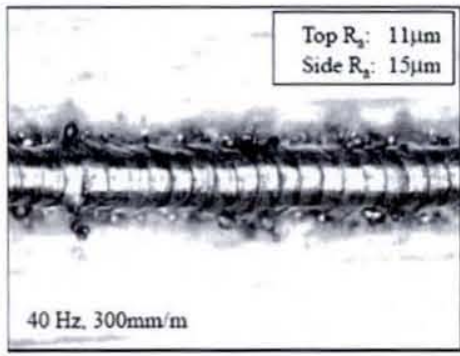

(c)

side $R_{2}$ improves top $R_{a}$ worsens. This is primarily due to surface tension forces that arise as a result of thermal variation within the melt pool. Low top $\mathrm{R}_{\mathrm{a}}$ is generally achieved using a high laser spot overlap (above 90 per cent) due to reduced variation in the peaks and troughs generated by individual overlapping spheres. Low processing scan speeds improve the top $R_{a}$ as melt pool is given more time to flatten before melt pool solidification. The melt pool is flattened by gravity and surface curvature forces that counteract the external shear forces generated by temperature difference caused by the moving laser. However, both a high laser spot overlap and low scan speed increase the volume of liquid produced within the melt pool. This has a tendency to widen the melt pool causing a larger variation in thermal properties across the melt pool. This subsequently generates a greater variation in surface tension and causes the melt pool to break off into smaller entities (balling) as the melt pool attempts to reduce surface tension differences. The entities solidify at the edge of the melt pool subsequently increasing surface $R_{a}$. Side $R_{2}$ can be reduced using high scan speeds and low laser spot overlap (below 85 per cent). This encourages quicker melt pool solidification and minimizes the volume of liquid within the melt pool. This produces relatively thin melt pools with less variation in the thermal properties across the melt pool than that of a larger melt pool. The melt pool is more stable and is less likely to suffer from balling. However, as explained earlier the fast melt pool solidification and low laser spot overlap will have a detrimental effect on top $R_{a}$.

The common factor that reduces both top and side surface $R_{a}$ is pulse energy, however it is believed that the increased peak power generated with the use of a higher pulse energy at a fixed pulse duration is the main reason for this to occur. High peak powers generate large recoil pressures. Work has shown that

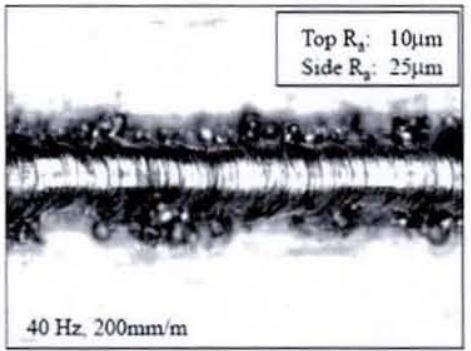

(b)

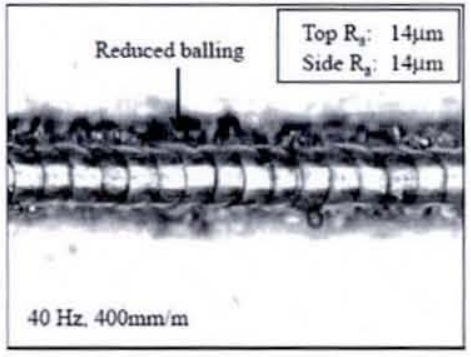

(d)

higher peak powers flatten out the melt pool (Morgan et al., 2004) improving interlayer connection and increasing the wettability of the melt (Mumtaz et al., 2008). Improved wettability reduces a melt pools tendency to undergo balling by relieving surface tension variations (Agarwala ct al., 1995, Kruth et al., 2004). Kruth et al. (2004) believes that high peak powers reduce the tendency for a melt pool to undergo balling due to a higher attained temperature within the melt. Another possible explanation for the decrease in balling with the use of higher peak powers is related to the increase in recoil pressurc causing any balling formation to completely detach from solidifying melt pool (and disperse onto the powder bed). However, if peak powers become too high large amounts of material vaporization can occur with recoil pressures that disrupt the melt pool surface and increase top $\mathrm{R}_{\mathrm{a}}$.

It has been shown that a variety of factors have a tendency to pull top and side $R_{a}$ in opposite directions. The best solution would be to compromise on optimum top/side roughness values and settle for values that combined produces the lowest value for top/side $R_{a}$. This is achieved with a pulse energy of $0.7 \mathrm{~J}$, repetition rate $40 \mathrm{~Hz}$ and a scan speed of $400 \mathrm{~mm} / \mathrm{min}$ is used (Figure $7(\mathrm{~b})$ ). This produces a part with a top $\mathrm{R}_{\mathrm{a}}$ of $9 \mu \mathrm{m}$ and side $\mathrm{R}_{\mathrm{a}}$ of $10 \mu \mathrm{m}$.

\section{Conclusions}

An Inconel 625 bonding region was identified. It was shown that shorter pulse durations enabled the production of parts to a higher resolution due to a reduced volume of liquid being produced due to a shorter laser on time.

It has been found that increasing the repetition rates will reduce top surface roughness (until material vaporization significantly increases) due to the centres of individual 
Figure 6 (a) Top $R_{a}$ processed at $100 \mathrm{~mm} / \mathrm{m}$ and (b) Side $R_{a}$ processed at $400 \mathrm{~mm} / \mathrm{m}$

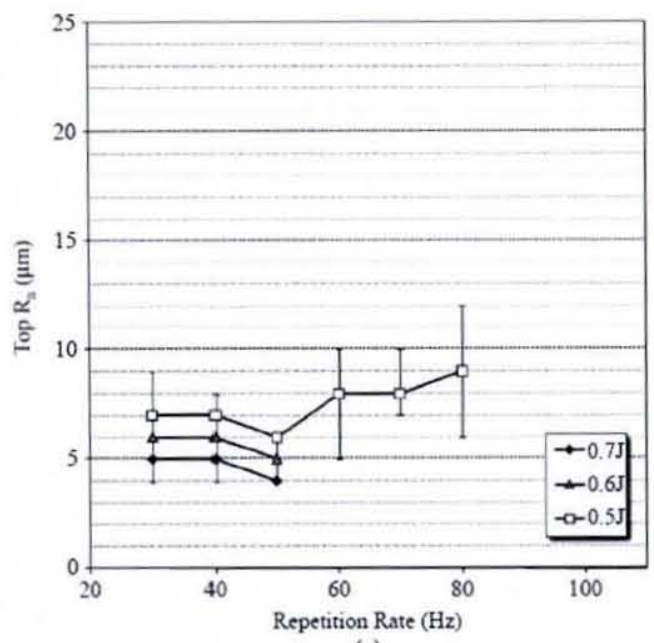

(a)

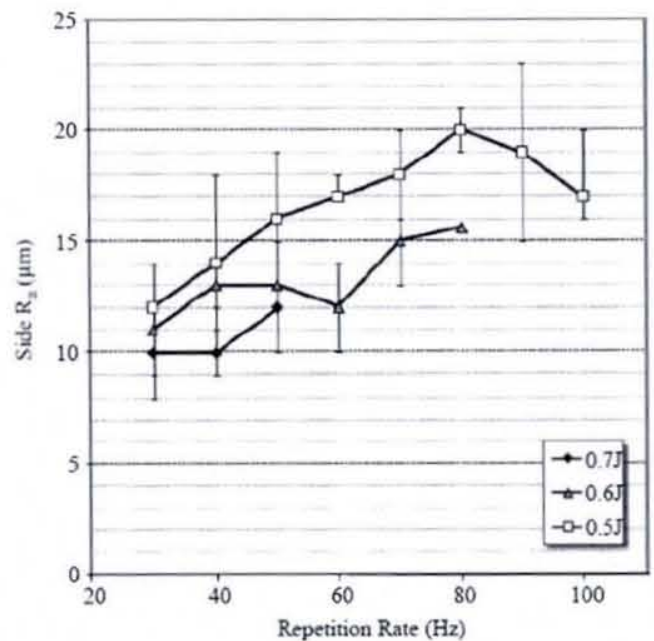

(b)

spherical masses becoming closer together subsequently reducing variation in top surface profile. Lower scan speeds produced parts with lower top $R_{\mathrm{a}}$ due to prolonged melt pool relaxation. It allows more time for gravity and surface curvature to counteract external shear forces due to temperature differences within melt pool. The melt pool flattens before complete solidification and subsequently reduces surface top roughness. It was found that a scan overlap of between 91-96 per cent produced the lowest top $\mathrm{R}_{\mathrm{a}}$.

Balling has been shown to directly affect the side roughness of parts. Balling occurs in an attempt to reduce the surface tension differences within the melt pool. It has been demonstrated that certain processing parameters within SLM promote balling formation. Controlling the volume of melt such that the length
Rapid Prototyping Journal

Wlume 15 - Number 2 $2009 \cdot 96-103$

Figure 7 (a) Lowest top $R_{a}$ and (b) lowest side $R_{a}$

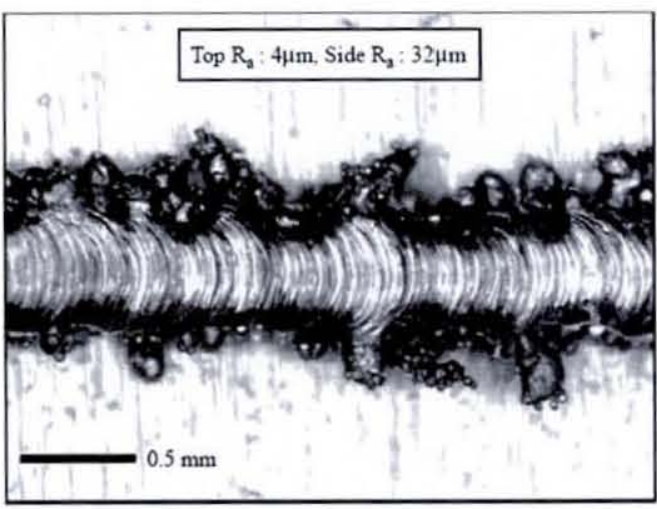

$0.5 \mathrm{~ms}, 0.7 \mathrm{~J}, 50 \mathrm{~Hz} \& 100 \mathrm{~mm} / \mathrm{s}$

(a)

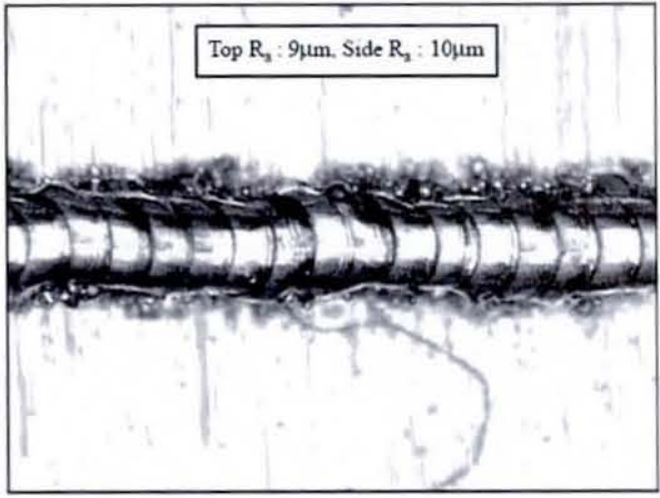

$0.5 \mathrm{~ms}, 0.7 \mathrm{~J}, 40 \mathrm{~Hz} \& 400 \mathrm{~mm} / \mathrm{s}$

(b)

to diameter ratio of melt is maximized was effectively regulated by increasing scan speed and reducing repetition rate. A smaller melt pool has less thermal variation than that of a larger melt pool. The surface tension variation between the centre and outer edges of the melt pool are therefore minimized. The melt pool is therefore more stable and less likely to break off into smaller entities in order to reduce surface tension variations. Processing at high scan speeds not only reduces side $R_{a}$ but also decreases part production time.

Achieving a minimum top and side $R_{a}$ are not currently possible as there are different mechanisms that affect each of these properties. Parameters that promote a reduction in top $\mathrm{R}_{\mathrm{a}}$ (increased overlap, increased repetition and reduced scan speed) tend to increase the balling effect and increase side $R_{a}$. Parameters that reduce side $R_{a}$ (reduced overlap, reduced repetition rate and increased scan speed) increase the top $R_{a}$ of parts. However, the parameter that aids a reduction in both top and side $R_{\mathrm{a}}$ is use of a higher peak power due to the flattening/smoothing of the melt pool surface (due to increased recoil pressure) and an improved wettability of the melt pool reducing its tendency to undergo the balling mechanism. 
Figure 8 Top/Side $R_{a}$ of thin wall parts

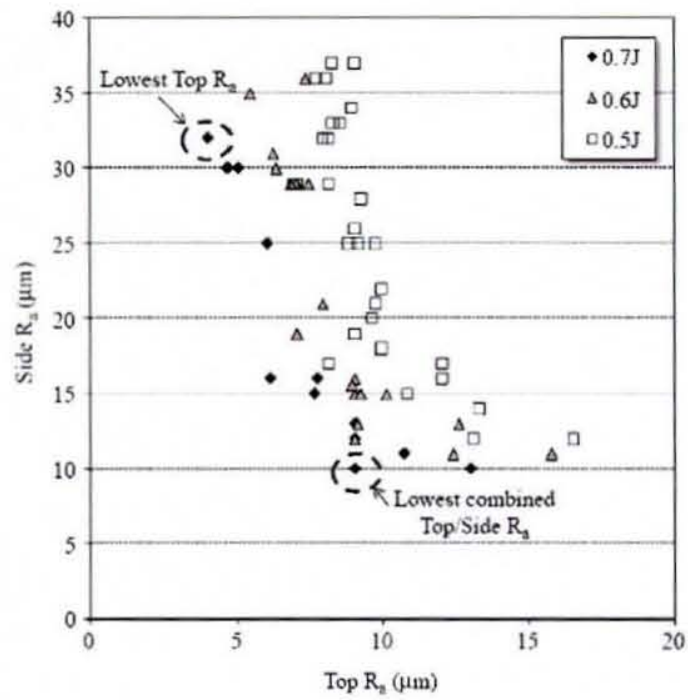

\section{References}

Agarwala, M., Bourell, D., Beaman, J., Marcus, H. and Barlow, J. (1995), "Direct selective laser sintering of metals", Rapid Prototyping Fournal, Vol. 1 No. 1, pp. 26-36.

Bourell, D.L., Wohlert, M., Harlan, N., Das, S. and Beaman, J.J. (2002), "Powder densification maps in selective laser sintering", Engineering Materials, Vol. 9 No. 4, pp. 663-9.

Childs, T.H.C., Hauser, C. and Badrossamay, M. (2005), "Selective laser sintering (melting) of stainless and tool steel powders: experiments and modelling", Proceedings of the IMech E Part B fournal of Engineering Manufacture, Vol. 219 No. 4 , p. 339.

Connolly, J. (2001), "Direct rapid manufacturing - is it possible?", Time Compression Technologies, Vol. 6 No. 4, pp. 46-7.

Dalgarno, K. (2007), "Materials research to support high performance RM parts", Rapid Manufacturing 2nd International Conference, Loughborough University, 9-12 fuly, pp. 147-56.

Das, S. (2003), "Physical aspects of process control in selective laser sintering of metals", Advanced Engineering Materials, Vol. 5 No. 10, pp. 701-11.

Fischer, P., Romano, V., Weber, H.P., Karapatis, N.P., Boillat, E. and Glardon, R. (2003), "Sintering of commercially pure titanium powder with an Nd:YAG laser source", Acta Materialia, Vol. 51 No. 6, pp. 1651-62.
Fuh, J.Y.H., Choo, Y.S., Nee, A.Y.C., Lu, L and Lee, K. (1995), "Improvement of the V curing process for the laser lithography technique", Materials of Design, Vol. 16 No. 1 , pp. 23-32.

Kruth, J.P., Mercelis, P., Vaerenbergh, J.V., Froyen, L. and Rombouts, M. (2005), "Binding mechanisms in selective laser sintering and selective laser melting", Rapid Prototyping Fournal, Vol. 11 No. 1, pp. 26-36.

Kruth, J.P., Froyen, L., Van Vaerenbergh, J., Mercelis, P., Rombouts, M. and Lauwers, B. (2004), "Selective laser melting of iron-based powder", fournal of Materials Processing Technology, Vol. 149 Nos 1/3, pp. 616-22.

Morgan, R., Sutcliffe, C.J. and O'Neill, W. (2004), "Density analysis of direct metal laser re-melted $316 \mathrm{~L}$ stainless steel cubic primitives", fournal of Matcrials Science, Vol. 39 No. 4 pp. 1195-205.

Mumtaz, K. and Hopkinson, N. (2008), "High density selective laser melting of waspaloy", Gournal of Material Processing, Vol. 195, pp. 77-87.

Mumtaz, K., Hopkinson, N. and Erasenthiran, P. (2007), "Selective laser melting functionally graded compositions of waspaloy and zirconia", fournal of Material Sciance, Vol. 42 No. 18 , pp. $7647-56$.

Niu, H.J. and Chang, I.T.H. (1998), "Liquid phase sintering of M3/2 high speed steel by selective laser sintering", Scripta Materialia, Vol. 30 No. 1, pp. 67-72.

Ramos, J.A., Bourell, D.L. and Beaman, J.J. (2003), "Surface over-melt during laser polishing of indirect-SLS metal parts", Materials Research Society Symposium, Vol. 758, pp. 53-61.

Ramos, J.A., Murphy, J., Wood, K., Bourell, D.L. and Beaman, J.J. (2001), "Surface roughness enhancement of indirect-SLS metal parts by laser surface polishing", Solid Freform Fabrication, Vol. 36 No. 3, pp. 28-38.

Rombouts, M., Kruth, J.P., Froyen, L and Mercelis, P (2006), "Fundamentals of selective laser melting of alloyed steel powders", CIRP Amals - Mamufacnwing Technology, Vol. 55 No. 1, pp. 187-92.

Santos, E.C., Osakada, K., Shiomi, M., Kitamura, Y. and Abe, F. (2004), "Microstructure and mechanical properties of pure titanium models fabricated by selective laser melting", Proceedings of the I Mech E Part C fournal of Mechanical Engineering Science, Vol. 218 No. 7, pp. 711-19. Schwendner, K.I., Baneriee, R., Collins, P.C., Brice, C.A and Fraser, H.L. (2001), "Direct laser deposition of alloys from elemental powder blends", Scripta Materialia, Vol. 45 No. 10, pp. 1123-9.

\section{Corresponding author}

Kamran Mumtaz can be contacted at: k.a.mumtazalboro.ac.uk

To purchase reprints of this article please e-mail: reprints $a$ emeraldinsight.com

Or visit our web site for further details: www.emeraldinsight.com/reprints 
